\title{
HEALTH TECHNOLOGY ASSESSMENT
}

VOLUME 17 ISSUE 12 MARCH 2013

ISSN 1366-5278

\section{Positron emission tomography/computerised tomography imaging in detecting and managing recurrent cervical cancer: systematic review of evidence, elicitation of subjective probabilities and economic modelling}

C Meads, P Auguste, C Davenport, S Małysiak, S Sundar, M Kowalska, A Zapalska, $P$ Guest, S Thangaratinam, P Martin-Hirsch, E Borowiack, $P$ Barton, $T$ Roberts and $K$ Khan 



\section{Positron emission tomography/ computerised tomography imaging in detecting and managing recurrent cervical cancer: systematic review of evidence, elicitation of subjective probabilities and economic modelling}

\section{Meads, ${ }^{1 *}$ P Auguste, ${ }^{2}$ C Davenport, ${ }^{3}$ S Małysiak, ${ }^{4}$ S Sundar, ${ }^{5}$ M Kowalska, ${ }^{4}$ A Zapalska, ${ }^{4}$ P Guest, ${ }^{6}$ S Thangaratinam, ${ }^{7}$ P Martin-Hirsch, ${ }^{8}$ E Borowiack, ${ }^{4}$ P Barton, ${ }^{2} \mathrm{~T}_{\text {Roberts }}^{2}$ and K Khan ${ }^{1}$}

\footnotetext{
${ }^{1}$ Centre for Primary Care and Public Health, Barts and The London School of Medicine and Dentistry, Queen Mary, University of London, London, UK ${ }^{2}$ Unit of Health Economics, University of Birmingham, Birmingham, UK ${ }^{3}$ Unit of Public Health, Epidemiology and Biostatistics, University of Birmingham, Birmingham, UK

${ }^{4}$ Arcana Institute, Kraków, Poland

${ }_{5}^{5}$ Pan Birmingham Gynaecological Cancer Centre, City Hospital and School of Cancer Science, University of Birmingham, Birmingham, UK

${ }^{6}$ University Hospitals Birmingham NHS Foundation Trust, Queen Elizabeth Hospital, Queen Elizabeth Medical Centre, Birmingham, UK

${ }^{7}$ School of Clinical and Experimental Medicine, University of Birmingham, Birmingham, UK

${ }^{8}$ Lancashire Teaching Hospitals NHS Trust, Royal Preston Hospital, Preston, UK

${ }^{*}$ Corresponding author
}

Declared competing interests of authors: none

Published March 2013

DOI: $10.3310 /$ hta17120 

This report should be referenced as follows:

Meads C, Auguste P, Davenport C, Małysiak S, Sundar S, Kowalska M, et al. Positron emission tomography/computerised tomography imaging in detecting and managing recurrent cervical cancer: systematic review of evidence, elicitation of subjective probabilities and economic modelling. Health Technol Assess 2013;17(12).

Health Technology Assessment is indexed and abstracted in Index Medicus/MEDLINE, Excerpta Medica/EMBASE, Science Citation Index Expanded (SciSearch ${ }^{\circledR}$ ) and Current Contents ${ }^{\circledast} /$ Clinical Medicine. 



\title{
Health Technology Assessment
}

\author{
ISSN 1366-5278 (Print)
}

ISSN 2046-4924 (Online)

Five-year impact factor: 5.596

Health Technology Assessment is indexed in MEDLINE, CINAHL, EMBASE, The Cochrane Library and the ISI Science Citation Index and is assessed for inclusion in the Database of Abstracts of Reviews of Effects.

This journal is a member of and subscribes to the principles of the Committee on Publication Ethics (COPE) (http://www.publicationethics.org/).

Editorial contact: nihredit@southampton.ac.uk

The full HTA archive is freely available to view online at http://www.hta.ac.uk/project/htapubs.asp. Print copies can be purchased from the individual report pages.

\section{Criteria for inclusion in the Health Technology Assessment journal}

Reports are published in Health Technology Assessment (HTA) if (1) they have resulted from work for the HTA programme, and (2) they are of a sufficiently high scientific quality as assessed by the reviewers and editors.

Reviews in Health Technology Assessment are termed 'systematic' when the account of the search appraisal and synthesis methods (to minimise biases and random errors) would, in theory, permit the replication of the review by others.

\section{HTA programme}

The HTA programme, part of the National Institute for Health Research (NIHR), was set up in 1993. It produces high-quality research information on the effectiveness, costs and broader impact of health technologies for those who use, manage and provide care in the NHS. 'Health technologies' are broadly defined as all interventions used to promote health, prevent and treat disease, and improve rehabilitation and long-term care.

The journal is indexed in NHS Evidence via its abstracts included in MEDLINE and its Technology Assessment Reports inform National Institute for Health and Clinical Excellence (NICE) guidance. HTA research is also an important source of evidence for National Screening Committee (NSC) policy decisions.

For more information about the HTA programme please visit the website: http://www.hta.ac.uk/

\section{This report}

The research reported in this issue of the journal was funded by the HTA programme as project number 09/29/02. The contractual start date was in February 2010. The draft report began editorial review in February 2012 and was accepted for publication in June 2012 . The authors have been wholly responsible for all data collection, analysis and interpretation, and for writing up their work. The HTA editors and publisher have tried to ensure the accuracy of the authors' report and would like to thank the reviewers for their constructive comments on the draft document. However, they do not accept liability for damages or losses arising from material published in this report.

This report presents independent research funded by the National Institute for Health Research (NIHR). The views and opinions expressed by authors in this publication are those of the authors and do not necessarily reflect those of the NHS, the NIHR, NETSCC, the HTA programme or the Department of Health.

(c) Queen's Printer and Controller of HMSO 2013. This work was produced by Meads et al. under the terms of a commissioning contract issued by the Secretary of State for Health. This issue may be freely reproduced for the purposes of private research and study and extracts (or indeed, the full report) may be included in professional journals provided that suitable acknowledgement is made and the reproduction is not associated with any form of advertising. Applications for commercial reproduction should be addressed to: NIHR Journals Library, National Institute for Health Research, Evaluation, Trials and Studies Coordinating Centre, Alpha House, University of Southampton Science Park, Southampton SO16 7NS, UK.

Published by the NIHR Journals Library, produced by Prepress Projects Ltd, Perth, Scotland (www.prepress-projects.co.uk). 


\section{Editor-in-Chief of Health Technology Assessment and NIHR Journals Library}

Professor Tom Walley Director, NIHR Evaluation, Trials and Studies and Director of the HTA Programme, UK

\section{NIHR Journals Library Editors}

Dr Andree Le May Chair of NIHR Journals Library Editorial Group

Professor Ken Stein Chair of HTA Editorial Board and Professor of Public Health, University of Exeter Medical School, UK

Dr Martin Ashton-Key Consultant in Public Health Medicine/Consultant Advisor, NETSCC, UK

Professor Matthias Beck Chair in Public Sector Management and Subject Leader (Management Group), Queen's University Management School, Queen's University Belfast, UK

Professor Aileen Clarke Professor of Health Sciences, Warwick Medical School, University of Warwick, UK

Dr Tessa Crilly Director, Crystal Blue Consulting Ltd, UK

Dr Peter Davidson Director of NETSCC, HTA, UK

Ms Tara Lamont Scientific Advisor, NETSCC, UK

Dr Tom Marshall Reader in Primary Care, School of Health and Population Sciences, University of Birmingham, UK

Professor William McGuire Professor of Child Health, Hull York Medical School, University of York, UK

Professor Geoffrey Meads Honorary Professor, Business School, Winchester University and Medical School, University of Warwick, UK

Professor Jane Norman Professor of Maternal and Fetal Health, University of Edinburgh, UK

Professor John Powell Senior Clinical Researcher, Department of Primary Care, University of Oxford, UK

Professor James Raftery Professor of Health Technology Assessment, Wessex Institute, Faculty of Medicine, University of Southampton, UK

Dr Rob Riemsma Reviews Manager, Kleijnen Systematic Reviews Ltd, UK

Professor Helen Roberts Professorial Research Associate, University College London, UK

Professor Helen Snooks Professor of Health Services Research, Institute of Life Science, College of Medicine, Swansea University, UK

Editorial contact: nihredit@southampton.ac.uk 


\title{
Abstract
}

\section{Positron emission tomography/computerised tomography imaging in detecting and managing recurrent cervical cancer: systematic review of evidence, elicitation of subjective probabilities and economic modelling}

\author{
C Meads, ${ }_{1}^{1 *}$ P Auguste, ${ }^{2}$ C Davenport, ${ }^{3}$ S Małysiak, ${ }^{4}$ S Sundar, ${ }^{5}$ \\ M Kowalska, ${ }^{4}$ A Zapalska, ${ }^{4}$ P Guest, ${ }^{6}$ S Thangaratinam, ${ }^{7}$ P Martin-Hirsch, ${ }^{8}$ \\ E Borowiack, ${ }^{4}$ P Barton, ${ }^{2}$ T Roberts ${ }^{2}$ and K Khan ${ }^{1}$
}

${ }^{1}$ Centre for Primary Care and Public Health, Barts and The London School of Medicine and Dentistry, Queen Mary, University of London, London, UK

${ }^{2}$ Unit of Health Economics, University of Birmingham, Birmingham, UK

${ }^{3}$ Unit of Public Health, Epidemiology and Biostatistics, University of Birmingham, Birmingham, UK

${ }^{4}$ Arcana Institute, Kraków, Poland

${ }^{5}$ Pan Birmingham Gynaecological Cancer Centre, City Hospital and School of Cancer Science,

University of Birmingham, Birmingham, UK

${ }^{6}$ University Hospitals Birmingham NHS Foundation Trust, Queen Elizabeth Hospital, Queen Elizabeth

Medical Centre, Birmingham, UK

${ }^{7}$ School of Clinical and Experimental Medicine, University of Birmingham, Birmingham, UK

${ }^{8}$ Lancashire Teaching Hospitals NHS Trust, Royal Preston Hospital, Preston, UK

*Corresponding author

Background: Cancer of the uterine cervix is a common cause of mortality in women. After initial treatment women may be symptom free, but the cancer may recur within a few years. It is uncertain whether it is more clinically effective to survey asymptomatic women for signs of recurrence or to await symptoms or signs before using imaging.

Objectives: This project compared the diagnostic accuracy of imaging using positron emission tomography/computerised tomography (PET-CT) with that of imaging using CT or magnetic resonance imaging (MRI) alone and evaluated the cost-effectiveness of adding PET-CT as an adjunct to standard practice.

Data sources: Standard systematic review methods were used to obtain and evaluate relevant test accuracy and effectiveness studies. Databases searched included MEDLINE, EMBASE, Science Citation Index and The Cochrane Library. All databases were searched from inception to May 2010.

Review methods: Study quality was assessed using appropriately modified Quality Assessment of Diagnostic Accuracy Studies (QUADAS) criteria. Included were any studies of PET-CT, MRI or CT compared with the reference standard of histopathological findings or clinical follow-up in symptomatic women suspected of having recurrent or persistent cervical cancer and in asymptomatic women a minimum of 3 months after completion of primary treatment. Subjective elicitation of expert opinion was used to supplement diagnostic information needed for the economic evaluation. The effectiveness of treatment with chemotherapy, radiotherapy, chemoradiotherapy, radical hysterectomy and pelvic exenteration was 
systematically reviewed. Meta-analysis was carried out in RevMan 5.1 (The Cochrane Collaboration, The Nordic Cochrane Centre, Copenhagen, Denmark) and Stata version 11 (StataCorp LP, College Station, Texas, USA). A Markov model was developed to compare the relative cost-effectiveness using TreeAge Pro software version 2011 (TreeAge Software Inc., Evanston, IL, USA).

Results: For the diagnostic review, a total of 7524 citations were identified, of which 12 test accuracy studies were included in the review: six studies evaluated PET-CT, two evaluated MRI, three evaluated CT and one evaluated both MRI and CT. All studies were small and the majority evaluated imaging in women in whom recurrence was suspected on the basis of symptoms. The PET-CT studies evaluated local and distant recurrence and most used methods similar to current practice, whereas five of the six CT and MRI studies evaluated local recurrence only and not all employed currently used methods. Meta-analysis of PETCT studies gave a sensitivity of $92.2 \%$ [95\% confidence interval (Cl) $85.1 \%$ to $96.0 \%$ ] and a specificity of $88.1 \%$ (95\% Cl $77.9 \%$ to $93.9 \%$ ). MRI sensitivities and specificities varied between $82 \%$ and $100 \%$ and between $78 \%$ and $100 \%$, respectively, and CT sensitivities and specificities varied between $78 \%$ and $93 \%$ and between $0 \%$ and $95 \%$, respectively. One small study directly compared PET-CT with older imaging methods and showed more true-positives and fewer false-negatives with PET-CT. The subjective elicitation from 21 clinical experts gave test accuracy results for asymptomatic and symptomatic women and the results for symptomatic women were similar to those from the published literature. Their combined opinions also suggested that the mean elicited increase in accuracy from the addition of PET-CT to MRI and/or CT was less than the elicited minimum important difference in accuracy required to justify the routine addition of PET-CT for the investigation of women after completion of primary treatment. For the effectiveness review, a total of 24,943 citations were identified, of which 62 studies were included (chemotherapy, 19 randomised controlled trials; radiotherapy or chemoradiotherapy, 16 case series; radical hysterectomy and pelvic exenteration, 27 case series). None provided the effectiveness of cisplatin monotherapy, the most commonly used chemotherapeutic agent in the NHS, compared with supportive care in a background of other treatment such as radiotherapy in recurrent and persistent cervical cancer. The model results showed that adding PET-CT to the current treatment strategy of clinical examination, MRI and/or CT scan was significantly more costly with only a minimal increase in effectiveness, with incremental cost-effectiveness ratios for all models being $>f 1 \mathrm{M}$ per quality-adjusted life-year (QALY) and the additional cost per additional case of recurrence being in the region of $f 600,000$.

Limitations: There was considerable uncertainty in many of the parameters used because of a lack of good-quality evidence in recurrent or persistent cervical cancer. The evidence on diagnostic and therapeutic impact incorporated in the economic model was poor and there was little information on surveillance of asymptomatic women.

Conclusions: Given the current evidence available, the addition of PET-CT to standard practice was not found to be cost-effective in the diagnosis of recurrent or persistent cervical cancer. However, although probabilistic sensitivity analysis showed that the main conclusion about cost-ineffectiveness of PET-CT was firm given the range of assumptions made, should more reliable information become available on accuracy, therapeutic impact and effectiveness, and the cost of PET-CT reduce, this conclusion may need revision. Current guidelines recommending imaging for diagnosis using expensive methods such as PET-CT need to be reconsidered in the light of the above.

Funding: The National Institute for Health Research Health Technology Assessment programme. 


\section{Contents}

List of abbreviations $\quad$ xi

Executive summary xiii

$\begin{array}{ll}\text { Chapter } 1 \text { Aims of the report } & 1\end{array}$

Chapter 2 Background 3

Description of the underlying health problem 3

Imaging to detect recurrence $\quad 6$

$\begin{array}{lr}\text { Objectives of this report } & 9\end{array}$

$\begin{array}{ll}\text { Chapter } 3 \text { Methods for systematic reviews and subjective elicitation } & 11\end{array}$

Protocol development and overview of review methods $\quad 11$

Methods for test accuracy and diagnostic and therapeutic impact reviews $\quad 11$

Methods of statistical analysis $\quad 13$

Methods for subjective elicitation $\quad 15$

$\begin{array}{ll}\text { Methods for effectiveness reviews } & 17\end{array}$

$\begin{array}{ll}\text { Methods of reporting and statistical analysis } & 19\end{array}$

$\begin{array}{ll}\text { Methods for systematic review of economic evaluations } & 19\end{array}$

$\begin{array}{ll}\text { Chapter } 4 \text { Diagnostic review results } & 21\end{array}$

$\begin{array}{ll}\text { Study selection } & 21\end{array}$

$\begin{array}{ll}\text { Characteristics of included studies } & 21\end{array}$

Chapter 5 Results of the elicitation of subjective probabilities 37

$\begin{array}{ll}\text { Prevalence of recurrence } & 37\end{array}$

Chapter 6 Effectiveness review $\quad 43$

Chemotherapy agents $\quad 43$

Radiotherapy or chemoradiotherapy 70

Surgery 86

Summary of accuracy and effectiveness results and inputs to economic evaluation 96

Chapter 7 Systematic review of economic evaluations 105

Chapter 8 Economic evaluation methods and results 107

$\begin{array}{ll}\text { Objective } & 107\end{array}$

$\begin{array}{ll}\text { Development of the model structure } & 107\end{array}$

Data required for the model 111

$\begin{array}{ll}\text { Results of modelling } & 118\end{array}$

$\begin{array}{lr}\text { Chapter } 9 \text { Discussion } & 125\end{array}$

$\begin{array}{ll}\text { Statement of principal findings } & 125\end{array}$

Strengths and limitations of the project 126

$\begin{array}{lr}\text { Uncertainties } & 128\end{array}$ 
Chapter 10 Conclusions

Implications for service provision

Implications for research

Acknowledgements

References

Appendix 1 Protocol

Appendix 2 Scoping search strategies and results

Appendix 3 Diagnostic review data extraction form

Appendix 4 Effectiveness review data extraction forms

Appendix 5 Case series quality assessment form

Appendix 6 Diagnostic systematic review search strategies

Appendix 7 Subjective elicitation questionnaire

Appendix 8 Effectiveness systematic review search strategies

Appendix 9 Economic evaluation systematic review search strategies and study categories

Appendix 10 Diagnostic review list of excluded studies with reasons for exclusion

Appendix 11 Diagnostic meta-analysis logistic regression results

Appendix 12 Subjective elicitation results

Appendix 13 Effectiveness review list of excluded studies with reasons for exclusion

Appendix 14 Baseline characteristics of chemotherapy randomised controlled trials

Appendix 15 Quality assessment of case series: radiotherapy and chemoradiotherapy 


\section{List of abbreviations}

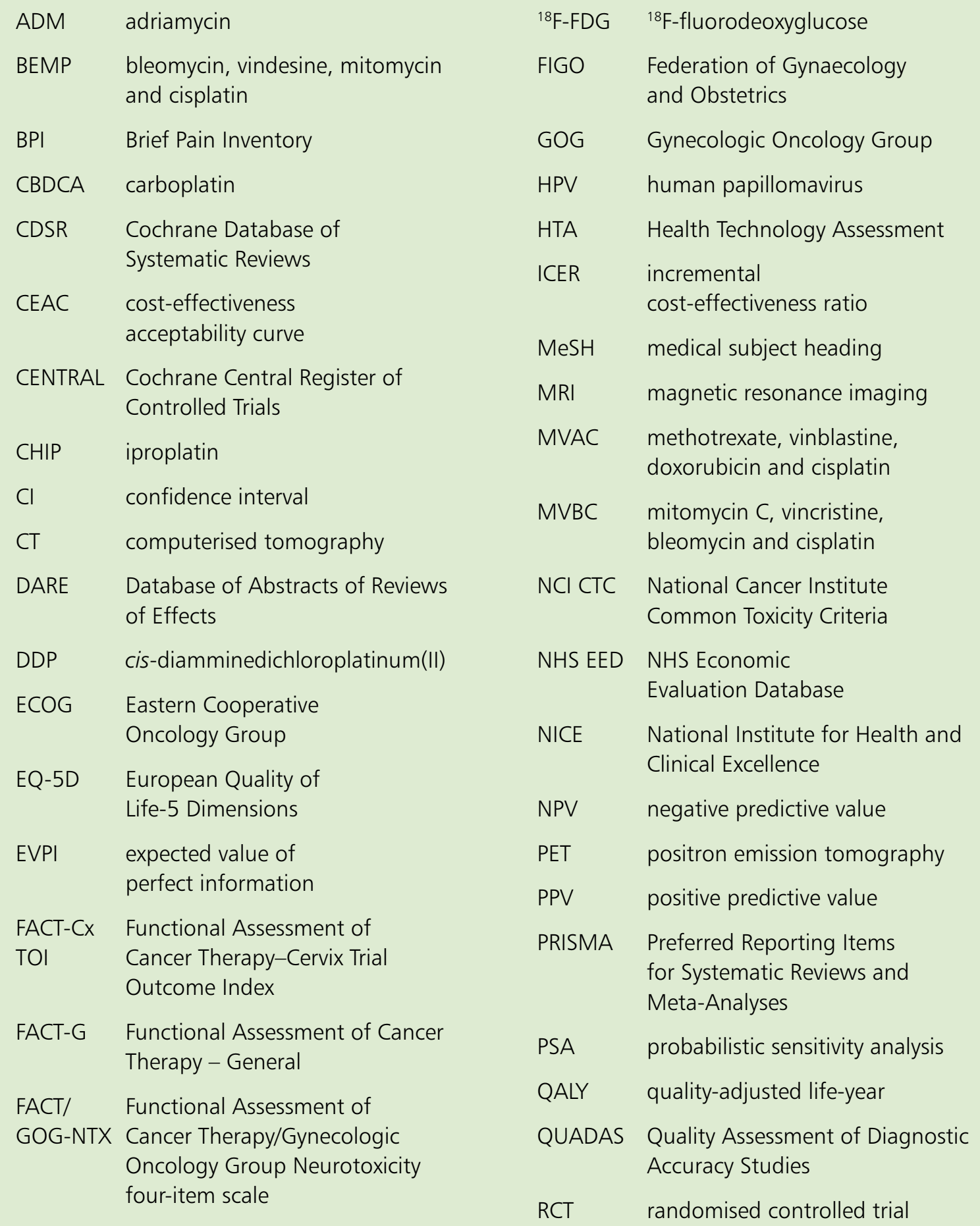


$\mathrm{RR} \quad$ relative risk

SD standard deviation

SIGN
SROC

VOI summary receiver operating characteristic

total pelvic exenteration

value of information

All abbreviations that have been used in this report are listed here unless the abbreviation is well known (e.g. NHS), or it has been used only once, or it is a non-standard abbreviation used only in figures/tables/appendices, in which case the abbreviation is defined in the figure legend or at the end of the table. 


\section{Executive summary}

\section{Background}

Cancer of the uterine cervix is a common cause of mortality in women. After initial treatment women may be symptom free, but the cancer may recur within a few years. It is uncertain whether it is more clinically effective to survey asymptomatic women for signs of recurrence or to await symptoms or signs before using imaging. This project compared the diagnostic accuracy of imaging using positron emission tomography/computerised tomography (PET-CT) with that of imaging using CT or magnetic resonance imaging (MRI) alone and evaluated the cost-effectiveness of adding PET-CT as an adjunct to standard practice.

\section{Methods}

Standard systematic review methods were used to obtain and evaluate relevant test accuracy and effectiveness studies. Databases searched included MEDLINE, EMBASE, Science Citation Index and The Cochrane Library. All databases were searched from inception to May 2010. Study quality was assessed using appropriately modified Quality Assessment of Diagnostic Accuracy Studies (QUADAS) criteria. Included were any studies of PET-CT, MRI or CT compared with the reference standard of histopathological findings or clinical follow-up in symptomatic women suspected of having recurrent or persistent cervical cancer and in asymptomatic women a minimum of 3 months after completion of primary treatment. Subjective elicitation of expert opinions was used to supplement diagnostic information needed for the economic evaluation. The effectiveness of treatment with chemotherapy, radiotherapy, chemoradiotherapy, radical hysterectomy and pelvic exenteration was systematically reviewed. Meta-analysis was carried out in RevMan 5.1 (The Cochrane Collaboration, The Nordic Cochrane Centre, Copenhagen, Denmark) and Stata version 11 (StataCorp LP, College Station, TX, USA). A Markov model was developed to compare the relative cost-effectiveness using TreeAge Pro software version 2011 (TreeAge Software, Inc., Evanston, IL, USA).

\section{Results}

From 7524 citations retrieved, 12 test accuracy studies were found: six studies evaluated PET-CT, two evaluated MRI, three evaluated CT and one evaluated both MRI and CT. All studies were underpowered and the majority evaluated imaging in women in whom recurrence was suspected on the basis of symptoms. The PET-CT studies evaluated local and distant recurrence and most used methods similar to current practice, whereas five of the six CT and MRI studies evaluated local recurrence only and were published between 1981 and 2000, and not all employed currently used methods.

Meta-analysis of PET-CT studies gave a sensitivity of 92.2\% [95\% confidence interval (CI) $85.1 \%$ to $96.0 \%$ ] and a specificity of $88.1 \%(95 \% \mathrm{Cl} 77.9 \%$ to $93.9 \%)$. MRI sensitivities and specificities varied between $82 \%$ and $100 \%$ and $78 \%$ and $100 \%$, respectively, and CT sensitivities and specificities varied between $78 \%$ and 93\% and $0 \%$ and $95 \%$ respectively. One small study directly compared PET-CT with older imaging methods and showed more true-positives and fewer false-negatives with PET-CT.

The subjective elicitation from 21 clinical experts gave test accuracy results for asymptomatic and symptomatic women and the results for symptomatic women were similar to those from the published literature. Their combined opinions also suggested that the mean elicited increase in accuracy from the addition of PET-CT to MRI and/or CT was less than the elicited minimum important difference in accuracy 
required to justify the routine addition of PET-CT for the investigation of women after completion of primary treatment.

From 24,943 citations, 62 effectiveness studies were included (chemotherapy, 19 randomised controlled trials; radiotherapy or chemoradiotherapy, 16 case series; radical hysterectomy and pelvic exenteration, 27 case series). None provided the effectiveness of cisplatin monotherapy, the most commonly used chemotherapeutic agent in the NHS, compared with supportive care in a background of other treatment such as radiotherapy in recurrent and persistent cervical cancer. The model results showed that adding PET-CT to the current treatment strategy of clinical examination, MRI and/or CT scan was significantly more costly with only a minimal increase in effectiveness, with incremental cost-effectiveness ratios for all models being $>f 1 \mathrm{M}$ per quality-adjusted life-year (QALY) and the additional cost per additional case of recurrence being in the region of $£ 600,000$.

\section{Conclusion}

Given the current evidence available, the addition of PET-CT to standard practice was not found to be costeffective in the diagnosis of recurrent or persistent cervical cancer. There was considerable uncertainty in many of the parameters used because of a lack of good-quality evidence in recurrent or persistent cervical cancer. The evidence on diagnostic and therapeutic impact incorporated in the economic model was poor and there was little information on surveillance of asymptomatic women. Although probabilistic sensitivity analysis showed that the main conclusion about cost-ineffectiveness of PET-CT was firm given the range of assumptions made, should more reliable information become available on accuracy, therapeutic impact and effectiveness, and the cost of PET-CT reduce, this conclusion may need revision. Current guidelines recommending imaging for diagnosis using expensive methods such as PET-CT need to be reconsidered in the light of the above.

\section{Funding}

The National Institute for Health Research Health Technology Assessment programme. 


\section{Chapter 1 Aims of the report}

he aims of this project were as follows:

1. To evaluate, through systematic review of the literature, the diagnostic accuracy of adding positron emission tomography/computerised tomography (PET-CT) to CT and/or magnetic resonance imaging (MRI) compared with the diagnostic accuracy of CT and/or MRI alone in women with suspected recurrent or persistent cervical cancer in identifying local recurrence, regional recurrence and nodal and distant metastases.

2. To evaluate, through systematic review of the literature, the diagnostic and therapeutic impact of the addition of PET-CT to CT and/or MRI compared with CT and/or MRI alone on recurrent and persistent cervical cancer.

3. To assess, through systematic review of the literature, the effectiveness of various interventions and combinations of interventions (surgery, radiotherapy, chemotherapy and chemoradiotherapy) for mortality, morbidity and quality of life in the management of recurrent and persistent cervical cancer.

4. To evaluate, using decision-analytic modelling, including value of information analysis, the costeffectiveness of adding PET-CT imaging to CT and/or MRI compared with CT and/or MRI alone, and with different follow-up strategies, for the detection and work-up of recurrent and persistent cervical cancer.

The original protocol for this report is provided in Appendix 1. 



\section{Chapter 2 Background}

\section{Description of the underlying health problem}

Cervical cancer is a malignancy originating in the female uterine cervix. Cervical cancer usually originates in the transformation zone of the cervix where the squamous epithelial cells of the ectocervix meet the columnar epithelium of the endocervix. Approximately $80 \%$ of cervical cancers are squamous cell carcinomas. This type of cancer originates in the thin, flat squamous cells on the surface of the ectocervix, the part of the cervix that is next to the vagina. Another $10 \%$ of cervical cancers are of the adenocarcinoma type. This cancer originates in the mucus-producing cells of the inner or endocervix, near the body of the uterus. Occasionally, the cancer may have characteristics of both types and is called adenosquamous carcinoma or mixed carcinoma. Cervical cancers can be locally invasive and also spread by metastases. Pelvic recurrence can be central at the cervix or vaginal vault and in the lymph nodes of the pelvic side wall. Distant metastases can be to supraclavicular lymph nodes, para-aortic lymph nodes and the lungs.

Staging of cervical cancer can use the tumour, node, and metastases parameters (Box 1), but much more often uses the Federation of Gynaecology and Obstetrics (FIGO) criteria' (Table 1).

\section{Aetiology}

Human papillomavirus (HPV) infection of the cervix is a sexually transmitted infection that is necessary for the development of cervical cancer. ${ }^{2}$ However, only a relatively small proportion of women who encounter persistent infection from high-risk genotypes (HPV 16 and 18, and some other strains) go on to develop cervical cancer. ${ }^{3}$ When HPV is detected, around $17 \%$ of women go on to develop cervical intraepithelial

\section{BOX 1 TNM classification for disease staging}

\section{T: size or direct extent of the primary tumour}

Tx: tumour cannot be evaluated

Tis: carcinoma in situ

TO: no signs of tumour

$\mathrm{T} 1, \mathrm{~T} 2, \mathrm{~T} 3, \mathrm{~T} 4$ : size and/or extension of the primary tumour

\section{$\mathrm{N}$ : degree of spread to regional lymph nodes}

Nx: lymph nodes cannot be evaluated

NO: tumour cells absent from regional lymph nodes

N1: regional lymph node metastasis present (at some sites, tumour spread to closest or small number of regional lymph nodes)

N2: tumour spread to an extent between N1 and N3 (N2 is not used at all sites)

N3: tumour spread to more distant or numerous regional lymph nodes (N3 is not used at all sites)

\section{M: presence of metastasis}

Mx: distant metastasis cannot be evaluated

M0: no distant metastasis

M1: metastasis to distant organs (beyond regional lymph nodes) 
TABLE 1 Revised FIGO criteria for disease staging

Stage Characteristic
Stage I The carcinoma is strictly confined to the cervix (extension to the uterine corpus would be disregarded)
Stage IA: invasive carcinoma that can be diagnosed only by microscopy, with deepest invasion $\leq 5 \mathrm{~mm}$
largest extension $\geq 7 \mathrm{~mm}$
Stage IA1: measured stromal invasion of $\leq 3 \mathrm{~mm}$ in depth and extension of $\leq 7 \mathrm{~mm}$
Stage IA2: measured stromal invasion $>3 \mathrm{~mm}$ and not $>5 \mathrm{~mm}$ with an extension of $\leq 7 \mathrm{~mm}$
Stage IB: clinically visible lesions limited to the cervix uteri or preclinical cancers greater than stage $\left.\right|^{\text {a }}$
Stage IB1: clinically visible lesion $\leq 4 \mathrm{~cm}$ in greatest dimension
Stage 1B2: clinically visible lesions $>4 \mathrm{~cm}$ in greatest dimension

Stage II Cervical carcinoma invades beyond the uterus but not to the pelvic wall or to the lower third of the vagina

Stage IIA: without parametrial invasion

Stage IIA1: clinically visible lesion $\leq 4 \mathrm{~cm}$ in greatest dimension

Stage IIA2: clinically visible lesions $>4 \mathrm{~cm}$ in greatest dimension

Stage IIB: with obvious parametrial invasion

Stage III The tumour extends to the pelvic wall and/or involves the lower third of the vagina and/or causes hydronephrosis or non-functioning kidney ${ }^{b}$

Stage IIIA: tumour involves lower third of the vagina, with no extension onto the pelvic wall

Stage IIIB: extension to the pelvic wall and/or causes hydronephrosis or non-functioning kidney

Stage IV The carcinoma has extended beyond the true pelvis or has involved (biopsy proven) the mucosa of the bladder or rectum. A bullous oedema, as such, does not permit a case to be allotted to stage IV

Stage IVA: spread of the growth to adjacent organs

Stage IVB: spread to distant organs

a All macroscopically visible lesions - even with superficial invasion - are allotted to stage IB carcinomas. Invasion is limited to a measured stromal invasion with a maximal depth of $5 \mathrm{~mm}$ and a horizontal extension of $\leq 7 \mathrm{~mm}$. Depth of invasion should be $\leq 5 \mathrm{~mm}$ taken from the base of the epithelium of the original tissue - superficial or glandular. The depth of invasion should always be reported in $\mathrm{mm}$, even in those cases with 'early (minimal) stromal invasion' $(\sim 1 \mathrm{~mm})$. The involvement of vascular/lymphatic spaces should not change the stage allotment.

b On rectal examination there is no cancer-free space between the tumour and the pelvic wall. All cases with hydronephrosis or non- functioning kidney are included, unless they are known to be due to another cause.

neoplasia grade II+ within 3 years. ${ }^{2}$ HPV infection is very common; it is estimated that $20 \%$ of sexually active girls will contract the virus by the age of 18 years. ${ }^{4}$ The risk of infection increases with the age at first sexual intercourse. ${ }^{5}$

There are a number of factors that can increase or decrease the risk of developing cervical cancer:

- Age. Cervical cancer is rare before the age of 20 years but the incidence increases rapidly with age, giving a peak incidence of around 17 per 100,000 between the ages of 30 and 39 years. ${ }^{6}$ Cervical cancer mortality rates generally increase with age, so that only about $7 \%$ of cervical cancer deaths occur in women under 35 years, with the highest rates in women over 70 years. ${ }^{7}$ Squamous cell tumours are more common, but the rates of both squamous cell tumours and adenocarcinomas rise sharply from age 20-40 years, after which they plateau until age 80 years. ${ }^{8}$

- Sexual behaviour. There is an increased risk of invasive cervical cancer with early age at first sexual intercourse, ${ }^{5,9}$ early pregnancy ${ }^{5}$ and current use of hormonal contraceptives. ${ }^{10}$

- Smoking. Current smoking intensity is an independent risk factor for high-grade cervical intraepithelial neoplasia in young women, after controlling statistically for cervical HPV infection, ${ }^{11}$ and may be a risk factor for developing cervical cancer. ${ }^{12}$

- HIV infection. HIV infection leads to an increased risk of advanced and early cervical pathology. ${ }^{13}$ 
- Socioeconomic status. Women living in the most deprived areas in the UK have cervical cancer rates that are more than three times as high as those women in the least deprived areas. Data from a longitudinal study, representing 1\% of the population from England and Wales, showed that cervical cancer incidence is considerably higher among women of working age in manual occupations than among women in non-manual occupations. ${ }^{14}$

\section{Epidemiology}

Cervical cancer is a common gynaecological malignancy, with an estimated 31,400 new cases diagnosed each year in the European Union. ${ }^{15}$ In the UK, approximately 2800 patients are diagnosed with cervical cancer per year, accounting for around $2 \%$ of all female cancer cases. ${ }^{6}$ In England, carcinoma of the cervix is rare in women $<20$ years of age. ${ }^{6}$ Cancer of the cervix is a leading cause of cancer death in women. In 2008, there were 1110 deaths from cervical cancer in the UK, giving a European age-standardised death rate of 2.7 per 100,000 person-years. ${ }^{15}$ In the UK population, the 5 -year disease-free survival rate for treated stage IA disease is almost $100 \%$, whereas it is $50-70 \%$ for stage IB2 and IIB, 30-50\% for stage III and $5-15 \%$ for stage IV disease. ${ }^{16}$ It is estimated that the median survival for stage IVB disease is around 9-10 months, with $30 \%$ of patients surviving 1 year and $2-5 \%$ surviving 2 years. ${ }^{17}$

\section{Initial treatment of cervical cancer}

When patients are initially diagnosed with cervical cancer they can be treated with surgery, a combination of chemotherapy and radiotherapy (chemoradiotherapy) or with palliative care. The treatment chosen is based on stage of tumour, fitness of the woman and tumour characteristics, for example greater than one-third stromal invasion, capillary lymphatic space involvement and large tumour diameter. ${ }^{18}$ Surgery is usually radical hysterectomy but can also be trachelectomy (if the tumour is small), which is the removal of the cervix only rather than the whole uterus and can be performed in younger women with early cervical cancer who wish to retain their fertility. ${ }^{3}$ Approximately $20-30 \%$ of women undergoing surgery also receive adjuvant postoperative chemoradiotherapy for positive tumour margins or positive lymph nodes or because of the tumour size, volume, lymphovascular space invasion or stromal invasion. ${ }^{19}$

\section{Recurrent or persistent cervical cancer}

Patients can be cured by initial treatment and approximately $70-80 \%$ of initially treated cases are cured with surgery. If surgery is not appropriate because of tumour characteristics or lack of fitness in the patient, chemoradiotherapy can be given. However, the initial treatment may not affect a cure and in approximately $15 \%$ of patients disease is detected 3 months after treatment, which is called persistent cervical cancer (rather than recurrent). Recurrence is more common within the first 24 months after the initial diagnosis, but can happen up to 15 years after initial treatment. ${ }^{20}$

The Scottish Intercollegiate Guidelines Network (SIGN) guideline ${ }^{3}$ found the rates of recurrence from the three studies reviewed in the guideline to be $13 \%,{ }^{21} 18.2 \%{ }^{22}$ and $29 \%{ }^{23}$ In another study, the proportion with recurrence in early-stage cervical cancer was 6\%; ${ }^{24}$ a further study of locally advanced cervical cancer reported 30\% recurrence. ${ }^{25}$ Recurrences are more common within the first 24 months after the initial diagnosis - the median disease-free interval was 17 months for symptomatic patients and 16 months for asymptomatic patients in one cohort ${ }^{21}$ and the median time from surgery to recurrence in another cohort was 17.6 months. ${ }^{22}$ The percentage recurrence was higher after radiotherapy $(17 \%)$ than after surgery $(13 \%),{ }^{21}$ but none of the studies compared recurrence after chemoradiotherapy with recurrence after surgery. The proportions of asymptomatic to symptomatic recurrences were 19:114 ${ }^{21}$ and $2: 5 .^{22}$

Patients with pelvic recurrence usually present with one or more of vaginal bleeding, discharge, pelvic pain and sciatic pain. Patients with disseminated recurrence eventually develop systemic symptoms associated with cachexia.

Risk factors for recurrence include disease stage, number of positive lymph nodes, parametrial involvement and depth of invasion of the tumour. ${ }^{24}$ The squamous cell carcinoma antigen is elevated in $28-88 \%$ of patients with cervical cancer and can precede clinical diagnosis of relapse in $46-92 \%$ of cases. $^{26}$ 
Patients with recurrence or persistence are described according to the stage they were when they were diagnosed originally, along with some further information on whether or not and how much the cancer has progressed since the original diagnosis. For example, a woman who presented with a stage IIA cancer who now has distant metastases does not become a stage IVB cancer, but is described as a stage IIA cancer with metastases. Occasionally, a new stage can be assigned in addition if the cancer has recurred, particularly in trials, in which case it will be described with a lower case $r$ in front of the new staging, for example stage rIVB. ${ }^{27}$

\section{Prognosis}

Survival with recurrent or persistent disease is poor - from 6 months to 2 years. ${ }^{3}$ Also, patients frequently experience substantial morbidity from local recurrence and distant spread. ${ }^{3}$ It is unclear whether or not earlier detection of recurrence (from clinical follow-up or scanning) leads to increased survival rates, but this is a reasonable assumption to make. Worse survival is associated with shorter disease-free interval, being symptomatic and poorer prognostic factors. ${ }^{28}$

\section{Imaging to detect recurrence}

This project investigates three imaging techniques: CT, MRI and PET-CT. These techniques allow noninvasive visualisation of anatomical structures and physiological functions of the body.

\section{Computerised tomography and magnetic resonance imaging scanning}

Computerised tomography scanning was introduced in the 1970s and is now widely used in the NHS. A CT scan is a series of tomographic radiographic images used to visualise two-dimensional 'slices' through the body. Because the X-ray beam emission and the receiving film-intensifying screen are both revolving around a focal point in the body, this focal point can be visualised much more clearly than in a standard radiography film. A very large number of focal points are visualised consecutively and then a computer is used to mathematically reconstruct a two-dimensional matrix to give a digital image of the part of the body being scanned. CT scanning is painless and takes 15-30 minutes. It is non-invasive unless contrast medium is being used. For most whole-body CT scans, intravenous iodinated contrast is now used and there is the risk of allergic reactions. The main disadvantage, however, is the dose of radiation that is absorbed during the scanning. It has been estimated that $40 \%$ of all diagnostic radiation exposure in patients comes from CT scanning. ${ }^{29} \mathrm{CT}$ scanning can also produce artefacts that impede interpretation of the images. These artefacts can come from motion (e.g. patients have to hold their breath when the chest is being scanned) and from high-density objects such as tooth fillings and orthopaedic hardware.

Magnetic resonance imaging scanning was introduced in the 1980s and is now also widely used in major centres in the NHS. It is also a tomographic imaging technique but uses the ability of hydrogen atoms to absorb and emit radio waves (at a similar frequency to FM radio) when placed in a strong magnetic field. Visualisation of tissues can occur because of the different concentrations of hydrogen atoms in different tissues and the characteristics of the atoms in different complex biochemical environments. MRI uses characteristics such as the density of hydrogen atoms, the speed at which they become magnetised and lose their magnetisation and the presence of flow or motion in a tissue. MRI does not use ionising radiation, which is an advantage compared with CT. However, patients are placed in a magnetic field and so metal objects inside and outside the body will be affected. Patients with pacemakers, cochlear implants, shotgun fragments, etc. should not have a MRI scan. The energy generated inside the body can cause hyperthermia, particularly in obese people. The size of the trolley and aperture (MRI machines are longer than CT machines and fit the whole body inside) mean that people who weigh $>20$ stone $(127 \mathrm{~kg})$ are unlikely to fit inside the machine. The machine is also noisy and a small proportion of patients have anxiety-related reactions. MRI scans can give false-positive results from motion artefacts, interfaces between fat and water and distortions due to magnetic objects inside the body. 
Computerised tomography and MRI are high-resolution anatomical imaging techniques that are commonly used in cancer to detect potential tumours. MRI and CT are currently considered first when recurrence is suspected. ${ }^{17}$ Whole-body CT and MRI scanning are now rarely performed; imaging for cervical cancer is frequently limited to the pelvis only. CT and MRI have limitations in differentiating recurrent tumours from postradiotherapy or surgical fibrosis and also have limitations in accurately identifying the extent of recurrence as small volume nodal metastasis. If CT or MRI of the pelvic area only is carried out, distant recurrence may not be identified. They can also be unreliable in determining the presence or absence of recurrent disease in the pelvis after radiotherapy, as radiotherapy-induced fibrosis makes tissues indurated and thus potentially conceals recurrent disease.

\section{Positron emission tomography/computerised tomography scanning}

Positron emission tomography is an imaging method that can be used to establish the functional parameters of tissue, allowing detection of metabolically active areas in tissues such as tumours. ${ }^{30}$ ${ }^{18} \mathrm{~F}$-fluorodeoxyglucose ( $\left.{ }^{18} \mathrm{~F}-\mathrm{FDG}\right)$ is the most widely used radiotracer and is intravenously injected 1-2 hours before imaging. It is a glucose analogue and is taken up and actively trapped in the enhanced glycolytic pathway of hypermetabolic areas, demonstrated by high-energy photons emitted as a result of annihilation of positrons emitted by the radioisotope, with nearby negatively charged electrons. PET provides anatomical image resolution of the order of $4-6 \mathrm{~mm}$, significantly better than conventional gamma cameras but inferior to the 1 - to $2-\mathrm{mm}$ resolution of CT or MRI. The size of lesion that can be detected by PET is limited by several factors, including the physics of positron emission, the spatial resolution of the scanner (typically $4.5-6.0 \mathrm{~mm}$ in the centre of the axial field) and the safe dosing limits of ${ }^{18} \mathrm{~F}-\mathrm{FDG} .{ }^{30}$

Positron emission tomography/computerised tomography is a combination of PET scanning and CT scanning on the same machine. It precisely aligns and combines metabolic PET imagines with anatomical CT images obtained immediately and consecutively without patient movement, and is being increasingly preferred over PET scanning alone as it allows more precise localisation of active disease sites than either technology separately. The CT scan usually has a lower radiation dose than standard CT scans and contrast media are rarely used. PET-CT in suspected recurrent or persistent cervical cancer can detect metabolically active metastatic lesions in normal-sized nodes and in postsurgical or radiotherapy fibrosis. PET-CT in the follow-up of cervical cancer patients can be used to identify recurrent or persistent disease, assess local tumour extension, evaluate pelvic nodal involvement, detect distant metastases (e.g. lung, supraclavicular lymph nodes and para-aortic lymph nodes), plan radiotherapy and assess response to therapy. ${ }^{31}$

There are several disadvantages to PET-CT scanning. First, the machine is very expensive (approximately $\mathrm{f} 2 \mathrm{M}$ ). Second, ${ }^{18} \mathrm{~F}-\mathrm{FDG}$ has a short half-life of around 2 hours and therefore can cause throughput difficulties. False-positives are relatively common because the technique is looking for metabolically active regions and not all are cancerous, for example sepsis and inflammation following surgery and radiotherapy may mimic metastases. False-negatives can also occur soon after chemotherapy because the drugs may slow the metabolism of the metastases but not eliminate them altogether. Therefore, PET-CT to find secondary spread is not recommended within 3 months of surgery and radiotherapy and within 6 weeks of chemotherapy.

\section{Current guidelines on imaging strategies in recurrent cervical cancer}

The SIGN guidelines ${ }^{3}$ state that evidence for the effectiveness of post-treatment surveillance is inconsistent and that there is no evidence to suggest that prior radiotherapy or chemotherapy alters the sensitivity of detection of recurrence. They suggest that patients should be followed up every 4 months for at least 2 years. In asymptomatic patients, a PET-CT scan is recommended at 9 months' follow-up in women who have had chemoradiotherapy. If positive, pelvic MRI should be considered for surgical planning if pelvic exenteration is appropriate. In symptomatic women, MRI or CT should be considered to assess potential clinical recurrence. If positive, a whole-body PET or PET-CT scan should be performed in patients in whom salvage therapy (pelvic exenteration or radiotherapy) is being considered. 
The Society of Gynecologic Oncologists recommendations state that there is insufficient data to support routine use of PET-CT in asymptomatic patients. ${ }^{32}$ It suggests that CT and/or PET should be used when recurrence is suspected at any time up to 5 years after treatment.

The UK Royal College of Radiologists guidelines used evidence that was not specific to recurrent cervical cancer. ${ }^{33}$ However, it suggests that PET-CT can be used for restaging patients with cervix carcinoma considered for exenterative surgery, and for suspected recurrence when other imaging is equivocal.

\section{Survival data from positron emission tomography/computerised tomography studies in cervical cancer}

There are two publications ${ }^{34,35}$ that contain useful information about survival in cervical carcinoma, using PET-CT to differentiate between different groups of patients, including those with persistent and recurrent cervical cancer. In Schwartz et al., 3592 women who had been treated with chemoradiotherapy for carcinoma of the cervix (FIGO stages IB1 to IVA) and who had whole-body PET-CT between 8 and 16 weeks after initial therapy were followed up clinically for at least 6 months (range 6-49 months). PET-CT was used to investigate prognosis, linking findings with progression-free survival and cause-specific survival. Among the 92 patients, PET-CT showed a complete response in $65(71 \%)$ and persistent tumour in $15(16 \%)$ and identified new abnormalities in 12 (13\%). The survival rates are shown in Figure 1. The 3 -year cause-specific rates were $96 \%$ for women with a complete response to treatment and $43 \%$ for patients with persistent disease, and the 2-year survival rate was 14\% for patients with any new sites of disease. The 3-year progression-free survival rates were $78 \%$ for patients with a complete response after therapy, 33\% for patients with persistent disease and $0 \%$ for those with new sites of tumour.

Brooks et al. ${ }^{34}$ investigated the usefulness of PET-CT imaging in 78 asymptomatic and 25 symptomatic patients following a complete response to initial chemoradiotherapy for cervical cancer. The post-therapy PET-CT was performed at 3 months after treatment completion and patients were followed up for a median of 13 months for asymptomatic patients and 8 months for symptomatic patients. Unfortunately, for the first 2 years only PET was used and for the remaining 4 years PET-CT was used. The number of women in each group is unclear. The survival curves are shown in Figure 2. The 3-year survival for patients with symptomatic recurrence was 19\% compared with $59 \%$ for patients with asymptomatic recurrence $(p=0.09)$.

(a) Cause-specific survival

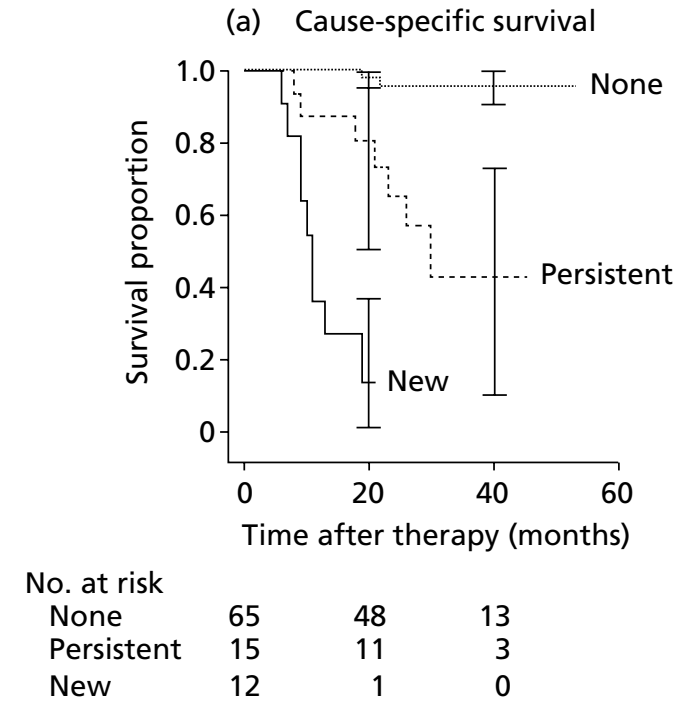

(b) Progression-free survival

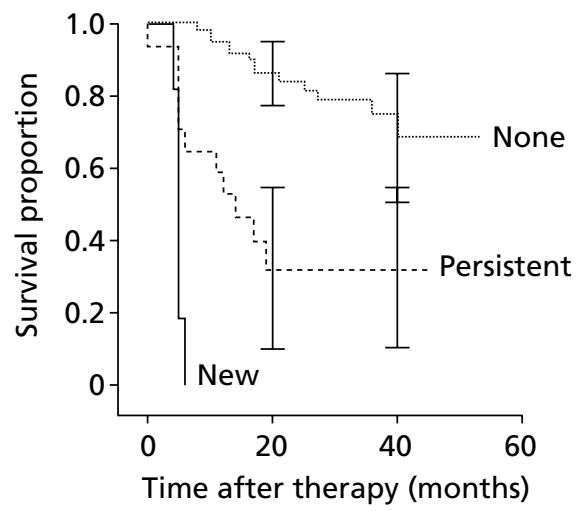

$\begin{array}{lrrr}\text { No. at risk } & & & \\ \text { None } & 65 & 43 & 11 \\ \text { Persistent } & 15 & 14 & 1 \\ \text { New } & 12 & 0 & 0\end{array}$

FIGURE 1 Cause-specific survival rates (a) and progression-free survival rates (b) for patients categorised by PET-CT as having no tumour, persistent tumour or new site of cervical cancer. 


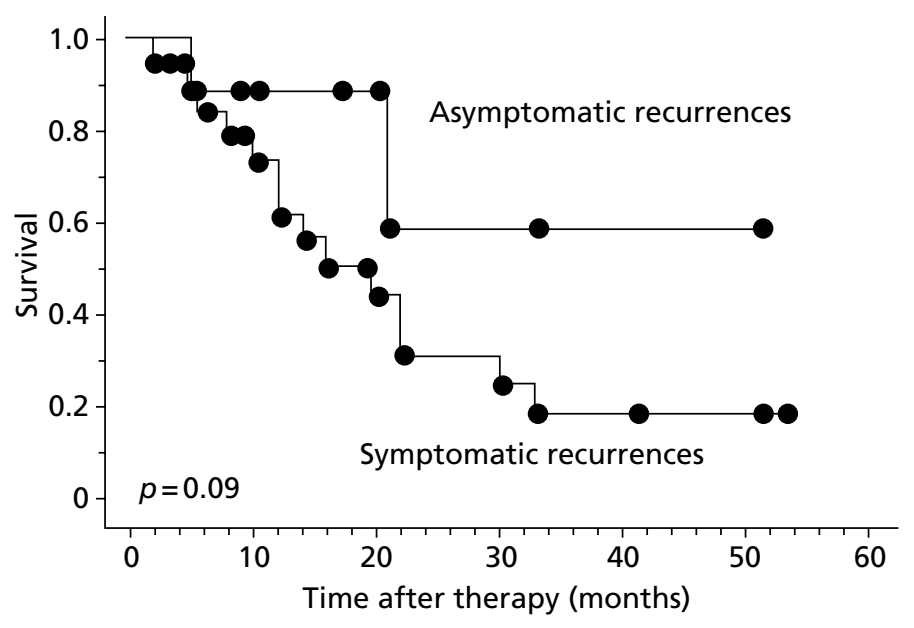

FIGURE 2 Survival in asymptomatic and symptomatic patients undergoing surveillance PET and PET-CT following one scan at 3 months.

\section{Treatment options for recurrent cervical cancer}

Treatment of recurrent cervical cancer depends on the site (central, pelvic, distant), extent of recurrence, type of previous treatment received (surgery, chemoradiotherapy, radiotherapy), time elapsed since primary treatment and patient fitness. Treatment intention is usually curative or palliative. Palliative treatment is used when there are distant metastases or multiple site recurrences and is usually chemotherapy.

Potentially curative disease is defined as:

- confirmed recurrence of the disease confined to the pelvis, provided that the patient has not received previous primary or adjuvant pelvic radiotherapy

- disease confined to the central pelvis, without pelvic side wall or extrapelvic involvement, provided that radiotherapy has been administered before recurrence

- distant recurrences at a single site (such as para-aortic lymph node) that could be completely resected or encompassed by a curative radiotherapy procedure.

In women with recurrence who had surgery for their primary tumour, radiotherapy is the treatment of choice. This may also include chemotherapy, which is often single-agent cisplatin. ${ }^{3}$ In women who had chemoradiotherapy or radiotherapy and who have persistent cervical cancer, salvage surgery is generally considered if the patient is sufficiently fit, if the disease is localised to the pelvis only and if surgery has a high chance of completely removing the disease with clear margins. ${ }^{3}$ Surgery can be radical hysterectomy or pelvic exenteration. Surgery for relapsed disease after radiotherapy is often associated with high morbidity as radiation fibrosis makes surgery difficult and, to enhance cure rates, surgical excision of disease often involves removal of the bladder, uterus, cervix and various amounts of the vagina (anterior exenteration) or the uterus, vagina and portions of the rectosigmoid colon and anus (posterior exenteration) or a complete pelvic clearance (exenteration). In a small number of patients, radical hysterectomy will suffice if the disease is highly localised. As exenterations are morbid surgical procedures resulting in alteration of body image and loss of bladder and/or bowel control, patients require extensive preoperative psychosocial counselling.

\section{Objectives of this report}

When this project was being defined there was some discussion around the exact focus, because the current UK imaging strategy using PET-CT is for selective use in symptomatic patients depending on symptoms and equivocal or negative findings on CT and/or MRI and to rule out the possibility of distant 
metastases when salvage surgery is being considered, rather than for routine use in all symptomatic patients with suspected recurrence and as routine follow-up in asymptomatic patients. In asymptomatic patients, clinical follow-up alone may also have been a useful comparator to routine CT, MRI or PET-CT.

This research project was undertaken to evaluate the clinical effectiveness and cost-effectiveness of strategies of imaging with MRI or CT with or without PET-CT in women with asymptomatic or symptomatic recurrent cervical cancer, and for their subsequent treatment with surgery, chemotherapy and/or radiotherapy. The relationship of our clinical objectives to the range of work required is shown in Figure 3 . The economic evaluation is in addition to these objectives and is described in Chapter 8.

Routine monitoring after primary treatment Suspicion of recurrent cervical cancer

\section{Current standard practice}

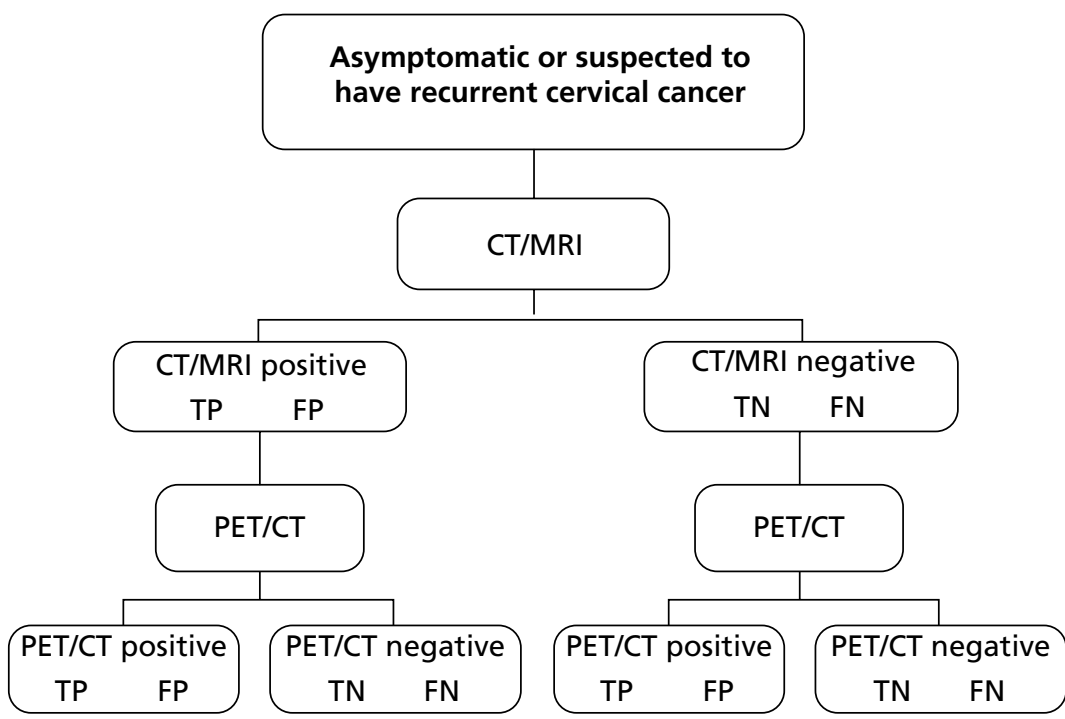

Reference standard

(Histopathology or clinical follow-up in 3-6 months)

Planned intervention

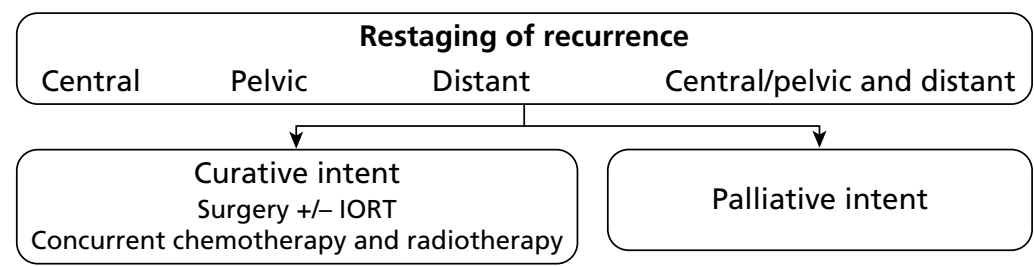

Outcome

Improvement in restaging with change in treatment plan 2-year survival

FIGURE 3 Imaging and treatment strategies in women with recurrent cervical cancer. FN, false-negative; FP, falsepositive; IORT, intraoperative radiotherapy; TN, true-negative; TP, true-positive. 


\section{Chapter 3 Methods for systematic reviews and subjective elicitation}

\section{Protocol development and overview of review methods}

A generic protocol was developed for undertaking the systematic reviews of test accuracy, diagnostic and therapeutic yield and effectiveness. Scoping searches for relevant systematic reviews were conducted in MEDLINE, EMBASE and The Cochrane Library (see Appendix 2).

Systematic reviews were carried out using established methods in line with the recommendations of the NHS Centre for Reviews and Dissemination ${ }^{36}$ and the Cochrane Collaboration, ${ }^{37}$ and, for diagnostic systematic reviews, using the latest methods from the Cochrane Diagnostic Test Accuracy Working Group. ${ }^{38}$ Presentation of systematic reviews is according to Preferred Reporting Items for Systematic Reviews and Meta-Analyses (PRISMA) guidelines. ${ }^{39}$

Inclusion of studies, data extraction and quality assessment were carried out in duplicate with differences resolved by consensus and/or arbitration involving a third reviewer. There were no language limitations on inclusion criteria. The selection process was piloted by applying the inclusion criteria to a sample of papers first, and then a two-stage process was used, first, by screening titles and abstracts. For all references categorised as 'include' or 'uncertain' by both reviewers, the full text was retrieved whenever possible and final inclusion decisions were made on the full paper. Reference Manager 12.0 software (Thomson ResearchSoft, San Francisco, CA, USA) was used to construct a database of citations for all systematic reviews.

Clinical, methodological and statistical data extraction was carried out using data extraction sheets by at least two reviewers and discrepancies were resolved through discussion. If consensus could not be reached, disagreements were resolved by arbitration by a third reviewer. For diagnostic studies, information was extracted regarding study design and methods, characteristics of participants, PET-CT and comparison tests, and outcomes of interest (see Appendix 3). For the effectiveness review, separate data extraction forms were used for different study designs: comparative experimental study (part $A$ ), comparative observational study (B) and non-comparative study (C) (see Appendix 4). The quality assessment questions for randomised controlled trials (RCTs) were included in the data extraction sheet, but a separate form was used for case series (see Appendix 5). Data extraction was managed with Microsoft Office 2003 Word and Excel (Microsoft Corporation, Redmond, WA, USA). Quality was also assessed independently by two reviewers. Any disagreements were resolved through discussion or by arbitration by the third reviewer.

\section{Methods for test accuracy and diagnostic and therapeutic impact reviews}

\section{Search strategy}

A sensitive search was conducted to identify all relevant published and unpublished studies and studies in progress. All databases were searched from inception to May 2010. Search strategies were designed from a series of test searches and discussions of the results of those searches among the review team. Both medical subject heading (MeSH) terms and text words were used and included 'cervical cancer', 'PET-CT', 'CT' and 'MRI'. The strategies from MEDLINE, EMBASE and The Cochrane Library can be found in Appendix 6. Literature was identified from several sources including: 
- general health and biomedical databases: MEDLINE (Ovid), EMBASE (Ovid), Science Citation Index, The Cochrane Library [Cochrane Central Register of Controlled Trials (CENTRAL)], Medion

- checking of reference lists of systematic and narrative review articles

- searching a range of relevant databases including ClinicalTrials.gov and the UK Clinical Research Network Portfolio to identify information about studies in progress, unpublished research or research reported in grey literature

- specialist search gateways (OMNI and the National Cancer Institute), general search engine (Google) and meta-search engine (Copernic) from March to May 2010

- hand-searching of Gynecologic Oncology from 1980 to May 2010

- authors of included studies contacted for information on relevant published or unpublished studies.

\section{Inclusion and exclusion criteria}

\section{Population}

Included:

- any women with clinical suspicion of persistent or recurrent cervical cancer after primary treatment, on the basis of one or more of clinical history, clinical examination and tests (including imaging and histology)

- any women who had had advanced-stage cervical cancer (IB2-IV) treated previously, for example with chemoradiotherapy, with a minimum gap between completion of treatment and imaging of 3 months, and who were currently asymptomatic and undergoing routine follow-up.

Excluded:

- studies in which the population contained women within 3 months of completion of treatment for primary disease were excluded because of problems associated with distinguishing treatment complications and inflammatory response from recurrence in this patient group.

\section{Index test}

Included:

- $\quad$ PET-CT using ${ }^{18}$ F-FDG as the radioisotope tracer.

Excluded:

- PET alone without concurrent CT.

\section{Comparator tests}

- CT (local or whole body).

- MRI (local or whole body).

\section{Reference standard}

Included:

- histopathological findings or clinical follow-up for $\geq 6$ months or both for all participants (differential reference standard was accepted because of the difficulty of biopsy when there was no indicated lesion to biopsy in test-negative patients).

Excluded:

- studies in which only some of the participants undergoing the index test also received any reference standard. 


\section{Outcome}

- Studies that provided numerical data sufficient to create $2 \times 2$ tables of test results comparing index or comparator tests with the reference standard to provide information on test accuracy, giving truepositive, true-negative, false-positive and false-negative results.

- Studies that provided any information on diagnostic impact: change in diagnosis and/or staging after PET-CT compared with existing tests or reference standard.

- Studies that provided therapeutic impact: change in treatment plan after PET-CT compared with existing tests or reference standard.

\section{Study design}

Included:

- any prospective or retrospective test accuracy studies

- any diagnostic before-and-after studies investigating diagnostic and therapeutic impact with or without concurrent assessment of test accuracy

- studies with $>10$ participants.

Excluded:

- studies on gynaecological cancers not providing separate data for the population with cervical cancer

- studies that described only lesion-based analysis rather than person-based analysis.

\section{Quality assessment}

Test accuracy quality assessment followed the Quality Assessment of Diagnostic Accuracy Studies (QUADAS) guidelines ${ }^{40}$ and diagnostic and therapeutic impact quality assessment followed guidelines suggested by Meads and Davenport. ${ }^{41}$ The items of methodological quality listed in the QUADAS guidelines ${ }^{40}$ are a representative spectrum, selection criteria clearly described, acceptable reference standard, acceptable delay between tests, partial verification avoided, differential verification avoided, reference standard independent of the index test, index test described in sufficient detail, reference standard described in sufficient detail, index test results blinded, reference standard results blinded, relevant clinical information available, uninterpretable results reported, and withdrawals explained.

These items were tailored to assess the included studies because different aspects of quality are applicable to different topic areas. The actual quality items used for this report are listed in Table 2. For acceptable delay between tests, this included delay between the index test and the comparator test (within 1 month) and between the index test and PET-CT (with 1 month). There will inevitably be a delay between the index test and clinical follow-up (as this had to be $>6$ months). Differential verification was omitted because it was inevitable that the test positives would have a different reference standard (histology) to the test negatives (clinical follow-up).

Study quality was summarised in a table. Additional issues (e.g. study design characteristics, method of patient enrolment, technique of data collection) were also collected. Technical quality was assessed by a consultant radiologist with considerable experience in current cancer imaging techniques.

\section{Methods of statistical analysis}

RevMan version 5.1 (The Cochrane Collaboration, The Nordic Cochrane Centre, Copenhagen, Denmark) and Stata version 11 (StataCorp LP, College Station, TX, USA) were used in the statistical analyses. Truepositives, false-positives, true-negatives and false-negatives were taken directly from the source papers and sensitivity and specificity calculated in RevMan. Equivocal results were used in sensitivity analyses by adding the total number of equivocal results to each of the true-positives, false-positives, true negatives and false-negatives in turn to derive maximum and minimum variation in sensitivity and specificity. Summary 
TABLE 2 Quality assessment items

\begin{tabular}{|c|c|c|c|}
\hline Item & Yes & No & Unclear \\
\hline $\begin{array}{l}\text { 1. Representative } \\
\text { spectrum }\end{array}$ & $\begin{array}{l}\text { If the stated characteristics } \\
\text { of the spectrum of patients } \\
\text { fulfilled the requirements of the } \\
\text { included population }\end{array}$ & $\begin{array}{l}\text { If the sample does not fit with } \\
\text { what was pre-specified as } \\
\text { acceptable or if groups with } \\
\text { and without the target disorder } \\
\text { were recruited together (e.g. } \\
\text { sample includes both primary } \\
\text { and recurrent cervical cancer } \\
\text { and results not given separately) }\end{array}$ & $\begin{array}{l}\text { If there is insufficient } \\
\text { information available to } \\
\text { make a judgement about } \\
\text { the spectrum }\end{array}$ \\
\hline $\begin{array}{l}\text { 2. Selection } \\
\text { criteria clearly } \\
\text { described }\end{array}$ & If the selection criteria described & $\begin{array}{l}\text { If the selection criteria not } \\
\text { described }\end{array}$ & $\begin{array}{l}\text { If there is insufficient } \\
\text { information available to } \\
\text { know clearly the selection } \\
\text { criteria }\end{array}$ \\
\hline $\begin{array}{l}\text { 3. Acceptable } \\
\text { reference } \\
\text { standard }\end{array}$ & $\begin{array}{l}\text { Both reference standards used } \\
\text { meet the pre-stated inclusion } \\
\text { criteria }\end{array}$ & $\begin{array}{l}\text { One or other reference } \\
\text { standards used do not meet the } \\
\text { pre-stated criteria }\end{array}$ & $\begin{array}{l}\text { It is unclear exactly what } \\
\text { reference standard was } \\
\text { used (particularly for clinical } \\
\text { follow-up) }\end{array}$ \\
\hline $\begin{array}{l}\text { 4. Acceptable } \\
\text { delay between } \\
\text { imaging tests }\end{array}$ & $\begin{array}{l}\text { If the time between tests was } \\
\text { shorter than } 1 \text { month, at } \\
\text { least for an acceptably high } \\
\text { proportion of patients }\end{array}$ & $\begin{array}{l}\text { If the time between tests was } \\
\text { longer than } 1 \text { month for an } \\
\text { unacceptably high proportion } \\
\text { of patients }\end{array}$ & $\begin{array}{l}\text { If information on timing of } \\
\text { tests is not provided }\end{array}$ \\
\hline $\begin{array}{l}\text { 5. Partial } \\
\text { verification } \\
\text { avoided }\end{array}$ & $\begin{array}{l}\text { If all patients, or a random } \\
\text { selection of patients, who } \\
\text { received the index test went on } \\
\text { to receive verification of their } \\
\text { disease status using a reference } \\
\text { standard, even if the reference } \\
\text { standard was not the same for } \\
\text { all patients }\end{array}$ & $\begin{array}{l}\text { If some of the patients who } \\
\text { received the index test did not } \\
\text { receive verification of their } \\
\text { true disease state, and the } \\
\text { selection of patients to receive } \\
\text { the reference standard was not } \\
\text { random }\end{array}$ & $\begin{array}{l}\text { If this information is not } \\
\text { reported by the study }\end{array}$ \\
\hline $\begin{array}{l}\text { 6. Reference } \\
\text { standard } \\
\text { independent of } \\
\text { the index test }\end{array}$ & $\begin{array}{l}\text { If the index test did not form } \\
\text { part of the reference standard }\end{array}$ & $\begin{array}{l}\text { If the reference standard } \\
\text { formally included the result of } \\
\text { the index test }\end{array}$ & $\begin{array}{l}\text { If it is unclear whether or } \\
\text { not the results of the index } \\
\text { test were used in the final } \\
\text { diagnosis }\end{array}$ \\
\hline $\begin{array}{l}\text { 7. Tests described } \\
\text { in sufficient } \\
\text { detail for } \\
\text { replication }\end{array}$ & $\begin{array}{l}\text { If both the index test(s) and } \\
\text { reference standard were fully } \\
\text { described to permit replication }\end{array}$ & If no tests described & If test descriptions unclear \\
\hline $\begin{array}{l}\text { 8. Reference } \\
\text { standard/index } \\
\text { test results } \\
\text { blinded }\end{array}$ & $\begin{array}{l}\text { If test results (index or reference } \\
\text { standard) were interpreted blind } \\
\text { to the results of the other test, } \\
\text { or blinding is dictated by the } \\
\text { test order, or meets the pre- } \\
\text { stated assumptions }\end{array}$ & $\begin{array}{l}\text { If it is clear that one set of test } \\
\text { results was interpreted with } \\
\text { knowledge of the other }\end{array}$ & $\begin{array}{l}\text { If it is unclear whether } \\
\text { blinding took place }\end{array}$ \\
\hline $\begin{array}{l}\text { 9. Relevant clinical } \\
\text { information }\end{array}$ & $\begin{array}{l}\text { If clinical data available on } \\
\text { previous operations and } \\
\text { previous imaging per patient }\end{array}$ & If clinical data not stated & $\begin{array}{l}\text { If information about clinical } \\
\text { data was unclear }\end{array}$ \\
\hline $\begin{array}{l}\text { 10. Uninterpretable } \\
\text { results reported }\end{array}$ & $\begin{array}{l}\text { If the number of uninterpretable } \\
\text { test results (equivocal results) } \\
\text { is stated, or if the number of } \\
\text { results reported agrees with the } \\
\text { number of patients recruited } \\
\text { (indicating no uninterpretable } \\
\text { test results). }\end{array}$ & $\begin{array}{l}\text { If it states that uninterpretable } \\
\text { test results occurred or were } \\
\text { excluded and does not report } \\
\text { how many }\end{array}$ & $\begin{array}{l}\text { If it is not possible to } \\
\text { work out whether or not } \\
\text { uninterpretable results } \\
\text { occurred }\end{array}$ \\
\hline
\end{tabular}


TABLE 2 Quality assessment items (continued)

\begin{tabular}{|c|c|c|c|}
\hline & Yes & No & Near \\
\hline $\begin{array}{l}\text { 11. Withdrawals } \\
\text { explained }\end{array}$ & $\begin{array}{l}\text { If it is clear what happened to } \\
\text { all patients who entered the } \\
\text { study, for example if a flow } \\
\text { diagram of study participants } \\
\text { is reported explaining any } \\
\text { withdrawals or exclusions, or } \\
\text { the numbers recruited match } \\
\text { those in the analysis }\end{array}$ & $\begin{array}{l}\text { If it appears that some of the } \\
\text { patients who entered the study } \\
\text { did not complete the study, i.e. } \\
\text { did not receive both the index } \\
\text { test and reference standard, } \\
\text { and these patients were not } \\
\text { accounted for }\end{array}$ & $\begin{array}{l}\text { If it is unclear how many } \\
\text { patients entered and, hence, } \\
\text { whether or not there were } \\
\text { any withdrawals }\end{array}$ \\
\hline 12. Technical quality & $\begin{array}{l}\text { If it is clear that the methods of } \\
\text { imaging described in the paper } \\
\text { are similar to those currently } \\
\text { used }\end{array}$ & $\begin{array}{l}\text { If it is clear that the methods of } \\
\text { imaging described in the paper } \\
\text { have since been superseded by } \\
\text { current imaging standards }\end{array}$ & $\begin{array}{l}\text { If the methods described } \\
\text { in the paper are close to } \\
\text { those currently in use and } \\
\text { should not noticeably affect } \\
\text { interpretation or results }\end{array}$ \\
\hline
\end{tabular}

estimates of sensitivity and specificity and summary receiver operating characteristic (SROC) curves were derived as appropriate using recognised methods for meta-analysis of test accuracy. Results were displayed graphically on forest and SROC plots. ${ }^{42}$ Meta-analyses were undertaken when adequate results were available. A bivariate model that included a random-effects term for variation in accuracy and threshold between studies was fitted. ${ }^{43}$ When the model failed to converge or a correlation could not be estimated properly the bivariate model was simplified to two univariate random-effects logistic regression models by assuming no correlation between sensitivity and specificity. Although no correlation between sensitivity and specificity was assumed, a confidence region is shown on the SROC plot as an indication of the uncertainty surrounding the point estimate of sensitivity and specificity.

\section{Methods for subjective elicitation}

\section{Rationale}

Subjective probabilities were elicited from clinicians representing the disciplines of radiology, oncology and gynaecology. Eliciting subjective probabilities from clinicians had three roles in the planned investigation of the clinical effectiveness of PET-CT imaging in the detection and management of recurrent cervical cancer:

1. Providing data to populate the economic model in the absence of information found in the literature.

2. Supplementing information found in the literature. Literature may be sparse, of poor quality or not transferable to the UK setting. Information gained from clinicians in the form of subjective probabilities may be used to supplement information found in the literature and to enable sensitivity analyses to be performed as part of the economic model.

3. Planning the dissemination strategy for the results of the research. If there is wide variation in accuracy estimates elicited from clinicians, or if elicited estimates of accuracy are very discrepant with those found in the literature, this may impact on the successful dissemination of the research findings to clinicians.

\section{Probabilities elicited}

Informed by the preliminary results of the systematic reviews of test accuracy (and effectiveness), the research team decided on the data priorities for elicitation as follows:

1. To determine the prevalence of recurrence in women with an initial diagnosis of stage IB-IVA cervical cancer, who are assumed to be disease free for a minimum of 3 months post completion of primary treatment:

i. presenting with symptoms suggestive of recurrence

ii. in the absence of symptoms 
2. To determine the test accuracy of chest, abdominal and pelvic CT and/or MRI performed at the discretion of clinicians in women with an initial diagnosis of stage IB-IVA cervical cancer, who are assumed to be disease free for a minimum of 3 months post completion of primary treatment:

i. presenting with symptoms suggestive of recurrence

ii. in the absence of symptoms (CT and/or MRI used for surveillance)

3. To determine the test accuracy of CT and/or MRI performed at the discretion of clinicians and of PET-CT (performed regardless of the result of initial imaging) in women with an initial diagnosis of stage IB-IVA cervical cancer, who are assumed to be disease free for a minimum of 3 months post completion of primary treatment:

i. presenting with symptoms suggestive of recurrence

ii. in the absence of symptoms (CT and/or MRI + PET-CT used for surveillance).

Information on rate of recurrence in women post completion of primary treatment as distinct from rate of recurrence in women following imaging was absent in the literature reviewed. Elicitation of accuracy data was necessary because of a lack of disaggregation of women with and without symptoms in the literature and because of the very limited accuracy data available. Elicitation also provided the opportunity to investigate the coherence of subjective probabilities elicited with estimates in the literature.

\section{Methods used}

Subjective probabilities were elicited by two project members (CD and $C M)$ during an educational meeting of the West Midlands Gynaecology Oncology Specialist Group on 1 July 2011 at the City Hospital, Birmingham, UK. Following the success of this initial elicitation, as judged by the face validity of the findings, the results were supplemented by purposive sampling by clinicians in the project team and by two further meetings - a gynae-oncology multidisciplinary meeting at Barts Hospital, London, UK, on 17 August 2011 and at the British Gynaecological Cancer Society Scientific Meeting at the International Convention Centre, Birmingham, UK, on 18 November 2011.

The initial elicitation exercise was preceded by a presentation outlining the aims of the project, the role of elicitation in the project, an overview of definitions of prevalence and test accuracy metrics to be elicited and a practice non-clinical elicitation exercise. Subsequent elicitations achieved by purposive sampling used a written description of the task and a printed elicitation example, except at the scientific meeting where a poster on the project was also displayed.

For the clinicians carrying out the first elicitations, the face-to-face pre-elicitation training, questions and discussion were conducted as a group to facilitate a common understanding of the problem and task and to allow participants to benefit from group discussion and interaction. Following the presentation and the non-clinical elicitation exercise (on estimated distance from London to Birmingham), participants were asked for written consent before undertaking the elicitation exercise. Participants were free to leave at any point in the exercise. Participants were instructed to undertake the elicitation exercise itself independently to ensure that variation within and across disciplines could be captured if there were sufficient numbers of respondents to allow subgroup analysis. In addition, mathematical aggregation (as opposed to behavioural aggregation) mitigates against the possibility of 'consensus' estimates being biased by the views of a minority. ${ }^{44}$

The elicitation exercise comprised an 11-page anonymous self-administered questionnaire (see Appendix 7). The questionnaire included background information on the length of time that participants had practised in their speciality, their use of current imaging techniques and their use of PET-CT. To be eligible participants did not have to have hands-on experience of using PET-CT. Use of PET-CT is not routine in this patient group and beliefs are shaped by factors other than first-hand experience, such as interaction with colleagues, published estimates of accuracy and knowledge of the technology. In addition to the probabilities elicited, participants were also asked to state the minimum important clinical difference in accuracy between imaging with $C T$ and/or MRI and imaging with $C T$ and/or MRI with the addition of PET-CT that they would require before choosing to use one or other imaging strategy routinely. 
Accuracy data were elicited in the form of the proportion of test errors (false-positives and false-negatives) that would be expected with the use of the combinations of imaging technologies outlined above. The choice of test errors as a metric of accuracy is based on research suggesting that test accuracy metrics with test result as reference class are more intuitive ${ }^{45}$ and that the clinical utility of a test is commonly conceptualised using test errors. ${ }^{46}$ Test errors were used to derive positive predictive values (PPVs) and negative predictive values (NPVs). Elicited estimates of prevalence in combination with PPVs and NPVs were used to derive estimates of sensitivity and specificity for use in the economic model.

Elicitation of prevalence and test accuracy information was undertaken using the allocation of points technique whereby respondents are asked to indicate the likelihood of a value range being a true estimate by allocating a proportion of 100 points to that value range (the sum of allocated points across each value range summing to 100). In this way probability functions were obtained for each individual and were aggregated mathematically to derive an average distribution for the sample. An aggregated mean value was estimated using the average distribution and the midpoint of each value range. The variability of this aggregated mean was estimated by calculating the standard deviation (SD) across the value ranges. Microsoft Excel was used for calculations and graphical display of results.

\section{Methods for effectiveness reviews}

\section{Search strategy}

A sensitive search was conducted to identify all relevant published and unpublished trials and trials in progress. All databases were searched from inception to August 2010. Search strategies were designed from a series of test searches. Both MeSH terms and text words were used and included a variety of synonyms for recurrent cervical cancer and the interventions (chemotherapy, radiotherapy, palliative treatment, surgery). Strategies for MEDLINE, EMBASE and The Cochrane Library can be found in Appendix 8. Trials were identified from several sources including:

- general health and biomedical databases: MEDLINE (Ovid), EMBASE (Ovid), CENTRAL

- database searches for systematic reviews, from which primary studies could be identified, including MEDLINE (Ovid), EMBASE (Ovid) and The Cochrane Library [Cochrane Database of Systematic Reviews (CDSR), Database of Abstracts of Reviews of Effects (DARE) and Health Technology Assessment (HTA) database]

- searches for studies in progress, unpublished research or research reported in the grey literature in a range of relevant databases including ClinicalTrials.gov and the UK Clinical Research Network Portfolio

- specialist search gateways (OMNI and the National Cancer Institute), general search engine (Google) and meta-search engine (Copernic) from March to May 2010

- hand-searches of Gynecologic Oncology from 1980 to May 2010

- reference lists of review articles and papers

- authors of the included studies, who were contacted for information on relevant published or unpublished studies.

\section{Inclusion/exclusion criteria}

\section{Population}

Included:

- Women with recurrent cervical cancer (i.e. initial treatment was apparently successful and patients now presenting after 3 months with new symptoms and signs indicating recurrence) or with persistent cervical cancer (stage IVB) at follow-up after initial treatment has been completed (i.e. patients have initial treatment that was completed and are now presenting after 3 months with symptoms and signs suggesting that the initial treatment had not been completely successful). The initial treatment could have been surgery, radiotherapy or chemotherapy or any combination of these. 
Excluded:

- women with advanced cervical cancer before initial treatment together with women with recurrent or persistent cervical cancer in which the results were not presented separately

- trials with a lack of information about the primary site of cancer (e.g. studies on gynaecological cancers in which the exact site is not specified)

- trials with a lack of information on the primary treatment of participants

- patients who had undergone a variety of different initial treatments in which the results for each treatment group were not presented separately

- patients who had undergone a variety of different types of surgery in which the results were not presented separately

- patients who had undergone surgery with radiotherapy for their initial treatment.

\section{Interventions and comparators}

Any of the following treatments for recurrence were included:

- $\quad$ surgery with curative intent (studies must have included $<10 \%$ surgery with palliative intent)

- chemotherapy with a variety of therapeutic agents

- radiation treatment

- combination of surgery with radiotherapy

- combination of surgery with chemotherapy

- combination of radiotherapy with chemotherapy.

Excluded:

- curative and palliative intent surgery presented together in which palliative intent was $\geq 10 \%$ of participants.

\section{Outcomes}

Included:

- survival or mortality

- morbidity, symptoms

- treatment success or failure rates

- quality of life.

Excluded:

- biochemical outcomes.

\section{Study design}

Included:

- RCTs, controlled clinical trials

- case series, cohort studies or case-control studies when RCTs or controlled clinical trials were not available.

Excluded:

- studies presenting results for $<10$ patients. 


\section{Quality assessment}

For the two designs found (RCTs and case series), quality assessment and presentation of results have been carried out separately.

\section{Randomised controlled trials}

Quality assessment of included RCTs was performed using the criteria outlined in the Cochrane Handbook for Systematic Reviews of Interventions. ${ }^{37}$ Each study was assessed for adequate sequence generation, adequate allocation concealment, all methods of blinding used and whether or not they were effective, whether or not there was incomplete outcome data presented (attrition and exclusions from analysis), non-selective outcome reporting, and freedom from other biases. In all cases 'yes' indicated a low risk of bias and 'no' indicated a high risk of bias. 'Unclear' was used if there was insufficient detail reported. The quality of studies was summarised in tables, which were then used to create quality diagrams.

\section{Case series}

Quality assessment of case series was performed using the checklist developed by the National Institute for Health and Clinical Excellence (NICE). ${ }^{47}$ Each study was then awarded an overall study quality grading for internal validity and an overall study quality grading for external validity:

- ++ : all or most of the checklist criteria have been fulfilled; where they have not been fulfilled the conclusions are very unlikely to alter.

- $\quad+$ : some of the checklist criteria have been fulfilled; where they have not been fulfilled, or not adequately described, the conclusions are unlikely to alter.

- - few or no checklist criteria have been fulfilled and the conclusions are likely or very likely to alter.

\section{Methods of reporting and statistical analysis}

Most results are reported in tables. Information was analysed based on the group to which the participants were allocated, regardless of whether or not they received the allocated intervention. For dichotomous data, results are presented as summary relative risks (RR) with 95\% confidence intervals (Cls). Separate analyses were performed on randomised and non-randomised data. RRs were calculated from numbers of patients, using StatsDirect version 2.7.8 (StatsDirect, Altrincham, UK) or RevMan version 5.0. For adverse events, only grade 3 and grade 4 events were reported.

RevMan version 5.0 was also used for meta-analyses. Any heterogeneity of results between studies was statistically and graphically assessed and potential causes explored. To explore causes of clinical heterogeneity, a priori subgroup analyses were conducted to see whether variations in clinical factors, for example populations, interventions, outcomes or study quality, affected the estimation of effect sizes. The $I^{2}$ statistic was used to assess heterogeneity between trials. In the absence of significant heterogeneity, results were pooled using a fixed-effects model. If substantial heterogeneity was detected $\left(I^{2}>50 \%\right)$, possible causes were explored and subgroup analyses for the main outcomes performed. Heterogeneity that was not explained by subgroup analyses was modelled using random-effects analysis where appropriate. For outcomes for which a meta-analysis was not appropriate, the RCT and non-randomised study results were presented, where possible, on a forest plot but without summary scores, allowing a visual presentation of the effects of each included trial. For case series, a narrative summary of the findings was given.

\section{Methods for systematic review of economic evaluations}

A systematic review was conducted to find published literature and work in progress on the economic evaluation of PET-CT for use in the detection of recurrent cervical cancer. The purpose of this review was to investigate the suitability of existing cost-effectiveness models and model designs and to identify 
information that could be used to populate the model subsequently developed for this project. The aim was also to identify economic studies that reported costs and consequences associated with recurrent cervical cancer detected by the use of PET-CT. Systematic reviews of the effectiveness of treatments, with meta-analysis of clinical studies, particularly RCTs, use well-established research methods but the approach for reviewing economic evaluations and costing studies is necessarily slightly different and more qualitative, primarily because of the heterogeneity that exists in economic studies, which means that formal data synthesis and meta-analyses are rarely possible. This systematic review was carried out using PRISMA guidelines with adaptations appropriate for systematic reviews of economic evaluation and costing studies. ${ }^{39}$ In addition to the systematic review of economic evaluations, a separate literature review was conducted to find suitable generic quality-of-life values [including quality-adjusted life-years (QALYS)] for use in the economic model.

Five electronic databases were searched [EMBASE, MEDLINE, NHS Economic Evaluation Database (NHS EED), DARE and HTA database] from 1980 to October 2011. Reference lists from relevant papers were also searched. Appendix 9 shows the detailed search strategies used. The inclusion criteria were:

- patients - those with recurrence or persistent cervical cancer who had previously completed treatment for their primary cervical cancer (primary cervical cancer alone was specifically excluded)

- intervention - PET-CT

- comparator - no PET-CT, other imaging

- outcomes - costs, cost-effectiveness, cost-utility, quality of life.

Studies were independently reviewed on the basis of their titles and abstracts by one researcher (PA). The screening process used followed established methods used to identify and categorise economic evaluation and costing studies. ${ }^{29}$ Briefly, a three-stage process was adopted. In stage 1, each study was categorised on the basis of its title and abstract (where available) into one of four groups. The two relevant groups for this review were group $A$ - studies suspected of being full economic evaluations on PET-CT recurrence of cervical cancer - and group B - cost studies, but not economic evaluations. Group A and group B studies would proceed to stage 2 where they would be read in full and, if confirmed in their classification, would proceed to stage 3 for quality assessment. Appendix 9 shows the full details of the three-stage process. 


\title{
Chapter 4 Diagnostic review results
}

\author{
Study selection
}

At the final update of May 2010 there were 7524 potentially relevant citations identified, of which 252 full-text articles were retrieved. Subsequently, 240 articles were excluded (see list of excluded studies in Appendix 10). The most common reason for exclusion was either that the study was on patients with newly diagnosed cervical cancer before primary treatment or that the study was of the incorrect design. The numbers of included and excluded citations are shown in Figure 4. The 12 included studies evaluated the test accuracy of PET-CT, MRI or CT imaging for persistent or recurrent cervical cancer compared with a reference standard of biopsy, clinical follow-up or both. Six studies evaluated PET-CT, 20,48-52 two evaluated MRI, ${ }^{53,54}$ three evaluated $\mathrm{CT}^{55-57}$ and one evaluated both MRI and $\mathrm{CT}^{58}$ Table 3 shows the basic characteristics of the included studies and Table 4 provides definitions of the reference standards used. There were no studies that directly compared PET-CT with MRI or CT separately. One of the included studies ${ }^{49}$ compared PET-CT with standard imaging (MRI, CT or both) and gave results for both PET-CT and standard imaging in the same table.

No additional papers were found that evaluated diagnostic or therapeutic yield. One of the included studies $^{20}$ gave information on diagnostic yield and also gave 2-year disease-free survival curves for participants with positive and negative PET-CT scans.

\section{Characteristics of included studies}

\section{Population characteristics}

The characteristics of the patient populations in the included studies are shown in Tables 5-7. The total number of patients in the studies ranged from 20 to 75 but some of the studies included any gynaecological cancers and others reported imaging results for both recurrent and primary cervical cancer. Therefore, the tables also report the number of patients with recurrent cervical cancer only and with imaging results. Many of the studies did not report summary patient characteristics for the patients with recurrent cervical cancer and imaging results only but for the full patient group, which is not relevant here and so has not been reported. When stated, most patients had squamous cell carcinoma; fewer had adenocarcinoma. In some studies, such as that by Chung et al. ${ }^{20}$ it was stated that histologically confirmed squamous cell carcinoma, adenocarcinoma or adenosquamous carcinoma of the uterine cervix was a requirement for study eligibility, but for others it was unclear.

All included studies except those by Mittra et al. ${ }^{51}$ and Hatano et al. ${ }^{53}$ described only women who had undergone treatment for histopathologically proven cervical cancer and who had suspected recurrence based on the presence of clinical signs and/or symptoms. The Mittra et al. study ${ }^{51}$ included both symptomatic and asymptomatic patients undergoing routine follow-up. The Hatano et al..$^{53}$ study verified whether MRI could provide accurate information to evaluate residual tumours after radiotherapy (persistent disease) and the MRI findings were compared with cytology/histopathology before and after radiotherapy.

Six studies ${ }^{20,49,50,52,56,58}$ described grounds on which the recurrence was suspected. Abnormal imaging and physical examination during follow-up were the main indications for performing PET-CT in the Chung et al. ${ }^{20}$ study. Each patient in the Grisaru et al..$^{49}$ study had undergone a comprehensive evaluation of her clinical status and was scheduled for routine staging or follow-up imaging studies for suspected recurrence (but results were given only for suspected recurrence). Recurrence in Kitajima et al. ${ }^{50}$ was suspected on the basis of physical examination, elevated levels of tumour markers and abnormal findings of conventional 
TABLE 3 Studies included in the diagnostic review

\begin{tabular}{|c|c|c|c|c|}
\hline Study & Diagnostic test(s) & Reference standard & $\begin{array}{l}\text { Suspected } \\
\text { recurrence/ } \\
\text { asymptomatic }\end{array}$ & $\begin{array}{l}\text { Number } \\
\text { evaluable } \\
\text { in study }\end{array}$ \\
\hline Amit $2006^{48}$ & $\mathrm{CT}$ then whole-body PET-CT & Histopathology & Suspected & $11^{\mathrm{a}}$ \\
\hline Chung $2007^{20}$ & $\begin{array}{l}\text { Imaging then whole-body } \\
\text { PET-CT }\end{array}$ & $\begin{array}{l}\text { Histopathology, radiology } \\
\text { and/or clinical follow-up for } \\
6 \text { months }\end{array}$ & $\begin{array}{l}\text { Suspected (but } \\
\text { possibly one or more } \\
\text { asymptomatic) }\end{array}$ & 52 \\
\hline \multirow[t]{2}{*}{ Grisaru $2004^{49}$} & $\begin{array}{l}\text { 1. CT and/or MRI plus PET- } \\
\text { CT (skull to mid-thigh) }\end{array}$ & $\begin{array}{l}\text { Histopathology, radiology and/ } \\
\text { or clinical follow-up }\end{array}$ & Suspected & 12 \\
\hline & 2. CT and/or MRI alone & & & \\
\hline Kitajima $2008^{50}$ & $\begin{array}{l}\text { Imaging then whole-body } \\
\text { PET-CT }\end{array}$ & $\begin{array}{l}\text { Histopathology, clinical follow- } \\
\text { up for }>1 \text { year, tumour marker } \\
\text { levels alone or with CT or } \\
\text { PET-CT }\end{array}$ & Suspected & 52 \\
\hline Mittra $2009^{51}$ & $\begin{array}{l}\text { Imaging then whole-body } \\
\text { PET-CT }\end{array}$ & $\begin{array}{l}\text { Histopathology or clinical } \\
\text { follow-up }\end{array}$ & $\begin{array}{l}\text { Suspected and } \\
\text { symptomatic } \\
\text { (disaggregation not } \\
\text { possible) }\end{array}$ & 30 \\
\hline Sironi $2007^{52}$ & $\begin{array}{l}\text { Imaging then whole-body } \\
\text { PET-CT }\end{array}$ & $\begin{array}{l}\text { Histopathology, clinical } \\
\text { follow-up with radiology for } \\
\text { >6 months }\end{array}$ & Suspected & 12 \\
\hline Hatano 199953 & MRI (pelvic) & Histopathology & Unclear & $35^{b}$ \\
\hline Weber $1995^{54}$ & MRI (pelvic) & $\begin{array}{l}\text { Histopathology, clinical follow- } \\
\text { up for up to } 4 \text { years }\end{array}$ & Suspected & $37^{b}$ \\
\hline Heron $1988^{55}$ & CT (abdomen) & $\begin{array}{l}\text { Histopathology, clinical follow- } \\
\text { up }\end{array}$ & Suspected & $70^{b}$ \\
\hline Park $2000^{56}$ & $\begin{array}{l}\mathrm{CT} \text { (chest, abdomen and } \\
\text { pelvis) }\end{array}$ & $\begin{array}{l}\text { Histopathology, tumour } \\
\text { marker, CT }\end{array}$ & Suspected & 36 \\
\hline Walsh $1981^{57}$ & CT (abdomen and pelvis) & Histopathology & Probably suspected & $33^{b}$ \\
\hline Williams $1989^{58}$ & CT, MRI (both pelvic) & Histopathology & Suspected & $20^{b}$ \\
\hline
\end{tabular}

imaging, including $\mathrm{CT}$ and/or MRI, or an abnormal cervical smear. In Sironi et al., ${ }^{52}$ suspicion of tumour recurrence was based on follow-up procedures (physical examination, serum tumour markers and morphological imaging studies, such as CT or MRI). In Park et al. ${ }^{56}$ recurrence was suspected also on the basis of increased levels of serum squamous cell carcinoma antigen and carcinoembryonic antigen, pain in the lower abdomen and back, oedema of the lower leg and oliguria. The suspicion of recurrence in Williams et al. ${ }^{58}$ was based on the clinical features of pelvic pain, vaginal discharge, vaginal bleeding, lower limb swelling or a palpable mass on pelvic examination.

\section{Imaging characteristics}

All six PET-CT studies ${ }^{20,48-52}$ were evaluations of PET-CT after patients had received conventional imaging (MRI and/or CT) or CT only. Of the PET-CT studies, only Amit et al. ${ }^{48}$ focused on extracervical lesions, whereas the other five studies evaluated any recurrence. Only Park et al. ${ }^{56}$ used CT to evaluate any recurrence and the other five MRI and CT studies evaluated local recurrence in the pelvis only. 
TABLE 4 Definitions of reference standards presented in included studies

\begin{tabular}{|c|c|c|c|}
\hline \multirow[b]{2}{*}{ Study } & \multirow[b]{2}{*}{ Histopathological findings } & \multicolumn{2}{|l|}{ Follow-up } \\
\hline & & Clinical & Radiological \\
\hline \multicolumn{4}{|l|}{ PET-CT } \\
\hline $\begin{array}{l}\text { Amit } \\
2006^{48}\end{array}$ & $\begin{array}{l}\text { Histopathological examination during } \\
\text { biopsy, random sampling of nodes }\end{array}$ & - & - \\
\hline $\begin{array}{l}\text { Chung } \\
2007^{20}\end{array}$ & $\begin{array}{l}\text { Histological tissue sampling during surgery } \\
\text { or biopsy }\end{array}$ & $\begin{array}{l}\text { Physical and gynaecological examination } \\
\text { over at least } 6 \text { months }\end{array}$ & $\begin{array}{l}\text { Serial imaging } \\
\text { studies over at } \\
\text { least } 6 \text { months }\end{array}$ \\
\hline $\begin{array}{l}\text { Grisaru } \\
2004^{49}\end{array}$ & $\begin{array}{l}\text { Histology during surgical exploration or } \\
\text { guided biopsies }\end{array}$ & $\begin{array}{l}\text { Clinical outcomes (all negative tissue } \\
\text { diagnoses were followed up to confirm } \\
\text { negative histology) }\end{array}$ & Radiological \\
\hline $\begin{array}{l}\text { Kitajima } \\
2008^{50}\end{array}$ & Histopathological examination $(n=21)$ & \multicolumn{2}{|c|}{$\begin{array}{l}\text { Clinical follow-up for periods }>1 \text { year on the basis of tumour } \\
\text { marker levels and contrast-enhanced CT findings }(n=14) \text {, } \\
\text { tumour marker levels and PET-CT findings }(n=12) \text { and } \\
\text { tumour marker levels }(n=5)\end{array}$} \\
\hline $\begin{array}{l}\text { Mittra } \\
2009^{51}\end{array}$ & Histological evaluation $(n=23)$ & Clinical follow-up $(n=7)$ & - \\
\hline $\begin{array}{l}\text { Sironi } \\
2007^{52}\end{array}$ & $\begin{array}{l}\text { Histopathological findings during surgery or } \\
\text { imaging-guided FNA biopsy in patients who } \\
\text { were positive on PET-CT }\end{array}$ & \multicolumn{2}{|c|}{$\begin{array}{l}\text { If negative on PET-CT: clinical outcomes with } \mathrm{CT} \text { or MR } \\
\text { imaging over at least } 6 \text { months }\end{array}$} \\
\hline \multicolumn{4}{|l|}{$M R I$} \\
\hline $\begin{array}{l}\text { Hatano } \\
1999^{53}\end{array}$ & $\begin{array}{l}\text { Histopathological findings during multiple } \\
\text { punch biopsies and cytology of tumour site } \\
\text { only }\end{array}$ & - & - \\
\hline $\begin{array}{l}\text { Weber } \\
1995^{54}\end{array}$ & $\begin{array}{l}\text { Histopathology and/or surgical outcomes } \\
(n=34)\end{array}$ & $\begin{array}{l}\text { Clinical follow-up for at least } 4 \text { years } \\
(n=3)\end{array}$ & - \\
\hline \multicolumn{4}{|l|}{$C T$} \\
\hline $\begin{array}{l}\text { Heron } \\
1988^{55}\end{array}$ & $\begin{array}{l}\text { Histological evaluation: at EUA }(n=4) \text {, by } \\
\text { laparotomy }(n=7) \text { and by CT-guided biopsy } \\
(n=3)\end{array}$ & $\begin{array}{l}\text { Unequivocal progressive clinical course } \\
(n=25) \text {, including post-mortem proof } \\
(n=2) \text { and supportive evidence of } \\
\text { deterioration on follow-up ( } n=17) \text {. For } \\
31 \text { patients with negative test, patients } \\
\text { considered to be free of recurrence only } \\
\text { if clinical condition remained stable for } \\
>2 \text { years and/or histology }\end{array}$ & - \\
\hline $\begin{array}{l}\text { Park } \\
2000^{56}\end{array}$ & $\begin{array}{l}\text { Percutaneous lymph node biopsy }(n=10) \text {, } \\
\text { biopsy of the pelvic mass }(n=3)\end{array}$ & $\begin{array}{l}\text { Tumour marker study and CT at 3- and 6-r } \\
(n=23)\end{array}$ & onth intervals \\
\hline $\begin{array}{l}\text { Walsh } \\
1981^{57}\end{array}$ & $\begin{array}{l}\text { Histological evaluation }(n=29) \text { : by } \\
\text { laparotomy }(n=10) \text {, parametrial biopsy } \\
(n=6) \text {, cervical and vaginal biopsy }(n=6) \text {, } \\
\text { perineal biopsy }(n=2) \text {, lymph node } \\
\text { aspiration }(n=2) \text {, autopsy }(n=2) \text { and bone } \\
\text { biopsy }(n=1)\end{array}$ & - & - \\
\hline
\end{tabular}

$M R I$ and $C T$

Williams Histological biopsies $(n=10)$, hysterectomy

$1989^{58} \quad$ specimens $(n=4)$, open biopsy at

laparotomy $(n=2)$, histological proof of

distant metastatic disease $(n=4)$

EUA, examination under anaesthetic; FNA, fine-needle aspiration. 


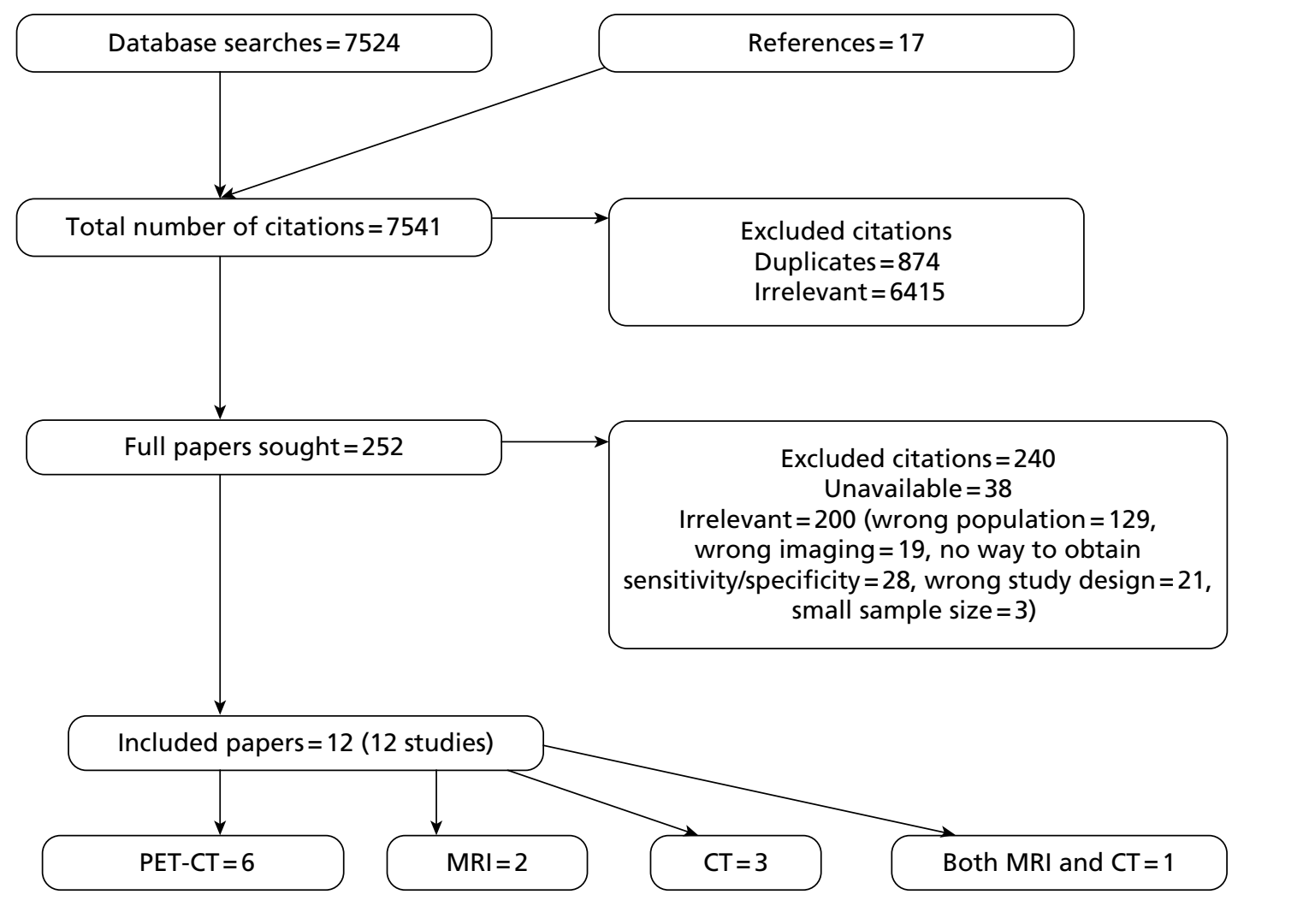

FIGURE 4 PRISMA diagram of selection process: diagnostic systematic review.

All six PET-CT studies used ${ }^{18} \mathrm{~F}-\mathrm{FDG}$ as a radioisotope tracer, with doses of $370-555 \mathrm{MBq}^{48} 555-740 \mathrm{MBq},{ }^{20}$ $370-666 \mathrm{MBq},{ }^{49} 4.0 \mathrm{MBq} / \mathrm{kg}^{50}{ }^{50} 400-555 \mathrm{MBq}^{51}$ and $370 \mathrm{MBq} \cdot{ }^{52}$ The time between injection of ${ }^{18} \mathrm{~F}-\mathrm{FDG}$ and the PET scan ranged from 30 minutes to 3 hours. The PET-CT scanning was performed mostly with a GE Discovery LS PET-CT scanner (GE Medical Systems, Milwaukee, WI, USA). In Amit et al., ${ }^{48}$ a hybrid PET-CT system combining a third-generation multislice spiral CT system [GE LightSpeed Plus (GE Medical Systems, Milwaukee, WI, USA)] with a dedicated full bismuth germanium oxide (BGO) ring PET scanner [GE Advance NXi (GE Medical Systems, Milwaukee, WI, USA)] was used. In Chung et al. ${ }^{20}$ a GEMINI PET-CT system (Philips, Guildford, UK) was used, and in Kitajima et al..$^{50}$ all imaging and data acquisitions were performed with a Biograph Sensation 16 PET-CT scanner (Siemens Systems, Erlangen, Germany). Two studies 48,50 measured glucose levels before administration of ${ }^{18} \mathrm{~F}-\mathrm{FDG}$.

In the three MRI studies 53,54,58 T1-weighted spin-echo and T2-weighted turbo spin-echo were used. Of the four CT studies, ${ }^{55-58}$ two $^{55,58}$ used optional intravenous contrast medium to elucidate problems identified on initial scans. Intravenous contrast medium was used routinely in the other two studies: non-ionic contrast $(150 \mathrm{mg})^{56}$ and Reno-M-DIP ${ }^{\circledR}$ contrast $(400 \mathrm{ml}$ of $4 \%$ oral meglumine diatrizoate) (Squibb, Princeton, NJ, USA)..$^{57}$

\section{Quality of studies}

The results of the quality assessment are provided in Table 8. Four studies ${ }^{48,49,52,53}$ collected patients' data prospectively (77 patients in total), seven studies ${ }^{20,50,51,54,56-58}$ collected data retrospectively (260 patients in total) and in one of the studies ${ }^{55}$ there was no information on the method of enrolment. Three studies ${ }^{20,51,52}$ clearly described their inclusion criteria such as presence of symptoms indicating recurrence, new lesions on surveillance imaging, elevated serum tumour markers with or without abnormal imaging and abnormal results on physical or cytological examination on routine surveillance. Relevant clinical information such as age, FIGO stage, histology type of tumour and primary treatment were described in all studies except for those by Amit et al. ${ }^{48}$ Grisaru et al. ${ }^{49}$ and Park et al. ${ }^{56}$ 


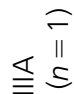

它邑

$\|$ in

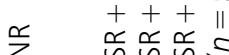

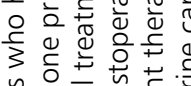

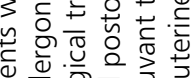

$\stackrel{\sim}{\sim} \stackrel{\dot{q}}{=} \subseteq$

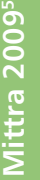

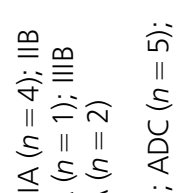

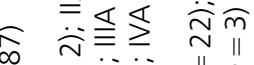

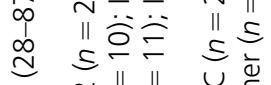

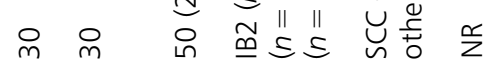

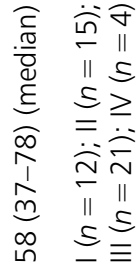

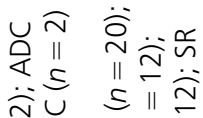

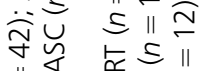

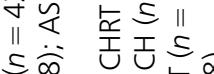

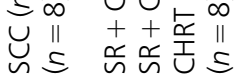

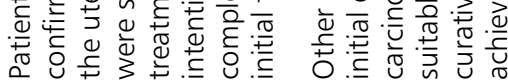

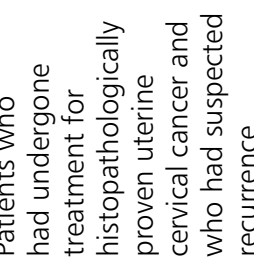

$\frac{\infty}{z}$

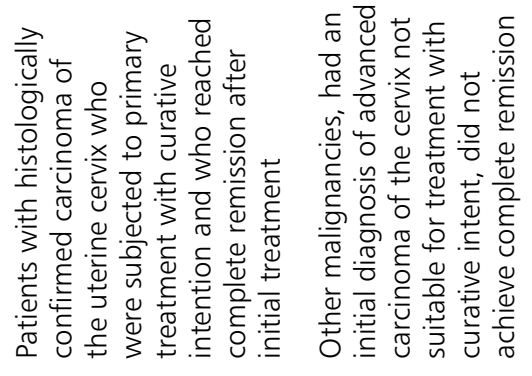

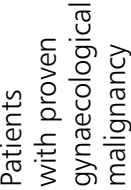

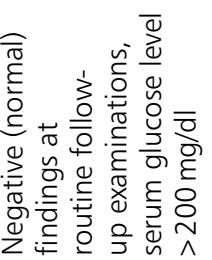

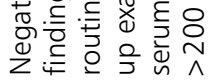

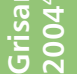

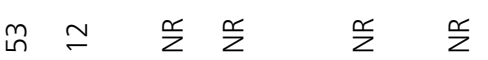

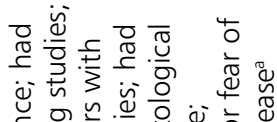

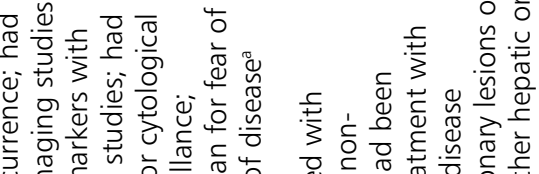

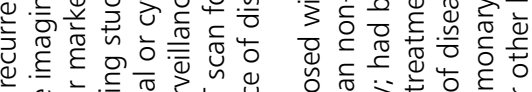

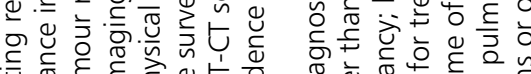

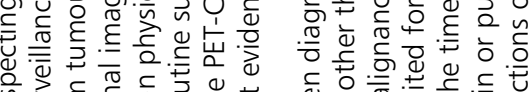

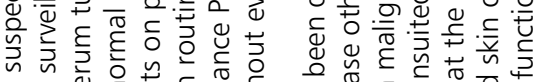

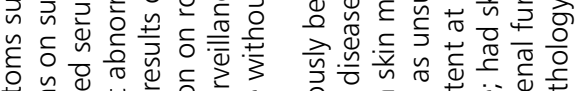

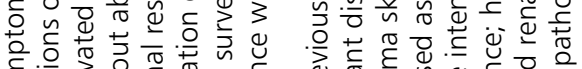

हो.

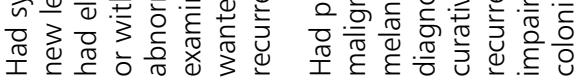

กิก กิ

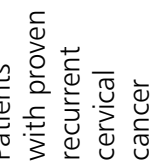

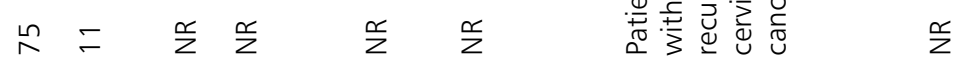

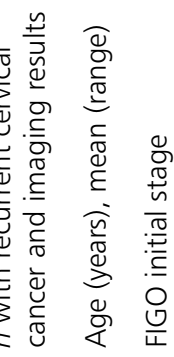

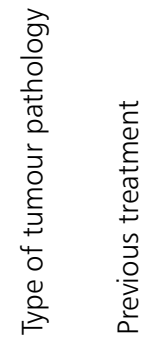

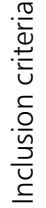

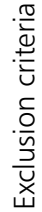

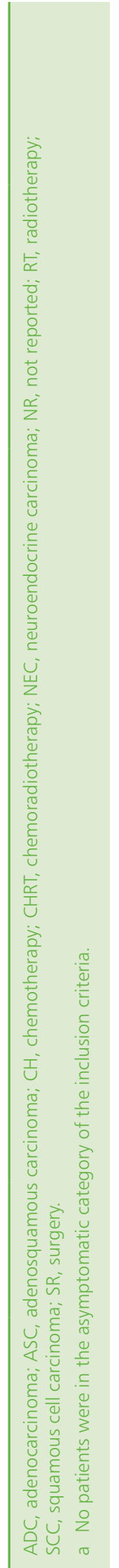


TABLE 6 Population characteristics of studies evaluating MRI and MRI+CT

\begin{tabular}{|c|c|c|c|}
\hline Characteristics & $\begin{array}{l}\text { Hatano } \\
1999^{53}\end{array}$ & Weber $1995^{54}$ & Williams $1989^{58}$ \\
\hline Imaging & MRI & MRI & $\mathrm{MRI}$ and $\mathrm{CT}$ \\
\hline Total $n$ in study & 42 & 37 & 20 \\
\hline $\begin{array}{l}n \text { with recurrent cervical } \\
\text { cancer and imaging results }\end{array}$ & 35 & 37 & 20 \\
\hline Age (years), mean (range) & 62.3 & $48(19-83)$ & NR \\
\hline FIGO initial stage & NR & $\begin{array}{l}\text { IB }(n=16) ; \| \mathrm{A}(n=2) ; \operatorname{IIB}(n=16) \\
\operatorname{III}(n=3)\end{array}$ & $\begin{array}{l}\mathrm{IB}(n=7) ;\|\mathrm{A}(n=2) ;\| \mathrm{B}(n=5) ; \operatorname{IIA}(n=3) ; \\
\mathrm{IIB}(n=3)\end{array}$ \\
\hline Type of tumour pathology & NR & $\operatorname{SCC}(n=33) ; \operatorname{ADC}(n=4)$ & $\operatorname{SCC}(n=18) ; \operatorname{AC}(n=1) ; \operatorname{ADC}(n=1)$ \\
\hline Previous treatment & NR & $\mathrm{RT}(n=37)$ & $\begin{array}{l}\text { Abdominal/Wertheim's hysterectomy } \\
(n=6) \text {; subtotal hysterectomy }(n=2) \text {; } \\
\text { anterior exenteration }(n=2) \text {; external-beam } \\
\text { irradiation }(n=10)\end{array}$ \\
\hline Inclusion criteria & $N R$ & $\begin{array}{l}\text { Patients with histopathological } \\
\text { diagnosis of cervical carcinoma, } \\
\text { who underwent primary RT and } \\
\text { then MRI after the initiation of RT }\end{array}$ & $\begin{array}{l}\text { Patients with a diagnosis of suspected } \\
\text { recurrent carcinoma of the cervix in whom } \\
\text { pathological verification of the imaging } \\
\text { results was available }\end{array}$ \\
\hline Exclusion criteria & $N R$ & NR & NR \\
\hline
\end{tabular}

TABLE 7 Population characteristics of studies evaluating CT

\begin{tabular}{|c|c|c|c|}
\hline Characteristics & Heron $1988^{55}$ & Park $2000^{56}$ & Walsh $1981^{57}$ \\
\hline Total $n$ in study & 70 & 36 & 36 \\
\hline $\begin{array}{l}n \text { with recurrent cervical } \\
\text { cancer and imaging results }\end{array}$ & 64 & 36 & 31 \\
\hline Age (years), mean (range) & $45(28-80)$ & 53 & $(23-68)$ \\
\hline FIGO initial stage & NR & NR & NR \\
\hline Type of tumour pathology & NR & NR & NR \\
\hline Previous treatment & NR & $\begin{array}{l}\mathrm{SR}(n=13) ; \mathrm{RT}(n=14) ; \\
\mathrm{SR}+\mathrm{RT}(n=9)\end{array}$ & NR \\
\hline Inclusion criteria & $\begin{array}{l}\text { Patients with suspected recurrent } \\
\text { carcinoma of the cervix }\end{array}$ & $\begin{array}{l}\text { Patients with uterine } \\
\text { cervical cancer }\end{array}$ & $\begin{array}{l}\text { Patients with previously } \\
\text { treated cervical } \\
\text { carcinoma }\end{array}$ \\
\hline Exclusion criteria & NR & NR & NR \\
\hline
\end{tabular}


TABLE 8 Quality of all diagnostic studies

\begin{tabular}{|c|c|c|c|c|c|c|c|c|c|c|c|c|c|c|}
\hline Study & Test & 1 & 2 & 3 & 4 & 5 & 6 & 7 & 8 & 9 & 10 & 11 & 12 & Comments \\
\hline Amit $2006^{48}$ & PET-CT & Y & N & Y & $U$ & Y & Y & N & $U$ & N & N & $\mathrm{N}$ & Y & Extrapelvic recurrence only \\
\hline Chung $2007^{20}$ & PET-CT & Y & Y & Y & $U$ & Y & $U$ & N & $U$ & Y & N & NA & Y & \\
\hline Grisaru $2004^{49}$ & $\begin{array}{l}\text { PET-CT } \\
\text { (CT and/ } \\
\text { or MRI) }\end{array}$ & $U$ & N & Y & $U$ & Y & Y & N & Y & N & N & NA & Y & \\
\hline Kitajima $2008^{50}$ & PET-CT & Y & Y & Y & $U$ & Y & Y & N & Y & Y & N & NA & Y & \\
\hline Mittra $2009^{51}$ & PET-CT & Y & Y & Y & $U$ & Y & $U$ & N & $U$ & Y & N & NA & Y & \\
\hline Sironi $2007^{52}$ & PET-CT & Y & Y & Y & Y & Y & Y & N & Y & Y & N & NA & Y & \\
\hline Hatano $1999^{53}$ & MRI & Y & $U$ & Y & $u$ & Y & Y & N & $U$ & Y & N & $\mathrm{N}$ & $\mathrm{N}$ & Tumour site only \\
\hline Weber $1995^{54}$ & MRI & U & $U$ & Y & $u$ & Y & Y & N & U & N & N & NA & N & Pelvic recurrence only \\
\hline Heron $1988^{55}$ & $\mathrm{CT}$ & Y & $U$ & N & U & $\mathrm{N}$ & Y & N & U & Y & N & NA & $\mathrm{N}$ & Local recurrence only \\
\hline Park $2000^{56}$ & CT & U & U & N & $u$ & Y & N & N & U & U & N & NA & N & \\
\hline Walsh $1981^{57}$ & $\mathrm{CT}$ & Y & Y & Y & Y & Y & Y & N & $U$ & N & Y & Y & $\mathrm{N}$ & Pelvic recurrence only \\
\hline Williams $1989^{58}$ & $\mathrm{MRI} / \mathrm{CT}$ & Y & U & Y & $u$ & Y & Y & N & Y & N & N & NA & N & $\begin{array}{l}\text { Local (central) recurrence } \\
\text { only }\end{array}$ \\
\hline
\end{tabular}

N, no; NA, not applicable; $U$, unclear; $Y$, yes.

1 - representative spectrum; 2 - selection criteria clearly described; 3 - acceptable reference standard; 4 - acceptable delay between imaging tests; 5 - partial verification avoided; 6 - reference standard independent of the index test; 7 - tests described in sufficient detail for replication; 8 - reference standard/index test blinded; 9 - relevant clinical information; 10 - uninterpretable results reported; 11 - withdrawals explained; 12 - technical quality.

In all of the included studies the reference standard for diagnosis of cervical cancer was histopathology with or without clinical/radiological follow-up. Four of the studies ${ }^{48,53,57,58}$ used only histopathology as the reference standard, whereas in the other studies diagnosis was supported by clinical follow-up. Selection bias (using the imaging study being investigated as part of the inclusion criteria into the study) was present in at least four studies. ${ }^{20,50-52}$

Information to judge the presence of incorporation bias (in which the index test forms part of the reference standard) was unclear in almost all of the studies, but in Kitajima et al..$^{50}$ the index test (PET-CT) was clearly part of the reference standard when the final diagnosis of 12 patients was based on the results of tumour marker level and PET-CT findings. Two studies reported the mean time between index test and reference standard, which was 2.3 weeks ${ }^{52}$ and 1 week. ${ }^{57}$ Readers of PET-CT, MRI and CT studies were reported to be blind to patients' clinical details and final diagnosis in only four studies. ${ }^{49,50,54,58}$

With regard to technical quality, the methods used in the more modern studies were similar to currently used imaging methods, whereas the methods used in the older studies were not. In the PET-CT studies there was slight variation found in whether or not and how much oral hydration was used as well as slight differences in acquisition times and injected doses. Chung et al. ${ }^{20}$ used oral contrast for $C T$, but this should not affect the PET interpretation or results. Heron et al. ${ }^{55}$ incorporated lymphangiography, which is now no longer used.

\section{Test accuracy}

The numerical results for all included studies are shown in Table 9. 
TABLE 9 Numerical results of imaging studies

\begin{tabular}{|c|c|c|c|c|c|c|}
\hline Study name, date & Diagnostic test(s) & TP & FP & FN & TN & Equivocal \\
\hline Amit $2006^{48}$ & PET-CT & 6 & 0 & 1 & 4 & - \\
\hline Chung $2007^{20}$ & PET-CT & 28 & 4 & 3 & 17 & - \\
\hline \multirow[t]{2}{*}{ Grisaru $2004^{49}$} & PET-CT & 10 & 0 & 0 & 2 & - \\
\hline & $\mathrm{CT}$ and/or MRI & 2 & 1 & 6 & 1 & $1^{\mathrm{a}}$ \\
\hline Kitajima $2008^{50}$ & PET-CT & 23 & 2 & 2 & 25 & - \\
\hline Mittra $2009^{51}$ & PET-CT & 22 & 2 & 1 & 5 & - \\
\hline Sironi $2007^{52}$ & PET-CT & 5 & 0 & 1 & 6 & - \\
\hline Hatano $1999^{53}$ & MRI & 1 & 0 & 0 & 34 & - \\
\hline Weber $1995^{54}$ & MRI & 18 & 1 & 3 & 15 & - \\
\hline Heron $1988^{55}$ & $\mathrm{CT}$ & 24 & 2 & 2 & 36 & 6 \\
\hline Park $2000^{56}$ & $\mathrm{CT}$ & 14 & 3 & 4 & 15 & - \\
\hline Walsh $1981^{57}$ & $\mathrm{CT}$ & 27 & 2 & 2 & 0 & 2 \\
\hline \multirow[t]{2}{*}{ Williams $1989^{58}$} & $\mathrm{CT}$ & 10 & 2 & 1 & 7 & - \\
\hline & MRI & 9 & 2 & 2 & 7 & - \\
\hline
\end{tabular}

\section{Positron emission tomography/computerised tomography}

Six PET-CT test accuracy studies were found..$^{20,48-52}$ Five studies ${ }^{20,49-52}$ evaluated local recurrence and distance metastasis and one study ${ }^{48}$ evaluated extrapelvic recurrence only. The sensitivities and specificities and their 95\% Cls are shown in Figure 5 and a SROC space plot is shown in Figure 6. The sensitivities and specificities of local and distant recurrence were $83-100 \%$ and $71-100 \%$, respectively, and the sensitivity and specificity of distant recurrence only were $86 \%$ and $100 \%$. The summary estimates of the sensitivity and specificity of PET-CT for the detection of cervical cancer recurrence were $92.2 \%(95 \% \mathrm{Cl} 85.1 \%$ to $96.0 \%$ ) and $88.1 \%$ (95\% Cl $77.9 \%$ to $93.9 \%$ ), respectively. Sensitivity analysis, omitting one study ${ }^{48}$ that reported accuracy for distant recurrence only, did not affect accuracy estimates to any significant degree [sensitivity $92.6 \%$ (95\% Cl 85.3\% to $96.4 \%$ ); specificity $87.3 \%$ (95\% Cl $76.6 \%$ to $93.5 \%)$ ]. The results tables of the univariate random-effects regression model for the meta-analysis and sensitivity analysis are in Appendix 11.

\section{Magnetic resonance imaging}

Three MRI test accuracy studies were found and all evaluated the pelvis only. ${ }^{53,54,58}$ Weber et al. ${ }^{54}$ and Williams et al. ${ }^{58}$ included women with clinical suspicion of recurrence and Hatano et al..$^{53}$ included women with residual, advanced-stage cervical cancer (stage IB2-IV). Previous treatment was radiotherapy in Hatano et al. ${ }^{53}$ and Weber et al. ${ }^{54}$ and $50 \%$ surgery and $50 \%$ radiotherapy in Williams et al. ${ }^{58}$ All three studies investigated local recurrence in the pelvis only. Distant recurrence was noted in Williams et al..$^{58}$ (4/20), but these women were not included in the numerical results for sensitivity and specificity. Distant metastases are also mentioned in Hatano et al. ${ }^{53}$ Because of clinical heterogeneity between these studies, no meta-analysis was conducted. The sensitivities and specificities and their 95\% Cls are shown in Figure 7 and a SROC space plot in Figure 8. The sensitivities and specificities of MRI in pelvic recurrence varied between $82 \%$ and $100 \%$ and $78 \%$ and $100 \%$ respectively. 


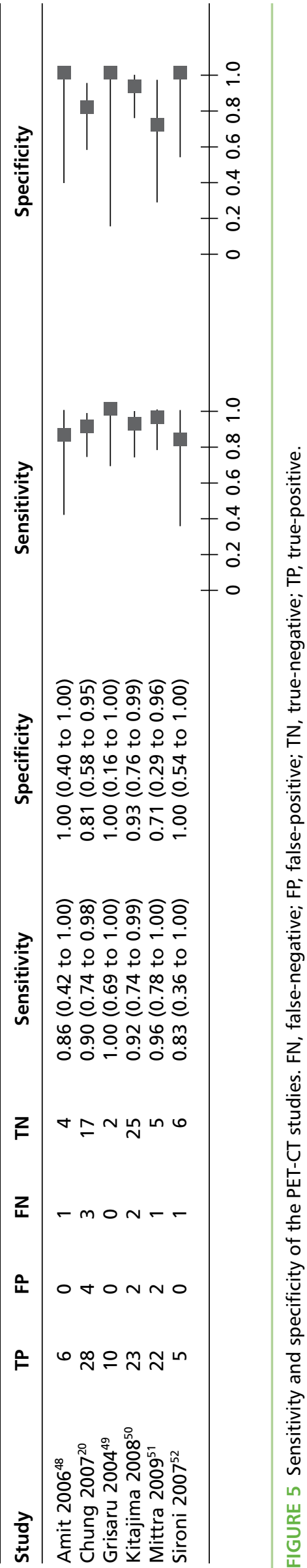




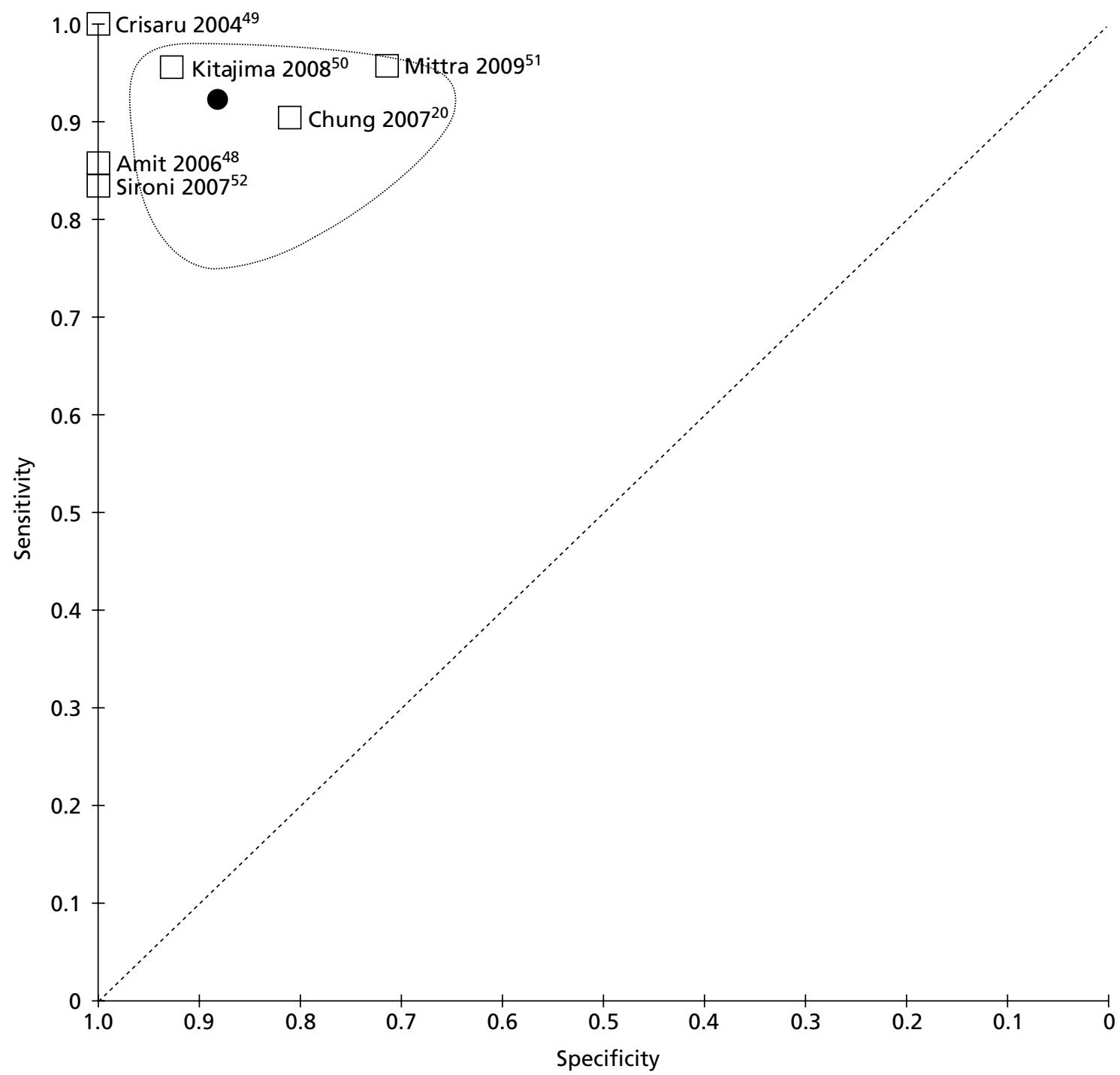

FIGURE 6 Summary receiver operating characteristic space plot for the PET-CT studies.

\section{Computerised tomography}

Four CT test accuracy studies were found. ${ }^{55-58}$ Heron et al., ${ }^{55}$ Walsh et al. ${ }^{57}$ and Williams et al. ${ }^{58}$ investigated local recurrence only, whereas Park et al. ${ }^{56}$ investigated local and distant recurrence. [As mentioned in the MRI section, Williams et al..$^{58}$ also mentioned 4 (of 20) women with distant recurrence, who were not included in the sensitivity and specificity statistics.] There is little information available on the patients included in Heron et al. ${ }^{55}$ and Walsh et al. ${ }^{57}$ Also, both Heron et al. ${ }^{55}$ and Walsh et al. ${ }^{57}$ have equivocal results. For six patients in the Heron et al. ${ }^{55}$ study, the CT findings were classified as equivocal; all of these patients had undergone radiotherapy, making differentiation between radiation fibrosis and recurrence difficult. For two patients in Walsh et al., ${ }^{57} \mathrm{CT}$ images could not differentiate radiation sequelae from tumour. The sensitivities and specificities and their 95\% Cls are shown in Figure 9 and a SROC space plot is shown in Figure 10. Because of clinical heterogeneity and lack of information about patients, no meta-analysis was conducted. The sensitivities and specificities of $C T$ in pelvic recurrence (excluding the equivocal results) varied between $78 \%$ and $93 \%$ and $0 \%$ and $95 \%$ respectively. Sensitivity analysis around the equivocal results for Heron et al. .55 varied the sensitivity from $75 \%$ to $94 \%$ and the specificity from $82 \%$ to $95 \%$. Sensitivity analysis around the equivocal results for Walsh et al. ${ }^{57}$ varied the sensitivity from $87 \%$ to $94 \%$ and the specificity from $0 \%$ to $50 \%$. 


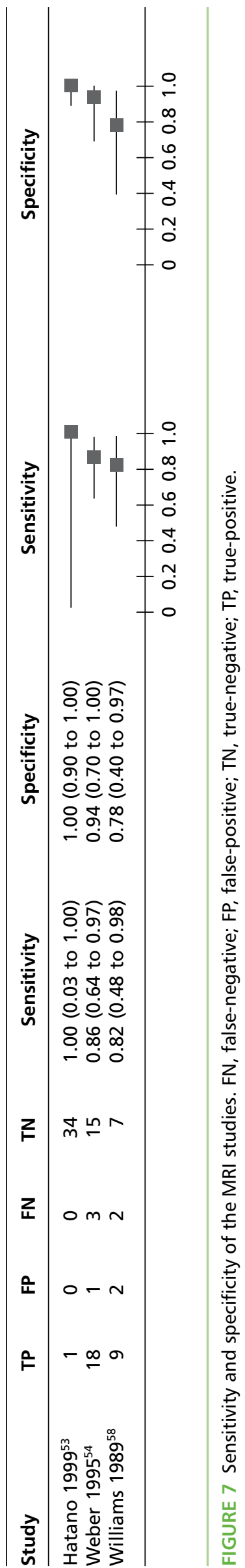




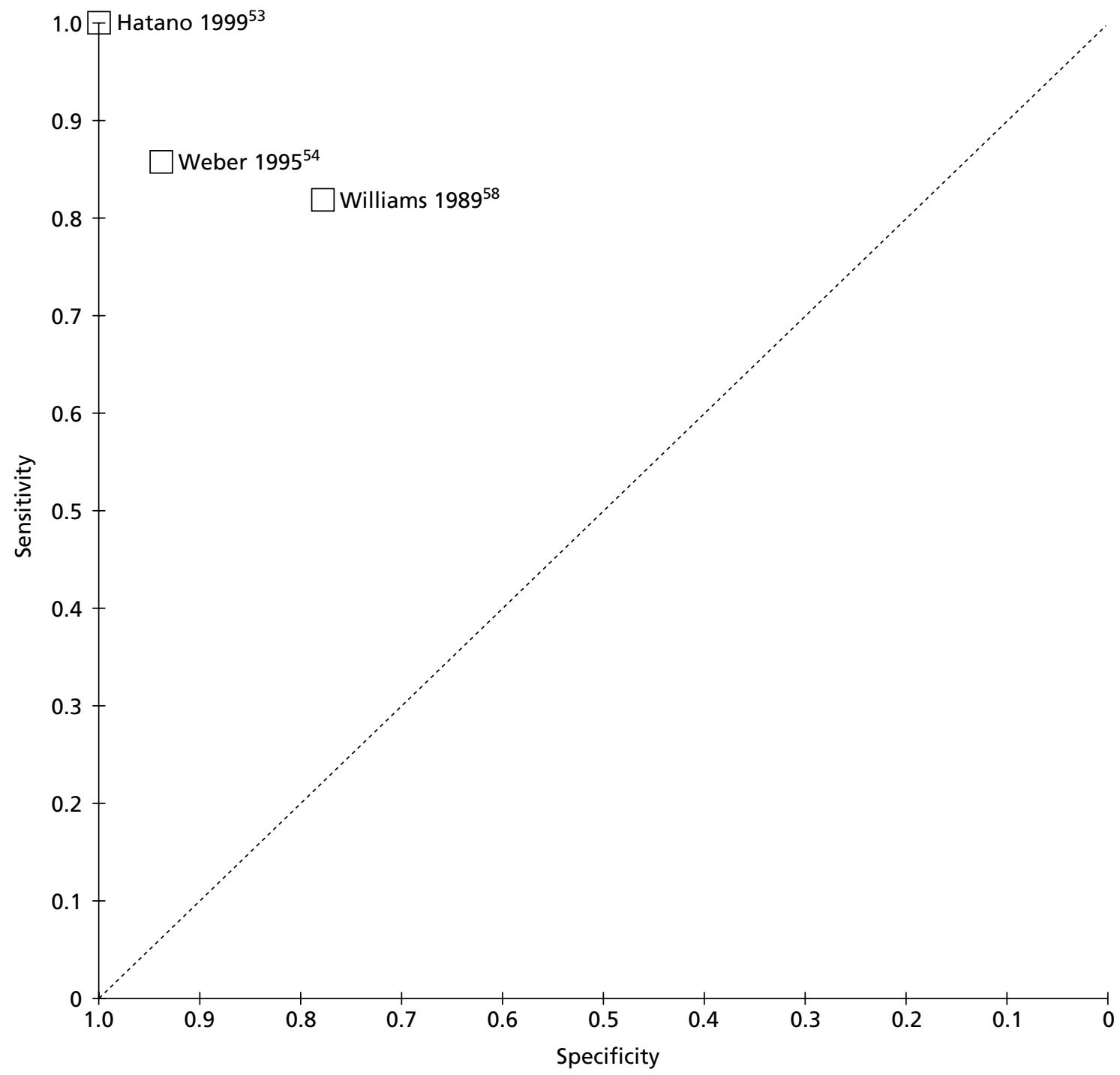

FIGURE 8 Summary receiver operating characteristic space plot for the MRI studies.

\section{Comparison of standard imaging followed by positron emission} tomography/computerised tomography with standard imaging only One study ${ }^{49}$ gave results in one table for both standard imaging alone and standard imaging with whole-body PET-CT with the same reference standard of histology or clinical evidence of disease, allowing comparisons to be made. Unfortunately, the part of the body imaged with standard imaging was not provided in the paper. The results are provided in Table 10. This shows that the PET-CT results are closer to the reference standard results than the standard imaging results.

\section{Diagnostic and therapeutic impact}

One included PET-CT study ${ }^{20}$ reported information on the diagnostic and therapeutic impact of the imaging. None of the included MRI or CT studies provided any details on whether or how the management of patients was altered by imaging.

In Chung et al., ${ }^{20}$ the mean age of patients was 53 years (range 32-77 years) and they had primarily stage I (50\%) and stage II (40\%) cancer. The results of PET-CT imaging were found to have an impact on the management of 12 patients (23\%) by initiating previously unplanned treatment (four patients), changing the previously planned therapeutic approach (five patients) or eliminating a previously planned 


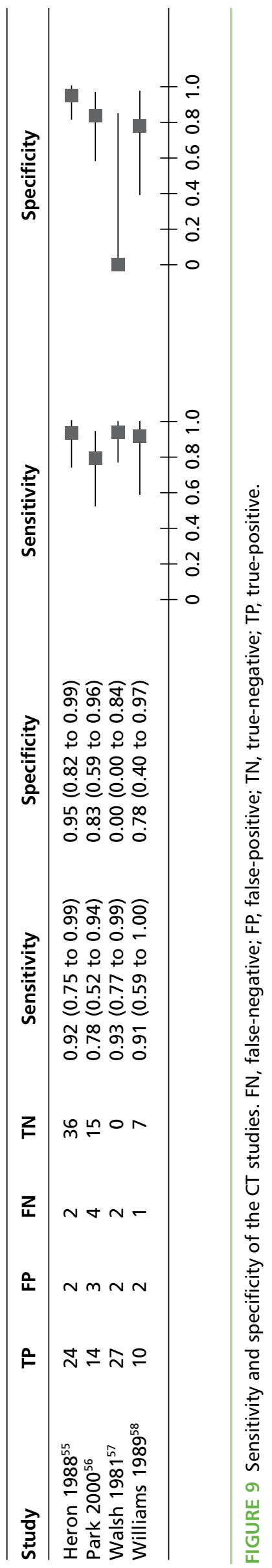




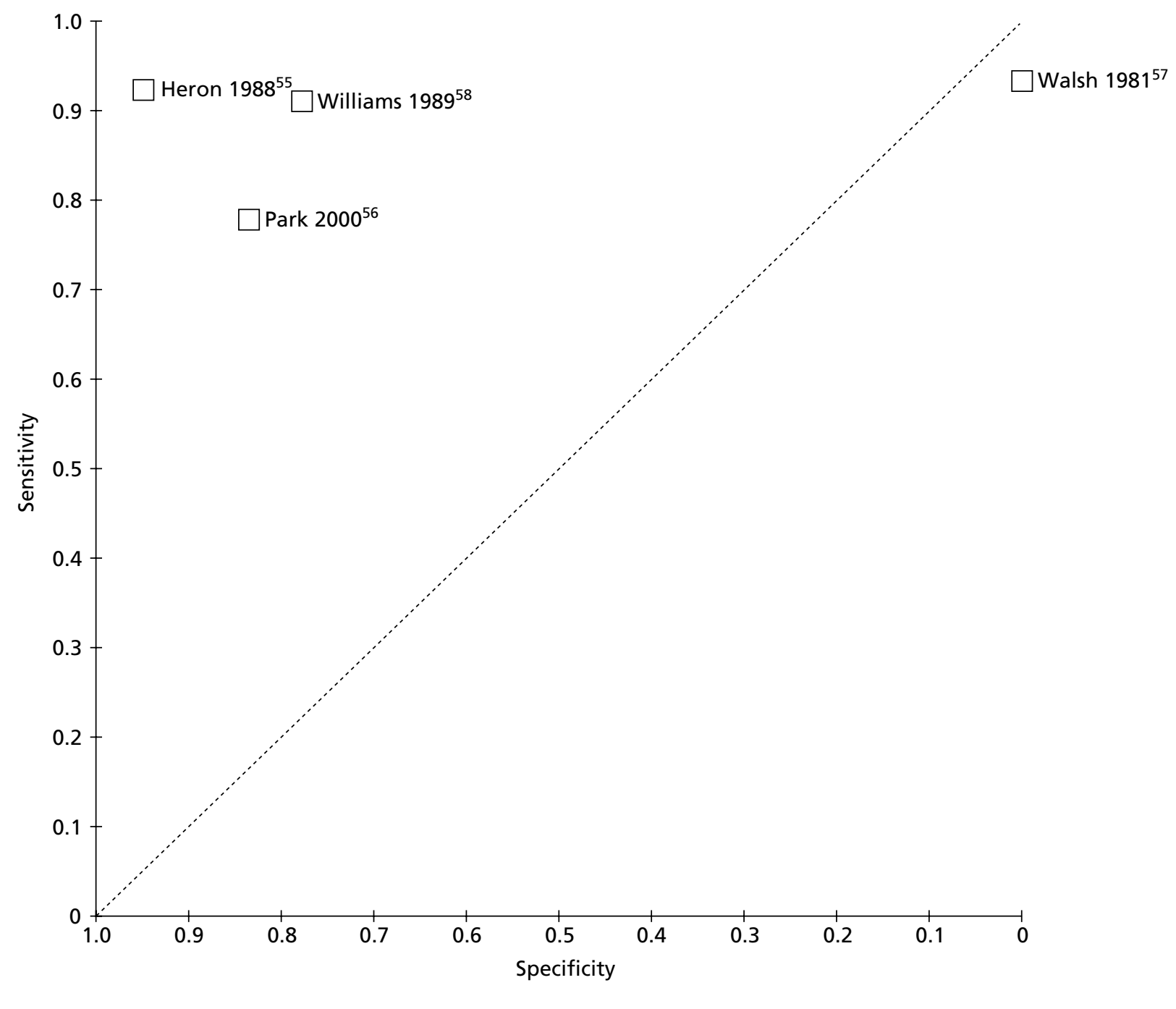

FIGURE 10 Summary receiver operating characteristic space plot for the CT studies.

diagnostic procedure (three patients). The PET-CT led to additional invasive diagnostic procedures in nine patients: mediastinoscopic biopsy in three patients, PET-CT-guided pelvic lymph node biopsy in three patients, supraclavicular lymph node biopsy in two patients and bone biopsy in one patient. The PET-CT assisted in the planning of the therapeutic strategy in nine patients.

Chung et al..$^{20}$ also reported the prognostic outcomes of patients undergoing PET-CT giving 2-year diseasefree survival rates and survival curves for women with positive and negative PET-CT results. The 2-year disease-free survival rates for women with a positive and a negative PET-CT result for recurrence were $10.9 \%$ and $85.0 \%$ respectively $(p=0002)$. The survival curves are reproduced in Figure 11 . 
TABLE 10 Comparison of standard imaging with PET-CT

\begin{tabular}{|c|c|c|c|}
\hline Patient & Standard imaging & $\begin{array}{l}\text { Standard imaging } \\
\text { followed by PET-CT }\end{array}$ & $\begin{array}{l}\text { Histology/clinical } \\
\text { follow-up }\end{array}$ \\
\hline 1 & - & + & + \\
\hline 2 & - & + & + \\
\hline 3 & + & - & - \\
\hline 4 & - & + & + \\
\hline 5 & $+/-$ & + & + \\
\hline 6 & - & - & - \\
\hline 7 & - & + & + \\
\hline 8 & + & + & + \\
\hline 9 & Not possible & + & + \\
\hline 10 & - & + & + \\
\hline 11 & - & + & + \\
\hline 12 & + & + & + \\
\hline
\end{tabular}

, presence of tumour; -, absence of tumour.

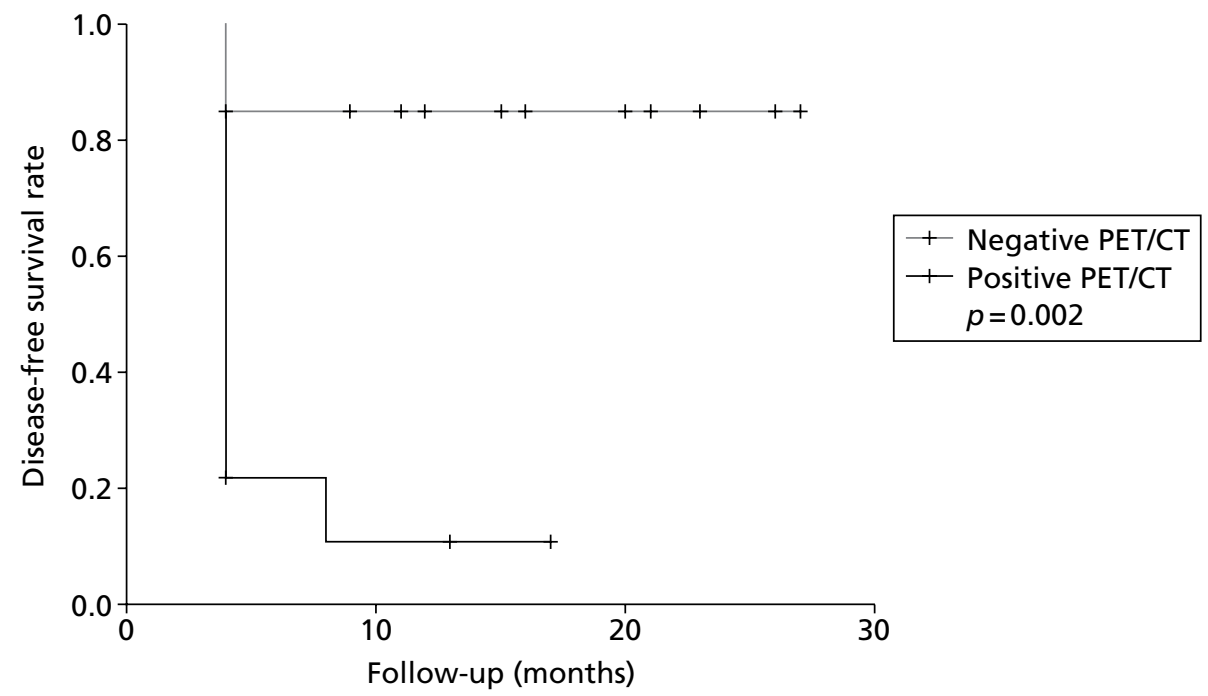

FIGURE 11 Two-year disease-free survival of patients with positive and negative PET-CT scans (from Chung et al. ${ }^{20}$ ). 



\title{
Chapter 5 Results of the elicitation of subjective probabilities
}

\begin{abstract}
The first face-to-face elicitation exercise resulted in responses from nine experts and subsequent sampling resulted in a further 12 completed elicited probabilities questionnaires. Prevalence of recurrence information was elicited from all respondents (21) and accuracy from 17-18 respondents. The selfreported characteristics of respondents and their reported use of imaging technologies are outlined in Table 11 and Figure 12.
\end{abstract}

\section{Prevalence of recurrence}

Individual respondents' prevalence of recurrence results are in Table 82 (symptomatic) and Table 83 (asymptomatic) in Appendix 12. The mean elicited prevalence of recurrence in women presenting with symptoms a minimum of 3 months after completion of primary treatment was $47.8 \%$ (SD 20.8) and that for asymptomatic women was $16.7 \%$ (SD 13.1).

\section{Accuracy}

Individual respondents' accuracy results (PPVs, NPVs) for MRI and/or CT and for MRI and/or CT with PET-CT for symptomatic and for asymptomatic women are given in Tables 85-92 in Appendix 12. Note that PPVs are the proportion of women who test positive on either $\mathrm{CT}$ or MRI at the discretion of a clinician (and PET-CT if used and performed regardless of the result of initial imaging) who are confirmed as having recurrence of cervical cancer on the basis of histology, and NPVs are the proportion of women who test negative on either CT or MRI at the discretion of a clinician (and PET-CT if used and performed regardless of the result of initial imaging) who are confirmed as not having recurrence on the basis of clinical follow-up. Summary results are shown in Table 12. These are shown graphically in Figures 13 and 14 for symptomatic and asymptomatic women respectively.

\section{Minimum important clinical difference in accuracy between imaging with computerised tomography and/or magnetic resonance imaging and imaging with computerised tomography and/or magnetic resonance imaging plus positron emission tomography/computerised tomography} The average minimum important increase in accuracy from the addition of PET-CT to CT and/or MRI that was considered necessary to warrant introduction of PET-CT as a routine investigation in this sample of clinical experts was similar for asymptomatic and symptomatic patients: a $7.7 \%$ reduction in false-positives and a $6.4 \%$ reduction in false-negatives for symptomatic women and an $8.7 \%$ reduction in false-positives and a $6.3 \%$ reduction in false-negatives for asymptomatic women. Mean elicited estimates of the differences in test accuracy between CT and/or MRI and CT and/or MRI plus PET-CT were 2.6 and 3.6 for PPV and NPV, respectively, for symptomatic women and 4.6 and 3.4 for PPV and NPV, respectively, for asymptomatic women.

The results suggest that, in our sample of experts, the elicited increase in accuracy as a result of the addition of PET-CT to MRI and/or CT is smaller than the elicited minimum important difference in accuracy required to justify the routine addition of PET-CT for the investigation of women post completion of primary treatment for cervical cancer, that is, all of the differences in false-positives and false-negatives in Table 12 are smaller than the minimum important clinical differences listed in the paragraph above.

\section{Comparison with systematic review results}

Comparison of elicited estimates of accuracy with those reported in the literature are complicated because of the age of the CT and MRI studies, the lack of disaggregation between symptomatic and asymptomatic 
TABLE 11 Characteristics of respondents to the elicitation exercise

\begin{tabular}{|c|c|c|c|c|}
\hline \multirow{2}{*}{$\begin{array}{l}\text { Speciality } \\
\text { and years of } \\
\text { experience }{ }^{a, b}\end{array}$} & \multicolumn{4}{|c|}{ Use of imaging technologies (\% of symptomatic consultations for recurrence) } \\
\hline & MRI & CT & MRI and CT & Experience with PET-CT \\
\hline $\begin{array}{l}\text { Gynaecological } \\
\text { oncology (8 years) }\end{array}$ & 20 & 20 & 60 & No \\
\hline $\begin{array}{l}\text { Gynaecological } \\
\text { oncology (15 years) }\end{array}$ & 70 & 90 & 60 & No \\
\hline Radiology (10 years) & NA & NA & NA & NA \\
\hline Radiology (20 years & NA & NA & NA & NA \\
\hline $\begin{array}{l}\text { Obstetrics and } \\
\text { gynaecology (SPR) } \\
\text { (5 years) }\end{array}$ & 30 & 60 & 10 & 1 year - 'To decide on treatment planning: Need surgery?' \\
\hline $\begin{array}{l}\text { Gynaecological } \\
\text { oncology ( } 5 \text { years) }\end{array}$ & 10 & 80 & 10 & 4 years - 'To decide on treatment planning: Prior to exenteration' \\
\hline $\begin{array}{l}\text { Gynaecological } \\
\text { oncology ( } 21 \text { years) }\end{array}$ & NS & NS & NS & No \\
\hline Not reported (7 years) & \multicolumn{3}{|c|}{$\begin{array}{l}\text { 'depends on } \\
\text { symptoms... MRI 100\% if } \\
\text { pelvic symptoms' }\end{array}$} & $\begin{array}{l}3 \text { years - 'To exclude distant recurrence in patients with proven } \\
\text { local recurrence' }\end{array}$ \\
\hline $\begin{array}{l}\text { Gynaecological } \\
\text { oncology ( } 10 \text { years as } \\
\text { a consultant })^{c}\end{array}$ & 50 & 30 & 30 & $\begin{array}{l}5 \text { years - 'Patients undergoing primary chemoradiation to } \\
\text { determine extent of any lymphadenopathy. Patients with local } \\
\text { recurrence after surgery prior to chemoradiation to determine } \\
\text { extent of lymphadenopathy. Prior to consideration of exenteration' }\end{array}$ \\
\hline $\begin{array}{l}\text { Gynaecological } \\
\text { oncology (15 years) }\end{array}$ & 70 & 30 & 0 & $\begin{array}{l}3 \text { years - 'Isolated central pelvic recurrence to confirm no } \\
\text { metastatic disease prior to exenteration' }\end{array}$ \\
\hline $\begin{array}{l}\text { Gynaecological } \\
\text { oncology ( } 3 \text { years as a } \\
\text { consultant) }\end{array}$ & 10 & 90 & 0 & $\begin{array}{l}3 \text { years - 'To clarify nature of lesions seen on CT or MRI and to rule } \\
\text { out other sites of disease if further surgery contemplated' }\end{array}$ \\
\hline $\begin{array}{l}\text { Gynaecological } \\
\text { oncology (15 years) }\end{array}$ & 25 & 50 & 25 & $\begin{array}{l}2 \text { years - 'Suspected recurrence. Consideration for exenterative } \\
\text { surgery' }\end{array}$ \\
\hline $\begin{array}{l}\text { Gynaecological } \\
\text { oncology (10 years) }\end{array}$ & 10 & 80 & 10 & $\begin{array}{l}3 \text { years - 'If recurrence suspected on the basis of clinical } \\
\text { examination/CT/MRI' }\end{array}$ \\
\hline
\end{tabular}

patients and a paucity of estimates of the combined accuracy of CT and MRI. Table 13 illustrates that elicited estimates of the accuracy of CT/MRI and CT/MRI plus PET-CT in symptomatic women are similar to estimates in the literature. For asymptomatic women the elicited specificities of CT/MRI and CT/MRI plus PET-CT are comparable to literature-based estimates but elicited estimates of sensitivity are lower. This is most likely to be a function of the spectrum of patients in included studies; inclusion of symptomatic patients in studies in the literature would be expected to result in higher sensitivity. 
TABLE 11 Characteristics of respondents to the elicitation exercise (continued)

\begin{tabular}{|c|c|c|c|c|}
\hline \multirow{2}{*}{$\begin{array}{l}\text { Speciality } \\
\text { and years of } \\
\text { experience }^{a, b}\end{array}$} & \multicolumn{4}{|c|}{ Use of imaging technologies (\% of symptomatic consultations for recurrence) } \\
\hline & MRI & СT & MRI and CT & Experience with PET-CT \\
\hline $\begin{array}{l}\text { Gynaecological } \\
\text { oncology ( } 28 \text { years) }\end{array}$ & 100 & 0 & 0 & 'Assessment of multiple site recurrence' \\
\hline $\begin{array}{l}\text { Gynaecological } \\
\text { oncology ( } 5 \text { years) }\end{array}$ & 20 & 30 & 50 & 2 years - 'Pre-exenteration or if biopsy difficult/inconclusive' \\
\hline $\begin{array}{l}\text { Gynaecological } \\
\text { oncology ( } 3 \text { years as a } \\
\text { consultant) }\end{array}$ & 60 & 10 & 30 & $\begin{array}{l}3 \text { years - 'After initial imaging to determine suitability for radical } \\
\text { salvage treatment to help exclude occult distant mets' }\end{array}$ \\
\hline Oncology (NS) & 20 & 60 & 20 & $\begin{array}{l}3 \text { years - '? local recurrence where MRI cannot differentiate } \\
\text { between recurrence and effects of radiotherapy. Proven local } \\
\text { recurrence for staging prior to exenteration' }\end{array}$ \\
\hline $\begin{array}{l}\text { Gynaecological } \\
\text { oncology ( } 34 \text { years) }\end{array}$ & 0 & 90 & 10 & $\begin{array}{l}8 \text { years - 'Those with advanced disease or recurrent disease. Those } \\
\text { requiring surgery following radiotherapy or chemoradiation' }\end{array}$ \\
\hline $\begin{array}{l}\text { Gynaecological } \\
\text { oncology (3 years) }\end{array}$ & 20 & 20 & 60 & $\begin{array}{l}1 \text { year - 'If CT/MRI positive for central recurrence and considering } \\
\text { exenteration as a management option' }\end{array}$ \\
\hline $\begin{array}{l}\text { Gynaecological } \\
\text { oncology ( } 30 \text { years) }\end{array}$ & 30 & 50 & 20 & $\begin{array}{l}3 \text { years - 'If further treatment is being considered - especially } \\
\text { exenteration' }\end{array}$ \\
\hline $\begin{array}{l}\text { Gynaecological } \\
\text { oncology ( } 30 \text { years) }\end{array}$ & 30 & 40 & 30 & 3 years - 'Exenteration candidates. Equivocal CT/MRI' \\
\hline
\end{tabular}

NA, not applicable; NS, not stated; SPR, specialist registrar.

a Years of experience were variably reported as years practising in a discipline or years practising as a consultant. When respondents clarified this it is indicated in the table.

b All respondents were consultants in their discipline with the exception of one SPR

c When numbers from a clinician did not sum to 100 , they were adjusted to $100 \%$.

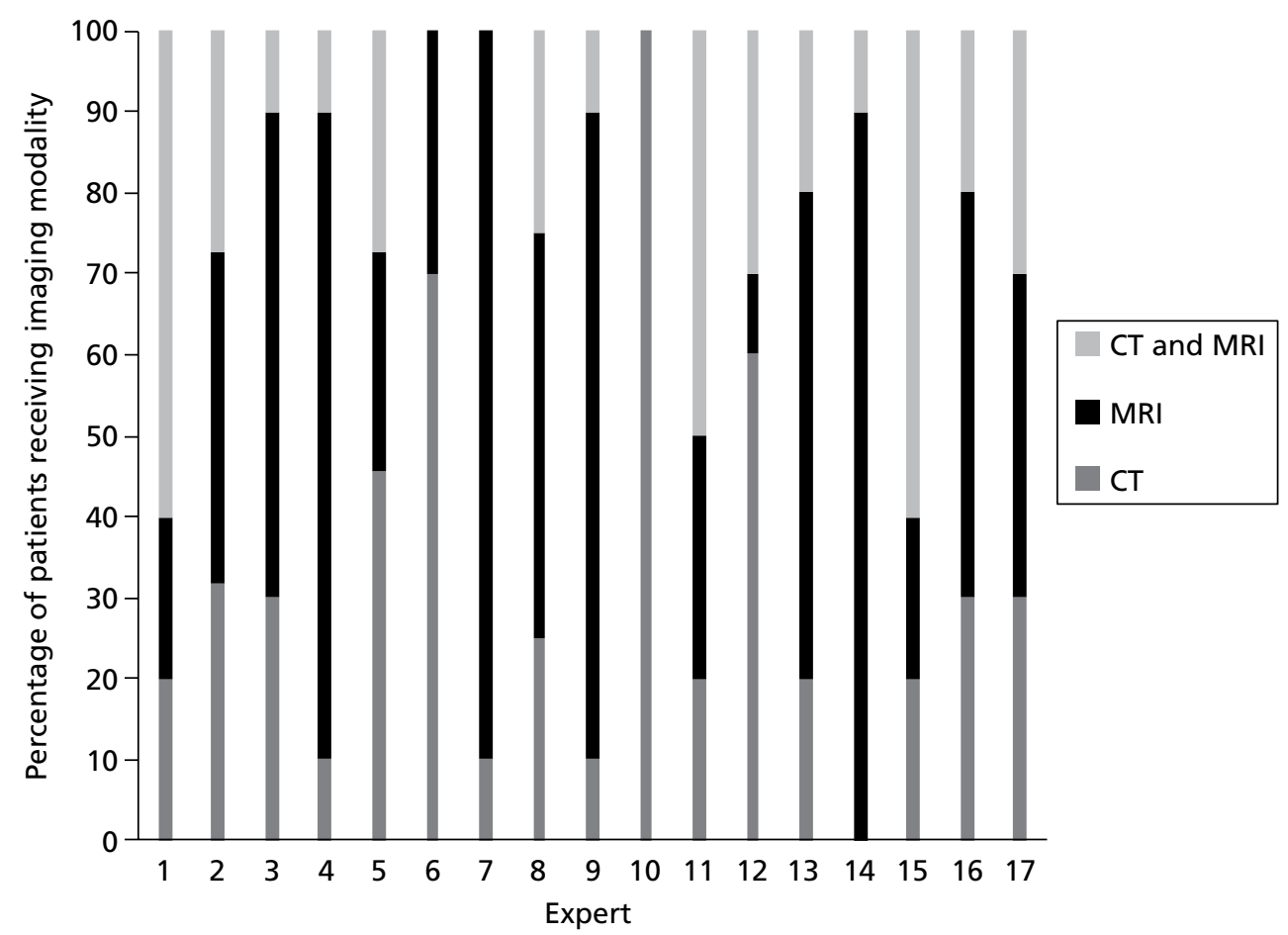

FIGURE 12 Use of imaging (MRI and/or CT) in patients presenting with suspected cervical cancer recurrence.

(c) Queen's Printer and Controller of HMSO 2013. This work was produced by Meads et al. under the terms of a commissioning contract issued by the Secretary of State for Health. This issue may be freely reproduced for the purposes of private research and study and extracts (or indeed, the full report) may be included in professional journals provided that suitable acknowledgement is made and the reproduction is not associated with any form of advertising. Applications for commercial reproduction should be addressed to: NIHR Journals Library, National Institute for Health Research, Evaluation, Trials and Studies Coordinating Centre, Alpha House, University of Southampton Science Park, Southampton SO16 7NS, UK. 
TABLE 12 Subjective elicitation summary accuracy results

\begin{tabular}{lllll} 
& & & MRI and/or CT & \\
Symptomatic & PPV (\%) & 88.4 (SD 9.2) & 91.0 (SD 8.2) & $\begin{array}{l}\text { Difference in false-positives } \\
\text { and false-negatives }\end{array}$ \\
& NPV (\%) & 86.8 (SD 8.7) & 90.7 (SD 7.2) & 2.6 \\
Asymptomatic & PPV (\%) & 85.6 (SD 9.8) & 90.2 (SD 7.7) & 3.6 \\
& NPV (\%) & 90.0 (SD 7.7) & 93.4 (SD 5.5) & 4.6 \\
\hline
\end{tabular}

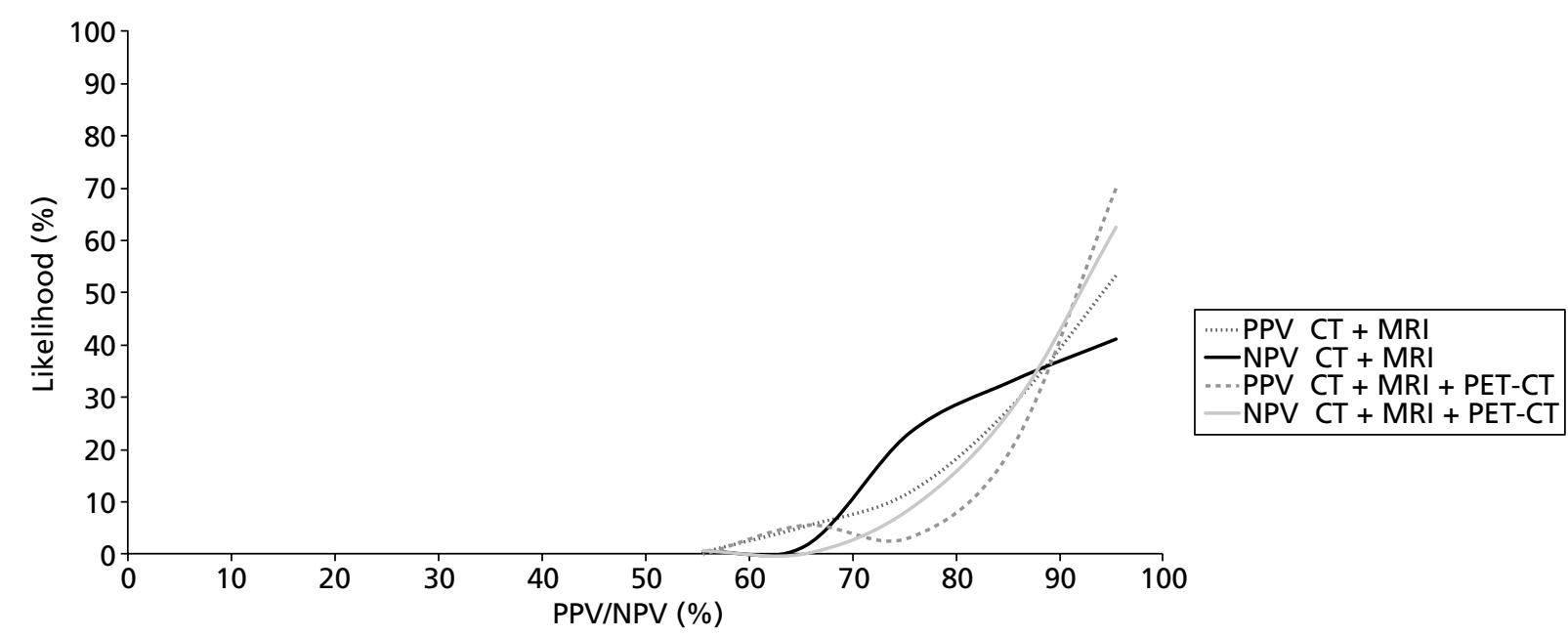

FIGURE 13 Elicited estimates of the accuracy of CT and/or MRI and CT and/or MRI with PET-CT in symptomatic women a minimum of 3 months after completion of primary treatment for cervical cancer.

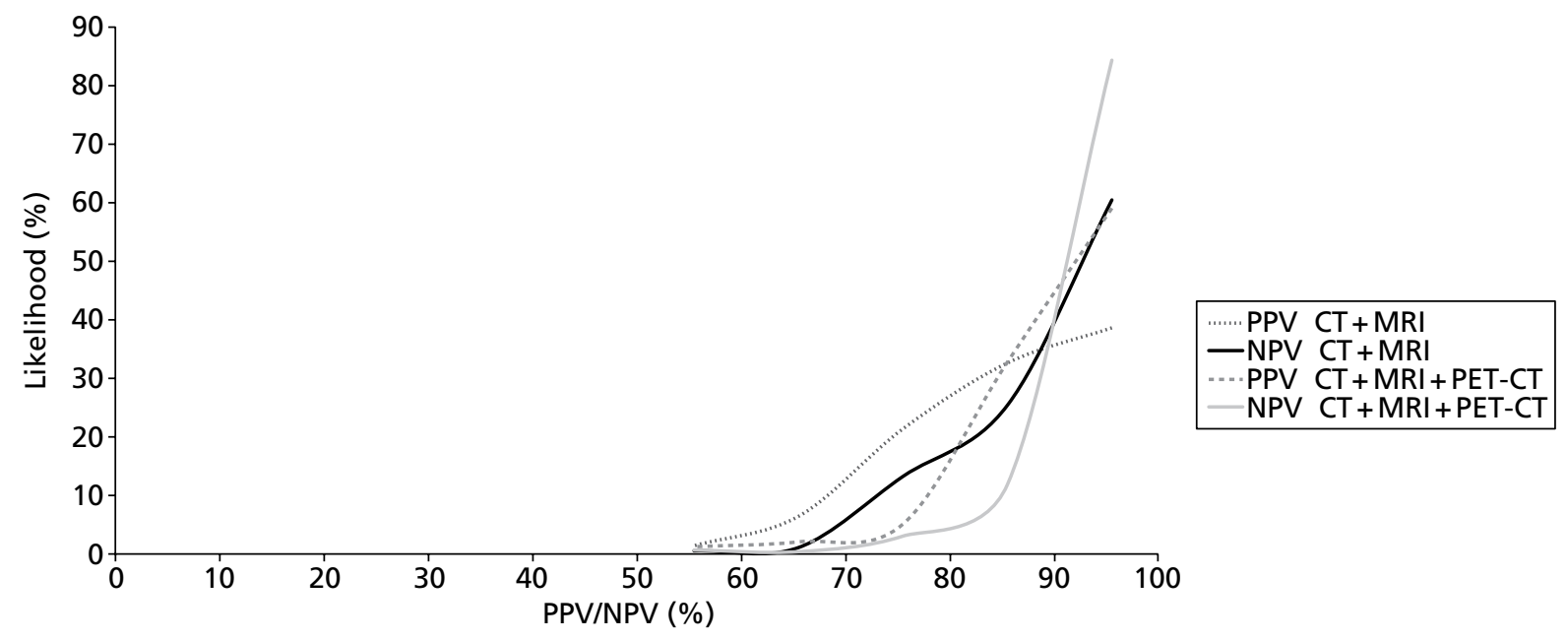

FIGURE 14 Elicited estimates of the accuracy of CT and/or MRI and CT and/or MRI with PET-CT in asymptomatic women a minimum of 3 months after completion of primary treatment for cervical cancer. 


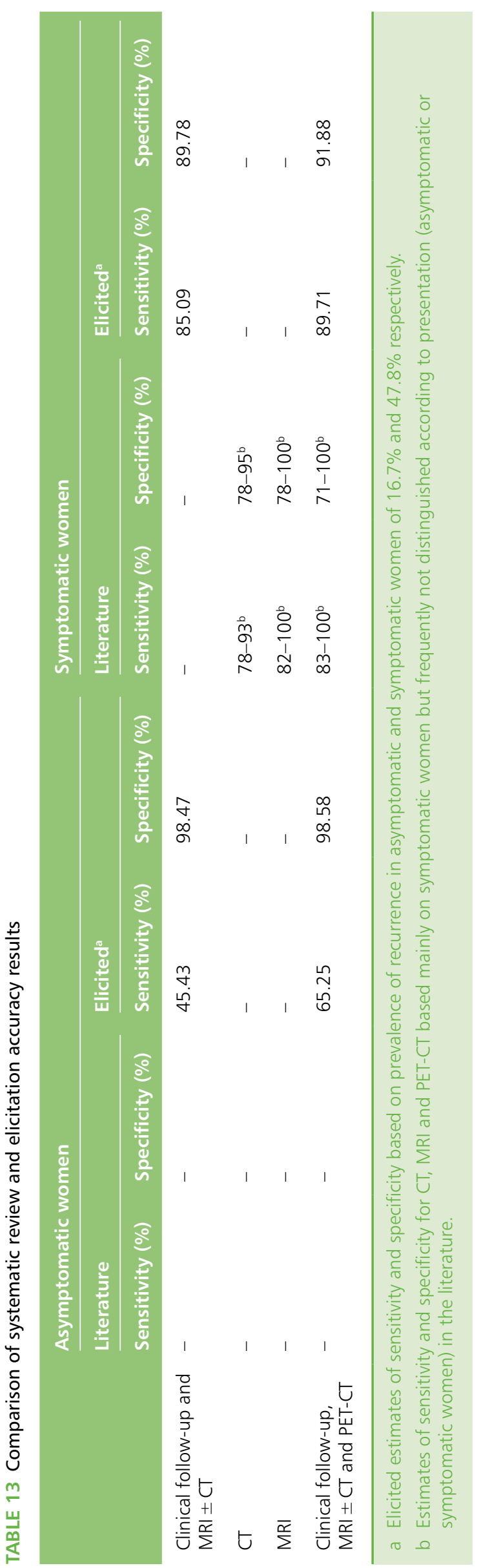
Park, Southampton SO16 7NS, UK. 



\title{
Chapter 6 Effectiveness review
}

\begin{abstract}
The database searches for primary studies identified 24,972 citations, 24,943 citations from the database searches and 29 citations from other sources such as reference lists. Of these, 4618 were duplicates, leaving 20,354 unique citations. Sifting of titles and abstracts excluded 19,994 citations, leaving 360 full-text articles to be assessed for eligibility. Of these, 42 papers were unavailable and 250 papers were excluded as irrelevant: 118 on the wrong population (many with primary and recurrent cervical cancer presented together), 24 on the wrong intervention, 33 with irrelevant outcomes and 75 with inadequate study designs. For a list of excluded papers, see Appendix 13. One existing systematic review $^{59}$ and a relevant guideline ${ }^{3}$ were found. The systematic review included 15 RCTs on chemotherapy in recurrent, metastatic or persistent cervical cancer. The searches for this systematic review were to 2006. Additional searches found four RCTs on chemotherapy. For surgery and radiotherapy, no systematic reviews or RCTs were found and all included studies were case series. In total, 68 papers were included: 19 RCTs of chemotherapy (25 papers), 27 case series in surgery and 16 case series in radiotherapy and chemoradiotherapy (Table 14 and Figure 15).
\end{abstract}

\section{Chemotherapy agents}

Nineteen RCTs (25 publications) compared one or more chemotherapeutic agents in women with recurrent or persistent or advanced (stage IVB) cervical cancer. There were eight RCTs with single-agent cisplatin regimens, four with cisplatin-based chemotherapy regimens, three with carboplatin (CBDCA)-based chemotherapy regimens and four with non-platinum-containing agents (Table 15). There were no RCTs investigating the effectiveness of cisplatin compared with placebo or no treatment in which both arms were given another chemotherapeutic agent. Baseline characteristics are shown in Appendix 14. The results for each category are given in the following sections.

\section{Effectiveness of single cisplatin agents}

\section{Characteristics of included studies}

Eight RCTs gave information about the effectiveness of single cisplatin agents as palliative treatment for recurrent, persistent or advanced cervical cancer (see Table 15). Baseline characteristics, including previous treatment, stage and site of disease, presented in Table 16, were well balanced between groups.

TABLE 14 Summary of identified studies: effectiveness review

\begin{tabular}{|c|c|c|c|}
\hline Characterisitics & Chemotherapy & Chemoradiotherapy & Surgery \\
\hline Population & $\begin{array}{l}\text { Population with multiple site } \\
\text { and distant recurrence }\end{array}$ & $\begin{array}{l}\text { Population with recurrence after } \\
\text { previous surgical treatment only }\end{array}$ & $\begin{array}{l}\text { Population with recurrence after } \\
\text { previous chemoradiotherapy or } \\
\text { radiotherapy only }\end{array}$ \\
\hline Intervention & Chemotherapy agents & $\begin{array}{l}\text { Radiotherapy, } \\
\text { chemoradiotherapy }\end{array}$ & $\begin{array}{l}\text { Surgery: pelvic exenteration, } \\
\text { radical hysterectomy }\end{array}$ \\
\hline $\begin{array}{l}\text { Number of } \\
\text { studies }\end{array}$ & $\begin{array}{l}\text { Single-agent cisplatin: } 8 \text {; } \\
\text { cisplatin-based chemotherapy: } \\
\text { 4; other platinum agents: } \\
\text { 3; non-platinum-containing } \\
\text { agents: } 4\end{array}$ & $\begin{array}{l}\text { Radiotherapy: 9; } \\
\text { chemoradiotherapy: } 7\end{array}$ & $\begin{array}{l}\text { Pelvic exenteration: 20; radical } \\
\text { hysterectomy: } 7\end{array}$ \\
\hline Type of evidence & RCTs & Non-comparative case series & Non-comparative case series \\
\hline
\end{tabular}




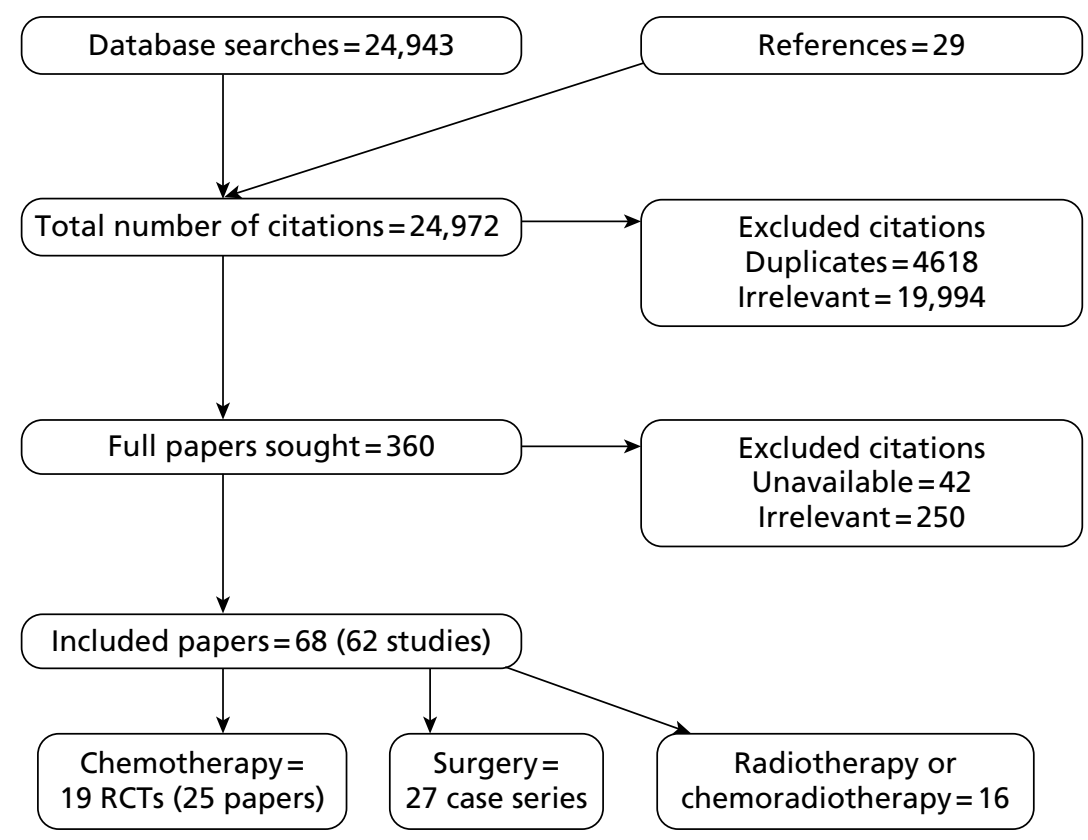

FIGURE 15 PRISMA diagram for effectiveness studies.

TABLE 15 Chemotherapy RCT treatment comparisons and outcomes measured

\begin{tabular}{|c|c|c|c|c|}
\hline Study & Population & Intervention(s) & Comparator(s) & $\begin{array}{l}\text { Outcomes } \\
\text { measured }\end{array}$ \\
\hline $\begin{array}{l}\text { Alberts } \\
198760\end{array}$ & $\begin{array}{l}\text { Advanced and recurrent } \\
\text { squamous cell }\end{array}$ & $\begin{array}{l}\text { Mitomycin C, vincristine, } \\
\text { bleomycin and cisplatin } \\
\text { Mitomycin C and cisplatin }\end{array}$ & Cisplatin & Response rates, AEs \\
\hline $\begin{array}{l}\text { Barlow } \\
1973^{61}\end{array}$ & Recurrent or prior & $\begin{array}{l}\text { Bleomycin } \\
\text { Adriamycin and bleomycin }\end{array}$ & Adriamycin & $\begin{array}{l}\text { Response rates, } \\
\text { OS, duration of } \\
\text { response }\end{array}$ \\
\hline $\begin{array}{l}\text { Bezwoda } \\
1986^{62}\end{array}$ & Recurrent or metastatic & $\begin{array}{l}\text { Cis- } \\
\text { diamminedichloroplatinum } \\
\text { plus methotrexate }\end{array}$ & Hydroxyurea & $\begin{array}{l}\text { OS, response rate, } \\
\text { AEs }\end{array}$ \\
\hline $\begin{array}{l}\text { Bloss } \\
2002^{63}\end{array}$ & $\begin{array}{l}\text { Advanced (stage IVB), } \\
\text { recurrent or persistent } \\
\text { squamous cell }\end{array}$ & $\begin{array}{l}\text { Cisplatin, ifosfamide and } \\
\text { bleomycin }\end{array}$ & Cisplatin and ifosfamide & $\begin{array}{l}\text { OS, PFS, response } \\
\text { rates, AEs }\end{array}$ \\
\hline $\begin{array}{l}\text { Bonomi } \\
1985^{64}\end{array}$ & Advanced squamous cell & Cisplatin $100 \mathrm{mg}$ & $\begin{array}{l}\text { Cisplatin } 20 \mathrm{mg} \\
\text { Cisplatin } 50 \mathrm{mg}\end{array}$ & $\begin{array}{l}\text { OS, PFS, duration of } \\
\text { response, response } \\
\text { rates, AEs }\end{array}$ \\
\hline $\begin{array}{l}\text { Cadron } \\
2005^{65}\end{array}$ & $\begin{array}{l}\text { Recurrent or with } \\
\text { distant metastases }\end{array}$ & $\begin{array}{l}\text { Cisplatin, ifosfamide and } \\
\text { 5-fluorouracil }\end{array}$ & Cisplatin & OS, response rates \\
\hline $\begin{array}{l}\text { aGarin } \\
2001^{66}\end{array}$ & Advanced (stage IVB) & $\begin{array}{l}\text { Irinotecan and cisplatin } \\
\text { Irinotecan and cisplatin } \\
\text { as first-line palliative } \\
\text { treatment }\end{array}$ & Irinotecan & Response rates, AEs \\
\hline $\begin{array}{l}\text { bGreenberg } \\
1977^{67}\end{array}$ & $\begin{array}{l}\text { Recurrent and advanced } \\
\text { (stage IVB) }\end{array}$ & $\begin{array}{l}\text { Bleomycin } \\
\text { Adriamycin and bleomycin }\end{array}$ & Adriamycin & OS, response rates \\
\hline $\begin{array}{l}\text { Lira-Puerto } \\
1991^{68}\end{array}$ & Recurrent & CBDCA & Iproplatin & $\begin{array}{l}\text { OS, PFS, response } \\
\text { rates, AEs }\end{array}$ \\
\hline
\end{tabular}


TABLE 15 Chemotherapy RCT treatment comparisons and outcomes measured (continued)

\begin{tabular}{|c|c|c|c|c|}
\hline Study & Population & Intervention(s) & Comparator(s) & $\begin{array}{l}\text { Outcomes } \\
\text { measured }\end{array}$ \\
\hline $\begin{array}{l}\text { Long } \\
2005^{69-73}\end{array}$ & $\begin{array}{l}\text { Advanced, recurrent or } \\
\text { persistent }\end{array}$ & $\begin{array}{l}\text { Methotrexate, vinblastine, } \\
\text { doxorubicin and cisplatin } \\
\text { Cisplatin and topotecan }\end{array}$ & Cisplatin & $\begin{array}{l}\text { OS, PFS, response } \\
\text { rates, QoL, AEs }\end{array}$ \\
\hline $\begin{array}{l}\text { McGuire } \\
1989^{74}\end{array}$ & Recurrent & CBDCA & Iproplatin & $\begin{array}{l}\text { OS, PFS, response } \\
\text { rates, AEs }\end{array}$ \\
\hline $\begin{array}{l}\text { Monk } \\
2010^{75}\end{array}$ & $\begin{array}{l}\text { Recurrent, advanced } \\
\text { (stage IVB) and } \\
\text { persistent }\end{array}$ & Pazopanib and lapatinib & $\begin{array}{l}\text { Lapatinib } \\
\text { Pazopanib }\end{array}$ & $\begin{array}{l}\text { OS, PFS, response } \\
\text { rates, AEs }\end{array}$ \\
\hline $\begin{array}{l}\text { Monk } \\
2009^{76}\end{array}$ & $\begin{array}{l}\text { Advanced (stage IVB), } \\
\text { recurrent or persistent }\end{array}$ & $\begin{array}{l}\text { Vinorelbine and cisplatin } \\
\text { Gemcitabine and cisplatin } \\
\text { Topotecan and cisplatin }\end{array}$ & Paclitaxel and cisplatin & $\begin{array}{l}\text { OS, PFS, response } \\
\text { rates, QoL, AEs }\end{array}$ \\
\hline $\begin{array}{l}\text { Moore } \\
2004^{77,78}\end{array}$ & $\begin{array}{l}\text { Recurrent or persistent, } \\
\text { advanced (stage IVB) } \\
\text { squamous cell }\end{array}$ & Cisplatin and paclitaxel & Cisplatin & $\begin{array}{l}\text { OS, PFS, response } \\
\text { rates, QoL, AEs }\end{array}$ \\
\hline $\begin{array}{l}\text { Mountzios } \\
2009^{79}\end{array}$ & $\begin{array}{l}\text { Primary metastatic or } \\
\text { recurrent }\end{array}$ & $\begin{array}{l}\text { Cisplatin, ifosfamide and } \\
\text { paclitaxel }\end{array}$ & Cisplatin and ifosfamide & $\begin{array}{l}\text { OS, PFS, response } \\
\text { rates, AEs }\end{array}$ \\
\hline $\begin{array}{l}\text { Omura } \\
1997^{80}\end{array}$ & $\begin{array}{l}\text { Recurrent or persistent, } \\
\text { advanced (stage IVB) } \\
\text { squamous cell }\end{array}$ & $\begin{array}{l}\text { Cisplatin and mitolacol } \\
\text { Cisplatin and ifosfamide }\end{array}$ & Cisplatin & $\begin{array}{l}\text { OS, PFS, duration of } \\
\text { response, response } \\
\text { rates, AEs }\end{array}$ \\
\hline $\begin{array}{l}\text { Thomsen } \\
1998^{81}\end{array}$ & Advanced or recurrent & CBDCA & Teniposide & $\begin{array}{l}\text { OS, PFS, response } \\
\text { rates, AEs }\end{array}$ \\
\hline $\begin{array}{l}\text { Vermorken } \\
2001^{82}\end{array}$ & $\begin{array}{l}\text { Recurrent, advanced } \\
\text { (stage IVB) squamous } \\
\text { cell }\end{array}$ & $\begin{array}{l}\text { Bleomycin, vindesine, } \\
\text { mitomycin, cisplatin }\end{array}$ & Cisplatin & $\begin{array}{l}\text { OS, PFS, duration of } \\
\text { response, response } \\
\text { rates, AEs }\end{array}$ \\
\hline $\begin{array}{l}\text { bWallace } \\
1978^{83}\end{array}$ & $\begin{array}{l}\text { Recurrent and advanced } \\
\text { (stage IVB) }\end{array}$ & $\begin{array}{l}\text { Adriamycin and vincristine } \\
\text { Adriamycin and } \\
\text { cyclophosphamide }\end{array}$ & Adriamycin & $\begin{array}{l}\text { OS, PFS, response } \\
\text { rates, AEs }\end{array}$ \\
\hline
\end{tabular}

AE, adverse event; OS, overall survival; PFS, progression-free survival; QoL, quality of life.

a Abstract only.

b Data from Hirte et al.'s systematic review. ${ }^{59}$

\section{Quality of studies}

All studies were RCTs with no blinding, but the description of randomisation was provided in only three trials..$^{71,78,80}$ In Long et al., ${ }^{71}$ patients were randomly assigned to the treatment regimens with equal probability using a fixed-block design; patients were stratified by treating institution only. In Moore et al. ${ }^{78}$ randomisation (with equal probability to each of the treatment arms) was carried out using a block design that balanced the sequence of assigned arms within parent institutions. In Omura et al., ${ }^{80}$ patients were prospectively stratified according to whether or not they had received previous radiation-sensitizer treatment (hydroxyurea, cisplatin or fluorouracil) and by Karnofsky performance score, and were then centrally randomised with equal probability to three groups.

Description of allocation concealment was not reported in any of the included studies. Several studies had methodological ambiguities. In Alberts et al., ${ }^{60}$ one of the treatment arms, cisplatin, was dropped early because of poor accrual; the number of patients in the cisplatin group was much lower than in the other two groups (9 vs 54 and 51). In Cadron et al., ${ }^{65}$ the intention had been to include 200 patients in the trial but because of poor accrual the trial was stopped prematurely and only 24 patients were included. In Long et al., ${ }^{71}$ the methotrexate, vinblastine, doxorubicin and cisplatin (MVAC) arm was closed by the 
TABLE 16 Characteristics of the populations in the included RCTs: single-agent cisplatin

\begin{tabular}{|c|c|c|c|c|c|c|c|c|}
\hline \multirow[b]{2}{*}{ Parameter } & \multicolumn{3}{|c|}{ Alberts $1987^{60}$} & \multicolumn{3}{|c|}{ Bonomi $1985^{64}$} & \multicolumn{2}{|c|}{ Cadron $2005^{65}$} \\
\hline & MVBC & $\mathrm{MC}$ & C & $\begin{array}{l}50 \mathrm{mg} \\
\mathrm{C}\end{array}$ & $\begin{array}{l}100 \mathrm{mg} \\
\mathrm{C}\end{array}$ & $\begin{array}{l}20 \mathrm{mg} \\
\mathrm{C}\end{array}$ & C & PIF \\
\hline $\begin{array}{l}\text { Number of patients } \\
\text { (randomised) }\end{array}$ & 54 & 51 & 9 & 167 & 185 & 145 & 13 & 11 \\
\hline $\begin{array}{l}\text { Age (years), median } \\
\text { (range) }\end{array}$ & $\begin{array}{l}47.5 \\
(20-77)\end{array}$ & $\begin{array}{l}51 \\
(23-78)\end{array}$ & $\begin{array}{l}51 \\
(29-63)\end{array}$ & $\begin{array}{l}49 \\
(21-78)\end{array}$ & $\begin{array}{l}53 \\
(22-85)\end{array}$ & $\begin{array}{l}49 \\
(22-79)\end{array}$ & $\begin{array}{l}53 \\
(40-80)\end{array}$ & $\begin{array}{l}56 \\
(45-66)\end{array}$ \\
\hline \multicolumn{9}{|l|}{ Previous treatment } \\
\hline Chemotherapy & $N R$ & NR & NR & NR & NR & $N R$ & NR & NR \\
\hline Radiotherapy & $N R$ & $N R$ & $N R$ & 156 & 170 & 134 & 5 & 5 \\
\hline Surgery & $N R$ & $N R$ & $N R$ & NR & $N R$ & $N R$ & $N R$ & NR \\
\hline $\begin{array}{l}\text { Chemotherapy and } \\
\text { radiotherapy }\end{array}$ & $N R$ & $N R$ & $N R$ & $N R$ & $N R$ & $N R$ & $N R$ & $N R$ \\
\hline $\begin{array}{l}\text { Surgery and } \\
\text { radiotherapy }\end{array}$ & $N R$ & NR & NR & NR & NR & $N R$ & 5 & 5 \\
\hline \multicolumn{9}{|l|}{ Stage } \\
\hline IVB & $100 \%$ & $100 \%$ & $100 \%$ & NR & NR & $N R$ & $N R$ & $N R$ \\
\hline Persistent & NR & NR & NR & NR & NR & NR & NR & NR \\
\hline Recurrent & $100 \%$ & $100 \%$ & $100 \%$ & $N R$ & $N R$ & $N R$ & $100 \%$ & $100 \%$ \\
\hline \multicolumn{9}{|l|}{ Site of disease } \\
\hline Pelvic & NR & NR & $N R$ & 96 & 103 & 78 & $64 \%^{a}$ & $60 \%^{\mathrm{a}}$ \\
\hline Distant & $\begin{array}{l}\text { Pulmonary } \\
31 \% \text {; lymph } \\
\text { nodes } 31 \%\end{array}$ & $\begin{array}{l}\text { Pulmonary } \\
14 \% \text {; Iymph } \\
\text { nodes } 25 \%\end{array}$ & $\begin{array}{l}\text { Pulmonary } \\
44 \% \text {; Iymph } \\
\text { nodes } 22 \%\end{array}$ & 71 & 82 & 67 & $36 \%^{\mathrm{a}}$ & $40 \%^{\mathrm{a}}$ \\
\hline Both & $35 \%$ & $37 \%$ & $22 \%$ & $N R$ & $N R$ & $N R$ & NR & NR \\
\hline \multicolumn{9}{|c|}{$\begin{array}{l}\text { C, cisplatin; CIFX, cisplatin, ifosfamide; CM, cisplatin, mitolactol; CP, cisplatin, paclitaxel; CT, cisplatin, topotecan; IC, } \\
\text { irinotecan, cisplatin; ICFL, irinotecan, cisplatin in first line; IR, irinotecan; MC, mitomycin C, cisplatin; MVBC, mitomycin C, } \\
\text { vincristine, bleomycin, cisplatin; NR, not reported; P, pazopanib; PIF, cisplatin, ifosfamide, 5-fluorouracil. } \\
\text { a If the tumour recurred both inside and outside an earlier irradiated area, the site of recurrence was recorded as inside. } \\
\text { b In both groups there was one patient with no site of disease recorded. }\end{array}$} \\
\hline
\end{tabular}




\begin{tabular}{|c|c|c|c|c|c|c|c|c|c|c|c|c|}
\hline \multicolumn{3}{|c|}{ Garin $2001^{66}$} & \multicolumn{3}{|c|}{ Long $2005^{71}$} & \multicolumn{2}{|c|}{ Moore $2004^{78}$} & \multicolumn{3}{|c|}{ Omura $1997^{80}$} & \multicolumn{2}{|c|}{$\begin{array}{l}\text { Vermorken } \\
2001^{82}\end{array}$} \\
\hline I & IC & C & C & $\mathrm{CT}$ & MVAC & C & $\mathrm{CP}$ & C & $\mathrm{CM}$ & CIFX & BEMP & $\mathbf{P}$ \\
\hline 39 & 27 & 31 & 146 & 147 & 63 & 134 & 130 & 140 & 147 & 151 & 143 & 144 \\
\hline 48 & 48 & 48 & $\begin{array}{l}48 \\
(27-76)\end{array}$ & $\begin{array}{l}46 \\
(22-84)\end{array}$ & - & $\begin{array}{l}46 \\
(22-84)\end{array}$ & $\begin{array}{l}48.5 \\
(21-77)\end{array}$ & $\begin{array}{l}47.3 \\
(24-85)\end{array}$ & $\begin{array}{l}48.8 \\
(22-84)\end{array}$ & $\begin{array}{l}46.3 \\
(23-83)\end{array}$ & $\begin{array}{l}53 \\
(25-72)\end{array}$ & $\begin{array}{l}52 \\
(28-76)\end{array}$ \\
\hline 0 & 0 & 0 & 82 & 85 & - & 40 & 31 & 36 & 45 & 38 & 3 & 0 \\
\hline NR & $N R$ & $N R$ & NR & $N R$ & - & 123 & 118 & 123 & 127 & 128 & 101 & 110 \\
\hline NR & NR & NR & NR & $N R$ & - & NR & NR & $N R$ & $N R$ & NR & 60 & 68 \\
\hline NR & $N R$ & NR & $N R$ & $N R$ & - & $N R$ & $N R$ & $N R$ & $N R$ & NR & $N R$ & NR \\
\hline NR & NR & NR & NR & $N R$ & - & NR & NR & $N R$ & $N R$ & NR & $N R$ & NR \\
\hline NR & $N R$ & $N R$ & 17 & 18 & - & 68 & 78 & 140 & 147 & 151 & 17 & 13 \\
\hline NR & $N R$ & $N R$ & 11 & 17 & - & 66 & 52 & $N R$ & $N R$ & NR & $N R$ & NR \\
\hline$N R$ & $N R$ & $N R$ & 118 & 112 & - & & & $N R$ & $N R$ & NR & $N R$ & $N R$ \\
\hline NR & $N R$ & $N R$ & $60^{b}$ & $68^{b}$ & - & 66 & 52 & 68 & 60 & 74 & $N R$ & NR \\
\hline NR & NR & NR & $63^{b}$ & $58^{b}$ & - & 49 & 61 & 63 & 70 & 62 & 69 & 68 \\
\hline NR & NR & NR & $22^{b}$ & $20^{b}$ & - & 19 & 17 & 9 & 17 & 15 & 69 & 71 \\
\hline
\end{tabular}


Data Safety Monitoring Board after four treatment-related deaths occurred among 63 patients, and results for the MVAC arm were not reported. In Vermorken et al. ${ }^{82} 45$ patients from the cisplatin group received bleomycin, vindesine, mitomycin and cisplatin (BEMP) as second-line treatment. The quality assessment results are shown in Figures 16 and 17.

\section{Effectiveness results}

\section{Overall survival, progression-free survival and overall response duration}

In Alberts et al. , ${ }^{60}$ median survival durations associated with receiving cisplatin, mitomycin $C$ and cisplatin and mitomycin $\mathrm{C}$, vincristine, bleomycin and cisplatin (MVBC) treatment were 17.0, 7.0 and 6.9 months respectively. However, because of the small number of patients in the cisplatin arm, meaningful comparison with other treatments cannot be made. Bonomi et al. ${ }^{64}$ found no appreciable differences in median survival duration and time to tumour progression for any of the cisplatin regimens. In Cadron et al. ${ }^{65}$ median survival in the cisplatin group amounted to 13 months and in the group treated with cisplatin, ifosfamide and 5-fluorouracil regimen to 12.3 months; data for progression-free survival were not provided. In Long et al. ${ }^{71}$ median survival was 6.5 months in the cisplatin-treated group and 9.4 months in the group receiving the cisplatin/topotecan combination. The unadjusted and adjusted RR estimates for survival were $0.76(95 \% \mathrm{Cl} 0.59$ to 0.98$)$ and 0.77 (95\% Cl 0.60 to 0.99$)$, respectively, favouring the combination. Statistically significant differences were also observed in progression-free survival, favouring the combination [unadjusted RR 0.76 (95\% Cl 0.60 to 0.97); adjusted for covariates 0.74 (95\% Cl 0.58 to 0.94)]. In Moore et al. ${ }^{78}$ the median progression-free survival for patients receiving cisplatin alone and cisplatin and paclitaxel was 2.8 and 4.8 months respectively $(p=0.001)$. There was no difference in median survival between patients receiving cisplatin alone and patients receiving cisplatin and paclitaxel (8.8 months and 9.7 months respectively). In Omura et al., ${ }^{80}$ progression-free survival was statistically significantly longer for cisplatin and ifosfamide than for cisplatin alone (median, 4.6 vs 3.2 months, $p=0.003$ ); however, there was no difference between cisplatin and mitolactol and cisplatin alone. There was no significant difference in survival between cisplatin and either of the combination regimens. In Vermorken et al. ${ }^{82}$ there was neither a significant difference in progression-free survival nor a significant difference in overall survival between BEMP and cisplatin although, according to the authors, for the former, a trend in favour of BEMP existed.

The results for median overall survival, progression-free survival and duration of response are given in Tables 17-19 respectively.

\section{Response rates}

Response rates, complete response rates and partial response rates for the RCTs are shown in Table 20. This shows that combinations are mostly more effective than single-agent cisplatin, but there is not always consistency in effect direction between the three response rates. For several $\mathrm{RCTS}^{65,66,80,82}$ the complete response rates were not statistically significant, whereas the response rates and/or partial response rates were significant.

\section{Quality of life}

Two RCTs had separate publications with quality-of-life data - quality-of-life data from the study by Long et al. ${ }^{71}$ were reported in Monk et al. ${ }^{72}$ and quality-of-life data from the study by Moore et al. ${ }^{78}$ were reported in McQuellon et al. ${ }^{77}$ For Long et al. ${ }^{71}$ patients completed quality-of-life assessments using the Functional Assessment of Cancer Therapy - General (FACT-G) questionnaire, the neurotoxicity (NTX) subscale, the Brief Pain Inventory (BPI), the Cx subscale and the UNISCALE at four time points during the study. However, there were no statistically significant differences in quality of life up to 9 months after randomisation between cisplatin plus topotecan and cisplatin. It should be noted that in the combination arm increased toxicity was observed.

In the study by Moore et al. ${ }^{78}$ patients were assessed at baseline and at three time points thereafter (prior to chemotherapy cycles 2, 3 and 4) on the FACT-G and subscales. Despite increased toxicity (grades 3-4 


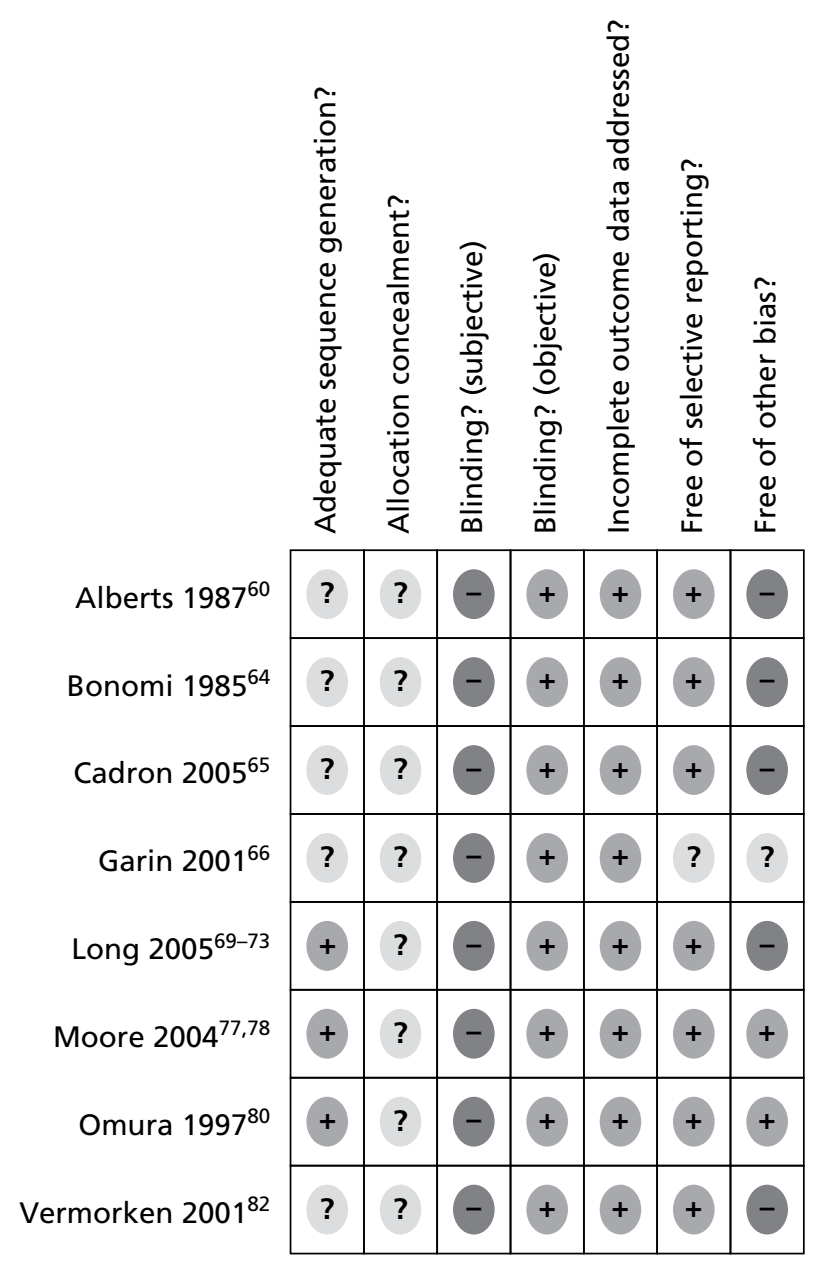

FIGURE 16 Methodological quality on individual items for the eight included RCTs: single-agent cisplatin.

Adequate sequence generation?

Allocation concealment?

Blinding? (subjective)

Blinding? (objective)

Incomplete outcome data addressed?

Free of selective reporting?

Free of other bias?
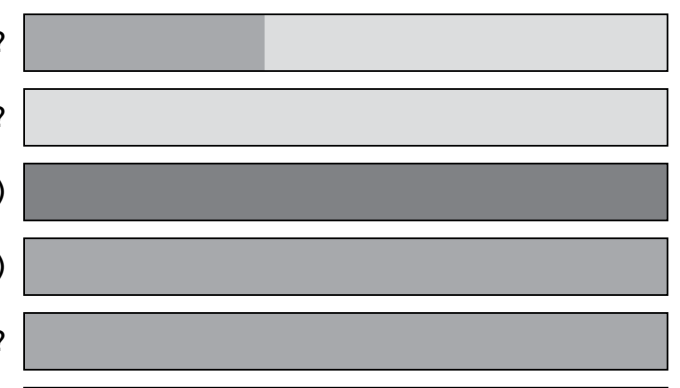

$\square$ Yes (low risk of bias) $\square$ Unclear

$\square$ No (high risk of bias)

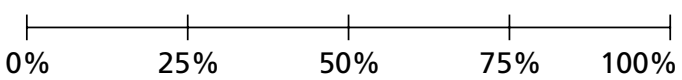

FIGURE 17 Summary of the quality and reporting assessment of the eight included RCTs: single-agent cisplatin. 
TABLE 17 Overall survival: single-agent cisplatin

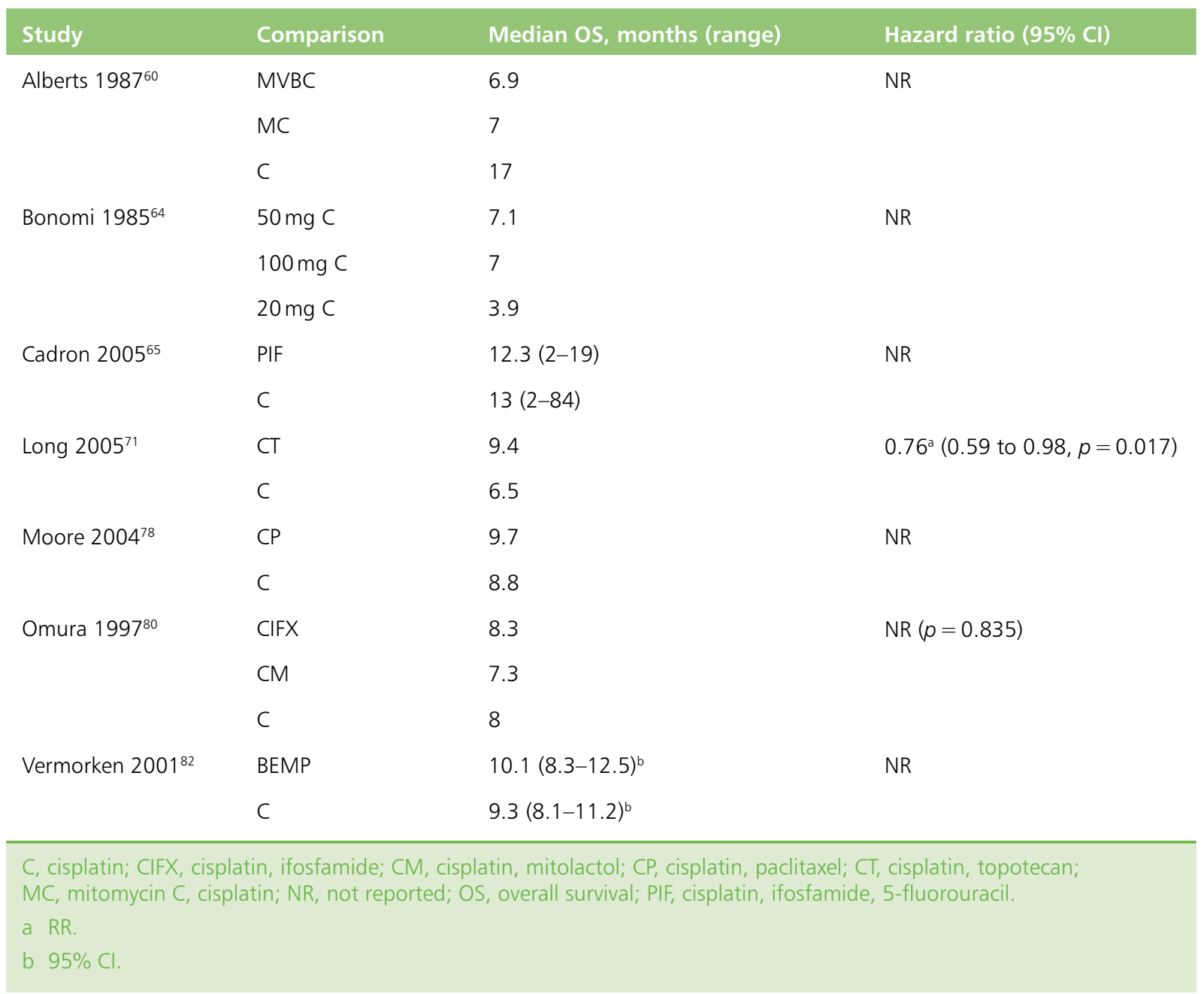

TABLE 18 Progression free-survival: single-agent cisplatin

\begin{tabular}{|c|c|c|c|}
\hline Study & Comparison & Median PFS, months (range) & Hazard ratio \\
\hline \multirow[t]{3}{*}{ Bonomi $1985^{64}$} & $50 \mathrm{mg} \mathrm{C}$ & 3.7 & \multirow[t]{3}{*}{ NR } \\
\hline & $100 \mathrm{mg} \mathrm{C}$ & 4.6 & \\
\hline & $20 \mathrm{mg} \mathrm{C}$ & 3.9 & \\
\hline \multirow[t]{2}{*}{ Long $2005^{71}$} & $\mathrm{CT}$ & 4.6 & \multirow[t]{2}{*}{$0.76^{\mathrm{a}}(0.60$ to $0.97, p=0.014)$} \\
\hline & $C$ & 2.9 & \\
\hline \multirow[t]{2}{*}{ Moore $2004^{78}$} & $\mathrm{CP}$ & 4.8 & \multirow[t]{2}{*}{ NR } \\
\hline & C & 2.8 & \\
\hline \multirow[t]{3}{*}{ Omura $1997^{80}$} & CIFX & 4.6 & \multirow[t]{3}{*}{$\operatorname{NR}(p=0.003)$} \\
\hline & $\mathrm{CM}$ & 3.3 & \\
\hline & $C$ & 3.2 & \\
\hline \multirow[t]{2}{*}{ Vermorken $2001^{82}$} & BEMP & $5.3(4.0-7.0)^{\mathrm{b}}$ & \multirow[t]{2}{*}{ NR } \\
\hline & PC & $4.5(4.0-5.0)^{b}$ & \\
\hline \multicolumn{4}{|c|}{$\begin{array}{l}\text { C, cisplatin; CIFX, cisplatin, ifosfamide; CM, cisplatin, mitolactol; CP, cisplatin, paclitaxel; CT, cisplatin, topotecan; NR, not } \\
\text { reported; PC, paclitaxel, cisplatin; PFS, progression-free survival. } \\
\text { a } R R \text {. } \\
\text { b } 95 \% \text { Cl. }\end{array}$} \\
\hline
\end{tabular}


TABLE 19 Overall duration of response: single-agent cisplatin

\begin{tabular}{|c|c|c|c|}
\hline Study & Comparison & $\begin{array}{l}\text { Median duration of response, } \\
\text { months (range) }\end{array}$ & Hazard ratio \\
\hline \multirow[t]{3}{*}{ Alberts $1987^{60}$} & MVBC & 5.4 & \multirow[t]{3}{*}{$N R$} \\
\hline & $M C$ & 7.2 & \\
\hline & C & 7.3 & \\
\hline \multirow[t]{3}{*}{ Bonomi $1985^{64}$} & $50 \mathrm{mg} \mathrm{C}$ & 4.9 & \multirow[t]{3}{*}{$N R$} \\
\hline & $100 \mathrm{mg} \mathrm{C}$ & 4.1 & \\
\hline & $20 \mathrm{mg} \mathrm{C}$ & 4.8 & \\
\hline \multirow[t]{3}{*}{ Omura $1997^{80}$} & CIFX & 10 & \multirow[t]{3}{*}{$N R$} \\
\hline & CM & 7.7 & \\
\hline & $C$ & 5.5 & \\
\hline \multirow[t]{2}{*}{ Vermorken $2001^{82}$} & BEMP & 9.2 & \multirow[t]{2}{*}{$N R$} \\
\hline & $\mathrm{C}$ & 7.1 & \\
\hline
\end{tabular}

TABLE 20 Response rates (RRs): single-cisplatin agent

\begin{tabular}{|c|c|c|c|c|}
\hline Study & Comparison & $\begin{array}{l}\text { Response rate } \\
(95 \% \mathrm{CI})\end{array}$ & $\begin{array}{l}\text { Complete response } \\
\text { rate }(95 \% \mathrm{Cl})\end{array}$ & $\begin{array}{l}\text { Partial response rate } \\
(95 \% \mathrm{Cl})\end{array}$ \\
\hline \multirow[t]{2}{*}{ Alberts $1987^{60}$} & $M C$ vs $C$ & 0.76 (0.32 to 2.29$)$ & 0.35 (0.05 to 2.63$)$ & 0.97 (0.32 to 3.69$)$ \\
\hline & MVBC vs MC & 1.15 (0.58 to 2.26$)$ & $0.53(0.12$ to 2.37$)$ & $1.46(0.65$ to 3.28$)$ \\
\hline \multirow[t]{3}{*}{ Bonomi $1985^{64}$} & $50 \mathrm{mg}$ C vs $100 \mathrm{mg} \mathrm{C}$ & $0.66(0.45$ to 0.97$)$ & $0.79(0.42$ to 1.48$)$ & $0.57(0.33$ to 1.00$)$ \\
\hline & $20 \mathrm{mg} \mathrm{C}$ vs $50 \mathrm{mg} \mathrm{C}$ & $1.21(0.79$ to 1.86$)$ & $0.86(0.41$ to 1.77$)$ & 1.54 (0.85 to 2.80$)$ \\
\hline & $20 \mathrm{mg} \mathrm{C}$ vs $100 \mathrm{mg} \mathrm{C}$ & 0.62 (0.43 to 0.88$)$ & $0.68(0.34$ to 1.33$)$ & 0.88 (0.53 to 1.44$)$ \\
\hline Cadron $2005^{65}$ & PIF vs $C$ & $4.40(0.81$ to 27.05$)$ & $0.36(0.00$ to 3.91$)$ & 9.82 (1.38 to infinity) \\
\hline \multirow[t]{2}{*}{ Garin $2001^{66}$} & IC vs $\mathrm{C}$ & 1.91 (0.80 to 4.57$)$ & 3.34 (0.15 to 80.83$)$ & $1.72(0.70$ to 4.21$)$ \\
\hline & $\mid C$ vs I & $2.89(1.11$ to 7.51$)$ & $4.29(0.18$ to 101.42$)$ & 2.60 (0.98 to 6.91$)$ \\
\hline Long $2005^{71}$ & CT vs $C$ & $1.99(1.20$ to 3.33$)$ & $3.48(1.24$ to 9.88$)$ & $1.56(0.84$ to 2.91$)$ \\
\hline Moore $2004^{78}$ & CP vs $\mathrm{C}$ & $1.86(1.24$ to 2.83$)$ & $2.58(1.21$ to 5.57$)$ & 1.55 (0.90 to 2.66$)$ \\
\hline \multirow[t]{2}{*}{ Omura $1997^{80}$} & $\mathrm{CM}$ vs $\mathrm{C}$ & $1.18(0.74$ to 1.90$)$ & $1.48(0.66$ to 3.31$)$ & 1.01 (0.54 to 1.92$)$ \\
\hline & CIFX vs C & 1.74 (1.14 to 2.67$)$ & $1.96(0.92$ to 4.18$)$ & 1.62 (0.92 to 2.86$)$ \\
\hline Vermorken $2001^{82}$ & BEMP vs $C$ & $1.76(1.08$ to 2.90$)$ & 1.51 (0.57 to 3.99) & 1.87 (1.03 to 3.42$)$ \\
\hline
\end{tabular}


anaemia and grades 3-4 neutropenia) in the combination arm (cisplatin and paclitaxel) there were no statistically significant differences in scores between the groups at any assessment point.

\section{Adverse events}

Haematological toxicity (neutropenia, febrile neutropenia, thrombocytopenia, leucopenia and anaemia) was generally more frequently associated with cisplatin in combination with other agents than with cisplatin monotherapy (Tables 21-24). Infections were more common with combination therapy than with single-agent cisplatin (cisplatin + topotecan arm: 26/147, cisplatin-only arm: 12/146; ${ }^{71}$ BEMP arm: 7/143, cisplatin-only arm: 3/14482). There was little difference in neuropathy between combination therapy and single-agent cisplatin (cisplatin + paclitaxel arm: 4/129, cisplatin-only arm: 6/130; ${ }^{78}$ BEMP arm: 7/143, cisplatin-only arm: $\left.3 / 144^{82}\right)$. Alopecia was also more common with combination therapy than with single-agent cisplatin (MVBC arm: 12/54, mitomycin C and cisplatin arm: 2/51, cisplatin-only arm: 0/9;60 BEMP arm: 81/143, cisplatin-only arm: $\left.31 / 144^{82}\right)$. The results for nausea and/or vomiting are shown in

TABLE 21 Neutropenia and febrile neutropenia: single-agent cisplatin

\begin{tabular}{|c|c|c|c|c|}
\hline Study & Comparison & Intervention $(n / N)$ & Control $(n / N)$ & $\operatorname{RR}(95 \% \mathrm{Cl})$ \\
\hline \multirow[t]{2}{*}{ Garin $2001^{66}$} & IC vs $C$ & $22 / 27$ & $3 / 31$ & 8.42 (2.83 to 25.05$)$ \\
\hline & $\mid C$ vs I & $22 / 27$ & $13 / 39$ & 2.44 (1.51 to 3.95$)$ \\
\hline aLong $2005^{71}$ & CT vs $\mathrm{C}$ & $103 / 147$ & $2 / 146$ & 51.15 (14.37 to 186.73$)$ \\
\hline bLong $2005^{71}$ & $\mathrm{CT}$ vs $\mathrm{C}$ & $27 / 147$ & $12 / 146$ & 2.23 (1.20 to 4.22$)$ \\
\hline Moore $2004^{78}$ & $\mathrm{CP}$ vs $\mathrm{C}$ & $86 / 129$ & $4 / 130$ & 21.63 (8.65 to 118.25$)$ \\
\hline${ }^{\mathrm{b}}$ Moore $2004^{78}$ & $\mathrm{CP}$ vs $\mathrm{C}$ & $86 / 129$ & $4 / 130$ & 21.63 (8.65 to 118.25$)$ \\
\hline \multirow[t]{2}{*}{ Omura $1997^{80}$} & CM vs $C$ & $19 / 145$ & $1 / 137$ & $17.95(2.44$ to 132.29$)$ \\
\hline & CIFX vs C & $55 / 146$ & $1 / 137$ & 51.61 (7.24 to 367.83 ) \\
\hline \multicolumn{5}{|c|}{$\begin{array}{l}\text { C, cisplatin; CIFX, cisplatin, ifosfamide; CM, cisplatin, mitolactol; CP, cisplatin, paclitaxel; CT, cisplatin, topotecan; } \\
\text { I, ifosfamide; IC, irinotecan, cisplatin. } \\
\text { a Grades } 3 \text { and } 4 \text { neutropenia only. }\end{array}$} \\
\hline
\end{tabular}

TABLE 22 Thrombocytopenia: single-agent cisplatin

\begin{tabular}{|c|c|c|c|c|}
\hline Study & Comparison & Intervention $(n / N)$ & Control $(n / N)$ & $\operatorname{RR}(95 \% \mathrm{Cl})$ \\
\hline \multirow[t]{2}{*}{ Alberts $1987^{60}$} & $M C$ vs $C$ & 9/51 & $0 / 9$ & 3.65 (0.54 to infinity) \\
\hline & MVBC vs MC & $13 / 54$ & $9 / 51$ & $1.36(0.64$ to 2.91$)$ \\
\hline \multirow[t]{3}{*}{ Bonomi $1985^{64}$} & $50 \mathrm{mg} \mathrm{C}$ vs $100 \mathrm{mg} \mathrm{C}$ & $2 / 162$ & $2 / 180$ & $1.11(0.20$ to 6.24$)$ \\
\hline & $20 \mathrm{mg} \mathrm{C}$ vs $50 \mathrm{mg} \mathrm{C}$ & $4 / 143$ & $2 / 162$ & 2.27 (0.49 to 10.47$)$ \\
\hline & $20 \mathrm{mg} \mathrm{C}$ vs $100 \mathrm{mg} \mathrm{C}$ & $4 / 143$ & $2 / 180$ & 2.24 (0.49 to 10.34$)$ \\
\hline Long $2005^{71}$ & CT vs $\mathrm{C}$ & $46 / 147$ & $4 / 146$ & 11.42 (4.45 to 29.99$)$ \\
\hline Moore $2004^{78}$ & CP vs $C$ & $5 / 129$ & $3 / 130$ & $1.68(0.45$ to 6.26$)$ \\
\hline \multirow[t]{2}{*}{ Omura $1997^{80}$} & $\mathrm{CM}$ vs $\mathrm{C}$ & $23 / 145$ & $1 / 137$ & $21.73(2.98$ to 158.73$)$ \\
\hline & CIFX vs $C$ & $28 / 146$ & $1 / 137$ & 26.27 (3.62 to 190.48$)$ \\
\hline
\end{tabular}

C, cisplatin; CIFX, cisplatin, ifosfamide; CM, cisplatin, mitolactol; CP, cisplatin, paclitaxel; CT, cisplatin, topotecan; MC, mitomycin C, cisplatin. 
Table 25. There were no significant differences between the combination therapy and the single-agent cisplatin arms.

\section{Effectiveness of cisplatin combinations}

\section{Characteristics of included studies}

Four RCTs contained relevant information about the effectiveness of cisplatin combinations as palliative treatment for recurrent, metastatic or persistent cervical cancer. ${ }^{62,63,76,79}$ Baseline characteristics (including previous treatment and stage or site of disease) presented in Table 26 were well balanced between the groups. However, not all relevant clinical information was presented in all publications.

TABLE 23 Leucopenia: single-agent cisplatin

\begin{tabular}{|c|c|c|c|c|}
\hline Study & Comparison & Intervention $(n / N)$ & Control $(n / N)$ & $\operatorname{RR}(95 \% \mathrm{CI})$ \\
\hline \multirow[t]{2}{*}{ Alberts $1987^{60}$} & $M C$ vs $C$ & $9 / 51$ & $0 / 9$ & 3.65 (0.54 to infinity) \\
\hline & MVBC vs MC & $10 / 54$ & $9 / 51$ & 1.05 (0.46 to 2.37 ) \\
\hline \multirow[t]{3}{*}{ Bonomi $1985^{64}$} & $50 \mathrm{mg} \mathrm{C}$ vs $100 \mathrm{mg} \mathrm{C}$ & $1 / 162$ & $12 / 180$ & 0.09 (0.01 to 0.70$)$ \\
\hline & $20 \mathrm{mg} C$ vs $50 \mathrm{mg} \mathrm{C}$ & $6 / 143$ & $1 / 162$ & $22.60(3.66$ to 140.53$)$ \\
\hline & $20 \mathrm{mg} \mathrm{C}$ vs $100 \mathrm{mg} \mathrm{C}$ & $6 / 143$ & $12 / 180$ & 2.09 (0.84 to 5.00$)$ \\
\hline Long $2005^{71}$ & CT vs $C$ & $93 / 147$ & $1 / 146$ & 92.37 (16.69 to 524.25$)$ \\
\hline Moore $2004^{78}$ & CP vs $C$ & $69 / 129$ & $4 / 130$ & 17.38 (6.90 to 44.98$)$ \\
\hline
\end{tabular}

C, cisplatin; CP, cisplatin, paclitaxel; CT, cisplatin, topotecan; MC, mitomycin C, cisplatin.

TABLE 24 Anaemia: single-agent cisplatin

\begin{tabular}{lllll} 
Study & Comparison & Intervention $(n / N)$ & Control $(n / N)$ & RR (95\% Cl) \\
Long $2005^{71}$ & CT vs C & $56 / 147$ & $34 / 146$ & $1.64(1.15$ to 2.35) \\
Moore 200478 & CP vs C & $39 / 129$ & $17 / 130$ & $2.31(1.33$ to 2.56) \\
\hline
\end{tabular}

C, cisplatin; CP, cisplatin, paclitaxel; CT, cisplatin, topotecan.

TABLE 25 Nausea and/or vomiting: single-agent cisplatin

$\begin{array}{lllll}\text { Study } & \text { Comparison } & \text { Intervention }(n / N) & \text { Control }(n / N) & \text { RR (95\% Cl) } \\ \text { Alberts 198760 } & \text { MC vs C } & 10 / 51 & 2 / 9 & 0.88(0.29 \text { to 3.38) } \\ & \text { MVBC vs MC } & 8 / 54 & 10 / 51 & 0.76(0.32 \text { to } 1.76) \\ \text { Garin 200166 } & \text { IC vs C } & 1 / 27 & 0 / 31 & 3.43(0.15 \text { to } 80.83) \\ & \text { IC vs I } & 1 / 27 & 2 / 39 & 0.72(0.07 \text { to } 7.57) \\ \text { Long 200571 } & \text { CT vs C } & 21 / 147 & 13 / 146 & 1.60(0.85 \text { to } 3.06) \\ \text { Moore 200478 } & \text { CP vs C } & 13 / 129 & 16 / 130 & 0.82(0.42 \text { to } 1.61) \\ \text { Omura 199780 } & \text { CM vs C } & 10 / 145 & 12 / 137 & 0.79(0.36 \text { to 1.76) } \\ & \text { CIFX vs C } & 17 / 146 & 12 / 137 & 1.33(0.66 \text { to 2.68) }\end{array}$

C, cisplatin; CIFX, cisplatin, ifosfamide; CM, cisplatin, mitolactol; CP, cisplatin, paclitaxel; CT, cisplatin, topotecan; I, ifosfamide; IC, irinotecan, cisplatin; MC, mitomycin C, cisplatin. 
高

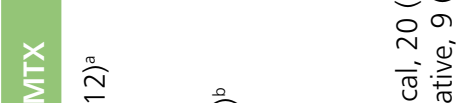

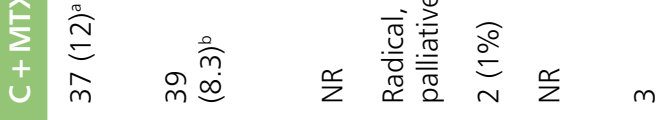

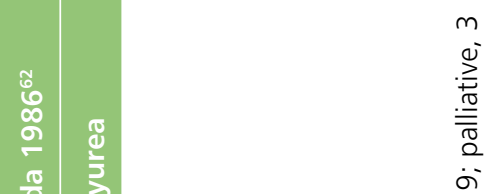

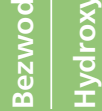

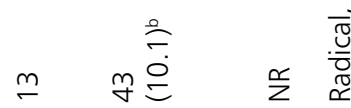

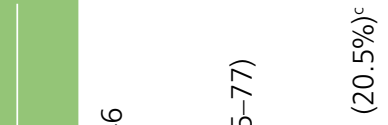

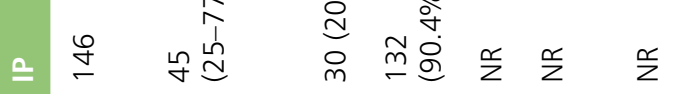

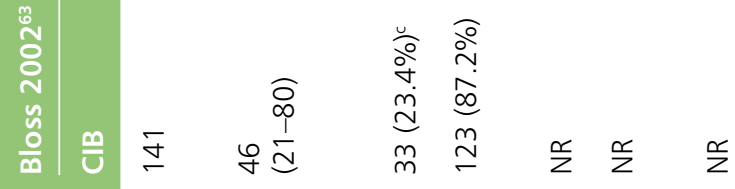

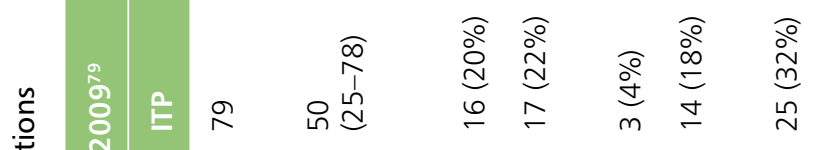

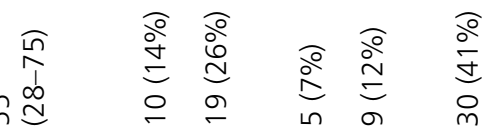

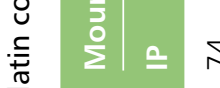

$\frac{n}{4}$

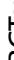

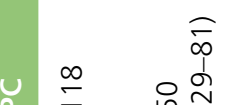

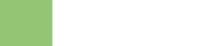

$\cup \stackrel{\infty}{\leftarrow} \quad \stackrel{\infty}{\sim}$

$\stackrel{\substack{n \\ \stackrel{n}{i}}}{\stackrel{n}{=}}$

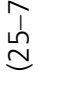

$\frac{a}{z}$

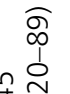

魚

잉

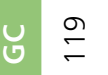

$\checkmark$

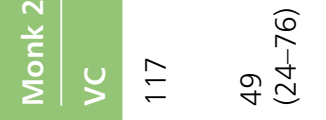

誉

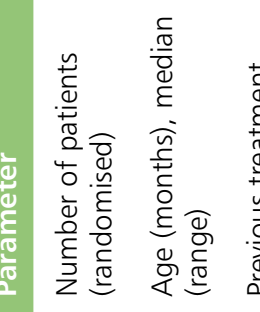

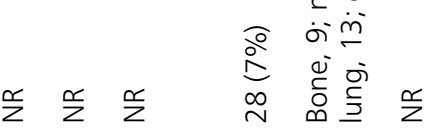

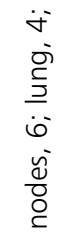

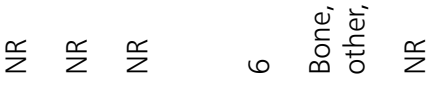

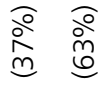

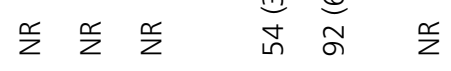

ஓ

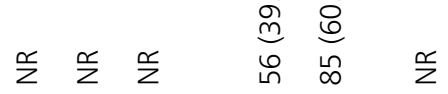

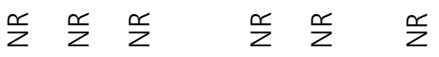

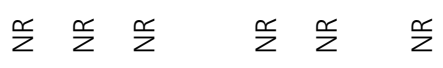
ニ

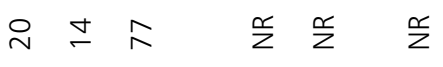

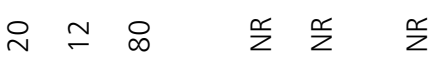

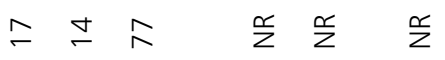

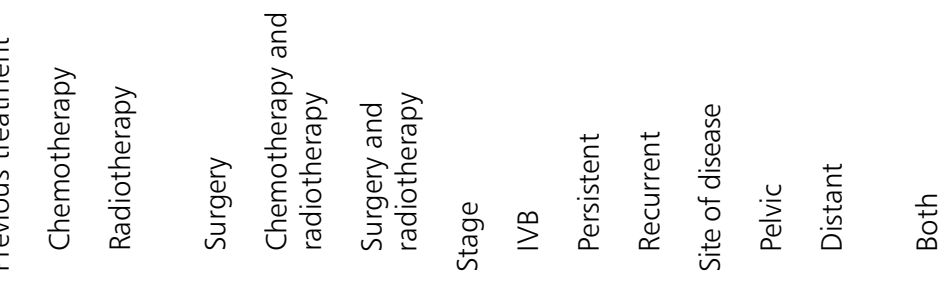




\section{Quality of studies}

Only two studies ${ }^{63,76}$ specified the method of randomisation in their reports. Description of allocation concealment was not reported in any of the included RCTs. Until January 2004, the Monk et al. ${ }^{76}$ study consisted of only two arms comparing cisplatin plus paclitaxel with cisplatin plus vinorelbine. Primary analyses excluded those 41 patients. In Bezwoda et al., ${ }^{62}$ after a preliminary analysis of the results, the hydroxyurea arm of the study was discontinued and a further 25 patients received the cis-diamminedichl oroplatinum(II) (DDP) plus methotrexate regimen. Figures 18 and 19 show the results of the quality assessment.

\section{Effectiveness results}

\section{Overall and progression-free survival}

Overall and progression-free survival results are presented in Tables 27 and 28 respectively. All four trials reported median overall survival, and values were highest for the cisplatin, ifosfamide and paclitaxel arm in Mountzios et al., ${ }^{79}$ reaching 15.4 months ( $95 \% \mathrm{Cl} 8.6$ to 22.3 months). The hazard ratio was given by Monk et al. ${ }^{76}$ and Mountzios et al. ${ }^{79}$ These results indicate that there were no statistically significant differences between chemotherapeutic schemes in any of the included studies.

The progression-free survival results were available in three $\mathrm{RCTs}^{63,76,79}$ and hazard ratios were provided by two. ${ }^{76,79}$ Multivariate Cox analysis for progression-free survival was performed in Mountzios et al. ${ }^{79}$ and indicated a statistically significantly longer progression-free survival for the cisplatin, ifosfamide and paclitaxel arm [hazard ratio 0.70 (95\% Cl 0.49 to 0.99$), p=0.046$ ].

\section{Response rate}

All of the RCTs reported response rates but complete and partial response rate was available only in three trials; $62,76,79$ Bloss et al. ${ }^{63}$ did not report complete and partial response rates. Response rates and risk ratios are presented in Table 29.

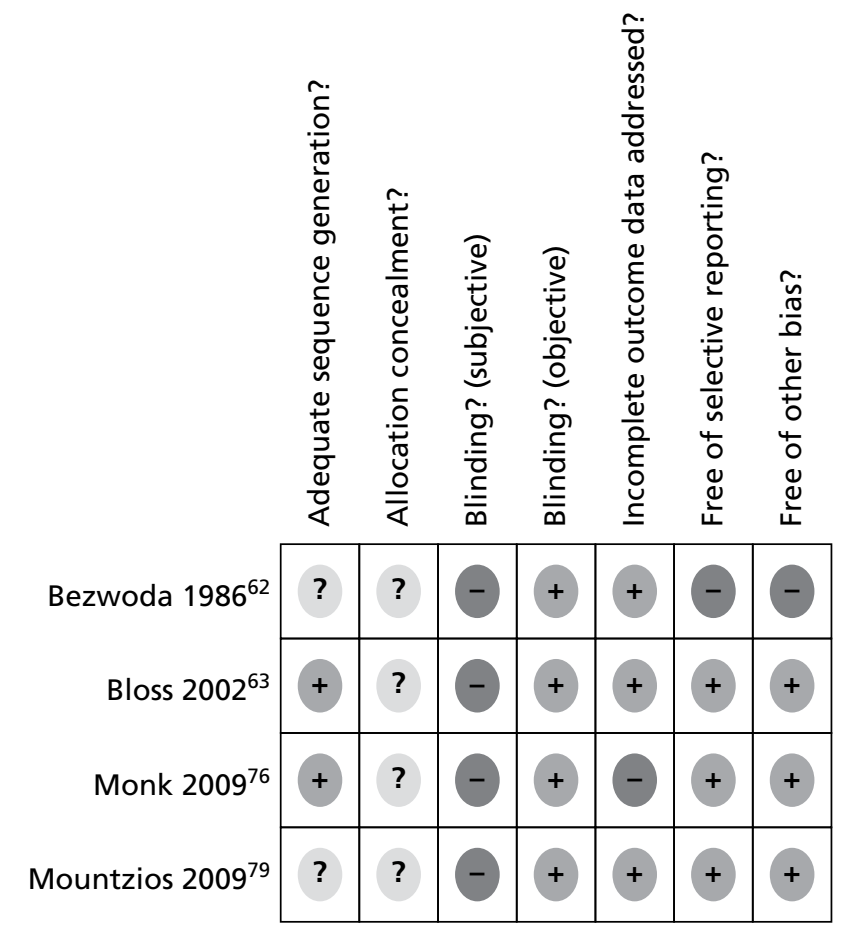

FIGURE 18 Methodological quality on individual items for the four included RCTs: ${ }^{62,63,76,79}$ cisplatin combinations. 
Adequate sequence generation?

Allocation concealment?

Blinding? (subjective)

Blinding? (objective)

Incomplete outcome data addressed?

Free of selective reporting?
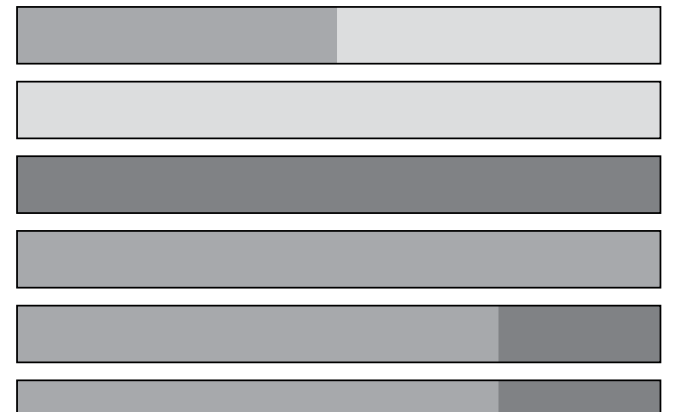

$\square$ Yes (low risk of bias)

$\square$ Unclear

$\square$ No (high risk of bias)

Free of other bias?

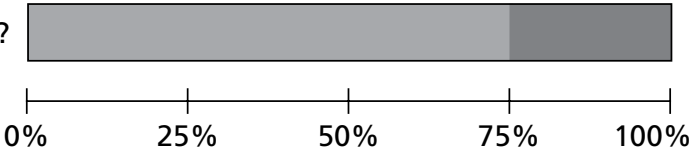

FIGURE 19 Summary of the quality and reporting assessment of the four included RCTs:52,63,76,79 cisplatin combinations.

TABLE 27 Overall survival: cisplatin combinations

$\begin{array}{llll}\text { Study } & \text { Comparison } & \text { Median OS, months (range) } & \text { Hazard ratio (95\% Cl) } \\ \text { Bezwoda 198622 } & \text { Hydroxyurea } & 4 & \text { NR } \\ & \text { C }+ \text { MTXa } & 9 & \\ \text { Bloss 200263 } & \text { C }+ \text { MTX } & 11 & \text { NR } \\ \text { Monk 200976 } & \text { IP } & 8.5 & \\ & \text { CIB } & 8.4 & 1.15(0.79 \text { to } 1.67)^{b} \\ & \text { VC } & 9.99(8.25-12.25) & 1.32(0.91 \text { to } 1.92)^{b} \\ & \text { GC } & 10.28(7.62-11.60) & 1.26(0.86 \text { to } 1.82)^{b} \\ \text { Mountzios 200979 } & \text { IP } & 10.25(8.61-11.66) & 0.75(0.53 \text { to } 1.08)\end{array}$

C, cisplatin; CIB, cisplatin, ifosfamide and bleomycin; GC, gemcitabine, cisplatin; IP, cisplatin, ifosfamide; ITP,

cisplatin, ifosfamide, paclitaxel; MTX, methotrexate; NR, not reported; OS, overall survival; PC, paclitaxel, cisplatin;

TC, topotecan, cisplatin; VC, vinorelbine, cisplatin.

a Initial 12 patients randomly allocated to receive DDP + MTX.

b Compared with PC reference arm.

c $95 \% \mathrm{Cl}$.

\section{Quality of life}

Monk et al. ${ }^{76}$ reported quality of life measured with the Functional Assessment of Cancer Therapy - Cervix Trial Outcome Index (FACT-Cx TOI), the Functional Assessment of Cancer Therapy/Gynecologic Oncology Group Neurotoxicity four-item scale (FACT/GOG-NTX) and the BPI but detailed data were not presented in the publication. After adjustment for baseline score, age and performance status at randomisation, there were no statistical differences between any of the experimental arms and the control arm. 
TABLE 28 Progression-free survival: cisplatin combinations

\begin{tabular}{|c|c|c|c|}
\hline Study & Comparison & Median PFS, months (range) & Hazard ratio $(95 \% \mathrm{Cl})$ \\
\hline \multirow[t]{2}{*}{ Bloss $2002^{63}$} & IP & 4.6 & \multirow[t]{2}{*}{ NR } \\
\hline & $\mathrm{CIB}$ & 5.1 & \\
\hline \multirow[t]{4}{*}{ Monk $2009^{76}$} & VC & $3.98(3.19-5.16)$ & $1.36(0.97 \text { to } 1.90)^{a}$ \\
\hline & GC & $4.70(3.58-5.59)$ & $1.39(0.99 \text { to } 1.96)^{\mathrm{a}}$ \\
\hline & TC & $4.57(3.71-5.75)$ & \multirow[t]{2}{*}{$1.27(0.90 \text { to } 1.78)^{\mathrm{a}}$} \\
\hline & PC & $5.82(4.53-7.59)$ & \\
\hline \multirow[t]{2}{*}{ Mountzios $2009^{79}$} & IP & $6.3(4.3-8.2)^{b}$ & \multirow[t]{2}{*}{0.70 (0.49 to 0.99$)$} \\
\hline & ITP & $7.9(6.1-9.8)^{b}$ & \\
\hline \multicolumn{4}{|c|}{$\begin{array}{l}\text { CIB, cisplatin, ifosfamide and bleomycin; GC, gemcitabine, cisplatin; IP, cisplatin, ifosfamide; ITP, cisplatin, ifosfam } \\
\text { paclitaxel; NR, not reported; PC, paclitaxel, cisplatin; PFS, progression-free survival; TC, topotecan, cisplatin; } \\
\text { VC, vinorelbine, cisplatin. } \\
\text { a Compared with PC reference arm. } \\
\text { b } 95 \% \mathrm{Cl} \text {. }\end{array}$} \\
\hline
\end{tabular}

TABLE 29 Response rates (RRs): cisplatin combinations

\begin{tabular}{|c|c|c|c|c|}
\hline Study & Comparison & $\begin{array}{l}\text { Response rate } \\
(95 \% \mathrm{Cl})\end{array}$ & $\begin{array}{l}\text { Complete response } \\
\text { rate }(95 \% \mathrm{CI})\end{array}$ & $\begin{array}{l}\text { Partial response rate } \\
(95 \% \mathrm{CI})\end{array}$ \\
\hline Bezwoda $1986^{62}$ & Hydroxyurea vs $\mathrm{C}+\mathrm{MTX}$ & 0.06 (0.00 to 0.97$)$ & 0.25 (0.01 to 4.18$)$ & 0.08 (0.01 to 1.28$)$ \\
\hline Bloss $2002^{63}$ & CIB vs IP & 0.97 (0.69 to 1.36$)$ & NR & NR \\
\hline \multirow[t]{3}{*}{ Monk $2009^{76}$} & VC vs $P C$ & $0.89(0.57$ to 1.38$)$ & $2.54(0.69$ to 9.32$)$ & $0.71(0.42$ to 1.18$)$ \\
\hline & GC vs PC & $0.77(0.48$ to 1.21$)$ & $0.31(0.03$ to 2.90$)$ & $0.82(0.51$ to 1.32$)$ \\
\hline & TC vs PC & $0.80(0.51$ to 1.26$)$ & 0.62 (0.11 to 3.63$)$ & $0.82(0.51$ to 1.33$)$ \\
\hline Mountzios $2009^{79}$ & IP vs ITP & 0.56 (0.38 to 0.81$)$ & 0.44 (0.20 to 0.94$)$ & 0.64 (0.38 to 1.09$)$ \\
\hline
\end{tabular}

\section{Adverse events}

Haematological adverse events were high in all RCTs (Tables 30-33). Bezwoda et al. ${ }^{62}$ did not specify the grade of reported adverse events. The authors mentioned that therapy was generally well tolerated but that all patients receiving high-dose hydroxyurea developed leucopenia with a nadir 10-14 days after the initial loading dose; however, all patients recovered rapidly. Haematological toxicity was rare in the cisplatin plus methotrexate-treated patients (two patients) and stomatitis occurred in only one patient. In Bloss et al. ${ }^{63}$ toxicity was graded according to standard Gynecologic Oncology Group (GOG) criteria, in Monk et al. ${ }^{76}$ the National Cancer Institute Common Toxicity Criteria (NCI CTC) version 2.0, was used for characterising adverse events and dose modifications and in Mountzios et al. ${ }^{79}$ The World Health Organization criteria were used in the assessment of toxicity. It was not appropriate to combine toxicity results because of differences in chemotherapy regimens in the RCTs.

Bezwoda et al. ${ }^{62}$ mentioned that three patients developed hypokalaemia and three developed symptomatic hypocalcaemia, two of whom also had hypomagnesaemia. Monk et al. ${ }^{76}$ reported a significantly smaller proportion of patients with adverse events such as vomiting and nausea in the topotecan/cisplatin and 
TABLE 30 Neutropenia and febrile neutropenia: cisplatin combinations

\begin{tabular}{|c|c|c|c|c|}
\hline Study & Comparison & Intervention $(n / N)$ & Control $(n / N)$ & $\operatorname{RR}(95 \% \mathrm{Cl})$ \\
\hline Bloss $2002^{63}$ & CIB vs IP & $117 / 137$ & $117 / 144$ & 1.05 (0.95 to 1.17$)$ \\
\hline \multirow[t]{3}{*}{ Monk $2009^{76}$} & VC vs PC & $83 / 106$ & 79/101 & $1.00(0.87$ to 1.16$)$ \\
\hline & GC vs PC & $46 / 109$ & 79/101 & $0.54(0.42$ to 0.69$)$ \\
\hline & TC vs PC & $90 / 109$ & 79/101 & $1.06(0.92$ to 1.21$)$ \\
\hline \multirow[t]{3}{*}{ Monk $2009^{76}$} & VC vs PC & $15 / 106$ & $13 / 101$ & $1.10(0.55$ to 2.19$)$ \\
\hline & GC vs PC & $7 / 109$ & 13/101 & $0.50(0.21$ to 1.20$)$ \\
\hline & TC vs PC & $11 / 109$ & 13/101 & $0.78(0.37$ to 1.67$)$ \\
\hline Mountzios $2009^{79}$ & IP vs ITP & $22 / 72$ & $20 / 77$ & $1.18(0.70$ to 1.97$)$ \\
\hline aMountzios $2009^{79}$ & IP vs ITP & $2 / 72$ & $7 / 77$ & $0.31(0.07$ to 1.42$)$ \\
\hline
\end{tabular}

TABLE 31 Thrombocytopenia: cisplatin combinations

\begin{tabular}{|c|c|c|c|c|}
\hline Study & Comparison & Intervention $(n / N)$ & Control $(n / N)$ & $\operatorname{RR}(95 \% \mathrm{Cl})$ \\
\hline Bloss $2002^{63}$ & CIB vs IP & 28/137 & $23 / 144$ & $1.28(0.78$ to 2.11$)$ \\
\hline \multirow[t]{3}{*}{ Monk $2009^{76}$} & VC vs PC & $8 / 106$ & $7 / 101$ & 1.09 (0.41 to 2.89$)$ \\
\hline & GC vs PC & $31 / 109$ & $7 / 101$ & 4.10 (1.89 to 8.90$)$ \\
\hline & TC vs PC & $38 / 109$ & $7 / 101$ & 5.03 (2.35 to 10.75$)$ \\
\hline Mountzios $2009^{79}$ & IP vs ITP & $8 / 72$ & $8 / 77$ & 1.07 (0.42 to 2.70$)$ \\
\hline
\end{tabular}

TABLE 32 Leucopenia: cisplatin combinations

\begin{tabular}{|c|c|c|c|c|}
\hline Study & Comparison & Intervention $(n / N)$ & Control $(n / N)$ & $\operatorname{RR}(95 \% \mathrm{Cl})$ \\
\hline Bloss $2002^{63}$ & CIB vs IP & $118 / 137$ & $121 / 144$ & 1.03 (0.93 to 1.13$)$ \\
\hline \multirow[t]{3}{*}{ Monk $2009^{76}$} & VC vs PC & $72 / 106$ & $64 / 101$ & 1.07 (0.88 to 1.31$)$ \\
\hline & GC vs PC & $47 / 109$ & $64 / 101$ & $0.68(0.52$ to 0.88$)$ \\
\hline & TC vs PC & $77 / 109$ & $64 / 101$ & $1.11(0.92$ to 1.35$)$ \\
\hline Mountzios 200979 & IP vs ITP & $1 / 72$ & $2 / 77$ & $0.53(0.05$ to 5.77$)$ \\
\hline
\end{tabular}


TABLE 33 Anaemia: cisplatin combinations

\begin{tabular}{|c|c|c|c|c|}
\hline Study & Comparison & Intervention $(n / N)$ & Control $(n / N)$ & $\operatorname{RR}(95 \% \mathrm{Cl})$ \\
\hline Bloss $2002^{63}$ & CIB vs IP & 29/137 & $32 / 144$ & $0.95(0.61$ to 1.49$)$ \\
\hline \multirow[t]{3}{*}{ Monk $2009^{76}$} & VC vs PC & $31 / 106$ & $17 / 101$ & $1.74(1.03$ to 2.94$)$ \\
\hline & GC vs $P C$ & $37 / 109$ & $17 / 101$ & $2.02(1.22$ to 3.35$)$ \\
\hline & TC vs PC & $38 / 109$ & $17 / 101$ & $2.07(1.25$ to 3.43$)$ \\
\hline Mountzios $2009^{79}$ & IP vs ITP & $6 / 72$ & $8 / 72$ & 0.75 (0.27 to 2.05$)$ \\
\hline
\end{tabular}

TABLE 34 Nausea and/or vomiting: cisplatin combinations

\begin{tabular}{|c|c|c|c|c|}
\hline Study & Comparison & Intervention $(n / N)$ & Control $(n / N)$ & $\operatorname{RR}(95 \% \mathrm{Cl})$ \\
\hline${ }^{a}$ Bloss $2002^{63}$ & CIB vs IP & $33 / 137$ & $31 / 144$ & $1.12(0.73$ to 1.72$)$ \\
\hline \multirow[t]{3}{*}{${ }^{\mathrm{b}}$ Monk $2009^{76}$} & VC vs PC & $14 / 106$ & 20/101 & $0.67(0.36$ to 1.25$)$ \\
\hline & GC vs PC & $11 / 109$ & $20 / 101$ & 0.51 (0.26 to 1.01$)$ \\
\hline & TC vs PC & $9 / 109$ & $20 / 101$ & $0.42(0.20$ to 0.87$)$ \\
\hline \multirow[t]{3}{*}{${ }^{c}$ Monk $2009^{76}$} & VC vs PC & $14 / 106$ & 20/101 & $0.67(0.36$ to 1.25$)$ \\
\hline & GC vs $P C$ & $11 / 109$ & $20 / 101$ & $0.51(0.26$ to 1.01$)$ \\
\hline & TC vs PC & $9 / 109$ & 20/101 & $0.42(0.20$ to 0.87$)$ \\
\hline aMountzios $2009^{79}$ & IP vs ITP & $11 / 72$ & $5 / 77$ & 2.35 (0.86 to 6.44$)$ \\
\hline \multicolumn{5}{|c|}{$\begin{array}{l}\text { CIB, cisplatin, ifosfamide and bleomycin; GC, gemcitabine, cisplatin; IP, cisplatin, ifosfamide; ITP, cisplatin, ifosfamide, } \\
\text { paclitaxel; PC, paclitaxel, cisplatin; TC, topotecan, cisplatin; VC, vinorelbine, cisplatin. }\end{array}$} \\
\hline \multicolumn{5}{|c|}{ a Nausea and vomiting. } \\
\hline \multicolumn{5}{|l|}{ b Nausea. } \\
\hline \multicolumn{5}{|l|}{ c Vomiting. } \\
\hline
\end{tabular}

gemcitabine/cisplatin arms compared with the paclitaxel/cisplatin arm (Table 34). No significant differences in frequency of non-haematological adverse drug reactions between the chemotherapeutic arms was observed in the other RCTs. In Mountzios et al. ${ }^{79}$ alopecia occurred in 48 out of 72 patients in the cisplatin and ifosfamide arm, and 52 out of 77 patients in the cisplatin, ifosfamide and paclitaxel arm.

\section{Effectiveness of other platinum agents}

\section{Characteristics of included studies}

Three RCTs evaluated the effectiveness of other platinum agents as palliative treatment for recurrent, persistent or advanced cervical cancer. ${ }^{68,74,81}$ Baseline characteristics including previous treatment and stage and site of disease are presented in Table 35 showing that the groups were comparable in each of the included studies. Chemotherapy as previous treatment was given in the study by Lira-Puerto et al. ${ }^{68}$ and radiotherapy and surgery were given in all trials.

\section{Quality of studies}

The description of randomisation was provided only in McGuire et al. ${ }^{74}$ None of the trials gave any details of the method of allocation concealment. In the study by Lira-Puerto et al., ${ }^{68}$ accrual was suspended in two institutions because of termination of support. The results of the quality assessment are provided in Figures 20 and 21. 
TABLE 35 Characteristics of the populations in the included RCTs: other platinum-agents

\begin{tabular}{|c|c|c|c|c|c|c|}
\hline \multirow[b]{2}{*}{ Parameter } & \multicolumn{2}{|c|}{ McGuire $1989^{74}$} & \multicolumn{2}{|c|}{ Lira-Puerto $1991^{68}$} & \multicolumn{2}{|c|}{ Thomsen $1998^{81}$} \\
\hline & CBDCA & CHIP & CBDCA & CHIP & CBDCA & $\mathrm{T}$ \\
\hline $\begin{array}{l}\text { Number of patients } \\
\text { (randomised) }\end{array}$ & 175 & 177 & 48 & 41 & 13 & 15 \\
\hline $\begin{array}{l}\text { Age (years), median } \\
\text { (range) }\end{array}$ & $47(23-74)$ & $49(25-94)$ & $48(26-67)$ & $44(30-59)$ & $52(33-62)$ & $52(31-71)$ \\
\hline \multicolumn{7}{|l|}{ Previous treatment } \\
\hline Chemotherapy & NR & NR & 5 & 2 & $N R$ & NR \\
\hline Radiotherapy & $159(91 \%)$ & $164(93 \%)$ & 47 & 41 & $83 \%$ & $86 \%$ \\
\hline Surgery & $108(62 \%)$ & $101(57 \%)$ & 2 & 4 & $25 \%$ & $29 \%$ \\
\hline $\begin{array}{l}\text { Chemotherapy and } \\
\text { radiotherapy }\end{array}$ & NR & NR & 4 & 2 & NR & NR \\
\hline $\begin{array}{l}\text { Surgery and } \\
\text { radiotherapy }\end{array}$ & NR & NR & $N R$ & NR & NR & NR \\
\hline \multicolumn{7}{|l|}{ Stage } \\
\hline IVB & $N R$ & NR & 0 & 1 & $N R$ & $N R$ \\
\hline Persistent & NR & NR & NR & NR & NR & NR \\
\hline Recurrent & $N R$ & $N R$ & $N R$ & $N R$ & $100 \%$ & $93 \%$ \\
\hline \multicolumn{7}{|l|}{ Site of disease } \\
\hline Pelvic & NR & $N R$ & 23 & 30 & NR & $N R$ \\
\hline Distant & NR & NR & $\begin{array}{l}\text { Lung, 1; } \\
\text { bone, 2; } \\
\text { inguinal } \\
\text { nodes, 6; } \\
\text { para-aortic } \\
\text { nodes, 1; } \\
\text { distant nodes, } \\
\text { 4; other, } \\
\text { 3; fibrosis } \\
\text { only, } 1\end{array}$ & $\begin{array}{l}\text { Lung, 7; } \\
\text { bone, 2; } \\
\text { inguinal } \\
\text { nodes, 8; } \\
\text { para-aortic } \\
\text { nodes, 3; } \\
\text { distant nodes, } \\
\text { 9; other, } \\
\text { 4; fibrosis } \\
\text { only, } 1\end{array}$ & NR & NR \\
\hline Both & NR & NR & 14 & 9 & NR & NR \\
\hline
\end{tabular}

\section{Effectiveness results}

\section{Overall and progression-free survival}

All RCTs reported overall survival and two reported progression-free survival (Tables 36 and 37). There was little difference in overall survival or progression-free survival between arms in each RCT. Hazard ratio results were not supplied for any of the included studies.

\section{Response rate}

Two of the RCTs 68,74 gave response rates, complete response rates and partial response rates for the same treatment comparisons and so meta-analysis was possible (Figures 22-24). There were no statistically significant differences in terms of frequency of response rate (overall, partial, complete) between these cisplatin agents. However, it should be noted that there were differences between studies in the frequency of response rate in the CBDCA arms (ranging from 15\% to 33\%) as well as in the iproplatin (CHIP) arms 


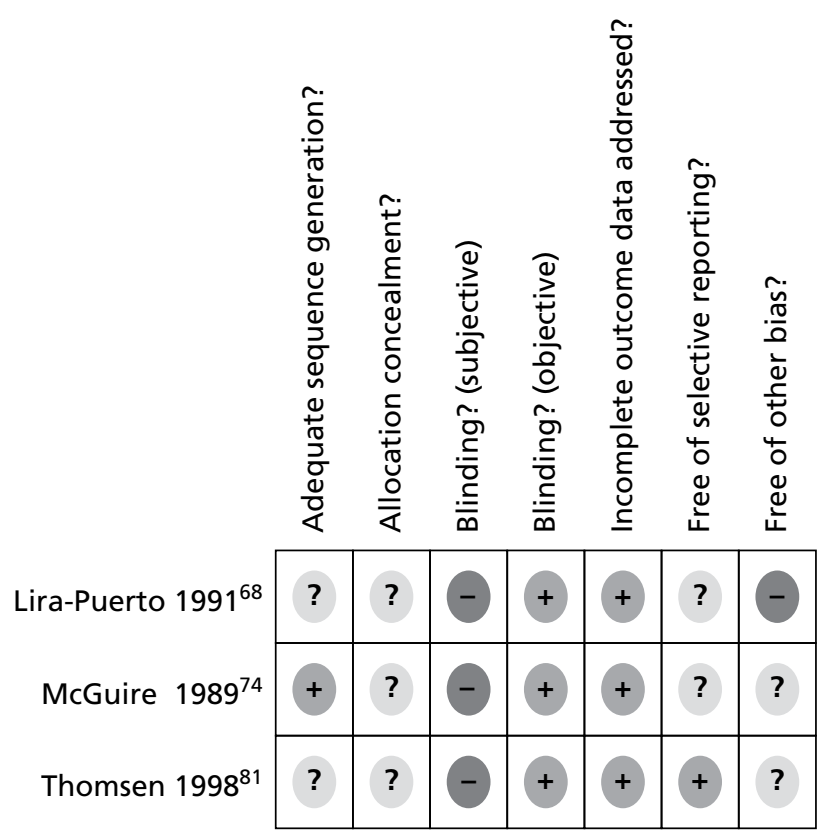

FIGURE 20 Methodological quality on individual items for the three included RCTs: ${ }^{68,74,81}$ other platinum agents.

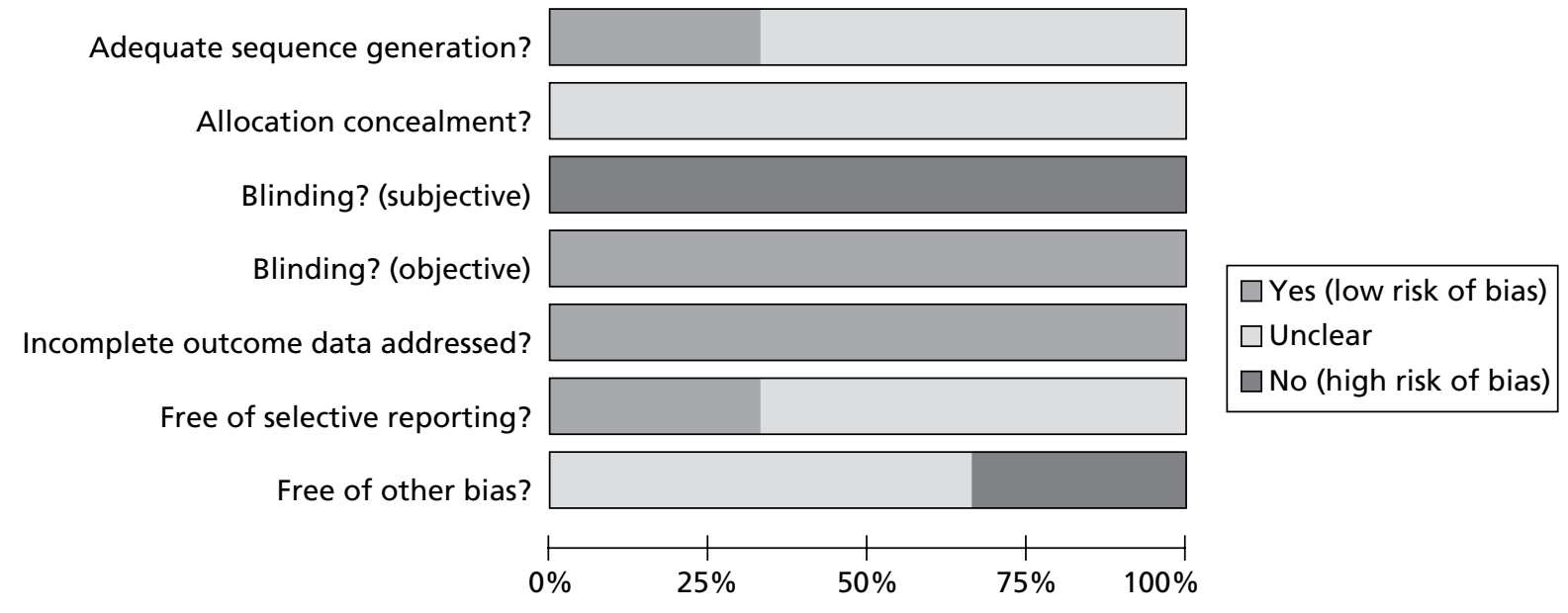

FIGURE 21 Summary of the quality and reporting assessment of the three included studies: ${ }^{68,74,81}$ other platinum agents.

$(11-30 \%)$, and in the frequency of partial response rate in the CBDCA arms (10-33\%) and the CHIP arms (7-25\%).

\section{Quality of life}

None of the studies assessed quality of life.

\section{Adverse events}

Data on haematological toxicity was supplied for all trials. Lira-Puerto et al. ${ }^{68}$ reported drug reactions of Eastern Cooperative Oncology Group (ECOG) grade 2 or more. Meta-analysis of thrombocytopenia rates comparing CBDCA with CHIP indicated no statistical differences between chemotherapeutic agents (Figure 25). There were no differences in leucopenia rates in two RCTs (CBDCA arm 17/176 vs CHIP arm $8 / 180 ; 7^{74}$ CBDCA arm 0/12 vs teniposide arm 1/14 ${ }^{81}$ ). Neurological adverse events (grades 2, 3 or 4 ) were 
TABLE 36 Overall survival: other platinum agents

\begin{tabular}{|c|c|c|c|}
\hline Study & Comparison & $\begin{array}{l}\text { Median OS, } \\
\text { months (range) }\end{array}$ & Hazard ratio $(95 \% \mathrm{Cl})$ \\
\hline \multirow[t]{2}{*}{ McGuire $1989^{74}$} & CBDCA & 6.2 & \multirow[t]{2}{*}{$N R$} \\
\hline & CHIP & 5.5 & \\
\hline \multirow[t]{2}{*}{ Lira-Puerto $1991^{68}$} & CBDCA & 7.5 & \multirow[t]{2}{*}{$N R$} \\
\hline & $\mathrm{CHIP}$ & 7.6 & \\
\hline \multirow[t]{2}{*}{ Thomsen $1998^{81}$} & CBDCA & $40(20-49)^{a}$ & \multirow[t]{2}{*}{ NR } \\
\hline & $\mathrm{T}$ & $41(34-56)^{a}$ & \\
\hline
\end{tabular}

TABLE 37 Progression-free survival: other platinum agents

\begin{tabular}{|c|c|c|c|}
\hline Study & Comparison & $\begin{array}{l}\text { Median PFS, } \\
\text { months (range) }\end{array}$ & Hazard ratio $(95 \% \mathrm{Cl})$ \\
\hline \multirow[t]{2}{*}{ McGuire $1989^{74}$} & CBDCA & 2.7 & \multirow[t]{2}{*}{ NR } \\
\hline & $\mathrm{CHIP}$ & 3 & \\
\hline \multirow[t]{2}{*}{ Thomsen $1998^{81}$} & CBDCA & $20(11-31)^{a}$ & \multirow[t]{2}{*}{ NR } \\
\hline & $T$ & $17(12-32)^{a}$ & \\
\hline
\end{tabular}

seen in 1 out of 47 patients treated with CBDCA and 6 out of 41 patients treated with CHIP in the study by Lira-Puerto et al. ${ }^{68}$ and in 6 out of 176 and 6 out of 180 patients, respectively, in the study by McGuire et al. ${ }^{74}$ Gastrointestinal adverse events such as nausea and vomiting were experienced less often in patients receiving CBDCA (57/176) than in patients receiving CHIP (95/180) [RR 0.61 (95\% Cl 0.48 to 0.79$)$ ] in the study by McGuire et al. ${ }^{74}$ but there was no difference in gastrointestinal adverse events between CBDCA $(2 / 12)$ and teniposide (2/14) in the study by Thomsen and Pfeiffer. ${ }^{81}$

\section{Effectiveness of non-platinum agents}

\section{Characteristic of included studies}

Four studies gave evidence on the effectiveness of non-platinum agents for the treatment of recurrent, persistent or advanced cervical cancer. ${ }^{61,67,75,83}$ Because two studies ${ }^{67,83}$ were, unfortunately, impossible to obtain, analysis was conducted on the basis of the systematic review by Hirte et al. ${ }^{59}$ Baseline characteristics including previous treatment and stage and site of disease are presented in Table 38; however, not all relevant clinical information was presented in all publications.

\section{Quality of studies}

As full texts were impossible to obtain for Greenberg et al. ${ }^{67}$ and Wallace et al. ${ }^{83}$ the quality assessment was based on the systematic review by Hirte et al. ${ }^{59}$ A description of the allocation concealment procedure was not reported in any of the RCTs and blinding was not used in any of the RCTs. In Barlow et al., ${ }^{61}$ two patients with squamous cell tumours were mistakenly randomised as non-squamous cell tumours and received adriamycin (ADM) alone (group of $21+2$ patients). In Monk et al., ${ }^{75}$ patients were initially randomly assigned to combination and monotherapy arms. The protocol was later amended after receiving 


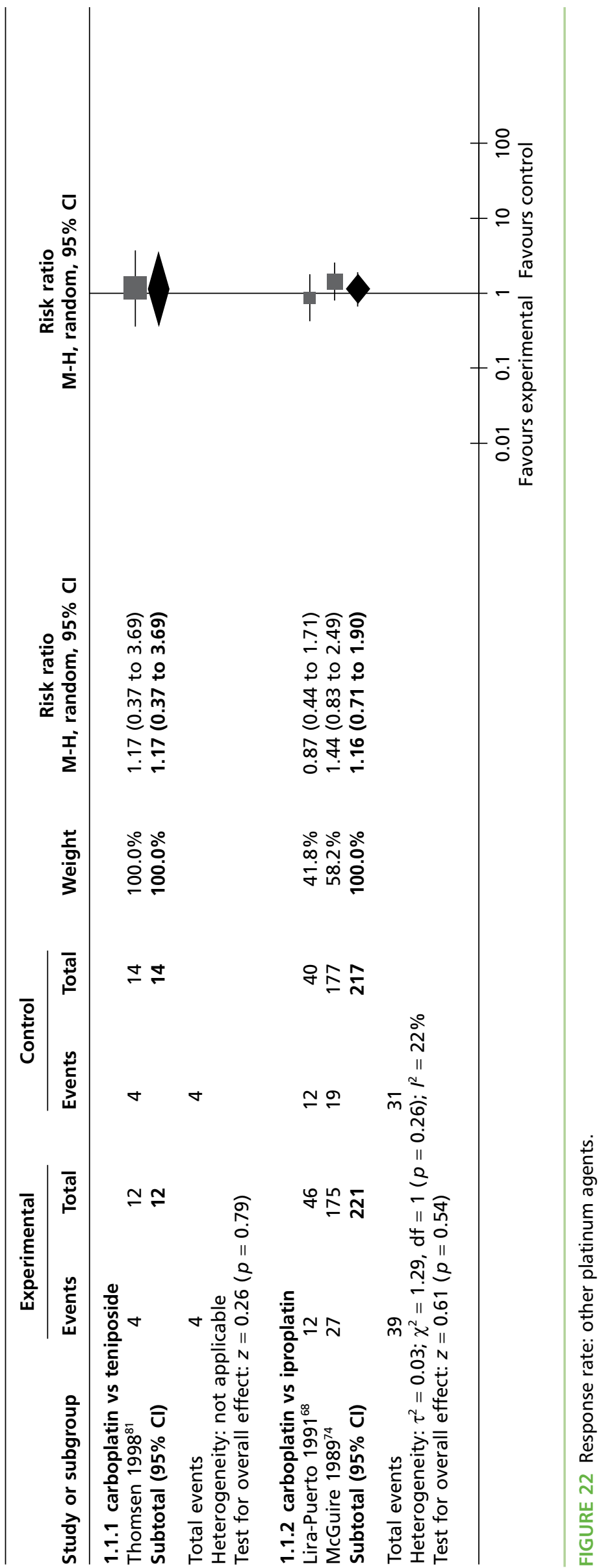




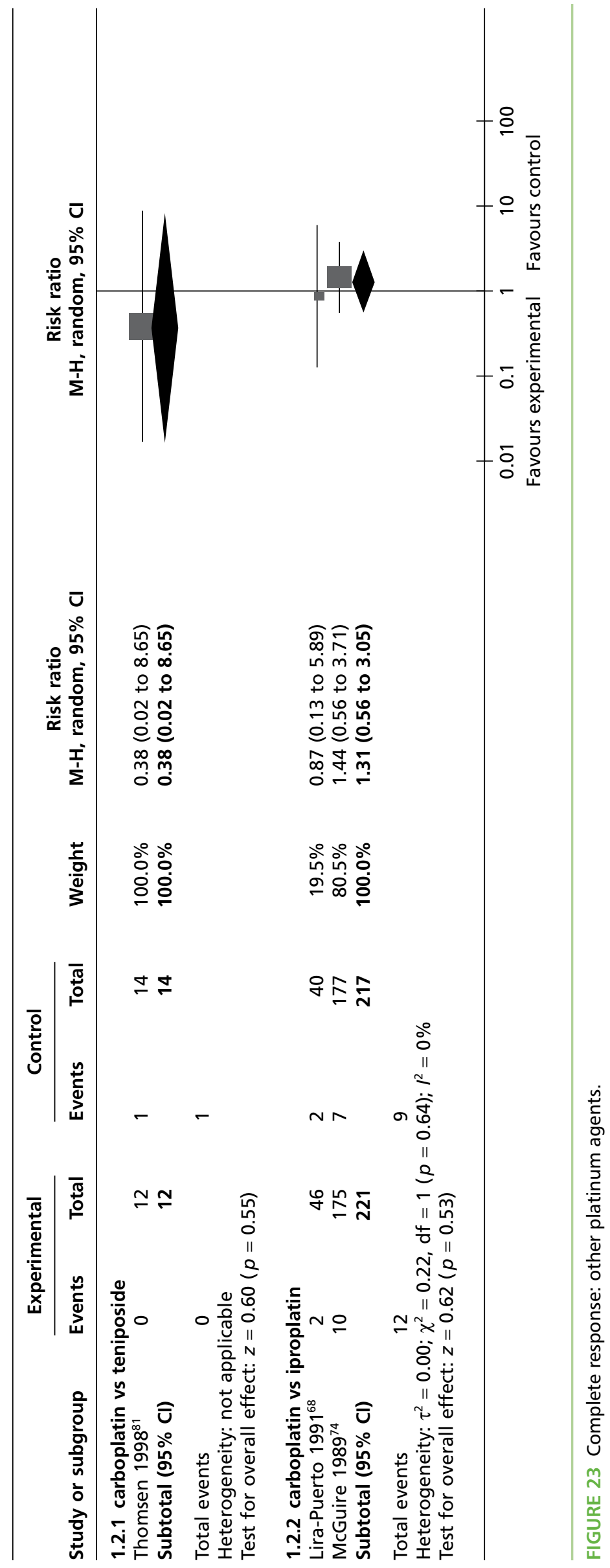




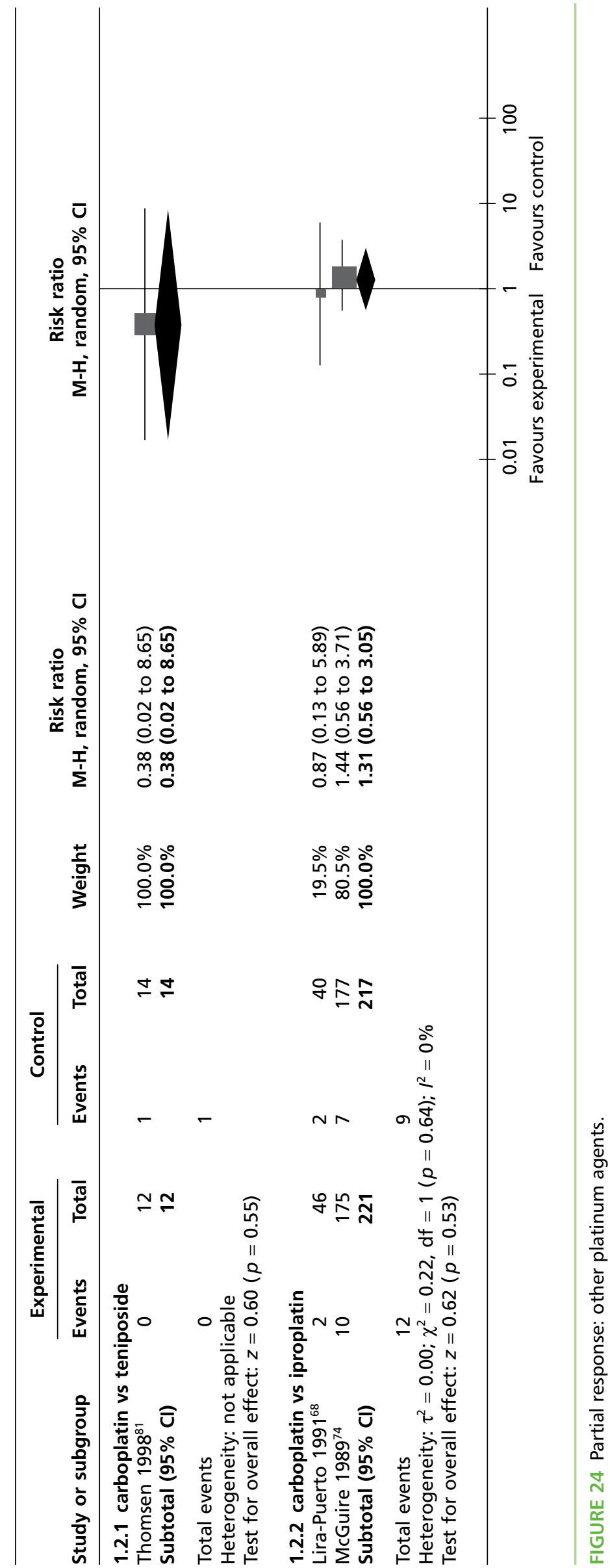




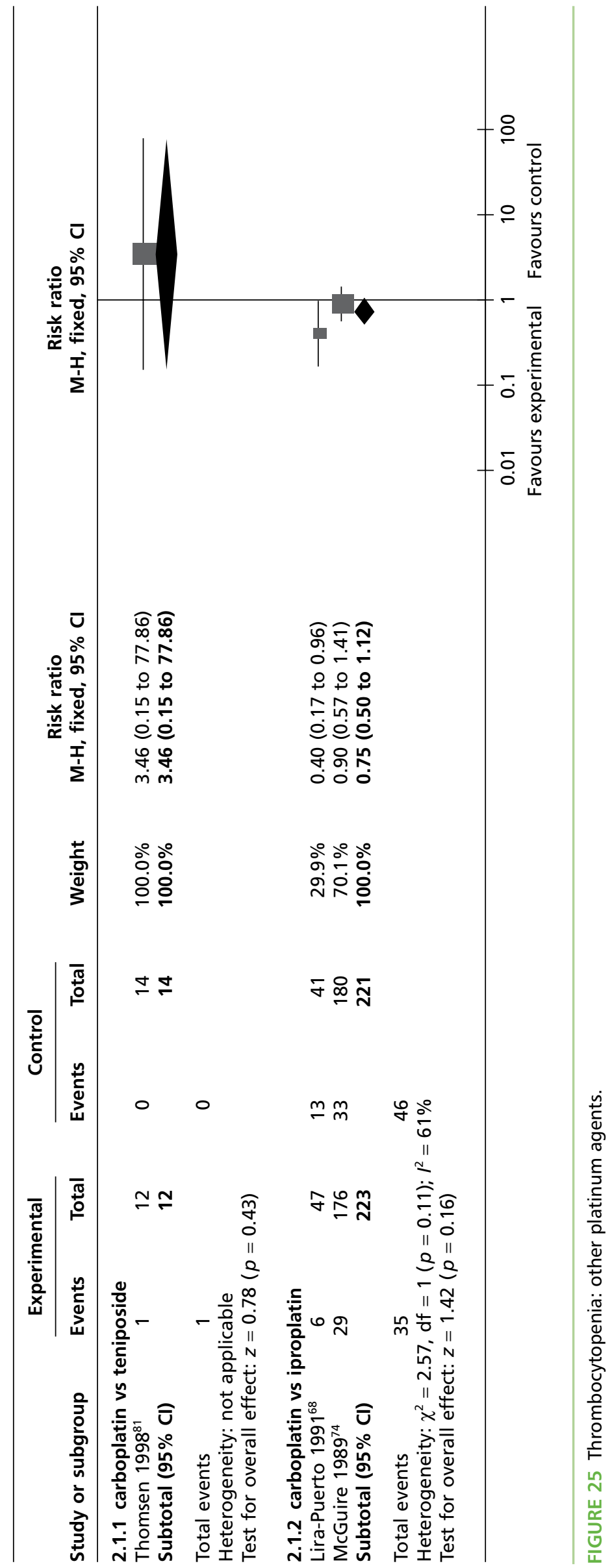




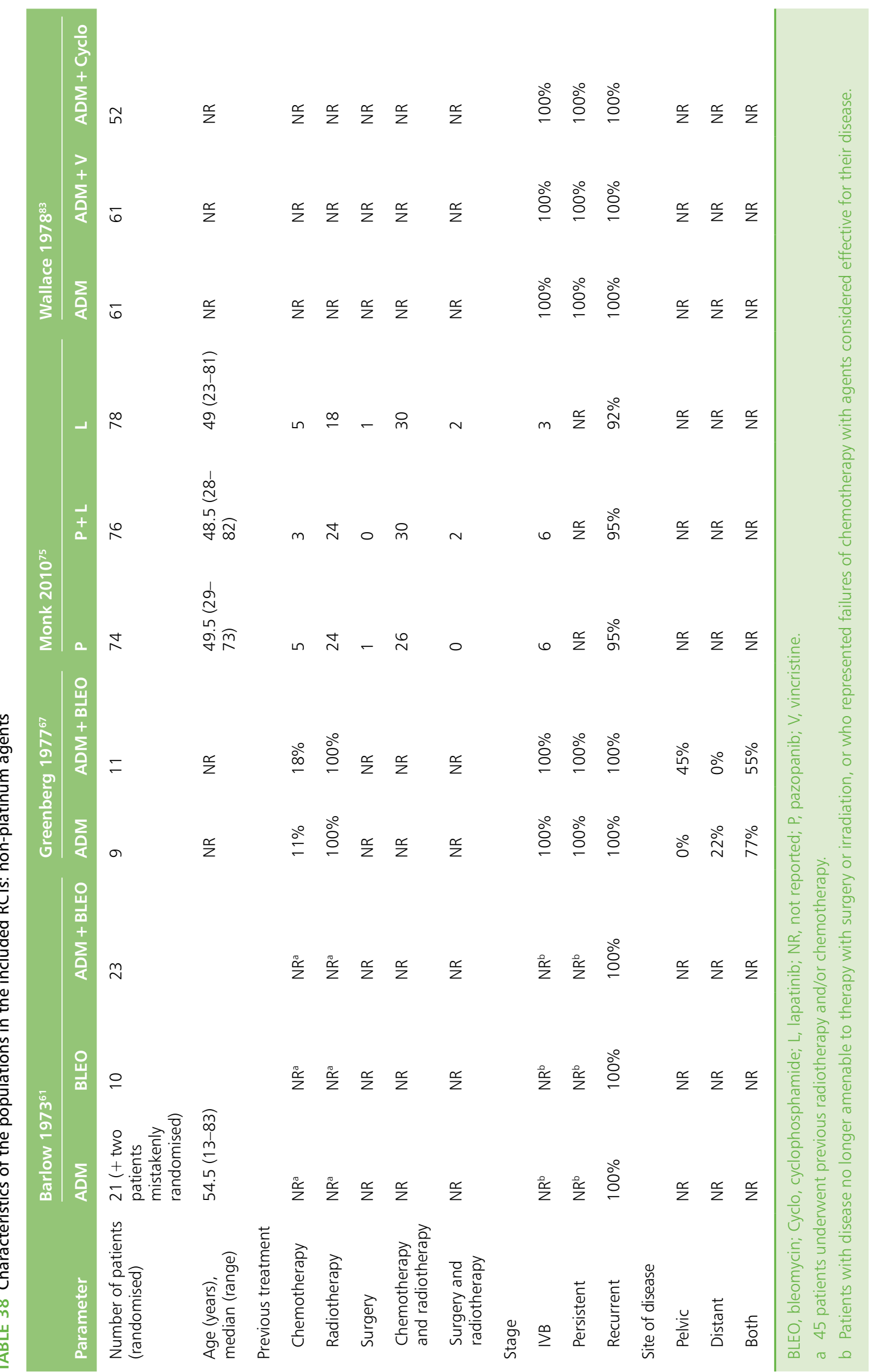

(c) Queen's Printer and Controller of HMSO 2013. This work was produced by Meads et al. under the terms of a commissioning contract issued by the Secretary of State for Health. This issue may be freely reproduced for the purposes of private research and study and extracts (or indeed, the full report) may be included in professional journals provided that suitable acknowledgement is made and the reproduction is not associated with any form of advertising. Applications for commercial reproduction should be addressed to: NIHR Journals Library, National Institute for Health Research, Evaluation, Trials and Studies Coordinating Centre, Alpha House, University of Southampton Science Park, Southampton SO16 7NS, UK. 
results of a formal interim analysis and combination therapy was discontinued. In the same study, the unconfirmed response rate (not verified on a second scan) was 19\% for pazopanib-treated patients and $9 \%$ for lapatinib-treated patients. The results of the quality assessment are shown in Figures 26 and 27.

\section{Effectiveness results}

\section{Overall and progression-free survival}

Barlow et al. ${ }^{61}$ did not report overall survival or progression-free survival and Greenberg et al. ${ }^{67}$ did not report progression-free survival. Monk et al. ${ }^{75}$ demonstrated better overall survival and progressionfree survival with pazopanib than with lapatinib. Hazard ratios were not supplied for any of the other RCTs. ${ }^{61,67,83}$ The overall survival results are shown in Table 39 and the progression-free survival results are shown in Table 40.

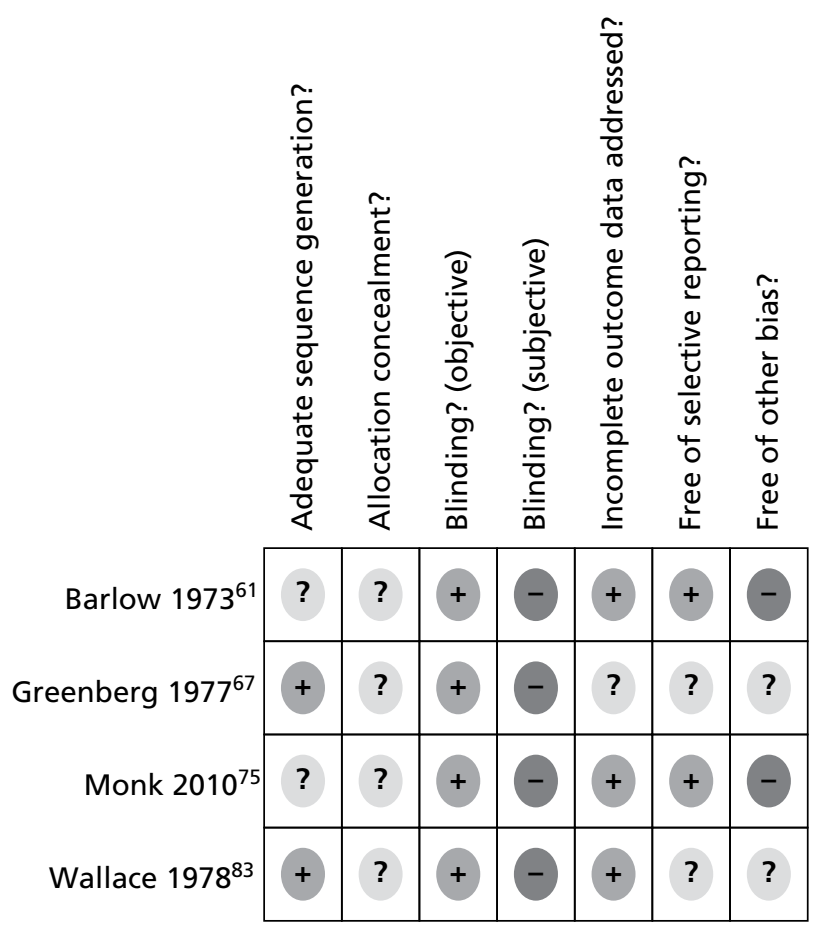

FIGURE 26 Methodological quality on individual items for the four included RCTs: ${ }^{61,67,75,83}$ non-platinum agents.

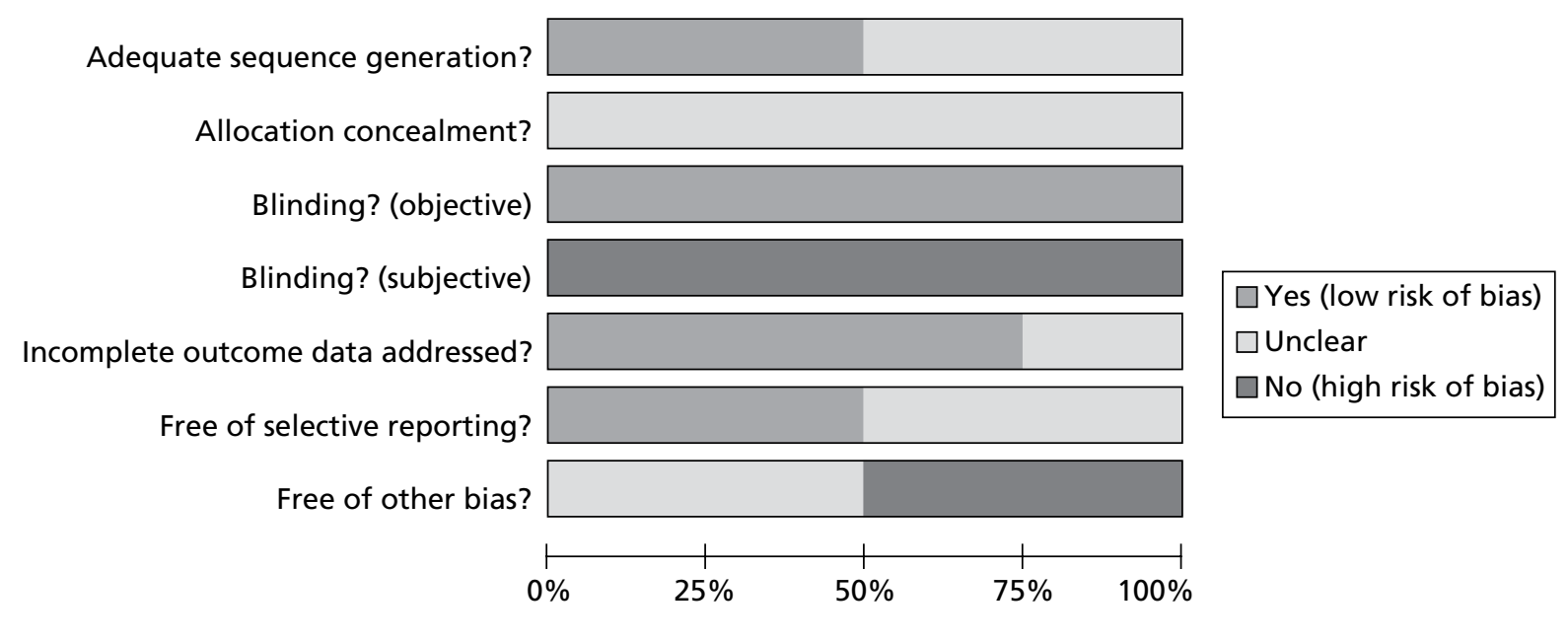

FIGURE 27 Summary of the quality assessment of the four included RCTs: ${ }^{61,67,75,83}$ non-platinum agents. 
TABLE 39 Overall survival: non-platinum agents

\begin{tabular}{|c|c|c|c|}
\hline Study & Comparison & Median OS, months (range) & Hazard ratio $(95 \% \mathrm{Cl})$ \\
\hline \multirow[t]{2}{*}{ Greenberg $1977^{67}$} & ADM & 4 & \multirow[t]{2}{*}{ NR } \\
\hline & $A D M+B L E O$ & 4.3 & \\
\hline \multirow[t]{3}{*}{ Monk $2010^{75}$} & $P$ & $50.7^{\mathrm{a}}$ & \multirow[t]{3}{*}{0.67 (0.56 to 0.99$)$} \\
\hline & $P+L$ & NR & \\
\hline & L & $39.1^{\mathrm{a}}$ & \\
\hline \multirow[t]{3}{*}{ Wallace $1978^{83}$} & ADM & 5.9 & \multirow[t]{3}{*}{ NR } \\
\hline & $A D M+V$ & 5.5 & \\
\hline & ADM + Cyclo & 7.3 & \\
\hline
\end{tabular}

TABLE 40 Progression-free survival: non-platinum agents

\begin{tabular}{|c|c|c|c|}
\hline Study & Comparison & Median PFS, months (range) & Hazard ratio $(95 \% \mathrm{Cl})$ \\
\hline \multirow[t]{3}{*}{ Monk $2010^{75}$} & $P$ & $18.1^{\mathrm{a}}$ & $0.66(0.48$ to 0.91$)$ \\
\hline & $P+L$ & NR & \\
\hline & L & $17.1^{\mathrm{a}}$ & \\
\hline \multirow[t]{3}{*}{ Wallace $1978^{83}$} & ADM & 3.3 & NR \\
\hline & $A D M+V$ & 3.4 & \\
\hline & ADM + Cyclo & 3.9 & \\
\hline
\end{tabular}

\section{Response rate}

There were no statistically significant differences between any of the non-platinum agents in the frequency of overall, complete and partial response rates (Table 41). In Monk et al., ${ }^{75} 9 \%$ of the pazopanib arm and four patients (5\%) in the lapatinib arm achieved a confirmed tumour response. It should be noted that the unconfirmed response rate was 19\% for pazopanib-treated patients and 9\% for lapatinib-treated patients.

\section{Quality of life}

None of the RCTs reported quality of life.

\section{Adverse events}

Haematological grade 3 or 4 adverse events were presented in Monk et al. ${ }^{75}$ and Wallace et al. ${ }^{83}$ No statistically significant differences were observed between the treatment arms for neutropenia (pazapanib arm $2 / 74$ vs lapatinib arm 0/76 75 ), thrombocytopenia ( $A D M+$ vincristine arm 2/61 vs ADM arm 4/61 vs $A D M+$ cyclophosphamide arm 1/5283), leucopenia (ADM + vincristine arm 15/61 vs ADM arm 15/61 vs $A D M+$ cyclophosphamide arm 12/5283) and anaemia (pazapanib arm 2/74 vs lapatinib arm 4/7675). The frequency of non-haematological adverse events (grade 3 or above) was low in patients receiving non-platinum agents. The most common adverse event was diarrhoea (pazapanib arm 8/74 vs lapatinib arm 10/76 ${ }^{75}$ ). In Monk et al. ${ }^{75}$ the results for nausea were 2 out of 74 patients in the pazapanib arm compared with 1 out of 76 patients in the lapatinib arm; for anorexia there were 2 out of 74 patients in 
TABLE 41 Response rates (RRs): non-platinum agents

\begin{tabular}{|c|c|c|c|c|}
\hline Study & Comparison & $\begin{array}{l}\text { Response rate } \\
(95 \% \mathrm{Cl})\end{array}$ & $\begin{array}{l}\text { Complete response } \\
\text { rate }(95 \% \mathrm{Cl})\end{array}$ & $\begin{array}{l}\text { Partial response rate } \\
(95 \% \mathrm{CI})\end{array}$ \\
\hline \multirow[t]{3}{*}{ Barlow $1973^{61}$} & ADM vs BLEO & $3.89(0.22$ to 68.67$)$ & 2.33 (0.11 to 48.99$)$ & 2.33 (0.11 to 48.99$)$ \\
\hline & $A D M+B L E O$ vs ADM & $0.53(0.09$ to 3.11$)$ & 0.53 (0.04 to 7.44$)$ & 0.53 (0.04 to 7.44$)$ \\
\hline & $A D M+B L E O$ vs BLEO & $2.19(0.12$ to 39.90$)$ & 1.31 (0.06 to 28.41$)$ & 1.31 (0.06 to 28.41$)$ \\
\hline $\begin{array}{l}\text { Greenberg } \\
1977^{67}\end{array}$ & $A D M+B L E O$ vs ADM & $N R$ & Not estimable & $0.08(0.00$ to 1.21$)$ \\
\hline Monk $2010^{75}$ & P vs L & 1.84 (0.56 to 6.04$)$ & 1.05 (0.07 to 16.55$)$ & $2.11(0.55$ to 8.12$)$ \\
\hline \multirow[t]{2}{*}{ Wallace $1978^{83}$} & $A D M+V$ vs $A D M$ & 0.75 (0.34 to 1.65$)$ & $0.14(0.02$ to 1.13$)$ & $1.60(0.55$ to 4.62$)$ \\
\hline & ADM + Cyclo vs ADM & $0.68(0.29$ to 1.61$)$ & $0.50(0.14$ to 1.85$)$ & $0.94(0.27$ to 3.31$)$ \\
\hline
\end{tabular}

BLEO, bleomycin; Cyclo, cyclophosphamide; L, lapatinib; NR, not reported; P, pazopanib; V, vincristine.

the pazapanib arm compared with 1 out of 76 patients in the lapatinib arm; and for vomiting there was 1 out of 74 patients in the pazapanib arm compared with none (out of 76 patients) in the lapatinib arm.

\section{Radiotherapy or chemoradiotherapy}

\section{Study selection}

Included in this section are studies in which participants have recurrent or persistent cervical cancer that was initially treated with surgery and who now have evidence of recurrence. The interventions are radiotherapy or radiotherapy with chemotherapy (chemoradiotherapy). The search for relevant studies did not identify any RCTs but 16 case series met the inclusion criteria: nine evaluated radiotherapy ${ }^{84-92}$ and seven evaluated chemoradiotherapy. ${ }^{93-99}$ The results of the quality assessment for the radiotherapy and chemoradiotherapy studies are presented in Appendix 15.

\section{Radiotherapy}

\section{Population characteristics}

The characteristics of the populations in the radiotherapy studies are presented in Table 42. Most of the nine case series ${ }^{84-92}$ included a small number of patients (median 82 cases, range 18-130 cases). Study locations included the UK, the USA, Japan, the Netherlands and Germany. The majority of women presented with early-stage cervical cancer, but in three studies ${ }^{86,87,91}$ there was no information about FIGO stage. The proportion of patients with recurrent or persistent disease in the pelvis as the only site of cancer (central recurrence) was lower than the proportion developing distant metastases. Patients with central recurrence, defined as confined to the vagina or paravaginal tissues not extending to the pelvis, constituted $44 \%$ of the total population in the included studies. Squamous cell carcinoma was the most common histological type of cancer, being present in $79 \%$ of patients; adenocarcinoma was present in $10.33 \%$. The histological type was not available in four studies.

\section{Description of the intervention}

Descriptions of the salvage radiotherapy in curative intent and previous surgery are provided in Table 43. Radical hysterectomy was the most common previous surgery type and was performed in $61.8 \%$ of patients. 


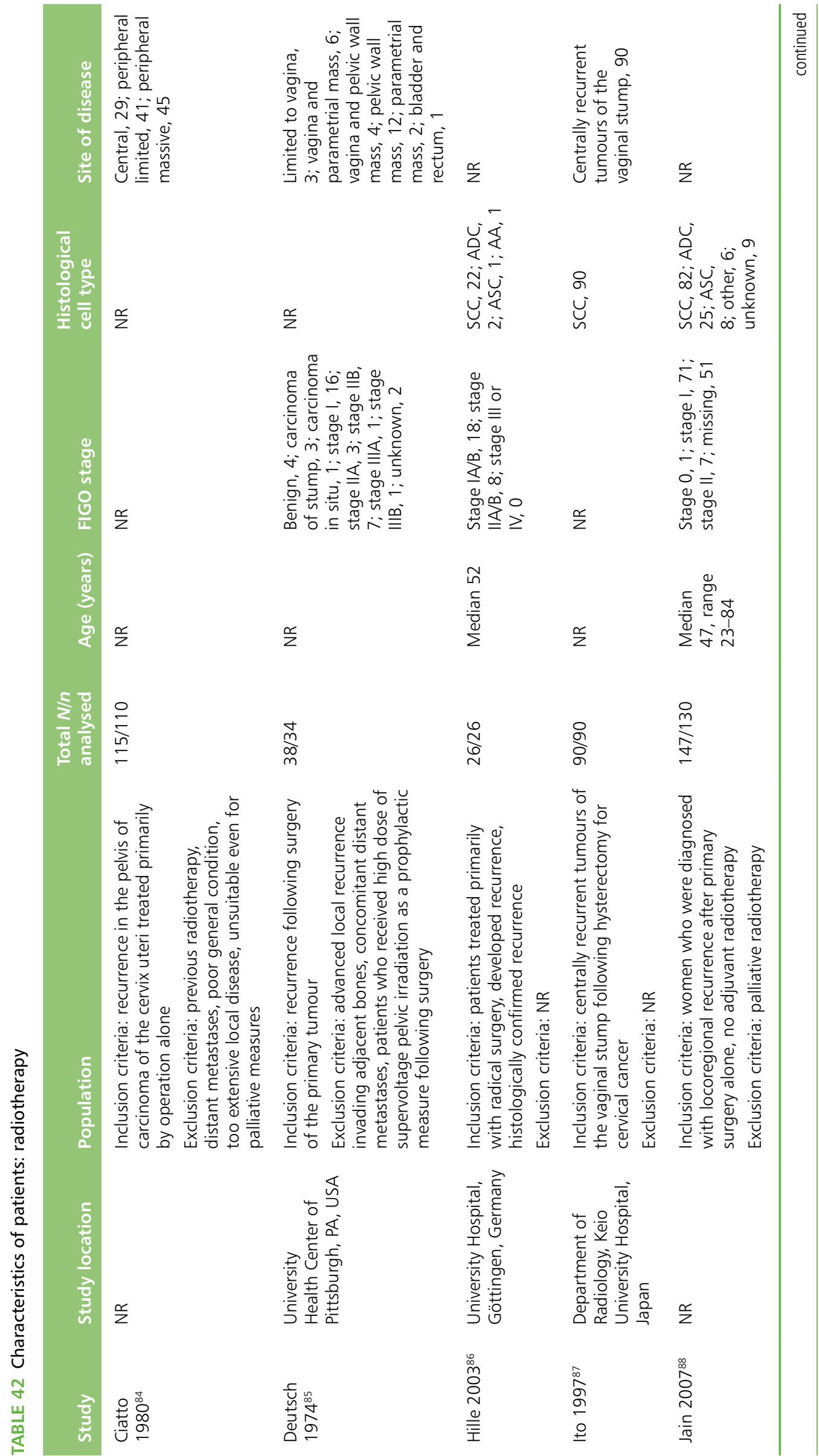




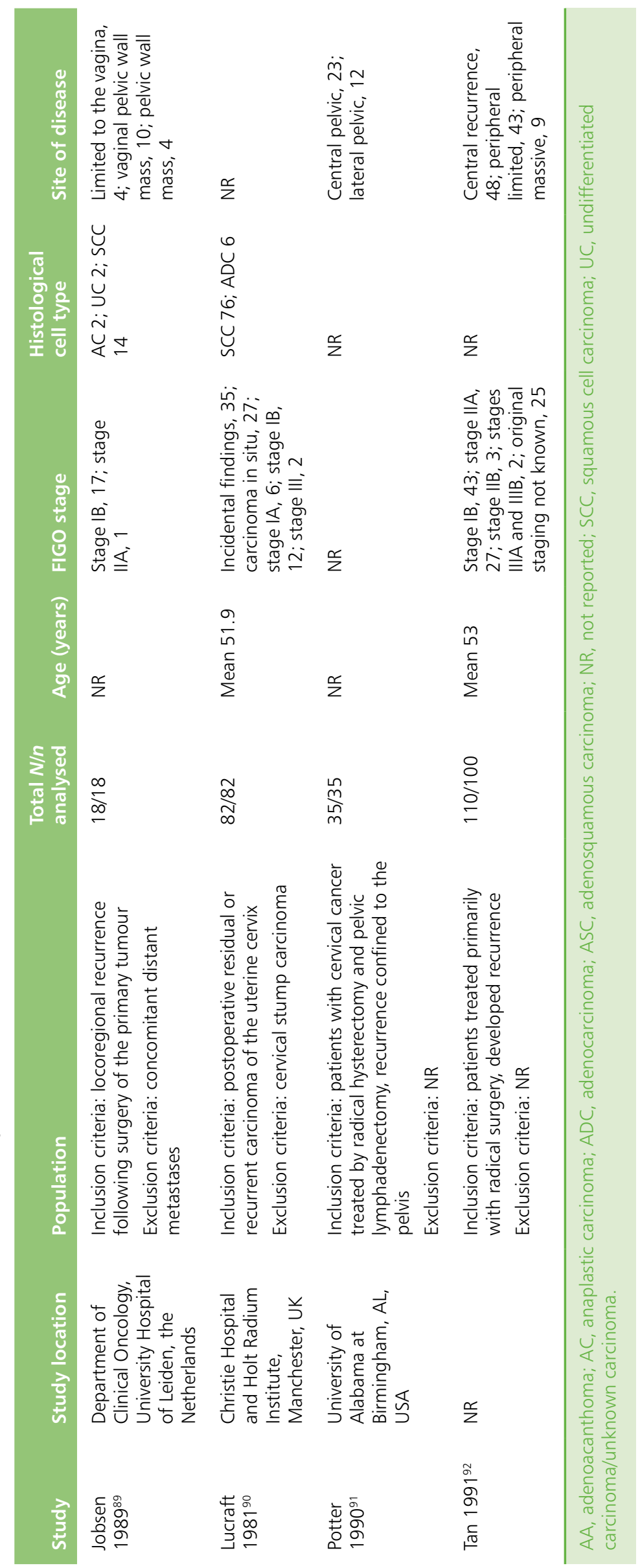



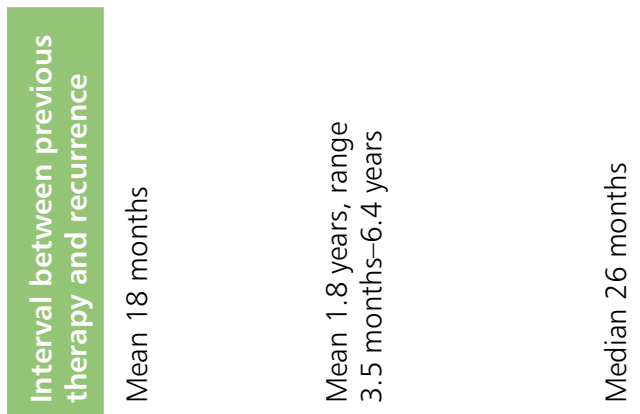

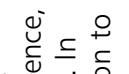

는

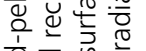

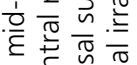

원 仓

प्रे

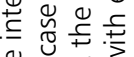

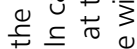

ทे घे 윙

¿

ขo mo

吾 ᄂ.

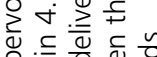

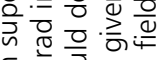

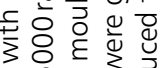

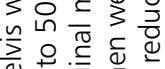

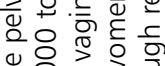

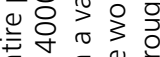

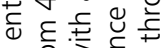

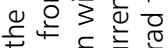

$\frac{1}{0} \frac{1}{\frac{1}{2}} \quad \frac{\pi}{0}$

离定

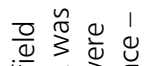

† ख्ये

웠ํㄹㄹ

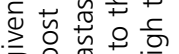

万⿺辶⿻

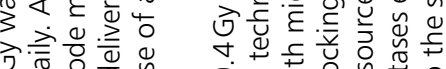

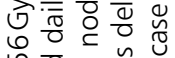

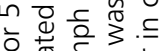

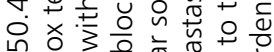

으운

.

음 으ㅇㅝㅗ

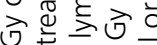

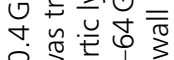

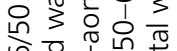

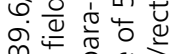

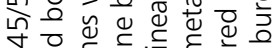

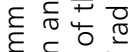

约紊亦

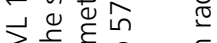

$\because$ 는

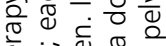

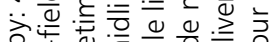

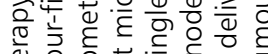

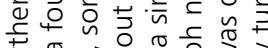

苍实产

论

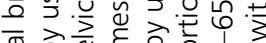

$>0$ 응요

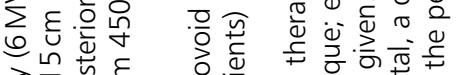

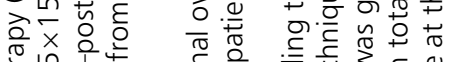

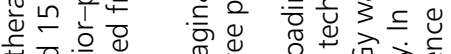

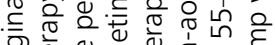

ग)

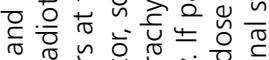

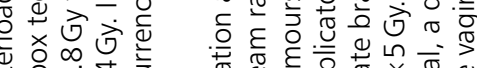

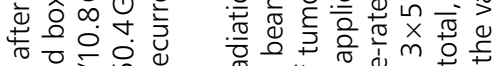

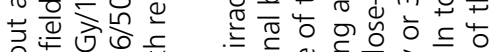

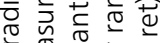

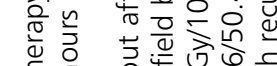

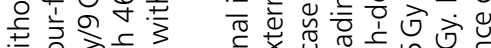

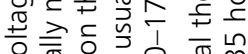

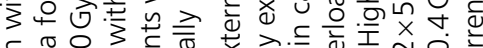

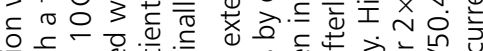

뭉ㅇㅇ

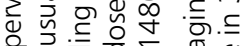

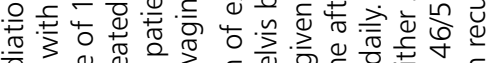

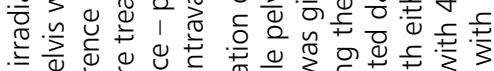

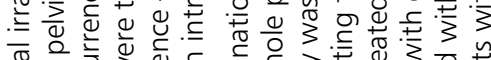

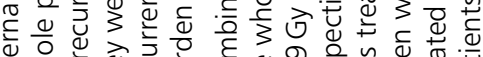

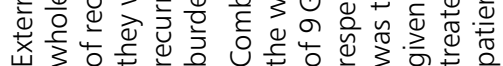
至

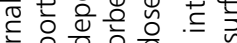

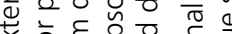

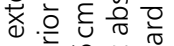

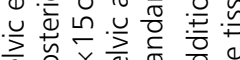

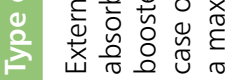

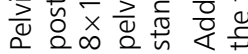

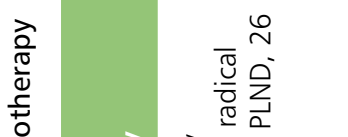

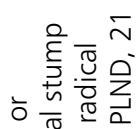

$\ddot{\sim}$

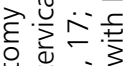

प्ष

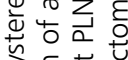

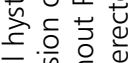

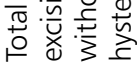

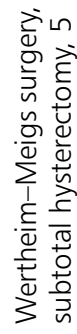

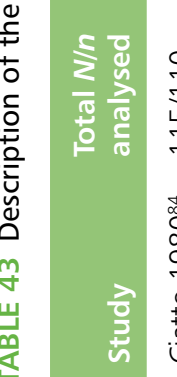

응 荠

$\underset{\substack{m \\ \infty}}{\substack{m \\ m}}$

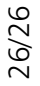

돈

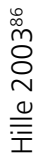

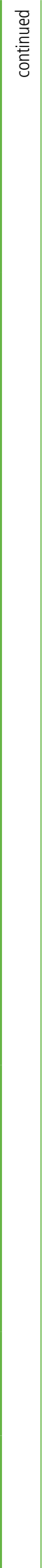




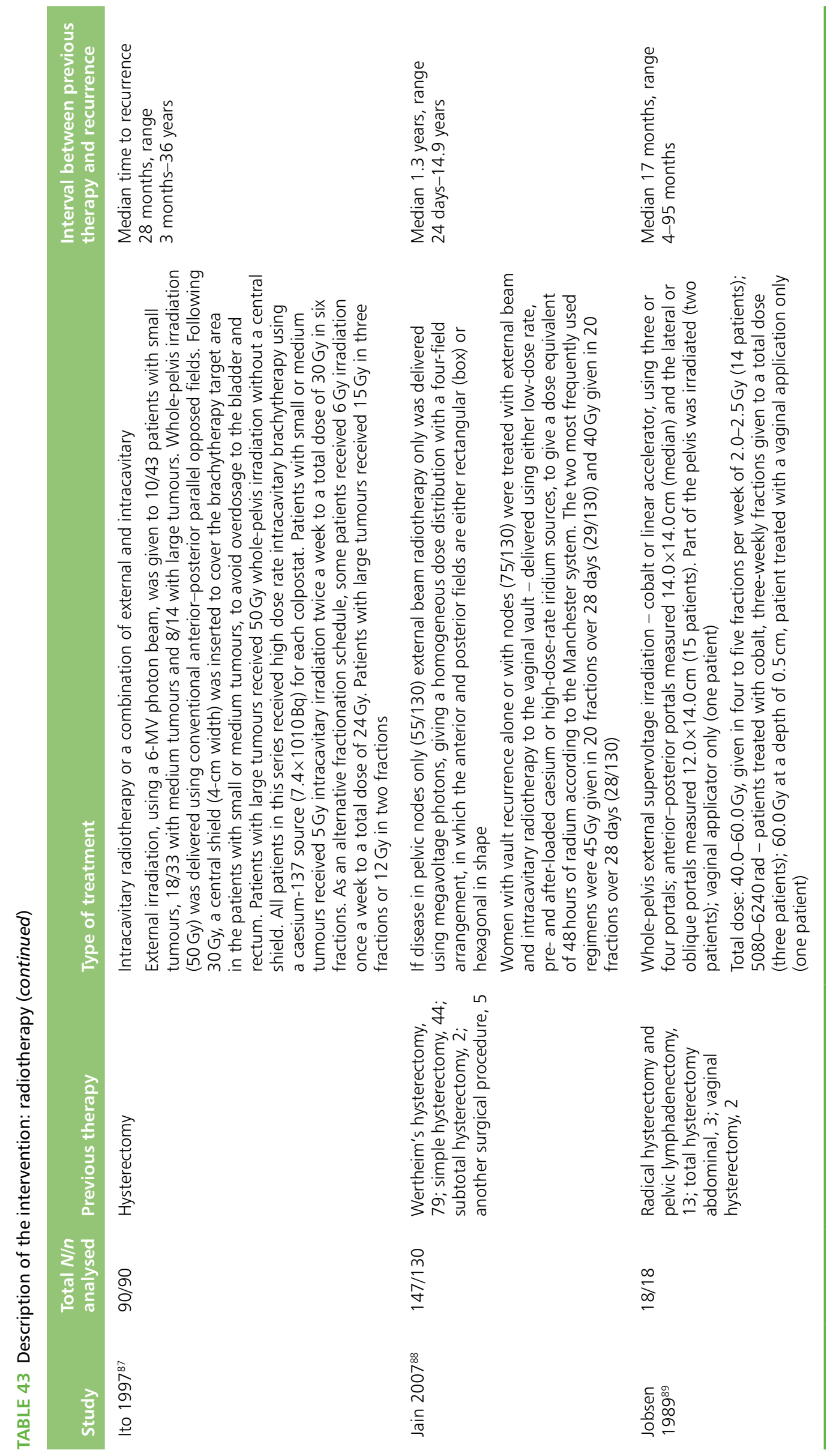


$\frac{\infty}{2}$

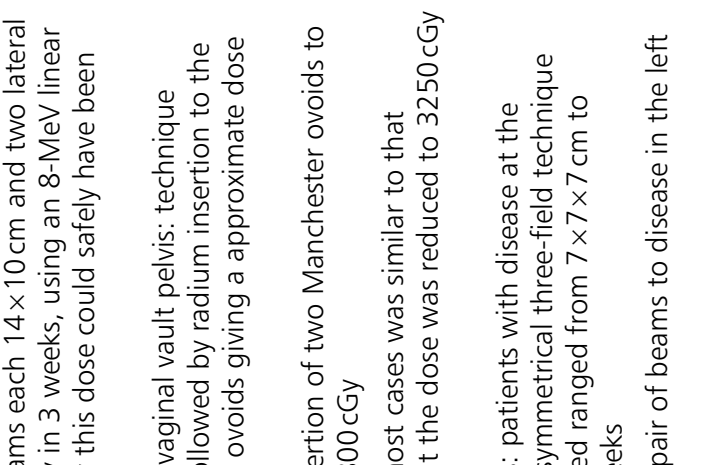

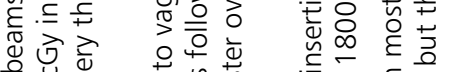

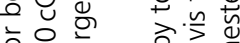

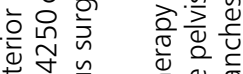

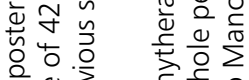

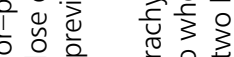

每

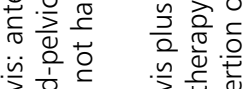

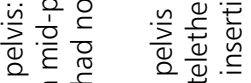

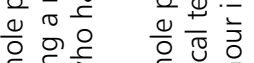

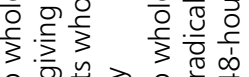

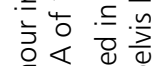

它.

o 은

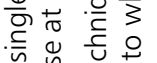

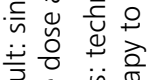

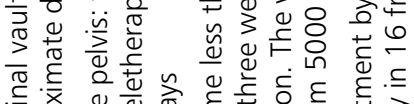

志

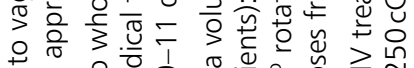

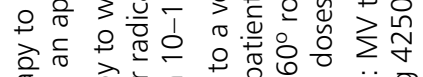

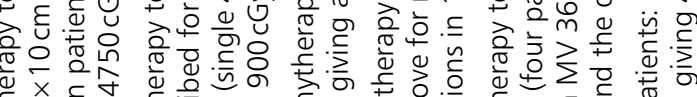

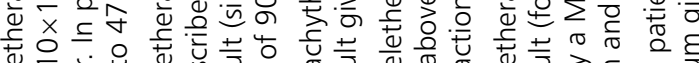

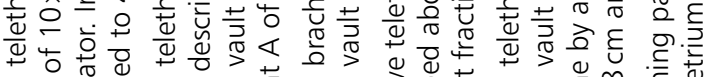

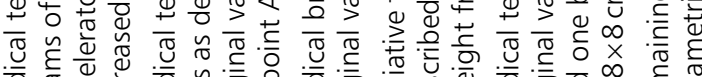

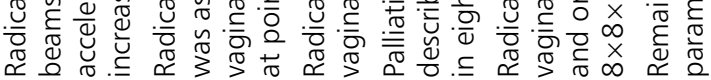

$\stackrel{\propto}{z}$

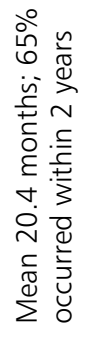

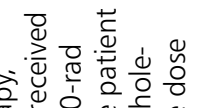

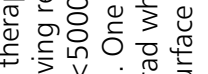

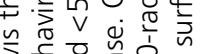

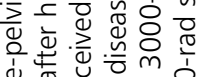
d

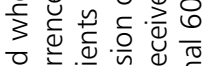

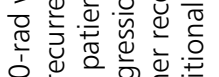

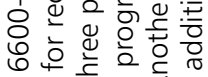

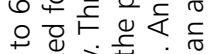

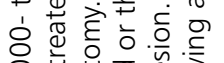

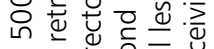

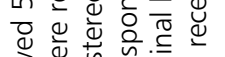

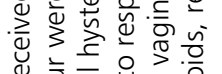

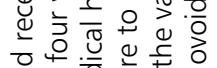

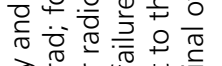

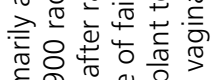

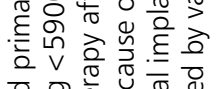

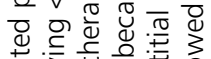

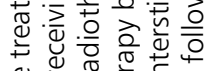

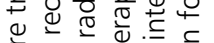

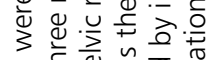

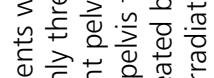
岕 m

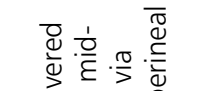

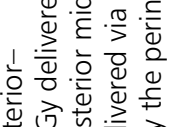

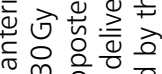

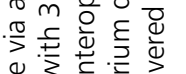

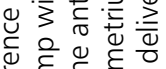

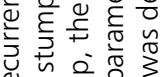

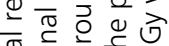

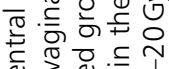

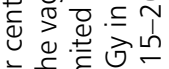

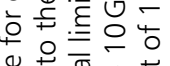

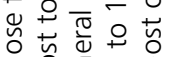

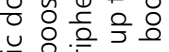

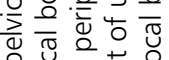

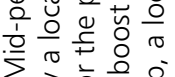

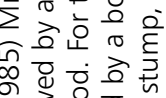

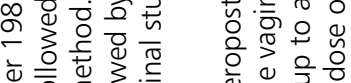

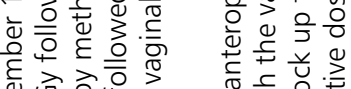

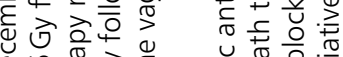

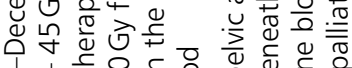
1)

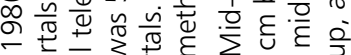

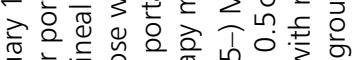

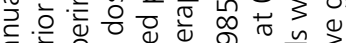

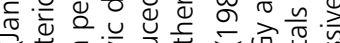

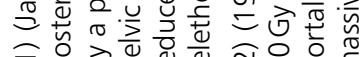
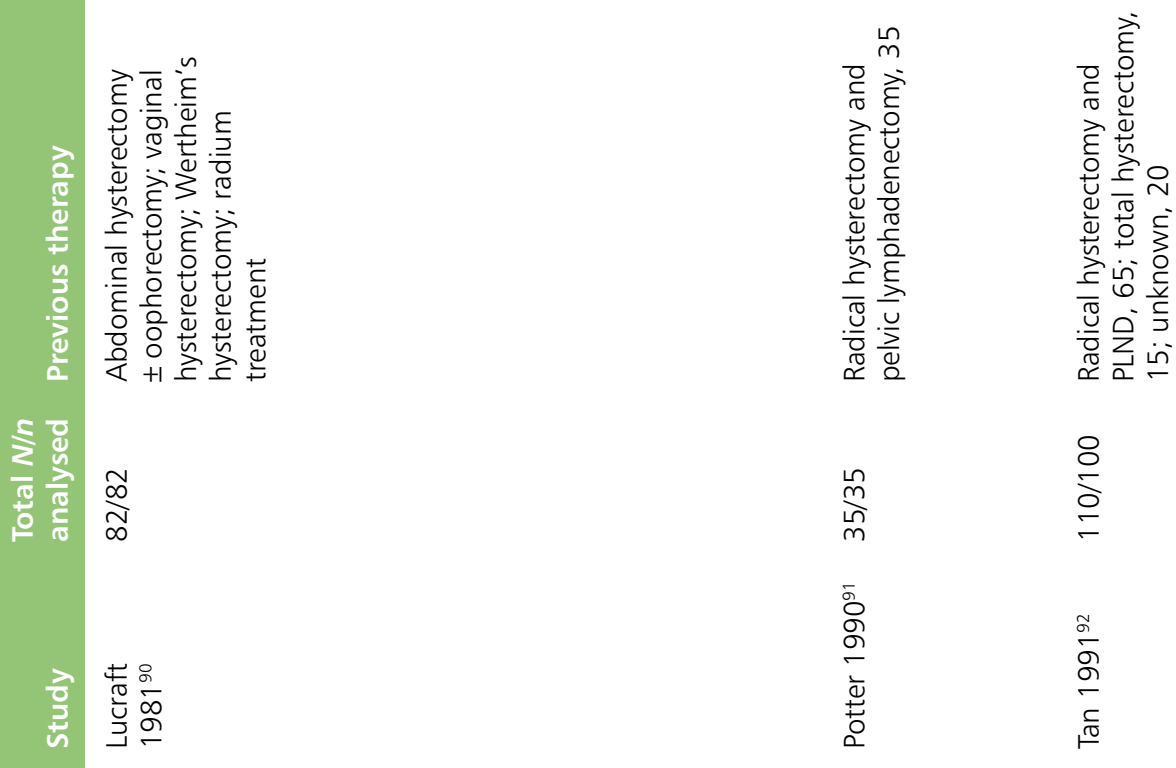

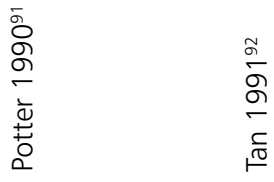




\section{Results}

Overall survival and progression-free survival The reported 2-year survival rates ranged from $12 \%$ to $85 \%$ and 5 -year survival rates ranged from $2 \%$ to $82 \%$. Patients with pelvic side wall recurrences were found to have poorer prognoses than patients with central recurrences only (range $2-15 \%$ and $42-82 \%$, respectively, for 5 -year survival). Data for 10-year overall survival were provided in Ito et al., ${ }^{87}$ with a $52 \%$ rate for patients with centrally recurrent tumours of the vaginal stump following hysterectomy for cervical cancer. Additionally, they showed that survival was greatly influenced by the tumour size of the vaginal stump so that the 10 -year survival rate of patients with small-sized tumours was $72 \%$, whereas the corresponding survival rate of patients with medium-sized tumours was $48 \%$ (Table 44).

Complications Five studies gave complication rates. ${ }^{86-88,90,92}$ In Hille et al., ${ }^{86}$ grade 3 late toxicity was observed in $8 \%$ of patients, including intestinal bleeding after 7 months and fistulae between the rectosigmoid colon and the vagina, removed subsequently by surgery. In Ito et al., ${ }^{87}$ late complications of radiotherapy, including intestinal and urinary complications of grade 2 and grade 3, occurred in $32 \%$ of patients. In Jain et al. ${ }^{88}$ one patient suffered from morbidity in the sigmoid colon requiring surgical resection and in Lucraft ${ }^{90}$ seven cases of complications were observed, including transient proctitis, intermittent haematuria and severe acute bowel reactions. In Tan et al., ${ }^{92}$ major complications included fistulae, proctitis and cystitis (see Table 44).

\section{Chemoradiotherapy}

\section{Population characteristics}

Seven studies ${ }^{93-99}$ were identified and their baseline patient characteristics are provided in Table 45 . Most included a small number of patients (median 30 cases, range 13-49 cases). Study location included the USA, the UK, Canada, Japan, Italy and the Netherlands. The majority of women presented with earlystage cervical cancer. In three studies ${ }^{93,94,97}$ there were no data on FIGO stage. In each of the studies the proportion of patients with disease recurrent or persistence in the pelvis as the only site of cancer (central recurrence) was lower than the proportion developing distant metastases. Patients with central recurrence constituted $37 \%$ of the total population in the included studies. Squamous cell carcinoma was the most common histological type of cancer, being present in $77 \%$ of patients. The histological type was not available in Haasbeek et al. ${ }^{94}$

\section{Description of the intervention}

Descriptions of the salvage chemotherapy and radiotherapy given are provided in Table 46. Radical hysterectomy was the most common previous surgery type and was performed in $81 \%$ of patients.

\section{Results}

Overall survival and progression-free survival Overall survival and progression-free survival results are presented in Table 47. Results for 2-year survival ranged between $44 \%$ and $93 \%$. Patients with central recurrences had a 63-69\% 5-year survival rate; the rate for studies with mixed recurrence was between $41 \%$ and $47 \%$; and the rate for patients with recurrence extending to the pelvic wall was between $18 \%$ and 28\%. Data for 10-year overall survival were provided in Grigsby et al. ${ }^{93}$ and Haasbeek et al. ${ }^{94}$ with a range of 33-35\% with central recurrence or recurrence extending to pelvic wall. Survival rates were also available for subpopulations of patients with central recurrence $(55 \%)$ and recurrence extending to the pelvic wall (15\%). In Grigsby et al., ${ }^{93} 15$-year overall survival for patients with central or with pelvic wall involvement was $35 \%$. Central recurrences had a higher 5 -year progression-free survival probability than those from all other patients (24-48\% vs $1-27 \%$ ).

Complications Grade 3/4 adverse events were observed in 27\% of patients in Grigsby et al., ${ }^{93} 17 \%$ in Haasbeek et al. ${ }^{94}$ and 31\% in Maneo et al. ${ }^{96}$ Tsuda et al. ${ }^{98}$ presented results for particular adverse events: grade 3/4 leucocytopenia/neutrocytopenia $-66.7 \%$; grade 4 haematological toxicity $-20 \%$; grade 3 


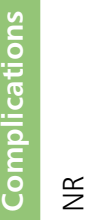

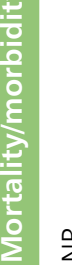

व

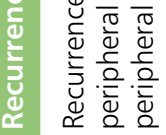

资

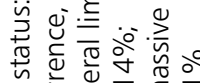

\&

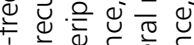

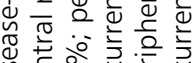

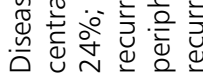

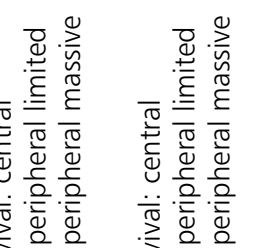

त.

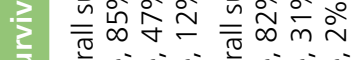
के बं बं के बं बं बं

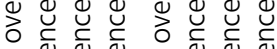
あ

$\stackrel{\stackrel{\circ}{\Sigma}}{\stackrel{\infty}{=}}$

\section{$=$}

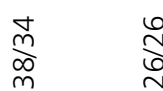

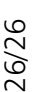

点点

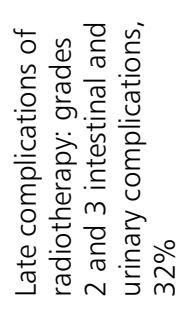

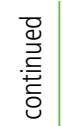

$\stackrel{\infty}{z}$

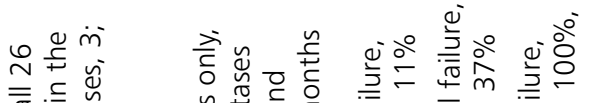

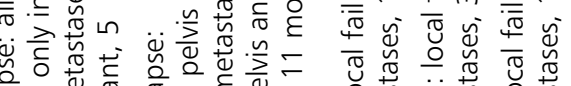

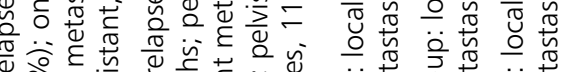

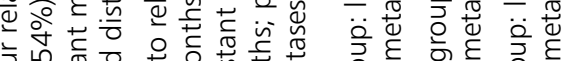

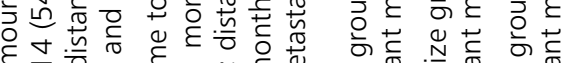

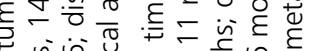

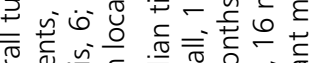

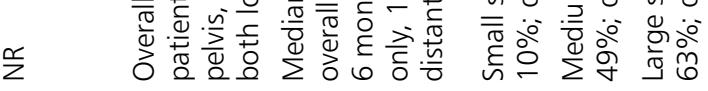

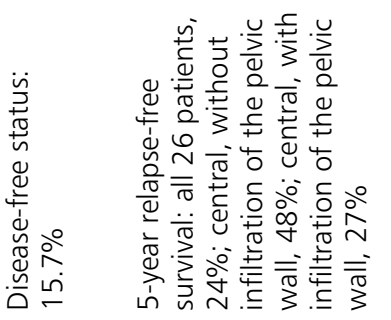

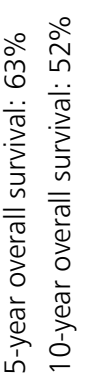

ஓ̊

$\infty$
$\stackrel{\infty}{\circ}$
$\stackrel{2}{\circ}$
\pm 


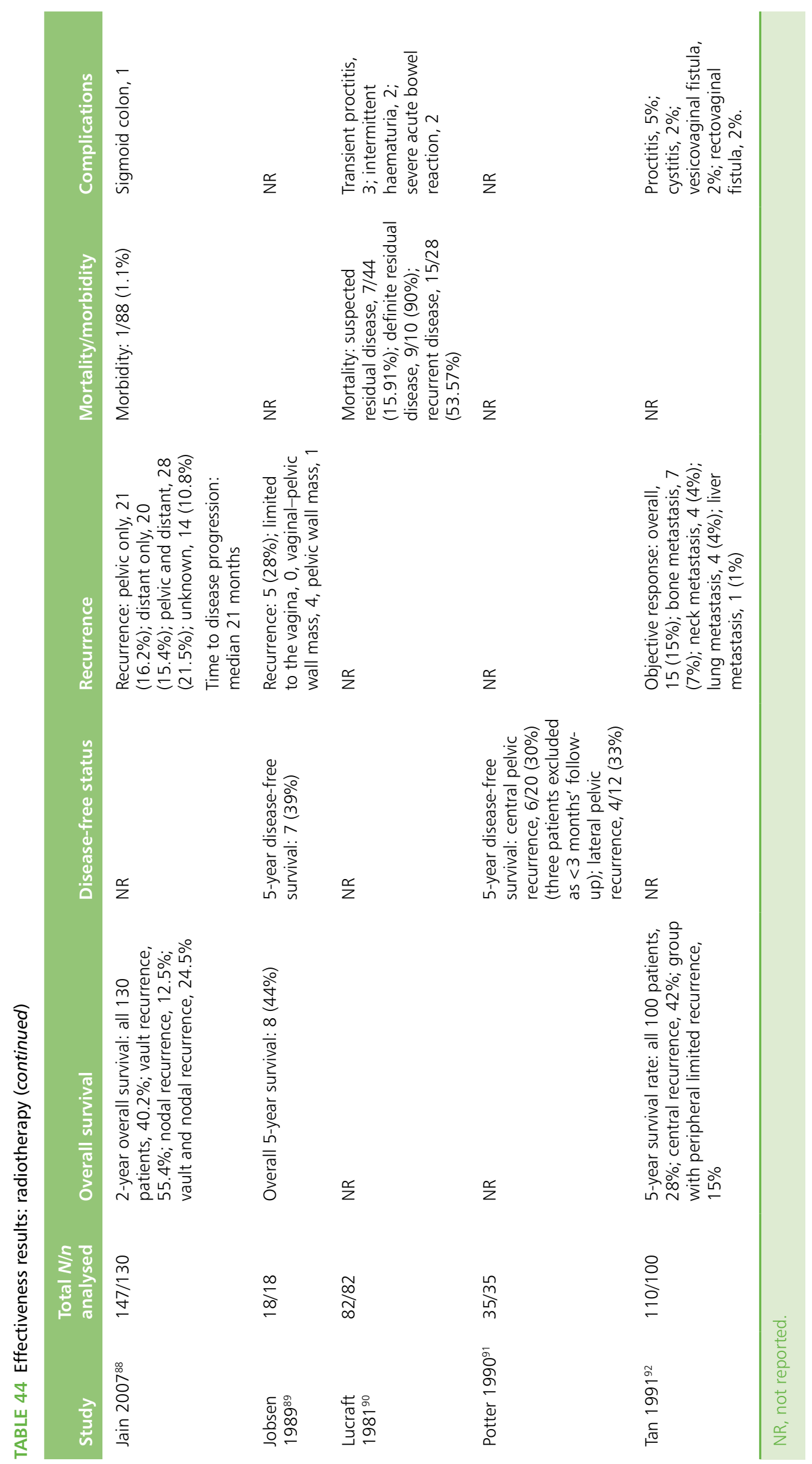



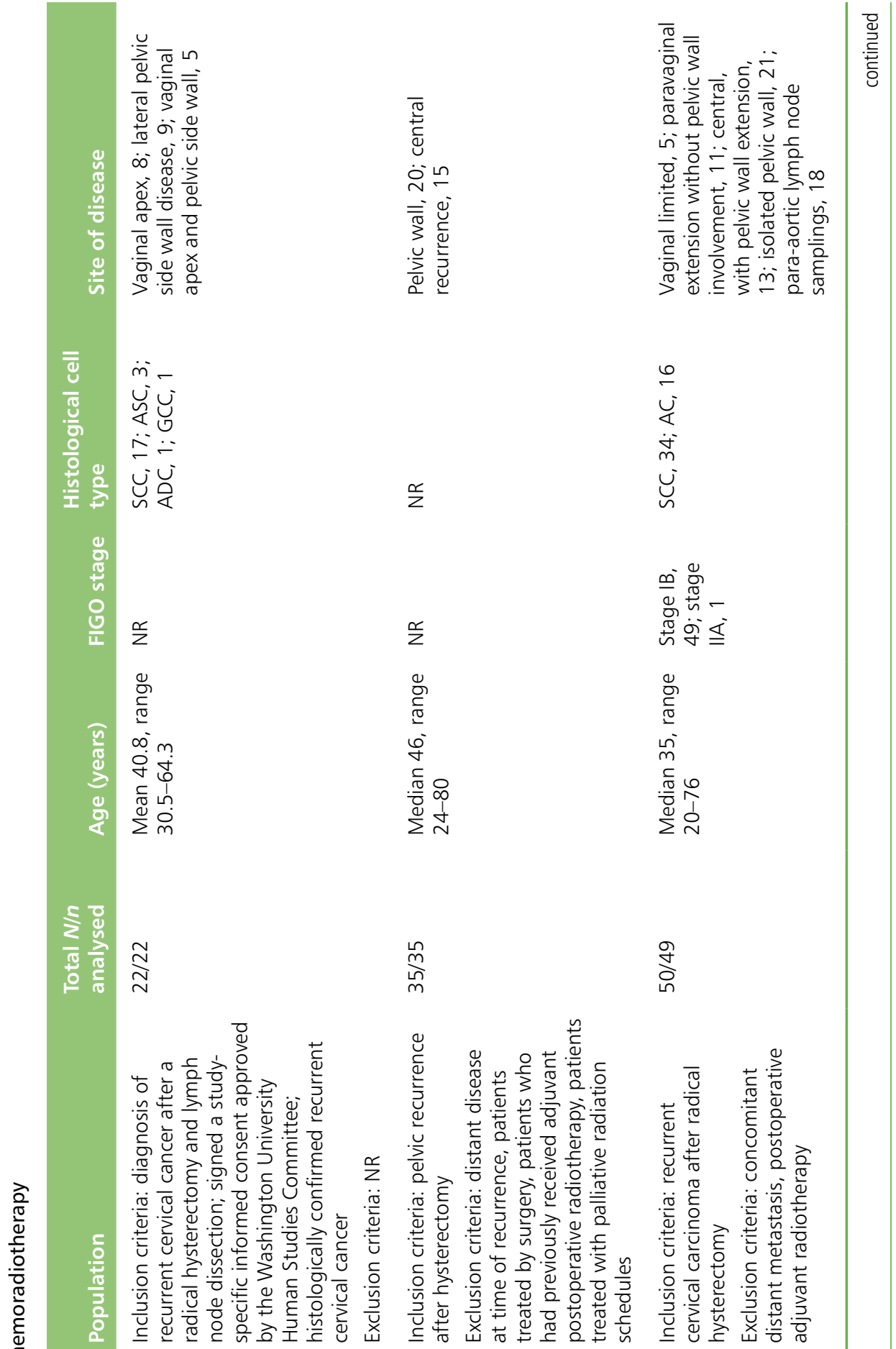

$\underset{\substack{\stackrel{m}{m} \\ m}}{\stackrel{n}{n}}$

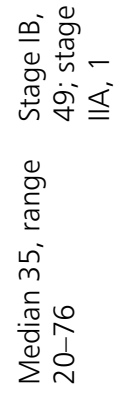

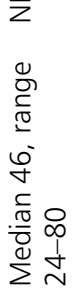
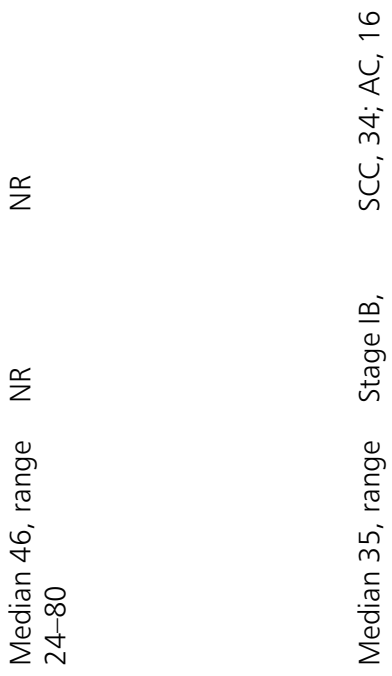

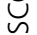

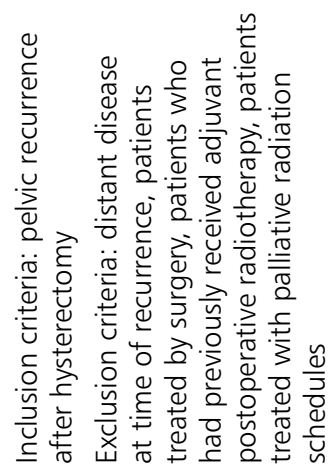

오ํ
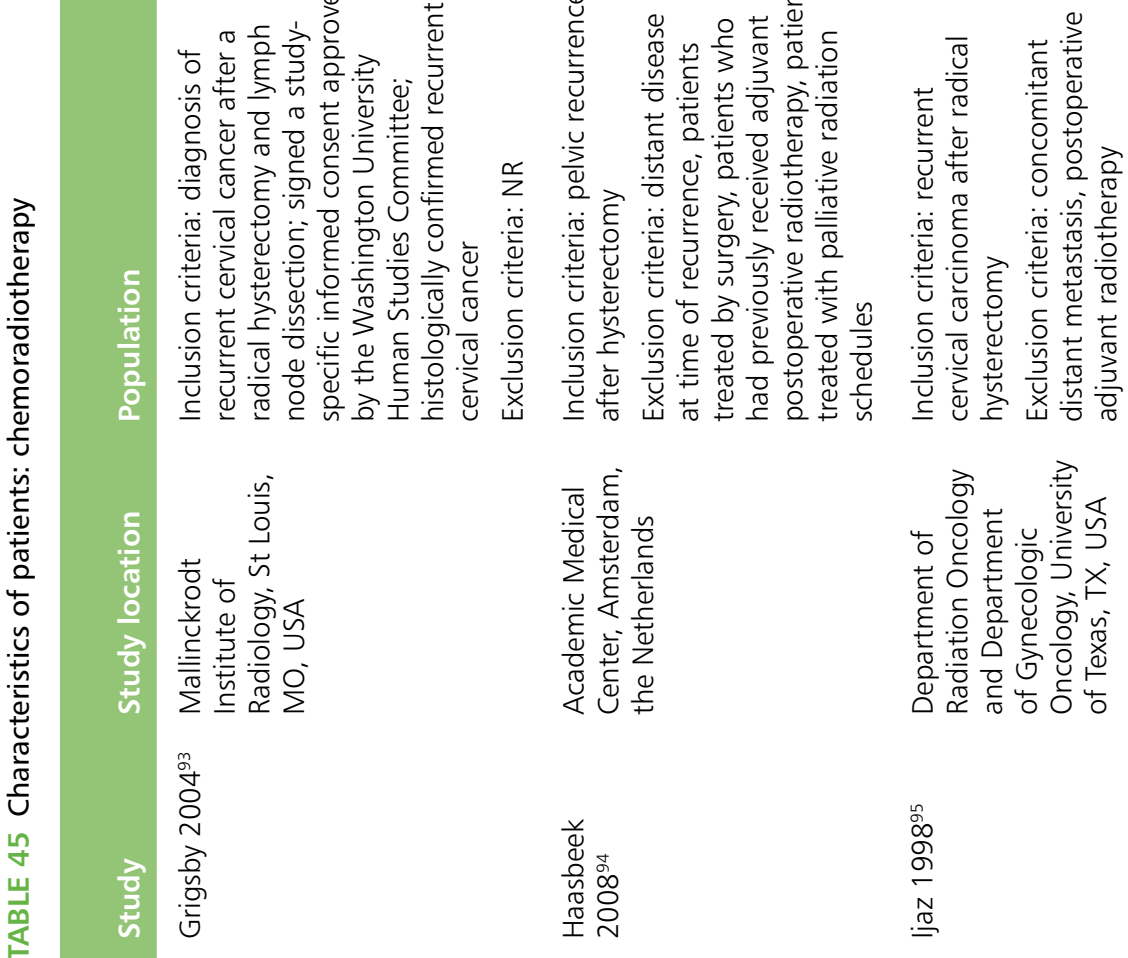

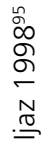




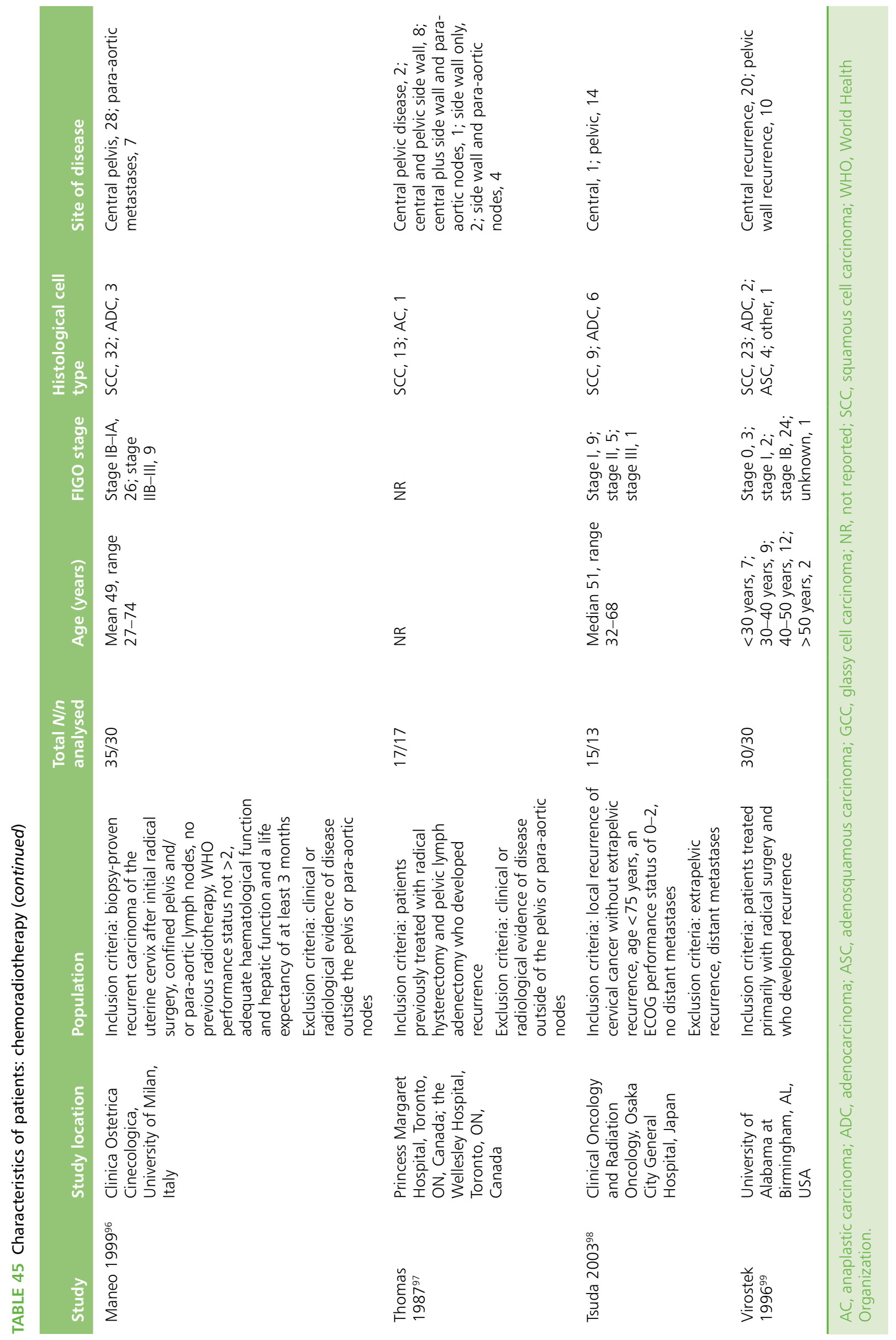




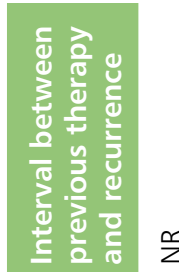

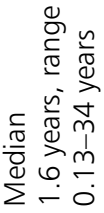

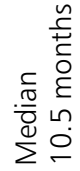

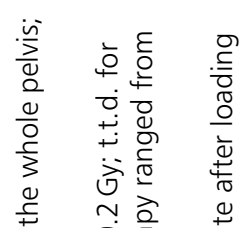

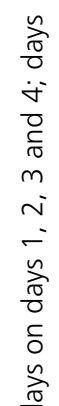

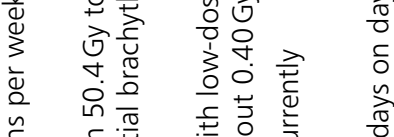

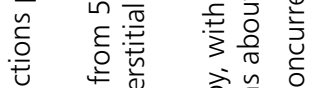

家管

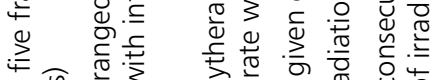

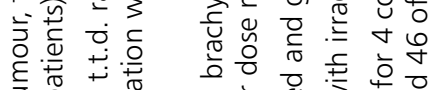

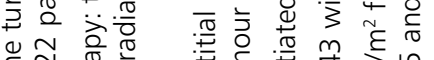

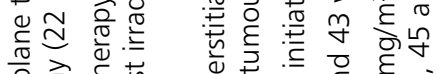

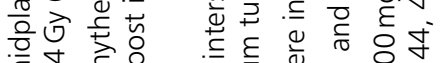

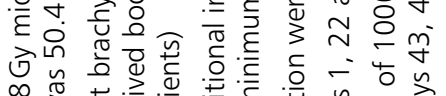

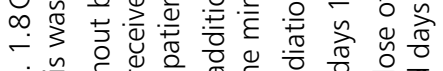

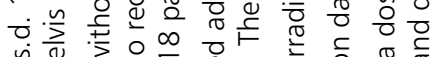

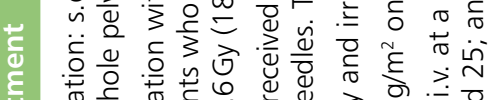

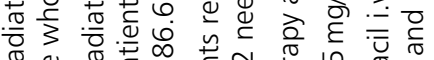

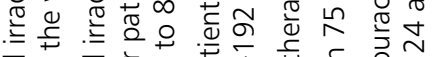

苋

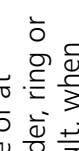

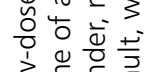

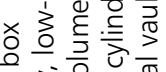

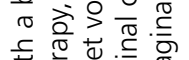

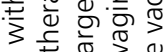

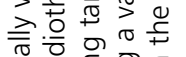

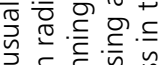

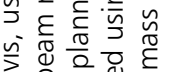

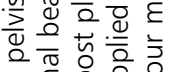

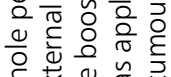

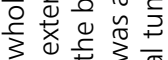

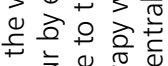

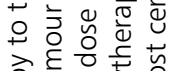

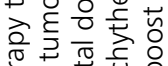

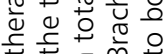

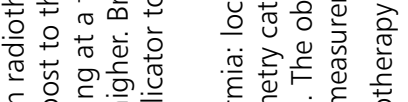

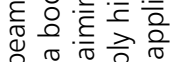

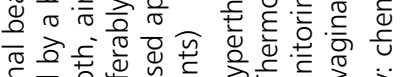

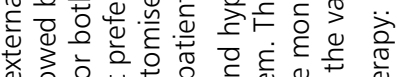

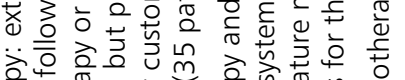

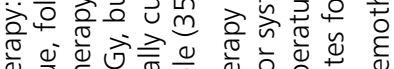

ᄒ

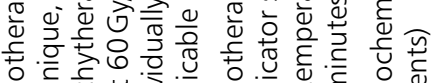

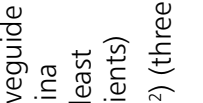

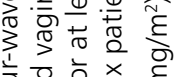

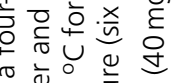

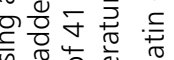

을. 일

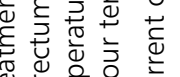

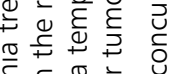

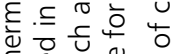

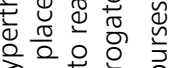

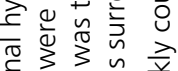

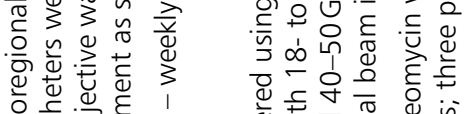

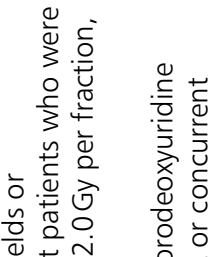

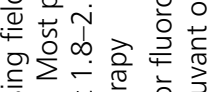

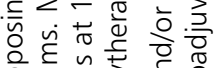

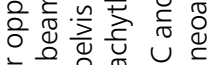

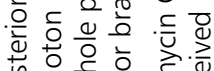

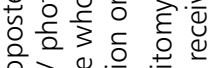

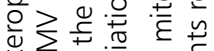

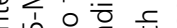

更

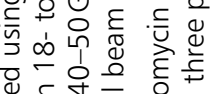

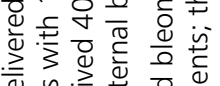

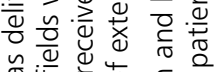

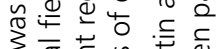

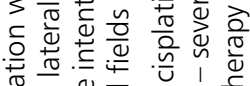

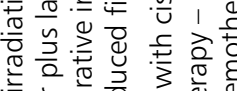

.

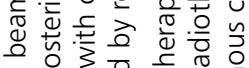

to

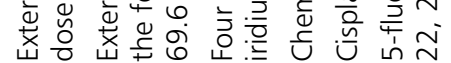

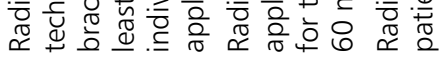
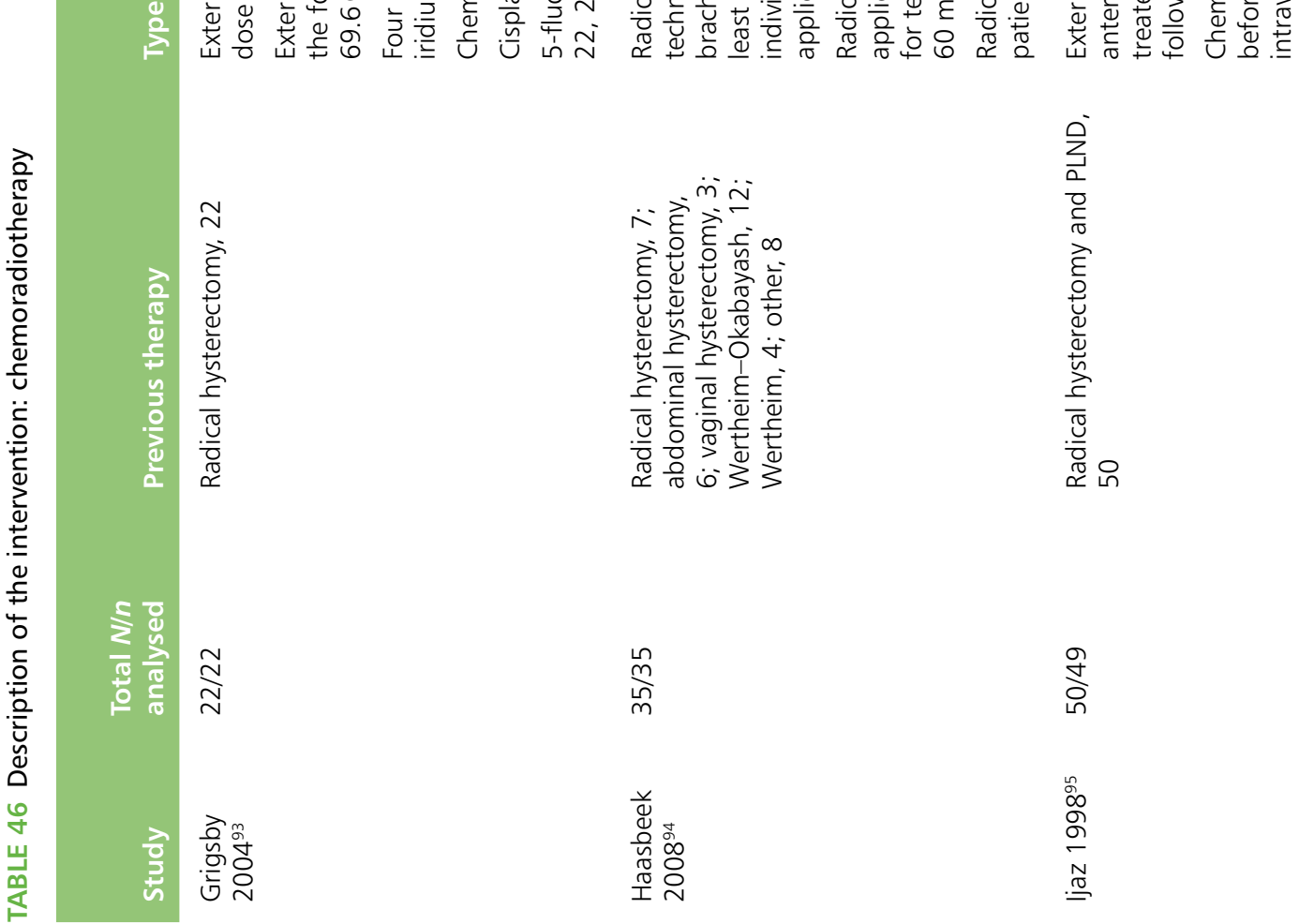

$\stackrel{\substack{m \\ \stackrel{n}{m}}}{m}$

守

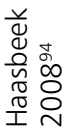

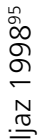




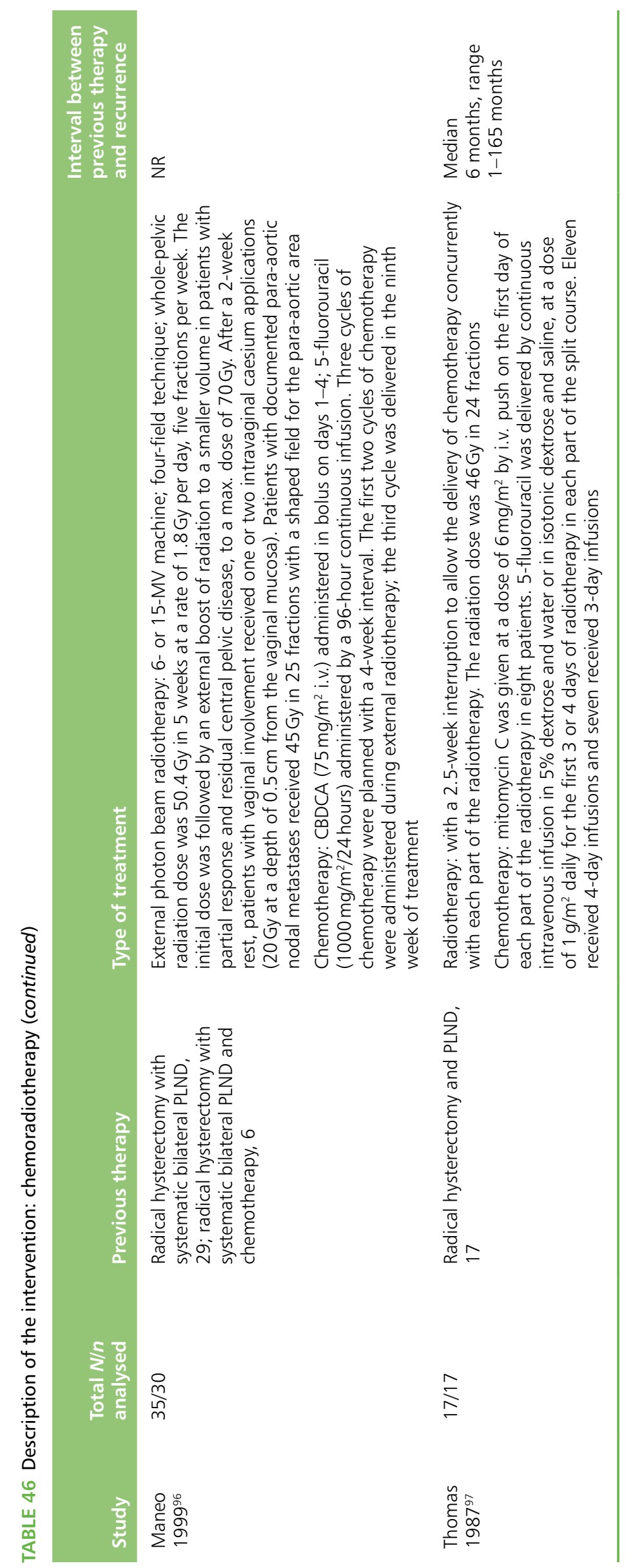


$\stackrel{\frac{c}{z}}{2}$

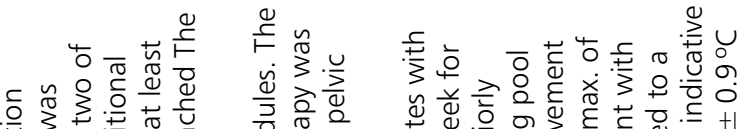

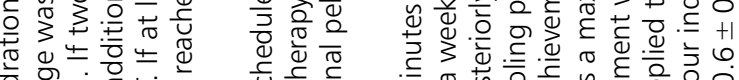

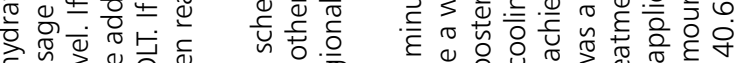

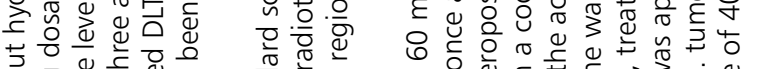

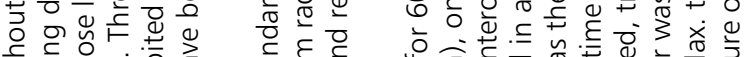

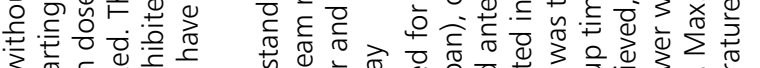

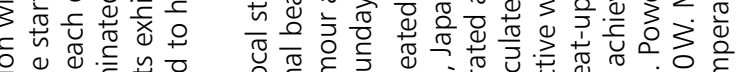

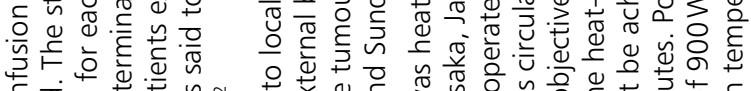

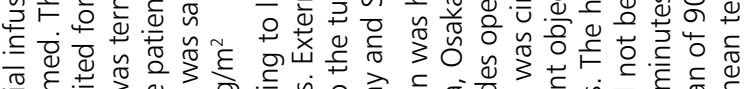

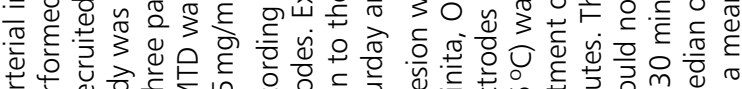

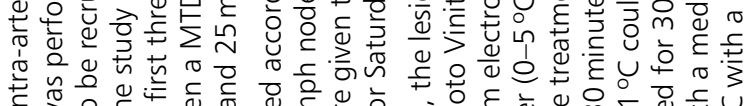

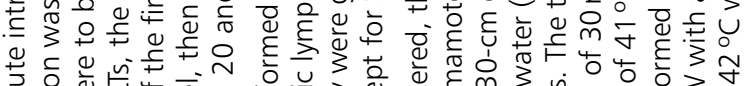

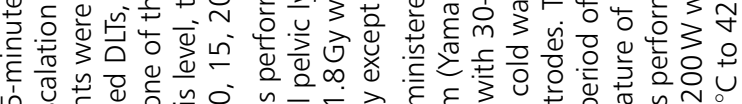

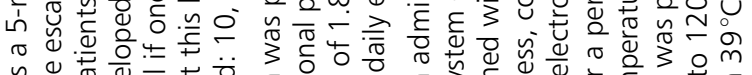

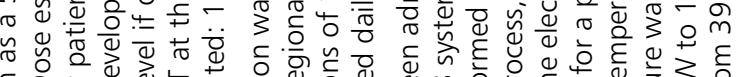

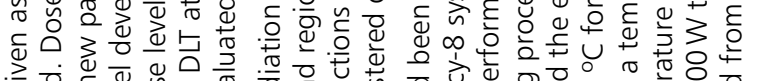

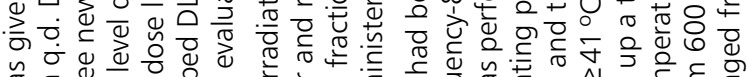

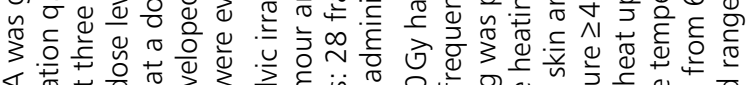

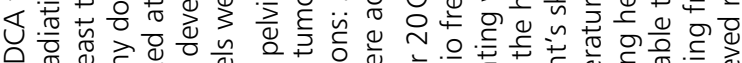

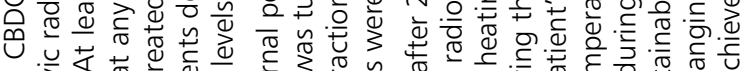

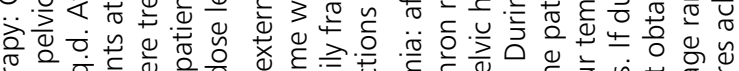

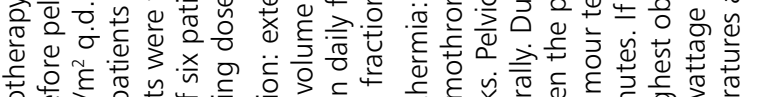
-

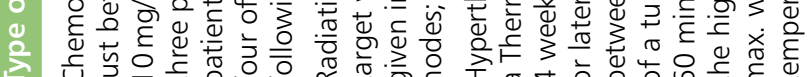

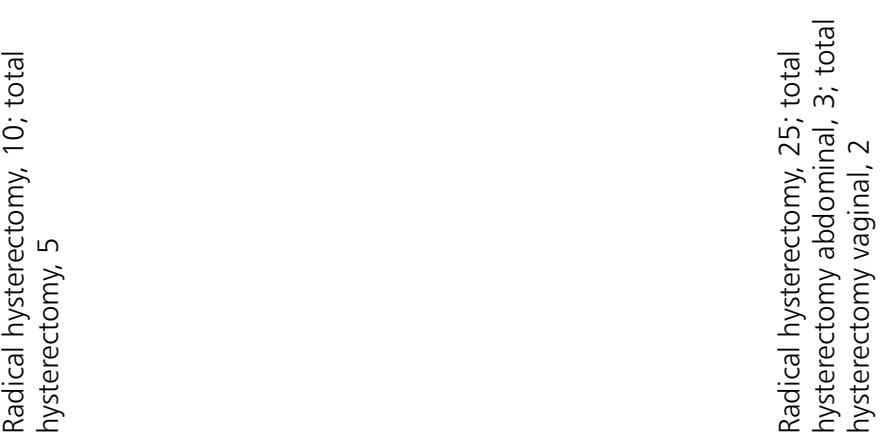

\section{$\frac{\alpha}{2}$}

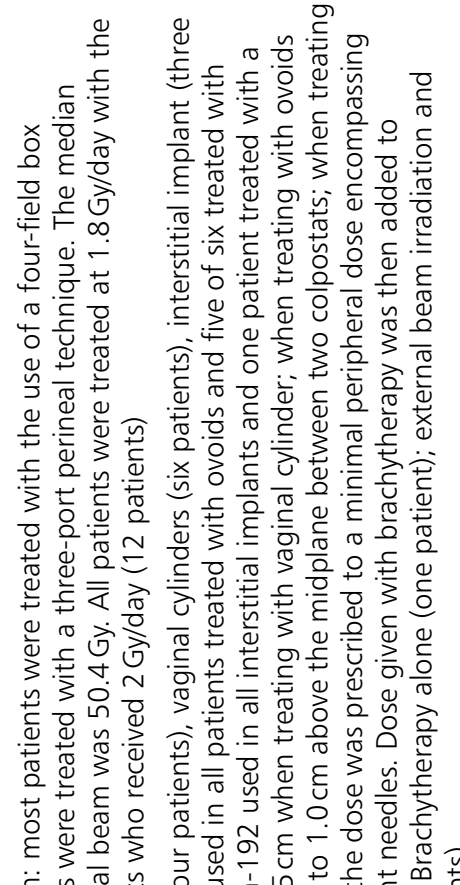

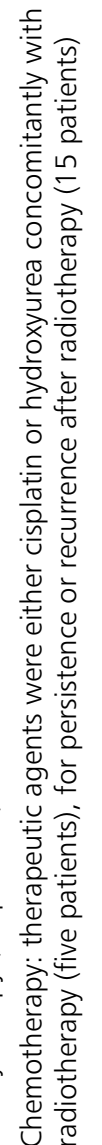

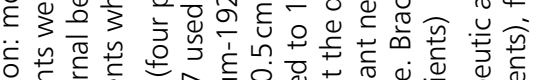

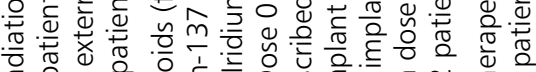

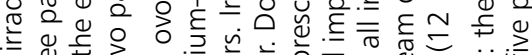

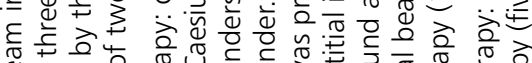
ه广

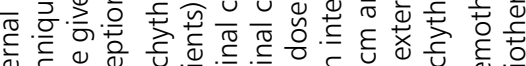

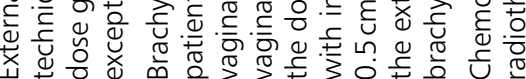
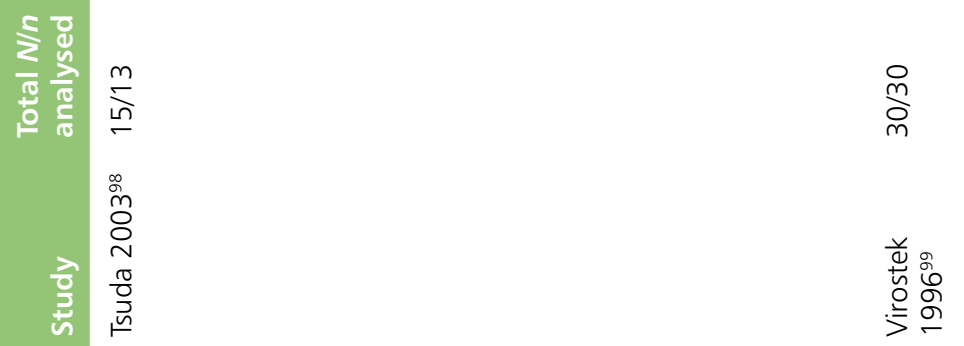


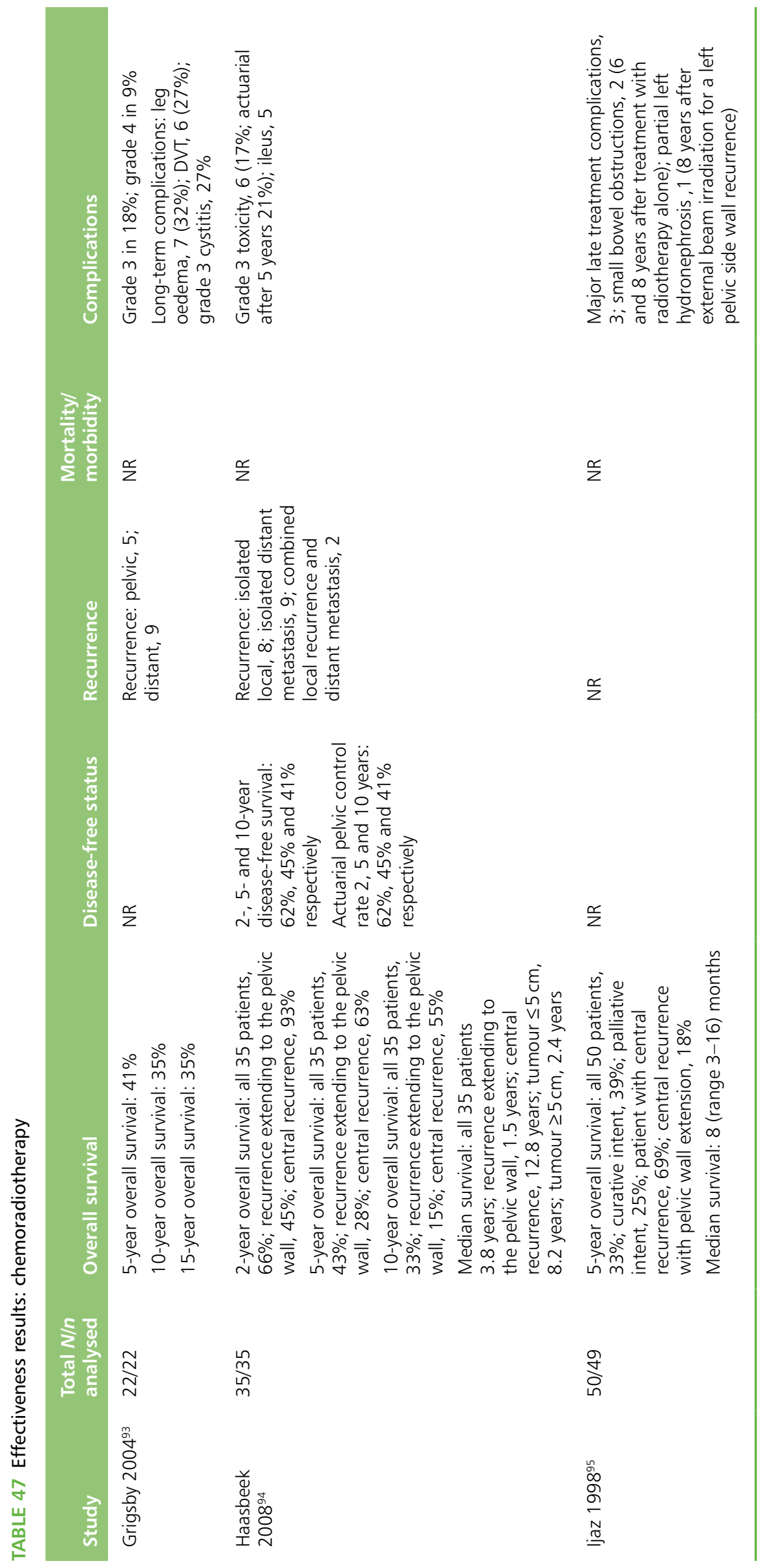


$\stackrel{\stackrel{c}{z}}{2}$

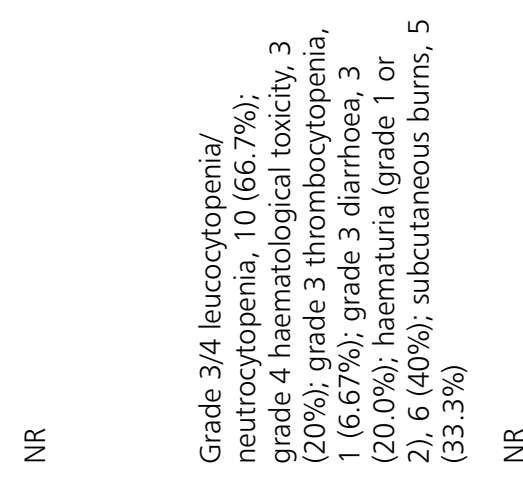

$\frac{\infty}{2}$

$\stackrel{\stackrel{c}{z}}{2}$

$\frac{\stackrel{c}{z}}{2}$

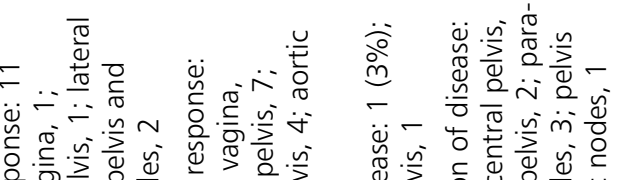

\&

先

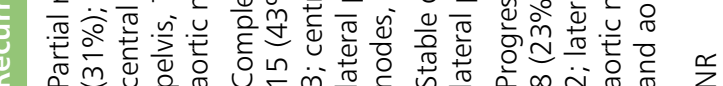

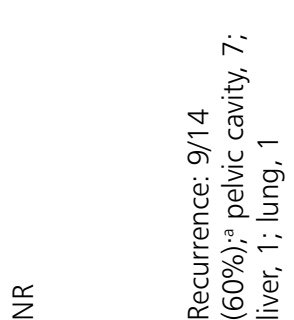

$\frac{\propto}{2}$

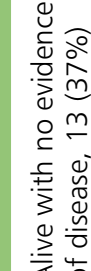

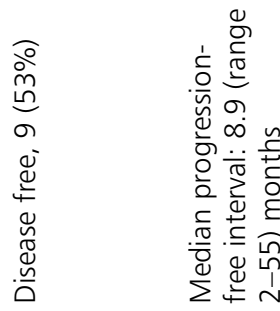

$\stackrel{\frac{c}{z}}{2}$

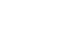

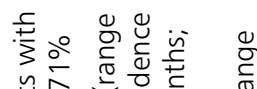

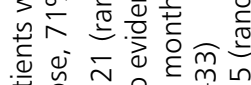

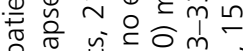

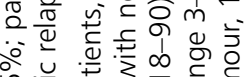

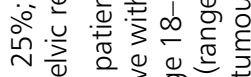

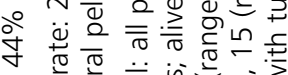

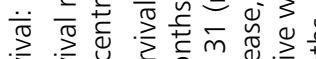

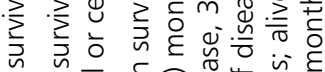

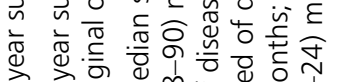

i

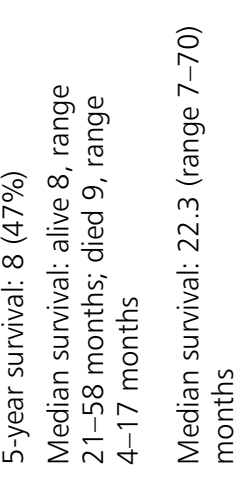

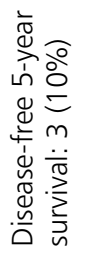

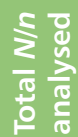

$\stackrel{\substack{m \\ m}}{\stackrel{m}{m}}$

$\underset{\Sigma}{\stackrel{N}{\Sigma}}$

$\stackrel{m}{\stackrel{m}{\leftrightarrows}}$

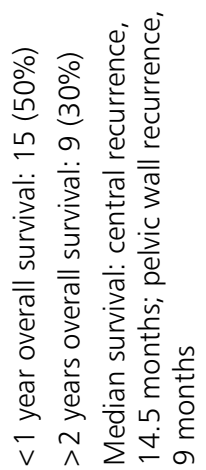

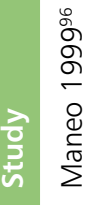

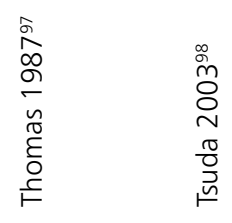

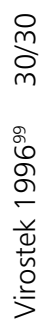


thrombocytopenia - 6.67\%; grade 3 diarrhoea - 20.0\%; haematuria (grade 1 or 2) - 40\%; subcutaneous burns $-33.3 \%$. In ljaz et al. , 95 major late treatment complications (small bowel obstructions, partial left hydronephrosis) were observed in 3 out of 49 patients. Long-term complications observed in Grigsby et al. ${ }^{93}$ included leg oedema in 32\% of patients, deep-vein thrombosis in $9 \%$ of patients and grade 3 cystitis in $27 \%$ of patients. In eight patients who survived beyond 5 years, the following grade 4 complications occurred: a vesicovaginal fistula (four patients), a rectovaginal fistula (three patients) and a life-threatening pelvic abscess (four patients) (see Table 47).

\section{Surgery}

\section{Study selection}

Included in this section are studies in which participants have recurrent or persistent cervical cancer that was initially treated with radiotherapy or chemoradiotherapy and who now have evidence of recurrence. The interventions are radical hysterectomy or Wertheim's operation and pelvic exenteration, and these two categories are described separately. The search found no relevant RCTs. Twenty-seven case series ${ }^{100-126}$ fulfilled the inclusion criteria, most of which were retrospective, based on chart reviews. Most of the excluded papers were case series of gynaecological cancers as a whole without giving separate results for cervical cancer patients, or studies of cervical cancer patients with primary and recurrent tumour characteristics with the results described together. The results of the quality assessment are presented in Appendix 16. No measure of quality of life was provided in any of the included studies.

\section{Radical hysterectomy population characteristics}

Seven case series ${ }^{100-106}$ gave information on radical hysterectomy (Table 48). They were published between 1965 and 1999 and were mostly from the USA, Canada and the European Union (Italy and Denmark). The number of participants ranged from 14 to 79. The mean or median age of patients was approximately 50 years. Most participants were classified at FIGO stage II and had squamous cell carcinoma.

\section{Pelvic exenteration population characteristics}

Twenty case series, ${ }^{107-126}$ published between 1953 and 2009, presented results on pelvic exenteration, mostly from the USA (Table 49). The number of patients varied between 14 and 263 . The mean or median age of the women was around 50 years (range 20-76 years). In many cases details about the baseline characteristics of the subpopulation of interest were incomplete but, in those publications in which the information was presented, most patients were classified as FIGO stage II and had squamous cell carcinoma.

\section{Radical hysterectomy intervention}

Descriptions of the interventions are provided in Table 50. In five studies, ${ }^{100,101,103-105}$ radical hysterectomy was conducted as salvage surgery with curative intent. Tupper ${ }^{106}$ described Wertheim's operation, and in Ibsen et al. ${ }^{102}$ both Wertheim's operation and pelvic exenteration (which could be total, anterior or posterior) were combined with pelvic lymph node dissection. In all case series the primary therapy was radiotherapy. In Maneo et al. ${ }^{103}$ chemotherapy with cisplatin was also used postoperatively. The median time from previous therapy to salvage surgery in curative intention was between 7.5 and 19 months.

\section{Pelvic exenteration intervention}

Descriptions of the interventions are provided in Table 51. All patients in the case series had radiotherapy as their primary treatment. Total pelvic exenteration (TPE) was conducted most often compared with anterior or posterior pelvic exenteration. Reconstructive procedures were performed frequently as part of the operations or scheduled at a time when the patient's condition allowed it.

\section{Radical hysterectomy results}

The results of the radical hysterectomy case series are presented in Table 52. Operative deaths occurred in Rubin et al. ${ }^{104}-10 \%$ (from sepsis); postoperative deaths were analysed in four studies and occurred 


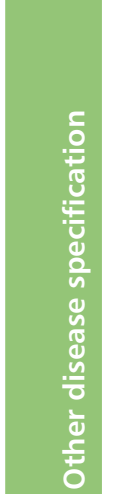

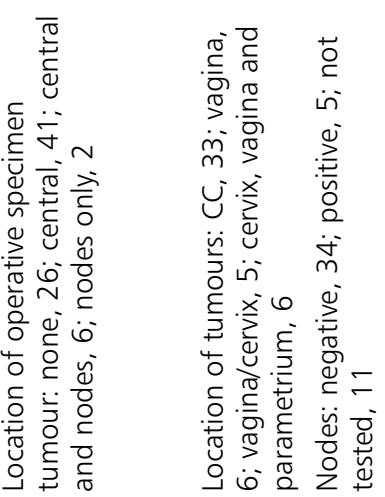

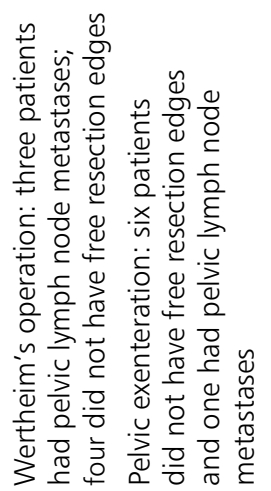

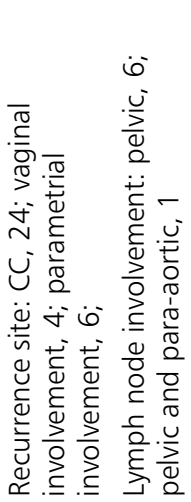

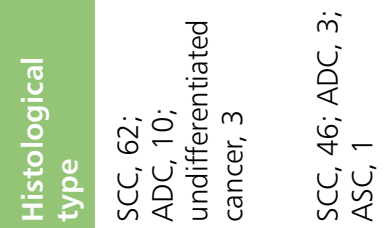

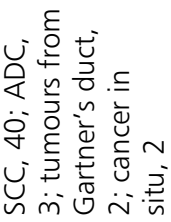

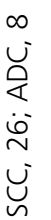

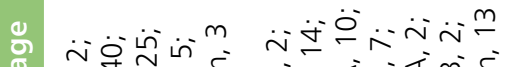

$\dot{\sim} \ddot{\sim} \ddot{\sim}$

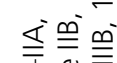

o \& $\%$

o 0 o

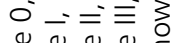

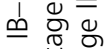

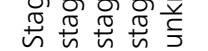

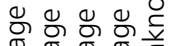

g)

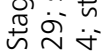

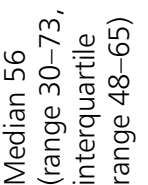

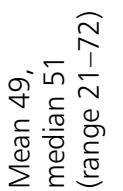

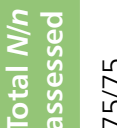

옹

$\underset{f}{\stackrel{f}{\&}}$

$\stackrel{\stackrel{f}{m}}{\stackrel{m}{m}}$

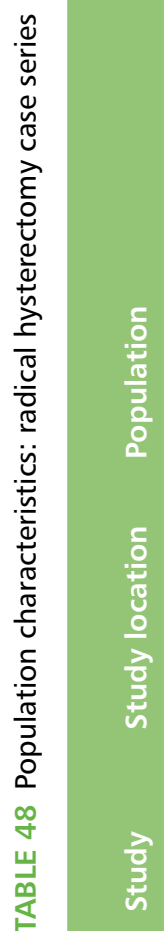

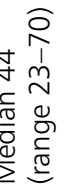

ํํ

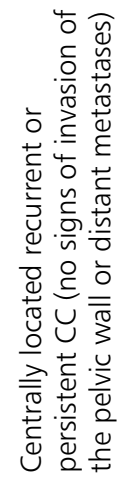

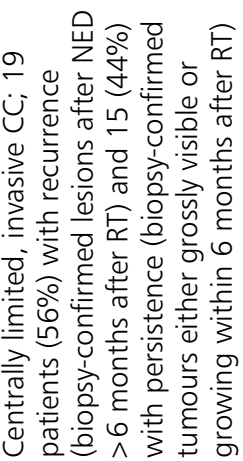

\begin{tabular}{|c|c|c|}
\hline 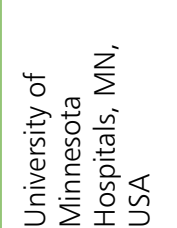 & 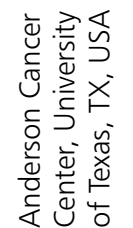 & 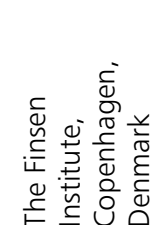 \\
\hline
\end{tabular}




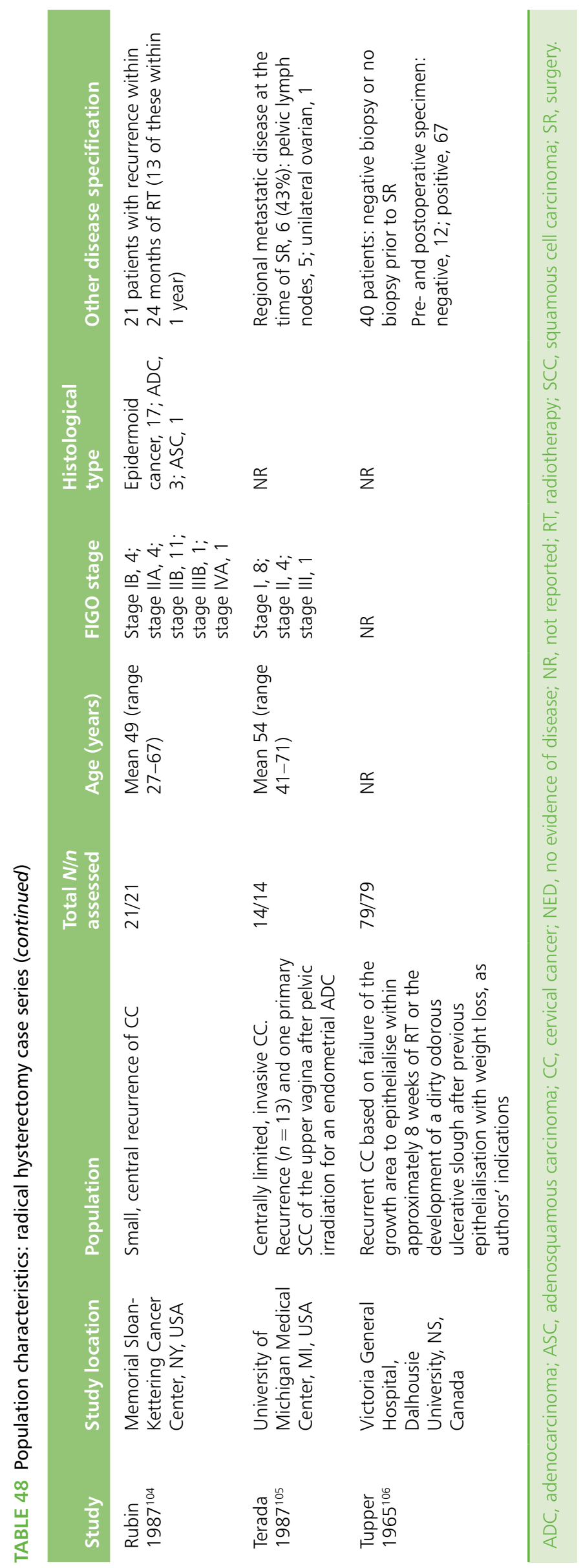



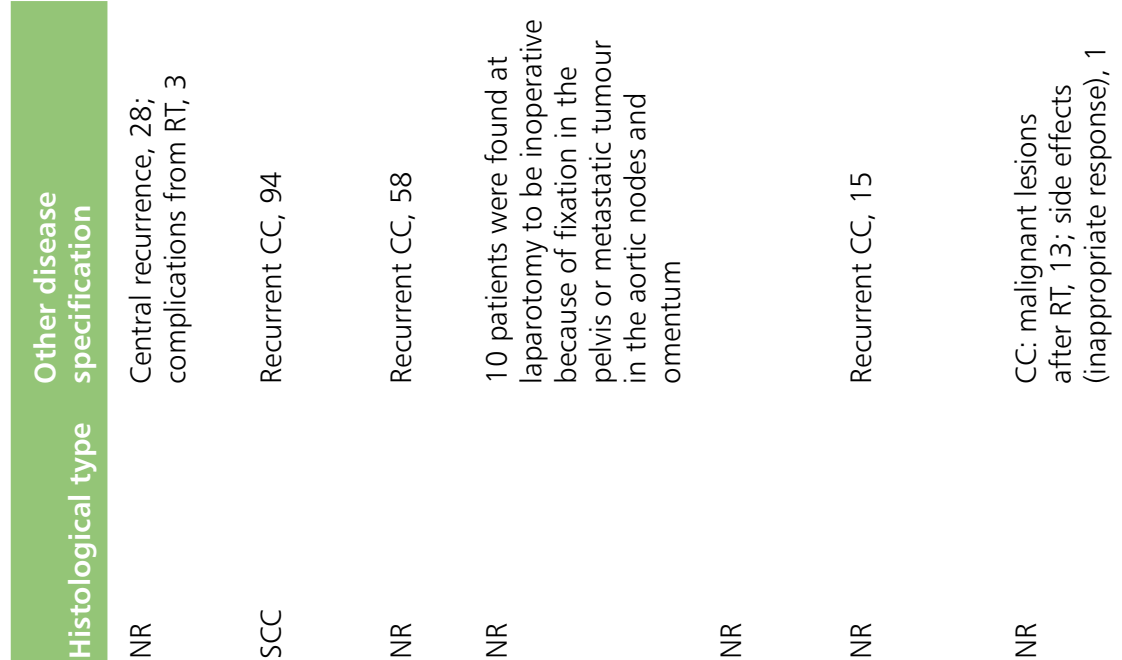

$\frac{\infty}{z} \quad \breve{u} \quad \frac{\infty}{z}$

$\stackrel{\infty}{2} \quad \frac{\infty}{2} \quad \frac{\infty}{z}$
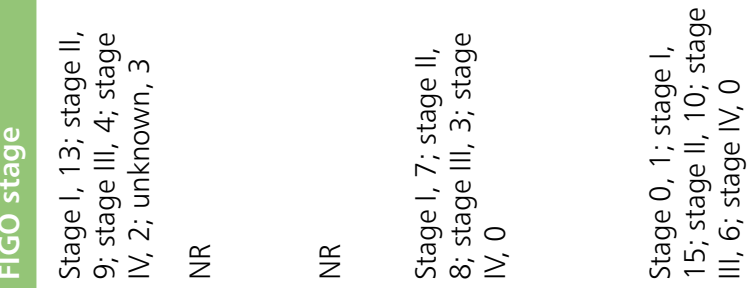

कृ

昰皆品 爱

a

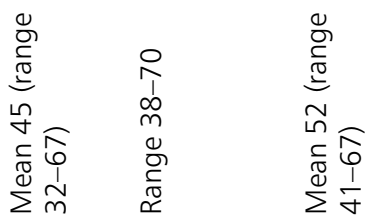

$\begin{array}{ll}3 & \frac{c}{0} \\ \frac{0}{0} & 0 \\ \frac{0}{2} & \frac{c}{0} \\ \frac{1}{2} & 0\end{array}$

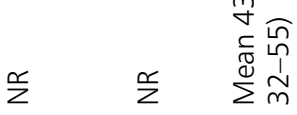

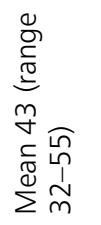

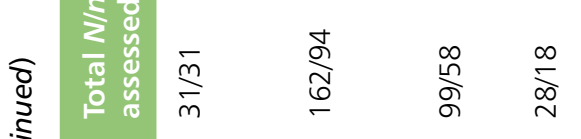

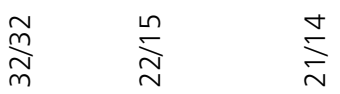

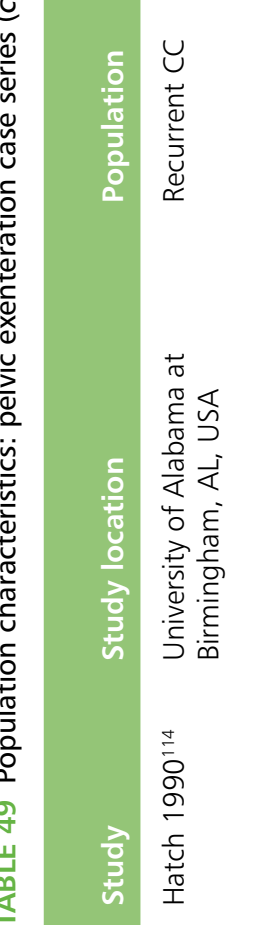

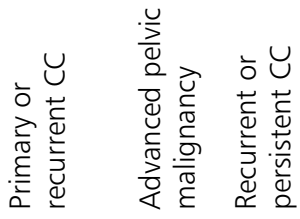
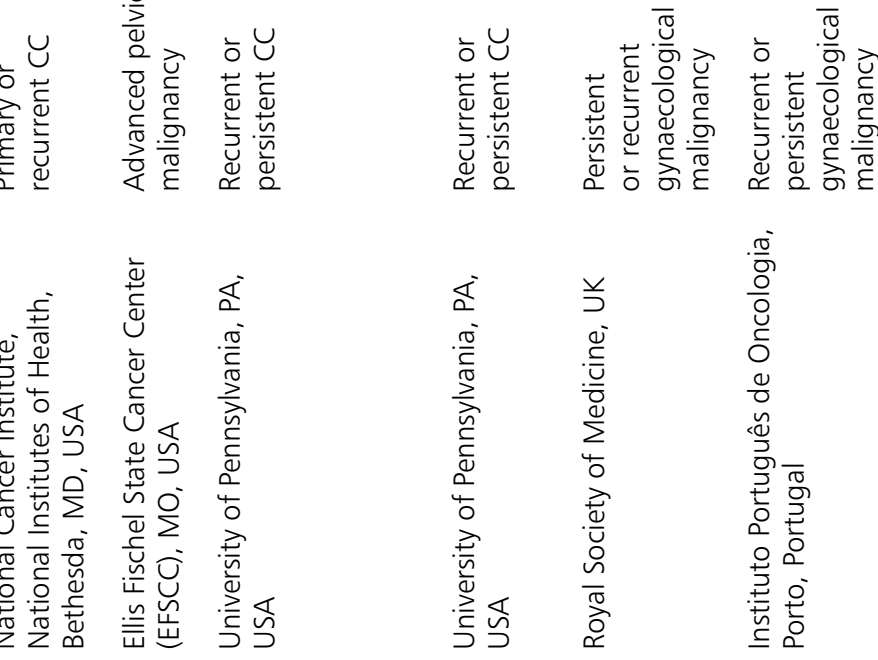


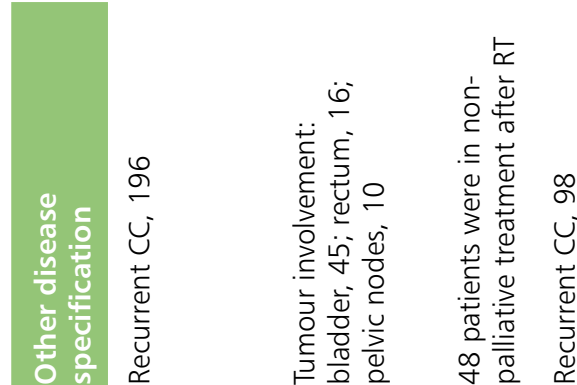

ชัช

U $\quad \dot{u}$

$\dot{\infty}$

这

$\stackrel{\stackrel{m}{m}}{\sim}$

à

这

$\stackrel{\infty}{\circ}$

$\frac{c}{z}$

岕䔏

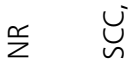

mं-

岁安

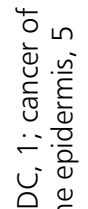

过

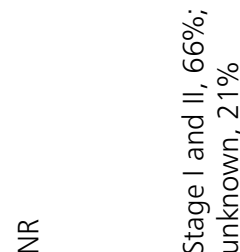

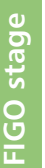

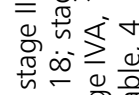

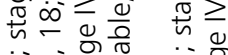

ヘ产焉的西

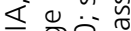

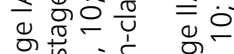

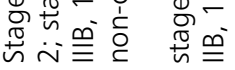

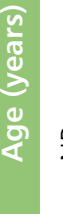

苍 。ำ

$\stackrel{\frac{c}{z}}{\frac{\alpha}{2}}$

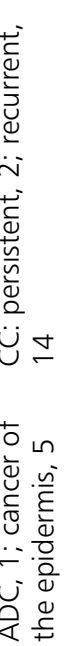

$\circ$

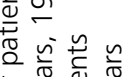

文离离离

$\frac{o}{2}$

원눙인

兴 爱

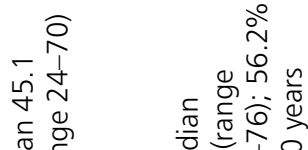

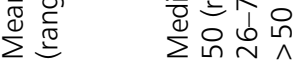

官

$\underset{\stackrel{m}{\stackrel{m}{q}}}{\stackrel{m}{\sim}}$

$\stackrel{\infty}{\stackrel{\infty}{ }} \stackrel{\stackrel{\infty}{\infty}}{\stackrel{\infty}{\sigma}}$

$\underset{\frac{1}{0}}{\stackrel{5}{2}}$

$\stackrel{6}{6}$

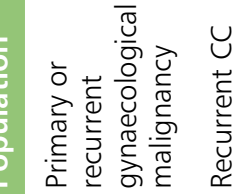

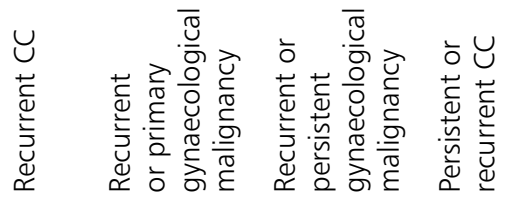

离

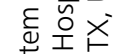

会

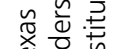

ำ

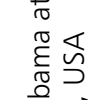

高芯

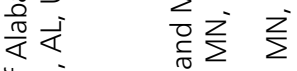

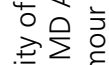

을

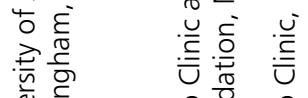

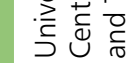

离

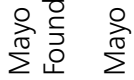

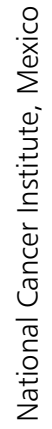

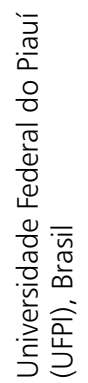

ते

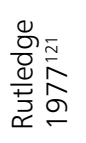

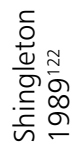

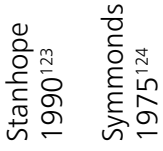

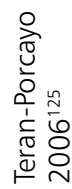

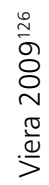




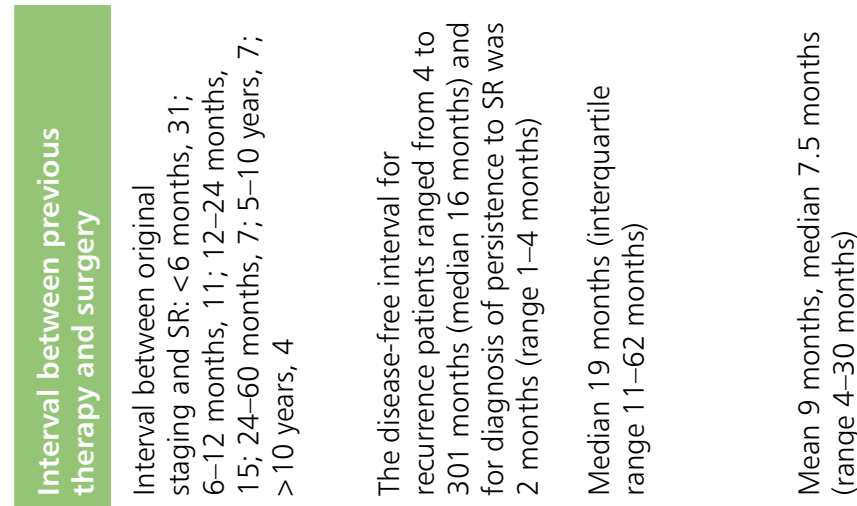

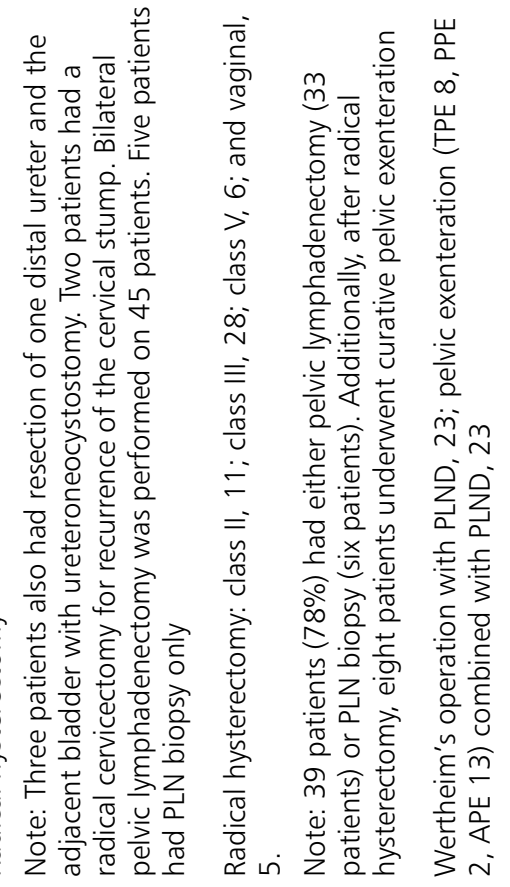
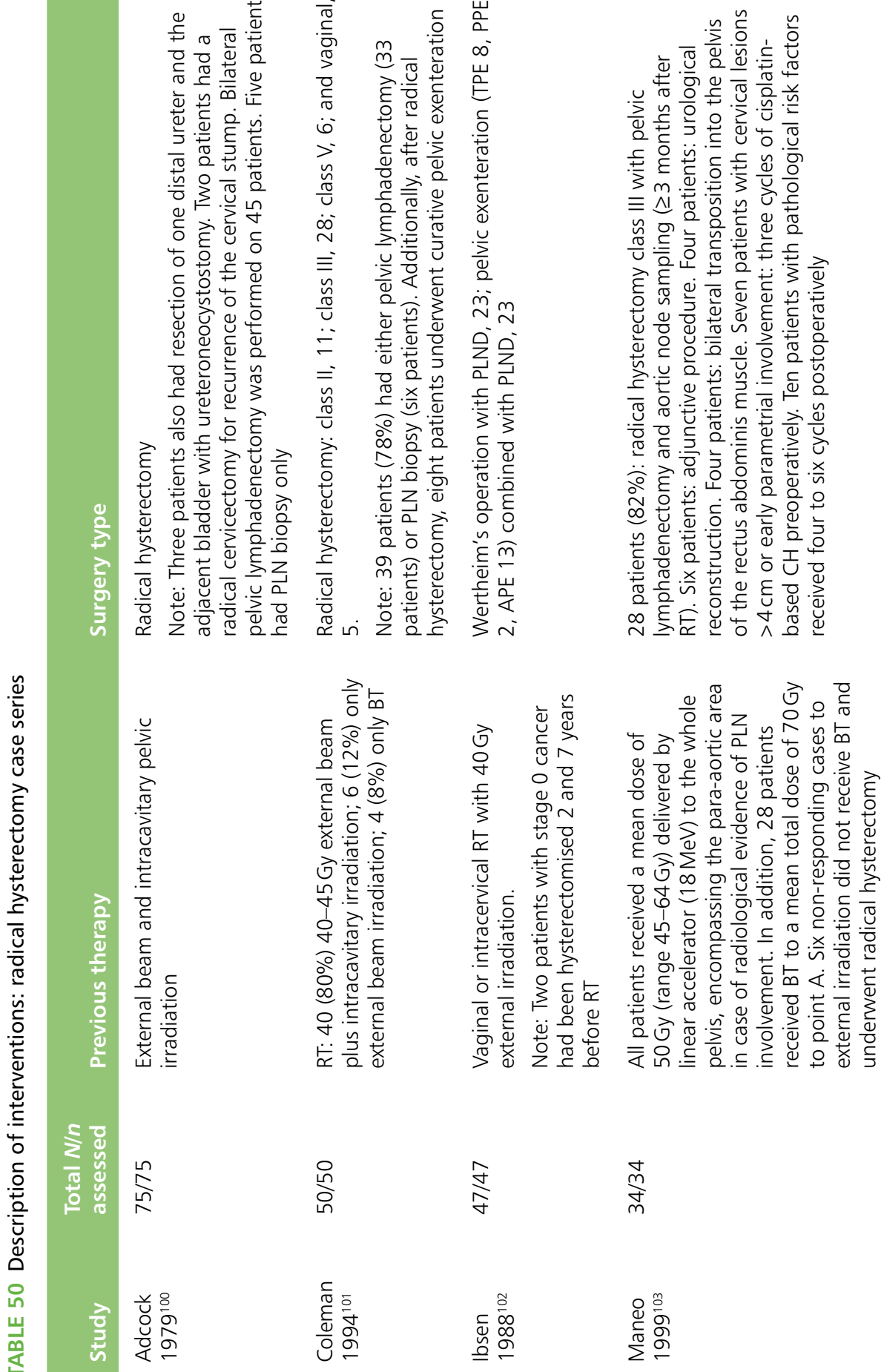

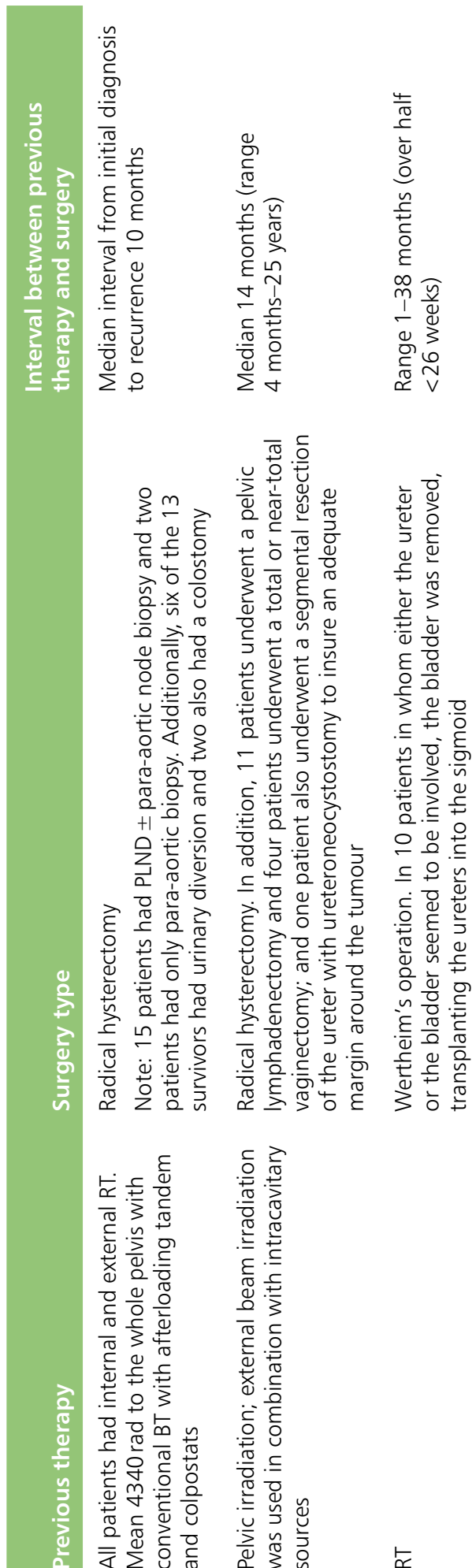

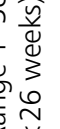

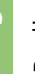

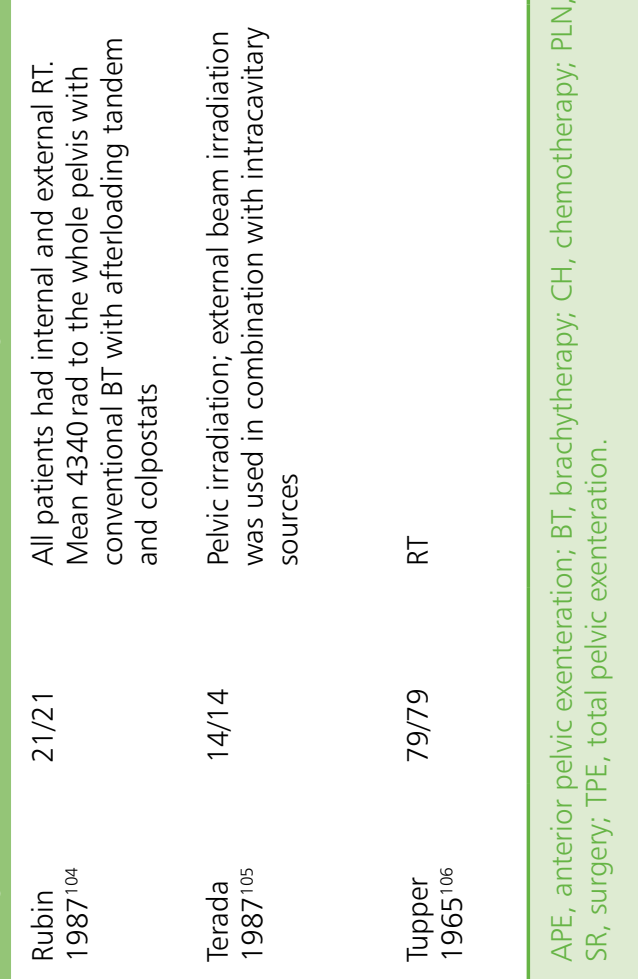
Park, Southampton SO16 7NS, UK. 


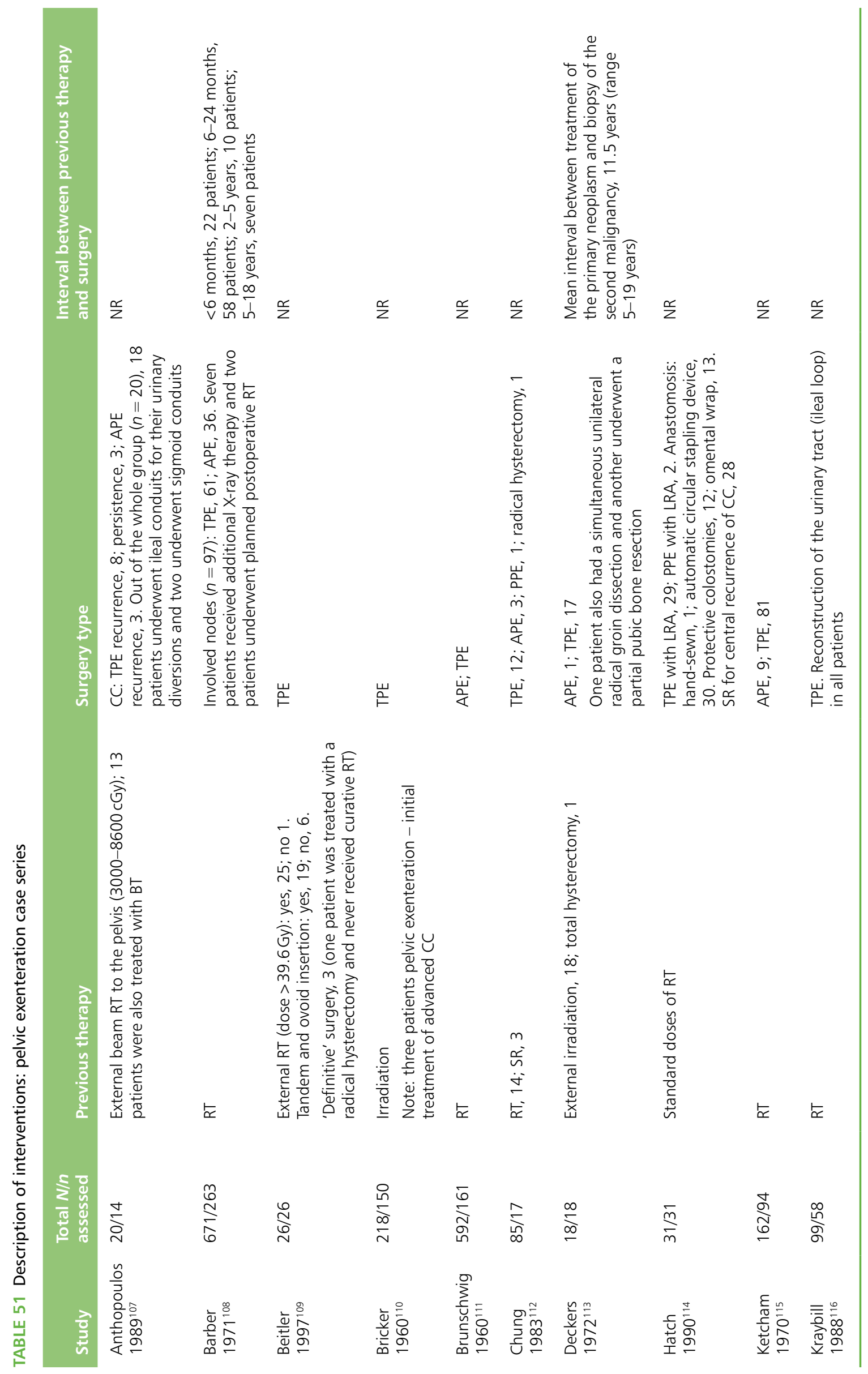




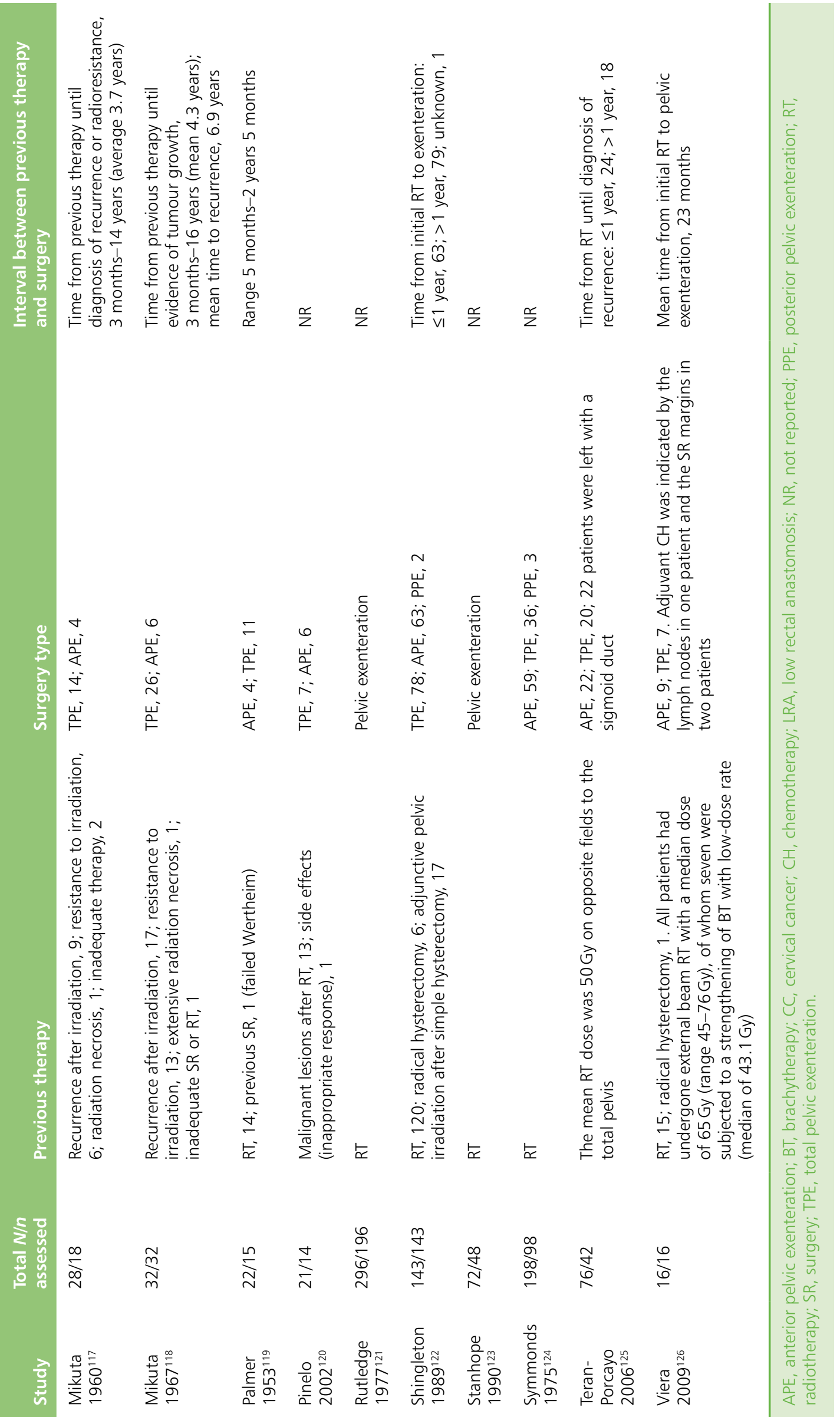


in $0 \%,{ }^{105} 2 \%$ (from sepsis), ${ }^{101} 2 \%{ }^{102}$ and $7.6 \%{ }^{106}$ of patients. Survival results were presented in all publications. Five-year survival rates ranged between $32 \%$ and $72 \%$ and 5 -year survival rates with no evidence of disease ranged between $27 \%$ and $65 \%$. In Adcock ${ }^{100}$ and Coleman et al. , ${ }^{101}$ 5-year survival rates were also available for subpopulations of patients with persistent cervical cancer (52\% and $82 \%$ respectively) and recurrent cervical cancer (65\% and $75 \%$ respectively). Ten-year survival rates were presented in Coleman et al. ${ }^{101}$ only and were $60 \%$ for the total population, $68 \%$ for the persistent subpopulation and $54 \%$ for the recurrent subpopulation. The rate of recurrence was between $32 \%$ and $59 \%$. Major complications included fistulae, which required further surgical interventions.

\section{Pelvic exenteration results}

The results of the pelvic exenteration case series are shown in Table 53. Operative mortality ranged from $0 \%$ to $22 \%$ and postoperative mortality from $15 \%$ to $33 \%$. The total percentage of complications varied between $50 \%$ and $69 \%$. Three studies ${ }^{103,107,124}$ gave 2 -year survival rates: Stanhope et al. ${ }^{103}$ for the complete population only (75\%), Anthopoulos et al. ${ }^{107}$ based on the type of surgery used (TPE 73\%, anterior pelvic exenteration $75 \%$ ) and Symonds et al. ${ }^{124}$ according to pelvic lymph node status (positive $29 \%$, negative $54 \%$ ). Five-year survival rates ranged from $33 \%$ to $66 \%$ with one very low exception (12\%) in Bricker et al. ${ }^{110}$ The 5 -year survival rates after specific types of exenteration were $58 \%{ }^{122}$ and $71.5 \%{ }^{125}$ for anterior exenteration and $42 \%{ }^{122}$ and $64.6 \%^{125}$ for total exenteration. When there were metastases to pelvic lymph nodes (positive status), 2-25\% of patients survived 5 years. For patients without metastases to pelvic lymph nodes (negative status), 5-year survival was between 17\% and 73\% (but 5-year survival for TPE was 7\%). The 10-year survival rate was presented in only one study (23\%). ${ }^{124}$ The rate of recurrence varied by type of exenteration and whether there was local or distant spread. General information about the incidence of complications was very scarce.

\section{Summary of accuracy and effectiveness results and inputs to economic evaluation}

\section{Statement of principal findings}

\section{Diagnostic studies and subjective elicitation}

- Six studies ${ }^{20,48-52}$ evaluating conventional imaging plus PET-CT, two studies ${ }^{53,54}$ evaluating MRI, three studies ${ }^{55-57}$ evaluating CT and one study ${ }^{58}$ evaluating both MRI and CT were included.

- The dates of the studies varied between 1981 and 2009.

- Most of the studies were small and several reported only a subset of results in a form that could be converted to a $2 \times 2$ table.

- The quality of the studies was poor. Although most probably included a representative spectrum of cervical cancer, very little clinical information about participants was given. Most studies did not report the time gap between the imaging test and the reference standard and most studies did not describe the reference standard clearly enough for replication.

- The later studies evaluated PET-CT, whereas the earlier studies evaluated CT and MRI. The technical imaging standards have changed since the early studies (reported in the 1980s) and so these are no longer valid. None of the MRI or CT studies used current standard methods.

- Five of the six PET-CT studies evaluated the whole body for recurrences and one reported extrapelvic recurrence only. Five of the six $C T$ and MRI studies evaluated pelvic recurrences only and the newest evaluated whole-body recurrences, but this study included only 36 participants.

- Meta-analysis was conducted on PET-CT studies, which gave a combined sensitivity of $92.2 \%(95 \%$ $\mathrm{Cl} 85.1 \%$ to $96.0 \%)$ and a specificity of $88.1 \%(95 \% \mathrm{Cl} 77.9 \%$ to $93.9 \%)$. Meta-analysis was not appropriate for the MRI and CT studies because of clinical heterogeneity.

- There was one study on the diagnostic and therapeutic impact of PET-CT, ${ }^{20}$ which found that it had an impact on management in 12 (out of 52) patients and additional invasive diagnostic procedures in nine patients and assisted in planning therapy in nine patients. 


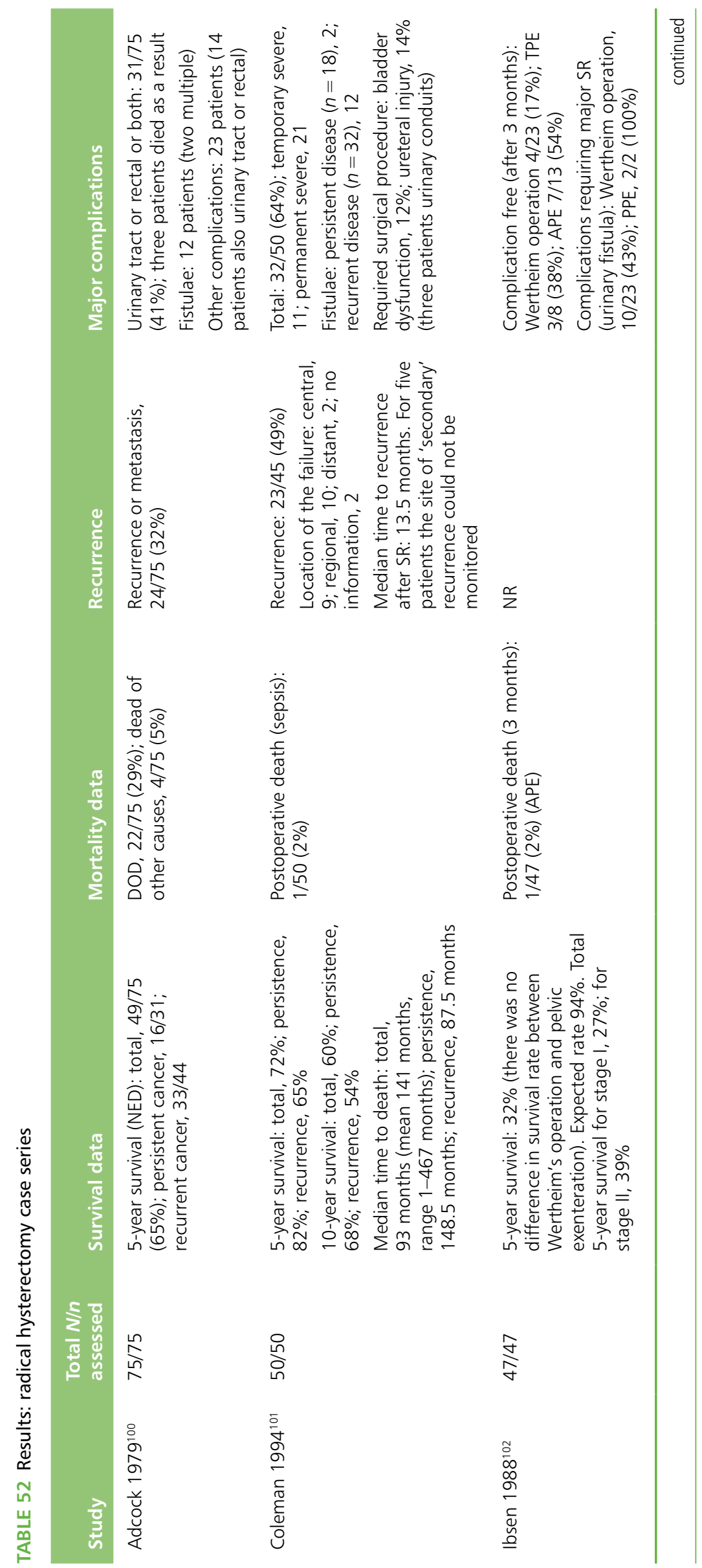



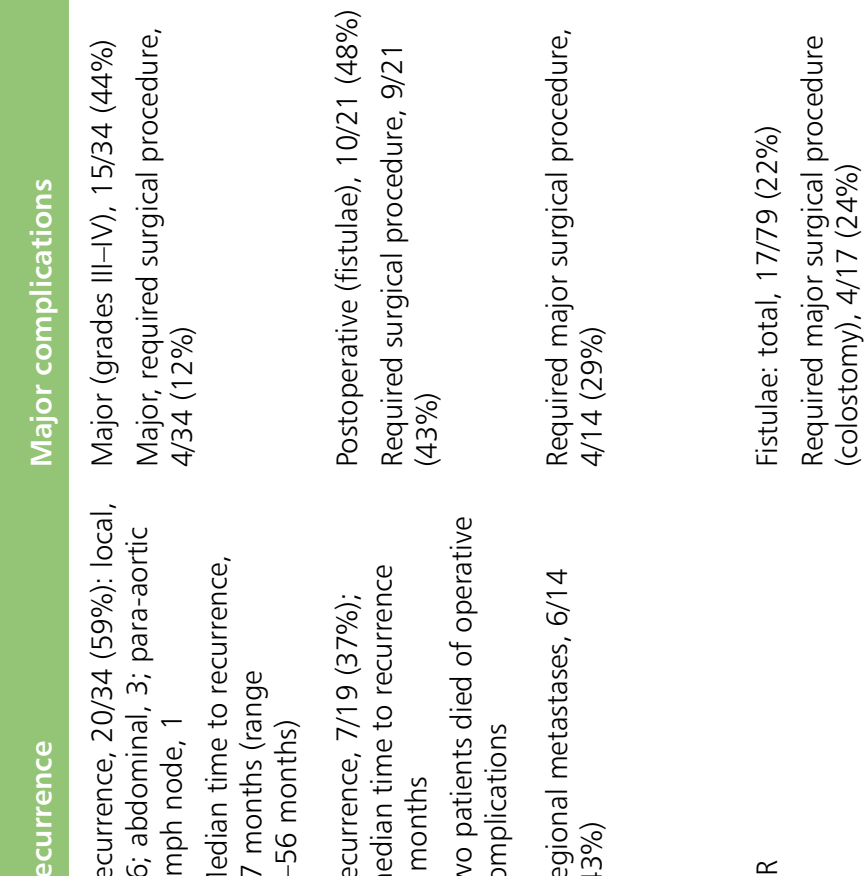

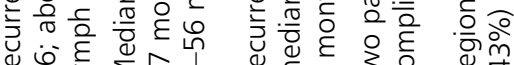

:

2m

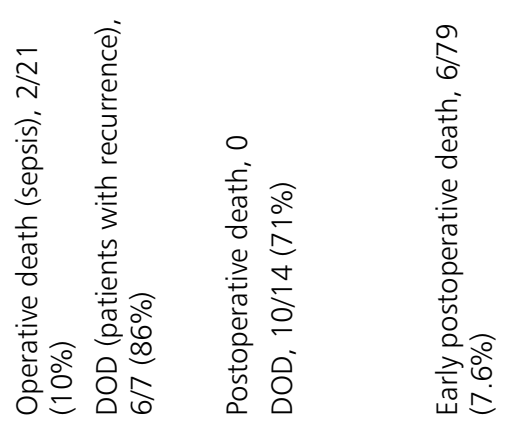

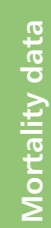

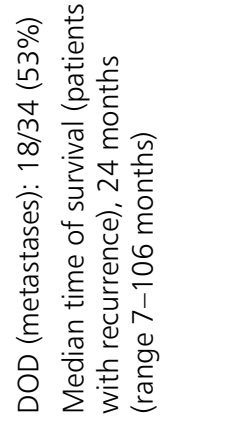

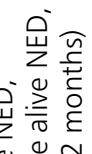

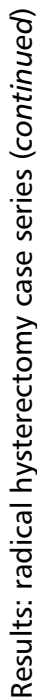

Z

$\therefore \div \frac{1}{m}$

ชัष ठั

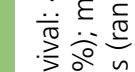

空导莣

ब্ं

ํํㄴำ

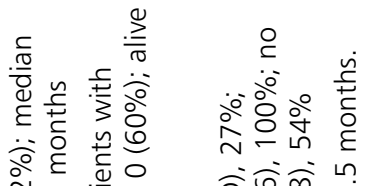

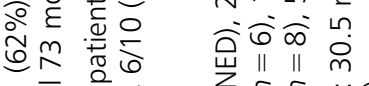

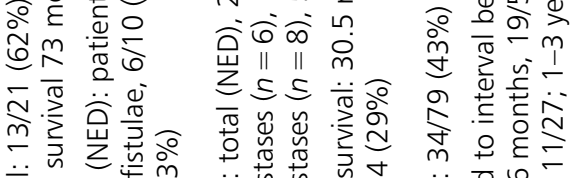

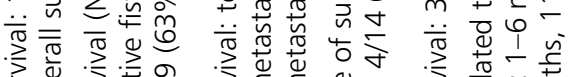

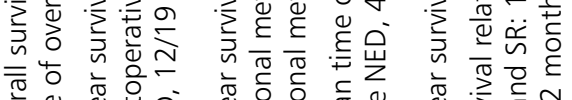

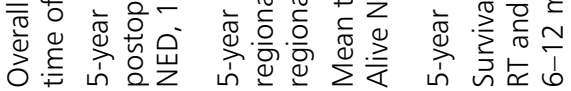

$n-\infty$

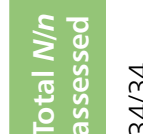

$\underset{\grave{N}}{\bar{N}}$

$\stackrel{Ð}{\stackrel{Ð}{\leftrightarrows}}$

$\frac{\sqrt{2}}{\frac{9}{2}}$ 

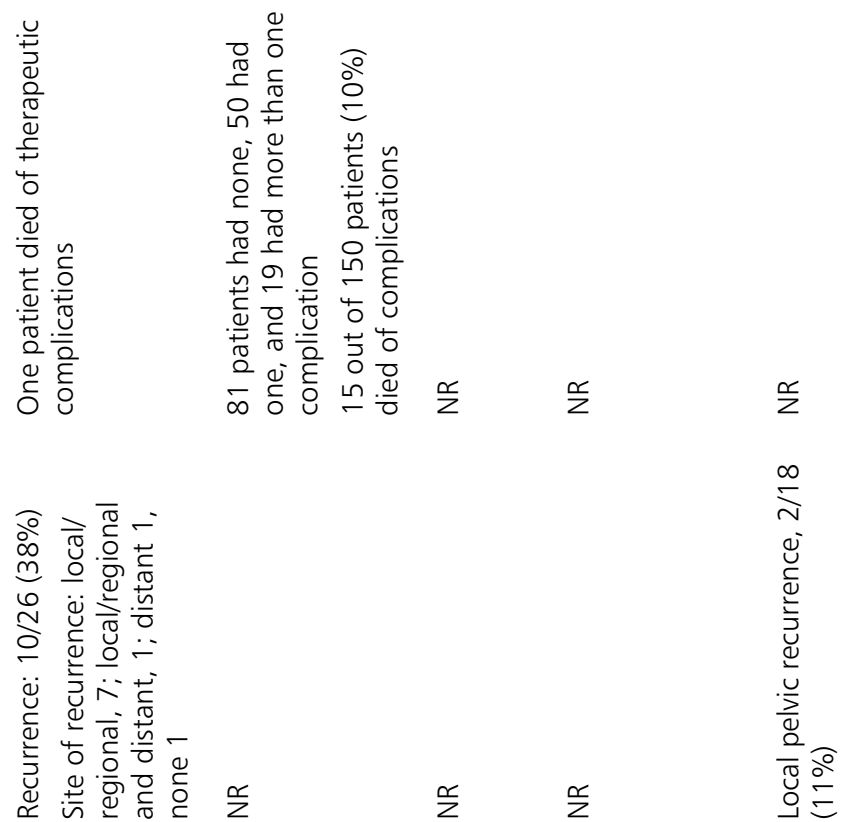

$\frac{\infty}{2}$

बे

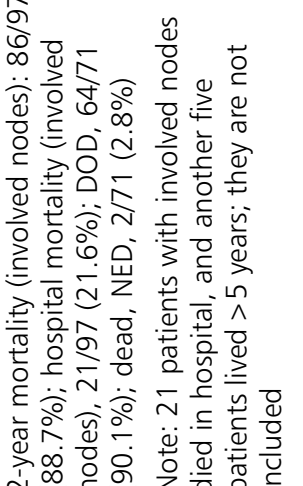

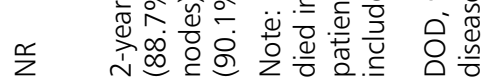

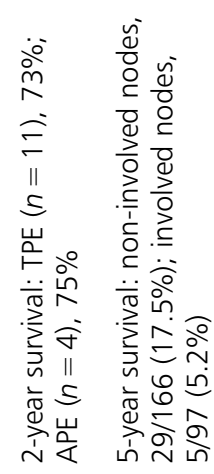

$\frac{}{0}$
$\frac{0}{0}$
$\frac{0}{0}$
$\frac{0}{5}$
$\frac{7}{8}$
$\frac{0}{0}$
$\frac{0}{0}$

ㅎํㅇㅇ

$\stackrel{2}{m} \stackrel{0}{i}$

$\stackrel{2}{i}$

ने

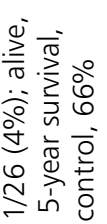

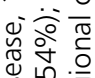

岁造

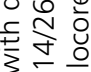

运影

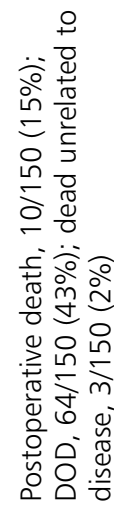

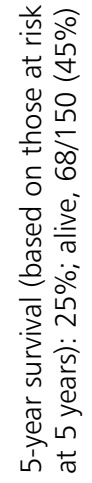

$\stackrel{\frac{c}{z}}{\frac{\alpha}{z}}$

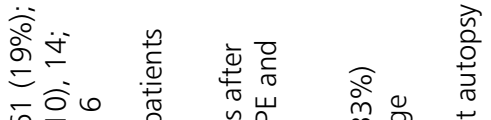

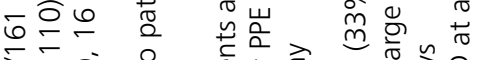
它

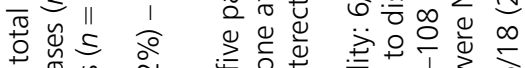
荡兽空

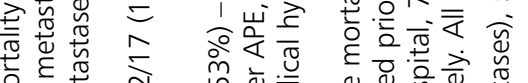

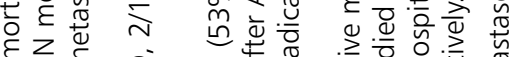

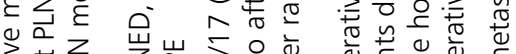

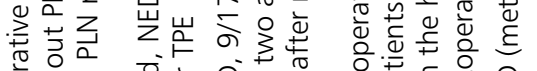

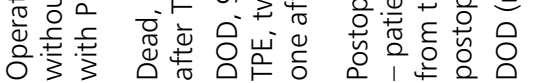

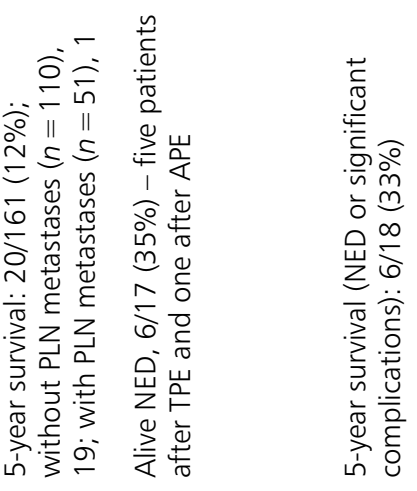

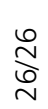

$\stackrel{\substack{n \\ \infty}}{\stackrel{n}{\infty}}$

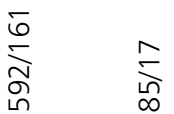

$\stackrel{\infty}{\infty}$

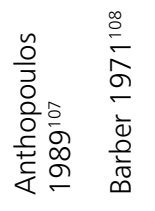

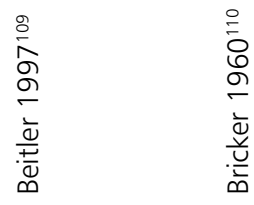

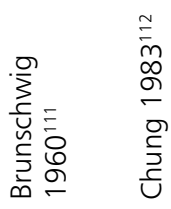

$\stackrel{m}{N}$
$\frac{n}{n}$
$\bar{u}$
$\frac{u}{\tilde{u}}$
0 


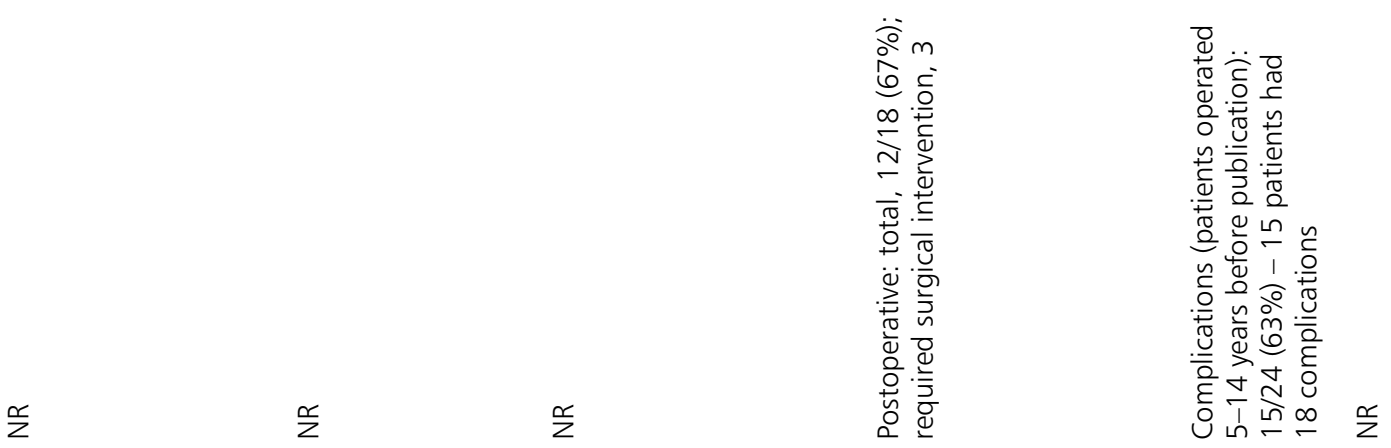

$\stackrel{\frac{c}{2}}{2}$

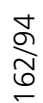

$\stackrel{\infty}{\stackrel{n}{\circ}}$

$\stackrel{\infty}{\infty}$

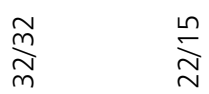

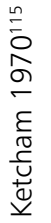

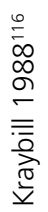

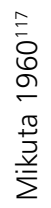

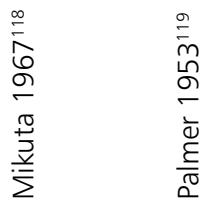



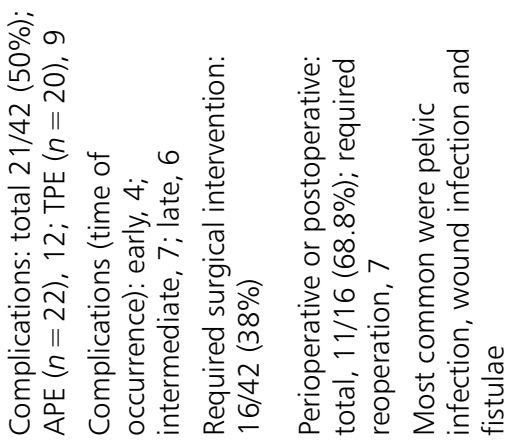

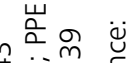

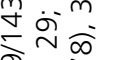

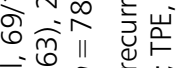

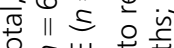

달

ن्य

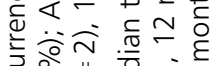

$\stackrel{\infty}{z}$

$\stackrel{\infty}{2}$

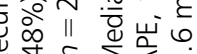

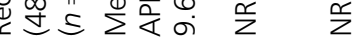

$\stackrel{\alpha}{z}$

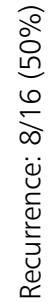

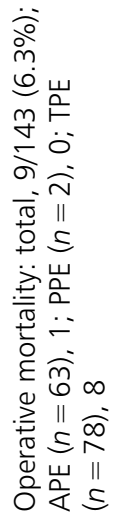

نำ

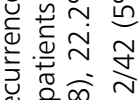

这 $\stackrel{0}{\circ}$

考家药

竞 $m=$

离离离离

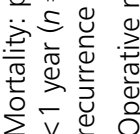

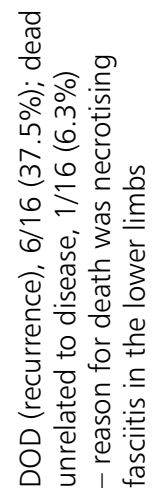

$\stackrel{\frac{c}{z}}{2}$

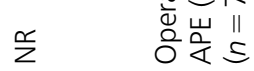

$\stackrel{\infty}{2} \frac{\infty}{2}$

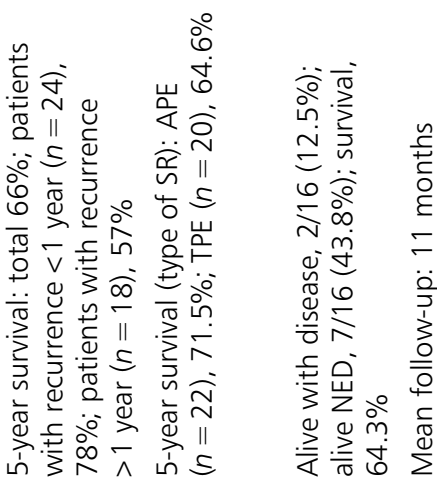

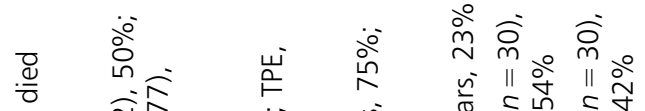

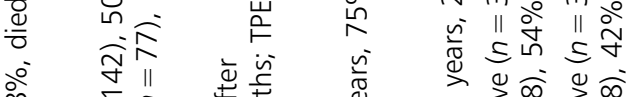

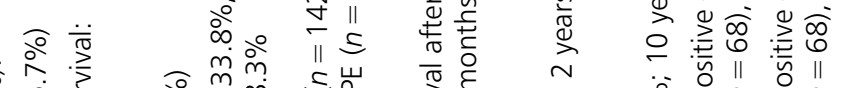

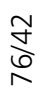

$\stackrel{6}{\leftrightarrows}$

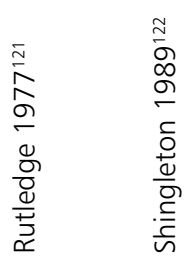

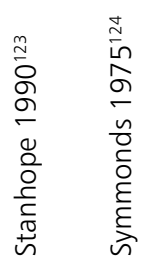

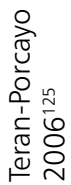

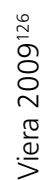


- The subjective elicitation exercise obtained opinions from 21 clinical experts using a structured questionnaire. The results for accuracy in symptomatic women were similar to those from the published test accuracy studies. No comparison was possible for asymptomatic women.

- There was insufficient information in the published literature to use the results as the base case for the economic evaluation and so the subjective elicitation results were used, with the published information in sensitivity analyses.

- The subjective elicitation found that the elicited increase in accuracy from adding PET-CT to CT or MRI was less than the elicited minimum important difference in accuracy required to justify its routine addition in clinical practice.

\section{Effectiveness}

- A total of 19 RCTs ${ }^{60-83}$ on chemotherapy (25 papers), 16 case series $^{84-99}$ on radiotherapy and chemoradiotherapy and 27 case series ${ }^{100-126}$ on radical hysterectomy and pelvic exenteration were included.

- The dates of the publications varied between 1953 and 2010.

- For chemotherapy, the quality of the RCTs was variable, with little information on allocation concealment and none using blinding of patients and outcome measurement.

- There was no information on the effectiveness of cisplatin used as a single therapeutic agent. In comparisons of cisplatin with multiple chemotherapy, cisplatin was associated with either similar or shorter overall survival and progression-free survival, but with fewer side effects.

- For the other chemotherapy comparisons there was too little information to be able to determine the most effective chemotherapeutic options.

- For radiotherapy, 2-year survival rates ranged between $12 \%$ and $85 \%$ and 5 -year survival rates varied between $2 \%$ and $82 \%$, depending on type and location of recurrence and TNM status. For chemoradiotherapy, 2-year survival rates varied between $44 \%$ and $93 \%$ and 5 -year survival rates varied between $30 \%$ and $71 \%$.

- For radical hysterectomy, 5-year survival rates varied between $32 \%$ and $100 \%$; for pelvic exenteration, 5 -year survival rates varied between $12 \%$ and $63 \%$. In general, the lower survival rates were in the earlier case series. Pelvic exenteration had high rates of complications, when results were given.

\section{Accuracy and effectiveness inputs to the economic evaluation}

\section{Accuracy inputs}

A key question of this project was whether PET-CT imaging would be useful as routine surveillance after primary cervical cancer treatment was successful in asymptomatic or symptomatic patients or whether or not it should be used at follow-up to plan management when patients become symptomatic. The systematic review of accuracy studies did not yield any information on routine follow-up of asymptomatic patients. Therefore, the subjective elicitation was used as the base case for the economic model. The test accuracy study results were used within sensitivity analyses for the symptomatic branch of the model and, when we had both, the published test accuracy results were similar to those from the subjective elicitation.

\section{Effectiveness inputs}

Assessment of the systematic review indicated that meta-analysis was not possible in almost all treatment areas. Key points were to ensure that recruitment of patients and treatment given occurred later than 1990 because of the changes in treatment since then. Other factors taken into account were the correct outcome measured and reported, the size of the study and the quality of the study. For some outcomes, little up-to-date information was available and so a pragmatic decision was made to use information from the best-quality studies for inputs to the economic model.

A wide range of chemotherapeutic agents was assessed in the systematic review, but not all are in current use. Clinical advice and the SIGN guideline ${ }^{3}$ suggested that cisplatin used on its own would be the best chemotherapeutic agent to incorporate into the model. A recent IMS Oncology Analyser data set (from 
October 2003 to September 2008) provides NHS clinical practice prescribing to women with recurrent or advanced cervical cancer (Table 54). ${ }^{17}$

Unfortunately, there were no RCTs investigating the effectiveness of cisplatin alone. The estimate of effectiveness was derived from an additional systematic review of cisplatin monotherapy compared with no treatment in any cervical cancer (as there was no evidence in recurrent cervical cancer). The methods and results from this systematic review can be found in Appendix 17. There was only one good-quality, relatively recent, $R C T$ with a large sample size and a survival curve for $\geq 5$ years. ${ }^{127}$ This compared cisplatin $\left(40 \mathrm{mg} / \mathrm{m}^{2}\right.$ weekly for 5 weeks) plus radiotherapy with radiotherapy alone in 259 women with cervical cancer of FIGO grades IB-IVA. The overall 5-year survival was approximately $63 \%$ in the cisplatin arm and $59 \%$ in the no cisplatin arm (log-rank test, $p=0.53$ ) (estimate derived from enlarging survival curve to A3 size).

TABLE 54 NHS prescribed drugs for recurrent or advanced cervical cancer

\begin{tabular}{|c|c|c|}
\hline Therapy & Number of patients & Percentage \\
\hline 5-Fluorouracil & 1 & 2 \\
\hline 5-Fluorouracil/cisplatin & 1 & 2 \\
\hline 5-Fluorouracil/mitomycin C & 1 & 2 \\
\hline Bleomycin/cisplatin/folinic acid/methotrexate & 2 & 4 \\
\hline Carboplatin & 4 & 7 \\
\hline Carboplatin/epirubicin & 1 & 2 \\
\hline Carboplatin/etoposide & 1 & 2 \\
\hline Carboplatin/gemcitabine & 1 & 2 \\
\hline Carboplatin/ifosfamide & 1 & 2 \\
\hline Carboplatin/paclitaxel & 10 & 18 \\
\hline Cisplatin & 22 & 39 \\
\hline Cisplatin/etoposide & 1 & 2 \\
\hline Cisplatin/ifosfamide & 1 & 2 \\
\hline Cisplatin/methotrexate & 2 & 4 \\
\hline Cisplatin/paclitaxel & 2 & 4 \\
\hline Cisplatin/topotecan & 1 & 2 \\
\hline Docetaxel/gemcitabine & 2 & 4 \\
\hline Mitoxantrone/paclitaxel & 1 & 2 \\
\hline Topotecan & 2 & 4 \\
\hline Total & 57 & 100 \\
\hline
\end{tabular}





\section{Chapter 7 Systematic review of economic evaluations}

The database searches identified 409 citations. No identified studies were considered to be relevant to the economic evaluation of PET-CT for the diagnosis of recurrent cervical cancer.

There were six published economic evaluations that were close to being relevant, ${ }^{128-133}$ but these were related to the diagnosis (using other methods) and treatment options for locally advanced cervical cancer and for the treatment of recurrent cervical cancer. Of the four studies ${ }^{128-130,132}$ on the diagnosis of recurrent cervical cancer, two ${ }^{129,130}$ investigated the surveillance of squamous cell carcinoma antigen levels, one ${ }^{128}$ focused on routine cytological surveillance following treatment for cervical cancer and the other ${ }^{132}$ investigated surveillance strategies after treatment for cervical intraepithelial neoplasia. Of the two studies ${ }^{131,133}$ related to the treatment of cervical cancer, one study's objective was to compare the cost-effectiveness of various treatment options for recurrent and stage IVB carcinoma of the cervix ${ }^{133}$ and the other's objective was to investigate the cost-effectiveness of concurrent chemoradiotherapy in comparison with the cost-effectiveness of radiotherapy alone in locally advanced cervical cancer. ${ }^{131}$ These six studies ${ }^{128-133}$ were reviewed in full, but no useful information was taken from them for the economic modelling. 



\section{Chapter 8 Economic evaluation methods and results}

\section{Objective}

The objective of the economic evaluation was to compare the cost-effectiveness of adding PET-CT imaging to standard practice with MRI and/or CT with that of standard practice with MRI and/or CT alone in the diagnosis of recurrent or persistent cervical cancer. Currently in the UK, patients with suspected recurrence will undergo the following investigations:

1. history taking and clinical examination (rectovaginal and speculum examination, assessment of inguinal/supraclavicular lymph nodes)

2. cross-sectional imaging by MRI or CT of chest, abdomen and pelvis

3. examination under anaesthesia, histological confirmation of any vaginal vault mass by biopsy.

The economic evaluation is intended to inform current diagnostic policy for suspected recurrent or persistent cervical cancer, and the value of information (VOI) was intended to highlight future research needs.

\section{Development of the model structure}

To assess the cost-effectiveness of the various diagnostic procedures, a state transition (Markov) model was developed using TreeAge Pro 2011 software (TreeAge Software Inc., Williamstown, MA, USA). A Markov model was the appropriate modelling approach for this evaluation because the time horizons available for both the imaging and the interventions were relatively long and because patients changed health states or experienced recurrent events over a long period of time. ${ }^{134}$

In the model, two diagnostic strategies were examined:

1. clinical examination, MRI and/or CT scan (which represents the standard practice that women receive during follow-up assessment)

2. clinical examination, MRI and/or CT scan with the addition of a PET-CT scan.

The starting point for the patients in the model was women who have previously been treated for primary cervical cancer with either surgery or chemoradiotherapy based on the cancer stage that was defined at diagnosis (Table 55). It was assumed in the model that women who were initially diagnosed with cervical cancer could receive three different management strategies, based on original stage at diagnosis, current development of the malignancy, tumour characteristics and fitness of the patient.

At 3 months' follow-up, if the results of the history and examination suggested the presence of malignancy-related abnormalities (from symptoms such as pain, vaginal bleeding, weight loss, neuropathy or swelling of the abdomen or legs), women will have undergone a biopsy to confirm the presence of persistent or recurrent cervical cancer. This means that a modelled cohort of women following a pathway for the detection and treatment of potential recurrent cervical cancer cannot be considered to be homogeneous. The accuracy of detection and the probability of treatment success in the recurrent stage were affected by the primary diagnosis and the treatment received previously. To address this issue, the same model structure was used for four separate analyses, to account for the following four cohorts of women based on their primary treatment: 
TABLE 55 Percentages of women receiving initial treatment strategies for cervical cancer

\begin{tabular}{|c|c|c|}
\hline Management & Percentage of women receiving care & Explanation \\
\hline \multirow[t]{3}{*}{ Surgery } & $30-40 \%{ }^{a}$ & $\begin{array}{l}\text { Surgery typically involves radical hysterectomy } \\
\text { or trachelectomy }\end{array}$ \\
\hline & Of these, $70-80 \%$ are cured & No further treatment needed \\
\hline & $\begin{array}{l}\text { The remaining } 20-30 \% \text { of women receive } \\
\text { adjuvant postoperative chemoradiotherapy }\end{array}$ & $\begin{array}{l}\text { This is because the histological examination of } \\
\text { the tumour has shown positive margins or there } \\
\text { are positive lymph nodes, or because of tumour } \\
\text { size or volume, lymphovascular space invasion } \\
\text { or stromal invasion }\end{array}$ \\
\hline \multirow[t]{3}{*}{ Chemoradiotherapy } & $50-60 \%{ }^{\mathrm{a}}$ & \\
\hline & Of these, $70 \%$ of the women are cured & No further treatment needed \\
\hline & $\begin{array}{l}\text { The remaining } 30 \% \text { of women are those who } \\
\text { have not responded to first-line treatment } \\
\text { (chemoradiotherapy) and may have persistent } \\
\text { disease }\end{array}$ & $\begin{array}{l}\text { Persistent disease can be detected at } 3 \text { months' } \\
\text { follow-up after initial course of treatment has } \\
\text { finished }\end{array}$ \\
\hline $\begin{array}{l}\text { Palliative treatment } \\
\text { with chemotherapy or } \\
\text { radiotherapy (or both) }\end{array}$ & $<5 \%$ a & \\
\hline
\end{tabular}

a Source: personal communication, Dr S Sundar, University of Birmingham, April 2011.

1. women who had undergone surgery for early-stage primary cervical cancer

2. women who, in addition to surgery as per cohort 1, had postoperative chemoradiotherapy for earlystage primary cervical cancer because of positive margins, etc.

3. women who had chemoradiotherapy for early-stage (stages I and IIA) primary cervical cancer but not surgery

4. women who had chemoradiotherapy for late-stage (stages IIB, III and IV) primary cervical cancer but not surgery.

For all cohorts of women, the clinical pathways and model structure are identical. Figure 28 shows the illustrative Markov model structure and the health-state transitions that are possible within the model. The full tree diagram is shown in Figures 37-43 in Appendix 18. Health states in the illustrative Markov model structure (see Figure 28) are shown in ovals and the arrows represent the transitions that can occur between health states. These 11 health states are described in Table 56. All women who had undergone treatment for primary cervical cancer will start in one of the following four groups: asymptomatic cancer at 3 months, asymptomatic without cancer, symptomatic without cancer or symptomatic cancer at 3 months. The transitions are as follows:

1. asymptomatic women with cancer at 3 months will move to
i. asymptomatic recurrence
ii. symptomatic recurrence
iii. post treatment: asymptomatic cancer at 3 months
iv. death

2. asymptomatic women without cancer will remain or move to

i. asymptomatic recurrence

ii. symptomatic recurrence

iii. symptomatic without cancer

iv. death

3. symptomatic women without cancer will remain or move to

i. asymptomatic without cancer 


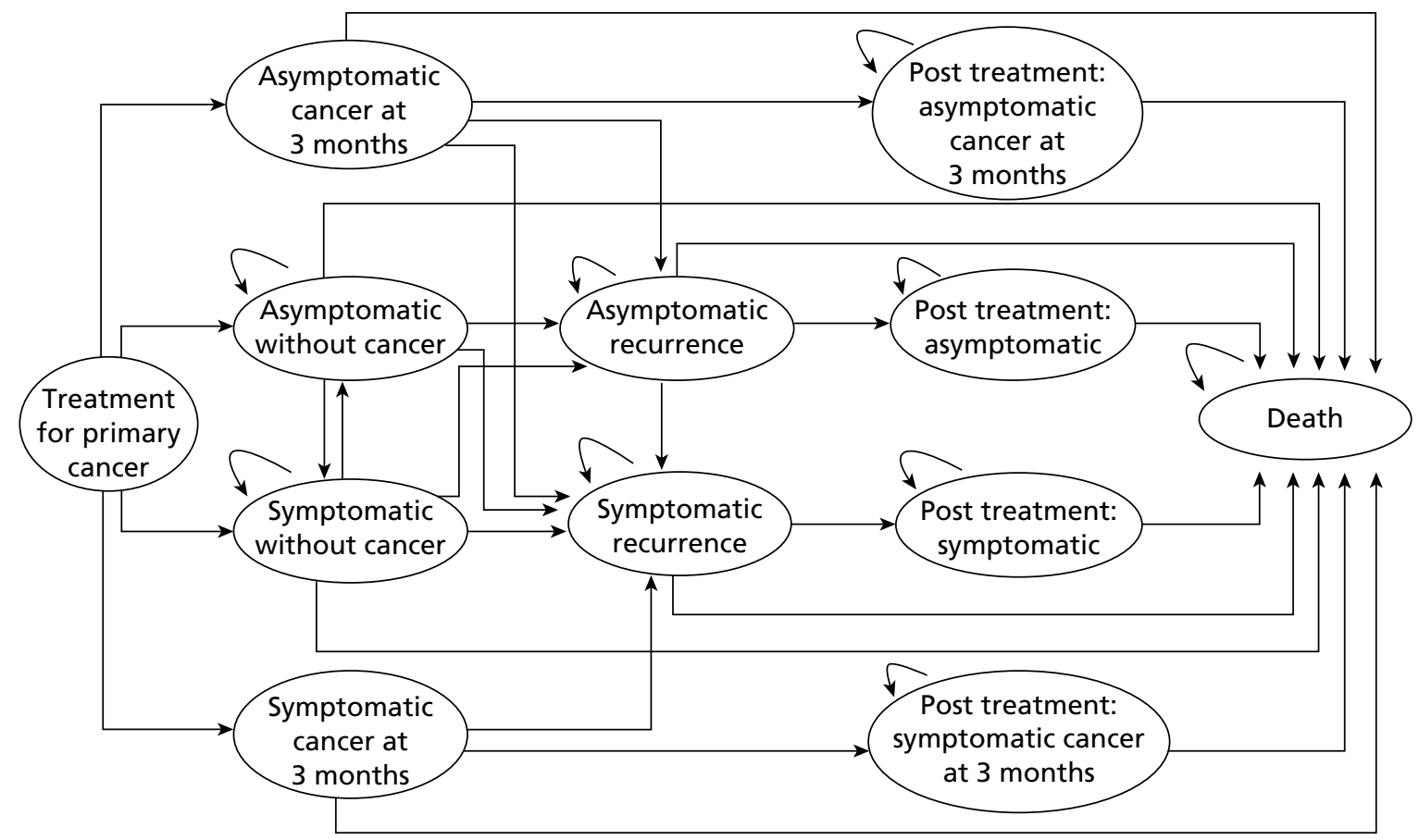

FIGURE 28 Markov model structure: health states and patient flow.
ii. asymptomatic recurrence
iii. symptomatic recurrence
iv. death

4. symptomatic women with cancer at 3 months will move to

i. symptomatic recurrence

ii. post treatment: symptomatic cancer at 3 months

iii. death

5. asymptomatic women with recurrence will remain or move to

i. post treatment: asymptomatic

ii. symptomatic recurrence

iii. death

6. symptomatic women with recurrence will remain or move to

i. post treatment: symptomatic

ii. death

7. post-treatment asymptomatic women with cancer at 3 months will remain or move to

i. death

8. post-treatment symptomatic women with cancer at 3 months will remain or move to

i. death

9. post-treatment asymptomatic women will remain then move to

i. death

10. post-treatment symptomatic women will remain then move to

i. death

11. death.

\section{Model assumptions}

A number of assumptions are required to develop a workable model structure and to enable the analysis to be carried out. These assumptions are: 
TABLE 56 Definition of 11 health states for recurrent cervical cancer pathways

\begin{tabular}{|c|c|c|}
\hline & Asymptomatic & Symptomatic \\
\hline $\begin{array}{l}\text { Cancer at } \\
3 \text { months }\end{array}$ & $\begin{array}{l}\text { Women without symptoms of cancer who are } \\
\text { likely to have recurrent or persistent cancer, } \\
\text { which may or may not be detected at } 3 \text { months' } \\
\text { follow-up }\end{array}$ & $\begin{array}{l}\text { Women with symptoms of cancer who have been } \\
\text { diagnosed with recurrent or persistent cancer, which } \\
\text { may or may not be detected at } 3 \text { months' follow-up } \\
\text { (i.e. symptoms may or may not be cancer) }\end{array}$ \\
\hline $\begin{array}{l}\text { Without } \\
\text { cancer }\end{array}$ & $\begin{array}{l}\text { Women who had previously been treated for } \\
\text { initial cervical cancer and are receiving follow- } \\
\text { up care, but are free of recurrent cervical cancer }\end{array}$ & $\begin{array}{l}\text { Women who experience symptoms that they assume } \\
\text { to be related to recurrent or persistent cervical cancer; } \\
\text { however, on follow-up and confirmatory testing these } \\
\text { women will be cleared of recurrent or persistent } \\
\text { cervical cancer }\end{array}$ \\
\hline Recurrence & $\begin{array}{l}\text { Women without symptoms of cancer who have } \\
\text { cancer that will not have been detected before } \\
\text { a potential follow-up appointment; this may } \\
\text { include women who may have had cancer not } \\
\text { detected during the first } 3 \text { months' follow-up }\end{array}$ & $\begin{array}{l}\text { Women with symptoms that are related to cancer } \\
\text { who received follow-up care and who are confirmed } \\
\text { as having recurrent or persistent cervical cancer }\end{array}$ \\
\hline $\begin{array}{l}\text { Post-treatment } \\
\text { cancer at } \\
3 \text { months }\end{array}$ & $\begin{array}{l}\text { Following diagnosis of cancer at first follow-up } \\
\text { having been asymptomatic, women will receive } \\
\text { new treatment (treatment type based on initial } \\
\text { treatment and location of cancer recurrence or } \\
\text { persistence) }\end{array}$ & $\begin{array}{l}\text { Following diagnosis of cancer at first follow-up having } \\
\text { been symptomatic, women will receive new treatment } \\
\text { (treatment type based on initial treatment and } \\
\text { location of cancer recurrence or persistence) }\end{array}$ \\
\hline Post treatment & $\begin{array}{l}\text { Following diagnosis of recurrent cervical cancer } \\
\text { after being asymptomatic, women will receive } \\
\text { new treatment (treatment type based on initial } \\
\text { treatment and location of cancer recurrence or } \\
\text { persistence) }\end{array}$ & $\begin{array}{l}\text { Following diagnosis of recurrent cervical cancer } \\
\text { after being symptomatic, women will receive new } \\
\text { treatment (treatment type based on initial treatment } \\
\text { and location of cancer recurrence or persistence) }\end{array}$ \\
\hline Death & Women may die from natural causes or may die & a result of recurrent or persistent cervical cancer \\
\hline \multicolumn{3}{|c|}{$\begin{array}{l}\text { The model does not distinguish between recurrence and persistence. Women who had previously been treated for } \\
\text { initial cancer with surgery and/or postoperative chemoradiotherapy or chemoradiotherapy are considered recurrent. } \\
\text { Women who were originally treated with chemoradiotherapy who are detected at this stage are considered to have } \\
\text { persistent disease. }\end{array}$} \\
\hline \multicolumn{3}{|c|}{ Treatment for primary cancer is not included. } \\
\hline
\end{tabular}

1. Women are followed up with examinations every 3 months for 2 years, then every 6 months for 2 years and then annually for 1 year, with the total follow-up being 5 years.

2. Women who were symptomatic at 3 months and whose cancer has not been detected cannot become asymptomatic.

3. Women with symptoms that they suspect are related to cervical cancer are usually given an urgent appointment or their pre-existing follow-up appointment is brought forward.

4. The sensitivity and specificity of the confirmatory biopsy test were $100 \%$ accurate.

5. Women who previously received chemoradiotherapy for primary cervical cancer and who are not diagnosed with persistence at 3 months' follow-up (i.e. not persistent or persistent cases missed) will be treated similarly to women with recurrent cervical cancer when detected.

6. The PET-CT procedure includes both the preparation and the scan of the patient; therefore, the preparation activity is implicit in the PET-CT scan resource use [NHS Reference Cost Team (anonymous) by email, pbrdatacollection@dh.gsi.gov.uk, 12 April 2011, personal communication].

7. Women who received treatment for primary cervical cancer and who have not survived at 5 years have died from recurrent cervical cancer only.

8. The utility for recurrent cervical cancer is equivalent to the average of the utilities for primary stage III and stage IV cervical cancer.

9. There is a constant hazard over 5 years for early-stage recurrent cervical cancer (i.e. the risk of recurrence is the same at 4 years as it is at 1 year). 
10. Women treated for recurrent cervical cancer will have the same quality of life following treatment as they had after treatment for initial cervical cancer.

\section{Data required for the model}

\section{Rates of recurrent cervical cancer}

The model was populated with the rates of recurrent cervical cancer derived from the literature and in consultation with clinical experts. The rates of recurrence were calculated using a two-stage process. First, the survival following treatment for primary cervical cancer was derived from disease-free survival curves from Landoni et al.,135 progression-free survival curves from Keys et al. ${ }^{136}$ and overall survival curves following initial treatment from Landoni et al. ${ }^{135}$ and Vale et al. ${ }^{137}$ Information from these curves was used with the standard assumption of an exponential survival function. Three-month survival results were calculated for women who received surgical treatment, based on the disease-free survival curve presented in Landoni et al. ${ }^{135}$ (Figure 29). Similar procedures were used to calculate survival following postoperative chemoradiotherapy and chemoradiotherapy. Second, the rates of recurrence were calculated, based on the initial survival of women in the branch of women who were symptomatic without cancer (see probabilities f1-f4 in Tables 98-101 in Appendix 18), using the conditional probabilities following survival and the formulae presented in Table 57. Table 58 shows the rates of recurrence used in the models.

Women enter the model at 3 months after initial treatment. If they have cancer at 3 months they enter the state 'Asymptomatic cancer at 3 months' or 'Symptomatic cancer at 3 months', but if they are free of cancer at this time they enter the state 'Asymptomatic without cancer' or 'Symptomatic without cancer'. In effect, the 3 months before entry in the model can be regarded as being represented by the probability tree shown in Figure 30.

Ideally, a separate data source would have been used to determine the proportions of women in each of these four states at the start of the model. In the absence of such a data source, it was necessary to make an assumption about these proportions. It was decided to use the probabilities for women moving from

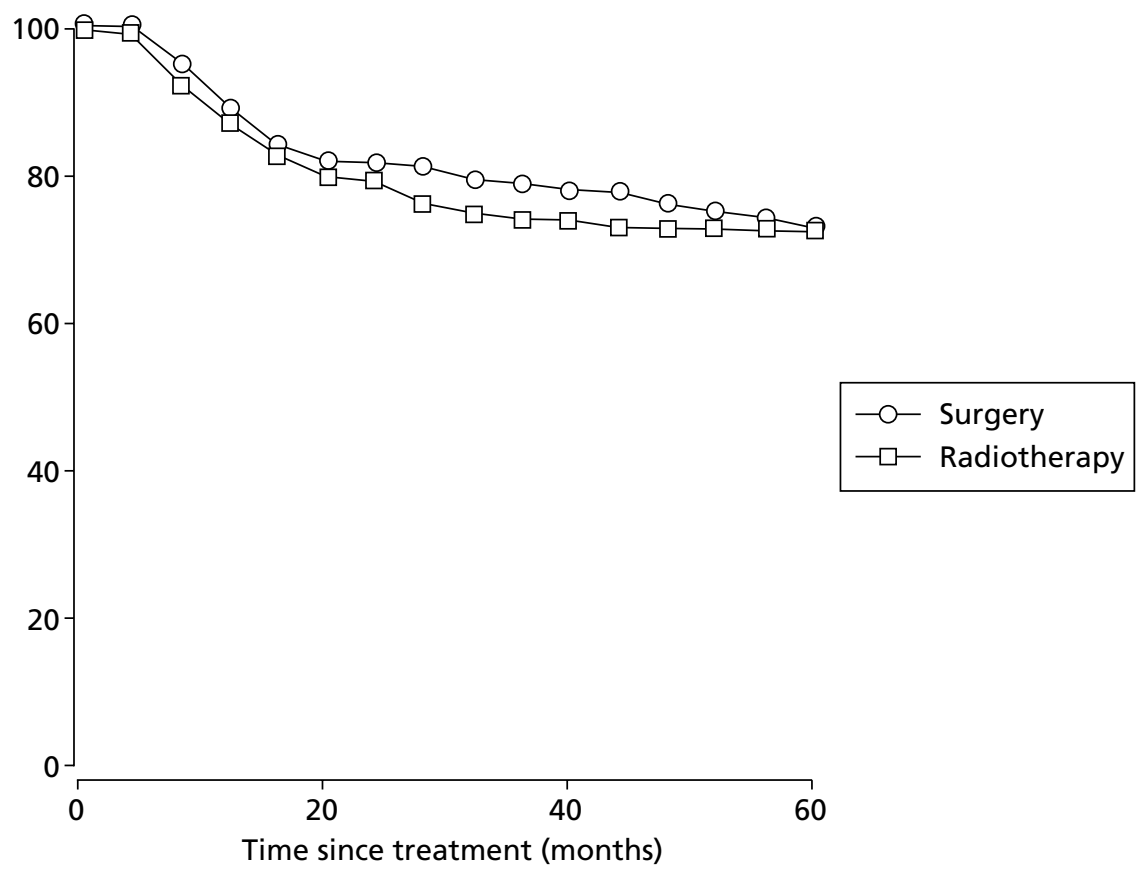




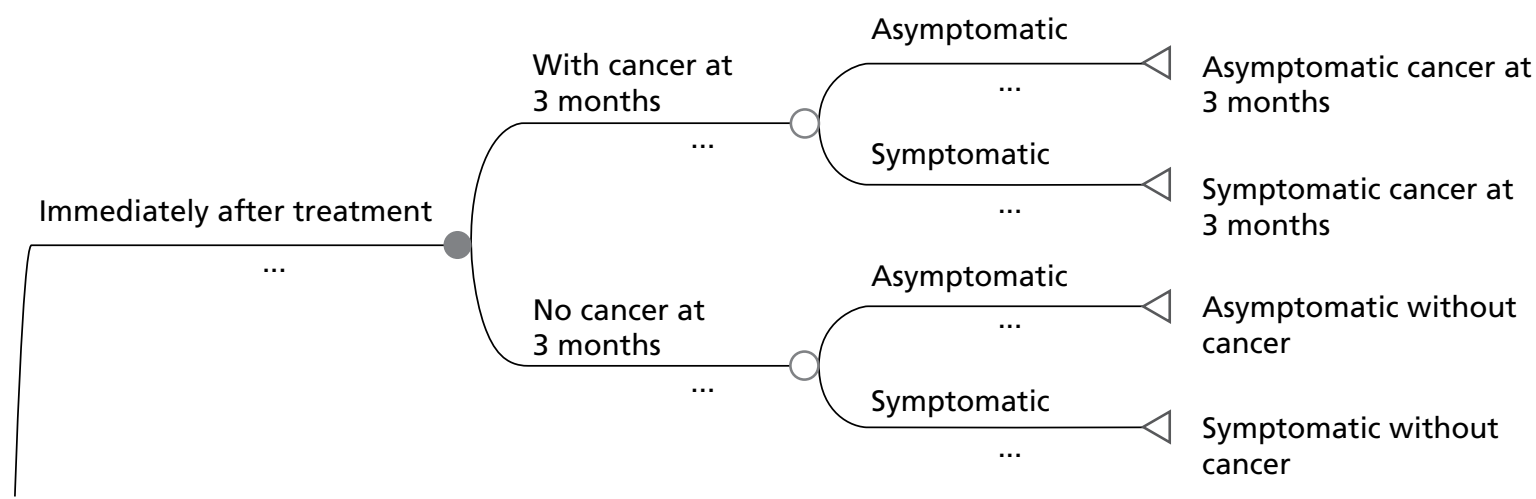

FIGURE 30 Initial 3 months following treatment (before entry into the model).

the state 'Symptomatic without cancer' (see Tables 98-101 in Appendix 18 for probabilities $\mathrm{f2-f4)}$ ) to give the necessary proportions. The way this was carried out is further detailed in Table 57.

\section{Test accuracy results}

Test accuracy results used in the model were based on the values estimated in the subjective elicitation exercise (see Chapter 5 and Table 14). The predictive values, 95\% Cls and probability distributions for MRI and/or CT and for PET-CT are shown in Tables 59 and 60 respectively. Using the appropriate formulae, predictive values were converted to sensitivities and specificities to be used in the models. Table 61 shows the accuracy data used in the base-case analysis. For sensitivity analysis, the uncertainty indicated in Tables 59 and 60 was applied to the predictive values before conversion to sensitivity and specificity.

\section{Survival following treatment}

The results used in the model for survival following treatment for recurrence or persistence are shown in Table 62. Survival was reported in the systematic review in Chapter 6. From the systematic review, survival data from studies that followed up women following treatment for recurrent cervical cancer prior to 1990 were excluded. In cases in which 5-year survival after 1990 was not reported, ${ }^{96} 2$ - and 3-year survival data were used. Note that these overall survival results are not given separately by the four FIGO stages.

From the results in Table 62, a weighted average of the 3-month survival following treatment was calculated, using weighting based on the percentages of people receiving the different treatments. Table 63 shows the 3 -month survival data used in the models.

\section{Costs and resources}

The costs of resources used were those that were directly incurred by the NHS. Costs for clinical examination, diagnostic imaging (PET-CT, MRI and CT), confirmatory biopsy and treatment were included (Table 64). Costs that were not considered were those incurred during the primary diagnosis and treatment of cervical cancer. Other costs not included were those for long-term and end-of-life care. In the models, recurrence was assumed to occur only once. Diagnostic procedure costs were taken from the NHS Reference Costs 2009-2010. ${ }^{139}$ Cost estimates for chemotherapy and radiotherapy treatment were taken from Clark et al., ${ }^{140}$ and estimates for chemoradiotherapy were taken from Clark et al. ${ }^{140}$ and were adjusted to 2010 prices using the Hospital and Community Health Services combined pay and price inflation index. ${ }^{141}$ Estimated costs for the diagnosis of recurrent cervical cancer included costs for clinical examination, PET-CT, MRI and CT. These cost estimates were taken from the NHS Reference Costs 2009$2010^{139}$ and published sources. ${ }^{141}$ As a result of a paucity of cost-effectiveness studies comparing PET-CT as an adjunct with standard practice, an additive procedural cost of PET-CT as an adjunct to standard practice was assumed, as shown in Table 64. All costs were adjusted to 2010 prices and were discounted at 3.5\% per annum. 
TABLE 57 Formulae used to calculate the rates of recurrence of cervical cancer used in the models

\begin{tabular}{|c|c|c|c|c|c|c|c|}
\hline Parameter & \multicolumn{6}{|c|}{ Written formula } & Formula $^{a}$ \\
\hline $\begin{array}{l}\text { Asymptomatic at } \\
3 \text { months }\end{array}$ & \multicolumn{6}{|c|}{$\begin{array}{l}\text { (Probability of becoming recurrent having been symptomatic without } \\
\text { cancer } \times \text { probability of being asymptomatic recurrence conditional on } \\
\text { recurrence) }\end{array}$} & $(\mathrm{f} 2 \times \mathrm{f3})$ \\
\hline $\begin{array}{l}\text { Asymptomatic } \\
\text { without cancer }\end{array}$ & \multicolumn{6}{|c|}{$\begin{array}{l}\text { [( } 1 \text { - probability of becoming recurrent having been symptomatic without } \\
\text { cancer }) \times \text { (probability of becoming asymptomatic without cancer conditional } \\
\text { on no recurrence)] }\end{array}$} & {$[(1-f 2) \times f 4]$} \\
\hline $\begin{array}{l}\text { Symptomatic at } \\
3 \text { months }\end{array}$ & \multicolumn{6}{|c|}{$\begin{array}{l}\text { [Probability of becoming recurrent having been symptomatic without } \\
\text { cancer } \times(1 \text { - probability of being asymptomatic recurrence conditional on } \\
\text { recurrence) }\end{array}$} & {$[f 2 \times(1-f 3)]$} \\
\hline $\begin{array}{l}\text { Symptomatic } \\
\text { without cancer }\end{array}$ & \multicolumn{6}{|c|}{$\begin{array}{l}\text { [( } 1 \text { - probability of becoming recurrent having been symptomatic without } \\
\text { recurrent cancer }) \times(1 \text { - probability of becoming asymptomatic without cancer } \\
\text { conditional on no recurrence })]\end{array}$} & {$[(1-f 2) \times(1-f 4)]$} \\
\hline \multicolumn{8}{|c|}{ a See Appendix 18 for tree diagram. } \\
\hline \multirow[b]{2}{*}{ Parameter } & \multicolumn{2}{|c|}{ Surgery } & \multicolumn{2}{|c|}{ Chemoradiotherapy } & \multicolumn{2}{|c|}{$\begin{array}{l}\text { Postsurgery } \\
\text { chemoradiotherapy }\end{array}$} & \multirow[b]{2}{*}{ Source } \\
\hline & Early & Late & Early & Late & Early & Late & \\
\hline $\begin{array}{l}\text { Asymptomatic at } \\
3 \text { months }\end{array}$ & 0.0041 & - & 0.0041 & 0.0041 & 0.0041 & - & \multirow{4}{*}{$\begin{array}{l}\text { Derived } \\
\text { from data } \\
\text { from the } \\
\text { literature } \\
\text { and clinical } \\
\text { experts }\end{array}$} \\
\hline $\begin{array}{l}\text { Asymptomatic } \\
\text { without cancer }\end{array}$ & 0.8907 & - & 0.8907 & 0.8907 & 0.8907 & - & \\
\hline $\begin{array}{l}\text { Symptomatic at } \\
3 \text { months }\end{array}$ & 0.0062 & - & 0.0062 & 0.0062 & 0.0062 & - & \\
\hline $\begin{array}{l}\text { Symptomatic without } \\
\text { cancer }\end{array}$ & 0.0990 & - & 0.0990 & 0.0990 & 0.0990 & - & \\
\hline
\end{tabular}

TABLE 59 Subjective elicitation summary accuracy results: MRI and/or CT

\begin{tabular}{lllll} 
Characteristic & Predictive value & MRI and/or CT & $95 \%$ Cl & $\begin{array}{l}\text { Probability } \\
\text { distribution }\end{array}$ \\
Symptomatic & PPV & 0.884 (SD 0.092) & 0.8415 to 0.9265 & $\operatorname{Beta}(188.77,24.77)$ \\
& NPV & 0.868 (SD 0.087) & 0.8308 to 0.9112 & $\operatorname{Beta}(226.38,33.53)$ \\
Asymptomatic & PPV & 0.856 (SD 0.098) & 0.8107 to 0.9013 & $\operatorname{Beta}(196.94,33.13)$ \\
& NPV & $0.900($ SD 0.077$)$ & 0.8644 to 0.9356 & $\operatorname{Beta}(237.14,26.35)$ \\
\hline
\end{tabular}


TABLE 60 Subjective elicitation summary accuracy results: MRI and/or CT with PET-CT

\begin{tabular}{lllll} 
Characteristic & Predictive value & $\begin{array}{l}\text { MRI and/or CT and } \\
\text { PET-CT }\end{array}$ & $95 \%$ Cl & $\begin{array}{l}\text { Probability } \\
\text { distribution }\end{array}$ \\
Symptomatic & PPV & $0.910($ SD 0.082$)$ & 0.8721 to 0.9479 & $\operatorname{Beta}(295.75,29.25)$ \\
& NPV & $0.907($ SD 0.072$)$ & 0.8737 to 0.9403 & $\operatorname{Beta}(299.31,30.69)$ \\
Asymptomatic & PPV & $0.902($ SD 0.077$)$ & 0.8664 to 0.9376 & $\operatorname{Beta}(270.6,29.4)$ \\
& NPV & $0.934($ SD 0.055$)$ & 0.9086 to 0.9594 & $\operatorname{Beta}(396.95,28.05)$ \\
\hline
\end{tabular}

TABLE 61 Accuracy results used in the models

\begin{tabular}{|c|c|c|c|c|c|}
\hline \multirow[b]{3}{*}{ Intervention } & \multicolumn{4}{|c|}{ Recurrent/persistent cervical cancer } & \multirow[b]{3}{*}{ Source } \\
\hline & \multicolumn{2}{|c|}{ Asymptomatic } & \multicolumn{2}{|c|}{ Symptomatic } & \\
\hline & $\begin{array}{l}\text { Sensitivity } \\
(\%)\end{array}$ & $\begin{array}{l}\text { Specificity } \\
(\%)\end{array}$ & $\begin{array}{l}\text { Sensitivity } \\
(\%)\end{array}$ & $\begin{array}{l}\text { Specificity } \\
(\%)\end{array}$ & \\
\hline \multicolumn{6}{|l|}{ Recurrence after initial treatment } \\
\hline Clinical follow-up and MRI $\pm \mathrm{CT}$ & 45.43 & 98.47 & 85.09 & 89.78 & \multirow{2}{*}{$\begin{array}{l}\text { Elicitation } \\
\text { exercise }\end{array}$} \\
\hline Clinical follow-up, MRI $\pm \mathrm{CT}$ and $\mathrm{PET}-\mathrm{CT}$ & 65.25 & 98.58 & 89.71 & 91.88 & \\
\hline
\end{tabular}

TABLE 62 Overall survival for treatment options following recurrent or persistent cervical cancer

\begin{tabular}{|c|c|c|c|c|}
\hline Treatment option & 2-year survival (\%) & 3-year survival (\%) & 5-year survival (\%) & Source \\
\hline Radiotherapy & - & - & $\begin{array}{l}40.2(95 \% \mathrm{Cl} 31.6 \text { to } 48.6) \\
\text { for whole group }\end{array}$ & Jain et al. ${ }^{88}$ \\
\hline Chemotherapy & _ & _- & 64 & Pearcey et al. ${ }^{127}$ \\
\hline Chemoradiotherapy & 44 & 25 & _- & Maneo et al. ${ }^{96}$ \\
\hline Pelvic exenteration & - & - & 63 & Beitler et al. ${ }^{109}$ \\
\hline Untreated & - & - & 3.1 & Adriano et al. ${ }^{138}$ \\
\hline
\end{tabular}

TABLE 63 Weighted 3-month survival data following recurrent cervical cancer

\begin{tabular}{|c|c|c|c|}
\hline Model (initial treatment) & $\begin{array}{l}\text { 3-month } \\
\text { survival }\end{array}$ & $95 \% \mathrm{Cl}$ & Source \\
\hline Model 1: Early stage, treated with surgery & 0.9307 & 0.8842 to 0.9772 & \multirow{4}{*}{$\begin{array}{l}\text { Derived from the survival literature } \\
\text { and the proportions of women } \\
\text { receiving treatment for recurrent } \\
\text { cervical cancer }\end{array}$} \\
\hline $\begin{array}{l}\text { Model 2: Early stage, treated with } \\
\text { chemoradiotherapy }\end{array}$ & 0.9778 & 0.8526 to 0.9968 & \\
\hline $\begin{array}{l}\text { Model 3: Late stage, treated with } \\
\text { chemoradiotherapy }\end{array}$ & 0.9779 & 0.8530 to 0.9969 & \\
\hline $\begin{array}{l}\text { Model 4: Early stage, treated with surgery and } \\
\text { postoperative chemoradiotherapy }\end{array}$ & 0.9778 & 0.8526 to 0.9968 & \\
\hline
\end{tabular}


TABLE 64 Cost data used in the model (all costs presented in 2010 UK pounds)

\begin{tabular}{|c|c|c|}
\hline Description & Unit cost (f) & Source \\
\hline \multicolumn{3}{|l|}{ Examination and imaging } \\
\hline Clinical examination & 28.17 & Curtis $2010^{141}$ \\
\hline PET-CT & 744.00 & NHS Reference Costs 2009-2010 139 \\
\hline MRI & 366.00 & NHS Reference Costs 2009-2010139 \\
\hline CT & 162.00 & NHS Reference Costs 2009-2010 \\
\hline \multicolumn{3}{|l|}{ Confirmatory test } \\
\hline Cone biopsy of cervix uteri NEC & 968.00 & NHS Reference Costs 2009-2010 139 \\
\hline \multicolumn{3}{|l|}{ Treatment } \\
\hline Surgical & 6723.00 & NHS Reference Costs 2009-2010 \\
\hline Chemoradiotherapy & $14,495.14$ & Brush et al., ${ }^{142}$ Curtis $2010^{141}$ \\
\hline \multicolumn{3}{|l|}{ Palliative } \\
\hline Chemotherapy & 356.56 & Clark et al.., ${ }^{140}$ Curtis $2010^{141}$ \\
\hline Radiotherapy & 1167.79 & Clark et al.., ${ }^{140}$ Curtis $2010^{141}$ \\
\hline \multicolumn{3}{|l|}{ Weighted treatment costs } \\
\hline Model 1 & $13,011.00$ & \multirow{4}{*}{$\begin{array}{l}\text { Derived from the literature and from consultation with clinical } \\
\text { experts }\end{array}$} \\
\hline Model 2 & 1629.85 & \\
\hline Model 3 & 993.20 & \\
\hline Model 4 & 1629.85 & \\
\hline
\end{tabular}

NEC, neuroendocrine carcinoma.

Treatment of recurrent cervical cancer depends on the site and extent of recurrence, the type of previous treatment received, time elapsed since primary treatment and the patient's performance status. Treatment options for recurrent cervical cancer include surgery (radical hysterectomy or pelvic exenteration), chemoradiotherapy and palliative treatment (which can be chemoradiotherapy or radiotherapy). Treatment costs are presented in Table 64. In the models, a weighted mean cost of treatment was calculated based on the proportion of women who would receive each treatment. In model 1, for women who had previously received surgery for early-stage cervical cancer, treatment for recurrence was likely to be chemoradiotherapy in $85 \%$ of cases, exenteration in $10 \%$ of cases and chemotherapy for palliative care in the remaining 5\%. The weighted treatment cost for model 1 was estimated at $f 13,011.00$. In model 2, for women who had previously received chemoradiotherapy for early-stage cervical cancer, treatment for recurrence was likely to be chemotherapy alone for $80 \%$ of cases and exenteration for the remaining $20 \%$. The weighted treatment cost for model 2 was estimated at $\mathrm{f} 1629.85$. In model 3 , for women who had previously received chemoradiotherapy for late-stage cervical cancer, treatment for recurrence was likely to be chemotherapy alone in $90 \%$ of cases and pelvic exenteration for the remaining $10 \%$. The weighted

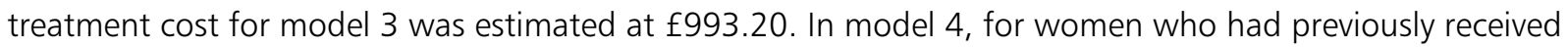
postoperative chemoradiotherapy for early-stage cervical cancer, treatment for recurrence was likely to be chemotherapy alone in $80 \%$ of cases and radical hysterectomy or pelvic exenteration for the remaining $20 \%$. The weighted treatment cost for model 4 was estimated at $f 1629.85$. (These percentage estimates were obtained in personal communication with Dr S Sundar, University of Birmingham, December 2011, as there was no published information available.) The proportions of women receiving treatment following recurrent cervical cancer, with their 95\% Cls and probability distributions, are provided in Tables 65-68 for models $1-4$ respectively. 
TABLE 65 Proportions of women receiving treatment following recurrent cervical cancer: model 1

\begin{tabular}{llll} 
Treatment following recurrence & Proportion & $95 \% \mathrm{Cl}$ & Probability distribution \\
Chemotherapy & 0.85 & 0.8075 to 0.8925 & $\operatorname{Beta}(226.23,39.99)$ \\
Surgery & 0.10 & 0.0500 to 0.1050 & $\operatorname{Beta}(44.65,401.82)$ \\
Palliative care & 0.05 & 0.0475 to 0.0525 & $\operatorname{Beta}(1840.04,34960.76)$ \\
\hline
\end{tabular}

TABLE 66 Proportions of women receiving treatment following recurrent cervical cancer: model 2

\begin{tabular}{llll} 
Treatment following recurrence & Proportion & $95 \% \mathrm{Cl}$ & Probability distribution \\
Chemotherapy & 0.80 & 0.7600 to 0.8400 & Beta $(309.98,77.50)$ \\
Surgery & 0.20 & 0.1900 to 0.2100 & Beta $(1183.93,4735.74)$ \\
\hline
\end{tabular}

TABLE 67 Proportions of women receiving treatment following recurrent cervical cancer: model 3

\begin{tabular}{llll} 
Treatment following recurrence & Proportion & $95 \% \mathrm{Cl}$ & Probability distribution \\
Chemotherapy & 0.90 & 0.8500 to 0.9500 & Beta $(123.47,13.72)$ \\
Surgery & 0.10 & 0.0500 to 0.1050 & $\operatorname{Beta}(44.65,401.82)$ \\
\hline
\end{tabular}

TABLE 68 Proportions of women receiving treatment following recurrent cervical cancer: model 4

\begin{tabular}{llll} 
Treatment following recurrence & Proportion & $95 \% \mathrm{Cl}$ & Probability distribution \\
Chemotherapy & 0.80 & 0.7600 to 0.8400 & Beta $(309.98,77.50)$ \\
Surgery & 0.20 & 0.1900 to 0.2100 & Beta $(1183.93,4735.74)$ \\
\hline
\end{tabular}

\section{Outcomes}

Three different effectiveness/outcome measures were used in the model: QALYs, recurrent case treated and death due to recurrent cervical cancer avoided. For the QALY calculations, utility weights for women who had been diagnosed with recurrent cervical cancer were obtained from Goldie et al. ${ }^{143}$ The authors reported utility weights for women with invasive cancer by FIGO stage. An average weight based on stages III (0.56) and IV (0.48) was calculated, giving a utility for recurrent cervical cancer of 0.52 . From the systematic review there were no studies that reported quality-of-life data following treatment for recurrent cervical cancer in a form that could be used in the model. It was assumed that women treated for recurrent cervical cancer would have the same quality of life as that following treatment for initial cervical cancer. Lang et al. ${ }^{144}$ measured the health-related quality of life of Taiwanese women who have been treated for cervical cancer - this was associated with a quality of life of 0.87 . In this paper the instruments used to measure health-related quality of life were the European Quality of Life-5 Dimensions (EQ-5D), Short Form questionnaire-8 items (SF-8) and the Karnofsky Performance Status (KPS). In the models, the results of the EQ-5D were used because it is recommended by NICE as the most appropriate measure to calculate QALY estimates. It is also useful because its responsiveness has been shown to be equal to that of the European Organisation for Research in the Treatment of Cancer (EORTC) Quality of Life Questionnaire Core 30 (QLQ C-30) global health status measure. ${ }^{144}$ The utilities, 95\% Cls and probability distributions used in the model are shown in Table 69.

\section{Analysis}

The recurrent cervical cancer model begins with a hypothetical cohort of women who have previously been treated for primary cervical cancer and who are now receiving follow-up assessment. The model estimates 
TABLE 69 Utility data used in the model

\begin{tabular}{llll} 
Recurrence & Utility & $95 \% \mathrm{Cl}$ & Probability distribution \\
Asymptomatic recurrence & 0.87 & 0.8564 to 0.8836 & Beta $(2175,325)$ \\
Symptomatic recurrence & 0.52 & 0.3900 to 0.6500 & Beta(28.6, 26.4) \\
\hline
\end{tabular}

the mean costs associated with the diagnostic procedure and assumes that women entering the model would be aged 50 years.

The model has a cycle length of 3 months. The follow-up pattern was every 3 months for 2 years and then twice a year for 3 years. This represents the follow-up pattern for women who were treated for initial cervical cancer. The model assumes a time period of 5 years; this represents the length of time that women are followed up after being diagnosed and treated and the time within which recurrent cervical cancer may be likely to occur. ${ }^{32}$

The model takes the form of a cost-utility analysis and was carried out from the UK NHS perspective in a secondary care setting. The primary outcome is cost per QALY, but a secondary outcome measure of cost per recurrent case treated was also estimated. The results of the cost-utility analysis are presented in terms of the incremental cost-effectiveness ratios (ICERs).

A deterministic sensitivity analysis was carried out on the 5-year survival rate for women who were untreated for recurrent cervical cancer $\left(3.0 \%^{138}-60 \%{ }^{127}\right)$. This wide range is because the estimate of $3 \%$ is from studies dated between 1906 and 1926 and it is likely that survival is now higher than this in untreated cervical cancer. As cervical cancer would now always be treated, it is unclear what the survival rate would be without treatment. The other inputs that were changed were the rates of symptomatic recurrence within 3 months of treatment and the utility values. Arbitrary values were used to explore the impact of changes on the results, given that the available data were poor. Rates of symptomatic recurrence within 3 months of treatment are given in Table $99(\mathrm{~d} 1=0.9778)$ and Table $100(\mathrm{~d} 2=0.9779)$ and these were changed to 0.9307 , which is the lowest available estimate for surviving within 3 months of testing. The utility values used in the model were halved.

Probabilistic sensitivity analysis (PSA) was undertaken to determine the uncertainty in the model input parameters of prevalence, sensitivity and specificity, treatment costs and expected QALYs. PSA was carried out based on an outcome of cost per QALY only. In PSA, each model parameter was assigned a distribution reflecting the amount and pattern of its variation, and cost-effectiveness results were calculated by simultaneously selecting random values from each distribution. The process was repeated 10,000 times in a Monte Carlo simulation of the model to give an indication of how variation in the model parameters led to variation in the ICERs for a given test combination.

\section{Value of information analysis}

When a decision is not robust to plausible variation in the input parameters, it is possible to estimate a statistic known as the expected value of perfect information (EVPI). This is determined as a function of the threshold ICER, which allows a conversion from QALYS to monetary value. The preferred decision under uncertainty is determined by maximising the mean net benefit across the distribution of input parameter values. For any specific parameter set that leads to the same decision, there is no value of information attached to those parameters. If, however, a parameter set leads to a change in the decision, then the value attached to that parameter set is the difference in net monetary benefit between the decision made under uncertainty and the decision made knowing those parameter values. The EVPI is obtained by calculating the value attached to each parameter set used in the PSA and averaging across all parameter sets, taking into account the weightings determined by the probabilistic calibration described in the previous section. 


\section{Results of modelling}

\section{Results in terms of cost per quality-adjusted life-year}

The base-case deterministic results of the strategies based on the cost per QALY are presented in Tables 70-73. The costs are adjusted to 2009/10 prices.

\section{Model 1: women who have been treated for early-stage cancer by surgery}

The results for model 1, women who have previously received treatment by surgery for early-stage cancer, are presented in Table 70. Standard practice had a mean cost of approximately 19169 with corresponding QALYs of 4.1086 compared with a mean cost of approximately f18,757 and 4.1096 QALYs for PET-CT together with standard practice. The estimated ICER for PET-CT together with standard practice compared with standard practice alone was $\mathrm{f9}, 254,000$ per QALY. This indicates that, for every additional QALY gained from the use of PET-CT as an adjunct to standard practice, there is an incremental cost of $f 9,254,000$.

\section{Model 2: women who have been treated for early-stage cancer by chemoradiotherapy}

The results for model 2, women who have previously received chemoradiotherapy for early-stage cancer, are presented in Table 71 . Standard practice had a mean cost of approximately $f 7695$ with corresponding QALYs of 4.1501 compared with a mean cost of approximately $f 17,122$ and corresponding QALYs of 4.1581 for PET-CT together with standard practice. The estimated ICER for PET-CT together with standard practice compared with standard practice alone was approximately $f 1,173,000$ per QALY. This indicates that, for every additional QALY gained from the use of PET-CT as an adjunct to standard practice, there is an incremental cost of $£ 1,173,000$.

Model 3: women who have been treated for late-stage cancer by chemoradiotherapy and model 4: women who have been treated for earlystage cancer by postoperative chemoradiotherapy

Similarly, for models 3 and 4, the results are presented in Tables 72 and 73 respectively. The mean costs for standard practice were $f 7612$ and $f 7695$ with QALYs of 4.1507 and 4.1501 respectively. The estimated ICERs for PET-CT together with standard practice compared with standard practice alone were approximately $£ 1,065,000$ per QALY for model 3 and $£ 1,173,000$ per QALY for model 4.

TABLE 70 Model 1 base-case results from the analysis based on cost per QALY

\begin{tabular}{lcllll} 
Strategy & $\begin{array}{l}\text { Mean cost per } \\
\text { strategy }(\mathrm{f})\end{array}$ & $\begin{array}{l}\text { Difference in } \\
\text { costs }(\mathrm{f})\end{array}$ & $\begin{array}{l}\text { Effectiveness } \\
\text { (QALYs) }\end{array}$ & $\begin{array}{l}\text { Incremental } \\
\text { QALYs }\end{array}$ & $\begin{array}{l}\text { ICER (f) } \\
\text { Standard practice }\end{array}$ \\
$\begin{array}{l}\text { PET-CT together with standard } \\
\text { practice }\end{array}$ & 18,759 & - & 4.1086 & - & - \\
\hline
\end{tabular}

TABLE 71 Model 2 base-case results from the analysis based on cost per QALY

$\begin{array}{lcllll}\text { Strategy } & \begin{array}{l}\text { Mean cost per } \\ \text { strategy }(\mathrm{f})\end{array} & \begin{array}{l}\text { Difference in } \\ \text { costs }(\boldsymbol{f})\end{array} & \begin{array}{l}\text { Effectiveness } \\ \text { (QALYs) }\end{array} & \begin{array}{l}\text { Incremental } \\ \text { QALYs }\end{array} & \text { ICER (f) } \\ \begin{array}{l}\text { Standard practice } \\ \begin{array}{l}\text { PET-CT together with standard } \\ \text { practice }\end{array}\end{array} & 7695 & - & 4.1501 & - & - \\ \end{array}$

a Apparent anomaly in subtraction is due to rounding effects. 
TABLE 72 Model 3 base-case results from the analysis based on cost per QALY

\begin{tabular}{lcllll} 
Strategy & $\begin{array}{l}\text { Mean cost per } \\
\text { strategy }(\mathrm{f})\end{array}$ & $\begin{array}{l}\text { Difference in } \\
\text { costs }(\mathrm{f})\end{array}$ & $\begin{array}{l}\text { Effectiveness } \\
(\text { QALYs })\end{array}$ & $\begin{array}{l}\text { Incremental } \\
\text { QALYs }\end{array}$ & ICER (f) \\
$\begin{array}{l}\text { Standard practice } \\
\begin{array}{l}\text { PET-CT together with } \\
\text { standard practice }\end{array}\end{array}$ & 17612 & - & 4.1507 & - & - \\
\hline
\end{tabular}

TABLE 73 Model 4 base-case results from the analysis based on cost per QALY

\begin{tabular}{lcllll} 
Strategy & $\begin{array}{l}\text { Mean cost per } \\
\text { strategy }(\mathrm{f})\end{array}$ & $\begin{array}{l}\text { Difference in } \\
\text { costs }(\mathrm{f})\end{array}$ & $\begin{array}{l}\text { Effectiveness } \\
\text { (QALY) }\end{array}$ & $\begin{array}{l}\text { Incremental } \\
\text { QALYs }\end{array}$ & ICER (f) \\
\hline $\begin{array}{l}\text { Standard practice } \\
\begin{array}{l}\text { PET-CT together with } \\
\text { standard practice }\end{array}\end{array}$ & 17,122 & - & 4.1501 & - & - \\
\hline
\end{tabular}

a Apparent anomaly in subtraction is due to rounding effects.

\section{Results in terms of cost per recurrent case treated}

The deterministic results for the cost per recurrent case treated were $>£ 600,000$ per case for all four models (Tables 74-77). In model 1 standard practice had a mean cost of approximately $f 9169$ with corresponding cases treated of 0.1296 compared with a mean cost of approximately $£ 18,757$ and corresponding cases treated of 0.1436 for PET-CT together with standard practice. The estimated ICER for PET-CT together with standard practice compared with standard practice alone was $£ 681,000$ per case treated. This indicates that, for every additional case treated with PET-CT as an adjunct to standard practice, there was an incremental cost of $£ 681,000$. Similar results can be seen for models $2-4$. PET-CT as an adjunct to standard practice was both more costly and more effective than standard practice alone, with an ICER of approximately $£ 670,000$ for each model.

\section{Deterministic sensitivity analysis results}

The deterministic results for the cost per recurrent case treated, presented in Tables 74-77, were $>£ 600,000$ per case for all four models. These results are summarised in Table 78.

\section{Results of the probabilistic sensitivity analysis for the base-case cost per quality-adjusted life-year outcome}

Figure 31 shows the Monte Carlo simulation for model 1. The scatterplot illustrates the uncertainty in the expected costs and QALYs based on PET-CT as an adjunct to standard practice compared with standard practice alone. For the 10,000 runs of the Monte Carlo simulation, the scatterplot shows considerable uncertainty about the additional expected costs and QALYs.

The scatterplots in Figures 32-35 show the uncertainty surrounding the incremental expected costs and incremental expected QALYs for models 1-4, respectively, based on PET-CT as an adjunct to standard practice in comparison with standard practice alone. In each figure, for the 10,000 runs of the Monte Carlo simulation, the scatterplot shows considerable uncertainty about the additional expected incremental costs and QALYS.

The results for model 1 are presented in the form of cost-effectiveness acceptability curves (CEACs) in Figure 36. Analogous results were observed for models 2-4 (not shown). CEACs give the probability that a screening strategy is cost-effective given society's willingness to pay for a QALY. In other words, the CEAC shows the probability that PET-CT as an adjunct to standard practice is cost-effective compared with standard practice alone at different values for society's maximum acceptable cost-effectiveness 
TABLE 74 Model 1 results from the analysis based on cost per recurrent case treated

\begin{tabular}{|c|c|c|c|c|c|}
\hline Strategy & $\begin{array}{l}\text { Mean cost per } \\
\text { strategy }(f)\end{array}$ & $\begin{array}{l}\text { Difference in } \\
\text { costs }(f)\end{array}$ & $\begin{array}{l}\text { Effectiveness } \\
\text { (cases treated) }\end{array}$ & $\begin{array}{l}\text { Incremental } \\
\text { cases treated }\end{array}$ & $\operatorname{ICER}(\mathbf{f})$ \\
\hline Standard practice & 9169 & - & 0.1296 & - & - \\
\hline $\begin{array}{l}\text { PET-CT together with } \\
\text { standard practice }\end{array}$ & 18,757 & 9588 & 0.1436 & $0.0141^{a}$ & 681,000 \\
\hline
\end{tabular}

TABLE 75 Model 2 results from the analysis based on cost per recurrent case treated

\begin{tabular}{|c|c|c|c|c|c|}
\hline Strategy & $\begin{array}{l}\text { Mean cost per } \\
\text { strategy }(f)\end{array}$ & $\begin{array}{l}\text { Difference in } \\
\text { costs }(f)\end{array}$ & $\begin{array}{l}\text { Effectiveness } \\
\text { (cases treated) }\end{array}$ & $\begin{array}{l}\text { Incremental } \\
\text { cases treated }\end{array}$ & $\operatorname{ICER}(\mathrm{f})$ \\
\hline Standard practice & 7695 & - & 0.1296 & - & - \\
\hline $\begin{array}{l}\text { PET-CT together with } \\
\text { standard practice }\end{array}$ & 17,122 & $9428^{a}$ & 0.1436 & $0.0141^{\mathrm{a}}$ & 670,000 \\
\hline
\end{tabular}

TABLE 76 Model 3 results from the analysis based on cost per recurrent case treated

\begin{tabular}{|c|c|c|c|c|c|}
\hline Strategy & $\begin{array}{l}\text { Mean cost per } \\
\text { strategy }(f)\end{array}$ & $\begin{array}{l}\text { Difference in } \\
\text { costs }(f)\end{array}$ & $\begin{array}{l}\text { Effectiveness } \\
\text { (cases treated) }\end{array}$ & $\begin{array}{l}\text { Incremental } \\
\text { cases treated }\end{array}$ & $\operatorname{ICER}(\mathrm{f})$ \\
\hline Standard practice & 7612 & - & 0.1296 & - & - \\
\hline $\begin{array}{l}\text { PET-CT together with } \\
\text { standard practice }\end{array}$ & 17,031 & 9419 & 0.1436 & $0.0141^{a}$ & 669,000 \\
\hline
\end{tabular}

TABLE 77 Model 4 results from the analysis based on cost per recurrent case treated

\begin{tabular}{lcllll} 
Strategy & $\begin{array}{l}\text { Mean cost per } \\
\text { strategy }(\mathrm{f})\end{array}$ & $\begin{array}{l}\text { Difference in } \\
\text { costs }(\mathrm{f})\end{array}$ & $\begin{array}{l}\text { Effectiveness } \\
\text { (cases treated) }\end{array}$ & $\begin{array}{l}\text { Incremental } \\
\text { cases treated }\end{array}$ & ICER $(\mathrm{f})$ \\
$\begin{array}{l}\text { Standard practice } \\
\begin{array}{l}\text { PET-CT together with } \\
\text { standard practice }\end{array}\end{array}$ & 7695 & - & 0.1296 & - & - \\
\hline
\end{tabular}

a Apparent anomaly in subtraction is due to rounding effects.

ratio. The threshold used by NICE is between $£ 20,000$ and $£ 30,000$ per QALY, that is, society is willing to pay $\mathrm{f20,000}$ per QALY for 1 year of life in full health. From Figure 36 it can be seen that the use of PET-CT as an adjunct to standard practice alone is not likely to be cost-effective given the data used in the model. This is illustrated by no PET-CT (standard practice) having a probability of being cost-effective of approximately $100 \%$ and PET-CT (PET-CT as an adjunct to standard practice) having a probability of being cost-effective of approximately $0 \%$. The implication of this result is that the VOI is necessarily zero across all thresholds, which means that further analysis of VOI was unnecessary. 
TABLE 78 Summary of deterministic sensitivity analysis cost-utility results

\begin{tabular}{|c|c|c|c|}
\hline & $\begin{array}{l}\text { Incremental } \\
\text { cost }(f)\end{array}$ & $\begin{array}{l}\text { Incremental } \\
\text { effectiveness }\end{array}$ & ICER (f) \\
\hline \multicolumn{4}{|l|}{ Model 1} \\
\hline Base case & 9588 & 0.0010 & $9,254,000$ \\
\hline $\begin{array}{l}\text { 1. Changing the } 5 \text {-year survival following untreated cervical cancer } \\
(3.0 \% \text { to } 60 \%)\end{array}$ & 9528 & -0.0072 & (Dominance) \\
\hline $\begin{array}{l}\text { 2. Halving the utility value for recurrent cervical cancer from } 0.5200 \\
\text { to } 0.2600\end{array}$ & 9588 & 0.0052 & $1,829,000$ \\
\hline 3. Changing the current follow-up schedule to annual follow-up & 4974 & 0.0008 & $6,091,000$ \\
\hline \multicolumn{4}{|l|}{ Model 2} \\
\hline Base case & 9428 & 0.0080 & $1,173,000$ \\
\hline $\begin{array}{l}\text { 1. Changing the } 5 \text {-year survival following untreated cervical cancer } \\
(3.0 \% \text { to } 60 \%)\end{array}$ & 9419 & -0.0015 & (Dominance) \\
\hline $\begin{array}{l}\text { 2. Halving the utility value for recurrent cervical cancer from } 0.5200 \\
\text { to } 0.2600\end{array}$ & 9428 & 0.0122 & 771,000 \\
\hline 3. Changing the current follow-up schedule to annual follow-up & 4824 & 0.0069 & 697,000 \\
\hline \multicolumn{4}{|l|}{ Model 3} \\
\hline Base case & 9419 & 0.0088 & $1,065,000$ \\
\hline $\begin{array}{l}\text { 1. Changing the } 5 \text {-year survival following untreated cervical cancer } \\
(3.0 \% \text { to } 60 \%)\end{array}$ & 9413 & -0.0007 & (Dominance) \\
\hline $\begin{array}{l}\text { 2. Halving the utility value for recurrent cervical cancer from } 0.5200 \\
\text { to } 0.2600\end{array}$ & 9419 & 0.0126 & 745,000 \\
\hline 3. Changing the 3 -month survival to 0.9307 & 9419 & 0.0027 & $3,527,000$ \\
\hline 4. Changing the current follow-up schedule to annual follow-up & 4815 & 0.0072 & 673,000 \\
\hline \multicolumn{4}{|l|}{ Model 4} \\
\hline Base case & 9428 & 0.0080 & $1,173,000$ \\
\hline $\begin{array}{l}\text { 1. Changing the } 5 \text {-year survival following untreated cervical cancer } \\
(3.0 \% \text { to } 60 \%)\end{array}$ & 9419 & -0.0015 & (Dominance) \\
\hline $\begin{array}{l}\text { 2. Halving the utility value for recurrent cervical cancer from } 0.5200 \\
\text { to } 0.2600\end{array}$ & 9428 & 0.0122 & 771,000 \\
\hline 3. Changing the current follow-up schedule to annual follow-up & 4824 & 0.0069 & 697,000 \\
\hline
\end{tabular}




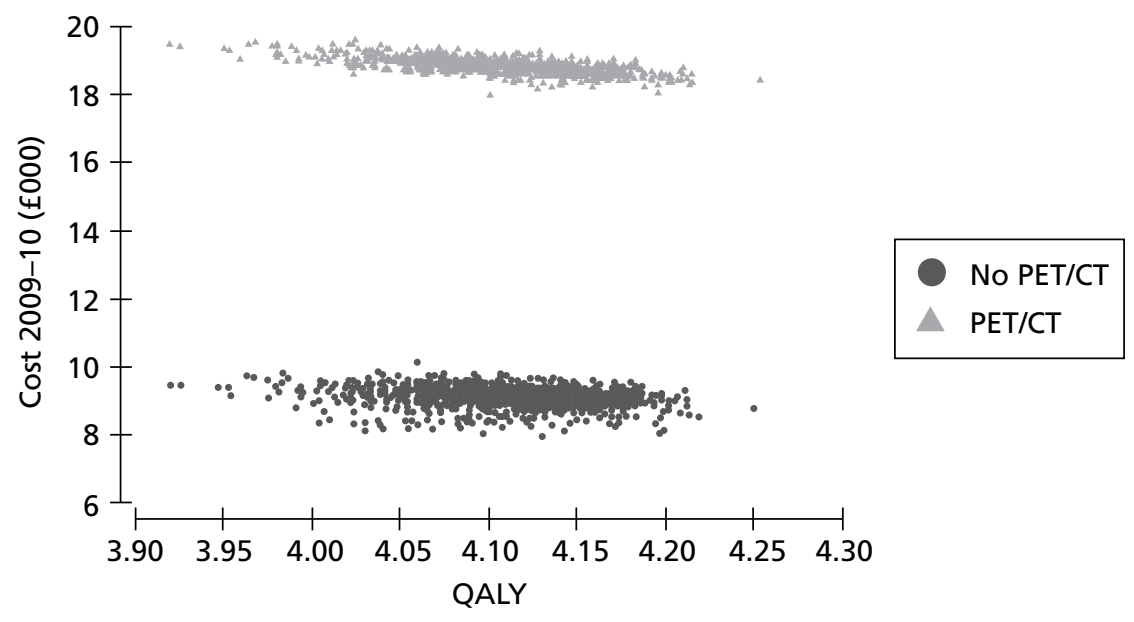

FIGURE 31 Scatterplot using distributions around the input parameters in model 1.

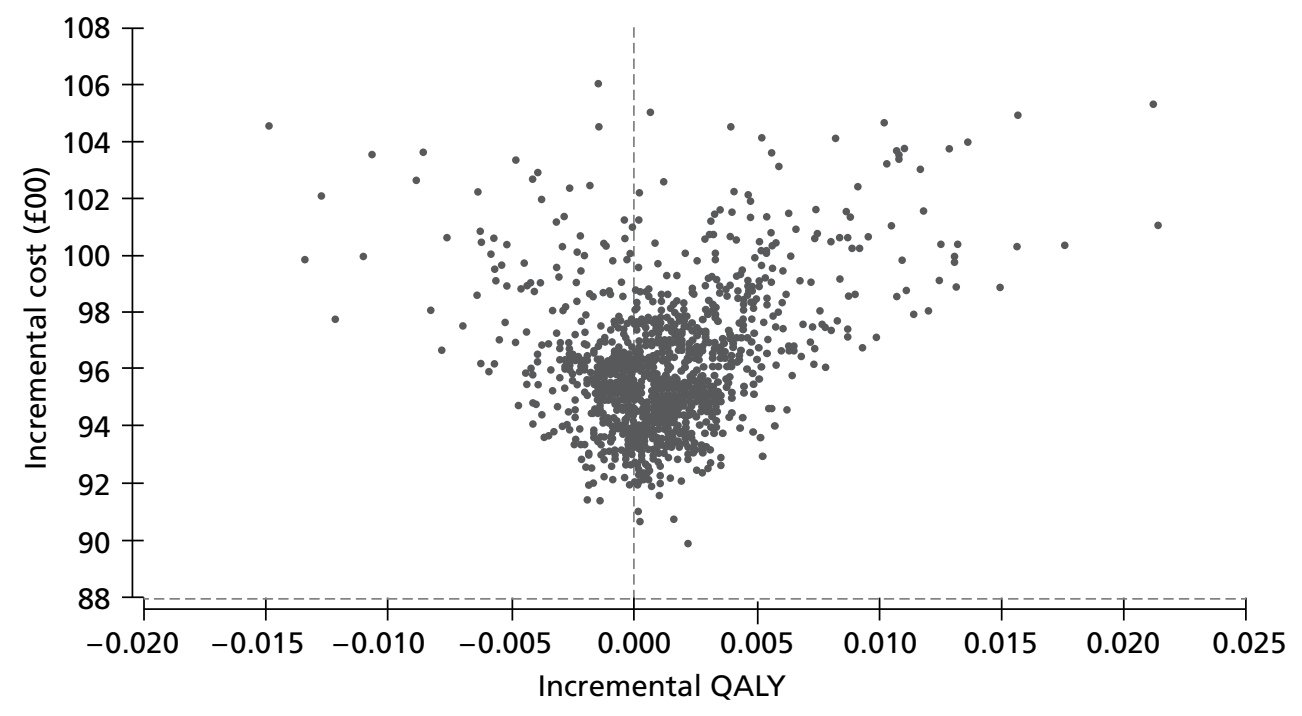

FIGURE 32 Scatterplot using distributions around the input parameters in model 1. 


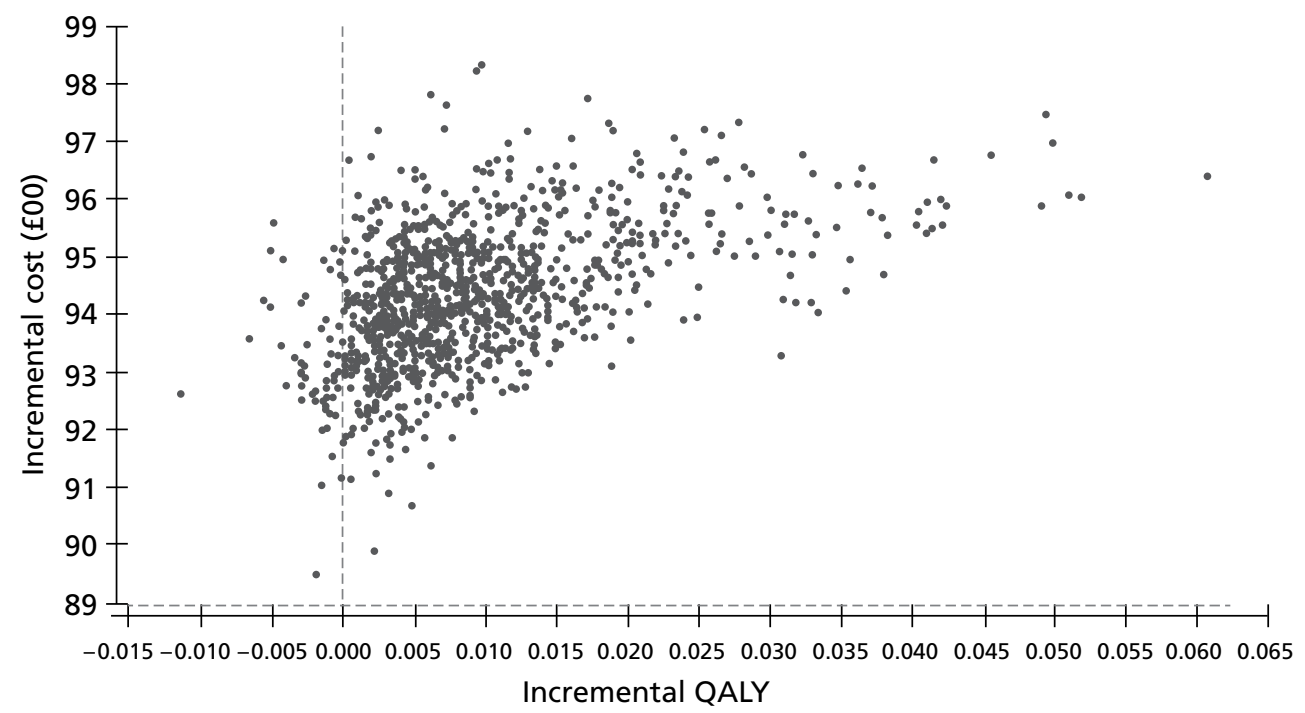

FIGURE 33 Scatterplot using distributions around the input parameters in model 2.

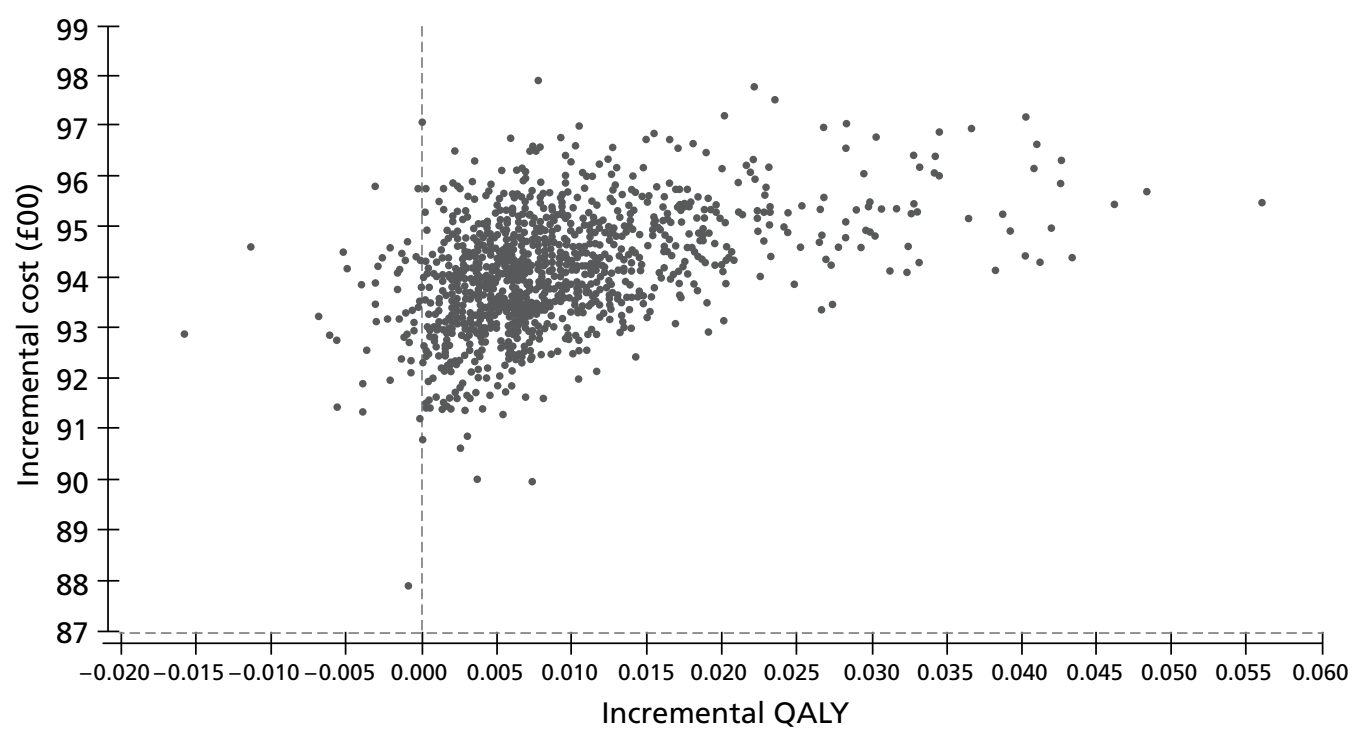

FIGURE 34 Scatterplot using distributions around the input parameters in model 3. 


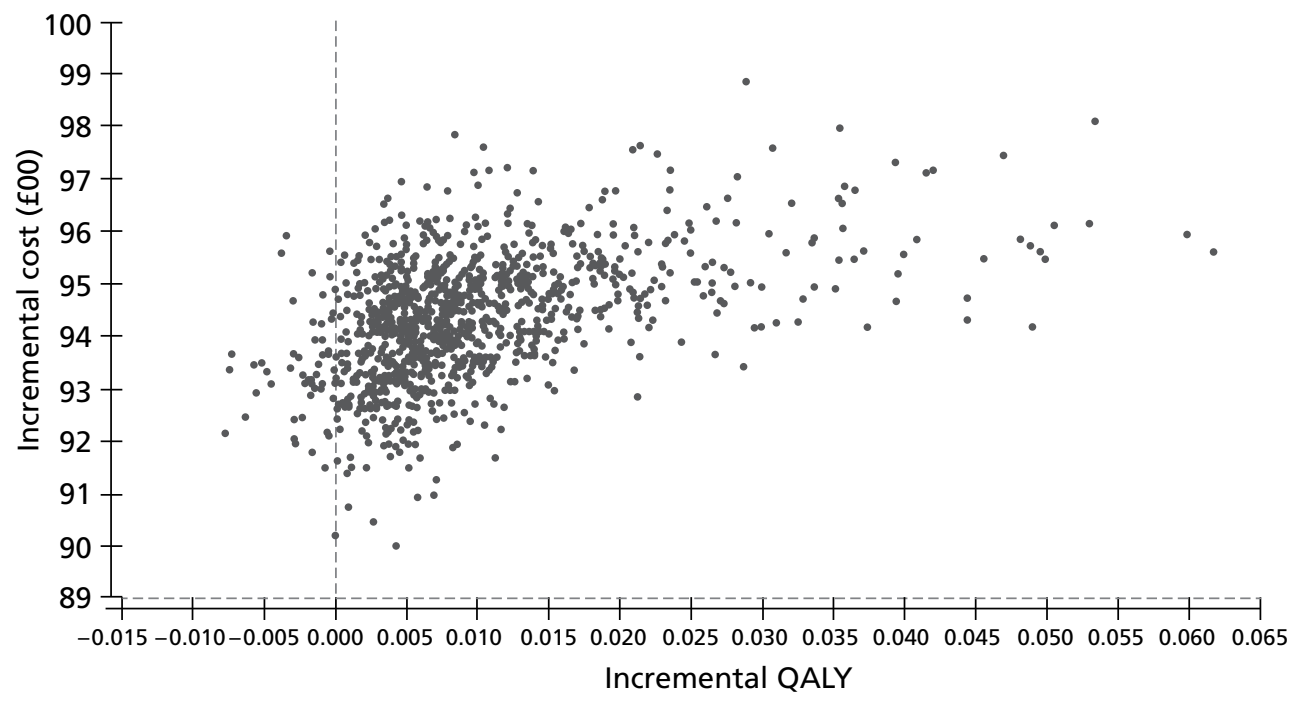

FIGURE 35 Scatterplot using distributions around the input parameters in model 4.

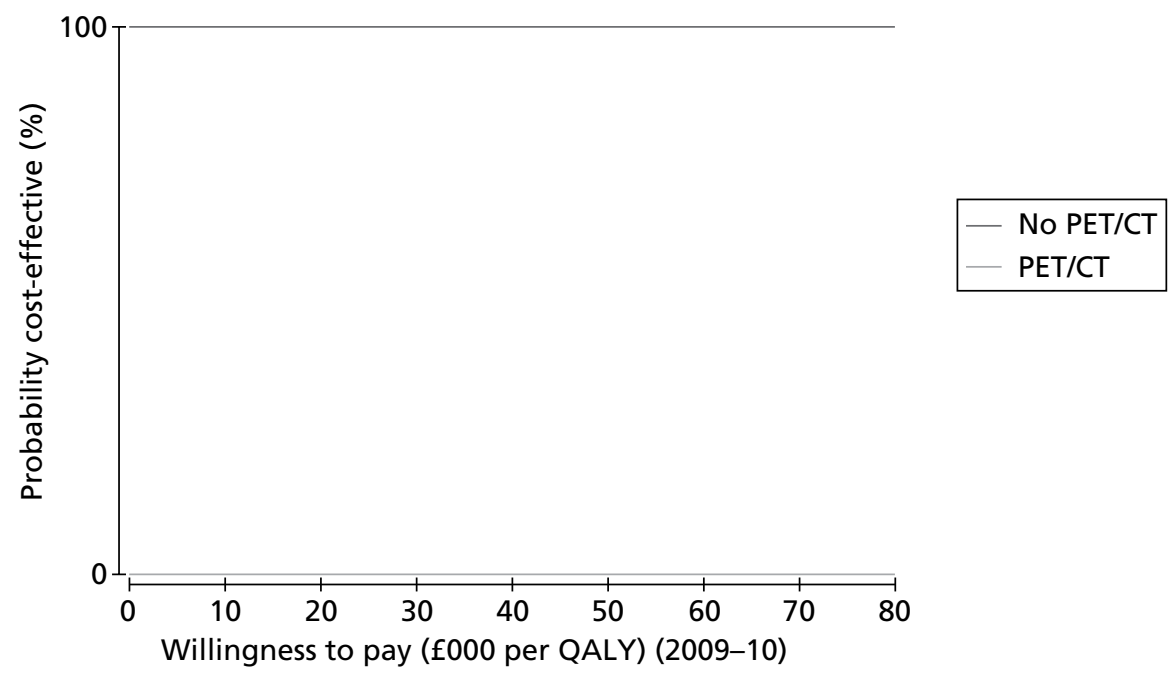

FIGURE 36 Cost-effectiveness acceptability curve using distributions around the outcomes. 


\section{Chapter 9 Discussion}

\section{Statement of principal findings}

\section{Test accuracy systematic review and subjective elicitation}

Twelve test accuracy studies ${ }^{20,48-58}$ were found that evaluated PET-CT $(n=6), \operatorname{MRI}(n=3), C T(n=2)$ and MRI and CT $(n=1)$ compared with histology and/or clinical follow-up. Most of the studies were underpowered and of poor quality. Most of the later studies evaluated PET-CT and earlier studies evaluated MRI and CT. Imaging practice has developed since the earlier studies so the MRI and CT studies did not reflect current practice standards, making it difficult to ascertain the value of PET-CT when current practice for CT/MRI is based on outdated research. Both symptomatic and asymptomatic patients were to be investigated in this project, but there was very little information on imaging as routine follow-up for asymptomatic patients. The subjective elicitation exercise obtained the opinions of 21 clinical experts and the results were similar to the published estimates of accuracy for symptomatic women. There was information from one study comparing PET-CT and CT and/or MRI in the same patient group ${ }^{49}$ which suggested that PET-CT imaging found many more true-positives and fewer false-negatives than CT or MRI. The subjective elicitation results suggested that the estimated increase in accuracy of adding PET-CT to MRI and/or CT was less than the elicited minimum important difference in accuracy required to justify the routine addition of PET-CT for the investigation of women after completion of primary treatment for cervical cancer.

\section{Effectiveness review}

Chemotherapy, radiotherapy, chemoradiotherapy and surgery (radical hysterectomy and pelvic exenteration) were reviewed. There were $19 \mathrm{RCTs}^{60-83}$ on chemotherapy but none evaluated the effectiveness of cisplatin compared with no cisplatin, which is the most commonly used drug in recurrent or stage IV cervical cancer and was needed for the economic evaluation. Therefore, another review was carried out to find this information from a RCT. The best-quality RCT found compared cisplatin with no cisplatin with both groups receiving radiotherapy, ${ }^{127}$ which gave an overall 5-year survival with cisplatin of $63 \%$ and without cisplatin of 59\%. Only case series were found on radiotherapy (nine studies ${ }^{84-92}$ ), chemoradiotherapy (seven studies ${ }^{93-99}$ ), radical hysterectomy (seven studies ${ }^{100-106}$ ) and pelvic exenteration (20 studies ${ }^{107-126}$ ). The survival rates varied considerably, depending on the date of publication, characteristics of patients and type of treatment given. It was noticeable that the pelvic exenteration results showed particularly high rates of perioperative mortality and morbidity and very low survival rates.

\section{Economic evaluation}

The results of the base-case deterministic analyses based on the outcome of cost per QALY show that adding PET-CT to the current treatment strategy of clinical examination, MRI and/or CT is significantly more costly with only a minimal increase in effectiveness. This result holds true for all four models that were used in the analyses to represent the alternative treatment paths that women followed for their treatment of primary cancer. These previous treatment paths were differentiated to ensure that the results of the current analysis were not influenced by previous treatment for primary cervical cancer.

The ICER for the strategy of PET-CT as an adjunct to the standard treatment strategy, which included clinical examination, MRI and/or CT, compared with usual treatment alone was $>f 1 \mathrm{M}$ per QALY in all four models:

- for women who had been treated for early-stage cancer by surgery (model 1) the ICER was f9.3M per QALY

- for women who had been treated for early-stage cancer by chemoradiotherapy (model 2) the ICER was f1.2M per QALY 
- for women who had been treated for late-stage cancer by chemoradiotherapy (model 3) the ICER was f1.1M per QALY

- for women who had been treated for early-stage cancer by postoperative chemoradiotherapy (model 4) the ICER was f1.2M per QALY.

For all models an exploration of the ICER based on the outcome of cost per additional case of recurrence treated was performed. For all four models, the additional cost per additional case of recurrence treated was in the region of $£ 600,000$ per case.

The acceptable ICER threshold used by NICE is $f 20,000-30,000$ per QALY. This means that an ICER has to be below this for a technology to be currently considered cost-effective. The PSA suggests that the strategy of PET-CT as an adjunct to standard practice is not likely to be considered cost-effective given current willingness-to-pay thresholds for any of the models and data used in this analysis.

The sensitivity analysis showed that there was nothing, in terms of the data used in the models, that could be changed within plausible estimates, based on the current available evidence, that would change the direction of the results sufficiently to provide any doubt about the results of the current analysis. Thus, based on the current available data and expert opinion used in the models, there is little doubt that PET-CT as an adjunct to standard treatment has been shown to be not cost-effective in the diagnosis of recurrent or persistent cervical cancer at this time.

\section{Strengths and limitations of the project}

\section{Strengths}

- Well-established systematic review methods were used for this technology assessment, which lends considerable strength to its validity and reliability.

- Searches for the diagnostic and effectiveness studies were conducted systematically using a sensitive search strategy and so it is unlikely that any useful information will have been missed.

- Throughout the project the focus has been to investigate recurrent and persistent cervical cancer, rather than merge this evidence with that for primary cervical cancer, even if it was advanced when first diagnosed.

- Elicited estimates of accuracy of CT, MRI and PET-CT are plausible and reflect the fact that the accuracy of imaging tests is likely to be greater in symptomatic than in asymptomatic women because of the more advanced stage of disease in the former. Elicited estimates of accuracy also reflect a greater likelihood of an improvement in NPV than in PPV in both symptomatic and asymptomatic women, which is consistent with the probability of a larger number of false-positives with the addition of PET-CT to current imaging practice.

- Importantly, elicited estimates of prevalence and accuracy had face validity as judged by feedback to clinical experts who participated in the face-to-face elicitation exercise. Probabilities elicited with and without pre-elicitation training appeared similar.

- There have been four recent systematic reviews and narrative reviews on recurrent, persistent metastatic and advanced cervical cancer ${ }^{59,145-147}$ and all have included RCTs on advanced primary cancer as well as cancer after primary treatment. They all investigated chemotherapy only and so the current project is the only one to incorporate information on radiotherapy, chemoradiotherapy and surgery in the same report.

- Considerable efforts were made to find appropriate input values for the decision-analytic model, for example conducting an additional systematic review on the effectiveness of single-agent cisplatin in (recurrent or primary) cervical cancer.

- The strength of the economic evaluation is that the analysis is based on the best available data. Systematic reviews showed that test accuracy evidence was severely limited.

- The subjective elicitation exercise was carried out using expert opinion before any economic analysis was undertaken. No assumption or item of data from the elicitation exercise was changed after the 
analysis started apart from in the sensitivity analysis. All assumptions used in the model were agreed by the team based on expert advice a priori.

\section{Limitations}

- There was no information on the selective use of PET-CT to guide management of patients when considering surgical procedures such as exenteration, as suggested in guidelines on the use of PET-CT in recurrent cervical cancer.

- The diagnostic systematic review is limited by the quantity and quality of the included studies. The studies had few participants and were underpowered and the quality was frequently poor. The reference standard was different for test-positive patients (histology) and test-negative patients (clinical follow-up) in eight of the studies. ${ }^{20,49-52,54-56}$ There was almost no information on the timing between the index tests and the reference standards. Also, imaging practice has changed and so the earlier studies do not reflect current practice; in particular, the CT and MRI studies were published between 1981 and 2000.

- There is a weakness in test accuracy studies in which the reference standard is not independent of the index text. In one study, ${ }^{50}$ PET-CT was incorporated in the reference standard. For some patients the final diagnosis was based on the results of tumour marker level and PET-CT findings. This means that these studies are unlikely to give an accurate estimate of the test specificity.

- There was very little information from published studies comparing PET-CT in addition to MRI or CT with MRI or CT alone in order to determine whether or not PET-CT use would enhance test accuracy and improve therapeutic impact.

- In most of the existing studies the results for recurrent and persistent cancer, and in some cases (particularly RCTs) for primary advanced cervical cancer, were analysed together. When possible, results are presented for the subgroup of patients with recurrence and persistence only.

- There was little evidence on the effectiveness of single-agent cisplatin in recurrent or persistent cervical cancer, and other information required for the analysis was also scarce.

- It is debateable whether or not effectiveness evidence for patients who had undergone surgery with radiotherapy for their initial treatment should have been excluded. It is likely that further treatment will be chemotherapy. The additional systematic review on cisplatin as a single agent did not exclude these studies, so it is unlikely that this exclusion from the main effectiveness systematic review will have had any impact on the subsequent project.

- The main systematic review of effectiveness studies did not include any information on the effectiveness of the most commonly used chemotherapy regimen in recurrent and persistent cervical cancer, single-agent cisplatin.

- Effectiveness studies with long-term follow-up are the most useful but if they have a long follow-up it is inevitable that recruitment happened earlier and so the treatment given at the time may not be as effective as that given more recently. This limits the generalisability of these studies.

- The evidence on radiotherapy, chemoradiotherapy and surgery was all from case series; no RCT or comparative studies were available. Comparison of patient populations between studies was difficult because of a lack of information on baseline characteristics such as patient age, FIGO stage, histological cell type and site of disease. Many of the case series were published years ago (1950s to 1970s) and treatment effectiveness has improved over time. It is debateable whether or not the systematic reviews should have included these early data. However, the economic modelling required estimates for a number of parameters and it was not clear at the outset how early the inclusion criterion needed to be to find estimates for some parameters. On the one hand, basing estimates on early research means that they are not likely to be accurate; however, at least the parameter estimates are based on some research, even if early, rather than clinicians' opinions only.

- There was no information about quality of life in recurrent and persistent cervical cancer and so information had to be taken from a quality-of-life study in patients with advanced primary cervical cancer. ${ }^{144}$

- With regards to the economic evaluation, there are some major limitations in the analysis that must be considered when interpreting the results. Any economic model is limited by the availability of suitable data to populate it. In addition to the absence of PET-CT accuracy data, which was overcome 
by the use of the preference elicitation exercise data, information on the effectiveness of appropriate treatments was also lacking. Thus, the data for the proportions of patients receiving treatment for recurrent cervical cancer were again provided by clinicians based on best clinical knowledge. Utility data for women diagnosed with recurrent cervical data were, with the approval of the clinicians on the team, calculated based on the average utility values for women who had been diagnosed with stage III and IV primary cervical cancer. Also, utility values for women treated were based on the utility values from Lang et al., ${ }^{144}$ which investigated primary cervical cancer but not recurrent or persistent cervical cancer. It is also worth clarifying that the data in the literature on survival did not report survival according to stage for women who have been treated for recurrent or persistent cervical cancer.

- When Cls were not reported in the literature, to conduct the PSA arbitrary \pm ranges were used. Limited availability of data also meant that any correlations that may exist between the sensitivity and the specificity data, for the range of diagnostic tests, have been ignored.

- Cost data for tests were available in very few published studies and only unit costs for relevant resource use were available.

\section{Uncertainties}

There are a number of uncertainties in the results of the economic model, mainly due to uncertainties in the clinical parameters, such as the lack of test accuracy information for asymptomatic women. This is due in turn to the poor-quality evidence that is available for some parameters and the lack of evidence for others.

The use of differential reference standards leads to overestimation of diagnostic test accuracy. ${ }^{148}$ However, on ethical grounds, clinical follow-up is an adequate way to evaluate test accuracy in patients with negative findings. Unfortunately, the different definitions of clinical follow-up in each study (from physical and gynaecological examination during at least 6 months to tumour marker levels and imaging findings) are problematic because it is uncertain whether or not the different studies are measuring the same imaging accuracy. When clinical follow-up is used as the reference standard it is inevitable that the condition of the patient will change. However, if no lesions are found on imaging it is unclear where biopsies for histology should be taken from.

There is a risk of under- or overestimation of diagnostic test accuracy depending on the change in a patient's condition, so information about the time period between the reference standard and the index test is important to be sure that the target condition did not change between the two tests.

With regard to the subjective elicitation, responses from individuals who received pre-elicitation education in the form of a lecture did not appear to differ from responses from those who did not. The data did not allow a formal investigation of the similarity of responses. Feedback from clinicians indicated that further disaggregation of women according to initial stage would have been ideal, reflecting variation in the prevalence of recurrence in women according to initial stage. However, this would have increased the number of accuracy elicitations from eight to 16 with an expected adverse impact on response rate and validity of responses.

Current practice in the UK does not include routine imaging surveillance of asymptomatic women post primary treatment for cervical cancer and therefore the elicited accuracy estimates for CT, MRI and PET-CT in this clinical population will not be based on the clinical experience of respondents, in contrast to the use of these imaging technologies in symptomatic women post primary treatment for cervical cancer. However, as discussed above, the pattern of estimates of accuracy in this population group is plausible given the lower prevalence and severity of any existing disease.

It is uncertain whether or not the addition of PET-CT is merited. One small published study ${ }^{49}$ suggested that PET-CT found more true-positives and fewer false-negatives than MRI and/or CT but the subjective 
elicitation suggested that the increase in accuracy was less than the minimum important clinical difference needed. PET-CT is recommended in the SIGN guidelines when CT or MRI has demonstrated recurrent or persistent disease, ${ }^{3}$ but the evidence upon which this recommendation is based is unclear.

There is considerable uncertainty around the comparative effectiveness of cisplatin monotherapy and for radiotherapy, chemoradiotherapy, radical hysterectomy and pelvic exenteration in recurrent and persistent cervical cancer. These are the mainstays of current treatment and, therefore, the lack of evidence regarding their effectiveness is worrying.

No studies were identified that had considered the relative cost-effectiveness of available technologies for the diagnosis of recurrent or persistent cervical cancer and, therefore, appropriate comparisons with other existing studies are not possible. Consequently, it is uncertain whether or not the approach taken here would be robust if other studies were conducted.

In terms of the EVPI, given that the probability calculated in the modelling never went above zero for the range of willingness-to-pay values plotted, the EVPI is necessarily zero at any such willingness to pay. The EVPI reflects the parameter uncertainty in the elicitation exercise and would be different should the test accuracy of PET-CT be measured directly. 



\section{Chapter 10 Conclusions}

Dased on the current model and given the limitations that have been highlighted in terms of availability Dof data, the results of the current analysis suggest that the use of PET-CT in the diagnosis of recurrent or persistent cervical cancer is not cost-effective for symptomatic or asymptomatic women. The results are not even close to the current willingness-to-pay thresholds that are accepted in the UK by decision-making bodies such as NICE. The results reflect enormous uncertainty at many levels and so a better expression of our current understanding is that the cost-effectiveness of PET-CT combined with usual tests and treatment for detecting recurrent cervical cancer is not proven. Although PSA showed that the main conclusion about the cost-ineffectiveness of PET-CT was firm given the range of assumptions made, should more reliable information become available on accuracy, therapeutic impact and effectiveness, and the cost of PET-CT reduce, the conclusion may need revision. Current guidelines recommending imaging for diagnosis using expensive methods such as PET-CT need to be reconsidered in light of the above.

\section{Implications for service provision}

- A diagnosis of recurrent cervical cancer must be an extremely distressing situation for women and their families. Current evidence suggests that there are huge knowledge gaps about women's quality of life and survival given such a diagnosis. Also, missing an early diagnosis of recurrence is very distressing. Adding an additional PET-CT test to the toolkit to confirm diagnosis of recurrence, or not, might add something in terms of reassurance and hope. However, given that the additional accuracy of such a test is currently not clear, as well as the lack of other necessary evidence, a case for its implementation in current practice cannot yet be supported. Much more robust evidence on test accuracy, survival and quality of life is required before any such case can be made.

- It is uncertain whether or not the addition of PET-CT in routine surveillance of asymptomatic women and diagnosis of symptomatic women is good value for money, given the current state of knowledge. This lack of information around the usefulness of routine surveillance with PET-CT does not help the women concerned.

- Patients should be informed that the effectiveness of single-agent cisplatin in recurrent and persistent cervical cancer is uncertain.

- The pelvic exenteration results showed high operative and postoperative mortality rates and the complication rates were also high. Considering the morbidity of pelvic exenteration, it could be argued that the NHS care of these women should be further centralised into supraregional centres.

\section{Implications for research}

- The key clinical question is whether it is better to evaluate asymptomatic women following primary treatment or to wait until symptoms occur. A RCT could be conducted in which women who had completed treatment for primary cervical cancer would be randomised to a policy of routine surveillance or current practice of symptomatic follow-up.

- It is necessary to conduct larger, good-quality studies directly comparing the test accuracy of the addition of PET-CT to MRI and/or CT imaging alone in a population of women with recurrent and persistent cervical cancer in order to evaluate whether or not the additional expenditure on PET-CT is merited. Population groups need to be distinguished between symptomatic presentation and asymptomatic women undergoing routine follow-up.

- There is also a need to compare current practice with CT or MRI and the use of PET-CT in terms of change in diagnosis, work-up and change in the treatment plan by response to treatment in a way that permits continuation or alteration of treatment. 
- To our knowledge this is the first example of the elicitation of test accuracy estimates. Use of predictive values and test errors resulted in consistent responses that had face validity in this sample. Further test accuracy elicitation exercises will be required to confirm the validity of this approach and for comparison of test accuracy elicitation using other test accuracy metrics.

- Investigation of the benefit of face-to-face pre-elicitation education for the validity of responses is warranted as this has an impact on the methods of elicitation that are possible (e.g. the use of postaland internet-based questionnaires), the resources required and the response rate.

- Generally, to obtain more reliable results for radiotherapy, chemoradiotherapy or surgery, there is a need to conduct prospective studies with a comparative group, preferably RCTs, that are sufficiently powered to present definitive results in the subpopulations of persistent and recurrent patients separately. These studies should collect information about long-term overall survival, disease-free status, recurrence, morbidity, hospital stay, late complications and, most importantly, generic quality of life using, for example, the EQ-5D, the assessment of which is crucial for the evaluation of the full impact of therapies on patients' well-being.

- It would be useful to have a UK register of pelvic exenterations for recurrent/persistent cervical cancer. This is a major operation with considerable implications for morbidity. Our searches demonstrate that current published data on outcomes from pelvic exenteration for cervical cancer are outdated. In the systematic review most pelvic exenteration case series were published before the year 2000 and the only one from the UK was published in $1953 .{ }^{119}$ This makes it impossible for the effectiveness of diagnostic work-ups or indeed exenterative surgery to be provided accurately. Such surgery - resulting in the loss of the bladder and/or bowel - has the potential for significant morbidity and mortality as well as having an impact on the patient's emotional well-being and body image. It is vital that we collect prospective good-quality data that can be used to improve care and establish standards and outcomes for women who require such surgery. Furthermore, this register may also help promote the rationalisation of service use by concentrating such services at centres that establish expertise in the preoperative, postoperative and long-term supportive care of these women. 


\title{
Acknowledgements
}

\author{
$\triangle$ nne Fry-Smith for helping to draft the original protocol and Paweł Chomiak for assistance with the \\ systematic reviews.
}

Subjective elicitation: Dr Indrajit Fernando, Consultant Clinical Oncologist; Mr KK Chan, Consultant Gynaecological Oncologist; Mr Janos Balega, Consultant Gynaecological Oncologist; Ahmed Elattar, Subspecialty Trainee in Gynaecological Oncology; Sudha Sundar, Consultant Gynaecological Oncologist; Raj Saha, Consultant Gynaecologist (unit lead); Martin Underwood, Specialist Trainee in Obstetrics and Gynaecology; Peter Guest, Consultant Radiologist; Dr Moji Balogun, Consultant Radiologist; Professor David Luesley, Consultant Gynaecologist (Director Pan Birmingham Gynaecological Cancer Centre); Pierre Martin-Hirsch, Consultant Gynaecological Oncologist; Raj Naik, Consultant Gynaecological Oncologist; Adam Rosenthal, Consultant Gynaecological Oncologist; Arjun Jeyarajam, Consultant Gynaecological Oncologist; John Butler, Subspecialty Trainee in Gynaecological Oncology; Alex Lawrence, Consultant Gynaecological Oncologist; NJ Wood, Consultant Gynaecological Oncologist; Mary Cairns, Consultant Gynaecological Oncologist; John Shepherd, Consultant Surgeon/Gynaecological Oncologist; and Miss Elizabeth Ball, Consultant Obstetrician and Gynaecologist.

\section{Contribution of authors}

Catherine Meads

Peter Auguste

Clare Davenport

Sylwia Małysiak

Sudha Sundar

Monika Kowalska

Anna Zapalska

Peter Guest

Shakila Thangaratinam

Pierre Martin-Hirsch
Supervised and co-ordinated the project and rewrote and edited the manuscript.

Designed the decision model and inputs, conducted the analysis, interpreted the results and provided detailed comments on the economic evaluation chapter.

Carried out the systematic review of test accuracy and subjective elicitation and commented on the final report.

Carried out the systematic review of test accuracy studies and commented on the final report.

Provided clinical orientation, contributed to the project design, interpreted the results and commented on the final report.

Carried out the systematic review of test accuracy studies and commented on the final report.

Carried out the systematic review of effectiveness and commented on the final report.

Carried out the quality assessment of test accuracy studies and had radiological input.

Preparation of the grant application and review of the final report.

Provided clinical comments on the final report. 
Ewa Borowiack

Pelham Barton

Tracy Roberts

Khalid Khan
Management of the original systematic reviews and the effectiveness systematic review.

Provided advice on all aspects of the economic evaluation and detailed comments on the economic evaluation chapter.

Management of the economic evaluation, designed and supervised the economic evaluation and wrote the first draft of the economic evaluation chapter and the discussion.

Design of the HTA and overall supervision of the project and commented on the final report. 


\section{References}

1. FIGO Committee on Gynecologic Oncology. Revised FIGO staging for carcinoma of the vulva, cervix and endometrium. Int J Gynecol Obstet 2009;105:103-4. http://dx.doi.org/10.1016/j. ijgo.2009.02.012

2. Castle PE, Rodriguez AC, Burk RD, Herrero R, Wacholder S, Alfaro M, et al. Short term persistence of human papillomavirus and risk of cervical precancer and cancer: population based cohort study. BMJ 1928;339:b2569. http://dx.doi.org/10.1136/bmj.b2569

3. Scottish Intercollegiate Guidelines Network. Management of cervical cancer. Guideline no. 99. Edinburgh: NHS Quality Improvement Scotland; 2008.

4. Jit M, Vyse A, Borrow R, Pebody R, Soldan K, Miller E. Prevalence of human papillomavirus antibodies in young female subjects in England. Br J Cancer 2008;97:989-91.

5. Louie KS, de Sanjose S, Diaz M, Castellsague X, Herrero R, Meijer CJ, et al. Early age at first sexual intercourse and early pregnancy are risk factors for cervical cancer in developing countries. $\mathrm{Br} \mathrm{J}$ Cancer 2007;100:1191-7. http://dx.doi.org/10.1038/sj.bjc.6604974

6. Cancer Research UK. Cervical cancer - UK incidence statistics. URL: http://info.cancerresearchuk.org/ cancerstats/types/cervix/incidence/ (accessed 16 January 2012).

7. Cancer Research UK. Cervical cancer - UK mortality statistics. URL: http://info.cancerresearchuk.org/ cancerstats/types/cervix/mortality/ (accessed 16 January 2012).

8. Reimers LL, Anderson WF, Rosenberg PS, Henson DE, Castle PE. Etiologic heterogeneity for cervical carcinoma by histopathologic type, using comparative age-period-cohort models. Cancer Epidemiol Biomarkers Prev 2009;18:792-800. http://dx. doi.org/1 0.1158/1055-9965.EPI-08-0965

9. International Collaboration of Epidemiological Studies of Cervical Cancer. Cervical carcinoma and sexual behavior: collaborative reanalysis of individual data on 15,461 women with cervical carcinoma and 29,164 women without cervical carcinoma from 21 epidemiological studies. Cancer Epidemiol Biomarkers Prev 2009;18:1060-9. http://dx.doi.org/10.1158/1055-9965.EPI-08-1186

10. International Collaboration of Epidemiological Studies of Cervical Cancer. Cervical cancer and hormonal contraceptives: collaborative reanalysis of individual data for 16,573 women with cervical cancer and 35,509 women without cervical cancer from 24 epidemiological studies. Lancet 2007;370:1609-21. http://dx.doi.org/10.1016/S0140-6736(07)61684-5

11. Collins S, Rollason TP, Young LS, Woodman CB. Cigarette smoking is an independent risk factor for cervical intraepithelial neoplasia in young women: a longitudinal study. Eur J Cancer 2010;46:40511. http://dx.doi.org/10.1016/j.ejca.2009.09.015

12. Fujita M, Tase T, Kakugawa Y, Hoshi S, Nishino Y, Nagase S, et al. Smoking, earlier menarche and low parity as independent risk factors for gynecologic cancers in Japanese: a case-control study. Tohoku J Exp Med 2008;216:297-307. http://dx. doi.org/10.1620/tjem.216.297

13. Holmes RS, Hawes SE, Toure P, Dem A, Feng Q, Weiss NS, et al. HIV infection as a risk factor for cervical cancer and cervical intraepithelial neoplasia in Senegal. Cancer Epidemiol Biomarkers Prev 2009;18:2442-6. http://dx.doi.org/10.1158/1055-9965.EPI-08-0956

14. Cancer Research UK. Cervical cancer - risk factors. URL: http://info.cancerresearchuk.org/cancerstats/ types/cervix/riskfactors/ (accessed 20 January 2012).

15. Ferlay J, Parkin DM, Steliarova-Foucher E. Estimates of cancer incidence and mortality in Europe in 2008. Eur J Cancer 2010;46:765-81. http://dx.doi.org/10.1016/j.ejca.2009.12.014 
16. Sundar S, Horne A, Kehoe S. Cervical cancer. Clinical Evid (Online) 2008. pii: 0818.

17. Anon. Topotecan $\left(\right.$ Hycamtin $\left.^{\circledR}\right)$ for the treatment of recurrent and stage IVB carcinoma of the cervix. Single Technology Appraisal (STA) submission to the National Institute for Health and Clinical Excellence. London: GlaxoSmithKline; 2009.

18. Sedlis A, Bundy BN, Rotman MZ, Lentz SS, Muderspach LI, Zaino RJ. A randomized trial of pelvic radiation therapy versus no further therapy in selected patients with stage IB carcinoma of the cervix after radical hysterectomy and pelvic lymphadenectomy: a Gynecologic Oncology Group Study. Gynecol Oncol 1999;73:177-83. http://dx.doi.org/10.1006/gyno.1999.5387

19. Peters WA, Liu PY, Barrett RJ, Stock RJ, Monk BJ, Berek JS, et al. Concurrent chemotherapy and pelvic radiation therapy compared with pelvic radiation therapy alone as adjuvant therapy after radical surgery in high-risk early-stage cancer of the cervix. J Clin Oncol 2000;18:1606-13.

20. Chung HH, Jo H, Kang WJ, Kim JW, Park NH, Song YS, et al. Clinical impact of integrated PET/CT on the management of suspected cervical cancer recurrence. Gynecol Oncol 2007;104:529-34. http:// dx.doi.org/10.1016/j.ygyno.2006.09.009

21. Bodurka-Bevers D, Morris M, Eifel PJ, Levenback C, Bevers MW, Lucas KR, et al. Posttherapy surveillance of women with cervical cancer: an outcomes analysis. Gynecol Oncol 2000;78:187-93. http://dx.doi.org/10.1006/gyno.2000.5860

22. Lim KC, Howells RE, Evans AS. The role of clinical follow up in early stage cervical cancer in South Wales. BJOG 2004;111:1444-8. http://dx.doi.org/10.1111/j.1471-0528.2004.00280.x

23. Hong JH, Tsai CS, Lai CH, Chang TC, Wang CC, Chou HH, et al. Recurrent squamous cell carcinoma of the cervix after definitive radiotherapy. Int J Radiat Oncol Biol Phys 2004;60:249-57. http:// dx.doi.org/10.1016/j.ijrobp.2004.02.044

24. Kim M-K, Jo H, Kong H-J, Kim HC, Kim JW, Kim Y-M, et al. Postoperative nomogram predicting risk of recurrence after radical hysterectomy for early-stage cervical cancer. Int J Gynecol Cancer 2010;20:1581-6.

25. Hirakawa M, Nagai Y, Inamine M, Ogawa K, Toita T, Murayama S, et al. Predictive factors of distant recurrence in locally advanced squamous cell carcinoma of the cervix treated with concurrent chemoradiotherapy. Gynecol Oncol 2008;108:126-9. http://dx.doi.org/10.1016/j. ygyno.2007.08.091

26. Gadducci A, Tana R, Fanucci A, Genazzani AR. Biochemical prognostic factors and the risk of relapses in patients with cervical cancer. Gynecol Oncol 2007;107:S23-26. http://dx.doi org/10.1016/j.ygyno.2007.07.033

27. American Cancer Society. Find support and treatment, the most reliable cancer treatment information. Staging. URL: www.cancer.org/Treatment/UnderstandingYourDiagnosis/staging (accessed January 2012).

28. Mabuchi S, Isohashi F, Yoshioka Y, Temma K, Takeda T, Yamamoto T, et al. Prognostic factors for survival in patients with recurrent cervical cancer previously treated with radiotherapy. Int J Gynecol Cancer 2010;20:834-40. http://dx.doi.org/10.1111/IGC.0b013e3181dcadd1

29. Albon E, Tsourapas A, Frew E, Davenport C, Oyebode F, Bayliss S, et al. Structural neuroimaging in psychosis: a systematic review and economic evaluation. Health Technol Assess 2008;12(18).

30. Facey K, Bradbury I, Laking G, Payne E. Overview of the clinical effectiveness of positron emission tomography imaging in selected cancers. Health Technol Assess 2007;11(44).

31. Jover R, Lourido D, Gonzalez C, Rojo A, Gorospe L, Alfonso JM. Role of PET/CT in the evaluation of cervical cancer. Gynecol Oncol 2008;110:S55-9. http://dx.doi.org/10.1016/j.ygyno.2008.05.023 
32. Salani R, Backes FJ, Fung Kee Fung M, Holschneider CH, Parker LP, Bristow RE, et al. Posttreatment surveillance and diagnosis of recurrence in women with gynecologic malignancies: Society of Gynecologic Oncologists recommendations. Am J Obstet Gynecol 2011;204:466-78. http://dx.doi. org/10.1016/j.ajog.2011.03.008

33. Barrington S, Scarsbrook A. Evidence based indications for the use of PET-CT in the United Kingdom 2012. London: Royal College of Physicians and Royal College of Radiologists; 2012.

34. Brooks RA, Rader JS, Dehdashti F, Mutch DG, Powell MA, Thaker PH, et al. Surveillance FDGPET detection of asymptomatic recurrences in patients with cervical cancer. Gynecol Oncol 2009;112:104-9. http://dx.doi.org/10.1016/j.ygyno.2008.08.028

35. Schwartz JK, Siegel BA, Dehdashti F, Grigsby PW. Association of posttherapy positron emission tomography with tumor response and survival in cervical carcinoma. JAMA 2012;298:2289-95. http://dx.doi.org/10.1001/jama.298.19.2289

36. NHS Centre for Reviews and Dissemination. Systematic reviews: CRD's guidance for undertaking reviews in health care. York: Centre for Reviews and Dissemination, University of York; 2009.

37. Higgins J, Green S. Cochrane handbook for systematic reviews of interventions. Chichester: WileyBlackwell; 2008. http://dx.doi.org/10.1002/9780470712184

38. Diagnostic Test Accuracy Working Group. Handbook for DTA reviews. URL: http://srdta.cochrane. org/handbook-dta-reviews (accessed January 2012).

39. Moher D, Liberati A, Tetzlaff J, Altman DG. Preferred reporting items for systematic reviews and meta-analyses: the PRISMA statement. J Clin Epidemio/ 2009;62:1006-12. http://dx. doi. org/10.1016/j.jclinepi.2009.06.005

40. Whiting P, Rutjes AW, Reitsma JB, Bossuyt PM, Kleijnen J. The development of QUADAS: a tool for the quality assessment of studies of diagnostic accuracy included in systematic reviews. BMC Med Res Methodol 2010;3:25. http://dx.doi.org/10.1186/1471-2288-3-25

41. Meads CA, Davenport CF. Quality assessment of diagnostic before-after studies: development of methodology in the context of a systematic review. BMC Med Res Methodol 2009;9:3.

http://dx.doi.org/10.1186/1471-2288-9-3

42. Harbord RM, Deeks JJ, Egger M, Whiting P, Sterne JA. A unification of models for meta-analysis of diagnostic accuracy studies. Biostatistics 2007;8:239-51. http://dx.doi.org/10.1093/biostatistics/ kxl004

43. Reitsma JB, Glas AS, Rutges AW, Scholten RJ, Bossuyt PM, Zwinderman AH. Bivariate analysis of sensitivity and specificity produces informative summary measures in diagnostic reviews. J Clin Epidemio/ 2005;58:982-90. http://dx.doi.org/10.1016/j.jclinepi.2005.02.022

44. O'Hagan A, Buck CE, Daneschkkah A, Eiser JR, Garthwaite PH, Jenkinson DJ, et al. Uncertain judgements: eliciting expert's probabilities. Chichester, UK: Wiley-Blackwell; 2006. http://dx.doi. org/10.1002/0470033312

45. Moons KG, Harrell FE. Sensitivity and specificity should be de-emphasized in diagnostic accuracy studies. Acad Radiol 2003;10:670-2. http://dx.doi.org/10.1016/S1076-6332(03)80087-9

46. Davenport CF. Systematic reviews of test accuracy: developing methods that meet practitioners' needs. PhD thesis. Birmingham: University of Birmingham; 2012.

47. National Institute for Health and Clinical Excellence. Methods for the development of NICE public health guidance. 2nd edn. London: NICE; 2009.

48. Amit A, Beck D, Lowenstein L, Lavie O, Bar S, Kedar Z, et al. The role of hybrid PET/CT in the evaluation of patients with cervical cancer. Gynecol Oncol 2006;100:65-9. http://dx.doi. org/10.1016/j.ygyno.2005.07.013 
49. Grisaru D, Almog B, Levine C, Metser U, Fishman A, Lerman H, et al. The diagnostic accuracy of ${ }^{18} \mathrm{~F}$-fluorodeoxyglucose PET/CT in patients with gynecological malignancies. Gynecol Oncol 2004;94:680-4. http://dx.doi.org/10.1016/j.ygyno.2004.05.053

50. Kitajima K, Murakami K, Yamasaki E, Domeki Y, Kaji Y, Sugimura K. Performance of FDG-PET-CT for diagnosis of recurrent uterine cervical cancer. Eur Radiol 2008;18:2040-7. http://dx. doi. org/10.1007/s00330-008-0979-9

51. Mittra E, El-Maghraby T, Rodriguez CA, Quon A, Ross McDougal I, Gambhir SS, et al. Efficacy of ${ }^{18} \mathrm{~F}-\mathrm{FDG}$ PET-CT in the evaluation of patients with recurrent cervical carcinoma. Eur J Nucl Med Mol Imaging 2009;36:1952-9. http://dx.doi.org/10.1007/s00259-009-1206-x

52. Sironi S, Picchio M, Landoni C, Galimberti S, Signorelli M, Bettinardi V, et al. Post-therapy surveillance of patients with uterine cancers: value of integrated FDG PET/CT in the detection of recurrence. Eur J Nucl Med Mol Imaging 2007;34:472-9. http://dx.doi.org/10.1007/ s00259-006-0251-y

53. Hatano K, Sekiya Y, Araki H, Sakai M, Togawa T, Narita Y, et al. Evaluation of the therapeutic effect of radiotherapy on cervical cancer using magnetic resonance imaging. Int J Radiat Oncol Biol Phys 1999;45:639-44. http://dx.doi.org/10.1016/S0360-3016(99)00228-X

54. Weber TM, Sostman HD, Spritzer CE, Ballard RL, Meyer GA, Clarke-Pearson DL, et al. Cervical carcinoma: determination of recurrent tumor extent versus radiation changes with MR imaging. Radiology 1995;194:135-9.

55. Heron CW, Husband JE, Williams MP, Dobbs J, Cosgrove DO. The value of CT in the diagnosis of recurrent carcinoma of the cervix. Clin Radiol 1988;39:501. http://dx.doi.org/10.1016/ s0009-9260(88)80211-3

56. Park DH, Kim KH, Park SY, Lee BH, Choi CW, Chin SY. Diagnosis of recurrent uterine cervical cancer: computed tomography versus positron emission tomography. Korean J Radiol 2000;1:51-5. http://dx.doi.org/10.3348/kjr.2000.1.1.51

57. Walsh JW, Amendola MA, Hall DJ, Tisnado J, Goplerud DR. Recurrent carcinoma of the cervix: CT diagnosis. AJR Am J Roentgenol 1981;136:117-22.

58. Williams MP, Husband JE, Heron CW, Cherryman GR, Koslin DB. Magnetic resonance imaging in recurrent carcinoma of the cervix. Br J Radiol 1989;62:544-50. http://dx.doi. org/10.1259/0007-1285-62-738-544

59. Hirte HW, Strychowsky JE, Oliver T, Fung-Kee-Fung M, Elit L, Oza AM. Chemotherapy for recurrent, metastatic or persistent cervical cancer: a systematic review. Int J Gynecol Cancer 2007;17:1194204. http://dx.doi.org/10.1111/j.1525-1438.2007.00900.x

60. Alberts DS, Kronmal R, Baker LH, Stock-Novack DL, Surwit EA, Boutselis JG, et al. Phase II randomized trial of cisplatin chemotherapy regimens in the treatment of recurrent or metastatic squamous cell cancer of the cervix: a Southwest Oncology Group Study. J Clin Oncol 1987;5:1791-5.

61. Barlow JJ, Piver MS, Chuang JT, Cortes EP, Onuma T, Holland JF. Adriamycin and bleomycin, alone and in combination, in gynecologic cancers. Cancer 1973;32:735-43. http://dx.doi. org/10.1002/1097-0142(197310)32:4<735::AID-CNCR2820320401>3.0.CO;2-K

62. Bezwoda WR, Nissenbaum M, Derman DP. Treatment of metastatic and recurrent cervix cancer with chemotherapy: a randomised trial comparing hydroxyurea with cisdiamminedichloro-platinum plus methotrexate. Med Pediatr Oncol 1986;14:17-19. http://dx.doi.org/10.1002/mpo.2950140105

63. Bloss JD, Blessing JA, Behrens BC, Mannel RS, Rader JS, Sood AK, et al. Randomized trial of cisplatin and ifosfamide with or without bleomycin in squamous carcinoma of the cervix: a Gynecologic Oncology Group study. J Clin Oncol 2002;20:1832-7. http://dx.doi.org/10.1200/JC0.2002.07.045 
64. Bonomi P, Blessing JA, Stehman FB, DiSaia PJ, Walton L, Major FJ. Randomized trial of three cisplatin dose schedules in squamous-cell carcinoma of the cervix: a Gynecologic Oncology Group study. J Clin Oncol 1985;3:1079-85.

65. Cadron I, Jakobsen A, Vergote I. Report of an early stopped randomized trial comparing cisplatin vs. cisplatin/ifosfamide/ 5-fluorouracil in recurrent cervical cancer. Gynecol Obstet Invest 2005;59:126-9.

66. Garin A, Moiseyenko VM, Roszak A, Valvere V, El Gueddari B, Fumoleau P, et al. Randomised Phase II study of irinotecan or irinotecan in combination with cisplatinum or cisplatinum in first line in patients with metastatic squamous cell carcinoma of the cervix. Proc Am Soc Clin Oncol $2001 ; 20$ :abstract 826 .

67. Greenberg BR, Kardinal CG, Pajak TF, Bateman JR. Adriamycin versus adriamycin and bleomycin in advanced epidermoid carcinoma of the cervix. Cancer Treat Rep 1977;61:1383-4.

68. Lira-Puerto V, Silva A, Morris M, Martinez R, Groshen S, Morales-Canfield F, et al. Phase II trial of carboplatin or iproplatin in cervical cancer. Cancer Chemother Pharmacol 1991;28:391-6. http://dx.doi.org/10.1007/BF00685695

69. Grendys EC Jr, Long HJ, Bundy BN, Benda J, McMeekin DS, Sorosky Jl, et al. Randomized phase III trial of cisplatin vs cisplatin plus topotecan vs the combination of methotrexate, vinblastine, doxorubicin and cisplatin (MVAC) in stage IVB, recurrent or persistent carcinoma of the uterine cervix: a Gynecologic Oncology Group study. Int J Gynecol Cancer 2004:14(Suppl. 1):12.

70. Long HJ, Bundy BN, Grendys EC Jr, Benda J, McMeekin DS, Sorosky JAU, et al. Randomized phase III trial of cisplatin vs cisplatin plus topotecan vs MVAC in stage IVB, recurrent or persistent carcinoma of the uterine cervix: a Gynecologic Oncology Group study. Gynecol Oncol 2004:92:397.

71. Long HJ III, Bundy BN, Grendys EC Jr, Benda JA, McMeekin DS, Sorosky J, et al. Randomized phase III trial of cisplatin with or without topotecan in carcinoma of the uterine cervix: a Gynecologic Oncology Group study. J Clin Oncol 2005;23:4626-33. http://dx.doi.org/1 0.1200/JCO.2005.10.021

72. Monk BJ, Huang H, Cella D, Long HJ. Quality of life outcomes from GOG 179: a phase III trial of cisplatin vs cisplatin/topotecan in recurrent or persistent carcinoma of the cervix. Int J Gynecol Cancer 2004:14(Suppl. 1):42.

73. Monk BJ, Huang HQ, Cella D, Long IIIH, Mackey D. Quality of life outcomes from a randomized phase III trial of cisplatin with or without topotecan in advanced carcinoma of the cervix: a Gynecologic Oncology Group study. J Clin Oncol 2005;23:4617-25. http://dx.doi.org/10.1200/ JCO.2005.10.522

74. McGuire WP III, Arseneau J, Blessing JA, DiSaia PJ, Hatch KD, Given FT Jr, et al. A randomized comparative trial of carboplatin and iproplatin in advanced squamous carcinoma of the uterine cervix: a Gynecologic Oncology Group study. J Clin Oncol 1989;7:1462-8.

75. Monk BJ, Mas LL, Zarba JJ, Oaknin A, Tarpin C, Termrungruanglert W, et al. Phase II, open-label study of pazopanib or lapatinib monotherapy compared with pazopanib plus lapatinib combination therapy in patients with advanced and recurrent cervical cancer. J Clin Oncol 2010;28:3562-9. http://dx.doi.org/10.1200/JCO.2009.26.9571

76. Monk BJ, Sill MW, McMeekin DS, Cohn DE, Ramondetta LM, Boardman CH, et al. Phase III trial of four cisplatin-containing doublet combinations in stage IVB, recurrent, or persistent cervical carcinoma: a Gynecologic Oncology Group study. J Clin Oncol 2009;27:4649-55. http://dx.doi. org/10.1200/JCO.2009.21.8909

77. McQuellon RP, Thaler HT, Cella D, Moore DH. Quality of life (QOL) outcomes from a randomized trial of cisplatin versus cisplatin plus paclitaxel in advanced cervical cancer: a Gynecologic Oncology Group study. Gynecol Oncol 2006;101:296-304. http://dx.doi.org/10.1016/j.ygyno.2005.10.039 
78. Moore DH, Blessing JA, McQuellon RP, Thaler HT, Cella D, Benda J, et al. Phase III study of cisplatin with or without paclitaxel in stage IVB, recurrent, or persistent squamous cell carcinoma of the cervix: a Gynecologic Oncology Group study. J Clin Oncol 2004;22:3113-19. http://dx.doi. org/10.1200/JCO.2004.04.170

79. Mountzios G, Dimopoulos MA, Bamias A, Vourli G, Kalofonos H, Aravantinos G, et al. Randomized multicenter phase II trial of cisplatin and ifosfamide with or without paclitaxel in recurrent or metastatic carcinoma of the uterine cervix: a Hellenic Cooperative Oncology Group (HeCOG) study. Ann Oncol 2009;20:1362-8. http://dx.doi.org/10.1093/annonc/mdn797

80. Omura GA, Blessing JA, Vaccarello L, Berman ML, Clarke-Pearson DL, Mutch DG, et al. Randomized trial of cisplatin versus cisplatin plus mitolactol versus cisplatin plus ifosfamide in advanced squamous carcinoma of the cervix: a Gynecologic Oncology Group study. J Clin Oncol 1997;15:165-71.

81. Thomsen TK, Pfeiffer P. Teniposide or carboplatin in patients with recurrent or advanced cervical carcinoma: a randomized phase II trial. Int J Gynecol Cancer 1998;8:310-14. http://dx.doi. org/10.1046/j.1525-1438.1998.09811.x

82. Vermorken JB, Zanetta G, de Oliveira CF, van der Burg ME, Lacave AJ, Teodorovic I, et al. Randomized phase III trial of bleomycin, vindesine, mitomycin-C, and cisplatin (BEMP) versus cisplatin $(P)$ in disseminated squamous-cell carcinoma of the uterine cervix: an EORTC Gynecological Cancer Cooperative Group study. Ann Oncol 2001;12:967-74. http://dx.doi. org/10.1023/A:1011165115426

83. Wallace HJ Jr, Hreshchyshyn MM, Wilbanks GD, Boronow RC, Fowler WC Jr, Blessing JA. Comparison of the therapeutic effects of adriamycin alone versus adriamycin plus vincristine versus adriamycin plus cyclophosphamide in the treatment of advanced carcinoma of the cervix. Cancer Treat Rep 1978;62:1435-41.

84. Ciatto S, Pirtoli L, Cionini L. Radiotherapy for postoperative failures of carcinoma of the cervix uteri. Surg Gynecol Obstet 1980;151:621-4.

85. Deutsch M, Parsons JA. Radiotherapy for carcinoma of the cervix recurrent after surgery. Cancer 1974;34:2051-5. http://dx.doi. org/10.1002/1097-0142(197412)34:6<2051::AID-CNCR2820340625>3.0.CO;2-G

86. Hille $A$, Weiss $E$, Hess CF. Therapeutic outcome and prognostic factors in the radiotherapy of recurrences of cervical carcinoma following surgery. Strahlenther Onkol 2003;179:742-7. http://dx.doi.org/10.1007/s00066-003-1100-6

87. Ito H, Shigematsu N, Kawada T, Kubo A, Isobe K, Hara R, et al. Radiotherapy for centrally recurrent cervical cancer of the vaginal stump following hysterectomy. Gynecol Oncol 1997;67:154-61. http://dx.doi.org/10.1006/gyno.1997.4855

88. Jain P, Hunter RD, Livsey JE, Coyle C, Swindell R, Davidson SE. Salvaging locoregional recurrence with radiotherapy after surgery in early cervical cancer. Clin Oncol 2007;19:763-8. http://dx.doi. org/10.1016/j.clon.2007.08.015

89. Jobsen JJ, Leer JW, Cleton FJ, Hermans J. Treatment of locoregional recurrence of carcinoma of the cervix by radiotherapy after primary surgery. Gynecol Oncol 1989;33:368-71. http://dx. doi. org/10.1016/0090-8258(89)90529-5

90. Lucraft HH. Radiotherapy following primary surgery for carcinoma of uterine cervix. Clin Radiol 1981;32:347-53. http://dx.doi.org/10.1016/S0009-9260(81)80062-1

91. Potter ME, Alvarez RD, Gay FL, Shingleton HM, Soong SJ, Hatch KD. Optimal therapy for pelvic recurrence after radical hysterectomy for early-stage cervical cancer. Gynecol Oncol 1990;37:74-7. http://dx.doi.org/10.1016/0090-8258(90)90311-8 
92. Tan R, Chung CH, Liu MT, Lai YL, Chang KH. Radiotherapy for postoperative recurrent uterine cervical carcinoma. Acta Oncol 1991;30:353-6. http://dx.doi.org/10.3109/02841869109092385

93. Grigsby PW. Prospective phase I/II study of irradiation and concurrent chemotherapy for recurrent cervical cancer after radical hysterectomy. Int I Gynecol Cancer 2004;14:860-4. http://dx. doi. org/10.1111/j.1048-891X.2004.14521.x

94. Haasbeek CJA, Uitterhoeve AU, van der Velden J, Gonzalez DG, Stalpers பA. Long-term results of salvage radiotherapy for the treatment of recurrent cervical carcinoma after prior surgery. Radiother Oncol 2008;89:197-204. http://dx.doi.org/10.1016/j.radonc.2008.01.004

95. Ijaz T, Eifel PJ, Burke T, Oswald MJ. Radiation therapy of pelvic recurrence after radical hysterectomy for cervical carcinoma. Gynecol Oncol 1998;70:241-6. http://dx.doi.org/10.1006/gyno.1998.5093

96. Maneo A, Landoni F, Cormio G, Colombo A, Placa F, Pellegrino A, et al. Concurrent carboplatin/5fluorouracil and radiotherapy for recurrent cervical carcinoma. Ann Oncol 1999;10:803-7. http://dx.doi.org/10.1023/A:1008356010556

97. Thomas GM, Dembo AJ, Black B, Bean HA, Beale FA, Pringle JR, et al. Concurrent radiation and chemotherapy for carcinoma of the cervix recurrent after radical surgery. Gynecol Oncol 1987;27:254-60. http://dx.doi.org/10.1016/0090-8258(87)90243-5

98. Tsuda H, Tanaka M, Manabe T, Ikeda H, Negoro S, Ishiko O, et al. Phase I study of combined radiation, hyperthermia and intra-arterial carboplatin for local recurrence of cervical cancer. Ann Oncol 2003;14:298-303. http://dx.doi.org/10.1093/annonc/mdg054

99. Virostek $\sqcup$, Kim RY, Spencer SA, Meredith RF, Jennelle RL, Soong SJ, et al. Postsurgical recurrent carcinoma of the cervix: reassessment and results of radiation therapy options. Radiology 1996;201:559-63.

100. Adcock LL. Radical hysterectomy preceded by pelvic irradiation. Gynecol Onco/ 1979;8:152-63. http://dx.doi.org/10.1016/0090-8258(79)90020-9

101. Coleman RL, Keeney ED, Freedman RS, Burke TW, Eifel PJ, Rutledge FN. Radical hysterectomy for recurrent carcinoma of the uterine cervix after radiotherapy. Gynecol Oncol 1994;55:29-35. http://dx.doi.org/10.1006/gyno.1994.1242

102. Ibsen TB, Sorensen BL, Knudsen JB, Sorensen HM. Operative treatment of recurrent cancer of the uterine cervix after radiotherapy. Acta Obst Gynecol Scand 1988;67:389-93. http://dx. doi. org/10.3109/00016348809004245

103. Maneo A, Landoni F, Cormio G, Colombo A, Mangioni C. Radical hysterectomy for recurrent or persistent cervical cancer following radiation therapy. Int J Gynecol Cancer 1999;9:295-301. http://dx.doi.org/10.1046/j.1525-1438.1999.99037.x

104. Rubin SC, Hoskins WJ, Lewis JU. Radical hysterectomy for recurrent cervical cancer following radiation therapy. Gynecol Oncol 1987;27:316-24. http://dx.doi. org/10.1016/0090-8258(87)90252-6

105. Terada K, Morley GW. Radical hysterectomy as surgical salvage therapy for gynecologic malignancy. Obstet Gynecol 1987;70:913-15.

106. Tupper $C$. Radical Wertheim as a salvage procedure. Patients with recurrence following initial definitive radiotherapy. Am J Obstet Gynecol 1965;91:364-8.

107. Anthopoulos AP, Manetta A, Larson JE, Podczaski ES, Bartholomew MJ, Mortel R. Pelvic exenteration: a morbidity and mortality analysis of a seven-year experience. Gynecol Oncol 1989;35:219-23. http://dx.doi.org/10.1016/0090-8258(89)90047-4

108. Barber HR, Jones W. Lymphadenectomy in pelvic exenteration for recurrent cervix cancer. JAMA 1971;215:1945-9. http://dx.doi.org/10.1001/jama.1971.03180250037008 
109. Beitler JJ, Anderson PS, Wadler S, Runowicz CD, Hayes MK, Fields AL, et al. Pelvic exenteration for cervix cancer: would additional intraoperative interstitial brachytherapy improve survival? Int J Radiat Oncol Biol Phys 1997;38:143-8. http://dx.doi.org/10.1016/50360-3016(96)00585-8

110. Bricker EM, Butcher J, Lawler J, McAfee CA. Surgical treatment of advanced and recurrent cancer of the pelvic viscera: an evaluation of ten years' experience. Ann Surg 1960;152:388-401. http://dx.doi.org/10.1097/00000658-196009000-00004

111. Brunschwig A, Daniel W. Pelvic exenteration operations: with summary of sixty-six cases surviving more than five years. Ann Surg 1960;151:571-6.

http://dx.doi.org/10.1097/00000658-196004000-00018

112. Chung CK, Nahhas WA, Stryker JA, Mortel R. Treatment outcome of recurrent cervical cancer. J Surg Oncol 1983;24:5-10. http://dx.doi.org/10.1002/jso.2930240103

113. Deckers PJ, Sugarbaker EV, Pilch YH, Ketcham AS. Pelvic exenteration for late second cancers of the uterine cervic after earlier irradiation. Ann Surg 1972;175:48-54. http://dx.doi. org/10.1097/00000658-197201000-00008

114. Hatch KD, Gelder MS, Soong SJ, Baker VV, Shingleton HM. Pelvic exenteration with low rectal anastomosis: survival, complications, and prognostic factors. Gynecol Oncol 1990;38:462-7. http://dx.doi.org/10.1016/0090-8258(90)90092-Y

115. Ketcham AS, Deckers PJ, Sugarbaker EV, Hoye RC, Thomas LB, Smith RR. Pelvic exenteration for carcinoma of the uterine cervix. A 15-year experience. Cancer 1970;26:513-21. http://dx.doi. org/10.1002/1097-0142(197009)26:3<513::AID-CNCR2820260304>3.0.C0;2-6

116. Kraybill WG, Lopez MJ, Bricker EM. Total pelvic exenteration as a therapeutic option in advanced malignant disease of the pelvis. Surg Gynecol Obstet 1988;166:259-63.

117. Mikuta JJ, Murphy JJ. The team approach to pelvic exenteration for cervical cancer. Am J Obstet Gynecol. 1960;80:795-801.

118. Mikuta JJ, Murphy JJ, Schoenberg HW. Pelvic exenteration for cervical cancer. Obstet Gynecol 1967;29:858-61.

119. Palmer AB, Blaikley JB. Discussion on pelvic exenteration. Proc $R$ Soc Med 1953;46:753-68.

120. Pinelo S, Petiz A, Domingues C, Lopes C, Alves A, Fael R. [Pelvic exenterations for gynaecological cancer - a 10 year institutional review.] Acta Med Port 2002;19:99-104.

121. Rutledge FN, Smith JP, Wharton JT, O'Quinn AG. Pelvic exenteration: analysis of 296 patients. Am J Obstet Gynecol 1977;129:881-92.

122. Shingleton HM, Soong SJ, Gelder MS, Hatch KD, Baker VV, Austin JMJ. Clinical and histopathologic factors predicting recurrence and survival after pelvic exenteration for cancer of the cervix. Obstet Gynecol 1989;73:1027-34. http://dx.doi.org/10.1097/00006250-198906000-00024

123. Stanhope CR, Webb MJ, Podratz KC. Pelvic exenteration for recurrent cervical cancer. Clin Obstet Gynecol 1990;33:897-909. http://dx.doi.org/10.1097/00003081-199012000-00026

124. Symmonds RE, Pratt JH, Webb MJ. Exenterative operations: experience with 198 patients. Am J Obstet Gynecol 1975;121:907-18.

125. Teran-Porcayo MA, Zeichner-Gancz I, del-Castillo RA, Beltran-Ortega A, Solorza-Luna G. Pelvic exenteration for recurrent or persistent cervical cancer: experience of five years at the National Cancer Institute in Mexico. Med Oncol 2006;23:219-23. http://dx.doi.org/10.1385/MO:23:2:219

126. Vieira SC, Costa DR, Meneses AD, Borges e Silva J, Oliveira AK, Sousa RB. [Post-radiotherapy pelvic exenteration in relapsed cervical cancer: experience of a tertiary health service in the northeast of Brazil.] Rev Bras Ginecol Obstet 2009;31:22-7. http://dx.doi.org/10.1590/ S0100-72032009000100005 
127. Pearcey $R$, Brundage $M$, Drouin P, Jeffrey J, Johnson D, Lukka $H$, et al. Phase III trial comparing radical radiotherapy with and without cisplatin chemotherapy in patients with advanced squamous cell cancer of the cervix. J Clin Oncol 2002;20:966-72. http://dx.doi.org/10.1200/JCO.20.4.966

128. Bliss P, Trott PA, Blake PR. Cost-effectiveness of routine cytological cervical surveillance following treatment for carcinoma of the cervix. J Clin Effect 1997;2:87-90.

129. Chan YM, Ng TY, Ngan HY, Wong LC. Monitoring of serum squamous cell carcinoma antigen levels in invasive cervical cancer: is it cost-effective? Gynecol Oncol 2002;84:7-11. http://dx.doi.org/10.1006/gyno.2001.6497

130. Forni F, Ferrandina G, Deodato F, Macchia G, Morganti AG, Smaniotto D, et al. Squamous cell carcinoma antigen in follow-up of cervical cancer treated with radiotherapy: evaluation of cost-effectiveness. Int J Radiat Oncol Biol Phys 2007;69:1145-9. http://dx.doi.org/10.1016/j. ijrobp.2007.04.055

131. Manusirivithaya S, Sripramote M, Tangjitgamol S, Sanjareonsuttikul N, Pisamturakit P. Cost effectiveness of concurrent chemoradiation in comparison with radiation alone in locally advanced cervical cancer. J Med Assoc Thail 2005;88:1035-44.

132. Melnikow J, Kulasingam S, Slee C, Helms J, Kuppermann M, Birch S, et al. Surveillance after treatment for cervical intraepithelial neoplasia. Obstet Gynecol 2010;116:1158-70. http://dx.doi. org/10.1097/AOG.0b013e3181f88e72

133. Paton F, Paulden M, Saramago P, Manca A, Misso K, Palmer S, et al. Topotecan for the treatment of recurrent and stage IVB carcinoma of the cervix. Health Technol Assess 2010;14(Suppl. 1):55-62.

134. Sonnenberg FA, Beck JR. Markov models in medical decision making: a practical guide. Med Dec Making 1993;13:332-8. http://dx.doi.org/10.1177/0272989X9301300409

135. Landoni F, Maneo A, Colombo A, Placa F, Milani R, Perego P, et al. Randomised study of radical surgery versus radiotherapy for stage Ib-lla cervical cancer. Lancet 1997;350:535-40. http://dx. doi. org/10.1016/S0140-6736(97)02250-2

136. Keys HM, Bundy BN, Stehman FB, Muderspach LI, Chafe WE, Suggs CL, et al. Cisplatin, radiation, and adjuvant hysterectomy compared with radiation and adjuvant hysterectomy for bulky stage IB cervical carcinoma. N Eng J Med 1999;340:1154-61. http://dx.doi.org/10.1056/ NEJM199904153401503

137. Vale CL, Tierney JF, Davidson SE, Drinkwater KJ, Symonds P. Substantial improvement in UK cervical cancer survival with chemoradiotherapy: results of a Royal College of Radiologists' audit. Clin Oncol 2010;22:590-601. http://dx.doi.org/10.1016/j.clon.2010.06.002

138. Adriano E, Jagoe J, Harrison T, Riffenburgh RH, Johnstone PA. Survival of patients with untreated cervical carcinoma. Am J Clin Oncol 2003;26:369-73. http://dx.doi.org/10.1097/01. COC.0000026909.73608.88

139. Department of Health (DoH). NHS reference costs 2009-10. London: DoH; 2011.

140. Clark J, Barton P, Gupta J, Khan K. Outpatient diagnosis of endometrial cancer in women with first episode of postmenopausal bleeding. Report no. 51. Birmingham: West Midlands Health Technology Assessment Collaboration, Department of Public Health and Epidemiology, University of Birmingham; 2002.

141. Curtis L. Unit costs of health and social care. Canterbury: Personal Social Services Research Unit, University of Kent; 2010.

142. Brush J, Boyd K, Chappell F, Crawford F, Dozier M, Fenwick E, et al. The value of FDG positron emission tomography/computerised tomography (PET/CT) in pre-operative staging of colorectal cancer: a systematic review and economic evaluation. Health Technol Assess 2011;15(35). 
143. Goldie SJ, Kohli M, Grima D, Weinstein MC, Wright TC, Bosch X, et al. Projected clinical benefits and cost-effectiveness of a human papillomavirus 16/18 vaccine. J Nat/ Cancer Inst 2004;96:604-15. http://dx.doi.org/10.1093/jnci/djh104

144. Lang $\mathrm{H}-\mathrm{C}$, Chang $\mathrm{K}$, Ying $\mathrm{Y}-\mathrm{H}$. Quality of life, treatments, and patients' willingness to pay for a complete remission of cervical cancer in Taiwan [published online ahead of print 9 September 2011]. Health Econ 2011.

145. Tewari K, Monk BJ. Gynecologic Oncology Group trials of chemotherapy for metastatic and recurrent cervical cancer. Curr Oncol Rep 2005;7:419-34. http://dx.doi.org/10.1007/ s11912-005-0007-z

146. Tzioras S, Pavlidis N, Paraskevaidis E, loannidis JP. Effects of different chemotherapy regimens on survival for advanced cervical cancer: systematic review and meta-analysis. Cancer Treat Rev 2007;33:24-38. http://dx.doi.org/10.1016/j.ctrv.2006.09.007

147. Lukka H, Hirte H, Fyles A, Thomas G, Elit L, Johnston M, et al. Concurrent cisplatin-based chemotherapy plus radiotherapy for cervical cancer - a meta-analysis. Clin Oncol 2002;14:203-12. http://dx.doi.org/10.1053/clon.2002.0076

148. Rutjes AW, Reitsma JB, Coomarasamy A, Khan KS, Bossyt PM. Evaluation of diagnostic tests when there is no gold standard. A review of methods. Health Technol Assess 2007;11(50).

149. Thigpen $T$, Shingleton $H$, Homesley H, Lagasse L, Blessing J. Cis-platinum in treatment of advanced or recurrent squamous cell carvinoma of the cervix: a phase II study of the Gynecologic Oncology Group. Cancer 1981;48:903. http://dx.doi. org/10.1002/1097-0142(19810815)48:4<899::AID-CNCR2820480406>3.0.C0;2-6

150. Lira-Puerto $V$, de la Huerta R, Cortes H, Fernandez A, Silva A, Martinez R, et al. Cisplatin (CDDP) plus radiotherapy (RT) vs radiotherapy alone in locally advanced cervical cancer. Proc Am Soc Clin Oncol 1990;9:abstract 633.

151. Pearcey R, Brundage M, Jeffrey J. A clinical trial comparing concurrent cisplatin and radiation therapy versus radiation alone for locally advanced squamous cell carcinoma of the cervix carried out by the National Cancer Institute of Canada Clinical Trials Group. Proc Am Soc Clin Oncol 2000;19:abstract 378a.

152. Chiara S, Bruzzone M, Merlini L, Bruzzi P, Rosso R, Franzone P, et al. Randomized study comparing chemotherapy plus radiotherapy versus radiotherapy alone in FIGO stage IIB-III cervical carcinoma. GONO (North West Oncologic Cooperative Group). Am J Clin Oncol 1994;17:294-7. http://dx.doi. org/10.1097/00000421-199408000-00003

153. Choo YC, Choy TK, Wong LC, Ma HK. Potentiation of radiotherapy by cis-dichlorodiammine platinum (II) in advanced cervical carcinoma. Gynecol Oncol 1986;23:94-100. http://dx.doi. org/10.1016/0090-8258(86)90120-4

154. Garipagaoglu M, Kayikcioglu F, Kose MF, Adli M, Gulkesen KH, Kocak Z, et al. Adding concurrent low dose continuous infusion of cisplatin to radiotherapy in locally advanced cervical carcinoma: a prospective randomised pilot study. Br J Radiol 2004;77:581-7. http://dx.doi.org/10.1259/ bjr/63967203

155. Wong LC, Choo YC, Choy D, Sham JS, Ma HK. Long term follow up of potentiation of radiotherapy by cis-platinum in advanced cervical cancer. Gynecol Oncol 1989;35:159-63. http://dx.doi. org/10.1016/0090-8258(89)90035-8 


\section{Appendix 1 Protocol}

\section{CLINICAL EFFECTIVENESS OF PET-CT IMAGING IN RESTAGING RECURRENT CERVICAL CANCER: SYSTEMATIC REVIEW OF EVIDENCE AND ECONOMIC MODELLING}

\section{Clinical background}

Cervical cancer is the most common gynaecological malignancy in the world with an estimated 493,000 new cases diagnosed worldwide each year. ${ }^{1}$ In the United Kingdom, approximately 2,800 patients are diagnosed with cervical cancer per year, accounting for around $2 \%$ of all female cancer cases ${ }^{2}$. In 2007 there were 941 deaths from cervical cancer which translates to a European age-standardised death rate of 2.4 per 100000 females. Early stage (stage 1A2-IB1) cervical cancer is treated with either surgery or chemo radiotherapy with equal survival rates whereas advanced stage cervical cancer (stage IB2-IV) is usually treated with chemo radiotherapy or chemotherapy alone ${ }^{3}$. Survival rates depend on stage at presentation and histology of tumour and the all-stage five-year survival rate is $64.1 \%{ }^{4}$. The risk of recurrence after primary treatment depends on the extent of the primary cancer at presentation. Approximately $10-20 \%$ of patients with stage IB-IIA cervical cancer with negative lymph nodes will recur, while those with nodal metastasis or locally advanced disease have an up to $70 \%$ risk of recurrence ${ }^{1-3}$.

Recurrences can be central at the cervix or vaginal vault, pelvic in the lymph nodes of the pelvic side wall or distant metastases (for e.g. lung, supraclavicular lymph nodes and para aortic lymph nodes). Recurrences are common within the first 24 months after the initial diagnosis and can be symptomatic or asymptomatic. Symptom status at time of recurrence is a significant predictor of survival; the median survival is 11 months for symptomatic recurrence and 42 months for asymptomatic recurrence detected at follow-up ${ }^{5}$. Routine clinical examination is not accurate in detecting recurrent disease as a high proportion of patients are found to be symptomatic at the time of detecting recurrence. Patients with pelvic recurrence usually present with vaginal bleeding, discharge, pelvic pain, and sciatic pain. Patient with disseminated recurrence will develop systemic symptoms associated with cachexia. Unfortunately 5 -year survival for recurrent or persistent cervical cancer evaluated with current imaging practices is between $3.2 \%$ and $13 \%{ }^{6}$. Identification of incurable metastases eliminates unnecessary salvage procedures and suffering, while more accurate delineation of tumour extent increases the probability of successful treatment. Survival with distant disease is poor ${ }^{3}$. The key issue is to correctly identify recurrent disease that is amenable to curative treatment, while also correctly identifying cases for palliation.

\subsection{Existing clinical practice}

Currently in the United Kingdom, patients with suspected recurrence will undergo ${ }^{4}$

- clinical examination (rectovaginal and speculum examination, assessment of inguinal/ supraclavicular lymph nodes)

- cross sectional imaging by MRI (Magnetic Resonance Imaging) or CT (Computed Tomography) of chest, abdomen and pelvis

- examination under anaesthesia, histological confirmation of any vaginal vault mass by biopsies.

In preparing this proposal, we have established that current imaging practice in England for the diagnosis and management of recurrence involves an MRI or CT scan of the chest or abdomen and pelvis ${ }^{4}$. A search of the cancer network guidelines of practice in South West, West Midlands and Lancashire confirms that this is standard practice; this is also supported by the evidence based Scottish intercollegiate network (SIGN) guidance governing practice in Scotland. Our conceptualisation of this bid (Fig 1) is therefore an assessment of the effectiveness of PET-CT over current practice. 


\subsection{Applications of PET-CT}

$\mathrm{CT}$ and $\mathrm{MRI}$ are high-resolution anatomical imaging techniques that are commonly used in cancer to detect potential tumours. MRI and CT are currently considered first when recurrence is suspected ${ }^{4}$ but have limitations in differentiating recurrent tumour from post-radiotherapy or surgical fibrosis. CT and MRI also have limitations in accurately identifying the extent of recurrence as small volume metastatic nodal disease and distant recurrence may not be identified. They can also be unreliable in determining the presence or absence of recurrent disease in the pelvis after radiotherapy as radiotherapy induced fibrosis makes tissues indurated thus potentially concealing recurrent disease. Incomplete excision of disease is associated with significantly reduced survival after surgery. Similarly, CT and MRI may not identify disease spread to regional and distant lymph nodes and other organ sites.

PET is an imaging method that can be used to establish the functional parameters of tissue allowing it to detect metabolically active tumours. ${ }^{7}{ }^{18} \mathrm{~F}$-fluorodeoxyglucose $\left({ }^{18} \mathrm{~F}-\mathrm{FDG}\right)$ is the most widely used radiotracer in the management of cancer patients. It is a glucose analogue and is taken up and actively trapped in the enhanced glycolytic pathway of cancer cells in particular. Positron emission tomography (PET) imaging utilises a coincidence camera system to detect the high energy photons emitted as a result of annihilation of positrons emitted by the radioisotope with nearby negatively charged electrons, thus providing anatomical localisation of the source i.e. area of pharmaceutical accumulation. PET provides anatomical image resolution of the order of 4-6 $\mathrm{mm}$, significantly better than conventional gamma-cameras, but inferior to the 1-2-mm resolution of CT or MRI. The size of lesion that can be detected by PET is limited by several factors, including the physics of positron emission, the spatial resolution of the scanner (typically $4.5-6 \mathrm{~mm}$ in the centre of the axial field) and safe dosing limits of ${ }^{18} \mathrm{~F}-\mathrm{FDG} .{ }^{7}$ PET-CT precisely aligns and combines metabolic PET mages with anatomical CT images obtained immediately consecutively on the same machine without patient movement, and is being increasingly preferred over PET scanning alone almost universally, as it allows precise localisation of active disease foci and recognition of normal variants.

The combination of PET-CT rather than PET alone is therefore used in cervical cancer in order to overcome limitations of either neuro-imaging technology alone. PET-CT detects metabolically active disease in primary tumours and metastatic lesions, and can demonstrate disease in normal sized nodes, and in post surgical or radiotherapy fibrosis. False positives are recognised following radiation and surgery as a result of radiotherapy and surgery induced inflammation and the general advice is to wait for 3 to 6 months after treatment. False positives can also occur in sepsis that cannot be always differentiated by PET-CT. False-negatives can also occur soon after chemotherapy and the advice is preferably to wait for 6 weeks with a minimum of 2 weeks. After allowing for important treatment effects, the detection capability of PET-CT is believed to be similar for detection of primary lesion and recurrence of tumour.

The applications of PET-CT in cervical cancer patients include: identification of persistent/recurrent disease, assessing local tumour extension, evaluating pelvic nodal involvement, detection of distant metastases (for example lung, supraclavicular lymph nodes and para-aortic lymph nodes, radiation therapy planning (in patients with PET scans positive for lymph nodes) and in assessing response to therapy ${ }^{8}$.

\subsection{Current treatment options for management of recurrent cervical cancer}

Treatment of recurrent cervical cancer depends upon the site (central, pelvic, distant), extent of recurrence, type of previous treatment received (surgery, chemoradiation, radiation), time elapsed since primary treatment and the patient's performance status. Treatment is usually curative or palliative in intent. Potentially curative disease is defined as a) confirmed recurrent disease confined to the pelvis, if the patient had not received previous primary or adjuvant pelvic RT (Radiotherapy) b) disease confined to the central pelvis, without pelvic side wall or extrapelvic involvement, if RT had been administered before recurrence c) distant recurrences at a single site (such as para aortic lymph node) that could be completely resected or encompassed by a curative RT procedure ${ }^{9}$. The treatment is palliative in intent if distant or multiple site recurrence. It is critical that the therapeutic intent (curative or palliative) is preceded by accurate diagnosis. 
The treatment options for recurrent cervical cancer varies according to the mode of treatment provided for primary cervical cancer and the extent of recurrence.

\subsubsection{Previous surgical treatment:}

In women who have had primary radical surgery and who have had a pelvic relapse, radiation is the treatment of choice ${ }^{3}$. This project will evaluate any evidence on whether early diagnosis of persistent or recurrent disease influences outcomes and whether clearly defining the extent of disease influences management and patient outcomes.

\subsubsection{Previous chemoradiation or radiation only:}

Salvage surgery is generally considered in women who have undergone chemoradiation or radiation treatment alone as primary treatment for cervical cancer and who have evidence of localised recurrence and surgery has a high chance of completely removing the disease ${ }^{4}$. Surgery for relapsed disease after radiation therapy is often associated with high morbidity as radiation fibrosis makes surgery difficult and to enhance cure rates surgical excision of disease often involves removal of bladder, uterus, cervix, and various amounts of the vagina (anterior exenteration) or uterus, vagina and portions of the rectosigmoid colon and anus (posterior exenteration) or a complete pelvic clearance (exenteration). In a small number of patients radical hysterectomy will suffice, if the disease is highly localised. As exenterations are morbid surgical procedures resulting in alteration of body image, patients require extensive preoperative psychosocial counselling.

The evidence base for outcomes after exenterative surgery is based on retrospective case series since the first advocate of this surgery by Alexander Brunschwig in 1946. With appropriate selection of patients, better pre- and postoperative care and improved operative techniques, the operative mortality varies from $16 \%$ to $2 \%^{7-8}$. The 5 -year survival of patients treated with pelvic exenteration is around $30 \%-60 \% \%^{8,10,11}$. Many of these reports vary in case selection, operative philosophy and technique. The objective of this aspect of the project will be to identify all published reports of salvage surgery after radiation and chemoradiation therapy for cervical cancer. Reports that include surgery for recurrent disease from other organs will be excluded. We will also endeavour to contact all cancer centres in the UK in case unpublished audits of salvage surgery have been undertaken. Once the evidence base has been established, we will endeavour to identify the optimum surgical approach from the evidence and quantify short and long term outcomes.

\subsubsection{Early Palliative Treatment}

For multiple site or distant recurrence, chemotherapy can be administered with a palliative intent. Treatment options must be balanced with good supportive care and often palliative care alone is appropriate to maintain quality of life towards the end of life. The project team will also review the evidence if early recognition of unresectable persistent or recurrent disease by imaging after chemoradiation influences patient outcomes.

\section{Work leading to the proposal}

We have conducted systematic reviews of accuracy PET, CT and MRI in primary cervical cancer staging with respect to diagnosis of lymphadenopathy through an MRC research training fellowship awarded under Prof Khalid Khan's supervision. In particular we have developed test accuracy studies comparing PET-CT and sentinel node technique with current imaging standards of CT and MRI to detect lymph node metastases in cervical cancer ${ }^{12}$. In our work, we have developed literature searching, data extraction procedures, and analytic strategies for this topic. We are familiar with the literature and the gaps therein.

There are only a few attempts to incorporate test accuracy evidence into therapeutic decision-making in cancer research. We have identified the need for and developed a decision analytical model for managing patients with vulval cancer which incorporates the accuracy of imaging techniques with the therapeutic 


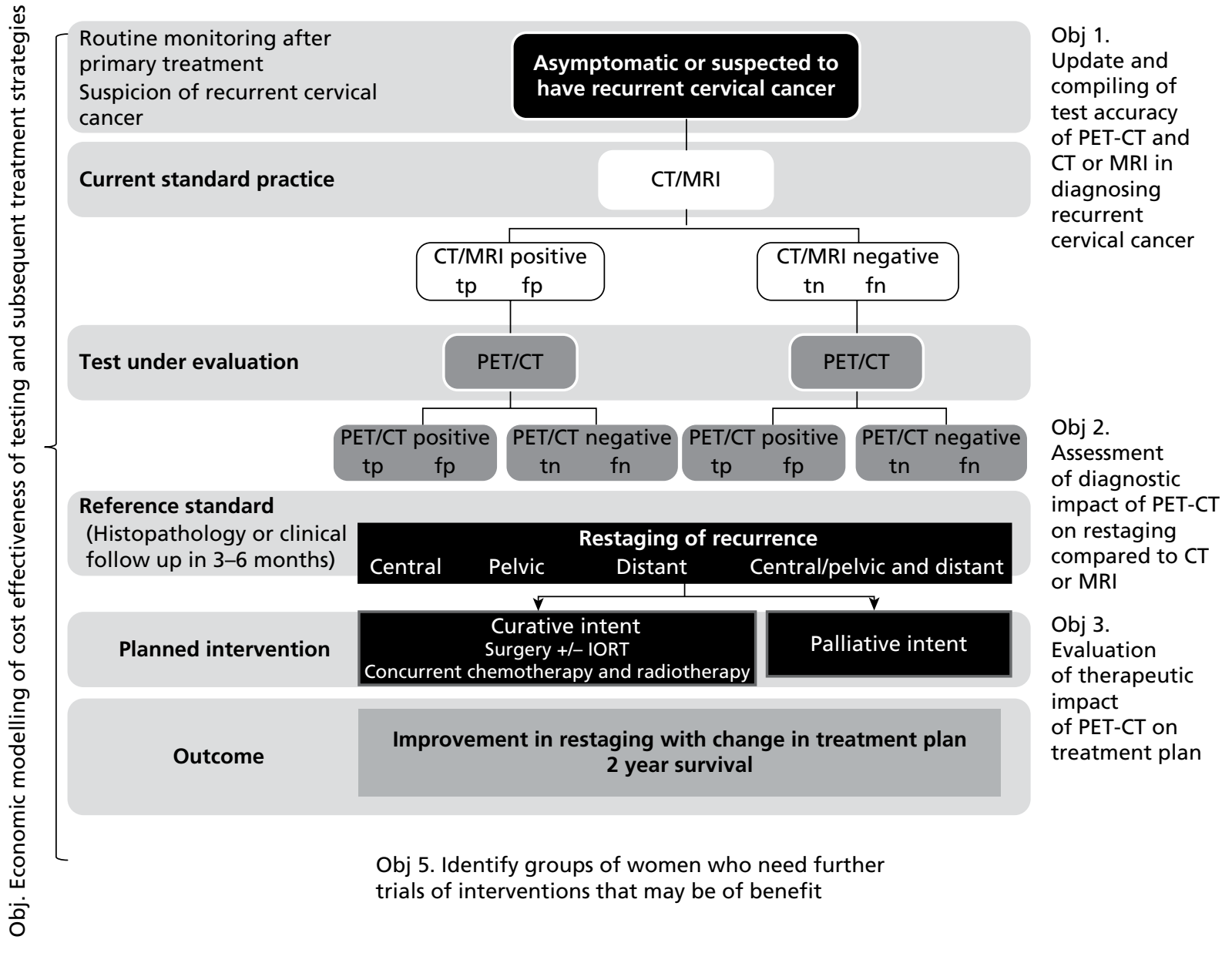

FIGURE 1 Imaging modalities and treatment strategies in women with recurrent cervical cancer.

evidence. A summary of data on accuracy of PET, CT and MRI for accurately staging primary cervical cancer from our published review is provided in Appendix 1.

We have also developed analytical techniques to compensate for the absence of gold standard histopathology for verification of test accuracy previously ${ }^{13}$. We are now in a position to use the above as the basis for developing a robust decision analytic model for recurrent cervical cancer. We have also conducted model based health economics evaluations in obstetrics using output from systematic review ${ }^{14}$. Therefore, we welcome the opportunity to bid for this call for proposals, which will allow us to consolidate and advance the work we have already undertaken in this field.

We are confident that given our knowledge and expertise in the relevant clinical and methodological fields, we can with appropriate resources, deliver a high quality HTA report.

\section{Research Objectives}

The commissioning brief is for an evidence synthesis of the added value of PET/CT for restaging women with recurrent cervical cancer. Our project will follow the key steps involved in health technology assessment and will meet the commissioned brief by fulfilling the following objectives: 
1. To determine the diagnostic accuracy of PET-CT compared to CT or MRI (current imaging) in women with suspected or confirmed recurrent cervical cancer in identifying (restaging) locoregional recurrence, nodal and distant metastasis

2. To evaluate the diagnostic impact of PET-CT resulting in change in diagnosis or restaging compared to CT or MRI

3. To assess the therapeutic impact of PET-CT in changing planned treatment improving mortality and morbidity through systematic review of effectiveness of various interventions (surgery, radiotherapy, chemotherapy, early palliative care) in the management of recurrent cervical cancer detected by current practice (CT or MRI) and PET-CT

4. To evaluate the cost-effectiveness of using PET-CT (in addition to standard practice) for the detection and restaging of recurrent disease and treatment response assessment and consequent treatment strategies in terms of both human and financial costs using decision-analytic modelling.

5. To identify groups of women with recurrent cervical cancer in whom it is possible to undertake future powerful trials of interventions to reduce mortality and morbidity, and to identify key areas and research questions requiring further primary research (in addition to identifying areas where evidence is strong enough to generate recommendations for clinical practice) using Value Of Information (VOI) analysis.

The relationship of our objectives to the clinical process is shown in Fig 1.

\section{Relevance to Commissioning Brief}

The title of the HTA commissioning brief (09/29) refers to 'The added value of PET-CT for restaging in recurrent cervical cancer'. It goes on to include the following in the scope of the work to be carried out: effect of staging on treatment planned, decision analysis and cost effectiveness of added value of PET-CT compared to current imaging practice. From this, we take it that the scope of the work is to be broad.

There is substantial literature on the diagnostic accuracy of PET-CT in primary diagnosis of cervical cancer but literature on recurrent disease is limited. In order to determine the value of PET-CT, information on diagnostic accuracy alone will not be sufficient. In addition information on diagnostic impact, therapeutic and patient outcomes will be needed. Thus, it is crucial to review effectiveness of various interventions in patients with recurrent cervical cancer in addition to accuracy of PET-CT and CT or MRI (current practice) in restaging to inform decision analytic modelling. The project team's interpretation of the scope of this commissioning brief is the added value of PET-CT in restaging in recurrent cervical cancer and not detection of recurrence alone. However the added value of PET-CT in detecting recurrence in asymptomatic women is being promoted as recommended best practice ${ }^{4}$ and the project team propose broadening the scope to include an assessment of the added value of PET-CT as surveillance for asymptomatic patients. Surveillance populations will be restricted to patients with advanced stage cervical cancer (IB2-IV) treated previously with chemoradiation as a sub-group in whom recurrence is most likely. We will obtain information on the treatment for primary cervical cancer for modelling as the mode of initial treatment will influence the accuracy of the test and subsequent treatment for recurrence.

We believe that it is feasible to undertake this work within the time scale with the resources we have requested. Our team has the necessary experience and expertise for fulfilling all the requirements in the HTA brief. We have a very strong, internationally renowned, group knowledgeable in systematic reviews of diagnostic and effectiveness data and in economic modelling. We have recently undertaken a large number of reviews for tests and treatments in cancer including primary cervical cancer and have developed a decision-making framework ${ }^{12,15,16}$. This background provides the basis for us to rapidly undertake the review work and the modelling within the time constraints specified in the brief. 


\section{Plan of research}

The plan of research will be to undertake a novel systematic review of the accuracy of PET-CT in recurrent cervical cancer and a systematic review of the effectiveness of treatments for recurrent cervical cancer. Simultaneously a decision analytic model will be developed and additional rapid systematic reviews will be undertaken as necessary to populate the emerging model. A scoping search of the literature on the test accuracy of PET-CT has been undertaken. This has identified 2 reviews in the literature at various levels of currency (2004-2005) $)^{17,18}$. Searches for these 2 reviews were conducted in 2004 and 2005 respectively and yielded a total of 14 citations of potential relevance to the assessment of accuracy of PET-CT in recurrent cervical cancer. On this basis we expect the volume of test accuracy literature to be small and we therefore propose to include triangulation of subjective probabilities of test accuracy elicited from clinical experts and information on the test accuracy of PET-CT in primary cervical cancer where appropriate ${ }^{12}$.

This project team will address the following structured question:

\section{Population}

1. Clinical suspicion of recurrence: women suspected to have persistent or recurrent cervical cancer after primary treatment, on the basis of one or more of clinical history, clinical examination, tests (including imaging and histology).

2. Surveillance in asymptomatic patients: patients with advanced stage cervical cancer (IB2-IV) treated previously with chemoradiation with a minimum gap between completion of treatment and imaging of 3 months.

\section{Tests}

PET-CT using FDG in addition to current imaging (CT or MRI) in comparison with current practice (CT or MRI) alone.

\section{Reference standard}

Disease status determined by histopathological findings, clinical follow up.

\section{Interventions}

Surgery, chemo radiation, radiation, palliative treatment.

\section{Outcomes}

Test accuracy: confirmation of stage of recurrence; incremental accuracy above existing tests in identifying potentially curable disease.

Diagnostic impact: change in diagnosis and/or staging after PET-CT compared to existing tests.

Therapeutic impact: change in treatment plan after PET-CT compared to existing tests by response to treatment that permits continuation or alteration of treatment.

Patient outcomes: morbidity, mortality, Quality of Life.

Costs: Use of resources.

\section{Study design}

Test accuracy studies.

Diagnostic before after studies investigating diagnostic and therapeutic impact with or without concurrent assessment of test accuracy.

Randomised controlled trials and non randomised controlled studies assessing effectiveness

of interventions.

Economic evaluations. 


\section{Exclusions}

Women within 3 months of completion of treatment for primary disease due to the problems associated with distinguishing treatment complications such as oedema and inflammatory response from recurrence in this patient group.

Systematic reviews of test accuracy, diagnostic and therapeutic impact and effectiveness will be carried out using established systematic review methodology in line with the recommendations of the NHS Centre for Reviews and Dissemination and the Cochrane Collaboration including those of Cochrane Methods Working Group on Screening and Diagnostic tests ${ }^{19,20}$. The systematic review of diagnostic test accuracy will be registered as a Cochrane review and as such will receive support from the Cochrane Methods Working Group on Screening and Diagnostic Tests based at the University of Birmingham. Inclusion, data extraction and quality assessment will be carried out in duplicate with differences resolved by consensus and/or arbitration involving a third reviewer.

\subsection{Reviews of diagnostic studies}

\subsubsection{Study identification and selection}

Evidence on the diagnostic accuracy of PET-CT, diagnostic impact of PET-CT and therapeutic impact of PET-CT in recurrent cervical cancer will be identified from a database of published and unpublished literature which will be assembled. Language restrictions will not be applied to electronic searches. The following databases will be searched: MEDLINE, EMBASE, Science Citation Index, and Cochrane Database of Systematic Reviews (CDSR), Cochrane Central Register of Controlled Trials (CENTRAL), Database of Abstracts of Reviews of Effects (DARE) and Health Technology Assessment Database (HTA). MEDION database has not been updated since 1998 and so would be irrelevant in these update searches. Information on studies in progress, unpublished research or research reported in the grey literature will be sought by searching a range of relevant databases including Clinical Trials.com and UK Clinical Research Network Portfolio. A draft MEDLINE strategy is included in Appendix 2. This strategy was devised in consultation with the information specialist at the Cochrane Methods Working Group on Screening and Diagnostic tests and consideration of the strategy compiled by Mijnhout ${ }^{21}$. Electronic searches will be supplemented by hand searching, contacting manufacturers and consultation with experts in the area. In addition authors of included studies will be contacted for information on relevant published or unpublished studies. Citations identified by the search will be selected for inclusion in the review in a two-stage process using predefined and explicit criteria regarding populations, tests, outcomes and study design. These criteria will be pilot tested using a sample of papers and agreement between reviewers will be measured.

Due to anticipated small numbers of studies we also plan to update existing reviews of the accuracy of PET-CT in primary cervical cancer using the search strategy outlined above, removing the terms for recurrent cervical cancer.

\subsubsection{Study quality assessment and data extraction}

Methodological quality of the selected primary studies will be assessed based on elements of study design, conduct and analysis included in a validated assessment tool, QUADAS, which will be adapted to the topic area as necessary ${ }^{22}$. No quality assessment tool exists for the assessment of diagnostic before after studies but members of the project team have experience of devising quality checklists for this particular study design based on existing knowledge in the area ${ }^{23}$. Data extraction will be performed using pre-designed, piloted data extraction forms, drawing on existing pro-formas used by the project team in previous, completed reviews in the topic area ${ }^{12}$. Missing information will be obtained from investigators if is crucial to subsequent stages of analysis and modelling. To avoid introducing bias, unpublished information will be treated in the same fashion as published information. In addition to using double data extraction to ensure the reproducibility of the overview, sensitivity analyses around important or questionable judgements regarding quality assessment and data extraction will be performed. 


\subsubsection{Data synthesis}

Sensitivity, specificity and likelihood ratios for individual studies comparing PET-CT and current imaging methods (CT or MRI) will be derived. Presence of a threshold effect will be examined by plotting sensitivity against 1 -specificity in a receiver operating-characteristic analysis (ROC analysis) and by calculating Spearman correlation coefficients ${ }^{24}$. Heterogeneity of results between studies will be investigated qualitatively by examining the distribution of sensitivities and specificities in (ROC) space and variability of estimates of diagnostic odds ratios (DOR) across studies using the forest plot $^{24}$. In addition heterogeneity will be investigated quantitatively using meta-regression and subgroup analyses if the volume of studies allow. Multivariable analysis will be undertaken to identify those criteria that have the most effect on our data set. Quantitative investigation will be undertaken based on variables defined a priori and including population characteristics, index and reference test characteristics and study quality ${ }^{25}$. It is anticipated that the following will be important sources of variation in test accuracy estimates:

- Population characteristics: initial staging of primary tumour, primary treatment received, interval between initial treatment and recurrence, symptomatic and asymptomatic recurrence.

- Index test characteristics: technical details of the PET CT scanner including imaging methodology and sequences used, skill and experience of the operator, healthcare setting (2y or $3 y)$, timing of scan post injection of tracer, does of tracer used.

- Reference test: histology or clinical follow up.

- Study quality: study design (prospective or case-control) and study quality (high: meeting all assessment criteria; medium: meeting at least one assessment criteria; low: meeting no quality criteria). High quality studies will be used as the reference category to determine whether medium and low quality studies have biased estimates of test accuracy.

Based on an investigation of heterogeneity summary estimates of sensitivity, specificity and likelihood ratios (LRs), and summary ROC curves will be derived as appropriate using recognised methods for metaanalysis of test accuracy ${ }^{26}$. Direct comparisons are more robust and will be distinguished from indirect comparisons $\mathbf{s}^{27,28}$. LRs are considered more clinically meaningful as measures of test accuracy and they allow estimation of probabilities for economic modelling. Post test probabilities can be used to tailor the absolute effectiveness estimates according to test results.

The risk of publication and related biases is expected to be high in reviews of test accuracy ${ }^{29,30}$. Publication bias will be investigated using funnel plots of DOR against corresponding variances. Qualitative investigation will be based on the premise that large gaps in the funnel indicate possible 'missing' publications. These omissions are usually due to small studies showing limited accuracy and are unlikely to be missing at random. Statistical investigation of publication bias will be undertaken in STATA based on templates of commands and instructions already developed by the project team.

\subsection{Reviews of effectiveness of interventions}

Once accurate imaging modality has identified the women with potentially curable disease by restaging, these patients may benefit from interventions effective in reducing mortality and morbidity. Existing reviews will be assessed for their quality and currency follow existing guidelines QUOROM and MOOSE ${ }^{30,31}$. Through this process we will identify gaps where reviews do not exist and where they need updating. Where necessary effectiveness reviews of RCTs of treatments for recurrent cervical cancer will be undertaken follow existing guidelines ${ }^{32}$ ensuring the output complies with the QUOROM statement ${ }^{30}$.

\subsubsection{Study identification and selection}

For evidence on the of effectiveness of treatments for recurrent cervical cancer we will begin by searching for exiting systematic reviews using the ARIF search protoco ${ }^{33}$. Any existing reviews will be examined for relevance and currency in order to inform further searching for primary studies. Searches for further primary studies will be performed. The following databases will be searched: MEDLINE, EMBASE, Science Citation Index and the Cochrane Library (all databases). On-going studies will be sought by searching Clinical Trials.com and the UK Clinical Research Network portfolio. Draft searches for MEDLINE are included 
in Appendix 2. Studies will be selected for inclusion in the review in a two-stage process using predefined and explicit criteria regarding populations, interventions and outcomes using procedures similar to the ones outlined in the previous section 5.1.1.

\subsubsection{Study quality assessment and data extraction}

The quality of included reviews will be assessed against a validated tool and a reporting checklist, QUOROM ${ }^{30}$. Methodological quality of randomised and non-randomised trials will be assessed based on accepted criteria. Information on the adequacy of randomisation, sequence generation, concealment, blinding, description of withdrawals, and follow-up rates would be sought as these are elements most likely to have a direct relationship to bias in a $\mathrm{RCT}^{34}$. Procedures for obtaining missing information and resolving disagreements will be similar to the ones outlined in section 5.2.1.

\subsubsection{Data synthesis}

Revman and Stata softwares will be used to conduct analyses. The former will allow uniformity with Cochrane reviews and the latter will allow the data analytic flexibility that we will need to examine issues not included in the Revman software. Heterogeneity of results between studies and investigation for publication bias will be statistically and graphically assessed using methods outlined in section 5.1.3. The decision to proceed to meta-analysis will depend on the degree of heterogeneity in the data set. It is anticipated that the following will be important sources of variation in the estimates of effectiveness:

- Population characteristics: initial staging of primary tumour, primary treatment received, interval between initial treatment and recurrence, symptomatic and asymptomatic recurrence, extent of recurrence, physical performance status of patient.

- Treatment characteristics: Type of intervention (surgery, radiotherapy or chemoradiotherapy), intention of treatment (curative or palliative) duration of therapy, healthcare setting (2y or 3y), timing of intervention.

- Outcome measures: Mortality, morbidity, Quality of life.

Conclusions regarding the typical estimate of an effect size of the intervention will be interpreted cautiously if there is significant heterogeneity.

\subsection{Eliciting subjective probabilities}

In anticipation of small numbers of test accuracy studies subjective probabilities will be elicited, using a group interview, from between 10 and 15 clinical experts in the fields of gynaecological cancer and radiology with no conflict of interest in the area, identified by clinicians in the project team and project advisors. The aim of the elicitation process will be to gather subjective views about the size and probability of incremental changes in test accuracy (true positives, false positives, true negatives and false negatives) from the addition of PET-CT to current imaging practice in the detection (surveillance in asymptomatic women) and restaging of recurrent cervical cancer.

Subjective probability estimates will be elicited concerning:

- The diagnostic accuracy of the addition of PET CT to current imaging practice (CT or MRI) and current imaging practice alone in recurrent cervical cancer.

- The diagnostic impact of the addition of PET CT to current imaging practice in recurrent cervical cancer (changes in diagnosis and treatment planning).

- The therapeutic impact of the addition of PET CT to current imaging practice in recurrent cervical cancer (the effects of changes in treatment).

Eliciting subjective probabilities from clinicians has three roles:

1. Providing data to populate the economic model in the absence of information found in the literature. 
2. Supplementing information found in the literature. Literature may be sparse, of poor quality or not transferable to the UK setting. Information gained from clinicians in the form of subjective probabilities may be used to supplement information found in the literature to enable sensitivity analyses to be performed as part of the economic model. For example subjective estimates of the therapeutic impact of the addition of PET-CT from clinicians in the UK may be different to results obtained by combining test accuracy and effectiveness evidence estimated in research settings (effectiveness versus efficacy).

3. Planning the dissemination strategy for the results of the research. If it is not possible for clinicians to reach agreement about their subjective estimates of the accuracy, diagnostic impact and therapeutic impact of PET-CT this information can be useful when developing a dissemination plan for this research project.

\section{The process of eliciting probabilities}

Subjective probabilities will be elicited using group interviews with between 10 and 15 clinical experts in the fields of gynaecology, oncology and radiology with no conflict of interest in the area. Experts will be identified by clinicians in the project team and project advisors. Based on experience of eliciting probabilities in other clinical topics it is anticipated that 2, half day interviews or one whole day interview will be necessary to elicit all the required information: test accuracy, diagnostic impact and therapeutic impact.

A face-face group interview (behavioural aggregation) will be used in preference to individual interviews as this facilitates a common understanding of the problem and task from experts and will allow us to benefit from group discussion and interaction leading to a consensus of opinion ${ }^{35}$. The expert group will be facilitated by both a clinical and non-clinical expert drawn from the project team with sufficient statistical expertise to provide probabilistic training to experts, validate their results and provide feedback. The expert group interview(s) will be facilitated by both a clinical and non-clinical expert drawn from the project team with sufficient statistical expertise to provide probabilistic training to experts, validate their results and provide feedback. The interview(s) will briefly comprise:

- Training of experts (probability, probability distributions, judgement heuristics and biases)

- Practising elicitations

In this part of the workshop participants will be presented with a non-related topic to allow evaluation of their understanding of the task to be completed (subjective probability estimates of the accuracy, diagnostic impact and therapeutic impact of the addition of PET-CT in recurrent cervical cancer) and a rehearsal of the process. In addition the results of the practice example will be fed back to participants to demonstrate how the outcome of the workshop will be integrated with the findings from the systematic review. Attachment: practice probabilities.xls provides an example of a practice elicitation exercise used successfully in previous research with clinicians.

\section{Eliciting probabilities about the use of PET-CT in recurrent cervical cancer}

Following completion of the practice exercise participants will be asked to provide separately their subjective estimates of the size and probability of test accuracy outcomes, diagnostic impact outcomes and therapeutic impact outcomes. The following sections detail how subjective probabilities about test accuracy will be elicited as an illustrative example. Participants will be presented with the prevalence of recurrent cervical cancer in the population of interest (the prior probability of having recurrent cervical cancer). The probability of recurrent cervical cancer will be modified by the results of the test(s) under investigation: CT or MRI versus CT or MRI and PET-CT. Respondents will be asked for their subjective estimates of the accuracy (expressed as the probability of true positives (TP) and true negatives (TN)) for each of the tests/test combinations under investigation. Uncertainty regarding estimates of test accuracy will result in a distribution of possible test accuracy estimates instead of a precise figure (see Appendix 3 in the illustrative example below). In Appendix 5 Tables 1-3 in the illustrative example a range of probabilities between 50 and $100 \%$ have been used. In practise in the workshop we plan to begin by asking 
respondents for their single most likely estimates of TP and TN and then present a range of probabilities around these estimates.

\section{Presentation of results back to experts.}

It is planned that the elicitation of subjective probabilities will be a paper based exercise and results will be analysed immediately following the elicitation exercise. In previous research it has been possible to analyse results from small numbers of participants in the period of one hour. The subjective probabilities of all participants will be combined and fed-back to participants. A combined probability distribution will be constructed (for example, see Appendix 3) by summing the frequency of points awarded to each probability presented to participants and presenting this graphically. If there is substantial disagreement within the group, individual subjective estimates will be presented and examination of agreement within sub-groups of respondents, for example according to speciality will be explored (for example see Appendix 4 in the illustrative example).

\section{Repeat elicitation of probabilities}

The elicitation process will be repeated following feedback of results from round 1 to ensure reliability. In the event of substantial variation suggesting construction of a combined probability would be inappropriate, a repeat of the exercise may result in greater agreement. In the event that it is not possible to construct a combined probability distribution for participants the degree of variability of estimates of test accuracy, diagnostic impact and therapeutic impact will be useful in informing the dissemination strategy for the results of the research.

\section{Updating the prior probability of disease (prevalence) using subjective probabilities and findings from the systematic review}

The probability of disease prior to testing (prevalence of cervical cancer) will be updated using the combined test accuracy distribution derived from respondents using a Bayesian updating formula to produce a posterior distribution of disease probability. This posterior distribution of disease derived from the elicitation process will be further updated with the results of the systematic review, again using a Bayesian updating formula to provide a final posterior distribution for use in probabilistic sensitivity analysis. In the event that it is not possible to derive a combined probability distribution from respondents the prior probability of disease (prevalence) will be updated using estimates from the systematic review only.

The elicitation process also aims to generate subjective probabilities concerning the diagnostic and therapeutic impact of the addition of PET-CT. Findings from the elicitation process will be triangulated with findings from the systematic reviews and probability distributions will assist with populating the decision analytic model. As well as expertise within the project team ${ }^{36}$ we have access to experts in the field, based at the University of Birmingham ${ }^{37}$.

\subsection{Model Based Economic Evaluation}

The objective of the economic evaluation is to compare the relative cost effectiveness of adding PET-CT imaging as an adjunct to standard practice against standard practice alone in re-staging recurrent cervical cancer.

\subsubsection{Perspective and data collection}

If PET-CT screening is shown to be an effective adjunct to the standard practice in re-staging recurrent cervical cancer then it is likely that important cost implications will be imposed on the health care sector. For example, PET-CT may detect additional evidence of the extent of metastasis compared to standard investigations which could increase the number and extent of subsequent tests and treatment required by the individual. But the additional associated costs associated with more accurate re-staging of the re-current cancer may lead to a reduction in costs associated with unnecessary or ineffective subsequent treatments and also prolong the life of the woman. Thus, if available data allow, the economic evaluation 
will be based on an outcome of cost per QALY and/or Cost per recurrent case treated; and/or cost per 'death due to recurrent cancer' avoided. The analysis will adopt the perspective of the NHS.

Therefore data collection required for the model based economic evaluation will include:

- The equipment, other resource use and costs associated with PET-CT

- Knock-on costs associated with further tests and treatments that are required as a result of the re-staging

- Equipment, resource use and costs associated with current practice

- Accuracy of the PET-CT test and current practice package compared to the accuracy of current practice tests alone

- Effectiveness of alternative intervention pathways that are followed as a result of the diagnosis

- Outcomes such as quality of life associated with cervical cancer at various disease stages; probability of death associated with various stages of the disease diagnosed.

A scoping search has already been undertaken to identify economic evaluations of cervical cancer. This search used terms for 'cervical cancer' in conjunction with an economic search filter in MEDLINE. The search identified 360 references. A systematic search for economic evaluations and any other data needed to populate the model will be undertaken in NHS EED, MEDLINE and EMBASE. The objective of searching the economic literature is to identify studies reporting costs and consequences associated with recurrent cervical cancer, which will provide estimates for a comparison with current practice. The review of economic studies will also try to identify quality-of-life information that could be used to estimate the proposed outcome of cost per QALY although our initial scoping search has not found many studies of this type. If relevant QALY data is unavailable for this type of recurrent cancer we will infer QALY values from other cancer studies.

Cost data will be collected from two principal sources. First, once the clinical evidence has been synthesised into the main strategies of diagnosis and treatment, relevant studies will be examined for their data on costs and resource use. These data will be subject to relevant quality criteria. Additional cost data will be available from other sources such as the National Schedule for Reference Costs. If necessary, primary cost and resource data will be collected from the Pan-Birmingham Gynaecology Cancer Network to complete any gaps in the information required for the modelling process.

Additional searches will be undertaken to help populate the decision model. The Information Specialist will work in close liaison with the health economist to identify the model questions. Information to answer these questions will be provided by focused searching of appropriate databases, including reference cost databases, statistical sources and other sources of relevant information.

The evidence found in the clinical accuracy and effectiveness reviews will provide the majority of the parameters required to carry out the economic evaluations of alternative test and treat packages. If information on the correlation between a package of tests and correlation between a package of treatments is available from the reviews, the framework will allow these more complex strategies to be evaluated as well as strategies that allow alteration in the form of repeated testing.

\subsubsection{Model and analysis}

The economic evaluation will involve the development of a decision analytic simulation model as a framework for conducting cost-effectiveness and associated value of information analyses ${ }^{38,39}$. The economic evaluations will inform current treatment policy in this clinical area, whilst the value of information component will serve to highlight future research needs and agendas, and inform possible future research funding decisions. A modelling framework is ideally suited to demonstrate and explore the importance of the inherent uncertainty. 
The risk of recurrence after primary treatment depends on the extent of primary cancer at presentation. Treatment of recurrent cervical cancer depends on the site and extent of recurrence, the type of previous treatment received, time elapsed since primary treatment and the patient's performance status. A Markov model is the appropriate modelling approach for this evaluation because the time horizons available for both the imaging and the interventions are relatively long. Markov models are also able to represent clinical situations where patients change health states or experience recurrent events over a long period of time $^{40}$. The Markov model will be constructed using TreeAge Pro software. This is a widely-used and highly user-friendly package ideally suited to the construction and analysis of decision trees and Markov models.

An incremental approach will be adopted with a focus on additional costs and gain in benefits associated with a move away from current practice to alternative test and treatment strategies. Using discounting, adjustments will be made to reflect the differential timing of costs and outcomes in terms of the extension to the length of life extend associated with the test and treat strategies. The base-case analysis will follow Treasury recommendations for public sector projects.

\subsubsection{Presentation of results and sensitivity analysis}

The results of these economic analyses will be presented using cost-effectiveness acceptability curves to reflect sampling variation and uncertainties in the appropriate threshold cost-effectiveness values.

For the Value of Information analysis we shall quantify the total uncertainty in terms of the value of removing that uncertainty. As appropriate, we shall include partial value of information analysis calculations. We shall also use both deterministic and probabilistic sensitivity analyses to explore the robustness of these results to plausible variations in key assumptions and variations in the analytical methods used, and to consider the broader issue of the generalisability of the results.

\section{Expertise in the team}

The applicants have a wide and appropriate range of expertise in systematic reviews, gynaecological oncology, medical oncology, radiology, clinical epidemiology, health measurement, medical statistics, information science and health technology assessment.

SK has successfully completed many HTA projects on systematic reviews of test and treatments including systematic reviews of tests for pre-eclampsia, systematic reviews of tests and treatment in pre term labour. In addition he has experience of the process of eliciting subjective probabilities ${ }^{36}$. He has been awarded MRC studentship fellowship to undertake systematic reviews of accuracy of tests and treatment in gynaecologic cancer ${ }^{12,15,16,41}$ including cervical cancer and for undertaking modelling and decision analytic economic evaluation and a grant on the methodology of evaluation of tests without gold standards by the NHS Research Methodology Programme. KSK and TR have a grant on evaluation of accuracy and cost effectiveness of intrapartum rapid tests for Group B streptococcus infection. TR has experience in cost-effectiveness analyses of tests and interventions in cancer. ST has undertaken many systematic reviews on tests and treatment in women with pre-eclampsia, preterm labour and epilepsy. SS and PM are both members of the gynaecological cancer clinical studies group of the NCRI (National Cancer Research Institute) - the national group responsible for selecting national trials for inclusion in the NCRI portfolio and supporting and directing clinical research in gynaecological cancer.

SS and PM are gynaecological oncologists involved in managing women with cervical cancer. PM has published systematic reviews on management in cervical pre cancer and compiled the evidence base for the Improving Outcomes Guidance (IOG) document in gynaecological cancer issued by the Department of Health. CD has considerable experience of undertaking and managing health technology assessments as part of the West Midlands Health Technology Assessment Collaboration (WMHTAC). Her experience includes HTAs concerned with diagnosis, and effectiveness, as well as a methodological review concerned with the use of on-going trials in health technology assessments HTA 8(24). Recent, relevant research 
includes development of a tool for assessment of quality in diagnostic before-after studies, identification of reviews of test accuracy and she is nearing the end of a programme of doctoral research concerned with communication of test accuracy outcome measures and evaluation of their diagnostic impact. AF has extensive experience as an information specialist in providing support to a diagnostic and effectiveness technology assessments as a member of the West Midlands Health Technology Assessment Collaboration and the Aggressive Research Intelligence Facility based at the University of Birmingham. She is currently undertaking a systematic review on PET and PET/CT in breast cancer recurrence (HTA no 08/34) concerned with the incremental diagnostic accuracy of PET and PET/CT compared to existing diagnostic strategies in recurrent breast cancer. PG is a consultant radiologist with expertise in PET CT in patients with gynaecological cancers. RM is the chair of Jo's Trust Fighting Cervical Cancer, the only UK dedicated to women, their families and friends affected by pre-cancer and cancer of the cervix.

The applicants will be supported in an advisory capacity by Dr Chris Hyde, Dr Jon J Deeks and Dr Chris Williams. JJD is an expert in test evaluation leading the NIHR funded Diagnostic evaluation and review support unit, and will provide input into the study design and in its output as a Cochrane review. CW is a Medical Oncologist (specialising in gynaecological cancer), with a particular interest in clinical trials methodology and systematic reviews. He is the Co-ordinating Editor of the Cochrane Gynaecological Cancer Review Group and a past Chair of the Cancer Therapy Committee and the Gynaecological Cancer Working party of the Medical Research Council. CH has been involved in projects like CASP (Critical Appraisal Skills for Purchasers) and the Cochrane Group on Effective Practice and Organisation of Care (EPOC). Through ARIF, his aim is to facilitate the use of research information, particularly systematic reviews of effectiveness in population level health care decision making within the West Midlands.

\section{Contribution to Collective Research Effort:}

This systematic review on the added value of PET-CT and cost effectiveness analysis of PET-CT imaging in comparison with current imaging fits comfortably with previously published HTA evaluations of PET-CT in other cancers. This research application complements existing NCRN (National cancer research network) portfolio research in gynaecological cancer. Members of the research team (PM, SS, KK, TR, AT, PG) are co-applicants in an NCRN endorsed primary investigation of PET-CT in endometrial cancer.

Due to the multiple methods employed by the proposed evidence synthesis the project team expect that the outputs of the work would be of interest to a broad research and clinical community including experts in the areas of evidence synthesis and in particular synthesis of test accuracy, gynaecological cancer, and decision making. Outputs would be submitted for presentation at national and international conferences such as Health Technology Assessment international, Medical Decision Making, European Society of Gynaecological Oncology (EGSO) and Society of Gynaecological Oncology (SGO). Similarly the outputs of this work would be of interest to a variety of peer reviewed journals and the project team would aim for a minimum of 3 peer reviewed publications in addition to publication as an HTA monograph. One of the outputs of this project would be a Cochrane review to be added to the Cochrane database of systematic reviews of test accuracy.

The project team have involved members of Jo's trust, a reputed national charity in cervical cancer and user representatives of the Pan Birmingham cancer research network (PBCRN) and the Lancashire cancer networks. Users will be represented in study conduct and planning of dissemination strategies. Experience from previous research conducted by the team (HTA no 01/64/04: Methods of prediction and prevention of pre-eclampsia: Systematic review of accuracy and effectiveness literature with economic modelling in preterm labour) has already indicated that publication and dissemination needs careful consideration from the outset. Publication strategy will also need to anticipate early the need for versions of the report, which can be, used by women themselves. For this we will seek input from relevant consumers. 


\section{Please provide details about any related (planned or active) grants held by any member of your research team in this or similar research areas.}

KSK has been awarded MRC studentship fellowship to undertake systematic reviews of accuracy of tests and treatment in gynaecologic cancer including cervical cancer. The information from the review on accuracy of PET CT over CT or MRI in diagnosing primary cervical cancer will be integrated in the modelling. We have also undertaking modelling and decision analytic economic evaluation for tests in vulval cancer and the experience will be utilised in modelling for this project. PM has been successfully awarded a NHS Cochrane grant application as a joint editor of the Cochrane Gynaecological Review Group for $f 380 \mathrm{~K}$ to support the generation of updated evidence based gynaecological oncology guidelines March 20007. He has also developed a joint project with the departments of epidemiology and psychology at UCLAN investigating the impact of cancer symptoms and being referred to secondary care. SS has 1 PhD student funded by the department of Health investigating the epigenetic changes induced by HPV in cervical cancer. The ongoing work by SS and PM in gynaecologic oncology will be of use in providing subjective probabilistic estimates for test accuracy and effectiveness. AF (information specialist) is currently working on an HTA assessing the value of PET-CT for recurrent breast cancer and her expertise in devising the search strategy and database management will be of benefit to this proposal.

\section{Summary for the non expert}

Every year in the UK, over 2,800 women are diagnosed with cervical cancer and 1,000 women will die from the disease. After breast cancer, cervical cancer is the second most common cancer in women aged 35 and under. Early stage cervical cancer is treated with either surgery or chemo radiotherapy with a cure rate of $80 \%$. Advanced stage cervical cancer is usually treated with chemo radiotherapy or chemotherapy alone and $30-50 \%$ of patients will have persistent or recurrent disease after treatment. The prognosis for recurrent cervical cancer is generally poor. The reported 5 year survival rates in recurrent cervical cancer are between $3.2 \%$ and $13 \%$ and the time to recurrence is short with $75 \%$ occurring before 3 years.

An accurate restaging of the extent of recurrent cancer (confined to the pelvis, spread to the lymph nodes or spread to distant organs) helps to plan subsequent treatment. Accurate identification of incurable spread of cancer avoids unnecessary treatment which itself is unpleasant and carries considerable risk, while more accurate delineation of tumour extent (restaging) increases the probability of receiving treatments appropriate to the extent of spread which may lead to improvements in survival and quality of life.

In current clinical practice, patients are monitored at regular intervals after primary treatment to detect persistent or recurrent disease. Present techniques of clinical examination and CT or MRI scans can be unreliable in detecting persistent or recurrent disease in the pelvis after radiotherapy as radiotherapy induced scarring can potentially conceal recurrent disease. Similarly, CT and MRI may not identify disease spread to lymph nodes and other organs. PET CT (Positron Emission Tomography with anatomical CT images) is an imaging method using radio labelled molecules to detect metabolically active tumours in the management of cancer patients. PET CT has been shown to improve the detection of cancer and its spread from $8 \%$ to $43 \%$ over conventional testing in patients with lung, colorectal cancer, lymphoma, melanoma, breast cancer, and thyroid cancer and it may have similar benefits for patients with recurrent cervical cancer.

For the proposed project our objectives are as follows:

In women who had undergone treatment for cervical cancer, under routine surveillance or with suspicion of recurrence 
- To assess if the addition of PET-CT to existing scans (CT or MRI) improves the detection of recurrent cervical cancer

- To evaluate if the use of PET-CT results in change in (re)staging i.e. extent of recurrence compared to CT or MRI

- To assess the impact of performing PET-CT on the planned treatment after diagnosis of recurrence and during subsequent monitoring

- To summarise the effectiveness of available treatments in women with recurrent cervical cancer

- To estimate the impact of PET CT findings patient outcomes and the costs associated with its routine use in this patient group.

We plan to fulfil the above objectives by systematically identifying the available evidence on the diagnostic accuracy of PET CT in recurrent cervical cancer compared to the diagnostic accuracy of exiting diagnostic tests used in this patients group and the effectiveness of treatments for recurrent cervical cancer. The evidence found will be used in an economic evaluation comparing existing testing and treatment strategies with PET CT guided treatment strategies. This evaluation will inform current treatment policy in this clinical area and highlight future research need.

\section{Project Timetable and Milestones}

Fig 2 shows the project timetable and milestones for the accuracy and effectiveness reviews and economic modelling. We have carefully evaluated the ongoing work and the level of staffing within our departments and feel that we would be able to commence the work in January 2010 for a period of 18 months, if funded.

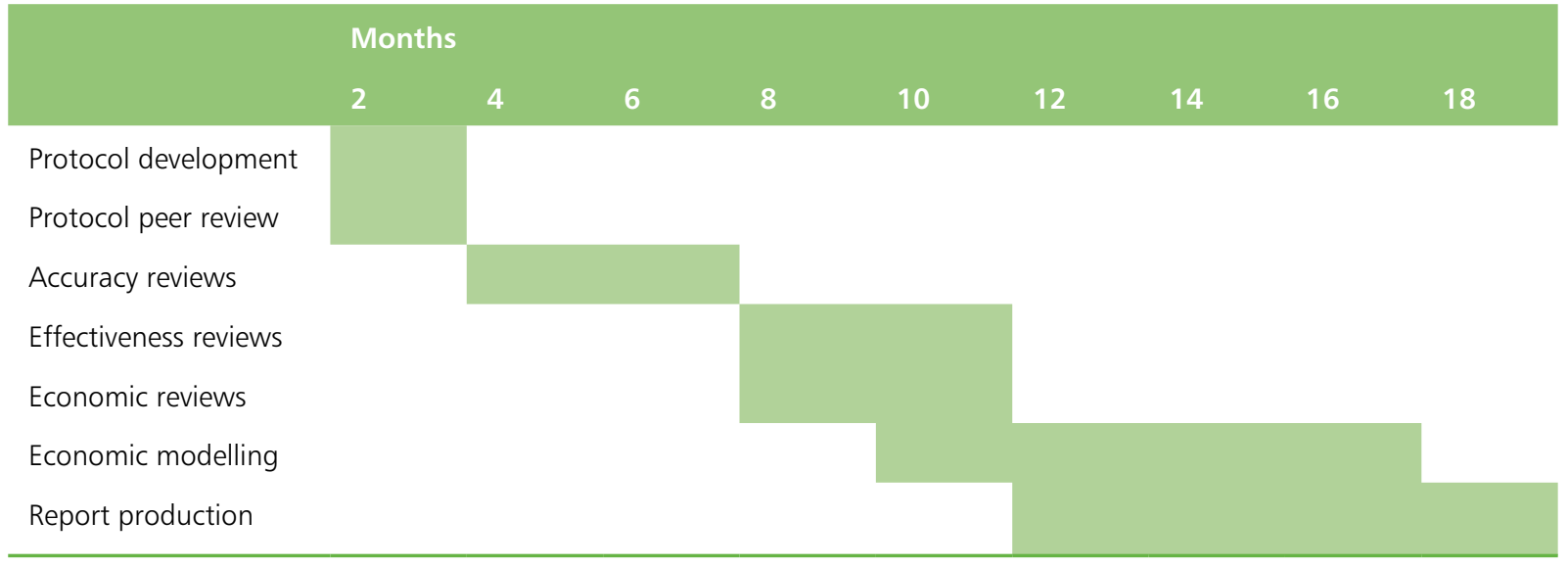

FIGURE 2 Timetable. 


\section{Justification for the support required}

Staff:

- Supervisor, also providing support for researchers, for example: double data extraction, assisting with inclusion decisions and being the lead for producing the final report - 1 day per week for the duration of the project.

- Researcher to perform systematic review of accuracy and effectiveness studies and to identify additional epidemiological and background information for input into the modelling exercise- 1 wte for 18 months.

- Health economist to perform systematic review of cost-effectiveness literature and modelling - 1wte for 12 months.

- Information support for searching and document retrieval - 20 days.

Equipment and consumables:

- two standard specification computers, printing cartridges, paper and photocopying,

- telephone and fax calls, postage,

- estimated 200 interlibrary loans.

Support:

- meeting room, refreshments and travel for the project team and consultants based on 4 face to face meetings over 12 months.

- administrative support, for steering group and preparation of final report - 10 days over 12 months.

We are in an excellent position to gauge the level of resources required to deliver this type of project (systematic review and cost-effectiveness analysis) with several years experience in their delivery. We are able to draw on additional in-house expertise if necessary. Travel costs are not estimated to be high. 


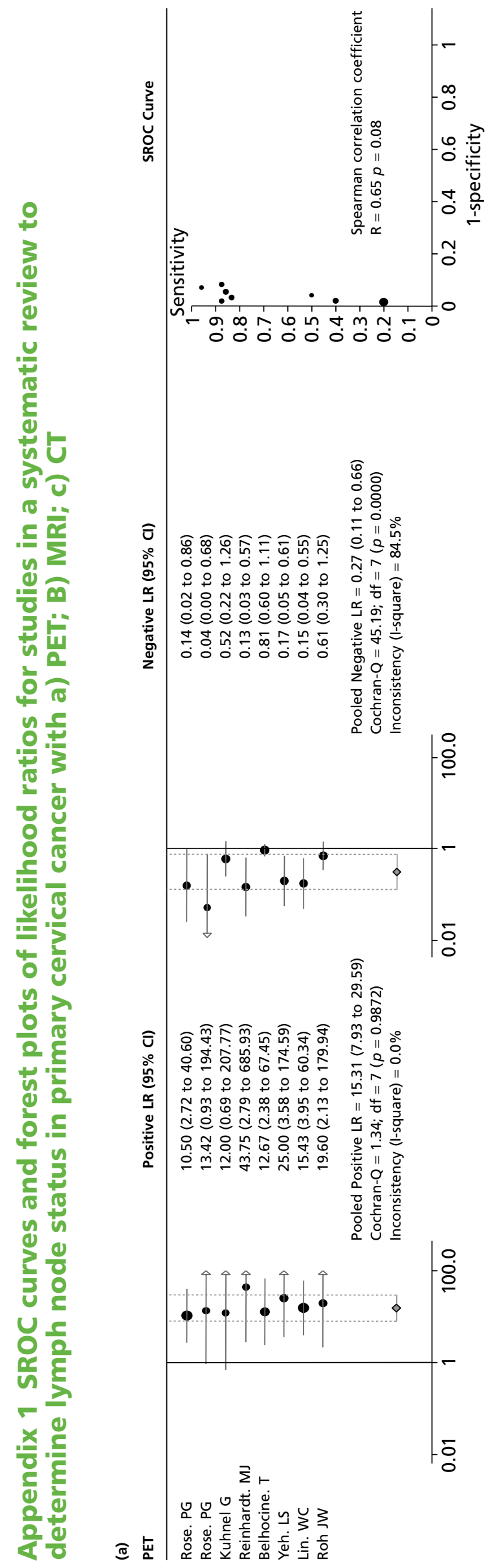




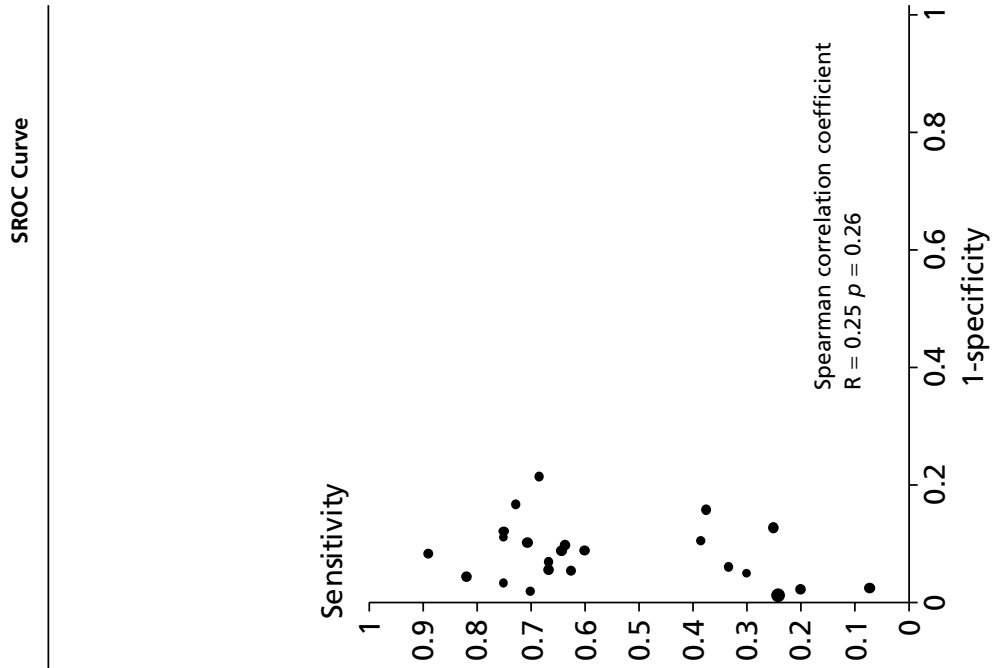

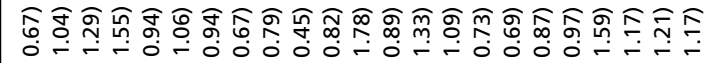
ธิ。

잉

mi

00000000000000000000000 L

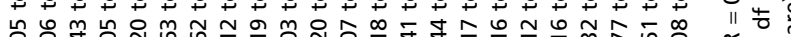

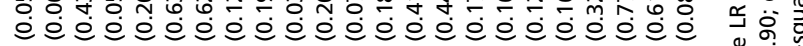

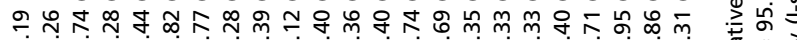

00000000000000000000000

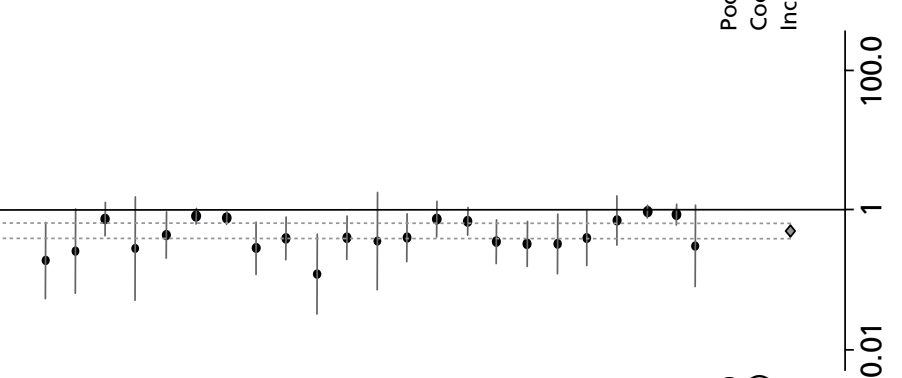

is

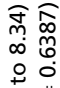

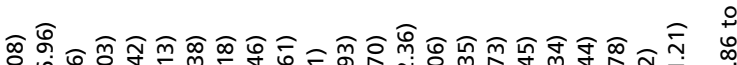

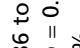

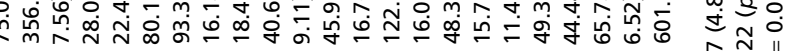

앙ㅇㅇㅇㅇㅇㅇㅇㅇㅇㅇㅇㅇㅇㅇㅇㅇㅇㅇㅇㅇㅇㅇㅇㅇㅇㅇㅇ

굿

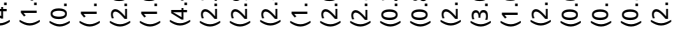

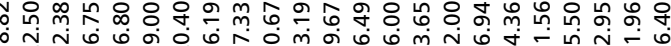

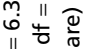

$\simeq \check{G}$

ه

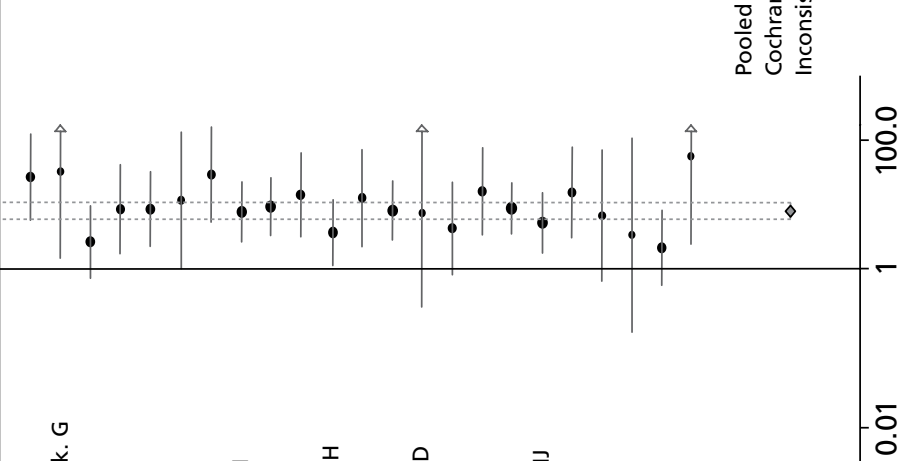

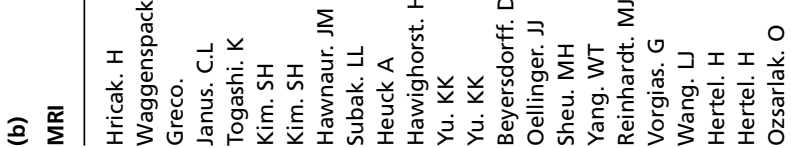




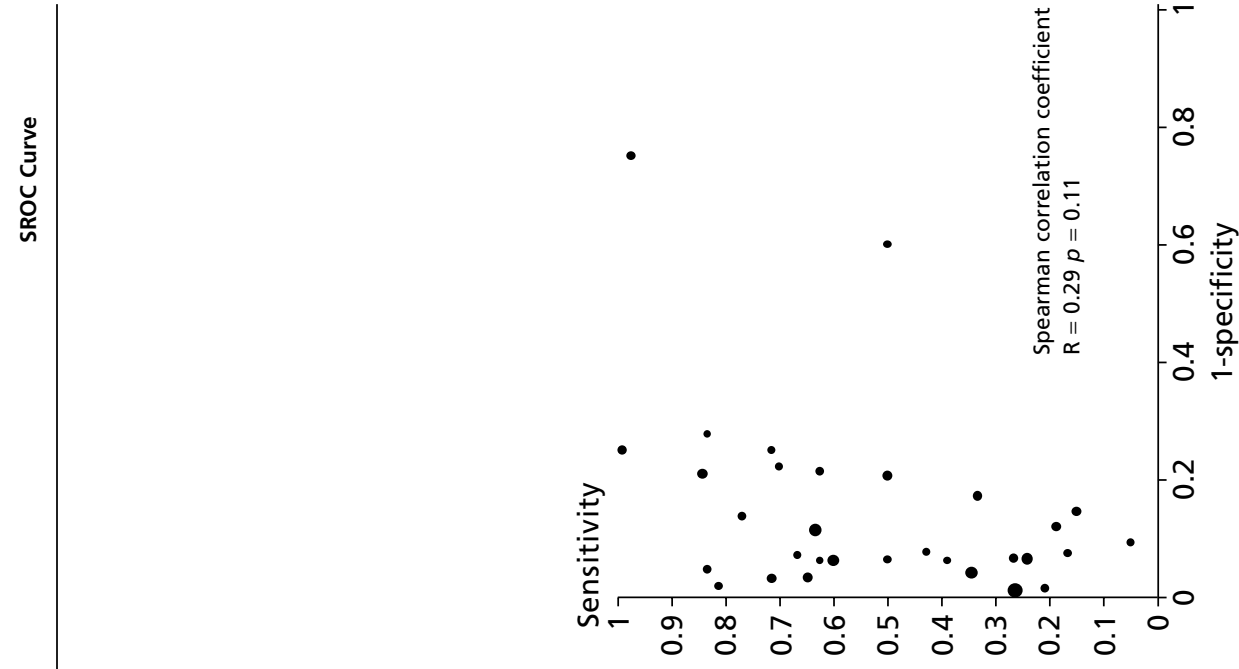

ธิ

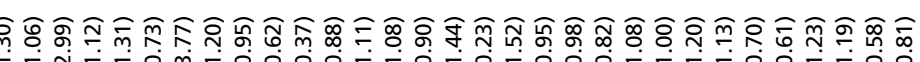

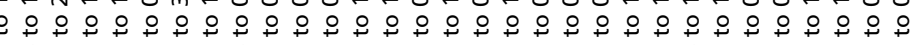
ळ

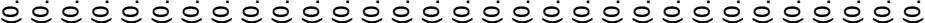
น

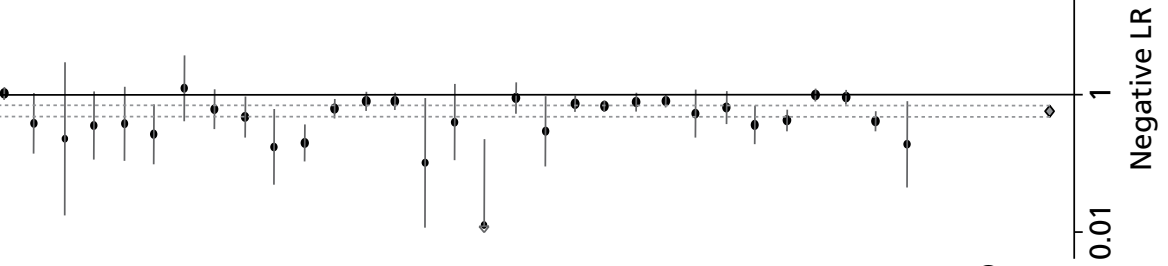

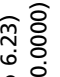

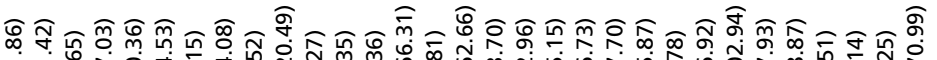

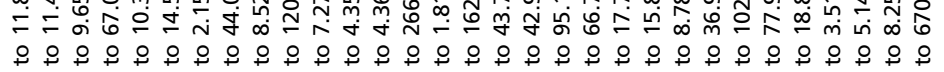
웅

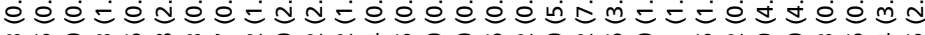

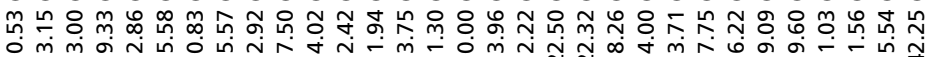

o 赵 닐 m. 琎 ฯ ทें 음

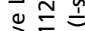
mon $N$ N
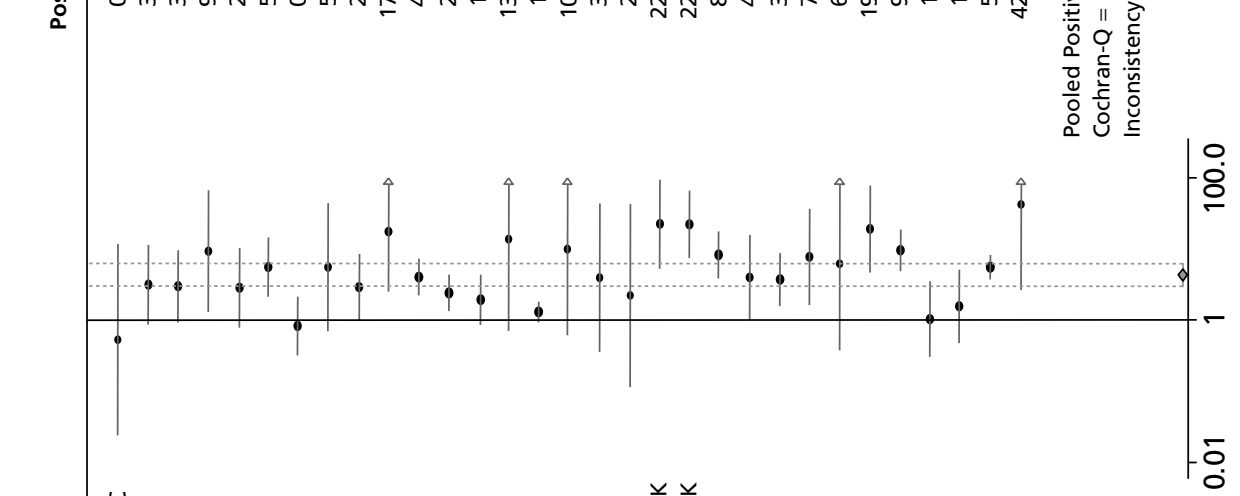

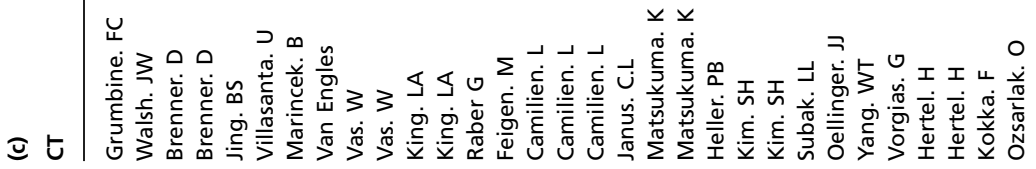




\section{Appendix 2 Proposed MEDLINE search strategy to identify the relevant studies}

\section{Test accuracy search - proposed MEDLINE strategy}

\section{Ovid MEDLINE(R) - 1950 to June week 12009}

1. exp tomography, emission-computed/ (53449)

2. (emission adj2 comput\$ adj2 tomograph\$).tw. (9829)

3. (tomograph\$ adj2 emission adj2 comput\$).tw. (10061)

4. (radionuclide-comput\$ adj2 tomograph\$).tw. (19)

5. (radionuclide adj2 cat scan\$).tw. (4)

6. (radionuclide adj2 ct scan\$).tw. (29)

7. (scintigraph $\$$ adj2 comput $\$$ adj2 tomograph $\$$ ).tw. (375)

8. (positron adj2 emission adj2 tomograph\$).tw. (21619)

9. (pet or petct).tw. (30569)

10. or/1-9 (66680)

11. (recur $\$$ or relaps $\$$ or metasta $\$$ or restag $\$$ or re-stag $\$) . m p . ~(638721)$

12. uterine cervical neoplasms/ (47784)

13. ((cervix or cervical) adj5 (cancer\$ or carcinoma $\$$ or adenocarcinoma $\$$ or carcinogen $\$$ or sarcoma $\$$ or malignan $\$$ or tumo? $\$$ or neoplas $\$)$ ).tw. (44525)

14. 12 or 13 (59939)

15. 11 and 10 and 14 (259)

\section{Effectiveness search (systematic reviews) - proposed MEDLINE strategy}

\section{Ovid MEDLINE(R) - 1950 to June week 12009}

1. (recur $\$$ or relaps $\$$ or metasta $\$$ or restag $\$$ or re-stag $\$$ ).mp. (638721)

2. uterine cervical neoplasms/ (47784)

3. ((cervix or cervical) adj5 (cancer $\$$ or carcinoma $\$$ or adenocarcinoma $\$$ or carcinogen $\$$ or sarcoma $\$$ or malignan\$ or tumo?r\$ or neoplas\$)).tw. (44525)

4. 2 or 3 (59939)

5. 1 and 4 (11331)

6. limit 5 to "reviews (specificity)" (66)

\section{Effectiveness search (RCTs) - proposed MEDLINE strategy}

\section{Ovid MEDLINE(R) -1950 to June week 12009}

1. (recur $\$$ or relaps $\$$ or metasta $\$$ or restag $\$$ or re-stag $\$$ ).mp. (638721)

2. uterine cervical neoplasms/ (47784)

3. ((cervix or cervical) adj5 (cancer $\$$ or carcinoma $\$$ or adenocarcinoma $\$$ or carcinogen $\$$ or sarcoma $\$$ or malignan $\$$ or tumo? $\$$ or neoplas $\$)$ ).tw. (44525)

4. 2 or $3(59939)$

5. 1 and 4 (11331)

6. limit 5 to "therapy (specificity)" (191) 
Appendix 3 Probability distributions of TP, TN, FP, FN derived from table 3 for CT or MRI used to detect recurrence of cervical cancer (stage IB2-IV)

The attached excel work sheet demonstrates an exercise in eliciting subjective probabilities on the estimated distance between London and Birmingham.

Frequency of estimation of probability of test outcomes (TP, FP, TN, FN)

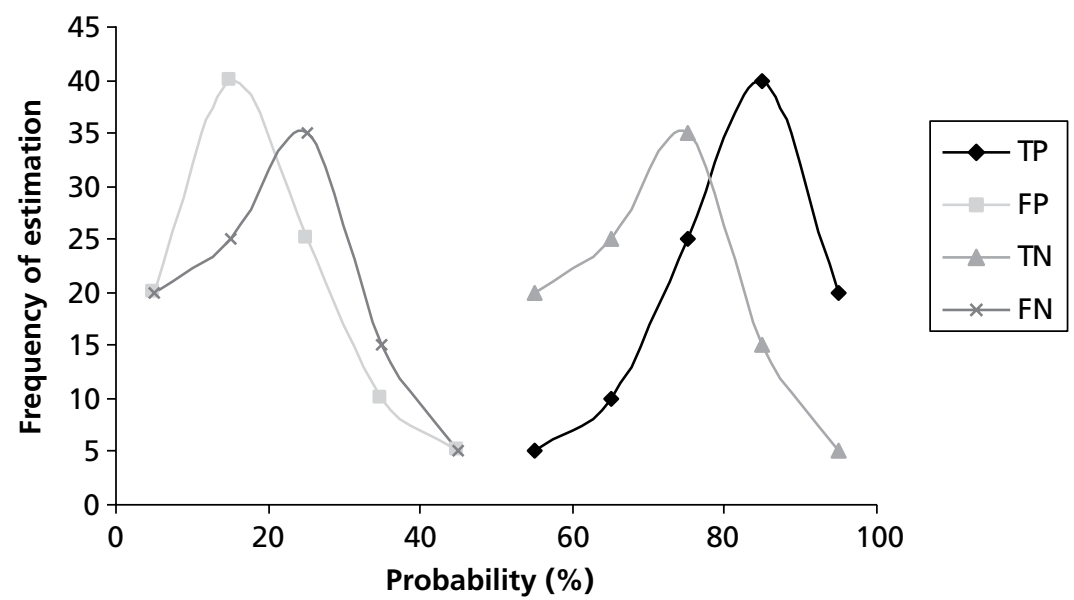



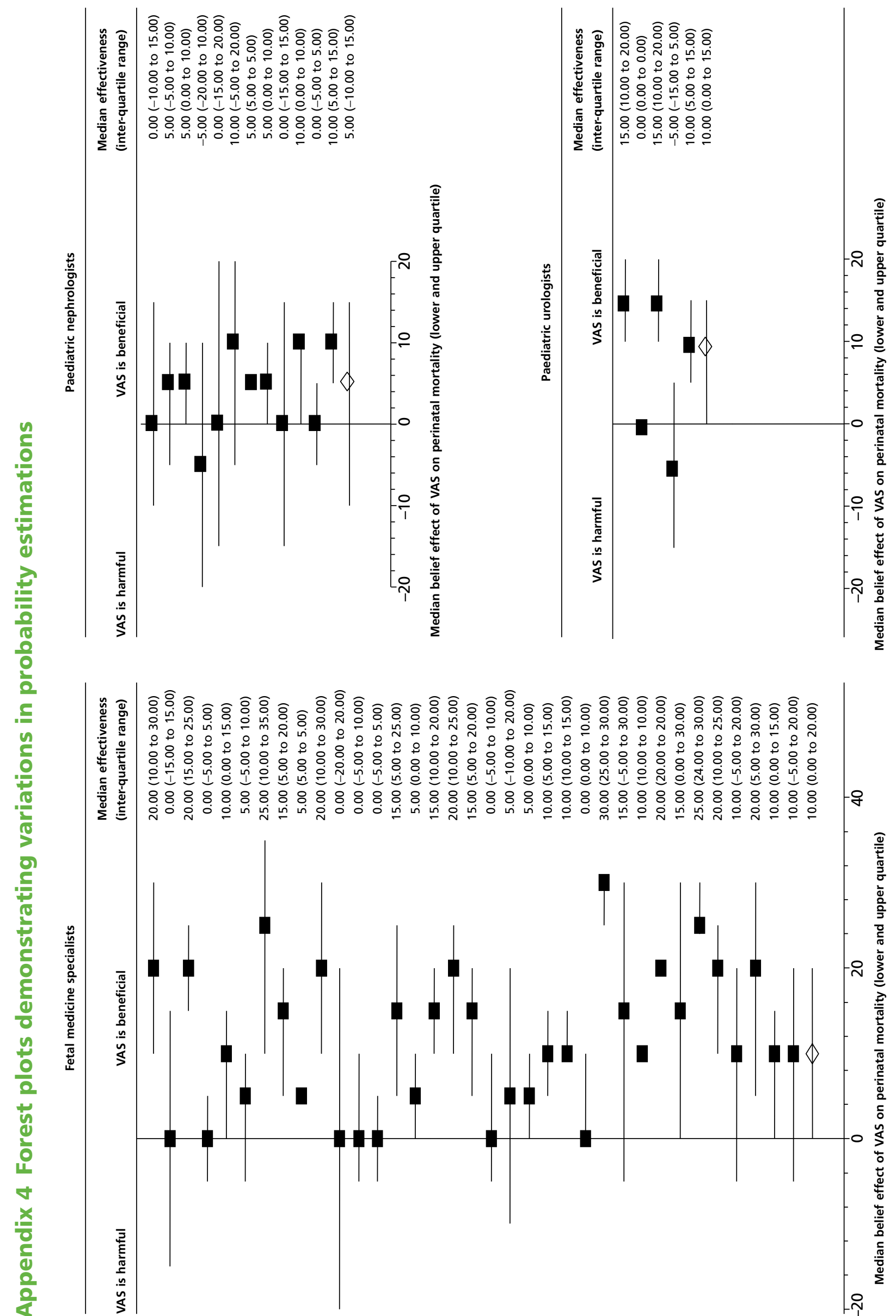

(c) Queen's Printer and Controller of HMSO 2013. This work was produced by Meads et al. under the terms of a commissioning contract issued by the Secretary of State for Health. This issue may be freely reproduced for the purposes of private research and study and extracts (or indeed, the full report) may be included in professional journals 


\section{Appendix 5 Illustrative example: eliciting subjective probabilities on test accuracy scenario 1}

Accuracy of CT/MRI in patients with a primary diagnosis of stage IB2-IV who are suspected to have recurrence on the basis of being symptomatic (assuming prevalence of recurrence of cervical cancer of $15 \%$ in this patient group)

Please indicate by allocating points to a sum of 100 how likely each estimate of the $\%$ of true-positives (as a $\%$ of all those with confirmed recurrent cervical cancer) and similarly how likely each estimate of the $\%$ of true-negatives (as a \% of those with no recurrence confirmed) is to be true when CT or MRI are used in the detection of recurrent cervical cancer in this population.

\begin{tabular}{|c|c|c|c|c|}
\hline & Recurrence confirmed D+ & & No recurrence confirmed D- & \\
\hline & True positive (TP) result & & False positive & \\
\hline \multirow[t]{6}{*}{$\mathrm{CT}$ or $\mathrm{MRI}+\mathrm{ve}$} & $\begin{array}{l}\text { Percent of TP detected: } \\
50-60 \% \text { of } 150\end{array}$ & & & \\
\hline & $\begin{array}{l}\text { Percent of TP detected: } \\
61-70 \% \text { of } 150\end{array}$ & & & \\
\hline & $\begin{array}{l}\text { Percent of TP detected: } \\
71-80 \% \text { of } 150\end{array}$ & & & \\
\hline & $\begin{array}{l}\text { Percent of TP detected: } \\
81-90 \% \text { of } 150\end{array}$ & & & \\
\hline & $\begin{array}{l}\text { Percent of TP detected: } \\
91-100 \% \text { of } 150\end{array}$ & & & \\
\hline & & Total: 100 points & & \\
\hline \multirow[t]{8}{*}{$\mathrm{CT}$ or MRI-ve } & & & True negative (TN) result & \\
\hline & & & $\begin{array}{l}\text { Percent of TN detected: } \\
50-60 \% \text { of } 850\end{array}$ & \\
\hline & & & $\begin{array}{l}\text { Percent of TN detected: } \\
61-70 \% \text { of } 850\end{array}$ & \\
\hline & & & $\begin{array}{l}\text { Percent of TN detected: } \\
71-80 \% \text { of } 850\end{array}$ & \\
\hline & & & $\begin{array}{l}\text { Percent of TN detected: } \\
81-90 \% \text { of } 850\end{array}$ & \\
\hline & & & $\begin{array}{l}\text { Percent of TN detected: } \\
91-100 \% \text { of } 850\end{array}$ & \\
\hline & & & & Total: 100 points \\
\hline & 150 & & 850 & \\
\hline
\end{tabular}


TABLE 2 Example of completed table

\begin{tabular}{|c|c|c|c|c|}
\hline & \multicolumn{2}{|l|}{ Recurrence confirmed D+ } & \multicolumn{2}{|c|}{ No recurrence confirmed D- } \\
\hline & True positive (TP) result & & False positive & \\
\hline \multirow[t]{6}{*}{$\mathrm{CT}$ or $\mathrm{MRI}$ + ve } & $\begin{array}{l}\text { Percent of TP detected: } \\
50-60 \% \text { of } 150\end{array}$ & 5 & & \\
\hline & $\begin{array}{l}\text { Percent of TP detected: } \\
61-70 \% \text { of } 150\end{array}$ & 1 & & \\
\hline & $\begin{array}{l}\text { Percent of TP detected: } \\
71-80 \% \text { of } 150\end{array}$ & 25 & & \\
\hline & $\begin{array}{l}\text { Percent of TP detected: } \\
81-90 \% \text { of } 150\end{array}$ & 40 & & \\
\hline & $\begin{array}{l}\text { Percent of TP detected: } \\
91-100 \% \text { of } 150\end{array}$ & 20 & & \\
\hline & & Total: 100 points & & \\
\hline \multirow[t]{8}{*}{$\mathrm{CT}$ or MRI -ve } & & & True negative (TN) result & \\
\hline & & & $\begin{array}{l}\text { Percent of TN detected: } \\
50-60 \% \text { of } 850\end{array}$ & 20 \\
\hline & & & $\begin{array}{l}\text { Percent of TN detected: } \\
61-70 \% \text { of } 850\end{array}$ & 25 \\
\hline & & & $\begin{array}{l}\text { Percent of TN detected: } \\
71-80 \% \text { of } 850\end{array}$ & 35 \\
\hline & & & $\begin{array}{l}\text { Percent of TN detected: } \\
81-90 \% \text { of } 850\end{array}$ & 15 \\
\hline & & & $\begin{array}{l}\text { Percent of TN detected: } \\
91-100 \% \text { of } 850\end{array}$ & 5 \\
\hline & & & & Total: 100 points \\
\hline & 150 & & 850 & \\
\hline
\end{tabular}


TABLE 3 Assuming the probability distribution of FP is the inverse of the TP distribution and the probability distribution of TN is the inverse of the FN distribution

\begin{tabular}{|c|c|c|c|c|}
\hline \multirow{3}{*}{ CT or MRI + ve } & \multirow{2}{*}{\multicolumn{2}{|c|}{$\begin{array}{l}\text { Recurrence confirmed D+ } \\
\text { True positive (TP) result }\end{array}$}} & \multicolumn{2}{|c|}{ No recurrence confirmed D- } \\
\hline & & & \multicolumn{2}{|l|}{ False positive (FP) result } \\
\hline & $\begin{array}{l}\text { Percent of TP detected: } \\
50-60 \% \text { of } 150\end{array}$ & 5 & $\begin{array}{l}\text { Percent of FP detected: } \\
40-49 \% \text { of } 850\end{array}$ & 5 \\
\hline & $\begin{array}{l}\text { Percent of TP detected: } \\
61-70 \% \text { of } 150\end{array}$ & 10 & $\begin{array}{l}\text { Percent of FP detected: } \\
30-39 \% \text { of } 850\end{array}$ & 10 \\
\hline & $\begin{array}{l}\text { Percent of TP detected: } \\
71-80 \% \text { of } 150\end{array}$ & 25 & $\begin{array}{l}\text { Percent of FP detected: } \\
20-29 \% \text { of } 850\end{array}$ & 25 \\
\hline & $\begin{array}{l}\text { Percent of TP detected: } \\
81-90 \% \text { of } 150\end{array}$ & 40 & $\begin{array}{l}\text { Percent of FP detected: } \\
10-19 \% \text { of } 850\end{array}$ & 40 \\
\hline & $\begin{array}{l}\text { Percent of TP detected: } \\
91-100 \% \text { of } 150\end{array}$ & 20 & $\begin{array}{l}\text { Percent of FP detected: } \\
0-9 \% \text { of } 850\end{array}$ & 20 \\
\hline & & Total: 100 points & & Total: 100 points \\
\hline \multirow[t]{8}{*}{$\mathrm{CT}$ or $\mathrm{MRI}-\mathrm{ve}$} & \multicolumn{2}{|l|}{ False negative (FN) result } & \multicolumn{2}{|l|}{ True negative (TN) result } \\
\hline & $\begin{array}{l}\text { Percent of FN detected: } \\
40-49 \% \text { of } 850\end{array}$ & 20 & $\begin{array}{l}\text { Percent of TN detected: } \\
50-60 \% \text { of } 850\end{array}$ & 20 \\
\hline & $\begin{array}{l}\text { Percent of FN detected: } \\
30-39 \% \text { of } 850\end{array}$ & 25 & $\begin{array}{l}\text { Percent of TN detected: } \\
61-70 \% \text { of } 850\end{array}$ & 25 \\
\hline & $\begin{array}{l}\text { Percent of FN detected: } \\
20-29 \% \text { of } 850\end{array}$ & 35 & $\begin{array}{l}\text { Percent of TN detected: } \\
71-80 \% \text { of } 850\end{array}$ & 35 \\
\hline & $\begin{array}{l}\text { Percent of FN detected: } \\
10-19 \% \text { of } 850\end{array}$ & 15 & $\begin{array}{l}\text { Percent of TN detected: } \\
81-90 \% \text { of } 850\end{array}$ & 15 \\
\hline & $\begin{array}{l}\text { Percent of FN detected: } \\
0-9 \% \text { of } 850\end{array}$ & 5 & $\begin{array}{l}\text { Percent of TN detected: } \\
91-100 \% \text { of } 850\end{array}$ & 5 \\
\hline & & Total: 100 points & & Total: 100 points \\
\hline & 150 & & 850 & \\
\hline
\end{tabular}

\section{Reference List}

1. Ferlay. Cancer Incidence, Mortality and Prevalence worldwide. IARC CancerBase 2004; No. 5 (Version 2.0).

2. Cancer Research UK: UK Cervical Cancer mortality statistics. http://info cancerresearchuk org/ cancerstats/types/cervix/mortality/ 2009.

3. Sundar SS, Horne A, Kehoe S. Cervical cancer. Clini EVID (Online) 20082008.

4. Scottish Intercollegiate Guidelines Network. Management of cervical cancer. Guideline No 992008.

5. Bodrka-Bevers D, Morris M, Eifel PJ, Levenback C, Bevers MW, et al. Posttherapy surveillance of women with cervical cancer: an outcomes analysis. Gynecol Oncol 2000;78:187-193.

6. Yen Tzu-Chen, Lai Chyong-Huey, Ma Shih-ya, Huang Kuan-Gen et al. Comparative benefits and limitations of 18F-FDG PET and CT-MRI in documented or suspected recurrent cervical cancer. European Journal of Nuclear Medicine and Molecular Imaging 2006; 33:1399-1407.

7. Facey K, Bradbury I, Laking G, Payne E. Overview of the clinical effectiveness of positron emission tomography imaging in selected cancers. Health Technol Assess 2007; 11(44). 
8. Jover R, Lorido D, Gonzalez C, Rojo A, Gorospe L, Alfonso JM. Role of PET/CT in the evaluation of cervical cancer. Gynecol Oncol 2008; 2008(110):S55-S59.

9. Wang $\mathrm{CJ}$, Lai $\mathrm{CH}$, Huang $\mathrm{HJ}$, Hong JH, et al. Recurrent cervical cancer after primary radical surgery. AmJOG 1999; 181:518-524.

10. Estape R, Angioli R. Surgical management of advanced and recurrent cervical cancer. Semin Surg Oncol 1999; 16:236-241.

11. Friedlander $\mathrm{M}$, Grogan $\mathrm{M}$. Guidelines for the treatment of recurrent and metastatic cervical cancer. The Oncologist 2002; 7:342-347.

12. Selman TJ, Mann C, Zamora J, Appleyard T, Khan K. Diagnostic accuracy of tests for lymph node status in primary cervical cancer: a systematic review and meta-analysis. CMAJ 2008; 178:855-862.

13. Reitsma JB, Rutjes AW, Khan KS, Coomarasamy AC, Bossuyt PM. A review of solutions for diagnostic accuracy studies with an imperfect or missing reference standard. J Clin Epidemio/ 2009; May 15 [Epub ahead of print].

14. Screening to prevent pre-term birth - systematic reviews of accuracy and effectiveness literature with economic modelling. HTA Ref: 05/03/01 (estimated pub date June 09).

15. Selman TJ, Luesley DM, Acheson N, Khan KS, Mann CH. A systematic review of the accuracy of diagnostic tests for inguinal lymph node status in vulvar cancer. Gynecol Oncol 2005; 99:206-214.

16. Selman TJ, Khan KS, Mann CH. An evidence-based approach to test accuracy studies in gynecologic oncology: the 'STARD' checklist. Gynecol Oncol 2005; 96:575-578.

17. Havrilesky L, Kulasingham SL, Matcher DB, Myers ER. FDG-PET for management of cervical and ovarian cancer. Gynecol Oncol 97, 183-191. 2005.

18. Matcher DB, Kulasingham SL, Havrilesky L, Mann LO, Myers ER, et al. Positron emission testing for six cancers (brain, cervical, small cell lung, ovarian, pancreatic and testicular). Agency for Healthcare Research and Quality (AHRQ) 221. 2004.

19. Cochrane Methods Working Group on Systematic Reviews of Screening and Diagnostic Tests: Recommended Methods. 6-6-1996.

20. CRD's guidance for undertaking reviews in healthcare. Systematic reviews. Centre for reviews and dissemination 2009.

21. Mijnhout GS, Riphagen II, Hoekstra OS. Update of the FDG PET search strategy. Nucl Med Comm 2004; 25:1187-1189.

22. Whiting P, Rutjes AWS, Reitsma JB, Bossuyt PM, Kleijnen J. The development of QUADAS: a tool for the quality assessment of studies of diagnostic accuracy included in systematic reviews. $B M C$ Medical Research Methodology 3. 2003.

23. Meads CA, Davenport CF. Quality assessment of diagnostic before-after studies: development of methodology in the context of a systematic review. BMC Medical Research Methodology 9. 2009.

24. Irwig LM, Tosteton AN, Gatsonis CA, Lao J, Colditz G, Chalmers TC et al. Guidelines for metaanalyses evaluating diagnostic tests. Annals of Internal Medicine 120, 667-676. 1994.

25. Lijmer JG, Bossuyt PM, Heistercamp SH. Exploring sources of heterogeneity in systematic reviews of diagnostic tests. Stat Med 21, 1525-1537. 2002.

26. Harbord RM, Deeks JJ, Egger M, Whiting P, Sterne JAC. A unification of models for meta-analysis of diagnostic accuracy studies. Biostatistics 2006; 1:1-21.

27. Deeks JJ, Altman D. Diagnostic Tests 4: Likelihood ratios. BMJ 329, 168. 2004. 
28. Song FJ, Khan KS, Dinnes J, Sutton AJ. Asymmetric funnel plots and publication bias in metaanalyses of diagnostic accuracy. International Journal of Epidemiology 31, 88-95. 2002.

29. Deeks JJ, Macaskill P, Irwig L. The performance of tests of publication bias and other sample size effects in systematic reviews of diagnostic test accuracy was assessed. Journal of Clinical Epidemiology 58, 882-893. 2005.

30. Moher D, Cook DJ, Eastwood S, et al. Improving the quality of reports of meta analyses of randomised controlled trials: the QUOROM statement. QUOROM group. Br J Surg 2000; 87:1448-1454.

31. Stroup DF, Berlin JA, Morton SC, et al. Meta analysis of observational studies in epidemiology:a proposal for reporting. Meta analysis Of Observational Studies in Epidemiology (MOOSE) group. JAMA 2000; 283:2008-2012.

32. Khan KS, ter Riet G, Glanville J, Sowden AJ, Kleijnen J. Undertaking Systematic Reviews of Research on Effectiveness. CRD's Guidance for Carrying Out or Commissioning Reviews. CRD Report Number 4 (2nd edition). 2nd ed. York: NHS Centre for Reviews and Dissemination, University of York; 2001.

33. http://www.arif.bham.ac.uk/strategy.shtml. Accessed 31/05/09 2009.

34. Jadad AR, Moore RA, et al. Assessing the quality of reports of randomized clinical trials: Is blinding necessary? Controlled Clinical Trials 1996; 17:1-12.

35. O'Hagan A, Buck CE, Daneschkkah Eiser R, et al. Uncertain Judgements: Eliciting Expert's Probabilties. Staistics in Practice 2006.

36. Lathe PM, Braunholtz DA, Hills RK, Khan KS, Lilford R. Measurement of beliefs about effectiveness of laparoscopic uterosacral nerve ablation. BJOG 2005; 112:243-246.

37. Lilford R. Formal measurements of clinical uncertainty: prelude to a trial in perinatal medicine. The Fetal Compromise Group. BMJ 1994; 308:111-112.

38. Claxton K, Neumann PJ, Araki S, Weinstein MC, et al. Bayesian Value-of-Information Analysis: An Application to a Policy Model of Alzheimer's Disease. International Journal of Technology Assessment in Health Care 2001; 17:38-55.

39. Felli JC, Hazen GB. Sensitivity Analysis and the Expected Value of Perfect Information. Med Dec Making 1998; 18:95-109.

40. Sonnenberg FA, Beck JR. Markov models in medical decision making: a practical guide. Med Dec Making 1993; 13:332-338.

41. Selman TJ, Mann C, Zamora J, Khan KS. A systematic review of tests for lymph node status in primary endometrial cancer. BMC Womens Health 2008; 8(8). 


\title{
Appendix 2 Scoping search strategies and results
}

\author{
The objective was a scoping search to identify published systematic reviews (for diagnostic accuracy, \\ yield and effectiveness). Searches were undertaken between May 2010 and August 2010. The following \\ databases would be searched: MEDLINE, EMBASE, Science Citation Index, The Cochrane Library (all \\ databases), UK Clinical Research Network Study Portfolio and ClinicalTrials.gov.
}

The search terms used are shown below.

In total, 468 citations for published studies and 12 citations for ongoing research were found. Of these, 50 full-text articles were assessed for eligibility and one systematic review was found. ${ }^{59}$ This was assessed using the form below.

\section{Searches}

\section{MEDLINE (Ovid Gateway) (May 2010)}

One hundred and thirty records were retrieved in MEDLINE.

1. exp Uterine Cervical Neoplasms/

2. (cervi\$ adj5 cancer\$).mp.

3. (cervi $\$$ adj5 carcinom $\$$ ).mp.

4. (cervi\$ adj5 adenocarcinom\$).mp.

5. (cervi\$ adj5 carcinogen\$).mp.

6. (cervi\$ adj5 sarcoma\$).mp.

7. (cervi\$ adj5 malignan\$).mp.

8. (cervi\$ adj5 tumor\$).mp.

9. (cervi\$ adj5 tumour\$).mp.

10. (cervi\$ adj5 neoplas\$).mp.

11. (cervi $\$$ adj5 metasta $\$$ ).mp.

12. 1 or 2 or 3 or 4 or 5 or 6 or 7 or 8 or 9 or 10 or 11

13. exp Recurrence/ or exp Neoplasm Recurrence, Local/

14. recur\$.mp.

15. relaps\$.mp.

16. repeat\$.mp.

17. repetitive\$.mp.

18. reappearance $\$ . m p$.

19. reoccurence $\$ . m p$.

20. return.mp.

21. exp Neoplasm Metastasis/

22. metasta\$.mp.

23. restag $\$ . m p$.

24. re-stag.mp.

25. 13 or 14 or 15 or 16 or 17 or 18 or 19 or 20 or 21 or 22 or 23 or 24

26. 12 and 25

27. ("review" or "review academic" or "review tutorial").pt.

28. (scisearch or psychinfo or psycinfo).tw, sh.

29. cinahl.tw,sh.

30. ((hand adj2 search\$) or (manual\$ adj2 search $\$)$ ).tw, sh.

31. (electronic database $\$$ or bibliographic database $\$$ or computeri? ed database $\$$ or online database $\$$ ). tw, sh. 
32. (pooling or pooled or mantel haenszel).tw,sh.

33. (retraction of publication or retracted publication).pt.

34. (peto or dersimonian or der simonian or fixed effect).tw, sh.

35. (medline or medlars or embase or pubmed).tw,sh.

36. 28 or 29 or 30 or 31 or 32 or 33 or 34 or 35

37. meta-analysis.pt.

38. meta-analysis.sh.

39. (meta-analys $\$$ or meta analys $\$$ or metaanalys $\$$ ).tw, sh.

40. (systematic\$ adj5 review\$).tw, sh.

41. (systematic $\$$ adj5 overview\$).tw, sh.

42. (quantitativ\$ adj5 overview\$).tw, sh.

43. (quantitativ\$ adj5 synthesis\$).tw, sh.

44. (methodologic\$ adj5 review\$).tw, sh.

45. (methodologic $\$$ adj5 overview\$).tw, sh.

46. (integrative research review $\$$ or research integration).tw.

47. (quantitativ $\$$ adj5 review $\$$ ).tw, sh.

48. 37 or 38 or 39 or 40 or 41 or 42 or 43 or 44 or 45 or 46 or 47

49. 27 and 36

50. 48 or 49

51. 26 and 50

\section{EMBASE (Ovid Gateway) (May 2010)}

Two hundred and three records were retrieved in EMBASE.

1. exp uterine cervix tumor/

2. (cervi $\$$ adj5 cancer\$).mp.

3. (cervi\$ adj5 carcinom $\$$ ). $m p$.

4. (cervi $\$$ adj5 adenocarcinom\$).mp.

5. (cervi\$ adj5 carcinogen\$).mp.

6. (cervi\$ adj5 malignan\$).mp.

7. (cervi\$ adj5 tumor\$).mp.

8. (cervi $\$$ adj5 tumour\$).mp.

9. (cervi $\$$ adj5 neoplas\$).mp.

10. (cervi\$ adj5 metasta\$).mp.

11. (cervi\$ adj5 cyst\$).mp.

12. 1 or 2 or 3 or 4 or 5 or 6 or 7 or 8 or 9 or 10 or 11

13. exp recurrent disease/

14. recur\$.mp.

15. relaps $\$ . m p$.

16. repeat\$.mp.

17. repetitive $\$ . m p$.

18. reappearance $\$ . m p$.

19. reoccurence\$.mp.

20. return.mp.

21. exp metastasis/

22. restag\$.mp.

23. re-stag.mp.

24. 13 or 14 or 15 or 16 or 17 or 18 or 19 or 20 or 21 or 22 or 23

25. 12 and 24

26. exp review/

27. (medline or medlars or embase or pubmed).ti,ab, sh.

28. (scisearch or psychlit or psyclit).ti,ab,sh.

29. (psycinfo or psychinfo).ti,ab,sh. 
30. cinahl.ti,ab,sh.

31. ((electronic adj database $\$$ ) or (bibliographic adj database $\$))$.tw.

32. ((pooled adj analys $\$$ ) or pooling).tw.

33. (peto or dersimonian or (fixed adj effect) or mantel haenszel).tw.

34. RETRACTED ARTICLE/

35. 27 or 28 or 29 or 30 or 31 or 32 or 33 or 34

36. 26 and 35

37. exp meta analysis/

38. meta?analys $\$$.tw, sh.

39. (systematic\$ adj5 review\$).tw, sh.

40. (systematic $\$$ adj5 overview\$).tw, sh.

41. (quantitativ\$ adj5 review\$).tw, sh.

42. (quantitativ\$ adj5 overview\$).tw, sh.

43. (methodologic\$ adj5 review\$).tw, sh.

44. (methodologic\$ adj5 overview\$).tw, sh.

45. ((integrative adj5 research adj5 review\$) or (research adj5 integration)).tw.

46. (quantitativ\$ adj5 synthesi\$).tw, sh.

47. 37 or 38 or 39 or 40 or 41 or 42 or 43 or 44 or 45 or 46

48. 36 or 47

49. 25 and 48

The Cochrane Library (all databases) (May 2010)

Six hundred and eleven records were retrieved in The Cochrane Library (all databases).

1. MeSH descriptor Uterine Cervical Neoplasms explode all trees

2. cervi* near/5 neoplas*

3. cervi* near $/ 5$ carcinom*

4. cervi* near/5 malignan*

5. cervi* near $/ 5$ tumor*

6. cervi* near/5 tumour*

7. cervi* near $/ 5$ cancer* $^{*}$

8. cervi* near $/ 5$ adenocarcinom*

9. cervi* near $/ 5$ carcinogen*

10. cervi* near $/ 5$ metasta*

11. \#1 OR \#2 OR \#3 OR \#4 OR \#5 OR \#6 OR \#7 OR \#8 OR \#9 OR \#10

12. recur*

13. relaps*

14. repeat*

15. reappearance*

16. reoccurence*

17. return

18. MeSH descriptor Neoplasm Metastasis explode all trees

19. restag*

20. re-stag

21. metasta*

22. \#12 OR \#13 OR \#14 OR \#15 OR \#16 OR \#17 OR \#18 OR \#19 OR \#21

23. \#12 AND \#23 


\section{Review Assessment Form - Effectiveness Part}

Ongoing review title

Date.

Reviewer ID

Assessed review ID.

\begin{tabular}{|l|l|}
\hline Assessed review title & \\
\hline First author & \\
\hline $\begin{array}{l}\text { Source of publication } \\
\text { Journal yy;vol(iss):pp }\end{array}$ & journal $\square$ abstract other (specify) $: \ldots \ldots \ldots \ldots \ldots \ldots \ldots \ldots \ldots \ldots \ldots$ \\
\hline Publication type & $\ldots \ldots \ldots \ldots \ldots \ldots \ldots \ldots$ \\
\hline
\end{tabular}

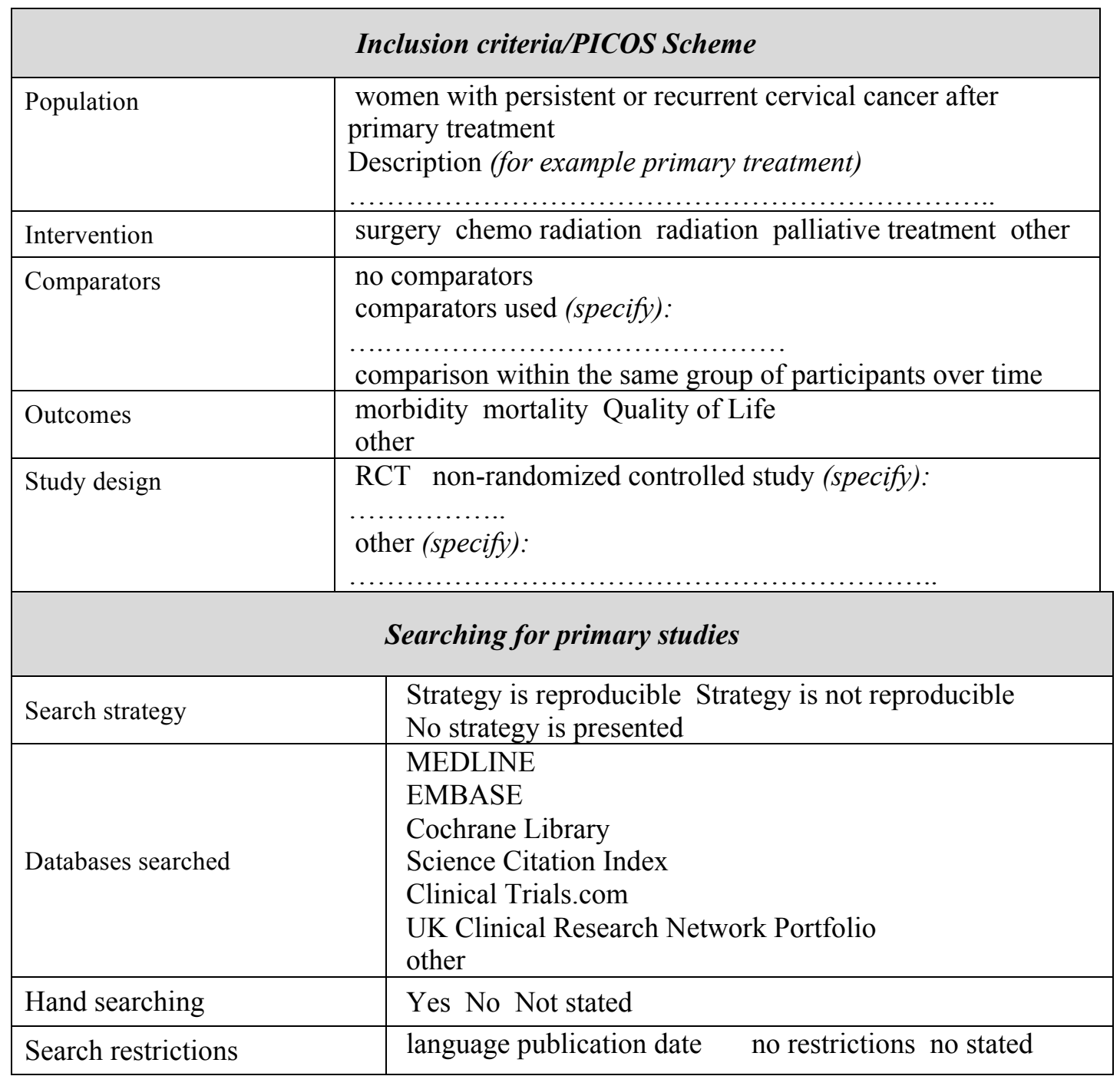




\section{Quality assessment of included studies}

Select the parameters which are included in quality assessment of particularly trials? method of randomization allocation concealment information about excluded patients intention-to-treat analysis blinding no quality assessment was conducted other parameters (specy):

\section{Data extraction}

Was extraction prepared independently by at least 2 reviewers?

Yes No Not stated

\section{Data synthesis}

Select the activities performed in data synthesis:

proper presentation of results (effect size and confidence intervals)

presentation of results for each treatment group in each trial for ich primary outcomes presentation of results as intention-to-treat analysis

assessment of heterogeneity

sensivity testing

biases assessment

Specify the method of combining results:

\section{Reviewer's assessment}

Do results and conclusions presented in the review need for:

updating?

filling gaps in information?

correction?

The review presents current, correct and valid information regarding clinical problem being concern.

\section{Reviewer's comments}





\section{Appendix 3 Diagnostic review data extraction form}




\section{Data Extraction Form - Diagnostic Part}

Review title.

Date.

(dd/mm/yy )

Reviewer ID

Study ID

\begin{tabular}{|l|l|}
\hline Study title & \\
\hline First author & \\
\hline $\begin{array}{l}\text { Source of publication } \\
\text { Journal yy;vol(iss):pp }\end{array}$ & \\
\hline Country of publication & journal $\square$ abstract other (specify): $\ldots \ldots \ldots \ldots \ldots \ldots \ldots \ldots \ldots \ldots \ldots$ \\
\hline Publication type &
\end{tabular}

\section{Study eligibility}

\begin{tabular}{|l|l|}
\hline \multirow{2}{*}{ Population } & $\begin{array}{l}\text { women suspected to have persistent or recurrent cervical cancer after } \\
\text { primary treatment }\end{array}$ \\
$\begin{array}{l}\text { patients with advanced stage cervical cancer (IB2-IV) treated } \\
\text { previously with chemoradiation with a minimum gap between } \\
\text { completion of treatment and imaging of } 3 \text { months }\end{array}$ \\
other
\end{tabular}

\section{Study characteristics}

\begin{tabular}{|l|l|}
\hline \multicolumn{2}{|l|}{ Population } \\
\hline Trial inclusion criteria & \\
\hline Trial exclusion criteria & \\
\hline Number of enrolled patients, $\mathrm{N}$ & \\
\hline $\begin{array}{l}\text { Number of patients who completed } \\
\text { the study, n (\%) }\end{array}$ & \\
\hline Age, in years; mean (range) & \\
\hline Type of initial treatment, $\mathrm{n}(\%)$ & \\
\hline Initial staging, n (\%) & \\
\hline Other main baseline parameters & \\
\hline Tests & \\
\hline $\begin{array}{l}\text { Type of index test used (short } \\
\text { description) }\end{array}$ & \\
\hline
\end{tabular}




\begin{tabular}{|c|c|}
\hline $\begin{array}{l}\text { Type of alternative test/comparator } \\
\text { (short description) }\end{array}$ & \\
\hline \multicolumn{2}{|l|}{$\begin{array}{l}\text { Type of reference standard (short } \\
\text { description) }\end{array}$} \\
\hline \multicolumn{2}{|l|}{$\begin{array}{l}\text { Duration of follow up in months } \\
\text { (range) }\end{array}$} \\
\hline \multicolumn{2}{|l|}{ Methods } \\
\hline Method of enrolment & consecutive arbitrary random not reported \\
\hline Data Collection & prospective retrospective not reported \\
\hline Information about drops out & $\begin{array}{l}\text { precise information inaccurate information } \\
\text { lack of information }\end{array}$ \\
\hline \multicolumn{2}{|l|}{ Statistical technique used } \\
\hline \multicolumn{2}{|l|}{ Sample size calculation } \\
\hline \multicolumn{2}{|l|}{ Funding source } \\
\hline \multicolumn{2}{|l|}{ Quality assessment } \\
\hline Representative spectrum? & Yes No Unclear \\
\hline Acceptable reference standard? & Yes No Unclear \\
\hline Acceptable delay between tests? & Yes No Unclear \\
\hline Partial verification avoided? & Yes No Unclear \\
\hline Differential verification avoided? & Yes No Unclear \\
\hline Incorporation avoided? & Yes No Unclear \\
\hline Reference standard results blinded? & Yes No Unclear \\
\hline Index test results blinded? & Yes No Unclear \\
\hline Relevant clinical information? & Yes No Unclear \\
\hline Uninterpretable results reported? & Yes No Unclear \\
\hline Withdrawals explained? & Yes No Unclear \\
\hline
\end{tabular}




\section{Results}

\begin{tabular}{|c|c|c|c|c|}
\hline & & \multicolumn{3}{|c|}{ Reference standard } \\
\hline & & Positive & Negative & Total \\
\hline \multirow{3}{*}{ PET-CT } & Positive & & & \\
\hline & Negative & & & \\
\hline & Total & & & \\
\hline
\end{tabular}

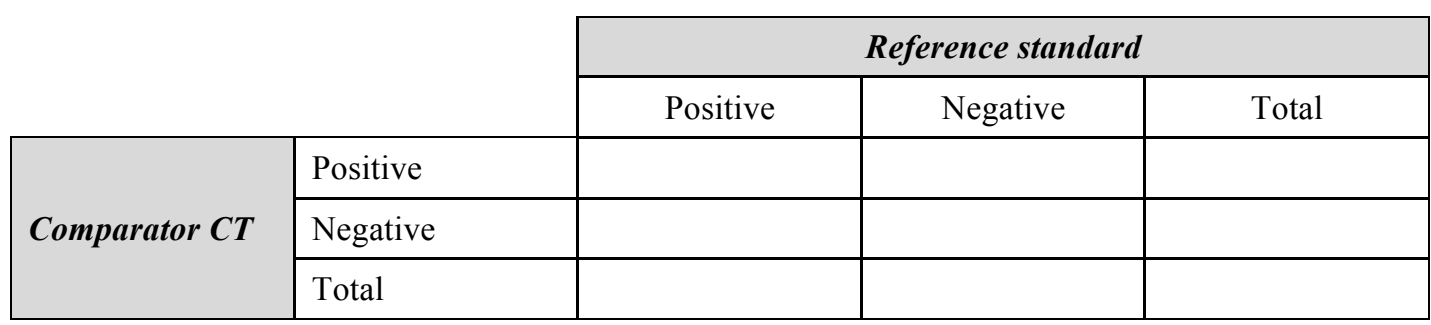

\section{Reviewer's comments}




\section{Appendix 4 Effectiveness review data extraction forms}




\section{Data Extraction Form - Effectiveness Part}

Review title

Date.

$(\mathrm{dd} / \mathrm{mm} / \mathbf{y y})$

Reviewer ID.

Study ID

\begin{tabular}{|l|l|}
\hline Study title & \\
\hline First author & \\
\hline $\begin{array}{l}\text { Source of publication } \\
\text { Journal yy;vol(iss):pp }\end{array}$ & \\
\hline Language & \\
\hline Publication type & journal $\square$ abstract other (specify) $\ldots \ldots \ldots \ldots \ldots \ldots \ldots \ldots \ldots \ldots \ldots \ldots \ldots \ldots \ldots \ldots \ldots \ldots \ldots \ldots \ldots$ \\
\hline
\end{tabular}

\section{Study eligibility/PICOS Scheme}

\begin{tabular}{|c|c|}
\hline Population & $\begin{array}{l}\text { women with persistent or recurrent cervical cancer after primary } \\
\text { treatment: radiation, chemoradiation } \\
\text { women with persistent or recurrent cervical cancer after primary } \\
\text { treatment: radical surgery } \\
\text { women with multiple site or distant reccurence (treatment in } \\
\text { palliative intent) } \\
\text { other }\end{array}$ \\
\hline Intervention & $\begin{array}{l}\text { Curative intent: } \\
\text { surgery chemo radiation radiation } \\
\text { Palliative intent: } \\
\text { palliative treatment other }\end{array}$ \\
\hline Comparison & 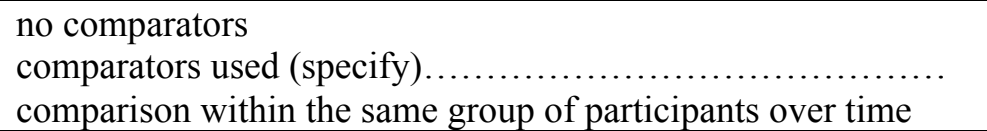 \\
\hline Outcomes & $\begin{array}{l}\text { morbidity mortality Quality of Life } \\
\text { none of the above }\end{array}$ \\
\hline Study design & $\begin{array}{l}\text { RCT non-randomized controlled study (specify): } \ldots \ldots \ldots \ldots \ldots \\
\text { other }(\text { specify) } \ldots \ldots \ldots \ldots \ldots \ldots \ldots \ldots \ldots \ldots \ldots \ldots \ldots \ldots \ldots \ldots \ldots \ldots \ldots\end{array}$ \\
\hline
\end{tabular}

If included study is comparative experimental study, then go to the point $A$,

If included study is comparative observational study, then go to the point $B$,

fincluded study is non-comparative study, then go to the point $C$ 


\section{PART A}

Comparative Experimental Studies:

1. Study characteristics

\begin{tabular}{|c|c|}
\hline \multicolumn{2}{|r|}{ Methods/methodological quality } \\
\hline Study design & RCT NRS \\
\hline \multicolumn{2}{|r|}{$R C T$} \\
\hline Method of randomization & $\begin{array}{l}\text { specify and assess the method: } \\
\ldots \ldots \ldots \ldots \ldots \ldots \ldots \ldots \ldots \ldots \ldots \ldots \ldots \\
\text { adequate inadequate unclear not reported }\end{array}$ \\
\hline Allocation concealment & $\begin{array}{l}\text { adequate inadequate unclear not reported } \\
\text { Describe } \ldots \ldots \ldots \ldots \ldots \ldots \ldots \ldots \ldots \ldots \ldots \ldots \ldots \ldots \ldots \ldots \ldots \ldots\end{array}$ \\
\hline Blinding & $\begin{array}{l}\text { select blinded subjects: } \\
\text { patients investigators/clinicians outcomes assessors no } \\
\text { blinding used } \\
\text { assess the method: } \\
\text { adequate inadequate unclear not reported }\end{array}$ \\
\hline Information about drop outs & $\begin{array}{l}\text { precise information (number of patients and reasons) } \\
\text { inaccurate information } \\
\text { lack of information }\end{array}$ \\
\hline \multicolumn{2}{|l|}{ Rate of loss to follow-up } \\
\hline \multicolumn{2}{|l|}{$\begin{array}{l}\text { Patients lost to follow-up } \\
\text { analyzed for adverse events }\end{array}$} \\
\hline $\begin{array}{l}\text { Was the follow-up adequate to } \\
\text { ascertain adverse effects? }\end{array}$ & $\begin{array}{l}\text { Yes No Unclear } \\
\text { If "yes", specify......................... }\end{array}$ \\
\hline \multicolumn{2}{|l|}{ Statistical technique used } \\
\hline $\begin{array}{l}\text { Was adequate statistical analysis } \\
\text { of potential confounders } \\
\text { performed? }\end{array}$ & Yes No Unclear \\
\hline $\begin{array}{l}\text { Intention-to-treat analysis } \\
\text { What was the definition of ITT } \\
\text { in the study? }\end{array}$ & 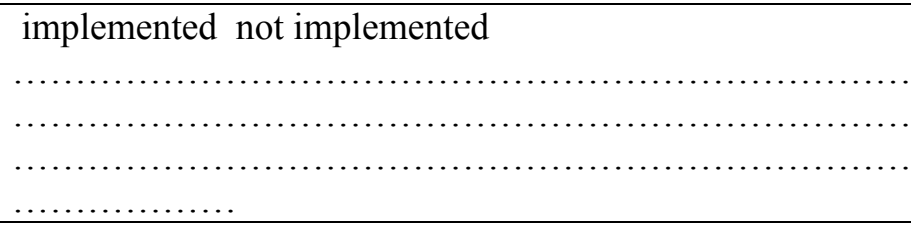 \\
\hline \multicolumn{2}{|l|}{ Sample size calculation } \\
\hline $\begin{array}{l}\text { Was the sensitivity analysis } \\
\text { performed? }\end{array}$ & Yes No Not applicable \\
\hline \multicolumn{2}{|l|}{$\begin{array}{l}\text { How problem with missing data } \\
\text { was resolved? }\end{array}$} \\
\hline $\begin{array}{l}\text { Were missing data accounted for } \\
\text { in the analyses? }\end{array}$ & Yes No \\
\hline
\end{tabular}




\begin{tabular}{|c|c|}
\hline \multicolumn{2}{|l|}{ Post hoc analysis } \\
\hline Funding source & \\
\hline \multicolumn{2}{|r|}{$N R S$} \\
\hline Control group selection & 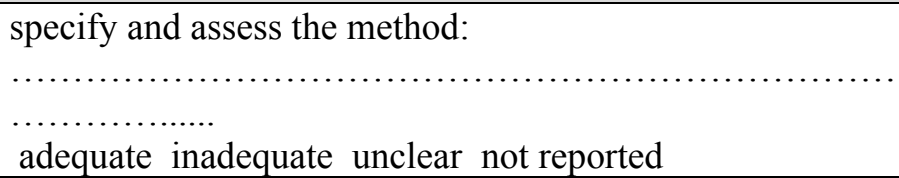 \\
\hline Allocation concealment & 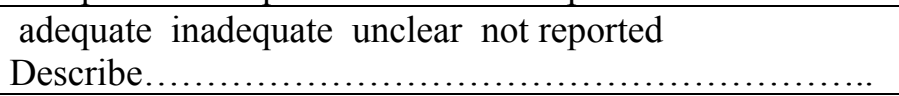 \\
\hline Blinding & $\begin{array}{l}\text { select blinded subjects: } \\
\text { patients investigators/clinicians outcomes assessors no } \\
\text { blinding used } \\
\text { assess the method: } \\
\text { adequate inadequate unclear not reported }\end{array}$ \\
\hline Information about drop outs & $\begin{array}{l}\text { precise information (number of patients and reasons) } \\
\text { inaccurate information } \\
\text { lack of information }\end{array}$ \\
\hline \multicolumn{2}{|l|}{ Rate of loss to follow-up } \\
\hline \multicolumn{2}{|l|}{$\begin{array}{l}\text { Patients lost to follow-up } \\
\text { analyzed for adverse events }\end{array}$} \\
\hline $\begin{array}{l}\text { Was the follow-up adequate to } \\
\text { ascertain adverse effects? }\end{array}$ & $\begin{array}{l}\text { Yes No Unclear } \\
\text { If "yes", specify } \ldots \ldots \ldots \ldots \ldots \ldots \ldots \ldots \ldots \ldots \ldots \ldots\end{array}$ \\
\hline \multicolumn{2}{|l|}{ Statistical technique used } \\
\hline $\begin{array}{l}\text { Was adequate statistical analysis } \\
\text { of potential confounders } \\
\text { performed? }\end{array}$ & Yes No Unclear \\
\hline $\begin{array}{l}\text { Intention-to-treat analysis } \\
\text { What was the definition of ITT } \\
\text { in the study? }\end{array}$ & $\begin{array}{l}\text { implemented not implemented } \\
\ldots \ldots \ldots \ldots \ldots \ldots \ldots \ldots \ldots \ldots \ldots \ldots \ldots \ldots \ldots \ldots \ldots \ldots \ldots \ldots \\
\ldots \ldots\end{array}$ \\
\hline \multicolumn{2}{|l|}{ Sample size calculation } \\
\hline $\begin{array}{l}\text { Was the sensitivity analysis } \\
\text { performed? }\end{array}$ & Yes No Not applicable \\
\hline \multicolumn{2}{|l|}{$\begin{array}{l}\text { How problem with missing data } \\
\text { was resolved? }\end{array}$} \\
\hline $\begin{array}{l}\text { Were missing data accounted for } \\
\text { in the analyses? }\end{array}$ & Yes No \\
\hline \multicolumn{2}{|l|}{ Post hoc analysis } \\
\hline Funding source & \\
\hline
\end{tabular}




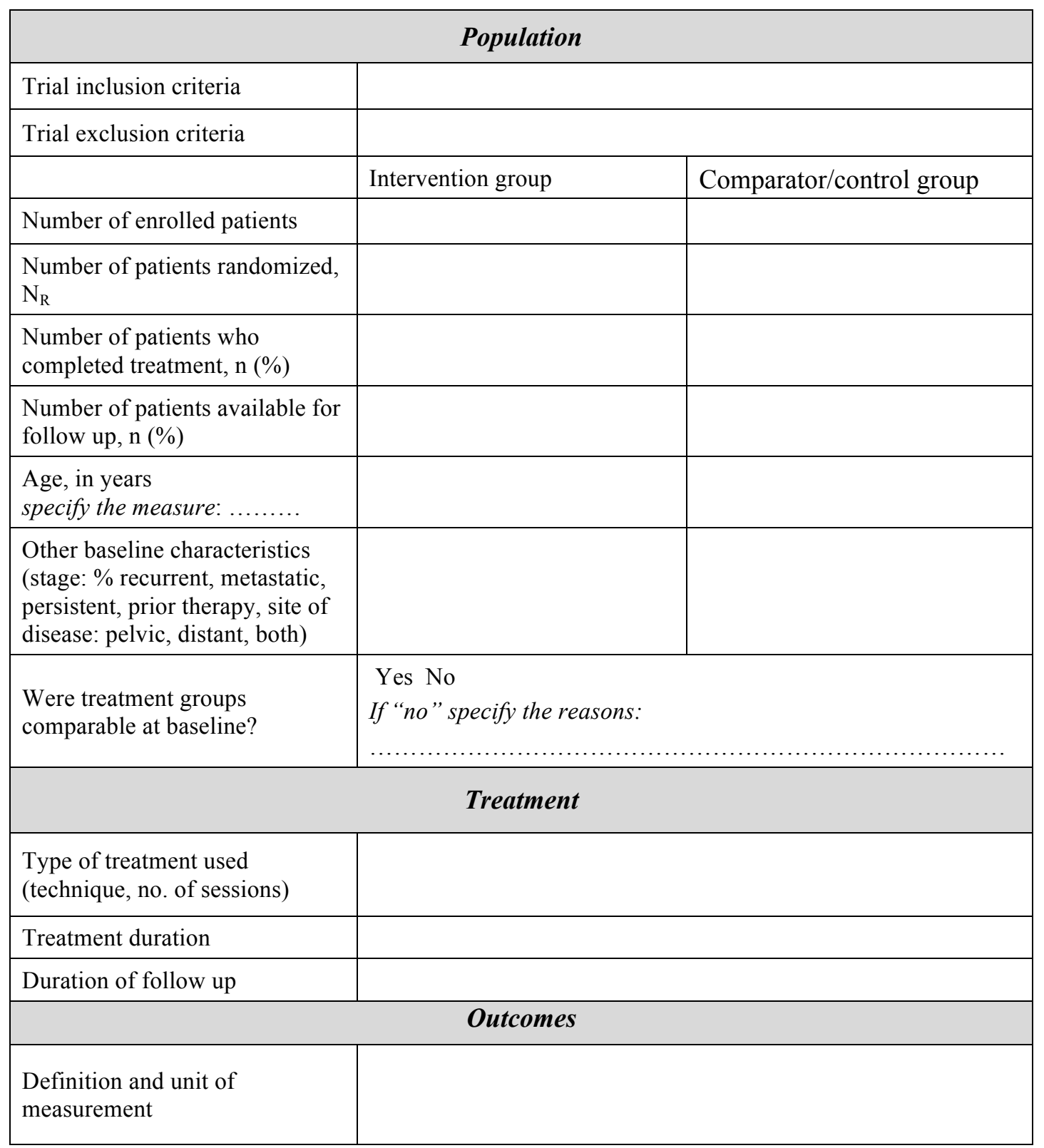




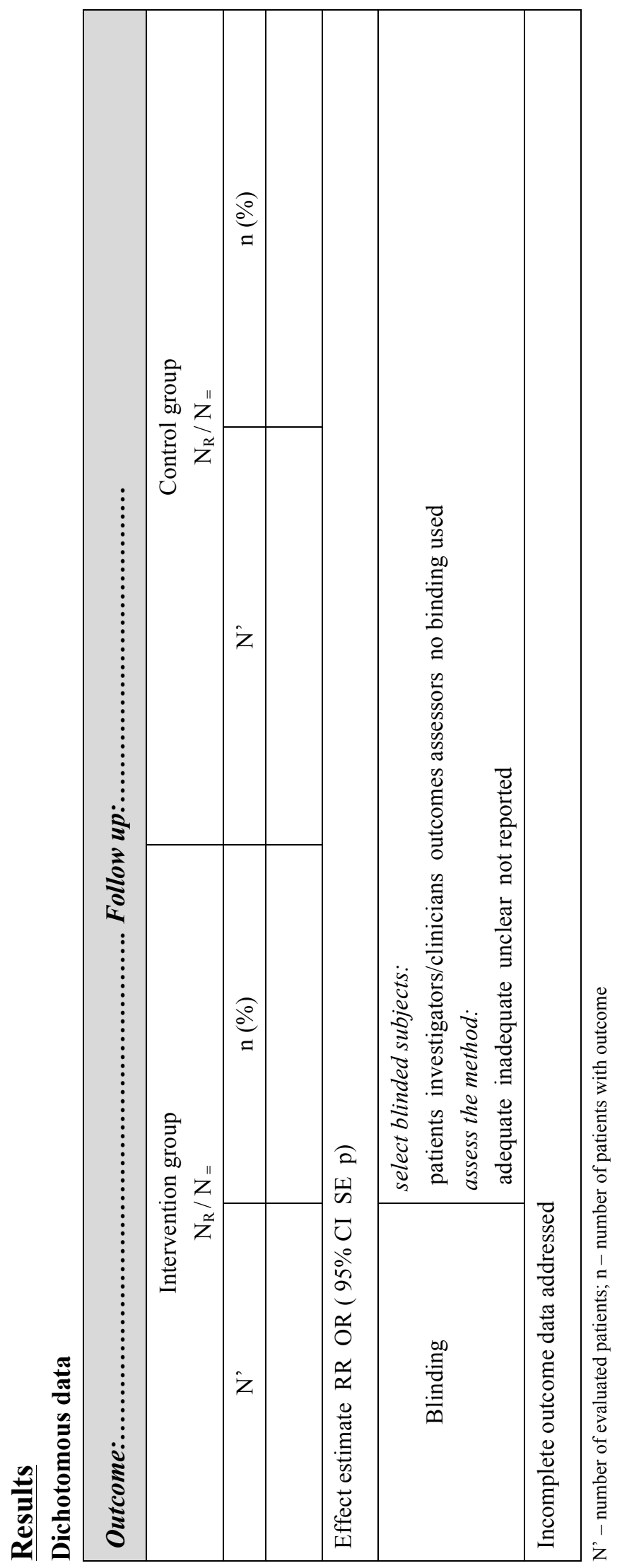




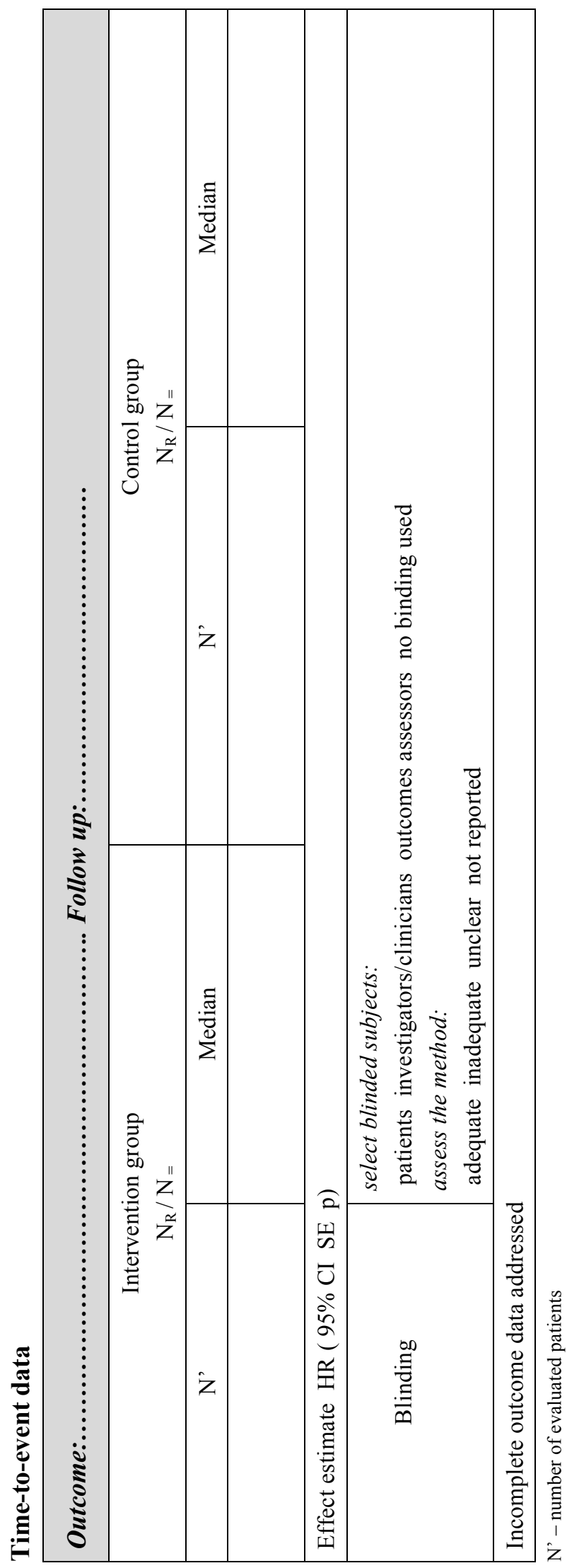




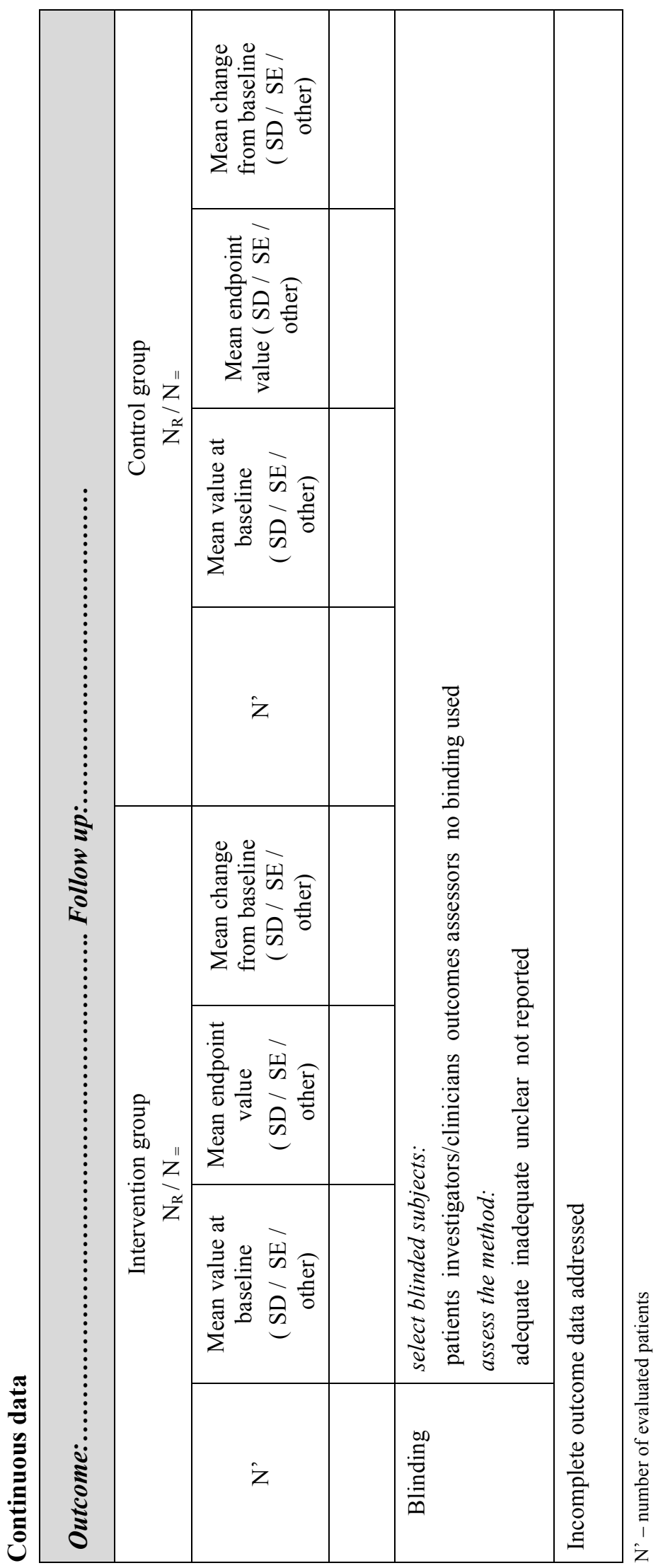




\section{PART B}

\section{B) Comparative Observational Studies:}

\section{Study characteristics}

\begin{tabular}{|c|c|}
\hline \multicolumn{2}{|r|}{ Methods/methodological quality } \\
\hline Study design & Case - control Cohort \\
\hline \multicolumn{2}{|r|}{ Case-Control } \\
\hline Is case definition adequate? & $\begin{array}{l}\text { independent validation record linkage self reported } \\
\text { none }\end{array}$ \\
\hline Are the cases representative? & $\begin{array}{l}\text { all cases arising from same population or group } \\
\text { not known }\end{array}$ \\
\hline Selection of controls & same population as cases not known or no \\
\hline Definition of controls & $\begin{array}{l}\text { outcome of interest not present in history } \\
\text { no mention of history of outcome }\end{array}$ \\
\hline $\begin{array}{l}\text { Comparability of cases } \\
\text { and controls }\end{array}$ & Yes No Unclear \\
\hline $\begin{array}{l}\text { Ascertainment of exposure } \\
\text { to intervention }\end{array}$ & $\begin{array}{l}\text { secure record } \\
\text { structured interview where blind to case/control status } \\
\text { interview not blinded to case/control status } \\
\text { written self report of medical record only } \\
\text { no description }\end{array}$ \\
\hline $\begin{array}{l}\text { Was the method of } \\
\text { ascertainment of exposure for } \\
\text { cases and controls the same? }\end{array}$ & Yes No Unclear \\
\hline Non-response rate & $\begin{array}{l}\text { same for both groups... non respondents described } \\
\text { rate different and no designation }\end{array}$ \\
\hline \multicolumn{2}{|r|}{ Cohort } \\
\hline Is the cohort representative & Yes No Unclear \\
\hline Selection of non-exposed cohort & same population as exposed cohort not known or no \\
\hline Ascertainment of exposure & $\begin{array}{l}\text { secure record structured interview } \\
\text { written self report } \\
\text { no description }\end{array}$ \\
\hline $\begin{array}{l}\text { Demonstration that outcome of } \\
\text { interest wasn't present at start of } \\
\text { study? }\end{array}$ & Yes No Unclear \\
\hline $\begin{array}{l}\text { Comparability of cohorts on the } \\
\text { basis of the design or analysis }\end{array}$ & Yes No Unclear \\
\hline Assessment of outcome & $\begin{array}{l}\text { independent or blind assessment } \\
\text { record linkage self-report no description }\end{array}$ \\
\hline $\begin{array}{l}\text { Was follow-up long enough for } \\
\text { outcomes to occur? }\end{array}$ & $\begin{array}{l}\text { Yes No Unclear } \\
\text { If "yes", specify } \ldots \ldots \ldots \ldots \ldots \ldots \ldots \ldots \ldots \ldots \ldots \ldots\end{array}$ \\
\hline
\end{tabular}




\begin{tabular}{|c|c|c|}
\hline $\begin{array}{l}\text { Was follow-up of cohorts } \\
\text { adequate? }\end{array}$ & \multicolumn{2}{|c|}{$\begin{array}{l}\text { complete follow-up } \\
\text { subjects lost to follow-up unlikely to introduce bias, small } \\
\text { number lost }(\ldots . . \%) \\
\text { follow-up rate } \ldots . . \% \text {, and no description of this lost } \\
\text { no statement }\end{array}$} \\
\hline \multicolumn{3}{|c|}{ Population } \\
\hline \multicolumn{3}{|l|}{ Trial inclusion criteria } \\
\hline \multicolumn{3}{|l|}{ Trial exclusion criteria } \\
\hline \multirow[t]{2}{*}{ Is the target population defined? } & Yes No & \\
\hline & Intervention group & Comparator/control group \\
\hline \multicolumn{3}{|l|}{ Number of included patients, $\mathrm{N}$} \\
\hline \multicolumn{3}{|l|}{$\begin{array}{l}\text { Number of patients who completed } \\
\text { treatment, } \mathrm{n}(\%)\end{array}$} \\
\hline \multicolumn{3}{|l|}{$\begin{array}{l}\text { Age, in years } \\
\text { specify the measure: }\end{array}$} \\
\hline \multicolumn{3}{|l|}{$\begin{array}{l}\text { Other baseline characteristics } \\
\text { (stage: \% recurrent, metastatic, } \\
\text { persistent, prior therapy, site of } \\
\text { disease: pelvic, distant, both) }\end{array}$} \\
\hline $\begin{array}{l}\text { Were treatment groups comparable } \\
\text { at baseline? }\end{array}$ & \multicolumn{2}{|c|}{$\begin{array}{l}\text { Yes No Not applicable } \\
\text { If "no" specify the reasons: }\end{array}$} \\
\hline \multicolumn{3}{|c|}{ Treatment } \\
\hline \multicolumn{3}{|l|}{$\begin{array}{l}\text { Type of treatment used (technique, } \\
\text { no. of sessions) }\end{array}$} \\
\hline \multicolumn{3}{|l|}{ Treatment duration } \\
\hline \multicolumn{3}{|l|}{ Duration of follow up } \\
\hline \multicolumn{3}{|c|}{ Outcomes } \\
\hline
\end{tabular}




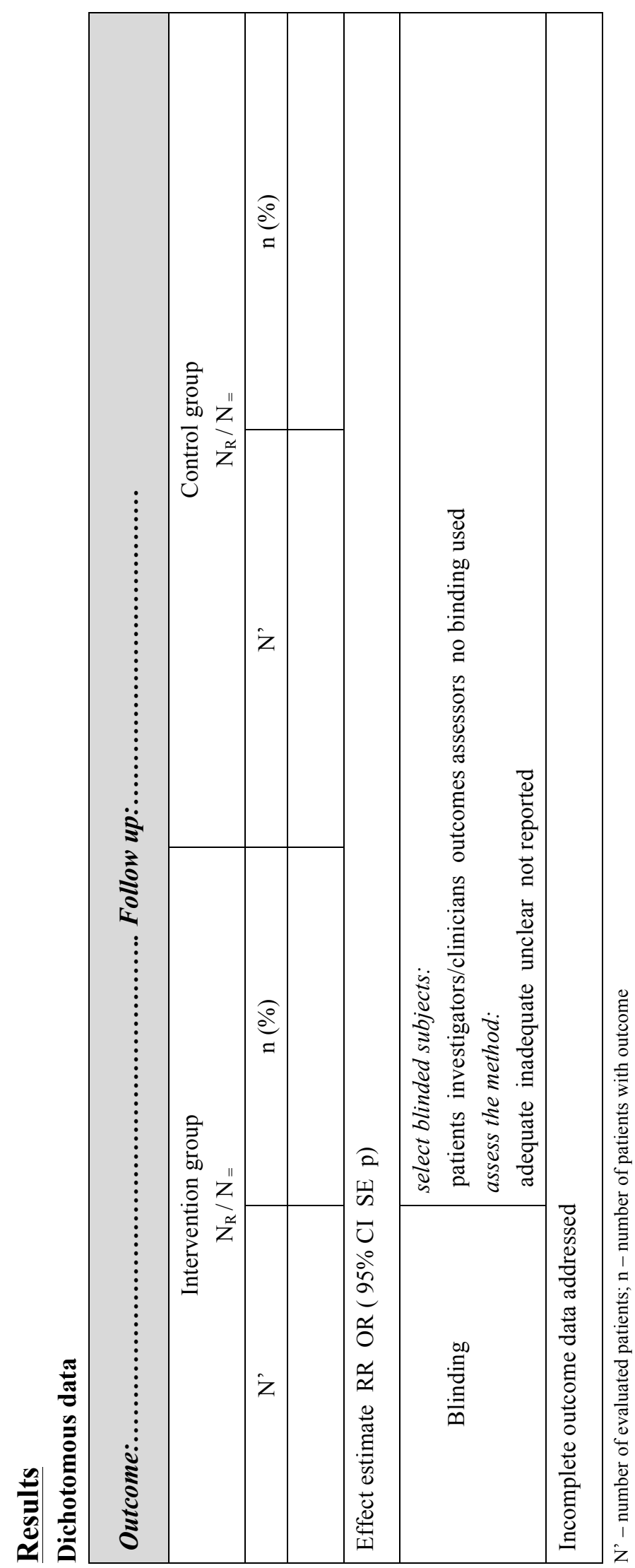




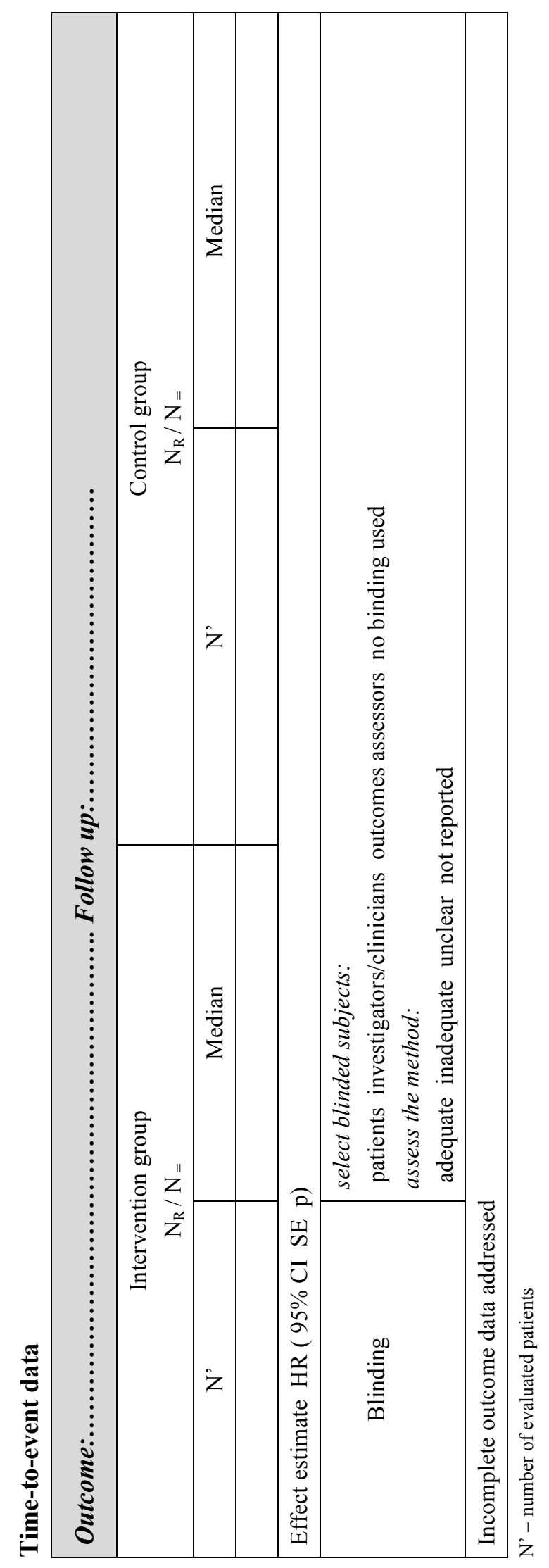




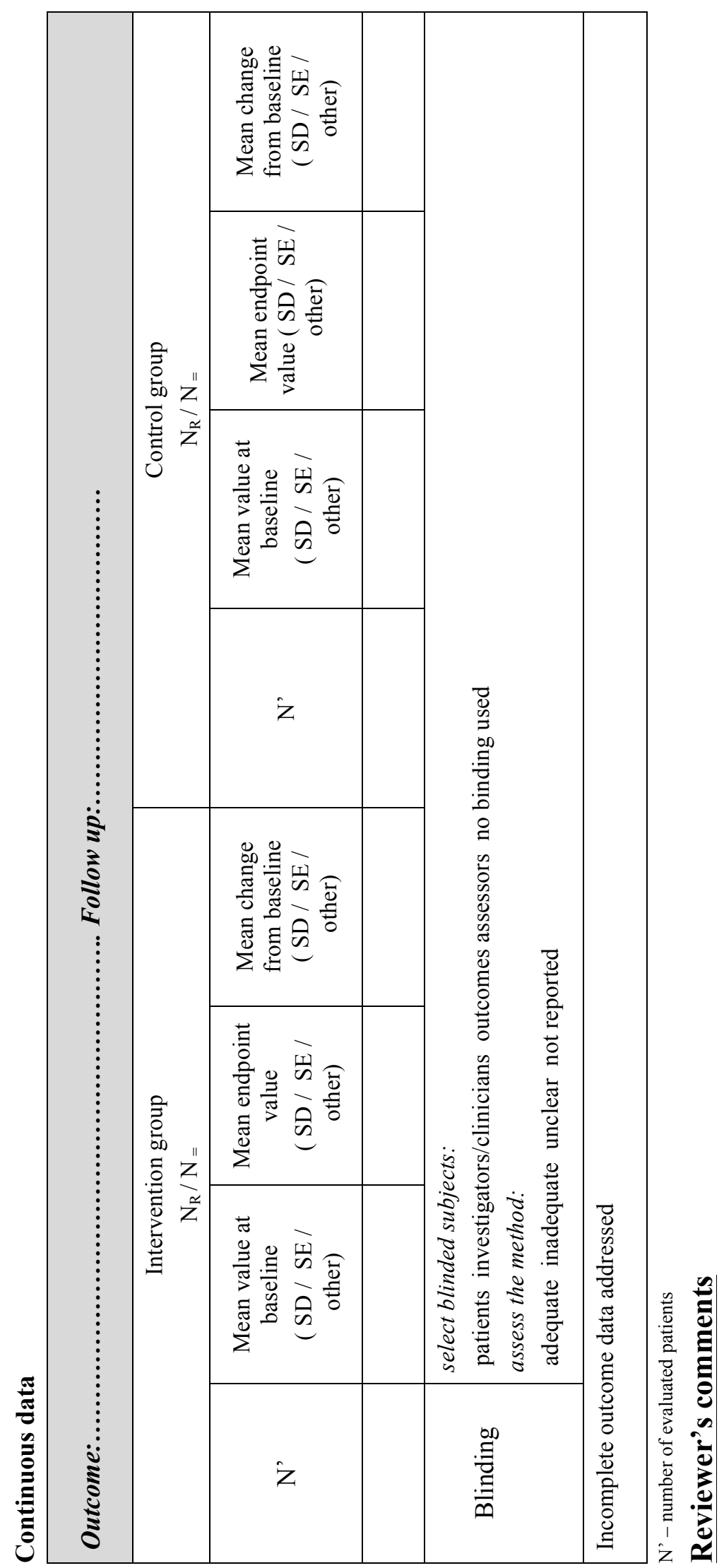




\section{PART C}

\section{Non-Comparative Studies:}

Quality assessment according checklist from "Methods for the development of NICE public health guidance (second edition)"

\section{Type of study,}

methodology decription

\begin{tabular}{|c|c|}
\hline \multirow{2}{*}{\multicolumn{2}{|c|}{\begin{tabular}{|l|l}
\multicolumn{2}{|c|}{ Population } \\
Trial inclusion criteria & \\
\end{tabular}}} \\
\hline & \\
\hline \multicolumn{2}{|l|}{ Trial exclusion criteria } \\
\hline \multicolumn{2}{|l|}{ Number of enrolled patients } \\
\hline \multicolumn{2}{|l|}{$\begin{array}{l}\text { Number of patients who } \\
\text { completed treatment, n (\%) }\end{array}$} \\
\hline \multicolumn{2}{|l|}{$\begin{array}{l}\text { Number of patients available for } \\
\text { follow up, } \mathrm{n}(\%)\end{array}$} \\
\hline \multicolumn{2}{|l|}{$\begin{array}{l}\text { Age, in years } \\
\text { specify the measure: ............... }\end{array}$} \\
\hline \multicolumn{2}{|l|}{$\begin{array}{l}\text { Other baseline characteristics } \\
\text { (stage: \% recurrent, metastatic, } \\
\text { persistent, prior therapy, site of } \\
\text { disease: pelvic, distant, both) }\end{array}$} \\
\hline \multicolumn{2}{|r|}{ Treatment } \\
\hline \multicolumn{2}{|l|}{$\begin{array}{l}\text { Type of treatment used } \\
\text { (technique, no. of sessions) }\end{array}$} \\
\hline \multicolumn{2}{|l|}{ Treatment duration } \\
\hline \multicolumn{2}{|l|}{ Duration of follow up } \\
\hline \multicolumn{2}{|r|}{ Outcomes } \\
\hline $\begin{array}{l}\text { Definition and unit of } \\
\text { measurement }\end{array}$ & \\
\hline
\end{tabular}




\section{Results}

Dichotomous data

Outcome: .............................................. Follow up:..........

\section{Intervention group}

\begin{tabular}{|l|l|}
\hline \multicolumn{1}{|c|}{$\mathrm{N}$} & $\mathrm{n}(\%)$ \\
\hline & \\
\hline$(95 \%$ CI SE $\mathrm{p})$ & \\
\hline Incomplete outcome data addressed & \\
\hline
\end{tabular}

$N^{\prime}$ - number of evaluated patients; $\mathrm{n}$ - number of patients with outcome

\section{Time to event data}

\begin{tabular}{|c|c|}
\hline \multicolumn{2}{|c|}{ 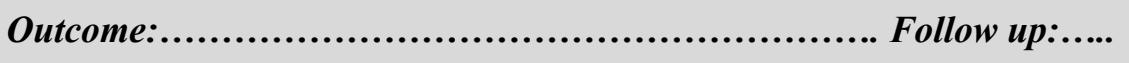 } \\
\hline \multicolumn{2}{|r|}{ Intervention group } \\
\hline $\mathrm{N}$ & Median \\
\hline \multicolumn{2}{|l|}{$(95 \%$ CI SE p) } \\
\hline Incomplete outcome data addressed & \\
\hline
\end{tabular}

$\mathrm{N}^{\prime}$ - number of evaluated patients; $\mathrm{n}$ - number of patients with outcome

\section{Continuous data}

\begin{tabular}{|c|c|c|c|}
\hline \multicolumn{4}{|c|}{ Outcome:.................................................. Follow up:................ } \\
\hline \multicolumn{4}{|c|}{ Intervention group } \\
\hline $\mathrm{N}$ & $\begin{array}{c}\text { Mean value at baseline } \\
\text { ( SD / SE / other) }\end{array}$ & $\begin{array}{l}\text { Mean endpoint value } \\
\text { ( SD / SE / other) }\end{array}$ & $\begin{array}{l}\text { Mean change from baseline } \\
\quad \text { ( SD / SE / other) }\end{array}$ \\
\hline \multicolumn{4}{|l|}{$\mathrm{p}$} \\
\hline Incomple & data addressed & & \\
\hline
\end{tabular}

N' - number of evaluated patients; $n$ - number of patients with outcome 



\section{Appendix 5 Case series quality assessment form}

Checklist used for quality assessment of case series 


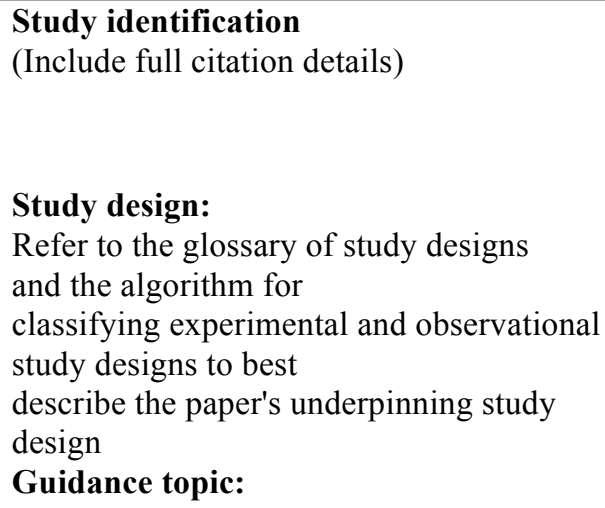

Assessed by:

\section{Section 1: Population}

1.1 Is the source population or source

area well described?

Was the country (e.g.

developed or nondeveloped,

type of health care system),

setting (primary schools,

community centres etc.),

location (urban, rural),

population demographics etc. $\square$ NA

adequately described?

1.2 Is the eligible population

or area

$\square++$

representative of the source

population

or area?

Was the recruitment of

individuals/clusters/areas well

defined (e.g. advertisement,

birth register)?

Was the eligible population $\square$ NA

representative of the source?

Were important groups under-

represented?

1.3 Do the selected

participants or

Comments:

areas represent the eligible

population

or area?

Was the method of selection of

participants from the eligible

population

Comments:

well described?

What $\%$ of selected

individuals/clusters agreed to 
participate? Were there any

sources of bias?

Were the in-/exclusion criteria

explicit and

appropriate?

Section 2: Method of Allocation to intervention (or comparison)

2.1 Allocation to intervention
(or
comparison). How was
selection bias
minimised?
Was allocation to exposure and
comparison randomised? Was it
truly
random ++ or pseudo-
randomised + (e.g.
consecutive admissions)?
If not randomised, was
significant
confounding likely (-) or not
(+)?
If a cross-over, was order of
intervention
randomised?

2.2 Were interventions (and comparisons) well described

and

appropriate?

Were intervention/s \&

comparison/s described in

sufficient detail (i.e. enough for

study to be replicated)?

Comments:

Was comparison/s appropriate

(e.g. usual practice rather than no intervention)?

\subsection{Was the allocation}

concealed?

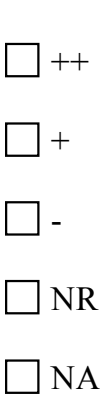

Comments:

Could the person(s)

determining allocation of

participants/clusters to

intervention or comparison

groups have influenced the

allocation?

Adequate allocation

concealment $(++)$ would

include centralised allocation or

computerised allocation

systems.

2.4 Were participants and/or investigators blind to

exposure and

comparison?

Were participants AND 


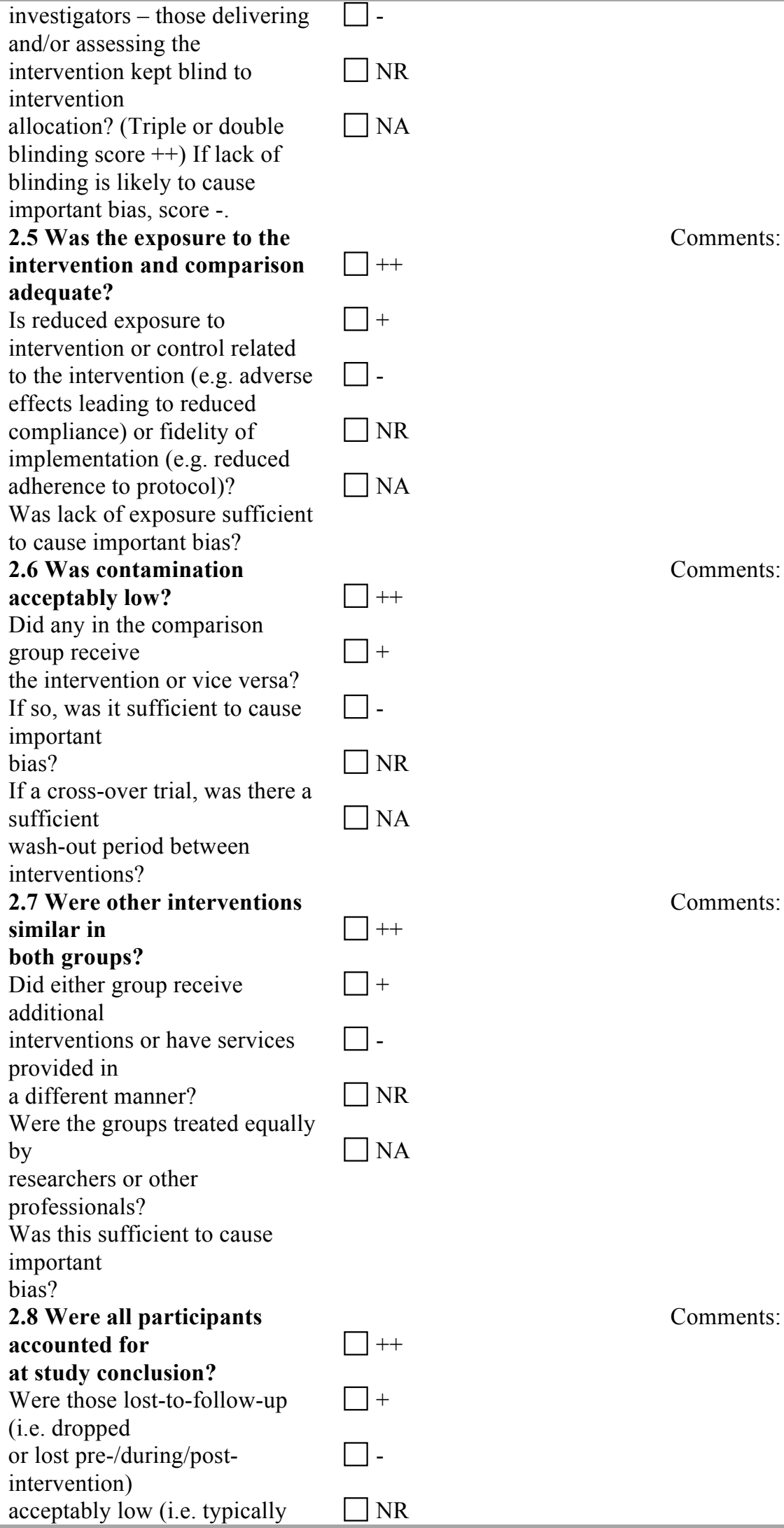

If a cross-over trial, was there a sufficient

wash-out period between

interventions?

2.7 Were other interventions similar in

both groups?

Did either group receive

additional

interventions or have services

provided in

a different manner?

Were the groups treated equally

by

researchers or other

professionals?

Was this sufficient to cause

important

bias?

2.8 Were all participants accounted for

at study conclusion?

Were those lost-to-follow-up $\quad \square+$

(i.e. dropped

or lost pre-/during/post-

intervention)

acceptably low (i.e. typically $\quad \square$ NR

Comments:

Comments:

Comments:

Comments: 
$<20 \%) ?$

Did the proportion dropped

NA

differ by

group? For example, were

drop-outs

related to the adverse effects of

the

intervention?

\subsection{Did the setting reflect}

\section{usual UK}

practice?

Did the setting in which the intervention or comparison was delivered differ

significantly from usual

practice in the UK?

For example, did participants

receive

intervention (or comparison)

condition in a

hospital rather than a

community-based

setting?

2.10 Did the intervention or

\section{control}

comparison reflect usual UK

practice?

Did the intervention or

comparison differ

significantly from usual

practice in the UK?

For example, did participants

receive

intervention (or comparison)

delivered by

specialists rather than GPs?

Were

participants monitored more

closely?

Comments:

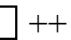

$\square+$

-

$\mathrm{NR}$

NA

Comments:
$++$
$+$
-
NR
NA

Section 3: Outcomes

3.1 Were outcome measures reliable?

Comments:

Were outcome measures

subjective or

objective (e.g. biochemically

validated

nicotine levels ++ vs self-

reported smoking

$-)$.

How reliable were outcome

measures (e.g.

inter- or intra-rater reliability

scores)? 
Was there any indication that

measures

had been validated (e.g.

validated against

a gold standard measure or

assessed for

content validity)

3.2 Were all outcome

measurements

complete?

Were all/most study

participants who met

the defined study outcome

definitions likely

to have been identified?

3.3 Were all important outcomes

assessed?

Were all important benefits and

harms

assessed? Was it possible to

determine the overall

balance of benefits and harms

of the

intervention versus

Comments:

comparison?

3.4 Were outcomes relevant?

Where surrogate outcome

measures were

used, did they measure what

they set out

to measure? (e.g. a study to

assess impact

on physical activity assesses

gym

membership - a potentially

objective

outcome measure - but is it a

reliable

predictor of physical activity?)

3.5 Were there similar follow-

up times

in exposure and comparison

groups?

If groups are followed for

different lengths

of time, then more events are

likely to

Comments:

Comments:

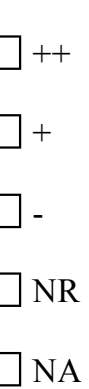


occur in the group followed-up

for longer

distorting the comparison.

Analyses can be adjusted to

allow for

differences in length of follow-

up (e.g.

using person-years)

3.6 Was follow-up time

meaningful?

Comments:

Was follow-up long enough to

assess longterm

benefits/harms?

Was it too long, e.g.

participants lost to

follow-up?

\author{
$++$ \\ NA
}

$+$

Section 4: Analyses

\title{
4.1 Were exposure and
}

comparison

Comments:

groups similar at baseline? If

not, were

these adjusted?

Were there any differences

between

groups in important

confounders at

baseline?

If so, were these adjusted for in

the

analyses (e.g. multivariate

analyses or

stratification).

Were there likely to be any

residual

differences of relevance?

\subsection{Was Intention to treat}

(ITT) analysis

conducted?

Were all participants (includin

those that

dropped out or did not fully

complete the

intervention course) analysed in

the

groups (i.e. intervention or

comparison) to 
which they were originally

allocated?

4.3 Was the study sufficiently

powered

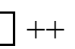

Comments:

to detect an intervention

effect (if one

exists)?

A power of 0.8 (i.e. it is likely

to see an

effect of a given size if one

exists, $80 \%$ of

the time) is the conventionally

accepted

standard.

Is a power calculation

presented? If not,

what is the expected effect

size? Is the

sample size adequate?

4.4 Were the estimates of

effect size

given or calculable?

Were effect estimates (e.g.

relative risks,

absolute risks) given or possible $\square$ -

calculate?

4.5 Were the analytical

Comments :

\section{methods \\ appropriate?}

Were important differences in

follow-up

time and likely confounders

adjusted for?

If a cluster design, were

analyses of

sample size (and power), and

effect size

performed on clusters (and not individuals)?

Were subgroup analyses prespecified?

4.6 Was the precision of

intervention

effects given or calculable?

Were they

meaningful?

Were confidence intervals

and/or p-values

for effect estimates given or

Comments

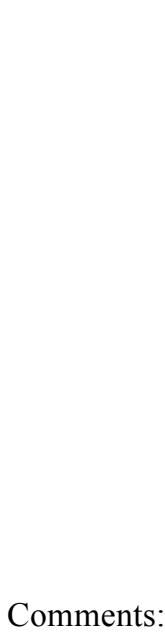


possible to calculate?

Were CI's wide or were they

sufficiently

precise to aid decision-making?

If

precision is lacking, is this

because the

study is under-powered?

Section 5: Summary

\subsection{Are the study results} internally valid

(i.e. unbiased)?

How well did the study

\section{NA}

minimise sources of

bias (i.e. adjusting for potential

confounders)?

Were there significant flaws in

the study

design?

5.2 Are the findings

generalisable to the

source population (i.e.

externally

valid)?

Are there sufficient details

given about the

study to determine if the

findings are

generalisable to the source

population?

Consider: participants,

interventions and

comparisons, outcomes,

resource and

policy implications. 



\section{Appendix 6 Diagnostic systematic review search strategies}

\section{MEDLINE (Ovid Gateway) (May 2010)}

Two thousand, five hundred and eighty-six records were retrieved in MEDLINE.

1. exp Uterine Cervical Neoplasms/

2. (cervi\$ adj5 cancer\$).mp.

3. (cervi\$ adj5 carcinom\$).mp.

4. (cervi $\$$ adj5 adenocarcinom $\$$ ).mp.

5. (cervi\$ adj5 carcinogen\$).mp.

6. (cervi\$ adj5 sarcoma\$).mp.

7. (cervi\$ adj5 malignan\$).mp.

8. (cervi\$ adj5 tumor\$).mp.

9. (cervi\$ adj5 tumour\$).mp.

10. (cervi\$ adj5 neoplas\$).mp.

11. (cervi $\$$ adj5 metasta $\$$ ).mp.

12. 1 or 2 or 3 or 4 or 5 or 6 or 7 or 8 or 9 or 10 or 11

13. exp Recurrence/ or exp Neoplasm Recurrence, Local/

14. recur\$.mp.

15. relaps $\$ . m p$.

16. repeat\$.mp.

17. repetitive $\$ . m p$.

18. reappearance $\$ . m p$.

19. reoccurence $\$$.mp.

20. return.mp.

21. exp Neoplasm Metastasis/

22. metasta \$.mp.

23. restag $\$ . m p$.

24. re-stag.mp.

25. 13 or 14 or 15 or 16 or 17 or 18 or 19 or 20 or 21 or 22 or 23 or 24

26. 12 and 25

27. exp Diagnostic Imaging/

28. exp Imaging, Three-Dimensional/

29. exp magnetic resonance imaging/ or exp diffusion magnetic resonance imaging/ or exp magnetic resonance imaging, cine/

30. exp Magnetic Resonance Spectroscopy/

31. magnetic resonance imaging.mp.

32. magnetic resonance.mp.

33. mri.mp.

34. $\mathrm{mr}$ imaging. $\mathrm{mp}$.

35. mri scan\$.mp.

36. exp Radionuclide Imaging/

37. exp tomography, emission-computed/ or exp positron-emission tomography/

38. (emission adj2 comput $\$$ adj2 tomograph\$).mp.

39. (tomograph $\$$ adj2 emission adj2 comput\$).mp.

40. (radionuclide adj2 cat scan\$).mp.

41. (scintigraph\$ adj2 comput\$ adj2 tomograph\$).mp. 
42. (positron adj2 emission adj2 tomograph\$).mp.

43. (radionuclide adj2 ct scan\$).mp.

44. (positron adj2 tomograph\$).mp.

45. (pet or petct).mp.

46. fdg-pet.mp.

47. exp tomography, x-ray computed/ or exp tomography/ or exp tomography, x-ray/

48. computer tomograph\$.mp.

49. computer tomogram $\$$.mp.

50. computer assisted tomograph\$.mp.

51. computer assisted tomogram $\$ . \mathrm{mp}$.

52. ct.mp.

53. $\mathrm{mr}$ scan\$.mp.

54. 27 or 28 or 29 or 30 or 31 or 32 or 33 or 34 or 35 or 36 or 37 or 38 or 39 or 40 or 41 or 42 or 43 or 44 or 45 or 46

55. or 47 or 48 or 49 or 50 or 51 or 52 or 53

56. 26 and 54

\section{EMBBASE (Ovid Gateway) (May 2010)}

Two thousand, six hundred and eighty-nine records were retrieved in EMBASE.

1. exp uterine cervix tumor/

2. (cervi\$ adj5 cancer\$).mp.

3. (cervi\$ adj5 carcinom\$).mp

4. (cervi $\$$ adj5 adenocarcinom\$).mp.

5. (cervi\$ adj5 carcinogen\$).mp.

6. (cervi\$ adj5 sarcoma\$).mp.

7. (cervi\$ adj5 malignan\$).mp.

8. (cervi\$ adj5 tumor\$).mp.

9. (cervi\$ adj5 tumour\$).mp.

10. (cervi $\$$ adj5 neoplas\$).mp.

11. (cervi\$ adj5 metasta\$).mp.

12. 1 or 2 or 3 or 4 or 5 or 6 or 7 or 8 or 9 or 10 or 11

13. exp recurrent disease/

14. recur\$.mp.

15. relaps\$.mp.

16. repeat\$.mp.

17. repetitive $\$ . m p$.

18. reappearance $\$ . m p$.

19. reoccurence $\$ . m p$.

20. return.mp.

21. exp metastasis/

22. metasta \$.mp.

23. restag\$.mp.

24. re-stag.mp.

25. 13 or 14 or 15 or 16 or 17 or 18 or 19 or 20 or 21 or 22 or 23 or 24

26. exp diagnostic imaging/

27. exp three dimensional imaging/

28. exp nuclear magnetic resonance imaging/

29. exp diffusion weighted imaging/

30. exp nuclear magnetic resonance imaging/ 
31. exp nuclear magnetic resonance spectroscopy/

32. magnetic resonance imaging.mp.

33. magnetic resonance. $\mathrm{mp}$.

34. mri.mp.

35. mr imaging.mp.

36. $\mathrm{mri}$ scan $\$ . m p$

37. $\mathrm{mr}$ scan\$.mp.

38. exp emission tomography/ or exp tomography/ or exp positron emission tomography/

39. (emission adj2 comput\$ adj2 tomograph\$).mp.

40. (radionuclide adj2 cat scan\$).mp.

41. (radionuclide adj2 ct scan\$).mp.

42. (scintigraph\$ adj2 comput\$ adj2 tomograph\$).mp.

43. (positron adj2 emission adj2 tomograph\$).mp.

44. (positron adj2 tomograph\$).mp.

45. (pet or petct).mp.

46. fdg-pet.mp.

47. exp computer assisted tomography/

48. tomography, x-ray.mp. or exp tomography/

49. computer tomograph\$.mp.

50. computer tomogram $\$ . \mathrm{mp}$.

51. computer assisted tomograph\$.mp.

52. computer assisted tomogram\$.mp.

53. ct.mp.

54. 26 or 27 or 28 or 29 or 30 or 31 or 32 or 33 or 34 or 35 or 36 or 37 or 38 or 39 or 40 or 41 or 42 or 43 or 44 or 45 or 46 or 47 or 48 or 49 or 50 or 51 or 52 or 53

55. 12 and 25

56. 54 and 55

\section{Cochrane Database of Systematic Reviews, Cochrane Central Register of Controlled Trials, Database of Abstracts of Reviews of Effects and Health Technology Assessment datalbase (May 2010)}

Eighty-six records were retrieved in CDSR, CENTRAL, DARE and HTA.

1. MeSH descriptor Uterine Cervical Neoplasms explode all trees

2. (cervi* near/5 neoplas*) in Cochrane Reviews, Other Reviews, Clinical Trials and Technology Assessments

3. (cervi ${ }^{\star}$ near/5 carcinom*) in Cochrane Reviews, Other Reviews, Clinical Trials and Technology Assessments

4. (cervi* near/5 malignan*) in Cochrane Reviews, Other Reviews, Clinical Trials and Technology Assessments

5. (cervi ${ }^{*}$ near/5 tumor*) in Cochrane Reviews, Other Reviews, Clinical Trials and Technology Assessments

6. (cervi* near/5 tumour*) in Cochrane Reviews, Other Reviews, Clinical Trials and Technology Assessments

7. (cervi* near/5 cancer*) in Cochrane Reviews, Other Reviews, Clinical Trials and Technology Assessments

8. (cervi* near/5 adenocarcinom*) in Cochrane Reviews, Other Reviews, Clinical Trials and Technology Assessments

9. (cervi* near/5 carcinogen*) in Cochrane Reviews, Other Reviews, Clinical Trials and Technology Assessments

10. (cervi* near/5 metasta*) in Cochrane Reviews, Other Reviews, Clinical Trials and Technology Assessments

11. (cervi* near/5 cyst*) in Cochrane Reviews, Other Reviews, Clinical Trials and Technology Assessments 
12. \#1 OR \#2 OR \#3 OR \#4 OR \#5 OR \#6 OR \#7 OR \#8 OR \#9 OR \#10 OR \#11

13. (recur $^{*}$ ) in Cochrane Reviews, Other Reviews, Clinical Trials and Technology Assessments

14. (relaps*) in Cochrane Reviews, Other Reviews, Clinical Trials and Technology Assessments

15. (repeat*) in Cochrane Reviews, Other Reviews, Clinical Trials and Technology Assessments

16. (reappearance*) in Cochrane Reviews, Other Reviews, Clinical Trials and Technology Assessments

17. (reoccurence* ${ }^{*}$ in Cochrane Reviews, Other Reviews, Clinical Trials and Technology Assessments

18. (return) in Cochrane Reviews, Other Reviews, Clinical Trials and Technology Assessments

19. MeSH descriptor Neoplasm Metastasis explode all trees

20. (restag*) in Cochrane Reviews, Other Reviews, Clinical Trials and Technology Assessments

21. (re-stag) in Cochrane Reviews, Other Reviews, Clinical Trials and Technology Assessments

22. (metasta*) in Cochrane Reviews, Other Reviews, Clinical Trials and Technology Assessments

23. \#13 OR \#14 OR \#15 OR \#16 OR \#17 OR \#18 OR \#19 OR \#20 OR \#21 OR \#22

24. \#12 AND \#23

25. (diagnostic imaging) in Cochrane Reviews, Other Reviews, Clinical Trials and Technology Assessments

26. MeSH descriptor Imaging, Three-Dimensional explode all trees

27. MeSH descriptor Magnetic Resonance Imaging explode all trees

28. MeSH descriptor Diffusion Magnetic Resonance Imaging explode all trees

29. MeSH descriptor Magnetic Resonance Imaging, Cine explode all trees

30. (magnetic resonance) in Cochrane Reviews, Other Reviews, Clinical Trials and Technology Assessments

31. (mri) in Cochrane Reviews, Other Reviews, Clinical Trials and Technology Assessments

32. (mr imaging) in Cochrane Reviews, Other Reviews, Clinical Trials and Technology Assessments

33. (mri scan*) in Cochrane Reviews, Other Reviews, Clinical Trials and Technology Assessments

34. (mr scan*) in Cochrane Reviews, Other Reviews, Clinical Trials and Technology Assessments

35. MeSH descriptor Tomography, Emission-Computed explode all trees

36. MeSH descriptor Tomography explode all trees

37. MeSH descriptor Positron-Emission Tomography explode all trees

38. (emission near/2 comput* near/2 tomograph*) in Cochrane Reviews, Other Reviews, Clinical Trials and Technology Assessments

39. (radionuclide near/2 cat scan*) in Cochrane Reviews, Other Reviews, Clinical Trials and Technology Assessments

40. (radionuclide near/2 ct scan*) in Cochrane Reviews, Other Reviews, Clinical Trials and Technology Assessments

41. (scintigraph* near/2 comput* near/2 tomograph*) in Cochrane Reviews, Other Reviews, Clinical Trials and Technology Assessments

42. (positron near/2 emission near/2 tomograph) in Cochrane Reviews, Other Reviews, Clinical Trials and Technology Assessments

43. (positron near/2 tomograph*) in Cochrane Reviews, Other Reviews, Clinical Trials and Technology Assessments

44. (pet or petct) in Cochrane Reviews, Other Reviews, Clinical Trials and Technology Assessments

45. (fdg-pet) in Cochrane Reviews, Other Reviews, Clinical Trials and Technology Assessments

46. MeSH descriptor Tomography, X-Ray explode all trees

47. MeSH descriptor Tomography, X-Ray Computed explode all trees

48. (computer tomograph*) in Cochrane Reviews, Other Reviews, Clinical Trials and Technology Assessments

49. (computer tomogram*) in Cochrane Reviews, Other Reviews, Clinical Trials and Technology Assessments

50. (computer assisted tomograph*) in Cochrane Reviews, Other Reviews, Clinical Trials and Technology Assessments

51. (computer assisted tomogram*) in Cochrane Reviews, Other Reviews, Clinical Trials and Technology Assessments

52. (ct) in Cochrane Reviews, Other Reviews, Clinical Trials and Technology Assessments 
53. \#25 OR \#26 OR \#27 OR \#28 OR \#29 OR \#30 OR \#31 OR \#32 OR \#33 OR \#34 OR \#35 OR \#36 OR \#37 OR \#38 OR \#40 OR \#41 OR \#42 OR \#43 OR \#44 OR \#45 OR \#46 OR \#46 OR \#47 OR \#48 OR \#49 OR \#50 OR \#51 OR \#52

54. \#24 AND \#53 



\section{Appendix 7 Subjective elicitation questionnaire}




\section{THE USE OF PETCT IN THE INVESTIGATION OF RECURRENT CERVICAL CANCER}

Currently in the United Kingdom, patients with suspected cervical cancer recurrence will undergo

- clinical examination (rectovaginal and speculum examination, assessment of inguinal/ supraclavicular lymph nodes)

- cross sectional imaging by MRI (Magnetic Resonance Imaging) or CT (Computed Tomography) of chest, abdomen and pelvis

- examination under anaesthesia, histological confirmation of any vaginal vault mass by biopsies.

The HTA project is evaluating the added value of PET/CT to current imaging practice for restaging women with recurrent cervical cancer. Information from the elicitation exercise will be used to complement the findings of a systematic review in order to achieve objective 1 in figure 1 below:

Fig 1: Imaging modalities and treatment strategies in women with recurrent cervical cancer

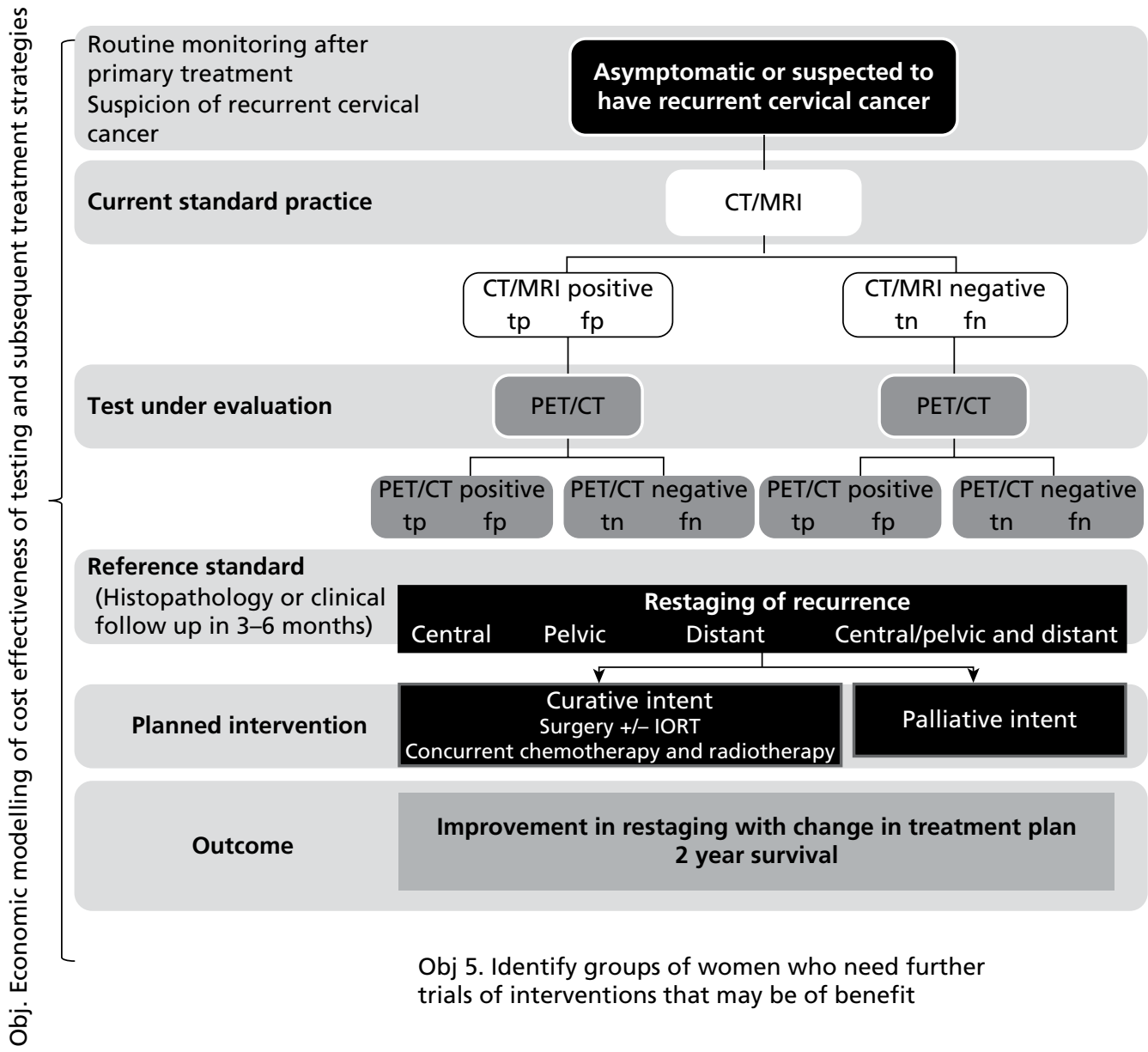

Obj 1. Update and compiling of test accuracy of PET-CT and CT or MRI in diagnosing recurrent cervical cancer

Obj 2.

Assessment of diagnostic impact of PET-CT on restaging compared to CT or MRI

Obj 3.

Evaluation of therapeutic impact of PET-CT on treatment plan

The accuracy of PETCT in addition to CT/MRI will be examined for women with initial stage I-IV disease presenting with symptoms and for surveillance of asymptomatic women with initial stage 1B2IV. 


\section{BACKGROUND INFORMATION}

The following information is to assist with interpretation of the information we are about to elicit. For example, your estimates of accuracy may vary according to your speciality or to your experience of using PETCT.

1) Speciality

2) Years working in your current speciality

3) In any one single follow up consultation for patients under surveillance following an initial diagnosis of cervical cancer, in what \% of patients do you estimate using MRI alone; CT alone; a combination of $\mathrm{CT}$ and MRI?

Indicate the \% of patients who you estimate receive (CT); (MRI );(CT and MRI)ensuring the total \% of patients sums to $100 \%$

\begin{tabular}{|c|c|}
\hline Imaging & \% of patients receiving tests in any one follow up consultation \\
\hline CT alone & \\
\hline MRI alone & \\
\hline CT + MRI & $100 \%$ \\
\hline TOTAL & \\
\hline
\end{tabular}

4) Do you currently use PETCT as part of the investigation of recurrent cervical cancer?

\section{Yes / No}

4 a) If you answered 'Yes' to Q.4, please state how long you have been using PETCT as part of the investigation of recurrent cervical cancer

4 b) If you answered 'Yes' to Q.4, please briefly describe in which patients or circumstances you use PETCT 
WHAT IS THE PREVALENCE OF RECURRENT DISEASE?

The first piece of information we would like to elicit from you is your estimate of the prevalence of recurrent cancer in symptomatic and asymptomatic women 3 months post completion of primary treatment.

4) Of women with a mix of initial stage I-IV cervical cancer presenting with symptoms suspicious for recurrence a minimum of 3 months post completion of treatment, what \% would you estimate to have recurrent disease?

Indicate the likelihood of each option by allocating a total of 100 points across the 5 options.

\begin{tabular}{|c|c|c|c|c|c|c|c|}
\hline $\begin{array}{c}\% \text { of symptomatic } \\
\text { women with recurrence } \\
\text { confirmed }\end{array}$ & $<50 \%$ & $51-60 \%$ & $61-70 \%$ & $71-80 \%$ & $81-90 \%$ & $90-100 \%$ & \\
\hline Points out of 100 & & & & & & & $\begin{array}{l}\text { Total } \\
=100\end{array}$ \\
\hline
\end{tabular}

5) Of asymptomatic women with a mix of initial stage IB2-IV cervical cancer a minimum of 3 months post completion of treatment, what \% would you estimate to have recurrent disease?

\begin{tabular}{|c|l|l|l|l|l|l|l|}
\hline $\begin{array}{c}\text { \% of asymptomatic } \\
\text { women with recurrence } \\
\text { confirmed }\end{array}$ & $0-10 \%$ & $11-20 \%$ & $21-30 \%$ & $31-40 \%$ & $41-50 \%$ & $>50 \%$ & \\
\hline \begin{tabular}{c} 
Points out of 100 \\
\hline
\end{tabular} & & & & & & $\begin{array}{c}\text { Total } \\
=100\end{array}$ \\
\hline
\end{tabular}




\section{ACCURACY OF IMAGING IN SYMPTOMATIC} INITIAL STAGE 1-IV CERVICAL CANCER 
The use of MRI and/or CT alone compared to the use of MRI and/or CT + PETCT in the diagnosis of recurrence in patients with an initial diagnosis of stage I to IV cervical cancer.

\section{SYMPTOMATIC PATIENTS}

-All patients are assumed to be a minimum of 3 months post completion of initial treatment (surgery+ chemotherapy or chemotherapy only).

-All patients are assumed to be symptomatic and have had a clinical examination which may be und anaesthesia (histological confirmation of any vaginal vault mass by biopsies) or not under general anaesthesia (rectovaginal and speculum examination, assessment of inguinal/ supraclavicular lymph nodes).

-Patients subsequently receive either:

-CT and/or MRI at the discretion of their physician and irrespective of the results of clinical examination. In other words clinical examination is not used to triage patients for further imaging with CT and/or MRI; CT and/or MRI are used as an add on to clinical examination.

OR

- CT and/or MRI at the discretion of their physician and irrespective of the results of clinical examination and PETCT. In other words CT and/or MRI are not used to triage patients for further imaging with PETCT ; PETCT is used as an add on to CT and/or MRI.

\section{ACCURACY OF CT and/or MRI}

-Of the patients who test positive following investigation with CT and/or MRI, what percentage । consider will subsequently be diagnosed as negative for recurrence following histology and / or । follow up as the gold standard tests. We are asking you to estimate the percentage of those who tı positive with CT and/or MRI who receive a false positive diagnosis (are actually disease negativ

Indicate the likelihood of each option by allocating a total of 100 points across the 5 options.

\begin{tabular}{|c|l|l|l|l|l|l|}
\hline \begin{tabular}{c} 
False positives (disease -ve) \\
\hline Test positives on CT and/or MRI
\end{tabular} & $0-9 \%$ & $\begin{array}{l}10 \\
19 \%\end{array}$ & $20-29 \%$ & $\begin{array}{l}30 \\
39 \%\end{array}$ & $\begin{array}{l}40 \\
49 \%\end{array}$ & - \\
\hline Points out of 100 & & & & & & $\begin{array}{l}\text { Total } \\
=100\end{array}$ \\
\hline
\end{tabular}

-Of the patients who test negative following investigation with CT and/or MRI, what percentage consider will subsequently be diagnosed as positive for recurrence following histology and / or $c$ follow up as the gold standard tests. We are asking you to estimate the percentage of those who tı negative with CT and/or MRI who receive a false negative diagnosis (are actually disease positi

Indicate the likelihood of each option by allocating a total of 100 points across the 5 options.

\begin{tabular}{|c|l|l|l|l|l|l|}
\hline $\begin{array}{c}\text { False negatives (disease +ve) } \\
\text { Test negatives on CT and/or } \\
\text { MRI }\end{array}$ & $0-9 \%$ & $\begin{array}{l}10 \\
19 \%\end{array}$ & $20-29 \%$ & $\begin{array}{l}30 \\
39 \%\end{array}$ & $\begin{array}{l}40 \% \\
49 \%\end{array}$ & \\
\hline Points out of 100 & & & & & & $\begin{array}{l}\text { Total } \\
=100\end{array}$ \\
\hline
\end{tabular}


The use of MRI and/or CT alone compared to the use of MRI and/or CT + PETCT in the diagnosis of recurrence in patients with an initial diagnosis of

stage I-IV cervical cancer.

\section{SYMPTOMATIC PATIENTS}

-All patients are assumed to be a minimum of 3 months post completion of initial treatment (surgery+/chemotherapy or chemotherapy only).

-All patients are assumed to be symptomatic and have had a clinical examination which may be under anaesthesia (histological confirmation of any vaginal vault mass by biopsies) or not under general anaesthesia (rectovaginal and speculum examination, assessment of inguinal/ supraclavicular lymph nodes).

-Patients subsequently receive either:

- CT and/or MRI at the discretion of their physician and irrespective of the results of clinical examination. In other words clinical examination is not used to triage patients for further imaging with CT and/or MRI; CT and/or MRI are used as an add on to clinical examination.

OR

- CT and/or MRI at the discretion of their physician and irrespective of the results of clinical examination and PETCT. In other words CT and/or MRI are not used to triage patients for further imaging with PETCT ; PETCT is used as an add on to CT and/or MRI.

\section{ACCURACY OF CT and/or MRI +PETCT}

-Of the patients who test positive following investigation with CT and/or MRI + PETCT, what percentage do you consider will subsequently be diagnosed as negative for recurrence following histology and / or clinical follow up as the gold standard tests. We are asking you to estimate the percentage of those who test positive with CT and/or MRI + PETCT who are false positives (are actually disease negative).

Indicate the likelihood of each option by allocating a total of 100 points across the 5 options.

\begin{tabular}{|c|l|l|l|l|l|l|}
\hline $\begin{array}{c}\text { False positives (disease-ve) } \\
\begin{array}{c}\text { Test positives on CT and/or MRI } \\
\text { +PETCT }\end{array}\end{array}$ & $0-9 \%$ & $\begin{array}{l}10 \\
19 \%\end{array}$ & $\mathbf{2 0 - 2 9 \%}$ & $\begin{array}{l}30 \\
39 \%\end{array}$ & $\begin{array}{l}\mathbf{4 0} \\
\mathbf{4 9 \%}\end{array}$ & - \\
\hline $\begin{array}{c}\text { Points out of } 100 \\
\end{array}$ & & & & & $\begin{array}{l}\text { Total } \\
=100\end{array}$ \\
\hline
\end{tabular}

-Of the patients who test negative following investigation with CT and/or MRI + PETCT, what percentage do you consider will subsequently be diagnosed as positive for recurrence following histology and / or clinical follow up as the gold standard tests. We are asking you to estimate the percentage of those who test negative with CT and/or MRI + PETCT who are false negatives (are actually disease positive).

Indicate the likelihood of each option by allocating a total of 100 points across the 5 options.

\begin{tabular}{|c|c|c|c|c|c|c|}
\hline $\begin{array}{l}\text { False negatives (disease +ve) } \\
\text { Test negatives on CT and/or } \\
\text { MRI } \\
\text { + PETCT }\end{array}$ & $0-9 \%$ & $\begin{array}{l}10 \\
19 \%\end{array}$ & $20-29 \%$ & $\begin{array}{l}30 \\
39 \%\end{array}$ & $\begin{array}{l}40 \\
49 \%\end{array}$ & \\
\hline Points out of 100 & & & & & & $\begin{array}{l}\text { Total } \\
=100\end{array}$ \\
\hline
\end{tabular}


The use of MRI and/or CT alone compared to the use of PETCT as an adjunct to

MRI and/or CT in the diagnosis of recurrence in patients with an initial diagnosis of stage I-IV cervical cancer.

\section{SYMPTOMATIC PATIENTS}

What do you consider the minimum important clinical reduction in the number of false positives (the difference in the percentage of those who test positive who are false positives (are actually disease negative) before introducing PETCT as an adjunct to CT and/or MRI?

\begin{tabular}{|c|c|c|c|c|c|}
\hline $\begin{array}{c}\text { False positives (disease-ve) } \\
\begin{array}{c}\text { Test positives on } \\
\text { CT and/or MRI } \\
\text { +PETCT }\end{array}\end{array}$ & $0-2 \%$ & $3-5 \%$ & $6-8 \%$ & $9-11 \%$ & $\begin{array}{l}>12 \% \\
\text { (please } \\
\text { specify) }\end{array}$ \\
\hline
\end{tabular}

What do you consider the minimum important clinical reduction in the number of false positives (the difference in the percentage of those who test negative who are false negatives (are actually disease positive) before introducing PETCT as an adjunct to CT and/or MRI?

\begin{tabular}{|c|c|c|c|c|c|}
\hline $\begin{array}{c}\text { False negatives (disease +ve) } \\
\begin{array}{c}\text { Test negatives on } \\
\text { CT and/or MRI } \\
+ \text { PETCT }\end{array}\end{array}$ & $0-2 \%$ & $3-5 \%$ & $6-8 \%$ & $9-11 \%$ & $\begin{array}{l}>12 \% \\
\text { (please } \\
\text { specify) }\end{array}$ \\
\hline
\end{tabular}




\section{ACCURACY OF IMAGING IN} ASYMPTOMATIC

INITIAL STAGE 1B2-IV CERVICAL CANCER 
The use of MRI and/or CT alone compared to the use of MRI and/or CT + PETCT in the diagnosis of recurrence in patients with an initial diagnosis of stage IB2-IV cervical cancer.

\section{ASYMPTOMATIC PATIENTS}

-All patients are assumed to be a minimum of 3 months post completion of initial treatment (surgery+/chemotherapy or chemotherapy only).

-All patients are assumed to be asymptomatic and have had a clinical examination which may be under anaesthesia (histological confirmation of any vaginal vault mass by biopsies) or not under general anaesthesia (rectovaginal and speculum examination, assessment of inguinal/ supraclavicular lymph nodes).

-Patients subsequently receive either:

- CT and/or MRI at the discretion of their physician and irrespective of the results of clinical examination. In other words clinical examination is not used to triage patients for further imaging with $\mathrm{CT}$ and/or MRI; CT and/or MRI are used as an add on to clinical examination.

OR

- CT and/or MRI at the discretion of their physician and irrespective of the results of clinical examination and PETCT. In other words CT and/or MRI are not used to triage patients for further imaging with PETCT ; PETCT is used as an add on to CT and/or MRI.

\section{ACCURACY OF CT and/or MRI}

-Of the patients who test positive following investigation with CT and/or MRI, what percentage do you consider will subsequently be diagnosed as negative for recurrence following histology and / or clinical follow up as the gold standard tests. We are asking you to estimate the percentage of those who test positive with $C T$ and/or MRI who receive a false positive diagnosis (are actually disease negative).

Indicate the likelihood of each option by allocating a total of 100 points across the 5 options.

\begin{tabular}{|c|c|c|c|c|c|c|}
\hline $\begin{array}{l}\text { False positives (disease -ve) } \\
\text { Test positives on CT and/or MRI }\end{array}$ & $0-9 \%$ & $\begin{array}{l}10 \\
19 \%\end{array}$ & $20-29 \%$ & $\begin{array}{l}30 \\
39 \%\end{array}$ & $\begin{array}{ll}40 & - \\
49 \% & \end{array}$ & \\
\hline Points out of 100 & & & & & & $\begin{array}{l}\text { Total } \\
=100\end{array}$ \\
\hline
\end{tabular}

-Of the patients who test negative following investigation with CT and/or MRI, what percentage do you consider will subsequently be diagnosed as positive for recurrence following histology and / or clinical follow up as the gold standard tests. We are asking you to estimate the percentage of those who test negative with CT and/or MRI who receive a false negative diagnosis (are actually disease positive).

Indicate the likelihood of each option by allocating a total of 100 points across the 5 options.

\begin{tabular}{|c|l|l|l|l|l|l|}
\hline $\begin{array}{c}\text { False negatives (disease +ve) } \\
\text { Test negatives on CT and/or } \\
\text { MRI }\end{array}$ & $0-9 \%$ & $\begin{array}{l}10 \\
19 \%\end{array}$ & $20-29 \%$ & $\begin{array}{l}30 \\
39 \%\end{array}$ & $\begin{array}{l}40 \\
49 \%\end{array}$ & \\
\hline Points out of 100 & & & & & $\begin{array}{l}\text { Total } \\
=100\end{array}$ \\
\hline
\end{tabular}


The use of MRI and/or CT alone compared to the use of MRI and/or CT + PETCT in the diagnosis of recurrence in patients with an initial diagnosis of stage IB2-IV cervical cancer.

\section{ASYMPTOMATIC PATIENTS}

-All patients are assumed to be a minimum of 3 months post completion of initial treatment (surgery+/chemotherapy or chemotherapy only).

-All patients are assumed to be asymptomatic and have had a clinical examination which may be under anaesthesia (histological confirmation of any vaginal vault mass by biopsies) or not under general anaesthesia (rectovaginal and speculum examination, assessment of inguinal/ supraclavicular lymph nodes).

-Patients subsequently receive either:

- CT and/or MRI at the discretion of their physician and irrespective of the results of clinical examination. In other words clinical examination is not used to triage patients for further imaging with CT and/or MRI; CT and/or MRI are used as an add on to clinical examination.

OR

- CT and/or MRI at the discretion of their physician and irrespective of the results of clinical examination and PETCT. In other words CT and/or MRI are not used to triage patients for further imaging with PETCT ; PETCT is used as an add on to CT and/or MRI.

\section{ACCURACY OF CT and/or MRI + PETCT}

-Of the patients who test positive following investigation with CT and/or MRI + PETCT, what percentage do you consider will subsequently be diagnosed as negative for recurrence following histology and / or clinical follow up as the gold standard tests. We are asking you to estimate the percentage of those who test positive with CT and/or MRI + PETCT who are false positives (are actually disease negative).

Indicate the likelihood of each option by allocating a total of 100 points across the 5 options.

\begin{tabular}{|c|l|l|l|l|l|l|}
\hline $\begin{array}{c}\text { False positives (disease-ve) } \\
\begin{array}{c}\text { Test positives on CT and/or MRI } \\
\text { +PETCT }\end{array}\end{array}$ & $0-9 \%$ & $\begin{array}{l}10 \\
19 \%\end{array}$ & $-20-29 \%$ & $\begin{array}{l}30 \\
39 \%\end{array}$ & $\begin{array}{l}40 \\
\mathbf{4 9} \%\end{array}$ & - \\
\hline \begin{tabular}{c} 
Points out of 100 \\
\hline
\end{tabular} & & & & & $\begin{array}{l}\text { Total } \\
=100\end{array}$ \\
\hline
\end{tabular}

-Of the patients who test negative following investigation with CT and/or MRI + PETCT, what percentage do you consider will subsequently be diagnosed as positive for recurrence following histology and / or clinical follow up as the gold standard tests. We are asking you to estimate the percentage of those who test negative with CT and/or MRI + PETCT who are false negatives (are actually disease positive).

Indicate the likelihood of each option by allocating a total of 100 points across the 5 options.

\begin{tabular}{|c|c|c|c|c|c|c|}
\hline $\begin{array}{l}\text { False negatives (disease +ve) } \\
\text { Test negatives on CT and/or } \\
\text { MRI } \\
\text { + PETCT }\end{array}$ & $0-9 \%$ & $\begin{array}{l}10 \\
19 \%\end{array}$ & $20-29 \%$ & $\begin{array}{l}30 \\
39 \%\end{array}$ & $\begin{array}{l}40 \\
49 \%\end{array}$ & \\
\hline Points out of 100 & & & & & & $\begin{array}{l}\text { Total } \\
=100\end{array}$ \\
\hline
\end{tabular}


The use of MRI and/or CT alone compared to the use of PETCT as an adjunct to MRI and/or CT in the diagnosis of recurrence in patients with an initial diagnosis of stage IB2-IV cervical cancer.

\section{ASYMPTOMATIC PATIENTS}

Before introducing PETCT as an adjunct to CT and/or MRI, what \% reduction in false positives (the percentage of those who test positive who are actually disease free) would you consider necessary?

\begin{tabular}{|c|l|l|l|l|l|}
\hline $\begin{array}{c}\text { False positives (disease-ve) } \\
\begin{array}{c}\text { Test positives on } \\
\text { CT and/or MRI } \\
\text { +PETCT }\end{array}\end{array}$ & $0-2 \%$ & $3-5 \%$ & $6-8 \%$ & $9-11 \%$ & $\begin{array}{l}>12 \% \\
\text { (please } \\
\text { specify) }\end{array}$ \\
\hline
\end{tabular}

Before introducing PETCT as an adjunct to CT and/or MRI, what \% reduction in false negatives (the percentage of those who test negative who actually have disease) would you consider necessary?

\begin{tabular}{|c|c|c|c|c|l|}
\hline $\begin{array}{c}\text { False negatives (disease +ve) } \\
\begin{array}{c}\text { Test negatives on } \\
\text { CT and/or MRI } \\
\text { + PETCT }\end{array}\end{array}$ & $0-2 \%$ & $3-5 \%$ & $6-8 \%$ & $9-11 \%$ & $\begin{array}{l}>12 \% \\
\text { (please } \\
\text { specify) }\end{array}$ \\
\hline
\end{tabular}




\section{Appendix 8 Effectiveness systematic review search strategies}

\section{Population: previous chemoradiotherapy or radiotherapy only}

MEDLINE (Ovid Gateway) (August 2010)

Four thousand, nine hundred and forty-one records were retrieved in MEDLINE.

1. exp Uterine Cervical Neoplasms/

2. (cervi\$ adj5 cancer\$).mp.

3. (cervi\$ adj5 carcinom\$).mp.

4. (cervi\$ adj5 adenocarcinom\$).mp.

5. (cervi\$ adj5 carcinogen \$).mp.

6. (cervi\$ adj5 sarcoma\$).mp.

7. (cervi\$ adj5 malignan\$).mp.

8. (cervi\$ adj5 tumor\$).mp.

9. (cervi\$ adj5 tumour\$).mp.

10. (cervi $\$$ adj5 neoplas\$).mp.

11. (cervi $\$$ adj5 metasta\$).mp.

12. 1 or 2 or 3 or 4 or 5 or 6 or 7 or 8 or 9 or 10 or 11

13. exp Recurrence/ or exp Neoplasm Recurrence, Local/

14. recur\$.mp.

15. relaps\$.mp.

16. repeat\$.mp.

17. repetitive $\$ . m p$.

18. reappearance $\$ . m p$.

19. reoccurence $\$ . m p$.

20. return.mp.

21. exp Neoplasm Metastasis/

22. metasta\$.mp.

23. restag\$.mp.

24. re-stag.mp.

25. 13 or 14 or 15 or 16 or 17 or 18 or 19 or 20 or 21 or 22 or 23

26. 12 and 25

27. exp Radiotherapy/

28. Radiotherapy.mp.

29. irradiation.mp.

30. radiation.mp.

31. brachytherapy.mp.

32. teletherapy.mp.

33. (chemoradiation or chemoradiotherapy). $\mathrm{mp}$.

34. (chemoradiation or chemoradiotherapy).mp.

35. radiochemotherapy. $\mathrm{mp}$.

36. 27 or 28 or 29 or 30 or 31 or 32 or 33 or 34 or 35

37. 26 and 36 


\section{EMBASE (Ovid Gateway) (August 2010)}

Eight thousand, seven hundred and seventy-nine records were retrieved in EMBASE.

1. (Uterine Cervical Neoplasms

2. cervi\$ adj5 cancer\$).mp.

3. (cervi\$ adj5 carcinom\$).mp.

4. (cervi\$ adj5 adenocarcinom\$).mp.

5. (cervi\$ adj5 carcinogen\$).mp.

6. (cervi $\$$ adj5 sarcoma $\$$ ).mp.

7. (cervi\$ adj5 malignan\$).mp.

8. (cervi\$ adj5 tumor\$).mp.

9. (cervi $\$$ adj5 tumour\$).mp.

10. (cervi $\$$ adj5 neoplas\$).mp.

11. (cervi $\$$ adj5 metasta $\$$ ).mp.

12. 1 or 2 or 3 or 4 or 5 or 6 or 7 or 8 or 9 or 10 or 11

13. exp Recurrence/ or exp Neoplasm Recurrence, Local/

14. recur\$.mp.

15. relaps\$.mp.

16. repeat\$.mp.

17. repetitive $\$ . m p$.

18. reappearance $\$ . m p$.

19. reoccurence $\$ . m p$.

20. return.mp.

21. exp Neoplasm Metastasis/

22. metasta \$.mp.

23. restag $\$ . m p$.

24. re-stag.mp.

25. 13 or 14 or 15 or 16 or 17 or 18 or 19 or 20 or 21 or 22 or 23

26. 12 and 25

27. exp Radiotherapy/

28. radiotherapy.mp.

29. irradiation.mp.

30. radiation.mp.

31. brachytherapy.mp.

32. teletherapy.mp.

33. (chemoradiation or chemoradiotherapy).mp.

34. (chemo-radiation or chemo-radiotherapy).mp.

35. radiochemotherapy.mp.

36. 27 or 28 or 29 or 30 or 31 or 32 or 33 or 34 or 35

37. 25 and 36

\section{Population: early palliative treatment}

\section{MEDLINE (Ovid Gateway) (August 2010)}

One thousand, six hundred and fifty records were retrieved in MEDLINE

1. exp Uterine Cervical Neoplasms/

2. (cervi\$ adj5 cancer\$).mp.

3. (cervi $\$$ adj 5 carcinom $\$$ ). $m p$.

4. (cervi\$ adj5 adenocarcinom\$).mp. 
5. (cervi\$ adj5 carcinogen\$).mp.

6. (cervi\$ adj5 sarcoma $\$$ ).mp.

7. (cervi\$ adj5 malignan\$).mp.

8. (cervi\$ adj5 tumor\$).mp.

9. (cervi $\$$ adj5 tumour\$).mp.

10. (cervi $\$$ adj5 neoplas\$).mp.

11. (cervi\$ adj5 metasta\$).mp.

12. 1 or 2 or 3 or 4 or 5 or 6 or 7 or 8 or 9 or 10 or 11

13. exp Neoplasm Recurrence, Local/

14. exp Recurrence/

15. recur\$.mp.

16. relaps\$.mp.

17. repeat\$.mp.

18. repetitive $\$ . m p$.

19. reappearance $\$ . m p$.

20. reoccurence $\$$.mp.

21. return.mp.

22. exp Neoplasm Metastasis/

23. metasta \$.mp.

24. restag \$.mp.

25. re-stag.mp. (0)

26. 13 or 14 or 15 or 16 or 17 or 18 or 19 or 20 or 21 or 22 or 23 or 24

27. 12 and 26

28. Randomized Controlled Trials as Topic

29. Randomized Controlled Trial

30. Random Allocation

31. Double-Blind Method

32. Clinical Trial

33. exp Clinical Trials as Topic

34. (clinic\$ adj trial\$1).tw.

35. ((singl\$ or doubl\$ or treb\$ or tripl\$) adj (blind $\$ 3$ or mask $\$ 3)$ ).tw.

36. Placebos/

37. Placebo\$.tw.

38. Randomly allocated.tw.

39. (allocated adj2 random).tw.

40. 28 or 29 or 30 or 31 or 32 or 33 or 34 or 35 or 36 or 37 or 38 or 39

41. $\exp$ Case-Control Studies/

42. $\exp$ Cohort Studies

43. Case control.tw.

44. (cohort adj (study or studies)).tw.

45. Cohort analy\$.tw.

46. (Follow up adj (study or studies)).tw.

47. (observational adj (study or studies)).tw.

48. Longitudinal.tw.

49. retrospective.tw.

50. Cross sectional.tw.

51. Cross-Sectional Studies

52. 41 or 42 or 43 or 44 or 45 or 46 or 47 or 48 or 49 or 50 or 51

53. 40 or 5227 and 53

54. limit 53 to $\mathrm{yr}=" 2004$-Current" 


\section{EMBASE (Ovid Gateway) (August 2010)}

One thousand, four hundred and eighty-seven records were retrieved in EMBASE

1. exp Uterine Cervical Neoplasms/

2. (cervi\$ adj5 cancer\$).mp.

3. (cervi\$ adj5 carcinom\$).mp.

4. (cervi $\$$ adj5 adenocarcinom\$).mp.

5. (cervi $\$$ adj5 carcinogen $\$$ ). $m p$.

6. (cervi\$ adj5 sarcoma\$).mp.

7. (cervi\$ adj5 malignan\$).mp.

8. (cervi $\$$ adj5 tumor\$).mp.

9. (cervi\$ adj5 tumour\$).mp.

10. (cervi $\$$ adj5 neoplas\$).mp.

11. (cervi $\$$ adj5 metasta $\$$ ).mp.

12. 1 or 2 or 3 or 4 or 5 or 6 or 7 or 8 or 9 or 10 or 11

13. exp Recurrence/ or exp Neoplasm Recurrence, Local/

14. recur\$.mp.)

15. relaps\$.mp.

16. repeat\$.mp.

17. repetitive $\$ . m p$.

18. reappearance $\$ . m p$.

19. reoccurence $\$$.mp.

20. return.mp.

21. exp Neoplasm Metastasis

22. metasta \$.mp.

23. restag $\$ . m p$.

24. re-stag.mp.

25. 13 or 14 or 15 or 16 or 17 or 18 or 19 or 20 or 21 or 22 or 23

26. 12 and 25

27. Randomized Controlled Trials as Topic

28. Randomized Controlled Trial/

29. Random Allocation

30. Double-Blind Method

31. Clinical Trial

32. exp Clinical Trials as Topic

33. (clinic $\$$ adj trial\$1).tw. (145860)

34. ((singl\$ or doubl\$ or treb\$ or tripl\$) adj (blind $\$ 3$ or mask $\$ 3)$ ).tw.

35. Placebos

36. Placebo\$.tw.

37. Randomly allocated.tw.

38. (allocated adj2 random).tw. (731)

39. 27 or 28 or 29 or 30 or 31 or 32 or 33 or 34 or 35 or 36 or 37 or 38

40. exp Case-Control Studies/

41. exp Cohort Studies/

42. Case control.tw.

43. (cohort adj (study or studies)).tw.

44. Cohort analy $\$$.tw.

45. (Follow up adj (study or studies)).tw.

46. (observational adj (study or studies)).tw.

47. Longitudinal.tw.

48. retrospective.tw.

49. Cross sectional.tw.

50. Cross-Sectional Studies 
51. 40 or 41 or 42 or 43 or 44 or 45 or 46 or 47 or 48 or 49 or 50

52. 39 or 51

53. 26 and 52

54. limit 53 to $\mathrm{yr}=$ "2004 -Current"

\section{Population: previous surgical treatment}

\section{MEDLINE (Ovid Gateway) (August 2010)}

Two thousand, two hundred and twenty-eight records were retrieved in MEDLINE

1. $\exp$ Uterine Cervical Neoplasms/

2. (cervi\$ adj5 cancer\$).mp.

3. (cervi\$ adj5 carcinom $\$$ ). $\mathrm{mp}$.

4. (cervi\$ adj5 adenocarcinom\$).mp.

5. (cervi\$ adj5 carcinogen\$).mp.

6. (cervi\$ adj5 sarcoma\$).mp.

7. (cervi\$ adj5 malignan\$).mp.

8. (cervi\$ adj5 tumor\$).mp.

9. (cervi\$ adj5 tumour\$).mp.

10. (cervi\$ adj5 neoplas\$).mp.

11. (cervi\$ adj5 metasta\$).mp.

12. 1 or 2 or 3 or 4 or 5 or 6 or 7 or 8 or 9 or 10 or 11

13. exp Recurrence/ or exp Neoplasm Recurrence, Local/

14. recur\$.mp.

15. relaps $\$ . \mathrm{mp}$.

16. repeat\$.mp.

17. repetitive $\$ . m p$.

18. reappearance $\$ . m p$.

19. reoccurence $\$ . m p$.

20. return.mp.

21. exp Neoplasm Metastasis/

22. metasta\$.mp.

23. restag\$.mp.

24. re-stag.mp.

25. 13 or 14 or 15 or 16 or 17 or 18 or 19 or 20 or 21 or 22 or 23 or 24

26. 12 and 25

27. exp Pelvic Exenteration/

28. (pelvi\$ adj3 exenteratio\$).mp.

29. (pelvi\$ adj3 evisceratio\$).mp.

30. (pelvi\$ adj3 Hysterectom $\$$ ).mp.

31. (pelvi $\$$ adj3 colpohysterectom $\$$ ).mp.

32. (pelvi $\$$ adj3 hysterocolpectom $\$$ ).mp.

33. (pelvi $\$$ adj3 panhysterectom $\$$ ).mp.

34. (uter\$ adj3 extirpatio\$).mp.

35. (uter\$ adj3 amputatio\$).mp.

36. salvage surgery.mp. or Salvage Therapy/

37. leer.mp.

38. hysterectomy.tw.

39. *Hysterectomy/

40. 38 or 39

41. 27 or 28 or 29 or 30 or 31 or 32 or 33 or 34 or 35 or 36 or 37 or 40

42. 26 and 41 


\section{EMBASE (Ovid Gateway) (August 2010)}

Three thousand, one hundred and seventy-nine records were retrieved in EMBASE

1. exp Uterine Cervical Neoplasms/

2. (cervi\$ adj5 cancer\$).mp.

3. (cervi\$ adj5 carcinom\$).mp.

4. (cervi\$ adj5 adenocarcinom\$).mp.

5. (cervi\$ adj5 carcinogen\$).mp.

6. (cervi $\$$ adj5 sarcoma $\$$ ).mp.

7. (cervi\$ adj5 malignan\$).mp.

8. (cervi $\$$ adj5 tumor\$).mp.

9. (cervi $\$$ adj5 tumour\$).mp.

10. (cervi $\$$ adj5 neoplas\$).mp.

11. (cervi $\$$ adj5 metasta $\$$ ).mp.

12. 1 or 2 or 3 or 4 or 5 or 6 or 7 or 8 or 9 or 10 or 11

13. exp Recurrence/ or exp Neoplasm Recurrence, Local/

14. recur\$.mp.

15. relaps\$.mp.

16. repeat\$.mp.

17. repetitive $\$ . m p$.

18. reappearance\$.mp.

19. reoccurence $\$ . m p$.

20. return.mp.

21. exp Neoplasm Metastasis/

22. metasta \$.mp.

23. restag $\$ . m p$.

24. re-stag.mp.

25. 13 or 14 or 15 or 16 or 17 or 18 or 19 or 20 or 21 or 22 or 23 or 24

26. 12 and 25

27. exp Pelvic Exenteration/

28. (pelvi\$ adj3 exenteratio\$).mp.

29. (pelvi\$ adj3 evisceratio\$).mp.

30. (pelvi $\$$ adj3 Hysterectom $\$$ ).mp.

31. (pelvi $\$$ adj3 colpohysterectom\$).mp.

32. (pelvi $\$$ adj3 hysterocolpectom\$).mp.

33. (pelvi\$ adj3 panhysterectom\$).mp.

34. (uter\$ adj3 extirpatio\$).mp.

35. (uter\$ adj3 amputatio \$).mp.

36. salvage surgery.mp. or Salvage Therapy/

37. leer.mp.

38. hysterectomy.tw.

39. *HYSTERECTOMY/

40. 38 or 39

41. 27 or 28 or 29 or 30 or 31 or 32 or 33 or 34 or 35 or 36 or 37 or 40

42. 26 and 4 


\section{Appendix 9 Economic evaluation systematic review search strategies and study categories}

The search strategy in this appendix was used to identify economic evaluation studies from the EMBASE database on the use of PET-CT to detect recurrent cervical cancer. Similar search strategies were used for MEDLINE and the ISI Web of Knowledge. NHS EED, DARE and HTA were searched within The Cochrane Library using the 'cervical cancer' search term.

\section{EMBASE (1980 to October 2011)}

1. Uterine cervical neoplasms.mp. or Uterine Cervical Neoplasms/

2. cancer.mp. or Neoplasms/

3. Carcinoma/ or carcinoma.mp.

4. Carcinogen\$.mp.

5. adenocarcinoma.mp. or Adenocarcinoma/

6. Cervi\$.mp.

7. 1 or 2 or 3 or 4 or 5

8. 6 and 7

9. Neoplasm Recurrence, Local/ or Recurrence/ or recur\$.mp.

10. 8 and 9

11. "Costs and Cost Analysis"/ or Cost-Benefit Analysis/ or Economic evaluation.mp.

12. cost-effectiveness analysis.mp.

13. "Quality of Life"/ or Quality-Adjusted Life Years/ or cost-utility analysis.mp.

14. 11 or 12 or 13

15. 10 and 14

\section{Study categories}

Stage I: selection of the papers.

(a) The study reports an economic evaluation based on primary (i.e. original data collected specifically for the study) or secondary (i.e. unoriginal data collected from already published articles or other sources) research on the costs and use of care and includes formal economic evaluation.

(b) The study discusses the economic aspects of recurrent cervical cancer and contains useful primary or secondary cost or use data but is not an economic evaluation.

(c) The study discusses economic aspects of policies for care but is neither A nor B.

(d) The study has no relevance to recurrent cervical cancer.

\section{Stage II: further categorisation of the relevant studies}

Studies that were considered relevant to the systematic review (A, B and C) were read in full and further classified according to the study type as outlined below:

1. economic evaluation studies that reported their results in terms of cost per QALY

2. other economic evaluation studies that did not report their results in terms of cost per QALY (e.g. recurrence detected)

3. studies not categorised as 1 or 2 .

All studies categorised as $A(1)$ and $A(2)$ were included in the quality assessment stage. Papers retrieved that were not classified as above were rejected at this stage. 



\section{Appendix 10 Diagnostic review list of excluded studies with reasons for exclusion}

\section{TABLE 79 Diagnostic review list of excluded studies with reasons for exclusion}

Reference

Adalsteinsson B, Påhlman L, Hemmingsson A, Glimelius B, Graffman S. Computed tomography in early diagnosis of local recurrence of rectal carcinoma. Acta Radiol 1987;28:41-7

Amano M, Kato T, Amano Y, Kumazaki T. Using MR imaging to predict and evaluate the response of invasive cervical carcinoma to systemic chemotherapy. AJR Am J Roentgenol 1998;171:1335-9

Babar S, Rockall A, Goode A, Shepherd J, Reznek R. Magnetic resonance imaging appearances of recurrent cervical carcinoma. Int J Gynecol Cancer 2007;17:637-45

Batka M, Staudach A, Haidinger M, Doringer E. [Magnetic resonance staging as a decision aid in therapy of cervix cancer.] Gynakol Rundsch 1991;31(Suppl. 2):239-41

Belhocine T, Thille A, Fridman V, Albert A, Seidel L, Nickers P, et al. Contribution of whole-body ${ }^{18}$ FDG PET imaging in the management of cervical cancer. Gynecol Oncol 2002;87:90-7

Bellomi M, Bonomo G, Landoni F, Villa G, Leon ME, Bocciolone L, et al. Accuracy of computed tomography and magnetic resonance imaging in the detection of lymph node involvement in cervix carcinoma. Eur Radiol 2005;15:2469-74

Beyersdorff D, Bahnsen J, Frischbier HJ. Nodal involvement in cancer of the uterine cervix: value of lymphography and MRI. Eur J Gynaecol Oncol 1995;16:274-7

Bjurberg M, Kjellén E, Ohlsson T, Ridderheim M, Brun E. FDG-PET in cervical cancer: staging, re-staging and follow-up. Acta Obstet Gyneco/ Scand 2007;86:1385-91

Bjurberg M, Kjellén E, Ohlsson T, Bendahl P-O, Brun E. Prediction of patient outcome with 2-deoxy-2-[18F] fluoro-D-glucose-positron emission tomography early during radiotherapy for locally advanced cervical cancer. Int J Gynecol Cancer 2009;9:1600-5

Boss EA, Massuger LF, Pop LA, Verhoef LC, Huisman H-J, Boonstra H, et al. Post-radiotherapy contrast enhancement changes in fast dynamic MRI of cervical carcinoma. J Magn Reson Imaging 2001;13:600-6

Boughanim M, Leboulkox S, Rey A, Pham CT, Zafrani Y, Haie-Meder C, et al. Histologic results of para-aortic lymphadenectomy in patients treated for stage IB2/II cervical cancer with negative [18F] fluorodeoxyglucose positron emission tomography scans in the para-aortic area. J Clin Oncol 2008;26:2558-61

Brenner DE, Whitley NO, Prempree T, Villasanta U. An evaluation of the computed tomographic scanner for the staging of carcinoma of the cervix. Cancer 1982;50:2323-8

Brooks RA, Rader JS, Dehdashti F, Mutch DG, Powell MA, Thaker PH, et al. Surveillance FDGPET detection of asymptomatic recurrences in patients with cervical cancer. Gynecol Oncol 2009;112:104-9

Brown JJ, Gutierrez ED, Lee JK. MR appearance of the normal and abnormal vagina after hysterectomy. AJR Am J Roentgenol 1992;158:95-9

Bruneton JN, Merran D, Balu-Maestro C, Rogopoulos A, Giordano P, Chauvel P, et al. [Echography and computed tomography in the evaluation and follow-up of uterine cancers]. Bull Cancer 1990;77:689-94

Chang TC, Law K-S, Hong J-H, Lai C-H, Ng K-K, Hsueh S, et al. Positron emission tomography for unexplained elevation of serum squamous cell carcinoma antigen levels during follow-up for patients with cervical malignancies: a phase II study. Cancer 2004;101:164-71
Reason for exclusion

Lack of full text

Wrong end points

Wrong end points

Wrong population

Wrong population

Lesion-based analysis

Wrong population

Wrong intervention

Wrong end points

Wrong end points

Wrong population

Wrong population

Wrong end points

No data

Lack of full text

Wrong population 
Chang WC, Hung YC, Lin CC, Shen YY, Kao C-H. Usefulness of FDG-PET to detect recurrent cervical cancer based on asymptomatically elevated tumor marker serum levels - a preliminary report. Cancer Invest 2004;22:180-4

Chang YC, Yen T-C, Ng K-K, See L-C, Lai C-H, Chang T-C, et al. Does diabetes mellitus influence the efficacy of FDG-PET in the diagnosis of cervical cancer? Eur J NuCl Med Mol Imaging 2005;32:647-52

Chao A, Ho K-C, Wang C-C, Cheng H-H, Lin G, Yen T-C, et al. Positron emission tomography in evaluating the feasibility of curative intent in cervical cancer patients with limited distant lymph node metastases. Gynecol Oncol 2008;110:172-8

Chen JT, Yamashiro T, Shimizu Y, Nakajama K, Teshima H, Hirai Y, et al. [Comparison of ultrasound and computed tomography (CT) for the diagnosis of paraaortic lymphnode metastasis in patients with gynecologic malignancies.] Acta Obst Gynaecol Jpn 1989:41:55-60

Choi EK, Kim JK, Choi HJ, Park SH, Park B-W, Kim N, et al. Node-by-node correlation between $M R$ and $P E T / C T$ in patients with uterine cervical cancer: diffusion-weighted imaging versus size-based criteria on T2WI. Eur Radiol 2009;19:2024-32

Choi HJ, Roi JW, Seo S-S, Lee S, Kim J-Y, Kim S-K, et al. Comparison of the accuracy of magnetic resonance imaging and positron emission tomography/computed tomography in the presurgical detection of lymph node metastases in patients with uterine cervical carcinoma: a prospective study. Cancer 2006;106:914-22

Choi SH, Kim S-H, Choi H-J, Park BK, Lee HJ. Preoperative magnetic resonance imaging staging of uterine cervical carcinoma: results of prospective study. J Comput Assist Tomogr 2004;:28:620-7

Chou HH, Chang T-C, Yen T-C, Ng K-K, Hsueh S, Ma SY, et al. Low value of [18F]-fluoro-2deoxy-D-glucose positron emission tomography in primary staging of early-stage cervical cancer before radical hysterectomy. J Clin Oncol 2006;24:123-8

Chung HH, Lee S, Sim J-S, Kim J-Y, Seo SS, Park S-Y, et al. Pretreatment laparoscopic surgical staging in locally advanced cervical cancer: preliminary results in Korea. Gynecol Oncol 2005:97:468-75

Chung HH, Kim S-K, Kim TH, Lee S, Kang KW, Kim J-Y, et al. Clinical impact of FDG-PET imaging in post-therapy surveillance of uterine cervical cancer: from diagnosis to prognosis. Gynecol Oncol 2006;103:165-70

Chung HH, Kang S-B, Cho JY, Kim JW, Park N-H, Song Y-S, et al. Can preoperative MRI accurately evaluate nodal and parametrial invasion in early stage cervical cancer? Jpn J Clin Oncol 2007:37:370-5

Chung HH, Kang WJ, Kim JW, Park N-H, Song Y-S, Chung J-K, et al. Characterization of surgically transposed ovaries in integrated PET/CT scan in patients with cervical cancer. Acta Obstet Gynecol Scand 2007;86:88-93

Chung HH, Park N-H, Kim JW, Song Y-S, Chung J-K, Kang S-B. Role of integrated PET-CT in pelvic lymph node staging of cervical cancer before radical hysterectomy. Gynecol Obstet Invest 2009;67:61-6

Crawford RA, Richards PJ, Reznek RH, Ngan HY, Shepherd JH. The role of CT in predicting the surgical feasibility of exenteration in recurrent carcinoma of the cervix. Int I Gynecol Cancer 1996:6:231-4

Dehdashti F, Grigsby PW, Lewis JS, LaForest R, Siegel BA, Welch MJ. Assessing tumor hypoxia in cervical cancer by PET with 60CU-labeled diacetyl-bis(N4-methylthiosemicarbazone). J Nucl Med 2008;49:201-5

Dehong L, Mulan S, Zhengang X, Wu N, Yao D, Hao Y, et al. Cervical lymph node metastasis: CT, ultrasound versus physical palpation. Chin J Oncol 1998;20:48-50

deSouza NM, Dina R, McIndoe GA, Soutter WP. Cervical cancer: value of an endovaginal coil magnetic resonance imaging technique in detecting small volume disease and assessing parametrial extension. Gynecol Oncol 2006;102:80-5

Wrong population

Wrong population

Lack of full text

Wrong population

Wrong population

Wrong population

Wrong population

Wrong population

Wrong intervention

Wrong population

Wrong population

Wrong population

Wrong study design

Wrong intervention

Lack of full text Wrong population 
TABLE 79 Diagnostic review list of excluded studies with reasons for exclusion (continued)

Reference

Dolezelova H, Slampa P, Ondrova B, Gombosova J, Sovadinova S, Novotny T, et al. The impact of PET with ${ }^{18} \mathrm{FDG}$ in radiotherapy treatment planning and in the prediction in patients with cervix carcinoma: results of pilot study. Neoplasma 2008;55:437-41

Donaldson SB, Buckley DL, O'Connor JP, Davidson SE, Carrington BM, Jones AP, et al. Enhancing fraction measured using dynamic contrast-enhanced MRI predicts disease-free survival in patients with carcinoma of the cervix. Br J Cancer 2010;102:23-6

Eiber M, Dütsch S, Gaa J, Fauser C, Rummeny EJ, Holzapfel K. [Diffusion-weighted magnetic resonance imaging (DWI-MRI): a new method to differentiate between malignant and benign cervical lymph nodes]. Laryngorhinootologie 2008;87:850-5

Esthappan J, Chaudhari S, Santanam L, Mutic S, Olsen J, MacDonald DM, et al. Prospective clinical trial of positron emission tomography/computed tomography image-guided intensitymodulated radiation therapy for cervical carcinoma with positive para-aortic lymph nodes. Int J Radiat Oncol Biol Phys 2008;72:1134-9

Ferdova E, Finek J, Ferda J. A role of ${ }^{18} \mathrm{~F}-\mathrm{FDG}-\mathrm{PET} / \mathrm{CT}$ in the treatment decisions of uterine and ovarian tumors, our clinical practice experience. Ceska Radio/ 2009;63:290-302

Fluckiger F, Ebner F, Poschauko H, Arian-Schad K, Einspieler E, Hausegger K. [Value of magnetic resonance tomography after primary irradiation of carcinoma of the cervix uteri: evaluation of therapeutic success and follow-up.] Strahlenther Onkol 1991;167:152-7

Flueckiger F, Ebner F, Poschauko H, Tamussino K, Einspieler R, Ranner G. Cervical cancer: serial MR imaging before and after primary radiation therapy - a 2-year follow-up study. Radiology 1992;184:89-93

Franchi M, La Fianza A, Babilonti L, Bolis PF, Alerci M, Di Giulio G, et al. Clinical value of computerized tomography (CT) in assessment of recurrent uterine cancers. Gynecol Oncol 1989;35:31-7

Genolet PM, Hanggi W, Dreher E. [Evaluation of tumor extension in invasive cancer of the uterine cervix. Diagnostic evaluation of cervix cancer.] Gynakol Geburtshilfliche Rundsch 1993;33:180-4

Ginaldi S, Wallace S, Jing B-S, Bernardino ME. Carcinoma of the cervix: lymphangiography and computed tomography. AJR Am J Roentgenol 1981;136:1087-91

Gochev G, Totsev N, Vasilev D, Simeonova L, lanev N, Elenchev L, et al. [The potentials of computed axial tomography (CAT) in the diagnosis of carcinoma of the cervix uteri.] Akush Ginekol 1994;33:25-6

Goff BA, Muntz HG, Paley PJ, Tamimi HK, Koh W-J, Greer BE. Impact of surgical staging in women with locally advanced cervical cancer. Gynecol Oncol 1999;74:436-42

Gong QY, Tan LT, Romanuik CS, Jones B, Brunt JN, Roberts N. Determination of tumour regression rates during radiotherapy for cervical carcinoma by serial MRI: comparison of two measurement techniques and examination of intraobserver and interobserver variability. $\mathrm{Br} \mathrm{J}$ Radiol 1999;72:62-72

Goudy G, Stoeckle E, Thomas L, Kind M, Guyon F, Brouste V, et al. [Prognostic impact of tumour volume and lymph node involvement in intermediate stage T1b1 to T2b cancer of the uterine cervix.] Bull Cancer 2009;96:685-94

Grigsby PW, Dehdashti F, Siegel BA. FDG-PET evaluation of carcinoma of the cervix. Clin Positron Imaging 1999;2:105-9

Grigsby PW, Siegel BA, Dehdashti F. Lymph node staging by positron emission tomography in patients with carcinoma of the cervix. J Clin Oncol 2001;19:3745-9

Grigsby PW, Singh AK, Siegel BA, Dehdashti F, Rader J, Zoberi I. Lymph node control in cervical cancer. Int J Radiat Oncol Biol Phys 2004;59:706-12

Grigsby PW, Siegel BA, Dehdashti F, Rader J, Zoberi I. Posttherapy [18F] fluorodeoxyglucose positron emission tomography in carcinoma of the cervix: response and outcome. J Clin Oncol 2004:22:2167-71
Reason for exclusion

Lack of gold standard

Wrong population

Lack of full text

Wrong study design

Lack of full text

No data

No data

No data

Lack of full text

Lack of gold standard

Lack of full text

Wrong population

Wrong population

Wrong population

Small sample size

No data

No data

Wrong population 
Hancke K, Heilmann V, Straka P, Kreienberg R, Kurzeder C. Pretreatment staging of cervical cancer: is imaging better than palpation?: role of CT and MRI in preoperative staging of cervical cancer: single institution results for 255 patients. Ann Surg Oncol 2008;15:2856-61

Hauth EA, Kuhl H, Kimmig R, Forsting M. Evaluation of MR imaging of the pelvis for the staging, follow-up and recurrence diagnosis of cervical cancer. Geburtshilfe Frauenheilkd 2006;66:1177-85

Havrilesky $\sqcup$, Wong TZ, Secord AA, Berchuck A, Clarke-Pearson DL, Jones EL. The role of PET scanning in the detection of recurrent cervical cancer. Gynecol Oncol 2003:90:186-90

Hawighorst $\mathrm{H}$, Knapstein PG, Schaeffer $U$, Brix G, Weikel P, Essig M, et al. [Diagnosis of recurrence of cervix carcinoma using dynamic MRI: correlation of pharmacokinetic analysis and histopathology.] Radiologe 1995;35:945-51

Hawighorst H, Knapstein PG, Schaeffer U, Knopp MV, Brix G, Hoffman U, et al. Pelvic lesions in patients with treated cervical carcinoma: efficacy of pharmacokinetic analysis of dynamic MR images in distinguishing recurrent tumors from benign conditions. AJR Am J Roentgenol 1996;166:401-8

Hawighorst H, Knapstein PG, Weikel P, Knopp MV, Schaeffer U, Essig M, et al. [Invasive cervix carcinoma (pT2b-pT4a). Value of conventional and pharmacokinetic magnetic resonance tomography (MRI) in comparison with extensive cross sections and histopathologic findings.] Radiologe 1997;37:130-8

Hawighorst H, Schoenberg SO, Knapstein PG. Staging of invasive cervical carcinoma and of pelvic lymph nodes by high resolution MRI with a phased-array coil in comparison with pathological findings. J Comput Assist Tomogr 1998;22:75-81

Hawighorst H, Knapstein PG, Knopp MV, Weikel P, Schaeffer U, Zuna I, et al. [Angiogenesis of cervix carcinoma. Contrast enhanced dynamic MRI, histologic quantification of capillary density and lymphatic system infiltration.] Radiologe 1998;38:50-7

Hawighorst H, Weikel P, Knapstein PG, Knopp MV, Zuna I, Schonberg SO, et al. Angiogenic activity of cervical carcinoma: assessment by functional magnetic resonance imaging-based parameters and a histomorphological approach in correlation with disease outcome. Clin Cancer Res 1998:4:2305-12

Hawnaur JM, Johnson RJ, Hunter RD, Jenkins PR, Isherwood I. The value of magnetic resonance imaging in assessment of carcinoma of the cervix and its response to radiotherapy. Clin Oncol 1992:4:11-17

Hawnaur JM, Johnson RJ, Buckley CH, Tindall V, Isherwood I. Staging, volume estimation and assessment of nodal status in carcinoma of the cervix: comparison of magnetic resonance imaging with surgical findings. Clin Radio/ 1994;49:443-52

Heller PB, Malfetano JH, Bundy BN, Barnhill DR, Okagaki T. Clinical-pathologic study of stage $I I B$, III, and IVA carcinoma of the cervix: extended diagnostic evaluation for paraaortic node metastasis - a Gynecologic Oncology Group study. Gynecol Oncol 1990;38:425-30

Heuck A, Scheidler J, Rimmig R, Muller-Lisse U, Steinborn M, Helmberger T, Reiser M. [Lymph node staging in cervix carcinomas: the results of high-resolution magnetic resonance tomography (MRT) with a phased-array body coil.] Rofo 1997;166:210-4

Heung-Tat NG, Shen-Li C, Jen-Chung W, Ming-Huei S. Preoperative examination with CT, MRI and comparison of both to histopathologic findings in cervical carcinoma. CME J Gynecol Oncol 1998:3:256-7

Ho CM, Chien TY, Jeng CM, Tsang YM, Shih BY, Chang SC. Staging of cervical cancer: comparison between magnetic resonance imaging, computed tomography and pelvic examination under anesthesia. J Formos Med Assoc 1992:91:982-90

Hope AJ, Saha P, Grigsby PW. FDG-PET in carcinoma of the uterine cervix with endometrial extension. Cancer 2001:106:196-200.
Wrong population

No data

Wrong intervention

Lesion-based analysis

Lesion-based analysis

Wrong population

Wrong population

Wrong population

Wrong end points

Wrong end points

Wrong population

Lack of full text

Wrong population

Lack of full text

Lack of full text

Wrong population 
TABLE 79 Diagnostic review list of excluded studies with reasons for exclusion (continued)

Hori M, Kim T, Murakami T, Imaoka I, Onishi H, Tomoda K, et al. Uterine cervical carcinoma: preoperative staging with 3.0-T MR imaging - comparison with 1.5-T MR imaging. Radiology 2009;251:96-104

Houvenaeghel G, Delpero JR, Rosello R, Resbeut M, Viens P, Jacquemier J, et al. Results of a prospective study with comparison of clinical, endosonographic, computed tomography, magnetic resonance imaging and pathologic staging of advanced gynecologic carcinoma and recurrence. Surg Gynecol Obstet 1993;177:231-6

Hricak H. Cancer of the uterus: the value of MRI pre- and post-irradiation. Int J Radiat Oncol Biol Phys 1991;21:1089-94

Hricak H, Swift PS, Campos Z, Quivey JM, Gildengorin V, Goranson H, et al. Irradiation of the cervix uteri: value of unenhanced and contrast-enhanced MR imaging. Radiology 1993;189:381-8

Hricak H, Quivey JM, Campos Z, Gildengorin V, Hindmarsh T, Bis KG, et al. Carcinoma of the cervix: predictive value of clinical and magnetic resonance (MR) imaging assessment of prognostic factors. Int J Radiat Oncol Biol Phys 1993;27:791-801

Hricak H, Mendelson E, Bohm-Velez M, Bree R, Finberg H, Fishman EK, et al. Role of imaging in cancer of the cervix. American College of Radiology. ACR Appropriateness Criteria.

Radiology 2000;215:925-30

Husain A, Akhurst T, Larson S, Alektiar K, Barakat RR, Chi DS. A prospective study of the accuracy of 18 Fluorodeoxyglucose positron emission tomography ( $\left.{ }^{8} \mathrm{FDG}-\mathrm{PET}\right)$ in identifying sites of metastasis prior to pelvic exenteration. Gynecol Oncol 2007;106:177-80

lizuka Y. [Clinical significance of magnetic resonance imaging (MRI) in evaluation of radiotherapeutic effect on uterine cervical cancer.] Acta Obstet Gynaecol Jpn 1996;48:37-44

Ishii C, Tada S, Tsukioka M, Tanaka H. [CT diagnosis of uterine cancer.] Gan to Kagaku Ryoho 1982;9:204-8

Ishii C, Tada S, Kato Y, Tanaka H. Computed tomographic evaluation of hydronephrosis in uterine carcinoma. Radiat Med 1983;1:42-5

Ito $\mathrm{H}$, et al. [Computed tomographic diagnosis in patients with recurrent cervical cancer invaded to the iliac bone (authors' translation).] Rinsho Hoshasen 1981;26:469-73

Kajiwara TH, Kataoka M, Hamamoto Y, Ikura M, Hosakawa A, Inoue T, et al. [Prediction of pelvic control using MRI for patients with cervical carcinoma treated with radiotherapy.] Nihon Igaku Hoshasen Gakkai Zasshi 2005;65:438-43

Kanehira C, Arai T, Suda Y, Suzuki M. [CT diagnosis and treatment of lymph node metastases from carcinoma of the cervix.] Rinsho Hoshasen 1983;28:285-92

Kecmanovic DM, Pavlov MJ, Kovacevic PA, Sepetkovski AV, Ceranic MS, Stamenkovic AB, et al. Management of advanced pelvic cancer by exenteration. Eur J Surg Oncol 2003;29:743-6

Keller TM, Michel SC, Frohlich J, Fink D, Caduff R, Marincek B, et al. USPIO-enhanced MRI for preoperative staging of gynecological pelvic tumors: preliminary results. Eur Radiol 2004;14:937-44

Kerr IG, Manji MF, Powe J, Bakheet S, Al Suhaibani H, Subhi J. Positron emission tomography for the evaluation of metastases in patients with carcinoma of the cervix: a retrospective review. Gynecol Oncol 2001;81:477-80

Kidd EA, Grigsby PW. Intratumoral metabolic heterogeneity of cervical cancer. Clin Cancer Res 2008; 14:5236-41

Kidd EA, Siegel BA, Dhdashti F, Rader J, Mutch DG, Powell MA, et al. Lymph node staging by positron emission tomography in cervical cancer: relationship to prognosis. $J$ Clin Oncol 2010;28:2108-13

Wrong population

No data

Lack of full text

Lesion-based analysis

No data

Lack of full text

Wrong intervention

Lack of full text

Lack of full text

Lack of full text

Lack of full text

Lack of full text

Lack of full text

Wrong end points

Wrong population

Lack of gold standard

Wrong end points

Wrong population 
Kilcheski TS, Arger PH, Mulhern CB, Coleman BG, Kressel HY, Mikuta JI. Role of computed tomography in the presurgical evaluation of carcinoma of the cervix. $J$ Comput Assist Tomogr 1981;5:378-83

Kim H, Kim W, Lee M, Song E, Loh JJ. Tumor volume and uterine body invasion assessed by $\mathrm{MRI}$ for prediction of outcome in cervical carcinoma treated with concurrent chemotherapy and radiotherapy. Jpn J Clin Oncol 2007;37:858-66

Kim MJ, Chung JJ, Lee YH, Lee JT, Yoo HS. Comparison ofthe use of the transrectal surface coil and the pelvic phased-array coil in MR imaging for preoperative evaluation of uterine cervical carcinoma. AJR Am J Roentgenol 1997;168:1215-21

Kim SH, Choi BI, Han JK, Kim HD, Lee HP, Kang SB, et al. Preoperative staging of uterine cervical carcinoma: comparison of CT and MRI in 99 patients. J Comput Assist Tomogr 1993; 17:633-40

Kim SH, Kim SC, Choi BI, Han MC. Uterine cervical carcinoma: evaluation of pelvic lymph node metastasis with MR imaging. Radiology 1994;190:807-11

Kim SK, Choi HJ, Park S-Y, Lee H-Y, Seo S-S, Yoo CW, et al. Additional value of MR/PET fusion compared with $\mathrm{PET} / \mathrm{CT}$ in the detection of lymph node metastases in cervical cancer patients. Eur J Cancer 2009;45:2103-9

King LA, Talledo OE, Gallup DG, El Gammal TA. Computed tomography in evaluation of gynecologic malignancies: a retrospective analysis. Am J Obstet Gynecol 1986;155:960-4

Kinkel K, Ariche M, Tardivon AA, Spatz A, Castaigne D, Lhomme C, et al. Differentiation between recurrent tumor and benign conditions after treatment of gynecologic pelvic carcinoma: value of dynamic contrast-enhanced subtraction MR imaging. Radiology 1997;204:55-63

Kitagaki H. [MR imaging-evaluation of therapeutic effect of radiotherapy for uterine cervix cancer.] Nihon Igaku Hoshasen Gakkai Zasshi 1995;55:215-21

Kitajima K, Murakami K, Yamasaki E, Kaji Y, Sugimura K. Accuracy of integrated FDG-PET/ contrast-enhanced $\mathrm{CT}$ in detecting pelvic and paraaortic lymph node metastasis in patients with uterine cancer. Eur Radiol 2009;19:1529-36

Kitajima K, Murakami K, Yamasaki E, Domeki Y, Kaji Y, Sugimura K. Performance of integrated FDG-PET/contrast-enhanced CT in the diagnosis of recurrent uterine cancer: comparison with PET and enhanced CT. Eur J Nucl Med Mol Imaging 2009;36:362-72

Klerkx WM, Heintz AP, Mali WP, de Kort GA, Takahara T, van Dorst EB, et al. Lymph node detection by MRI before and after a systematic pelvic lymphadenectomy. Gynecol Oncol 2009;114:315-18

Kodaira T, Fuwa N, Toita T, Nomoto Y, Kazuya K, Tachibana H, et al. Comparison of prognostic value of MRI and FIGO stage among patients with cervical carcinoma treated with radiotherapy. Int J Radiat Oncol Biol Phys 2001;56:769-77

Kodaira T, Fuwa N, Toita T, Nomoto Y, Kuzuya K, Tachibana K, et al. Clinical evaluation using magnetic resonance imaging for patients with stage III cervical carcinoma treated by radiation alone in multicenter analysis: its usefulness and limitations in clinical practice. Am J Clin Oncol 2003;26:574-83

Kolesnikova EK. [Computed tomography in the diagnosis of cervical cancer.] Akush Ginekol 1986;(11):18-23

Kühnel G, Horn L-C, Fischer U, Hesse S, Seese A, Georgi P, et al. [ ${ }^{18} \mathrm{~F}-\mathrm{FDG}$ positron-emissiontomography in cervical carcinoma: preliminary findings.] Zentralb Gynakol 2001;123:229-35

Kumar R, Dadparvar S. 18F-fluoro-2-deoxy-D-glucose-positron emission tomography (PET)/ PET-computed tomography in carcinoma of the cervix. Cancer 2007;110:1650-3

La Fianza A, Dore R, Di Giulio G, Alerci M, Di Maggio EM, Franchi M, et al. [Lymph node metastasis of carcinoma of the cervix uteri. Role of lymphography and computerized tomography.] Radiol Med 1990;80:486-91
No data

Wrong population

Wrong population

Wrong population

Wrong population

Wrong population

Wrong population

Wrong population

Lack of full text

Wrong population

No data

No data

Wrong end points

Wrong population

Lack of full text

Wrong population

Wrong study design

No data 
TABLE 79 Diagnostic review list of excluded studies with reasons for exclusion (continued)

La Fianza A, Campani R, Dore R, Babilonti L, Tateo S, Calliada F. [CT in the diagnosis and treatment of lymphoceles following gynecologic cancer surgery.] Radiol Med 1993;86:106-15

Lai CH, Huang K-G, See L-C, Yen T-C, Tsai C-S, Chang T-C, et al. Restaging of recurrent cervical carcinoma with dual-phase [18F]fluoro-2-deoxy-D-glucose positron emission tomography. Cancer 2001;100:544-52

Lai PH, Yang CF, Pan HB, Wu MT, Chu ST, Ger LP, et al. Recurrent inverted papilloma: diagnosis with pharmacokinetic dynamic gadolinium-enhanced MR imaging. AJNR Am J Neuroradiol 1999:20:1445-51

Lien HH, Blomlie V, Kjørstad K, Abeler V, Kaalhus O. Clinical stage I carcinoma of the cervix: value of MR imaging in determining degree of invasiveness. AJR Am J Roentgenol 1991;156:1191-4

Lin CT, Yen T-C, Chang T-C, Ng K-K, Tsai C-S, Ho K-C, et al. Role of [18F]fluoro-2-deoxy-Dglucose positron emission tomography in re-recurrent cervical cancer. Int I Gynecol Cancer 2006;16:1994-2003

Lin G, Ho K-C, Wang J-J, Ng K-K, Wai Y-Y, Chen Y-T, et al. Detection of lymph node metastasis in cervical and uterine cancers by diffusion-weighted magnetic resonance imaging at $3 \mathrm{~T}$. J Magn Reson Imaging 2008;28:128-35

Lin WC, Hung YC, Yeh LS, Kao CH, Yen RF, Shen YY. Usefulness of (18)F-fluorodeoxyglucose positron emission tomography to detect para-aortic lymph nodal metastasis in advanced cervical cancer with negative computed tomography findings. Gynecol Oncol 2003;89:73-6

Liu FY, Yen T-C, Chen M-Y, Lai C-H, Chang T-C, Chou H-H, et al. Detection of hematogenous bone metastasis in cervical cancer: 18F-fluorodeoxyglucose-positron emission tomography versus computed tomography and magnetic resonance imaging. Cancer 2009;115:5470-80

Liu Y, Bai R, Sun H, Liu H, Zhao X, Li Y. Diffusion-weighted imaging in predicting and monitoring the response of uterine cervical cancer to combined chemoradiation. Clin Radiol 2009;64:1067-74

Loft A, Berthelsen AK, Roed H, Ottosen C, Lundvall L, Knudsen J, et al. The diagnostic value of PET/CT scanning in patients with cervical cancer: a prospective study. Gynecol Oncol 2007;106:29-34

Lorenzen M, Nicolas V, Kopp A. [MRT diagnosis of recurrence of gynecologic tumors]. Rofo 1994:161:526-30

Lorenzen M, Braun J, Gehrckens A, Nicolas V. [Value of MRI, CT and findings in staging of gynecologic malignancies.] Aktuelle Radio/ 1998;8:266-72

Luo D, Shi M, Xu Z. [Cervical lymph node metastasis: CT, ultrasound versus physical palpation.] Chung-Hua Chung Liu Tsa Chih - Chin J Oncol 1998;20:48-50

Ma SY, See L-C, Lai C-H, Chou H-H, Tsai C-S, Ng K-K, et al. Delayed (18)F-FDG PET for detection of paraaortic lymph node metastases in cervical cancer patients. J Nucl Med 2003;44:1775-83

Manfredi R, Maresca G, Smaniotto D, Greggi S, Andrulli D, Rabitti C, et al. Cervical cancer response to neoadjuvant therapy: MR imaging assessment. Radiology 1998;209:819-24

Manfredi R, Baltieri S, Tognolini A, Graziani R, Smaniotto D, Cellini N, et al. Recurrent uterine cancer after surgery: magnetic resonance imaging patterns and their changes after concomitant chemoradiation. Radiol Med 2008:113:1143-56

Manfredi R, Gui B, Giovanzana A, Marini S, Di Stefano M, Zannoni G, et al. Localized cervical cancer (stage < IIB): accuracy of MR imaging in planning less extensive surgery. Radiol Med 2009;114:960-75

Marano P, Summaria V, Smaniotto D, Danza FM, Speca S, Valantini AL, et al. [Experience with the combined diagnosis and therapy of locally advanced carcinoma of the uterine cervix (stage FIGO IIB-III). Transrectal ultrasonography and CT in the staging and in follow-up after therapy. Preliminary results.] Radiol Med 1993;86:630-8
No data

No data

Lesion-based analysis

Wrong study design

Wrong intervention

Wrong population

Wrong intervention

Lack of gold standard

Wrong end points

Wrong population

Wrong population

Lack of full text

Lack of full text

Wrong population

Wrong population

Wrong population

Wrong population

Wrong population 
Matsubara M. [Clinical significance of magnetic resonance imaging (MRI) in evaluation of the extension of uterine cervical cancer]. Acta Obstet Gynaecol Jpn 1993;45:1115-22

Matsukuma K, Tsukamoto N, Matsuyama T, Ono M, Nakano H. Preoperative CT study of lymph nodes in cervical cancer - its correlation with histological findings. Gynecol Oncol 1989;33:168-71

Matsukuma K, Tsukamoto N, Jo S, Imachi M, Kamura T, Matsuyama T, et al. [An evaluation of scalene lymph node metastasis in patients with gynecologic malignancies.] Gan No Rinsho 1989;35:275-9

Mayr NA, Yuh WT, Magnotta VA, Erhardt TC, Wheeler JA, Sorosky Jl, et al. Tumor perfusion studies using fast magnetic resonance imaging technique in advanced cervical cancer: a new noninvasive predictive assay. Int I Radiat Oncol Biol Phys 1996;36:623-33

Mayr NA, Magnotta VA, Erhardt TC, Wheeler JA Sorosky JI, Wen B-C, et al. Usefulness of tumor volumetry by magnetic resonance imaging in assessing response to radiation therapy in carcinoma of the uterine cervix. Int J Radiat Oncol Biol Phys 1996;35:915-24

Mayr NA, Yuh WT, Zheng J, Erhardt TC, Magnotta VA, Sorosky Jl, et al. Prediction of tumor control in patients with cervical cancer: analysis of combined volume and dynamic enhancement pattern by MR imaging. AJR Am J Roentgenol 1998;170:177-82

Mayr NA, Taoka T, Yuh WT, Denning LM, Zhen WK, Paulino AC, et al. Method and timing of tumor volume measurement for outcome prediction in cervical cancer using magnetic resonance imaging. Int J Radiat Oncol Biol Phys 2001;52:14-22

Mayr NA, Yu WT, Jajoura D, Wang JZ, Lo SS, Montebello JF, et al. Ultra-early predictive assay for treatment failure using functional magnetic resonance imaging and clinical prognostic parameters in cervical cancer. Cancer 2010;116:903-12

Meanwell CA, Rolfe EB, Blackledge G, Docker MF, Lawton FG, Mould JJ. Recurrent female pelvic cancer: assessment with transrectal ultrasonography. Radiology 1987;162:278-81

Miller TR, Grigsby PW. Measurement of tumor volume by PET to evaluate prognosis in patients with advanced cervical cancer treated by radiation therapy. Int J Radiat Oncol Biol Phys 2001:53:353-9

Miller TR, Pinkus E, Dehdashti F, Grigsby PW. Improved prognostic value of ${ }^{18} \mathrm{~F}-\mathrm{FDG}$ PET using a simple visual analysis of tumor characteristics in patients with cervical cancer. J Nucl Med 2003;44:192-7

Mitchell DG, Snyder B, Coakley F, Reinhold C, Thomas G, Amendola MA, et al. Early invasive cervical cancer: MRI and CT predictors of lymphatic metastases in the ACRIN 6651/GOG 183 intergroup study. Gynecol Oncol 2009;112:95-103

Monzen Y, Mori H, Matsumoto A, Yoshida S, Wakisaka M, Komatsu E, et al. [Uterine cervical cancer: usefulness of MR imaging after the initial radiation therapy.] Nihon Igaku Hoshasen Gakkai Zasshi 1995;55:745-50

Moore DH, Dotters DJ, Fowler WCJ. Computed tomography: does it really improve the treatment of cervical carcinoma? Am J Obstet Gynecol 1992;167:768-21

Mortier DG, Stroobants S, Amant F, Neven P, Van Limbergen E, Vergote I. Laparoscopic paraaortic lymphadenectomy and positron emission tomography scan as staging procedures in patients with cervical carcinoma stage IB2-IIIB. Int J Gynecol Cancer 2008;18:723-9

Nakai G, Matsuki M, Inada Y, Tatsugami F, Tanikake M, Narabayashi I, et al. Detection and evaluation of pelvic lymph nodes in patients with gynecologic malignancies using body diffusion-weighted magnetic resonance imaging. J Comput Assist Tomogr 2008;32:764-8

Nakamoto Y, Eisbruch A, Achtyes ED, Sugawara Y, Reynolds KR, Johnston CM, et al. Prognostic value of positron emission tomography using F-18-fluorodeoxyglucose in patients with cervical cancer undergoing radiotherapy. Gynecol Oncol 2002;84:289-95

Lack of full text

Wrong population

Wrong population

Wrong end points

Wrong population

Wrong end points

Wrong population

Wrong population

Wrong population

Wrong population

Lack of full text

Wrong population

Wrong population

Wrong population

Wrong intervention Wrong study design 
TABLE 79 Diagnostic review list of excluded studies with reasons for exclusion (continued)

Reference

Narayan K, Hicks RJ, Jobling T, Bernshaw D, McKenzie AF. A comparison of MRI and PET scanning in surgically staged loco-regionally advanced cervical cancer: potential impact on treatment. Int J Gynecol Cancer 2001;11:263-71

Narayan K, McKenzie AF, Hicks RJ, Fisher R, Bernshaw D, Bau S. Relation between FIGO stage, primary tumor volume, and presence of lymph node metastases in cervical cancer patients referred for radiotherapy. Int J Gynecol Cancer 2003;13:657-63

Narayan K, Fisher RJ, Bernshaw D. Patterns of failure and prognostic factor analyses in locally advanced cervical cancer patients staged by positron emission tomography and treated with curative intent. Int J Gynecol Cancer 2009:19:912-18

Newton WA, Roberts WS, Marsden DE, Cavanagh D. Value of computerized axial tomography in cervical cancer. Oncology 1987;44:124-7

Oberoi R, Vohra S, Jain P, Jena A. Staging of carcinoma cervix with MRI and histopathological correlation in 105 cases. Asian Oceanian J Radiol 2002; 7:88-94

Odunsi KO, Lele S, Ghamande S, Seago P, Driscoll DL. The impact of pre-therapy extraperitoneal surgical staging on the evaluation and treatment of patients with locally advanced cervical cancer. Eur J Gynaecol Oncol 2001;22:325-30

Oellinger JJ, Blohmer JU, Michniewicz K, Siewert C, Wust P, Guthberlet M, et al. Pre-operative staging of cervical cancer: comparison of magnetic resonance imaging (MRI) and computed tomography (CT) with histologic results. Zentralb/ Gynakol 2000;122:82-91

Ogino I, Okamoto N, Andoh K, Kitamura T, Okajima H, Matsubara S. Analysis of prognostic factors in stage IIB-IVA cervical carcinoma treated with radiation therapy: value of computed tomography. Int J Radiat Oncol Biol Phys 1997;37:1071-7

Ohara K, Tanaka YO, Tsunoda H, Nishida M, Sugahara S, Itai Y. Assessment of cervical cancer radioresponse by serum squamous cell carcinoma antigen and magnetic resonance imaging. Obstet Gynecol 2002;100:781-7

Page JE, Constant $\mathrm{O}$, Parsons $\mathrm{C}$. The role of abdominal computed tomography in the assessment of patients with malignant tumours of the cervix and body of the uterus. Clin Radiol 1988;39:273-7

Pakkal MV, Rudralingam V, McCluggage WG, Kelly BE. MR staging in carcinoma of the endometrium and carcinoma of the cervix. Ulster Med J 2004;73:20-4

Park W, Park YJ, Huh SJ, Kim BG, Bae DS, Lee J, et al. The usefulness of MRI and PET imaging for the detection of parametrial involvement and lymph node metastasis in patients with cervical cancer. Jpn J Clin Oncol 2005;35:260-4

Parker LA, McPhail AH, Yankanskas BC, Mauro MA. Computed tomography in the evaluation of clinical stage IB carcinoma of the cervix. Gynecol Oncol 1990;37:332-4

Pellegrino A, Cormio G, Maneo A, Vanzulli A, Villa G, Lissoni A, et al. [Nuclear magnetic resonance imaging in the staging of adenocarcinoma of the uterine cervix.] Minerva Ginecol 1995;47:523-6

Potter R, Dimopoulos J, Georg P, Lang S, Waldha C, Wachter-Gerstner N, et al. Clinical impact of MRI assisted dose volume adaptation and dose escalation in brachytherapy of locally advanced cervix cancer. Radiother Oncol 2007;83:148-55

Qiu JT, Ho KC, Lai CH, Yen TC, Huang YT, Chao A, et al. Supraclavicular lymph node metastases in cervical cancer. Eur J Gynaecoll Oncol 2007;28:33-8

Reinhardt MJ, Eritt-Braun C, Vogelgesang D, Ihling C, Hogerle S, Mix M, et al. Metastatic lymph nodes in patients with cervical cancer: detection with MR imaging and FDG PET. Radiology 2001;218:776-82

Reinhardt MJ, Technau-Ihling K, Altehoefer C, Vogelgesang D, Krause TM. Lymphangiography causes false-positive findings on 18F-FDG PET imaging. Anticancer Res 2003;23:2941-4
Reason for exclusion

Wrong population

Wrong population

Wrong study design

Wrong population

Lack of full text

Lack of full text

Wrong population

Wrong end points

Wrong end points

Lack of gold standard

Wrong population

Wrong population

Wrong population

Wrong population

Wrong end points

Wrong intervention

Wrong population

Lack of full text 
Rockall AG, Sohaib SA, Harisinghani MG, Babar SA, Singh N, Jeyarajah AR, et al. Diagnostic performance of nanoparticle-enhanced magnetic resonance imaging in the diagnosis of lymph node metastases in patients with endometrial and cervical cancer. J Clin Oncol 2005;23:2813-21. [Erratum published in J Clin Oncol 2005;23:4808]

Roh JW, Seo SS, Lee S, Kang KW, Kim S-K, Sim JS, et al. Role of positron emission tomography in pretreatment lymph node staging of uterine cervical cancer: a prospective surgicopathologic correlation study. Eur J Cancer 2005;41:2086-92

Rose PG, Adler LP, Rodriguez PF, Abdul-Karim FW, Miraldi F. Positron emission tomography for evaluating para-aortic nodal metastasis in locally advanced cervical cancer before surgical staging: a surgicopathologic study. J Clin Oncol 1999;17:41-5

Roy C, le Bras Y, Mangold L, Saussinej C, Tuchmann C, Pfleger D, et al. Small pelvic lymph node metastases: evaluation with MR imaging. Clin Radiol 1997;52:437-40

Rubens D, Thornbury JR, Angel C, Stoler MH, Weiss SL, Lerner RM, et al. Stage IB cervical carcinoma: comparison of clinical, MR, and pathologic staging. AJR Am J Roentgenol 1988:150:135-8

Russell AH, Walter JP, Anderson MW, Zukowski CL. Sagittal magnetic resonance imaging in the design of lateral radiation treatment portals for patients with locally advanced squamous cancer of the cervix. Int J Radiat Oncol Biol Phys 1992;23:449-55

Ryu SY, Kim M-H, Choi C-S, Choi C-W, Lee K-H. Detection of early recurrence with ${ }^{18} \mathrm{~F}-\mathrm{FDG}$ PET in patients with cervical cancer. J Nucl Med 2003;44:347-52

Sahdev A, Sohaib SA, Wenaden AE, Shepherd JH, Reznek RH. The performance of magnetic resonance imaging in early cervical carcinoma: a long-term experience. Int I Gynecol Cancer 2007;17:629-36

Sakurai $H$, Suzuki $Y$, Nonaka T, Ishikawa $H$, Shioya M, Kiyohara $H$, et al. FDG-PET in the detection of recurrence of uterine cervical carcinoma following radiation therapy - tumor volume and FDG uptake value. Gynecol Oncol 2006;100:601-7

Schaffer U, Hawighorst H, Pilch H, Welkel W, Zuna I, Knapstein PG. [Value of clinically established MRI procedures concerning the pretherapeutic evaluation of maximal tumor diameter in primary or recurrent cervix cancer in relation to palpation findings and histopathologic whole mount specimens.] Zentralb/ Gynakol 1999;121:131-6

Schwarz JK, Seigel BA, Dehdashti F, Grigsby PW. Association of posttherapy positron emission tomography with tumor response and survival in cervical carcinoma. JAMA 2007:298:2289-95

Schwarz JK, Grigsby PW, Dehdashti F, Delbeke D. The role of 18F-FDG PET in assessing therapy response in cancer of the cervix and ovaries. J Nucl Med 2009;50:64S-73S

Semple SIK, Harry VN, Parkin DE, Gilbert FJ. A combined pharmacokinetic and radiologic assessment of dynamic contrast-enhanced magnetic resonance imaging predicts response to chemoradiation in locally advanced cervical cancer. Int J Radiat Oncol Biol Phys 2009;75:611-17

Shatov AV. [Potentialities of low-field magnetic resonance tomography in the diagnosis and treatment of invasive cancer of cervix uteri.] Vestn Rentgenol Radiol 2003;3:48-53

Sheu M, Chang C-Y, Wang J-H, Yen M-S. MR staging of clinical stage I and Ila cervical carcinoma: a reappraisal of efficacy and pitfalls. Eur J Radiol 2001;38:225-31

Sheu MH, Chang CY, Wang JH, Yen MS. Cervical carcinoma: assessment of parametrial invasion and lymph node metastasis with magnetic resonance imaging. Chung Hua i Hsueh Tsa Chih - Chin Med J 2000;63:634-40

Shiraiwa M, Joja I, Asakawa T, Okuno K, Shibutani O, Akamatsu N, et al. Cervical carcinoma: efficacy of thin-section oblique axial T2-weighted images for evaluating parametrial invasion. Abdom Imaging 1999;24:514-19

Silberer H, Wölber L, Fuchs L, Schwarz J. Pre-therapeutic determination of tumor stage in patients with cervical carcinoma - a comparison of clinical evaluation, NMR and CT. Geburtshilfe Frauenheilkd 2007:67:837-42

Wrong population

Wrong population

Wrong population

Wrong study design

Wrong population

Wrong population

Wrong population

Wrong intervention

No data

Wrong end points

Wrong study design

Wrong population

Wrong population

Wrong population

Lack of full text

Wrong population

Wrong population 
TABLE 79 Diagnostic review list of excluded studies with reasons for exclusion (continued)

Reference

Singh AK, Grigsby PW, Dehdashti F, Herzog TJ, Siegel BA. FDG-PET lymph node staging and survival of patients with FIGO stage IIIb cervical carcinoma. Int J Radiat Oncol Biol Phys 2001;56:489-93

Sironi S, Belloni C, Taccagni GL, DelMaschio A. Carcinoma of the cervix: value of MR imaging in detecting parametrial involvement. AJR Am J Roentgenol 1991;156:753-6

Sironi S, Buda A, Picchio M, Perego P, Moreni R, Pellegrino A, et al. Lymph node metastasis in patients with clinical early-stage cervical cancer: detection with integrated FDG PET/CT. Radiology 2006;238:272-9

Smaniotto D, Smaniotto D, Andrulli AD, Tortoreto F, Niespolo RM, Valentini V. Organ preservation in locally advanced carcinoma of the uterine cervix. Rays 1997;22:472-7

Soeters RP, Beningfield SJ, Dehaeck K, Levin W, Bloch B. The value of magnetic resonance imaging in patients with carcinoma of the cervix (a pilot study). Eur J Surg Oncol 1991;17:119-24

Soutter WP, Hanoch J, D'Arcy T, Dina R, McIndoe GA, deSouza NM. Pretreatment tumour volume measurement on high-resolution magnetic resonance imaging as a predictor of survival in cervical cancer. BJOG 2004;111:741-7

Steinbrich W, Rohde U, Friedmann G. [Importance of computed tomography for the diagnosis of tumors of the uterus and recurrent lesions.] Radiologe 1982;22:154-61

Steinkamp HJ, Heim T, Schubeus P, Schorner W, Felix R. [The magnetic resonance tomographic differential diagnosis between reactively enlarged lymph nodes and cervical lymph node metastases.] Rofo 1992;157:406-13

Steinkamp HJ, Zwicker C, Langer M, Mathe M, Ehritt C, Neumann K, et al. [Reactive enlargement of cervical lymph nodes and cervical lymph node metastases: sonography (M/Q quotient) and computed tomography.] Aktuelle Radiol 1992;2:188-95

Stryker JA, Mortel R. Survival following extended field irradiation in carcinoma of cervix metastatic to para-aortic lymph nodes. Gynecol Oncol 2000;79:399-405

Stummvoll W, Holbock E, Schoissengeier A. [Diagnostic value of abdominal computerized tomography. After-care of gynecologic malignancies.] Gynakol Geburtshilfliche Rundsch 1994;34:55-7

Subak LL, Hricak H, Powell B, Azizi L, Stern JL. Cervical carcinoma: computed tomography and magnetic resonance imaging for preoperative staging. Obstet Gynecol 1995;86:43-50

Sugawara Y, Eisbruch A, Kosuda S, Recker BE, Kison PV, Wahl RL. Evaluation of FDG PET in patients with cervical cancer. J Nucl Med 1999;40:1125-31

Sugimura K, Carrington BM, Quivey JM, Hricak H. Postirradiation changes in the pelvis: assessment with MR imaging. Radiology 1990;175:805-13

Sun SS, Chen T-Z, Yen R-F, Shen Y-Y, Changlai S-P, Kao A. Value of whole body 18F-fluoro-2deoxyglucose positron emission tomography in the evaluation of recurrent cervical cancer. Anticancer Res 2001;21:2957-61

Tardivon AA, Kinkel K, Lartigau E, Masselot J, Gerbaulet AP, Vanel D. MR imaging during intracavitary brachytherapy of vaginal and cervical cancer: preliminary results. Radiographics 1996;16:1363-70

Tatsumi M, Cohade C, Bristow RE, Wahl RL. Imaging uterine cervical cancer with FDG-PET/CT: direct comparison with PET. Mol Imaging Biol 2009;11:229-35

Taylor MB, Carrington BM, Davidson SE, Swindell R, Lawrance JA. Staging of advanced cervical carcinoma using MRI-predictors of outcome after radical radiotherapy. Clin Radiol 2003;58:532-41

Testa AC, Ludovisi M, Manfredi R, Zanoni G, Gui B, Basso D, et al. Transvaginal ultrasonography and magnetic resonance imaging for assessment of presence, size and extent of invasive cervical cancer. Ultrasound Obstet Gynecol 2009;34:335-44
Reason for exclusion

Wrong population

Wrong population

Wrong population

Lack of full text

Wrong population

Wrong population

Lack of full text

Wrong population

Lack of full text

Wrong end points

Lack of full text

Wrong population

Wrong intervention

Wrong end points

Wrong intervention

Wrong population

Lesion-based analysis

Wrong population

Wrong population 
Thomas L, Chacon B, Kind M, Lasbareilles O, Muyldermans P, Chemin A, et al. Magnetic resonance imaging in the treatment planning of radiation therapy in carcinoma of the cervix treated with the four-field pelvic technique. Int J Radiat Oncol Biol Phys 1997;37:827-32

Thorvinger B. Diagnostic and interventional radiology in gynecologic neoplasms. Acta Radiol Supp/ 1992;378:93-108

Togashi K, Nishimura K, Itoh K, Fujisawa I, Asato R, Nakano Y, et al. Uterine cervical cancer: assessment with high-field MR imaging. Radiology 1986;160:431-5

Thurnher S, McPhillips M, von Schulthess GK, Maricek B. [Cervical carcinoma staging with magnetic resonance tomography: the use of gadolinium-DOTA with 31 patients.] Rofo 1991;154:643-9

Toita T, Nakano M, Higashi M, Sakumto K, Kanazawa K. Prognostic value of cervical size and pelvic lymph node status assessed by computed tomography for patients with uterine cervical cancer treated by radical radiation therapy. Int J Radiat Oncol Biol Phys 1995;33:843-9

Tran BN, Grigsby PW, Dehdashti F, Herzog TJ, Siegel BA. Occult supraclavicular lymph node metastasis identified by FDG-PET in patients with carcinoma of the uterine cervix. Gynecol Oncol 2003;90:572-6

Trinci M, Raffeto N, Petrozza V, Melis M, Biagini C. Pretreatment scalene node biopsy in cervical carcinoma. Eur J Gynaecol Oncol 1988;9:308-12

Tsai CS, Chang T-C, Lai C-H, Tsai C-C, Ng K-K, Hsueh S, et al. Preliminary report of using FDGPET to detect extrapelvic lesions in cervical cancer patients with enlarged pelvic lymph nodes on MRI/CT. Int J Radiat Oncol Biol Phys 2004;58:1506-12

Tsai CS, Lai C-H, Chang C-C, Yen T-C, Ng K-K, Hsueh S, et al. A prospective randomized trial to study the impact of pretreatment FDG-PET for cervical cancer patients with MRI-detected positive pelvic but negative para-aortic lymphadenopathy. Int J Radiat Oncol Biol Phys 2010;76:477-84

Umesaki N, Tanaka T, Miyama M, Kawabe J, Okamura T, Koyama K, et al. The role of 18F-fluoro-2-deoxy-D-glucose positron emission tomography ( ${ }^{18} \mathrm{~F}-\mathrm{FDG}$-PET) in the diagnosis of recurrence and lymph node metastasis of cervical cancer. Oncol Rep 2000;7:1261-4

Unger JB, Ivy JJ, Connor P, Charrier A, Ramaswamy MR, Ampil FL, et al. Detection of recurrent cervical cancer by whole-body FDG PET scan in asymptomatic and symptomatic women. Gynecol Oncol 2004;94:212-16

Unger JB, Ivy JJ, Ramaswamy MR, Charrier A, Connor P. Whole-body [ $\left.{ }^{18} \mathrm{~F}\right]$ fluoro-2deoxyglucose positron emission tomography scan staging prior to planned radical hysterectomy and pelvic lymphadenectomy. Int J Gynecol Cancer 2005;15:1060-4

Unger JB, Lilien DL, Caldito G, Ivy JJ, Charrier A, Bellaire B. The prognostic value of pretreatment 2-[18F]-fluoro-2-deoxy-D-glucose positron emission tomography scan in women with cervical cancer. Int J Gynecol Cancer 2007;17:1062-7

van der Veldt AA, Hooft L, van Diest PJ, Berkhof J, Buist MR, Comans EF, et al. Microvessel density and p53 in detecting cervical cancer by FDG PET in cases of suspected recurrence. Eur J Nucl Med Mol Imaging 2006;33:1408-16

van der Veldt AA, Buist MR, van Baal MW, Comans EF, Hoekstra OS, Molthoff CF. Clarifying the diagnosis of clinically suspected recurrence of cervical cancer: impact of ${ }^{18} \mathrm{~F}-\mathrm{FDG}$ PET. J NuCl Med 2008:49:1936-43

Van Engelshoven J, Versteege CWM, Ruys JHJ. Computed tomography in staging untreated patients with cervical cancer. Gynecol Obstet Invest 1984;18:289-95

Vergote I, Tsolakidis D, Mortier D, Neven P, Amant F. Value of positron emission tomography of the para-aortic lymph nodes in cervical carcinoma stage IB2-IIIB. J Clin Oncol 2008;26:5654-5

Villasanta U, Whitley NO, Haney PJ, Brenner D. Computed tomography in invasive carcinoma of the cervix: an appraisal. Obstet Gynecol 1983;62:218-24

Wrong end points

Wrong study design

Lack of full text

Wrong population

Wrong end points

Wrong population

Wrong population

Wrong population

Wrong population

Lack of full text

Differential verification of reference standard

Wrong intervention

Wrong population

Wrong intervention

Wrong intervention

Wrong population

Wrong study design

Wrong population 
TABLE 79 Diagnostic review list of excluded studies with reasons for exclusion (continued)

Reference

Voss $\mathrm{AC}$, Hubener $\mathrm{KH}$, Metzger $\mathrm{H}$. [The value of radiometry in the treatment planning of inoperable carcinomas of the cervix (authors' translation).] Rofo 1981;135:225-9

Wagenaar HC, Trimbos JB, Postema S, Anastasopoulou A, van der Geest RJ, Reiber JH, et al. Tumor diameter and volume assessed by magnetic resonance imaging in the prediction of outcome for invasive cervical cancer. Gynecol Oncol 2001;82:474-82

Walsh JW, Amendola MA, Konerding KF, Tisnado J, Hazra TA. Computed tomographic detection of pelvic and inguinal lymph-node metastases from primary and recurrent pelvic malignant disease. Radiology 1980;137:157-66

Walton LA, McCartney WH, Vesterinen E. The use of computerized tomography to obviate celiotomy in recurrent carcinoma of the cervix. Gynecol Oncol 1981;12:166-76

Wang $\sqcup$, Wong Y-C, Chen C-J, Huang K-G, Hsueh S. Cervical carcinoma: MR imaging with integrated endorectal/phased-array coils: a pilot study. Eur Radiol 2001;11:1822-7

Weiss E, Eberlein K, Pradier O, Schmidberger H, Hess CF. The impact of patient positioning on the adequate coverage of the uterus in the primary irradiation of cervical carcinoma: a prospective analysis using magnetic resonance imaging. Radiother Oncol 2002;63:83-7

Whitley NO, Brenner DE, Francis A, Villasanta V, Aisner J, Wiernik PH. Computed tomographic evaluation of carcinoma of the cervix. Radiology 1982;142:439-46

Wong TZ, Jones EL, Coleman RE. Positron emission tomography with 2-deoxy-2-[(18)F]fluoroD-glucose for evaluating local and distant disease in patients with cervical cancer. Mol Imaging Biol 2004;6:55-62

Wright JD, Dehdashti F, Herzog TJ, Mutch DG, Huettner PC, Rader JS. Preoperative lymph node staging of early-stage cervical carcinoma by [18F]-fluoro-2-deoxy-D-glucose-positron emission tomography. Cancer 2005;104:2484-91

Xue F, Lin LL, Dehdashti F, Miller TR, Siegel BA, Grigsby PW. F-18 fluorodeoxyglucose uptake in primary cervical cancer as an indicator of prognosis after radiation therapy. Gynecol Oncol 2006;101:147-51

Xue HD, Li SL, Sun F, Sun H-Y, Jin Z-Y, Yang J-X, et al. Clinical application of body diffusion weighted MR imaging in the diagnosis and preoperative $\mathrm{N}$ staging of cervical cancer. Chin Med Sci J 2008;23:133-7

Yamashita Y, Harada M, Torashima M, Takahashi M, Miyazaki K, Tanaka N, et al. Dynamic MR imaging of recurrent postoperative cervical cancer. J Magn Reson Imaging 1996;6:167-71

Yang WT, Lam WW, Yu MY, Cheung TH, Metreweli C. Comparison of dynamic helical CT and dynamic MR imaging in the evaluation of pelvic lymph nodes in cervical carcinoma. AJR Am J Roentgenol 2000;175:759-66

Yeh LS, Hung Y-C, Shen Y-Y, Kao C-H, Lin C-C, Lee C-C. Detecting para-aortic lymph nodal metastasis by positron emission tomography of $18 \mathrm{~F}$-fluorodeoxyglucose in advanced cervical cancer with negative magnetic resonance imaging findings. Oncol Rep 2002;9:1289-92

Yen TC, Ng K-K, Ma S-Y, Chou H-H, Tsai C-S, Hsueh S, et al. Value of dual-phase 2-fluoro2-deoxy-D-glucose positron emission tomography in cervical cancer. J Clin Oncol

2003:21:3651-8

Yen TC, See L-C, Chang T-C, Huang K-G, Ng K-K, Tang SG, et al. Defining the priority of using ${ }^{18}$ F-FDG PET for recurrent cervical cancer. J Nucl Med 2004;45:1632-9

Yen TC, Lai CH. Positron emission tomography in gynecologic cancer. Semin Nucl Med 2006;36:93-104

Yen TC, Lai C-H, Ma S-Y, Huang K-G, Huang H-J, Hong J-H, et al. Comparative benefits and limitations of ${ }^{18} \mathrm{~F}-\mathrm{FDG}$ PET and CT-MRI in documented or suspected recurrent cervical cancer. Eur J Nucl Med Mol Imaging 2006;33:1399-407
Reason for exclusion

Wrong end points

Wrong population

Small sample size

Wrong study design

Wrong population

Wrong end points

Wrong population

Wrong intervention

Wrong population

Wrong end points

Wrong population

No data

Wrong population

Wrong intervention

Wrong intervention

Wrong intervention

Wrong study design

Wrong intervention 
TABLE 79 Diagnostic review list of excluded studies with reasons for exclusion (continued)

Yildirim Y, Sehirali S, Avci ME, Yilmaz C, Ertopcu K, Tinar S, et al. Integrated PET/CT for the evaluation of para-aortic nodal metastasis in locally advanced cervical cancer patients with negative conventional CT findings. Gynecol Oncol 2008;108:154-9

Yokoyama T, Hiura M, Myoga H, Yorishima M, Tanaka M, Chiba T. [Computed tomography for the assessment of pelvic lymph node metastasis in cases of uterine cervical carcinoma.] Gan No Rinsho 1990;36:495-8

Yoo SC, Kim WY, Yoon JH, Kim HY, Lee EJ, Chang SJ, et al. Accuracy of preoperative magnetic resonance imaging in assessing lymph node metastasis and myometrial invasion in patients with uterine cancer. Eur J Gynaecol Oncol 2009;30:167-70

Yousem DM, Sorn PM, Hackney DB, Schwaibold F, Hendrix RA. Central nodal necrosis and extracapsular neoplastic spread in cervical lymph nodes: MR imaging versus CT. Radiology 1992;182:753-9

Yu KK, Hricak H, Subak LL, Zaloudek CJ, Powell CB. Preoperative staging of cervical carcinoma: phased array coil fast spin-echo versus body coil spin-echo T2-weighted MR imaging. AJR Am J Roentgenol 1998;171:707-11

Zanetta G, Pellegrino A, Vanzulli A, Di Lelio A, Milani R, Mangioni C. Magnetic resonance imaging of cervical cancer in pregnancy. Int J Gynecol Cancer 1998;8:265-9

Wrong population

Lack of full text

Wrong population

Wrong population

Wrong population

Zapf S, Halbsguth A, Schweden F, Klose K, Lochner B, Beck T, et al. [Problem of pretherapeutic staging of cervical carcinoma. Studies of the diagnostic value of computerized tomography and magnetic resonance tomography in comparison with gynecologic palpation findings and pathologic-anatomic diagnosis.] Geburtshilfe Frauenheilkd 1987;47:838-42

Zeisler $\mathrm{H}$, Joura EA, Moeschl P, Maier U, Koebl H. Preoperative evaluation of tumor extension in patients with recurrent cervical cancer. Acta Obstet Gynecol Scand 1997;76:474-7 


\section{Appendix 11 Diagnostic meta-analysis logistic regression results}

TABLE 80 Results of univariate random-effects logistic regression models of all PET-CT studies

\begin{tabular}{lllll} 
Summary & Estimate & SE & $95 \%$ lower limit & $95 \%$ upper limit \\
Sensitivity & 0.9215686 & 0.02662 & 0.850943 & 0.9602922 \\
Specificity & 0.880597 & 0.0396149 & 0.7789671 & 0.9391483 \\
\hline SE, standard error. & & &
\end{tabular}

TABLE 81 Results of univariate random-effects logistic regression models of PET-CT studies: sensitivity analysis omitting Amit et al. ${ }^{48}$

\begin{tabular}{lllll} 
Summary & Estimate & SE & $95 \%$ lower limit & $95 \%$ upper limit \\
Sensitivity & 0.9263158 & 0.0268043 & 0.8534262 & 0.9644673 \\
Specificity & 0.8730159 & 0.0419484 & 0.7660703 & 0.9352045 \\
\hline
\end{tabular}

$\mathrm{SE}$, standard error. 



\section{Appendix 12 Subjective elicitation results}

TABLE 82 Elicitation: prevalence of recurrent disease in symptomatic patients a minimum of 3 months post completion of primary treatment

\begin{tabular}{|c|c|c|c|c|c|c|c|}
\hline & \multicolumn{6}{|c|}{ Percentage of symptomatic women with recurrence } & \multirow{2}{*}{$\begin{array}{l}\text { Mean prevalence } \\
(\%)\end{array}$} \\
\hline & $\leq \mathbf{5 0}$ & $51-60$ & $61-70$ & $71-80$ & $81-90$ & $91-100$ & \\
\hline Midpoint (\%) & 25.5 & 55.5 & 65.5 & 75.5 & 85.5 & 95.5 & \\
\hline Likelihood - clinician 1 & 50 & 50 & 0 & 0 & 0 & 0 & 40.5 \\
\hline Likelihood - clinician 2 & 44 & 22 & 22 & 11 & 0 & 0 & 46.6 \\
\hline Likelihood - clinician 3 & 57 & 14 & 10 & 8 & 7 & 5 & 44.7 \\
\hline Likelihood - clinician 4 & 10 & 80 & 10 & 0 & 0 & 0 & 53.5 \\
\hline Likelihood - clinician 5 & 0 & 10 & 10 & 70 & 10 & 0 & 73.5 \\
\hline Likelihood - clinician 6 & 10 & 10 & 50 & 30 & 0 & 0 & 63.5 \\
\hline Likelihood - clinician 7 & 0 & 100 & 0 & 0 & 0 & 0 & 55.5 \\
\hline Likelihood - clinician 8 & 100 & 0 & 0 & 0 & 0 & 0 & 25.5 \\
\hline \multicolumn{8}{|l|}{ Likelihood - clinician 9} \\
\hline Likelihood - clinician 10 & 80 & 20 & 0 & 0 & 0 & 0 & 31.5 \\
\hline Likelihood - clinician 11 & 90 & 10 & 0 & 0 & 0 & 0 & 28.5 \\
\hline Likelihood - clinician 12 & 95 & 0 & 0 & 0 & 0 & 5 & 29.0 \\
\hline Likelihood - clinician 13 & 100 & 0 & 0 & 0 & 0 & 0 & 25.5 \\
\hline Likelihood - clinician 14 & 0 & 0 & 50 & 50 & 0 & 0 & 70.5 \\
\hline Likelihood - clinician 15 & 0 & 20 & 60 & 20 & 0 & 0 & 65.5 \\
\hline Likelihood - clinician 16 & 0 & 10 & 20 & 40 & 20 & 10 & 75.5 \\
\hline Likelihood - clinician 17 & 70 & 20 & 10 & 0 & 0 & 0 & 35.5 \\
\hline Likelihood - clinician 18 & 0 & 0 & 100 & 0 & 0 & 0 & 65.5 \\
\hline Likelihood - clinician 19 & 20 & 80 & 0 & 0 & 0 & 0 & 49.5 \\
\hline Likelihood - clinician 20 & 40 & 25 & 15 & 10 & 5 & 5 & 50.5 \\
\hline Likelihood - clinician 21 & 100 & 0 & 0 & 0 & 0 & 0 & 25.5 \\
\hline $\begin{array}{l}\text { Mean prevalence } \\
\text { symptomatic }\end{array}$ & 43.3 & 23.6 & 17.8 & 11.9 & 2.1 & 1.2 & 47.8 \\
\hline SD prevalence & & & & & & & 20.8 \\
\hline
\end{tabular}


TABLE 83 Elicitation: prevalence of recurrent disease in asymptomatic patients a minimum of 3 months post completion of primary treatment

\begin{tabular}{|c|c|c|c|c|c|c|c|}
\hline & \multicolumn{6}{|c|}{ Percentage of asymptomatic women with recurrence } & \multirow{2}{*}{$\begin{array}{l}\text { Mean prevalence } \\
(\%)\end{array}$} \\
\hline & $0-10$ & $11-20$ & $21-30$ & $31-40$ & $41-50$ & $>50$ & \\
\hline Midpoint (\%) & 5.5 & 15.5 & 25.5 & 35.5 & 45.5 & 75.5 & \\
\hline Likelihood - clinician 1 & 30 & 30 & 40 & 0 & 0 & 0 & 16.5 \\
\hline Likelihood - clinician 2 & 100 & 0 & 0 & 0 & 0 & 0 & 5.5 \\
\hline Likelihood - clinician 3 & 10 & 5 & 50 & 30 & 5 & 0 & 27.0 \\
\hline Likelihood - clinician 4 & 90 & 10 & 0 & 0 & 0 & 0 & 6.5 \\
\hline Likelihood - clinician 5 & 90 & 10 & 0 & 0 & 0 & 0 & 6.5 \\
\hline Likelihood - clinician 6 & 20 & 20 & 20 & 20 & 15 & 5 & 27.0 \\
\hline Likelihood - clinician 7 & 0 & 100 & 0 & 0 & 0 & 0 & 15.5 \\
\hline Likelihood - clinician 8 & 10 & 80 & 10 & 0 & 0 & 0 & 15.5 \\
\hline \multicolumn{8}{|l|}{ Likelihood - clinician 9} \\
\hline Likelihood - clinician 10 & 100 & 0 & 0 & 0 & 0 & 0 & 5.5 \\
\hline Likelihood - clinician 11 & 0 & 10 & 90 & 0 & 0 & 0 & 24.5 \\
\hline Likelihood - clinician 12 & 95 & 0 & 0 & 0 & 0 & 5 & 9.0 \\
\hline Likelihood - clinician 13 & 100 & 0 & 0 & 0 & 0 & 0 & 5.5 \\
\hline Likelihood - clinician 14 & 0 & 0 & 50 & 50 & 0 & 0 & 30.5 \\
\hline Likelihood - clinician 15 & 10 & 60 & 30 & 0 & 0 & 0 & 17.5 \\
\hline Likelihood - clinician 16 & 10 & 20 & 40 & 20 & 10 & 0 & 25.5 \\
\hline Likelihood - clinician 17 & 100 & 0 & 0 & 0 & 0 & 0 & 5.5 \\
\hline Likelihood - clinician 18 & 100 & 0 & 0 & 0 & 0 & 0 & 5.5 \\
\hline Likelihood - clinician 19 & 10 & 80 & 10 & 0 & 0 & 0 & 15.5 \\
\hline Likelihood - clinician 20 & 5 & 5 & 40 & 30 & 10 & 10 & 34.0 \\
\hline Likelihood - clinician 21 & 0 & 6 & 22 & 56 & 11 & 6 & 35.5 \\
\hline $\begin{array}{l}\text { Mean prevalence } \\
\text { asymptomatic }\end{array}$ & 44 & 21.8 & 20.1 & 10.3 & 2.6 & 1.3 & 16.7 \\
\hline SD prevalence & & & & & & & 13.1 \\
\hline
\end{tabular}


TABLE 84 Elicitation: accuracy (PPV) - symptomatic women investigated using CT and/or MRI

\begin{tabular}{|c|c|c|c|c|c|c|}
\hline & \multicolumn{5}{|c|}{ Mid-point PPV (\%) } & \multirow[b]{2}{*}{ Mean PPV (\%) } \\
\hline & 95.5 & 85.5 & 75.5 & 65.5 & 55.5 & \\
\hline Likelihood - clinician 1 & 0 & 0 & 80 & 20 & 0 & 73.5 \\
\hline Likelihood - clinician 2 & 50 & 50 & 0 & 0 & 0 & 90.5 \\
\hline Likelihood - clinician 3 & 40 & 40 & 10 & 10 & 0 & 86.5 \\
\hline Likelihood - clinician 4 & 90 & 10 & 0 & 0 & 0 & 94.5 \\
\hline Likelihood - clinician 5 & 70 & 30 & 0 & 0 & 0 & 92.5 \\
\hline Likelihood - clinician 6 & 95 & 5 & 0 & 0 & 0 & 95 \\
\hline \multicolumn{7}{|l|}{ Likelihood - clinician 7} \\
\hline \multicolumn{7}{|l|}{ Likelihood - clinician 8} \\
\hline \multicolumn{7}{|l|}{ Likelihood - clinician 9} \\
\hline Likelihood - clinician 10 & 100 & 0 & 0 & 0 & 0 & 95.5 \\
\hline Likelihood - clinician 11 & 50 & 50 & 0 & 0 & 0 & 90.5 \\
\hline Likelihood - clinician 12 & 100 & 0 & 0 & 0 & 0 & 95.5 \\
\hline Likelihood - clinician 13 & 0 & 0 & 50 & 50 & 0 & 70.5 \\
\hline Likelihood - clinician 14 & 50 & 50 & 0 & 0 & 0 & 90.5 \\
\hline Likelihood - clinician 15 & 15 & 70 & 15 & 0 & 0 & 85.5 \\
\hline Likelihood - clinician 16 & 10 & 80 & 10 & 0 & 0 & 85.5 \\
\hline Likelihood - clinician 17 & 90 & 10 & 0 & 0 & 0 & 94.5 \\
\hline Likelihood - clinician 18 & 10 & 80 & 10 & 0 & 0 & 85.5 \\
\hline Likelihood - clinician 19 & 90 & 10 & 0 & 0 & 0 & 94.5 \\
\hline Likelihood - clinician 20 & 10 & 20 & 40 & 20 & 10 & 75.5 \\
\hline Likelihood - clinician 21 & 90 & 10 & 0 & 0 & 0 & 94.5 \\
\hline Mean PPV & 53.3 & 28.6 & 11.9 & 5.6 & 0.6 & 88.4 \\
\hline SD PPV & & & & & & 9.2 \\
\hline
\end{tabular}


TABLE 85 Elicitation: accuracy (NPV) - symptomatic women investigated using CT and/or MRI

\begin{tabular}{|c|c|c|c|c|c|c|}
\hline & \multicolumn{5}{|c|}{ Mid-point NPV (\%) } & \multirow[b]{2}{*}{ Mean NPV (\%) } \\
\hline & 95.5 & 85.5 & 75.5 & 65.5 & 55.5 & \\
\hline Likelihood - clinician 1 & 0 & 80 & 20 & 0 & 0 & 83.5 \\
\hline Likelihood - clinician 2 & 70 & 30 & 0 & 0 & 0 & 92.5 \\
\hline Likelihood - clinician 3 & 30 & 40 & 20 & 10 & 0 & 84.5 \\
\hline Likelihood - clinician 4 & 5 & 90 & 5 & 0 & 0 & 85.5 \\
\hline Likelihood - clinician 5 & 80 & 20 & 0 & 0 & 0 & 93.5 \\
\hline Likelihood - clinician 6 & 95 & 5 & 0 & 0 & 0 & 95 \\
\hline \multicolumn{7}{|l|}{ Likelihood - clinician 7} \\
\hline \multicolumn{7}{|l|}{ Likelihood - clinician 8} \\
\hline \multicolumn{7}{|l|}{ Likelihood - clinician 9} \\
\hline Likelihood - clinician 10 & 80 & 20 & 0 & 0 & 0 & 93.5 \\
\hline \multicolumn{7}{|l|}{ Likelihood - clinician 11} \\
\hline Likelihood - clinician 12 & 10 & 0 & 90 & 0 & 0 & 77.5 \\
\hline Likelihood - clinician 13 & 0 & 0 & 100 & 0 & 0 & 75.5 \\
\hline Likelihood - clinician 14 & 0 & 50 & 50 & 0 & 0 & 80.5 \\
\hline Likelihood - clinician 15 & 80 & 10 & 10 & 0 & 0 & 92.5 \\
\hline Likelihood - clinician 16 & 10 & 80 & 10 & 0 & 0 & 85.5 \\
\hline Likelihood - clinician 17 & 90 & 10 & 0 & 0 & 0 & 94.5 \\
\hline Likelihood - clinician 18 & 0 & 10 & 80 & 10 & 0 & 75.5 \\
\hline Likelihood - clinician 19 & 20 & 80 & 0 & 0 & 0 & 87.5 \\
\hline Likelihood - clinician 20 & 40 & 30 & 10 & 10 & 10 & 83.5 \\
\hline Likelihood - clinician 21 & 90 & 10 & 0 & 0 & 0 & 94.5 \\
\hline Mean NPV & 41.2 & 33.2 & 23.2 & 1.8 & 0.6 & 87.1 \\
\hline SD NPV & & & & & & 8.7 \\
\hline
\end{tabular}


TABLE 86 Elicitation: accuracy (PPV) - symptomatic women investigated using CT and/or MRI and PET-CT

\begin{tabular}{|c|c|c|c|c|c|c|}
\hline & \multicolumn{5}{|c|}{ Mid-point PPV (\%) } & \multirow[b]{2}{*}{ Mean PPV (\%) } \\
\hline & 95.5 & 85.5 & 75.5 & 65.5 & 55.5 & \\
\hline Likelihood - clinician 1 & 0 & 0 & 50 & 50 & 0 & 70.5 \\
\hline Likelihood - clinician 2 & 20 & 50 & 30 & 0 & 0 & 84.5 \\
\hline Likelihood - clinician 3 & 50 & 40 & 5 & 5 & 0 & 89.0 \\
\hline Likelihood - clinician 4 & 100 & 0 & 0 & 0 & 0 & 95.5 \\
\hline Likelihood - clinician 5 & 90 & 10 & 0 & 0 & 0 & 94.5 \\
\hline Likelihood - clinician 6 & 10 & 90 & 0 & 0 & 0 & 86.5 \\
\hline \multicolumn{7}{|l|}{ Likelihood - clinician 7} \\
\hline \multicolumn{7}{|l|}{ Likelihood - clinician 8} \\
\hline \multicolumn{7}{|l|}{ Likelihood - clinician 9} \\
\hline Likelihood - clinician 10 & 100 & 0 & 0 & 0 & 0 & 95.5 \\
\hline Likelihood - clinician 11 & 5 & 90 & 5 & 0 & 0 & 85.5 \\
\hline Likelihood - clinician 12 & 95 & 0 & 0 & 0 & 5 & 93.5 \\
\hline Likelihood - clinician 13 & 0 & 100 & 0 & 0 & 0 & 85.5 \\
\hline Likelihood - clinician 14 & 0 & 50 & 50 & 0 & 0 & 80.5 \\
\hline Likelihood - clinician 15 & 11.1 & 22.2 & 33.3 & 22.2 & 11.1 & 75.5 \\
\hline Likelihood - clinician 16 & 0 & 10 & 80 & 10 & 0 & 75.5 \\
\hline Likelihood - clinician 17 & 90 & 10 & 0 & 0 & 0 & 94.5 \\
\hline Likelihood - clinician 18 & 100 & 0 & 0 & 0 & 0 & 95.5 \\
\hline Likelihood - clinician 19 & 20 & 80 & 0 & 0 & 0 & 87.5 \\
\hline Likelihood - clinician 20 & 5 & 30 & 40 & 20 & 5 & 76.5 \\
\hline Likelihood - clinician 21 & 0 & 5 & 90 & 5 & 0 & 75.5 \\
\hline Mean PPV & 38.7 & 32.6 & 21.3 & 6.2 & 1.2 & 85.6 \\
\hline SD PPV & & & & & & 9.8 \\
\hline
\end{tabular}


TABLE 87 Elicitation: accuracy (NPV) - symptomatic women investigated using CT and/or MRI and PET-CT

\begin{tabular}{|c|c|c|c|c|c|c|}
\hline & \multicolumn{5}{|c|}{ Mid-point NPV (\%) } & \multirow[b]{2}{*}{ Mean NPV (\%) } \\
\hline & 95.5 & 85.5 & 75.5 & 65.5 & 55.5 & \\
\hline Likelihood - clinician 1 & 80 & 20 & 0 & 0 & 0 & 93.5 \\
\hline Likelihood - clinician 2 & 40 & 40 & 20 & 0 & 0 & 87.5 \\
\hline Likelihood - clinician 3 & 90 & 10 & 0 & 0 & 0 & 94.5 \\
\hline Likelihood - clinician 4 & 90 & 10 & 0 & 0 & 0 & 94.5 \\
\hline Likelihood - clinician 5 & 95 & 5 & 0 & 0 & 0 & 95.0 \\
\hline Likelihood - clinician 6 & 100 & 0 & 0 & 0 & 0 & 95.5 \\
\hline \multicolumn{7}{|l|}{ Likelihood - clinician 7} \\
\hline \multicolumn{7}{|l|}{ Likelihood - clinician 8} \\
\hline \multicolumn{7}{|l|}{ Likelihood - clinician 9} \\
\hline Likelihood - clinician 10 & 90 & 10 & 0 & 0 & 0 & 94.5 \\
\hline Likelihood - clinician 11 & 0 & 50 & 50 & 0 & 0 & 80.5 \\
\hline Likelihood - clinician 12 & 90 & 0 & 0 & 0 & 10 & 91.5 \\
\hline Likelihood - clinician 13 & 0 & 100 & 0 & 0 & 0 & 85.5 \\
\hline Likelihood - clinician 14 & 0 & 50 & 50 & 0 & 0 & 80.5 \\
\hline Likelihood - clinician 15 & 80 & 20 & 0 & 0 & 0 & 93.5 \\
\hline Likelihood - clinician 16 & 10 & 80 & 10 & 0 & 0 & 85.5 \\
\hline Likelihood - clinician 17 & 90 & 10 & 0 & 0 & 0 & 94.5 \\
\hline Likelihood - clinician 18 & 90 & 10 & 0 & 0 & 0 & 94.5 \\
\hline Likelihood - clinician 19 & 0 & 80 & 20 & 0 & 0 & 83.5 \\
\hline Likelihood - clinician 20 & 80 & 10 & 5 & 3 & 2 & 91.8 \\
\hline Likelihood - clinician 21 & 100 & 0 & 0 & 0 & 0 & 95.5 \\
\hline Mean NPV & 62.5 & 28.1 & 8.6 & 0.2 & 0.7 & 90.7 \\
\hline SD NPV & & & & & & 7.2 \\
\hline
\end{tabular}


TABLE 88 Elicitation: accuracy (PPV) - asymptomatic women investigated using CT and/or MRI

\begin{tabular}{|c|c|c|c|c|c|c|}
\hline & \multicolumn{5}{|c|}{ Mid-point PPV (\%) } & \multirow{2}{*}{$\begin{array}{l}\text { Mean PPV } \\
(\%)\end{array}$} \\
\hline & 95.5 & 85.5 & 75.5 & 65.5 & 55.5 & \\
\hline Likelihood - clinician 1 & 0 & 0 & 50 & 50 & 0 & 70.5 \\
\hline Likelihood - clinician 2 & 20 & 50 & 30 & 0 & 0 & 84.5 \\
\hline Likelihood - clinician 3 & 50 & 40 & 5 & 5 & 0 & 89.0 \\
\hline Likelihood - clinician 4 & 100 & 0 & 0 & 0 & 0 & 95.5 \\
\hline Likelihood - clinician 5 & 90 & 10 & 0 & 0 & 0 & 94.5 \\
\hline Likelihood - clinician 6 & 10 & 90 & 0 & 0 & 0 & 86.5 \\
\hline \multicolumn{7}{|l|}{ Likelihood - clinician 7} \\
\hline \multicolumn{7}{|l|}{ Likelihood - clinician 8} \\
\hline \multicolumn{7}{|l|}{ Likelihood - clinician 9} \\
\hline Likelihood - clinician 10 & 100 & 0 & 0 & 0 & 0 & 95.5 \\
\hline Likelihood - clinician 11 & 5 & 90 & 5 & 0 & 0 & 85.5 \\
\hline Likelihood - clinician 12 & 95 & 0 & 0 & 0 & 5 & 93.5 \\
\hline Likelihood - clinician 13 & 0 & 100 & 0 & 0 & 0 & 85.5 \\
\hline Likelihood - clinician 14 & 0 & 50 & 50 & 0 & 0 & 80.5 \\
\hline Likelihood - clinician 15 & 11.1 & 22.2 & 33.3 & 22.2 & 11.1 & 75.5 \\
\hline Likelihood - clinician 16 & 0 & 10 & 80 & 10 & 0 & 75.5 \\
\hline Likelihood - clinician 17 & 90 & 10 & 0 & 0 & 0 & 94.5 \\
\hline Likelihood - clinician 18 & 100 & 0 & 0 & 0 & 0 & 95.5 \\
\hline Likelihood - clinician 19 & 20 & 80 & 0 & 0 & 0 & 87.5 \\
\hline Likelihood - clinician 20 & 5 & 30 & 40 & 20 & 5 & 76.5 \\
\hline Likelihood - clinician 21 & 0 & 5 & 90 & 5 & 0 & 75.5 \\
\hline Mean PPV & 38.7 & 32.6 & 21.3 & 6.2 & 1.2 & 38.7 \\
\hline SD PPV & & & & & & 9.8 \\
\hline
\end{tabular}


TABLE 89 Elicitation: accuracy (NPV) - asymptomatic women investigated using CT and/or MRI

\begin{tabular}{|c|c|c|c|c|c|c|}
\hline & \multicolumn{5}{|c|}{ Mid-point NPV (\%) } & \multirow{2}{*}{$\begin{array}{l}\text { Mean NPV } \\
(\%)\end{array}$} \\
\hline & 95.5 & 85.5 & 75.5 & 65.5 & 55.5 & \\
\hline Likelihood - clinician 1 & 100 & 0 & 0 & 0 & 0 & 95.5 \\
\hline Likelihood - clinician 2 & 40 & 40 & 20 & 0 & 0 & 87.5 \\
\hline Likelihood - clinician 3 & 80 & 15 & 5 & 0 & 0 & 93 \\
\hline Likelihood - clinician 4 & 100 & 0 & 0 & 0 & 0 & 95.5 \\
\hline Likelihood - clinician 5 & 90 & 10 & 0 & 0 & 0 & 94.5 \\
\hline Likelihood - clinician 6 & 10 & 90 & 0 & 0 & 0 & 86.5 \\
\hline \multicolumn{7}{|l|}{ Likelihood - clinician 7} \\
\hline \multicolumn{7}{|l|}{ Likelihood - clinician 8} \\
\hline \multicolumn{7}{|l|}{ Likelihood - clinician 9} \\
\hline Likelihood - clinician 10 & 80 & 20 & 0 & 0 & 0 & 93.5 \\
\hline Likelihood - clinician 11 & 5 & 90 & 5 & 0 & 0 & 85.5 \\
\hline Likelihood - clinician 12 & 5 & 0 & 95 & 0 & 0 & 76.5 \\
\hline Likelihood - clinician 13 & 0 & 100 & 0 & 0 & 0 & 85.5 \\
\hline Likelihood - clinician 14 & 100 & 0 & 0 & 0 & 0 & 95.5 \\
\hline Likelihood - clinician 15 & 80 & 10 & 10 & 0 & 0 & 92.5 \\
\hline Likelihood - clinician 16 & 0 & 10 & 80 & 10 & 0 & 75.5 \\
\hline Likelihood - clinician 17 & 90 & 10 & 0 & 0 & 0 & 94.5 \\
\hline Likelihood - clinician 18 & 90 & 10 & 0 & 0 & 0 & 94.5 \\
\hline Likelihood - clinician 19 & 80 & 20 & 0 & 0 & 0 & 93.5 \\
\hline Likelihood - clinician 20 & 40 & 30 & 20 & 5 & 5 & 85 \\
\hline Likelihood - clinician 21 & 100 & 0 & 0 & 0 & 0 & 95.5 \\
\hline Mean NPV & 60.6 & 25.3 & 13.1 & 0.81 & 0.28 & 90 \\
\hline SD NPV & & & & & & 7.7 \\
\hline
\end{tabular}


TABLE 90 Elicitation: accuracy (PPV) - asymptomatic women investigated using CT and/or MRI and PET-CT

\begin{tabular}{|c|c|c|c|c|c|c|}
\hline & \multicolumn{5}{|c|}{ Mid-point PPV (\%) } & \multirow{2}{*}{$\begin{array}{l}\text { Mean PPV } \\
(\%)\end{array}$} \\
\hline & 95.5 & 85.5 & 75.5 & 65.5 & 55.5 & \\
\hline Likelihood - clinician 1 & 80 & 20 & 0 & 0 & 0 & 93.5 \\
\hline Likelihood - clinician 2 & 10 & 40 & 40 & 10 & 0 & 80.5 \\
\hline Likelihood - clinician 3 & 80 & 20 & 0 & 0 & 0 & 93.5 \\
\hline Likelihood - clinician 4 & 100 & 0 & 0 & 0 & 0 & 95.5 \\
\hline Likelihood - clinician 5 & 95 & 5 & 0 & 0 & 0 & 95.0 \\
\hline Likelihood - clinician 6 & 20 & 80 & 0 & 0 & 0 & 87.5 \\
\hline \multicolumn{7}{|l|}{ Likelihood - clinician 7} \\
\hline \multicolumn{7}{|l|}{ Likelihood - clinician 8} \\
\hline \multicolumn{7}{|l|}{ Likelihood - clinician 9} \\
\hline Likelihood - clinician 10 & 100 & 0 & 0 & 0 & 0 & 95.5 \\
\hline Likelihood - clinician 11 & 90 & 10 & 0 & 0 & 0 & 94.5 \\
\hline Likelihood - clinician 12 & 0 & 95 & 0 & 0 & 5 & 84.0 \\
\hline Likelihood - clinician 13 & 0 & 100 & 0 & 0 & 0 & 85.5 \\
\hline Likelihood - clinician 14 & 50 & 50 & 0 & 0 & 0 & 90.5 \\
\hline Likelihood - clinician 15 & 20 & 30 & 20 & 20 & 10 & 78.5 \\
\hline Likelihood - clinician 16 & 10 & 80 & 10 & 0 & 0 & 85.5 \\
\hline Likelihood - clinician 17 & 100 & 0 & 0 & 0 & 0 & 95.5 \\
\hline Likelihood - clinician 18 & 100 & 0 & 0 & 0 & 0 & 95.5 \\
\hline Likelihood - clinician 19 & 80 & 20 & 0 & 0 & 0 & 93.5 \\
\hline Likelihood - clinician 20 & 40 & 30 & 20 & 5 & 5 & 85.0 \\
\hline Likelihood - clinician 21 & 90 & 10 & 0 & 0 & 0 & 94.5 \\
\hline Mean PPV & 59.2 & 32.8 & 5.0 & 1.9 & 1.1 & 90.2 \\
\hline SD PPV & & & & & & 7.7 \\
\hline
\end{tabular}


TABLE 91 Elicitation: accuracy (NPV) - asymptomatic women investigated using CT and/or MRI and PET-CT

\begin{tabular}{|c|c|c|c|c|c|c|}
\hline & \multicolumn{5}{|c|}{ Mid-point NPV (\%) } & \multirow{2}{*}{$\begin{array}{l}\text { Mean NPV } \\
(\%)\end{array}$} \\
\hline & 95.5 & 85.5 & 75.5 & 65.5 & 55.5 & \\
\hline Likelihood - clinician 1 & 100 & 0 & 0 & 0 & 0 & 95.5 \\
\hline Likelihood - clinician 2 & 50 & 30 & 20 & 0 & 0 & 88.5 \\
\hline Likelihood - clinician 3 & 90 & 10 & 0 & 0 & 0 & 94.5 \\
\hline Likelihood - clinician 4 & 100 & 0 & 0 & 0 & 0 & 95.5 \\
\hline Likelihood - clinician 5 & 95 & 5 & 0 & 0 & 0 & 95 \\
\hline Likelihood - clinician 6 & 90 & 10 & 0 & 0 & 0 & 94.5 \\
\hline \multicolumn{7}{|l|}{ Likelihood - clinician 7} \\
\hline \multicolumn{7}{|l|}{ Likelihood - clinician 8} \\
\hline \multicolumn{7}{|l|}{ Likelihood - clinician 9} \\
\hline Likelihood - clinician 10 & 90 & 10 & 0 & 0 & 0 & 94.5 \\
\hline Likelihood - clinician 11 & 90 & 10 & 0 & 0 & 0 & 94.5 \\
\hline Likelihood - clinician 12 & 95 & 0 & 0 & 0 & 5 & 93.5 \\
\hline Likelihood - clinician 13 & 100 & 0 & 0 & 0 & 0 & 95.5 \\
\hline Likelihood - clinician 14 & 100 & 0 & 0 & 0 & 0 & 95.5 \\
\hline Likelihood - clinician 15 & 90 & 7 & 3 & 0 & 0 & 94.2 \\
\hline Likelihood - clinician 16 & 90 & 10 & 0 & 0 & 0 & 94.5 \\
\hline Likelihood - clinician 17 & 100 & 0 & 0 & 0 & 0 & 95.5 \\
\hline Likelihood - clinician 18 & 100 & 0 & 0 & 0 & 0 & 95.5 \\
\hline Likelihood - clinician 19 & 10 & 80 & 10 & 0 & 0 & 85.5 \\
\hline Likelihood - clinician 20 & 30 & 40 & 20 & 5 & 5 & 84 \\
\hline Likelihood - clinician 21 & 100 & 0 & 0 & 0 & 0 & 95.5 \\
\hline Mean NPV & 84.4 & 11.8 & 2.9 & 0.3 & 0.6 & 93.4 \\
\hline SD NPV & & & & & & 5.5 \\
\hline
\end{tabular}


TABLE 92 Minimum increase in accuracy required before PET-CT is introduced as a routine investigation in symptomatic women with initial stage IB-IVA cervical cancer

\begin{tabular}{|c|c|c|c|c|}
\hline Clinician & $\begin{array}{l}\text { Minimum decrease } \\
\text { in FP (\%) }\end{array}$ & $\begin{array}{l}\text { Mid-point decrease } \\
\text { in FP }(\%)\end{array}$ & $\begin{array}{l}\text { Minimum decrease } \\
\text { in FN (\%) }\end{array}$ & $\begin{array}{l}\text { Mid-point decrease } \\
\text { in FN }(\%)\end{array}$ \\
\hline 1 & 9-11 & 10 & $9-11$ & 10 \\
\hline 2 & 20 & 20 & $9-11$ & 10 \\
\hline 3 & $9-11$ & 10 & $6-8$ & 7 \\
\hline 4 & $3-5$ & 4 & $3-5$ & 4 \\
\hline 5 & $3-5$ & 4 & $3-5$ & 4 \\
\hline 6 & 9-11 & 10 & $3-5$ & 4 \\
\hline 7 & NS & NA & NS & NA \\
\hline 8 & NS & NA & NS & NA \\
\hline 9 & $9-11$ & 10 & $9-11$ & 10 \\
\hline 10 & $0-2$ & 1 & $0-2$ & 1 \\
\hline 11 & $9-11$ & 10 & $9-11$ & 10 \\
\hline 12 & $0-2$ & 1 & $0-2$ & 1 \\
\hline 13 & $6-8$ & 7 & $6-8$ & 7 \\
\hline 14 & $3-5$ & 4 & $3-5$ & 4 \\
\hline 15 & $6-8$ & 7 & $6-8$ & 7 \\
\hline 16 & $9-11$ & 10 & $9-11$ & 10 \\
\hline 17 & $6-8$ & 7 & $6-8$ & 7 \\
\hline 18 & $6-8$ & 7 & $6-8$ & 7 \\
\hline 19 & $6-8$ & 7 & $9-11$ & 10 \\
\hline 20 & $6-8$ & 7 & $6-8$ & 7 \\
\hline 21 & $9-11$ & 10 & $0-2$ & 1 \\
\hline Average & & 7.7 & & 6.4 \\
\hline
\end{tabular}

FN, false-negative; FP, false-positive; NA, not applicable; NS, not stated. 
TABLE 93 Minimum increase in accuracy required before PET-CT is introduced as a routine investigation in asymptomatic women with initial stage IB-IVA cervical cancer

\begin{tabular}{|c|c|c|c|c|}
\hline Clinician & $\begin{array}{l}\text { Minimum decrease } \\
\text { in FP }(\%)\end{array}$ & $\begin{array}{l}\text { Mid-point decrease } \\
\text { in FP }(\%)\end{array}$ & $\begin{array}{l}\text { Minimum decrease } \\
\text { in FN }(\%)\end{array}$ & $\begin{array}{l}\text { Mid-point decrease } \\
\text { in FN }(\%)\end{array}$ \\
\hline 1 & 9-11 & 10 & 9-11 & 10 \\
\hline 2 & 30 & 30 & $2-6$ & 4 \\
\hline 3 & $9-11$ & 10 & $3-5$ & 4 \\
\hline 4 & $0-2$ & 1 & $0-2$ & 1 \\
\hline 5 & $3-5$ & 4 & $3-5$ & 4 \\
\hline 6 & 20 & 20 & 20 & 20 \\
\hline 7 & NS & NA & NS & NA \\
\hline 8 & NS & NA & NS & NA \\
\hline 9 & 30 & 30 & $6-8$ & 7 \\
\hline 10 & $0-2$ & 1 & $0-2$ & 1 \\
\hline 11 & $3-5$ & 4 & $9-11$ & 10 \\
\hline 12 & $0-2$ & 1 & $0-2$ & 1 \\
\hline 13 & $3-5$ & 4 & $3-5$ & 4 \\
\hline 14 & 9-11 & 10 & $9-11$ & 10 \\
\hline 15 & $6-8$ & 7 & $6-8$ & 7 \\
\hline 16 & 9-11 & 10 & 9-11 & 10 \\
\hline 17 & $3-5$ & 4 & $6-8$ & 7 \\
\hline 18 & $3-5$ & 4 & $3-5$ & 4 \\
\hline 19 & $3-5$ & 4 & $3-5$ & 4 \\
\hline 20 & 9-11 & 10 & 9-11 & 10 \\
\hline 21 & $0-2$ & 1 & $0-2$ & 1 \\
\hline Average & & 8.7 & & 6.3 \\
\hline
\end{tabular}

FN, false-negative; FP, false-positive; NA, not applicable; NS, not stated. 


\section{Appendix 13 Effectiveness review list of excluded studies with reasons for exclusion}

TABLE 94 Effectiveness review list of excluded studies with reasons for exclusion

Reference $\quad$ Reason for exclusion

\section{Chemotherapy}

Atahan IL, Yildiz F, Ozyar E, Pehlivan B, Genc M, Kose MF, et al. Radiotherapy in the adjuvant setting of cervical carcinoma: treatment, results, and prognostic factors. Int J Gynecol Cancer 2007;17:813-20

Benjapibal M, Thirapakawong C, Leelaphatanadit C, Therasakvichya S, Inthasorn P. A pilot phase II study of capecitabine plus cisplatin in the treatment of recurrent carcinoma of the uterine cervix. Oncology 2007;72:33-8

Bigler LR, Tate Thigpen J, Blessing JA, Fiorica J, Monk BJ; Gynecologic Oncology Group. Evaluation of tamoxifen in persistent or recurrent nonsquamous cell carcinoma of the cervix: a Gynecologic Oncology Group study. Int J Gynecol Cancer 2004;14:871-4

Brave M, Dagher R, Farrell A, Abraham S, Ramchandani R, Gobburu J. Topotecan in combination with cisplatin for the treatment of stage IVB, recurrent, or persistent cervical cancer. Oncology 2006;20:1401-11

Brewer CA, Blessing JA, Nagourney RA, McMeekin DS, Lele S, Zweizig SL. Cisplatin plus gemcitabine in previously treated squamous cell carcinoma of the cervix: a phase II study of the Gynecologic Oncology Group. Gynecol Oncol 2006;100:385-8

Brooks RA, Rader JS, Dehdashti F, Mutch DG, Powell MA, Thaker PH. Surveillance FDGPET detection of asymptomatic recurrences in patients with cervical cancer. Gynecol Oncol 2009;112:104-9

Candelaria M, Arias-Bonfill D, Chávez-Blanco A, Chanona J, Cantú D, Pérez C, et al. Lack in efficacy for imatinib mesylate as second-line treatment of recurrent or metastatic cervical cancer expressing platelet-derived growth factor receptor alpha. Int J Gynecol Cancer 2009;19:1632-7

Chen SW, Liang JA, Hung YC, Yeh LS, Chang WC, Lin WC, et al. Concurrent weekly cisplatin plus external beam radiotherapy and high-dose rate brachytherapy for advanced cervical cancer: a control cohort comparison with radiation alone on treatment outcome and complications. Int J Radiat Oncol Biol Phys 2006;66:1370-7

Dobrowsky W, Huigol NG, Jayatilake RS, Kizilbash NI, Okkan S, Kagiya VT, et al. AK2123 (Sanazol) as a radiation sensitizer in the treatment of stage III cervical cancer: results of an IAEA multicentre randomised trial. Radiother Oncol 2007;82:24-9

Duenas-Gonzalez A, Cetina-Perez L, Lopez-Graniel C, Gonzalez-Enciso A, GómezGonzalez E, Rivera-Rubi L, et al. Pathologic response and toxicity assessment of chemoradiotherapy with cisplatin versus cisplatin plus gemcitabine in cervical cancer: a randomised Phase II study. Int J Radiat Oncol Biol Phys 2005;61:817-23

Eifel PJ, Winter K, Morris M, Levenback C, Grigsby PW, Cooper J, et al. Pelvic irradiation with concurrent chemotherapy versus pelvic and para-aortic irradiation for high-risk cervical cancer: an update of radiation therapy oncology group trial (RTOG) 90-01. J Clin Oncol 2001;22:872-80

Elst P, Ahankour F, Tjalma WAA. Management of recurrent cervical cancer. Review of the literature and case report. Eur J Gynaecol Oncol 2007;28:435-41
Wrong intervention - adjuvant radiotherapy

Wrong study design - not a RCT

Wrong study design - not a RCT

Wrong study design - not a RCT

Wrong study design - not a RCT

Wrong study design - diagnostic study

Wrong study design - not a RCT

Waiting to be received

Wrong population - primary treatment

Wrong population - primary treatment

Wrong population - primary treatment

Wrong study design - not a RCT 


\section{Reference}

Gold MA, Tian C, Whitney CW, Rose PG, Lanciano R. Surgical versus radiographic determination of para-aortic lymph node metastases before chemoradiation for locally advanced cervical carcinoma: a Gynecologic Oncology Group study. Cancer 1954;112:1954-63

Long H III, Nelimark RA, Podratz KC, Suman V, Keeney GL, Nikcevich DA, et al. Phase III comparison of methotrexate, vinblastine, doxorubicin, and cisplatin (MVAC) vs. doxorubicin and cisplatin $(A C)$ in women with advanced primary or recurrent metastatic carcinoma of the uterine endometrium. Gynecol Oncol 2006;100:501-5

Mabuchi S, Morishige K, Isohashi F, Yoshioka Y, Takeda T, Yamamoto T, et al. Postoperative concurrent nedaplatin-based chemoradiotherapy improves survival in early-stage cervical cancer patients with adverse risk factors. Gynecol Oncol 2000;115:482-7

Mabuchi S, Morishige K, Fujita M, Tsutsui T, Sakata M, Enomoto T, et al. The activity of carboplatin and paclitaxel for recurrent cervical cancer after definitive radiotherapy. Gynecol Oncol 2009;113:200-4

Maluf FC, Leiser AL, Aghajanian C, Sabbatini P, Pezzulli S, Chi DS, et al. Phase II study of tirapazamine plus cisplatin in patients with advanced or recurrent cervical cancer. Int J Gynecol Cancer 2006;16:1165-71

Martinez-Monge R, Jurado M, Cambeiro M, Valero J, Villafranca E, Alcázar JL. Perioperative high-dose-rate brachytherapy in locally advanced and recurrent gynecologic cancer: initial results of a phase II trial. Brachytherapy 2003;5:203-10

Matulonis UA, Campos S, Duska L, Krasner CN, Atkinson T, Penson RT, et al. Phase I/II dose finding study of combination cisplatin and gemcitabine in patients with recurrent cervix cancer. Gynecol Oncol 2006;103:160-4

Micha JP, Goldstein BH, Rettenmaier MA, Brown JV 3rd, John CR, Markman M. Surgery alone or surgery with a combination radiation or chemoradiation for management of patients with bulky-stage IB2 cervical carcinoma. Int I Gynecol Cancer 2006;16:1147-51

Miglietta L, Franzone P, Centurioni MG, Boni L, Tacchini L, Cosso M, et al. A phase II trial with cisplatin-paclitaxel cytotoxic treatment and concurrent external and endocavitary radiation therapy in locally advanced or recurrent cervical cancer. Oncology 2006;70:19-24

Motton S, Houvenaeghel G, Delannes M, Querleu D, Soulé-Tholy M, Hoff J, et al. Results of surgery after concurrent chemoradiotherapy in advanced cervical cancer: comparison of extended hysterectomy and extrafascial hysterectomy. Int I Gynecol Cancer 2010:20:268-75

Nagy V, Coza O, Ordeanu C, Traila A, Rancea A, Todor N, et al. Radiotherapy versus concurrent 5-day cisplatin and radiotherapy in locally advanced cervical carcinoma: long-term results of a phase III randomised trial. Strahlenther Onkol 2009;185:177-83

Noda K, Ohashi Y, Sugimori H, Ozaki M, Niibe H, Ogita S, et al. Phase III double-blind randomised trial of radiation therapy for stage IIIB cervical cancer in combination with low- or high-dose Z-100: treatment with immunomodulator, more is not better. Gynecol Oncol 2006;101:455-63

Piura B, Rabinovich A, Friger M. Recurrent cervical carcinoma after radical hysterectomy and pelvic lymph node dissection: a study of 32 cases. Eur J Gynaecol Oncol

2008;29:31-6

Poolkerd S, Leelahakorn S, Manusirivithaya S, Tangjitgamol S, Thavaramara T, Sukwattana $\mathrm{P}$, et al. Survival rate of recurrent cervical cancer patients. J Med Assoc Thail 2006:89:275-82

\section{Reason for exclusion}

Wrong study design - not a RCT

Wrong population - endometrial cancer

Wrong study design - not a RCT

Wrong study design - not a RCT

Wrong study design - not a RCT

Waiting to be received

Wrong study design - not a RCT

Wrong study design - not a RCT

Wrong study design - not a RCT

Waiting to be received

Wrong population - primary treatment

Wrong population - primary treatment

Waiting to be received

Wrong study design - not a RCT 
TABLE 94 Effectiveness review list of excluded studies with reasons for exclusion (continued)

\section{Reference}

Saito I, Kitagawa R, Fukuda H, Shibata T, Katsumata N, Konishi I, et al. A phase III trial of paclitaxel plus carboplatin versus paclitaxel plus cisplatin in stage IVB, persistent or recurrent cervical cancer: Gynecologic Cancer Study Group/Japan Clinical Oncology Group Study (JCOG0505). Jpn J Clin Oncol 2010;40:90-3

Smaniotto D, D'Agostino G, Luzi S, Valentini V, Macchia G, Mantini G, et al. Concurrent 5-fluorouracil, mitomycin $\mathrm{C}$ and radiation with or without brachytherapy in recurrent cervical cancer: a scoring system to predict clinical response and outcome. Tumori 2005;91:295-301

Tacev T, Vacek A, Ptácková B, Strnad V. Hypoxic versus normoxic externalbeam irradiation of cervical carcinoma combined with californium-252 neutron brachytherapy. Comparative treatment results of a 5-year randomised study. Strahlenther Onkol 2005;181:273-84

Tan LT, Zahra M. Long-term survival and late toxicity after chemoradiotherapy for cervical cancer - the Addenbrooke's experience. Clin Oncol 2008;20:358-64

Tewari KS, Monk BJ. Recent achievements and future developments in advanced and recurrent cervical cancer: trials of the Gynecologic Oncology Group. Semin Oncol 2009;36:170-80

Tran PT, Su Z, Hara W, Husain A, Teng N, Kapp DS. Long-term survivors using intraoperative radiotherapy for recurrent gynecologic malignancies. Int J Radiat Oncol Biol Phys 2007;69:504-11

Vasishta S, Varghese A, Ragheb A. Patterns of failure in cervical carcinoma and outcome of salvage therapy: a retrospective study. Gulf J Oncol 2007;1:43-9

Vieira SC, Costa DR, Meneses AD, Borges e Silva J, Oliveira AK, Sousa RB. [Postradiotherapy pelvic exenteration in relapsed cervical cancer: experience of a tertiary health service in the northeast of Brazil.] Rev Bras Ginecol Obstet 2009;31:22-7

Vorgias G, Profitis E, Sarris G, Strigou S, Kosmas C, Katsoulis M, et al. Evaluation of the possible benefits of post-radiotherapy surgery after concomitant chemoradiotherapy with a new radio-sensitizing regimen (irinotecan/CPT-11, interferon $\mathrm{A} 2 \mathrm{~b}$ and amifostine) for advanced-stage cervical carcinoma. Preliminary results of a pilot phase-II study. J BUON 2007;14:197-202

\section{Radiotherapy/chemoradiotherapy}

Badakh DK, Grover AH. Reirradiation with high-dose-rate remote afterloading brachytherapy implant in patients with locally recurrent or residual cervical carcinoma. J Cancer Res Ther 2009;5:24-30

Bellotti JE, Kagan AR, Wollin M, Olch A. Application of the ICRU Report 38 reference volume concept to the radiotherapeutic management of recurrent endometrial and cervical carcinoma. Radiother Oncol 1993;26:254-9

Bignardi M, Bardelli D, Bertoni F, Tordiglione M. Treatment by radiotherapy alone of uterine cervix carcinoma recurrent in the pelvis. Radiol Med 1988;75:540-4

Blake PR, Branson AN, Lambert HE. Combined radiotherapy and chemotherapy for advanced carcinoma of the cervix. Clin Radiol 1986;37:465-9

Boyce J, Fruchter RG, Nicastri AD, Ambiavagar PC, Reinis MS, Nelson JH Jr. Prognostic factors in stage I carcinoma of the cervix. Gynecol Oncol 1981;12:154-65
Reason for exclusion

Ongoing study - description of methodology

Waiting to be received

Wrong population - primary treatment

Wrong study design - not a RCT

Wrong study design - not a RCT

Wrong population - population with gynecological malignancies

Wrong study design - not a RCT

Wrong study design - not a RCT

Wrong population - primary treatment

Wrong population re-irradiation

Irrelevant or inadequate presented outcomes - results presented separately for each patient

Irrelevant or inadequate presented outcomes - results presented altogether for patients with different types of primary treatment (radiotherapy and/or chemoradiotherapy with surgery)

Wrong population - primary treatment

Wrong population - primary treatment 
TABLE 94 Effectiveness review list of excluded studies with reasons for exclusion (continued)

\section{Reference}

Chung CK, Nahhas WA, Stryker JA, Mortel R. Treatment outcome of recurrent cervical cancer. J Surg Oncol 1983;24:5-10

Eifel PJ, Jhingran A, Brown J, Levenback C, Thames $\mathrm{H}$. Time course and outcome of central recurrence after radiation therapy for carcinoma of the cervix. Int I Gynecol Cancer 2006;16:1106-11

Evans RA. Radical hysterectomy for recurrent carcinoma of the uterine cervix following radiotherapy. Gynecol Oncol 1995;59:162-3

Evans SR Jr, Hilaris BS, Barber HRK. External vs. interstitial irradiation in unresectable recurrent cancer of the cervix. Cancer 1971;28:1284-8

Fang FM, Yeh CY, Lai YL, Chiou JF, Chang KH. Radiotherapy following simple hysterectomy in patients with invasive carcinoma of the uterine cervix. J Formos Med Assoc 1993;92:420-5

Friedman M, Pearlman AW. Carcinoma of the cervix: radiation salvage of surgical failures. Radiology 1965;84:801-11

Grigsby PW. Radiotherapy for pelvic recurrence after radical hysterectomy for cervical cancer. Radiat Med 2005;23:327-30

Guttmann R. Significance of postoperative irradiation in carcinoma of the cervix: a ten year survey. Am J Roentgenol Radium Ther Nucl Med 1970;108:102-8

Heaton D, Yordan E, Reddy S, Bonomi P, Lee MS, Lincoln S, et al. Treatment of 29 patients with bulky squamous cell carcinoma of the cervix with simultaneous cisplatin 5-fluorouracil, and split-course hyperfractionated radiation therapy. Gynecol Oncol 1990;38:323-7

Hogan WM, Littman P, Griner L, Miller CL, Mikuta JJ. Results of radiation therapy given after radical hysterectomy. Cancer 1982;49:1278-85

Hong JH, Tsai CS, Lai CH, Chang TC, Wang CC, Chou HH, et al. Recurrent squamous cell carcinoma of cervix after definitive radiotherapy. Int J Radiat Oncol Biol Phys $2001: 60: 249-57$

Ito $H$, Kumagaya H, Shigematsu N, Nishiguchi I, Nakayama T, Hashimoto S. High dose rate intracavitary brachytherapy for recurrent cervical cancer of the vaginal stump following hysterectomy. Int J Radiat Oncol Biol Phys 1991;20:927-32

Kaneyasu Y, Okawa MK, Kokubo N, Takemoto M, Karasawa K, Fukuhara N, et al. [Clinical evaluation of intra-arterial infusion chemotherapy for advanced or recurrent pelvic tumors with or without radiotherapy.] Gan to Kagaku Ryoho 1996;23:1486-93

Karlan BY, Chamorro T, Fowler JM, Muderspach LI, Greenberg S, Lagasse LD. Concurrent interstitial radiotherapy and infusional chemotherapy for recurrent gynecologic malignancies. Int J Gynecol Cancer 1993;3:304-10

Kucera $\mathrm{H}$, Riss $\mathrm{P}$, Weghaupt K. [Irradiation therapy of recurrent cervical carcinoma (authors' translation).] Geburtshilfe Frauenheilkd 1980;40:1000-5

\section{Reason for exclusion}

Irrelevant or inadequate presented outcomes - results presented altogether for patients with or without previous irradiation

Wrong population - primary treatment

Wrong study design - letter

Irrelevant or inadequate presented outcomes - results presented altogether for different types of primary treatment [radiation $(60 \%$ of patients) and/or surgery]

Waiting to be received

Irrelevant or inadequate presented outcomes - not all patients were analysed, results separate for each patient

Waiting to be received

Wrong intervention - adjuvant radiotherapy

Wrong population - primary treatment

Irrelevant or inadequate presented outcomes - results presented altogether for patients with and without previous irradiation

Wrong population - primary radiotherapy

Wrong study design - letter

Waiting to be received

Waiting to be received

Irrelevant or inadequate presented outcomes - results presented together for patients with and without previous irradiation 
TABLE 94 Effectiveness review list of excluded studies with reasons for exclusion (continued)

Reference

Kumagaya $\mathrm{H}$, Ito $\mathrm{H}$, Hashimoto $\mathrm{S}$. [High-dose intracavitary brachytherapy of a recurrent cervical cancer following surgery.] Gan No Rinsho 1990;36:51-6

Lanciano R. Radiotherapy for the treatment of locally recurrent cervical cancer. J Natl Cancer Inst Monogr 1996;96:113-15

Larson DM, Copeland $\sqcup$, Stringer CA, Gershenson DM, Malone JM Jr, Edwards CL. Recurrent cervical carcinoma after radical hysterectomy. Gynecol Oncol 1988;30:381-7

Macia M, Novo A, Ces J, Gonzalez M, Huidobro C, Yuste J, et al. Neoadjuvant and salvage chemotherapy with cisplatin (CDDP) and 5-fluorouracil (5-FU) in cervical carcinoma. Eur J Gynaecol Oncol 1993;14:192-6

Mahe MA, Gerard JP, Dubois JB, Roussel A, Bussieres E, Delannes M, et al. Intraoperative radiation therapy in recurrent carcinoma of the uterine cervix: report of the French intraoperative group on 70 patients. Int J Radiat Oncol Biol Phys 1996:34:21-6

Malfetano J, Keys H, Kredentser D, Cunningham M, Kotlove D, Weiss L. Weekly cisplatin and radical radiation therapy for advanced, recurrent, and poor prognosis cervical carcinoma. Cancer 1993;71:3703-6

Martinez Monge R, Jurado M, Azinovic I, Aristu JJ, Tangco E, Viera JC, et al. Intraoperative radiotherapy in recurrent gynecological cancer. Radiother Oncol 1993;28:127-33

Martinez-Monge R, Jurado M, Aristu JJ, Moreno M, Cambeiro M, Perez-Ochoa A, et al. Intraoperative electron beam radiotherapy during radical surgery for locally advanced and recurrent cervical cancer. Gynecol Oncol 2001;82:538-43

Martino M, Houvenaeghel G, Hardwigsen J, Moutardier V, Resbeut M, Delpero JR. Pelvic recurrence of cancers of the uterine cervix. A study of a series of 49 cases. Ann Chir 1997; 51:36-45

Miglietta L, Franzone P, Centurioni MG, Boni L, Tacchini L, Cosso M, et al. A phase II trial with cisplatin-paclitaxel cytotoxic treatment and concurrent external and endocavitary radiation therapy in locally advanced or recurrent cervical cancer. Oncology 2006;70:19-24

Monk BJ, Walker JL, Tewari K, Ramsinghani NS, Nisar Syed AM, DiSaia PJ. Open interstitial brachytherapy for the treatment of local-regional recurrences of uterine corpus and cervix cancer after primary surgery. Gynecol Oncol 1994;52:222-8

Nakano T, Gomi H, Morita S, Arai T. Interstitial radiotherapy for recurrent cancer of the uterine cervix. Jpn J Cancer Clin 1986;32:481-4

Niibe $Y$, Kenjo M, Kazumoto T, Michimoto K, Takayama M, Yamauchi C, et al. Multiinstitutional study of radiation therapy for isolated para-aortic lymph node recurrence in uterine cervical carcinoma: 84 subjects of a population of more than 5,000. Int J Radiat Oncol Biol Phys 2006;66:1366-9

Nori D, Hilaris BS. Interstitial irradiation in recurrent cervical cancer. Indian J Cancer 1980;17:253-7

Poolkerd S, Leelahakorn S, Manusirivithaya S, Tangjitgamol S, Thavaramara T, Sukwattana $\mathrm{P}$, et al. Survival rate of recurrent cervical cancer patients. J Med Assoc Thail 2006;89:275-82

\section{Reason for exclusion}

Waiting to be received

Waiting to be received

Wrong population $-26 \%$ of patients received adjuvant radiotherapy - lack of separate results

Wrong intervention neoadjuvant chemotherapy, or salvage therapy after radiotherapy

Wrong intervention radiotherapy plus surgery

Wrong population - mixed primary and recurrence - results presented together

Irrelevant or inadequate presented outcomes - results presented together for different types of primary cancer site

Wrong intervention - surgery

Wrong intervention - surgery plus radiotherapy

Irrelevant or inadequate presented outcomes - results presented together for different populations

Irrelevant or inadequate presented outcomes - results presented together for different types of primary cancer site

Waiting to be received

Not adequate population results presented together for patients with and without previous irradiation

Wrong population, results presented together for patients with and without previous irradiaition

Irrelevant or inadequate presented outcomes - results presented together for different types of intervention 
TABLE 94 Effectiveness review list of excluded studies with reasons for exclusion (continued)

\section{Reference}

Prempree T, Amornmarn R, Villasanta U. Retreatment of very late recurrent invasive squamous cell carcinoma of the cervix with irradiation. II. Criteria for patients' selection to achieve the success. Cancer 1984;54:1950-5

Roth TM, Secord AA, Havrilesky $\sqcup$, Jones E, Clarke-Pearson DL. High dose rate intraoperative radiotherapy for recurrent cervical cancer and nodal disease. Gynecol Oncol 2003;91:258-60

Sakurai H, Mitsuhashi N, Takahashi M, Akimoto T, Muramatsu H, Ishikawa H, et al. Analysis of recurrence of squamous cell carcinoma of the uterine cervix after definitive radiation therapy alone: patterns of recurrence, latent periods, and prognosis. Int J Radiat Oncol Biol Phys 2001;50:1136-44

Schulz-Wendtland R, Kramer S, Sabel M, Heller F, Keilholz L, Jager W, et al. [Pelvic wall recurrence of cervix carcinomas. Combined surgical-radio-chemotherapeutic procedure (CORCT).] Strahlenther Onkol 1998;174:279-83

Singh AK, Grigsby PW, Rader JS, Mutch DG, Powell MA. Cervix carcinoma, concurrent chemoradiotherapy, and salvage of isolated paraaortic lymph node recurrence. Int J Radiat Oncol Biol Phys 2001;61:450-5

Sommers GM, Grigsby PW, Perez CA, Camel HM, Kao MS, Galakatos AE, et al. Outcome of recurrent cervical carcinoma following definitive irradiation. Gynecol Oncol 1989;35:150-5

Stelzer KJ, Koh WJ, Greer BE, Cain JM, Tamimi HK, Figge DC, et al. The use of intraoperative radiation therapy in radical salvage for recurrent cervical cancer: outcome and toxicity. Am J Obstet Gynecol 1995;172:1881-8

Tan D, Wan H, Peng X. Treatment of recurrent carcinoma of the uterine cervix. Chin J Oncol 1995;17:47-9

Tan LT, Zahra M. Long-term survival and late toxicity after chemoradiotherapy for cervical cancer - the Addenbrooke's experience. Clin Oncol 2008;20:358-64

Thomas G, Dembo A, Beale F. Concurrent radiation, mitomycin C and 5-fluorouracil in poor prognosis carcinoma of cervix: preliminary results of a phase I-II study. Int J Radiat Oncol Biol Phys 1984;10:1785-90

Thomas G, Dembo A, Fyles A, Gadalla T, Beale F, Bean H, et al. Concurrent chemoradiation in advanced cervical cancer. Gynecol Oncol 1990;38:446-51

Thomas GM, Dembo AJ, Myhr T, Black B, Pringle JF, Rawlings G. Long-term results of concurrent radiation and chemotherapy for carcinoma of the cervix recurrent after surgery. Int J Gynecol Cancer 2003;3:193-8

Vasishta S, Varghese A, Ragheb A. Patterns of failure in cervical carcinoma and outcome of salvage therapy: a retrospective study. Gulf J Oncol 2007;1:43-9

Wang $\mathrm{CJ}$, Lai $\mathrm{CH}$, Huang $\mathrm{HJ}$, Hong $\mathrm{JH}$, Chou $\mathrm{HH}$, Huang KG, et al. Recurrent cervical carcinoma after primary radical surgery. Am J Obstet Gynecol 1999;181:518-24

Windschall A, Ott OJ, Sauer R, Strnad V. Radiation therapy and simultaneous chemotherapy for recurrent cervical carcinoma. Strahlenther Onkol 2005;181:545-50

Xiang E, Shu-mo C, Ya-qin D, Ke W. Treatment of late recurrent vaginal malignancy after initial radiotherapy for carcinoma of the cervix: an analysis of 73 cases. Gynecol Oncol 1998;69:125-9

Yang MG. Radiotherapy for locoregional recurrent cervix cancer after surgery. J Korean Soc Ther Radiol 1994;12:377-86.

\section{Reason for exclusion}

Wrong population re-irradiation

Wrong study design - case study, one patient

Wrong population - primary radiotherapy

Wrong intervention - surgicalradio-chemotherapeutic procedure

Wrong population - primary radiotherapy

Wrong population - primary radiotherapy

Wrong intervention radiotherapy plus surgery

Waiting to be received

Wrong population - primary and recurrent together

Irrelevant or inadequate presented outcomes - results presented together for different types of primary treatment (radiotherapy and/or chemoradiotherapy, surgery)

Wrong population - primary treatment

Waiting to be received

Irrelevant or inadequate presented outcomes - results presented together for different types of salvage therapy

Wrong population - patients received adjuvant radiotherapy

Irrelevant or inadequate presented outcomes - results presented together for different types of primary treatment (radiotherapy and/or chemotherapy with surgery)

Wrong population - primary radiotherapy

Waiting to be received 
TABLE 94 Effectiveness review list of excluded studies with reasons for exclusion (continued)

Reference

\section{Salvage surgery}

Allum WH, Ambrose NS, Fielding JW, Chan KK. Selective salvage surgery in gastrointestinal and gynaecological cancer. Ann R Coll Surg Engl 1990;72:2-5

Ayhan A, Otegen U, Guven S, Kucukali T. Radical reoperation for invasive cervical cancer found in simple hysterectomy. J Surg Oncol 2006;94:28-34

Azria E, Morice P, Haie-Meder C, Thoury A, Pautier P, Lhomme C, et al. Results of hysterectomy in patients with bulky residual disease at the end of chemoradiotherapy for stage IB2/II cervical carcinoma. Ann Surg Oncol 2005;12:332-7

Bader AA, Petru E, Winter R. Long-term follow-up after neoadjuvant chemotherapy for high-risk cervical cancer during pregnancy. Gynecol Oncol 2007;105:269-72

Barber HR. Pelvic exenteration. Cancer Invest 1987;5:331-8

Barber HRK, Roberts S, Brunschwig A. Prognostic significance of preoperative nonvisualized kidney in patients receiving pelvic exenteration. Cancer 1963;16:614-15

Barber HRK, Brunschwig A. Pelvic exenteration for extensive visceral necrosis following radiation therapy for gynecologic cancer. Obstet Gynecol 1965;25:575-8

Barber HRK, Graber EA. Treatment of advanced cancer of the cervix by pelvic exenteration. Bull N Y Acad Med 1973;49:870-86

Berek JS, Howe C, Lagasse LD, Hacker NF. Pelvic exenteration for recurrent gynecologic malignancy: survival and morbidity analysis of the 45-year experience at UCLA. Gynecol Oncol 2005;99:153-9

Bjornstahl H, Johnsson JE, Lindberg LG. Hysterectomy in central recurrence of carcinoma of the uterine cervix. Acta Obstet Gynecol Scand 1977;56:227-31

Bobin JY, Romestaing P, Gerard JP. [Treatment of loco-regional recurrences of cervix cancers.] Ann Chir 1999;53:904

Bochner BH, McCreath WA, Aubey JJ, Levine DA, Barakat RR, Abu-Rustum N, et al. Use of an ureteroileocecal appendicostomy urinary reservoir in patients with recurrent pelvic malignancies treated with radiation. Gynecol Oncol 2004;94:140-6

Bolla M, Berland E, Salvat J, Artignan X, de Cornulier J, Colonna M. Fast growing cervical carcinomas. A retrospective analysis of 20 IB-IIB FIGO. Eur J Obstet Gynecol Reprod Biol 2000;90:81-5

Bompiani A, Benedetti Panici P, Greggi S, Margariti PA, Di Roberto P. Pelvic exenteration in gynaecologic oncology: analysis of 44 cases. Eur J Gynaecol Oncol 1985;6:165-9

Brand $\mathrm{E}$. Cecal rupture after continent ileocecal urinary diversion during total pelvic exenteration. Obstet Gynecol 1991;78:570-2

Bricker EM. Radical evisceration of the pelvis for advanced and recurring carcinoma. Arch Gynakol 1967;2:1-19

Brodsky JT, Sloane BB, Khanna OP. Total pelvic exenteration with preservation of fecal continence. J Surg Oncol 1993;53:261-4

Brunschwig A. The possibilities of radical surgery, in cancer of the cervix uteri recurrent after radiation therapy. Am J Roentgenol 1951;65:720-5

Brunschwig A. The surgical treatment of cancer of the cervix. Cancer 1953;6:980-6

Brunschwig A. Surgical treatment of stage I cancer of the cervix. Cancer 1960;13:34-6

\section{Reason for exclusion}

Small number of patients

Wrong intervention - radical reoperation

Wrong study design - case report

Wrong study design - case report

Wrong study design - literature review

Wrong study design inadequate aim of study, advanced cervical cancer

Wrong population - patients with visceral necrosis

Wrong study design - literature review

Wrong population - different types of cancer

Waiting to be received

Waiting to be received

Wrong study design - surgical techniques

Wrong study design inadequate aim of study, fastgrowing cervical cancer

Waiting to be received

Wrong study design - case report

Wrong study design - literature review

Wrong study design - case report

Waiting to be received

Wrong population - unclear primary treatment, not directly recurrent or persistent

Wrong population - primary treatment 
TABLE 94 Effectiveness review list of excluded studies with reasons for exclusion (continued)

\section{Reference}

Brunschwig A, Daniel WW. The surgery of pelvic lymph node metastases from carcinoma of the cervix. Am J Obstet Gynecol 1962;83:389-92

Brunschwig A. Surgical treatment of carcinoma of the cervix, recurrent after irradiation or combination of irradiation and surgery. Am J Roentgenol Radium Ther Nucl Med 1967;99:365-70

Brunschwig A. The surgical treatment of cancer of the cervix stages I and II. Minerva Ginecol 1968;102:147-51

Brunschwig A, Daniel WW. Surgical treatment of cancer of the cervix recurrent after previous radiation therapy. Surg Gynecol Obstet 1957;105:186-90

Brunschwig A, Barber HR. Extended pelvic exenteration for advanced cancer of the cervix. Long survivals following added resection of involved small bowel. Cancer 1964;17:1267-70

Brunschwig A, Barber HRK. Surgical treatment of carcinoma of the cervix. Obstet Gynecol 1966;27:21-9

Caceres A, Mourton SM, Bochner BH, Gerst SR, Liu L, Alektiar KM, et al. Extended pelvic resections for recurrent uterine and cervical cancer: out-of-the-box surgery. Int $J$ Gynecol Cancer 2008;18:1139-44

Cantrell LA, Mendivil A, Gehrig PA, Boggess JF. Survival outcomes for women undergoing type III robotic radical hysterectomy for cervical cancer: a 3-year experience. Gynecol Oncol 2010;117:260-5

Carter J, Chi DS, Abu-Rustum N, Brown CL, McCreath W, Barakat RR. Brief report: total pelvic exenteration - a retrospective clinical needs assessment. Psychooncology 2004;13:125-31

Cetina L, Garcia-Arias A, Candelaria M, Cantú D, Rivera L, Coronel J, et al. Brachytherapy versus radical hysterectomy after external beam chemoradiation: a non-randomised matched comparison in IB2-IIB cervical cancer patients. World J Surg Oncol 2009;7:19

Chang HK, Lo KY, Chiang HS. Complications of urinary diversion after pelvic exenteration for gynecological malignancy. Int Urogynecol J 2000;11:358-60

Cheewakriangkrai C, Srisomboon J, Chitapanarux I, Suprasert P, Phongnarisorn C, Siriaree $S$, et al. Concurrent cisplatin-based chemoradiation and adjuvant hysterectomy for bulky stage IB-IIA cervical cancer. J Med Assoc Thai 2005;88:1331-7

Chiva LM, Lapuente F, González-Cortijo L, González-Martín A, Rojo A, García JF, et al. Surgical treatment of recurrent cervical cancer: state of the art and new achievements. Gynecol Oncol 2008;110:S60-6

Chou HH, Wang CC, Lai CH, Hong JH, Ng KK, Chang TC, et al. Isolated paraaortic lymph node recurrence after definitive irradiation for cervical carcinoma. Int J Radiat Oncol Biol Phys 2001;51:442-8

Colombo PE, Bertrand MM, Gutowski M, Mourregot A, Fabbro M, Saint-Aubert B, et al. Total laparoscopic radical hysterectomy for locally advanced cervical carcinoma (stages IIB, IIA and bulky stages IB) after concurrent chemoradiation therapy: surgical morbidity and oncological results. Gynecol Oncol 2009;114:404-9

Cox EF, Ketchum AS, Villasanta U, Munford RS. Patient evaluation for pelvic exenteration. Am Surg 1964;30:574-7

Creasman WT, Rutledge F. Preoperative evaluation of patients with recurrent carcinoma of the cervix. Gynecol Oncol 1972;1:11-18

Crozier M, Morris M, Levenback C, Lucas KR, Atkinson EN, Wharton JT. Pelvic exenteration for adenocarcinoma of the uterine cervix. Gynecol Oncol 1995;58:74-8
Reason for exclusion

No relevant outcomes reported

Waiting to be received

Wrong population - primary treatment

Waiting to be received

Wrong study design - case report

Wrong intervention - palliative treatment

Wrong intervention - extended surgical treatment

Wrong population - patients after surgical procedure

Wrong population gynecological cancers, unclear primary therapy

Wrong intervention - externalbeam chemoradiotherapy plus radical hysterectomy vs externalbeam chemoradiotherapy plus brachytherapy

Wrong population - patients with different gynecological cancers, radiotherapy and surgery as primary treatment

Wrong population - previously untreated, chemoradiotherapy plus adjuvant hysterectomy

Wrong study design - literature review

Wrong population - para-aortic recurrence

Wrong population neoadjuvant chemoradiotherapy plus radical hysterectomy

No relevant outcomes reported lack of adequate clinical data

Wrong study design - literature review

Wrong population - primary radiotherapy or radiotherapy plus surgery 
TABLE 94 Effectiveness review list of excluded studies with reasons for exclusion (continued)

\section{Reference}

Curry SL, Nahhas WA, Jahshan AE. Pelvic exenteration: a 7-year experience. Gynecol Oncol 1981;11:119-23

Deckers PJ, Olsson C, Williams LA, Mozden PJ. Pelvic exenteration as palliation of malignant disease. Am J Surg 1976;131:509-15

Delmore JE, Turner DA, Gershenson DM, Horbelt DV. Perineal hernia repair using human dura. Obstet Gynecol 1987;70:507-8

Dem A. [Evaluation of surgical resection in the locally advanced cervical carcinomas after neoadjuvant external beam radiation therapy.] Bull Cancer 2008;95:235-40

DePasquale SE, Mylonas I, Falkenberry SS. Fatal recurrent ureteroarterial fistulas after exenteration for cervical cancer. Gynecol Oncol 2001;82:192-6

deSouza NM, Soutter WP, Rustin G, Mahon MM, Jones B, Dina R, et al. Use of neoadjuvant chemotherapy prior to radical hysterectomy in cervical cancer: monitoring tumour shrinkage and molecular profile on magnetic resonance and assessment of 3-year outcome. Br J Cancer 2004;90:2326-31

Di Saia PJ, Morrow CP. Pelvic exenteration. Calif Med 1973;118:13-17

Distefano M, Ferrandina G, Smaniotto D, Margariti AP, Zannoni G, Macchia G, et al. Concomitant radiochemotherapy plus surgery in locally advanced cervical cancer: update of clinical outcome and cyclooxygenase-2 as predictor of treatment susceptibility. Oncology 2004;67:103-11

Dottino PR, Segna RA, Jennings TS, Mandeli JP, Konsker K, Cohen CJ. Pelvic exenteration in gynecologic oncology: experience at the Mount Sinai Center, 1975-1992. Mt Sinai J Med 1995;62:431-5

Durrance FY, Fletcher GH, Rutledge FN. Analysis of central recurrent disease in stages I and II squamous cell carcinomas of the cervix on intact uterus. Am J Roentgenol 1969;106:831-8

El-Lamie IK. Preliminary experience with Mainz type II pouch in gynecologic oncology patients. Eur J Gynaecol Oncol 2001;22:77-80

Elst P, Ahankour F, Tjalma W. Management of recurrent cervical cancer. Review of the literature and case report. Eur J Gynaecol Oncol 2007;28:435-41

Ferenschild FT, Vermaas M, Verhoef C, Ansink AC, Kirkels WJ, Eggermont AM, et al. Total pelvic exenteration for primary and recurrent malignancies. World J Surg 2009;33:1502-8

Ferron G, Querleu D, Martel P, Letourneur B, Soulié M. Laparoscopy-assisted vaginal pelvic exenteration. Gynecol Oncol 2006;100:551-5

Fleisch MC, Pantke P, Beckmann MW, Schnuerch HG, Ackermann R, Grimm MO, et al. Predictors for long-term survival after interdisciplinary salvage surgery for advanced or recurrent gynecologic cancers. J Surg Oncol 2007;95:476-84

Fotopoulou C, Neumann U, Kraetschell R, Schefold JC, Weidemann H, Lichtenegger $W$, et al. Long-term clinical outcome of pelvic exenteration in patients with advanced gynecological malignancies. J Surg Oncol 2010;101:507-12

Friedberg V. Results of 108 exenteration operations in advanced gynaecological carcinomas. Geburtshilfe Frauenheilkd 1989;49:423-7

Füller J, Guderian D, Köhler C, Schneider A, Wendt TG. Lymph edema of the lower extremities after lymphadenectomy and radiotherapy for cervical cancer. Strahlenther Onkol 2008;184:206-11

\section{Reason for exclusion}

Wrong population - unclear primary treatment

Wrong population - patients with different gynecological malignancies

Wrong study design - case report

Wrong population - patients after neoadjuvant radiotherapy plus surgery

Wrong study design - case report

Wrong intervention -

neoadjuvant chemotherapy plus radical hysterectomy plus partial radiotherapy

Wrong study design - literature review

Wrong population - patients with locally advanced cervical cancer, chemoradiotherapy plus hysterectomy

Waiting to be received

No relevant outcomes reported

Wrong population gynecological cancers

Wrong study design - literature review

Wrong population - different primary treatment

Wrong population - different primary treatment

Wrong population - diffrent types of cancer

Wrong population - diffrent types of cancer

Wrong population - diffrent types of cancer

Wrong intervention patients after surgery and adjuvant radiotherapy or chemoradiotherapy 
TABLE 94 Effectiveness review list of excluded studies with reasons for exclusion (continued)

Reference

Galante M, Hill EC. Pelvic exenteration: a critical analysis of a ten-year experience with the use of the team approach. Trans Pac Coast Obstet Gynecol Soc 1970;38:59-68

Gemignani ML, Alektiar KM, Leitao M, Mychalczak B, Chi D, Venkatraman E, et al. Radical surgical resection and high-dose intraoperative radiation therapy (HDRIORT) in patients with recurrent gynecologic cancers. Int I Radiat Oncol Biol Phys 2001;50:687-94

Gillitzer R, Hampel C, Thuroff JW. Pelvic exenteration for gynecologic malignancies. Urological reconstructive armamentarium. Gynakologe 2007;40:883-90

Goldberg GL, Sukumvanich P, Einstein MH, Smith HO, Anderson PS, Fields AL. Total pelvic exenteration: the Albert Einstein College of Medicine/Montefiore Medical Center Experience (1987 to 2003). Gynecol Oncol 2006;101:261-8

Green AE, Escobar PF, Neubaurer N, Michener CM, Vongruenigen VE. The Martius flap neovagina revisited. Int J Gynecol Cancer 2005;15:964-6

Guimarães GC, Baiocchi G, Rossi BM, Ferreira FO, Aguiar S, Nakagawa WT, et al. The use of silicone expander and cecal transposition after pelvic exenteration. Eur J Surg Oncol 2007;33:586-9

Hatch KD, Shingleton HM, Potter ME, Baker VV. Low rectal resection and anastomosis at the time of pelvic exenteration. Gynecol Oncol 1988;31:262-7

Hatch KD, Shingleton HM, Soong SJ, Baker VV, Gelder MS. Anterior pelvic exenteration. Gynecol Oncol 1988;31:205-16

Hawighorst-Knapstein S, Fusshoeller C, Franz C, Trautmann K, Schmidt M, Pilch H, et al. The impact of treatment for genital cancer on quality of life and body image results of a prospective longitudinal 10-year study. Gynecol Oncol 2004;94:398-403

Hays DM, Raney RB Jr, Lawrence W Jr, Gehan EA, Soule EH, Tefft M, et al. Rhabdomyosarcoma of the female urogenital tract. J Pediatr Surg 1981;16:828-34

Hill EC, Galante M. Radical surgery in the management of clear cell adenocarcinoma of the cervix and vagina in young women. Am J Obstet Gynecol 1981;140:221-6

Ho YH, Cheng C, Tay SK. Total pelvic exenteration: results from a multispecialty team approach to complex cancer surgery. Int Surg 2001;86:107-11

Höckel M. Laterally extended endopelvic resection: surgical treatment of infrailiac pelvic wall recurrences of gynecologic malignancies. Am J Obstet Gynecol 1999;180:306-12

Höckel M. Laterally extended endopelvic resection (LEER) for surgical treatment of pelvic wall recurrences of cervical carcinoma. Onkologe 2001;7:875-9

Höckel M. Laterally extended endopelvic resection: novel surgical treatment of locally recurrent cervical carcinoma involving the pelvic side wall. Gynecol Oncol 2003;91:369-77

Höckel M. Laterally extended endopelvic resection for surgical treatment of gynecological malignancies with infiltration of the pelvic wall. Gynakol Praxis 2003;27:369-77

Höckel M. New developments in the surgical therapy of cervical carcinoma. J Turkish German Gynecol Assoc 2005;6:2-11

Höckel M. Pelvic exenteration for gynaecological tumours: achievements and unanswered questions. Lancet Oncol 2006;7:837-47

Höckel M, Dornhofer N. How to manage locally advanced primary and recurrent cancer of the uterine cervix: the surgeon's view. Rev Gynaecol Pract 2005;5:212-20

\section{Reason for exclusion}

Waiting to be received

Wrong population - inadequte primary treatment (surgery $+/-$ radiotherapy)

Wrong study design - literature review

Wrong population - different types of cancer

Wrong study design - case report

Wrong population - women and men, reconstruction procedures

Wrong study design - case reports

Waiting to be received

Wrong intervention - surgery +/- adjuvant radiotherapy/ chemotherapy

Wrong population - different types of cancer

Wrong population - inadequate primary treatment (surgery)

Waiting to be received

Wrong population - inadequate primary treatment (radiotherapy, radiotherapy/surgery), adjuvant postoperative radiotherapy

Wrong population - inadequate previous treatment, LEER

Wrong population - some patients had inadequate primary treatment or received adjuvant radiotherapy

Waiting to be received

Wrong study design - literature review

Wrong study design - literature review

Wrong study design - literature review 
TABLE 94 Effectiveness review list of excluded studies with reasons for exclusion (continued)

\section{Reference}

Hoffman MS, Fiorica JV, Roberts WS, Hewitt S, Shepherd JH, Owens S, et al. Williams' vulvovaginoplasty after supralevator total pelvic exenteration. South Med J $1991 ; 84: 43-5$

Hong JH, Tsai CS, Lai CH, Chang TC, Wang CC, et al. Recurrent squamous cell carcinoma of cervix after definitive radiotherapy. Int J Radiat Oncol Biol Phys 2004;60:249-57

Houvenaeghel G, Moutardier V, Karsenty G, Bladou F, Lelong B, Buttarelli M, et al. Major complications of urinary diversion after pelvic exenteration for gynecologic malignancies: a 23-year mono-institutional experience in 124 patients. Gynecol Oncol 2004:92:680-3

Houvenaeghel G, Lelievre L, Gonzague-Casabianca L, Buttarelli M, Moutardier V, Goncalves A, et al. Long-term survival after concomitant chemoradiotherapy prior to surgery in advanced cervical carcinoma. Gynecol Oncol 2006;100:338-43

Houvenaeghel G, Lelievre L, Buttarelli M, Jacquemier J, Carcopino X, Viens P, et al. Contribution of surgery in patients with bulky residual disease after chemoradiation for advanced cervical carcinoma. Eur J Surg Oncol 2007;33:498-503

Huang WY, Huang CY, Chen CA, Hsieh CY, Cheng WF. Ruptured pseudoaneurysm of the external iliac artery in an advanced cervical cancer patient treated by endovascular covered stent placement. J Formos Med Assoc 2008;107:348-51

Huguet F, Cojocariu OM, Levy P, Lefranc JP, Darai E, Jannet $D$, et al. Preoperative concurrent radiation therapy and chemotherapy for bulky stage IB2, IIA, and IIB carcinoma of the uterine cervix with proximal parameterial invasion. Int J Radiat Oncol Biol Phys 2008;72:1508-15

Husain A, Curtin J, Brown C, Chi D, Hoskins W, Poynor E, et al. Continent urinary diversion and low-rectal anastomosis in patients undergoing exenterative procedures for recurrent gynecologic malignancies. Gynecol Oncol 2000;78:208-11

Ingersoll FM, Ulfelder $\mathrm{H}$. Pelvic exenteration for carcinoma of the cervix. N Eng/ J Med 1966:274:648-51

Ingiulla W, de Laurentiis G. [Considerations on 241 pelvic eviscerations.] Riv Ostet Ginecol 1967:22:85-92

Jakowatz JG, Porudominsky D, Riihimaki DU, Kemeny M, Kokal WA, Braly PS, et al. Complications of pelvic exenteration. Arch Surg 1985;120:1261-5

Janser JC, Rodier JF, Rodier D, Vergnes Y. Current place of pelvis exenteration in treatment of recurrent cervix carcinoma. A 41 case report. Chirurgie 1995;120:409-15

Jaspers KD, Reck G. [Unusual course of metastatic cancer of the uterine cervix.] Geburtshilfe Frauenheilkd 1989;49:64-6

Jurado Chacon M, Berian JM, Zudaire JJ. Role of pelvic exenteration in the treatment of some advanced or recurrent gynecological cancers. Prog Obstet Ginecol 1995;38:121-8

Jurado M, Alcazar JL, Martinez-Monge R. Resectability rates of previously irradiated recurrent cervical cancer (PIRCC) treated with pelvic exenteration: is still the clinical involvement of the pelvis wall a real contraindication? A twenty-year experience. Gynecol Oncol 2010;116:38-43

Karlen JR, Piver MS. Reduction of mortality and morbidity associated with pelvic exenteration. Gynecol Oncol 1975;3:164-7

\section{Reason for exclusion}

No relevant outcomes reported

Wrong intervention salvage surgery included total hysterectomy, radical hysterectomy, anterior pelvic exenteration, posterior pelvic exenteration, TPE and other

Wrong population - different types of cancer

Wrong population - patients with locally advanced cervical cancer

Wrong population - patients with locally advanced cervical cancer

Wrong study design - case report

Wrong intervention - some patients underwent adjuvant radiotherapy after surgery

Wrong population - different primary treatment

No relevant outcomes reported

Waiting to be received

Waiting to be received

Waiting to be received

Wrong study design - case report

Waiting to be received

Wrong population - primary treatment (radiotherapy or surgery/radiotherapy)

Wrong population - primary treatment (radiotherapy or surgery/radiotherapy) 
TABLE 94 Effectiveness review list of excluded studies with reasons for exclusion (continued)

\section{Reference}

Kasamatsu T, Onda T, Yamada T, Tsunematsu R. Clinical aspects and prognosis of pelvic recurrence of cervical carcinoma. Int J Gynaecol Obstet 2005;89:39-44

Kecmanovic DM, Pavlov MJ, Kovacevic PA, Sepetkovski AV, Ceranic MS, Stamenkovic $A B$. Management of advanced pelvic cancer by exenteration. Eur J Surg Oncol 2003;29:743-6

Keys HM, Bundy BN, Stehman FB, Muderspach LI, Chafe WE, Suggs CL III, et al. Cisplatin, radiation, and adjuvant hysterectomy compared with radiation and adjuvant hysterectomy for bulky stage IB cervical carcinoma. N Engl J Med 1999;340:1154-61. [Erratum published in N Eng/ J Med 1999;341:708.]

Khoury GG, Bulman AS, Joslin CA. Long term results of Cathetron high dose rate intracavitary radiotherapy in the treatment of carcinoma of the cervix. $\mathrm{Br} J$ Radiol 1991;64:1036-43

Kinney WK, Egorshin EV, Ballard DJ, Podratz KC. Long-term survival and sequelae after surgical management of invasive cervical carcinoma diagnosed at the time of simple hysterectomy. Gynecol Oncol 1992;44:24-7

Kirova YM, Bourhaleb Z, Alran S, Campitelli M, Plancher C, Fourchotte V, et al. [Preoperative concomitant radiochemotherapy in bulky carcinoma of the cervix: Institut Curie experience.] Cancer Radiother 2009;13:291-7

Kiselow M Butcher HR Jr, Bricker EM. Results of the radical surgical treatment of advanced pelvic cancer: a fifteen-year study. Ann Surg 1967;166:428-36

Kneale B, Anderson B. T-tube suction drainage and radical pelvic surgery. Aust N Z J Obstet Gynaecol 1963;24:178-81

Kohler C, Klemm P, Schau A, Possover M, Krause N, Tozzi R, et al. Introduction of transperitoneal lymphadenectomy in a gynecologic oncology center: analysis of 650 laparoscopic pelvic and/or paraaortic transperitoneal lymphadenectomies. Gynecol Oncol 2004;95:52-61

Kunkler IH, Kerr GR, Ludgate SM. The value of follow-up in stage II carcinoma of the cervix. Clin Oncol 1991:3:28-31

Lambaudie E, Narducci F, Leblanc E, Bannier M, Houvenaeghel G. Robotically-assisted laparoscopic anterior pelvic exenteration for recurrent cervical cancer: report of three first cases. Gynecol Oncol 2010;116:582-3

Larciprete G, Casalino B, Segatore MF, Jarvis S, Catarinella V, Cirese E. Pelvic lymphadenectomy for cervical cancer: extraperitoneal versus laparoscopic approach. Eur J Obstet Gynecol Rep Biol 2006;126:259-63

Lawhead RA Jr, Clark DG, Smith DH, Pierce VK, Lewis JL Jr. Pelvic exenteration for recurrent or persistent gynecologic malignancies: a 10-year review of the Memorial Sloan-Kettering Cancer Center experience (1972-1981). Gynecol Oncol 1989;33:279-82

Lawson JB, Nwosu SSO, Olafimihan KA. Carcinoma of the cervix in Nigeria. A review of 246 cases seen in Ibadan in the ten years 1953-1962. J Obst Gynaecol Br Commonw $1964 ; 71: 701-6$

Leath CA, Wilder JL, Decherd ME. Panniculectomy concurrent with anterior pelvic exenteration for recurrent cervical cancer. Gynecol Oncol 2008;110:268-9

Lim PC. Robotic assisted total pelvic exenteration: a case report. Gynecol Oncol 2009:115:310-11

Lim SW, Lim SB, Park JY, Park SY, Choi HS, Jeong SY. Outcomes of colorectal anastomoses during pelvic exenteration for gynaecological malignancy. $\mathrm{Br} J$ Surg 2008;95:770-3

\section{Reason for exclusion}

Wrong population - primary treatment (radiotherapy or surgery/radiotherapy)

Wrong population - unclear primary treatment

Wrong population - primary teratment

Wrong study design - literature review

Wrong population - inadequate primary treatment (surgery)

Wrong population neoadjuvant chemoradiotherapy plus extended radical hysterectomy

Wrong population - different types of cancer

No relevant outcomes reported

Wrong study design description of investigation protocols, laparoscopy as method of diagnosis

No relevant outcomes reported

Wrong study design - case report

Wrong population - patients with advanced cervical cancer

Wrong population - different types of cancer

Wrong population - surgery only

Wrong study design - letter

Wrong study design - case report

No relevant outcomes reported 
TABLE 94 Effectiveness review list of excluded studies with reasons for exclusion (continued)

\section{Reference}

Lin MY, Fan EW, Chiu AW, Tian YF, Wu MP, Liao AC. Laparoscopy-assisted transvaginal total exenteration for locally advanced cervical cancer with bladder invasion after radiotherapy. J Endourol 2004;18:867-70

Lindsey WF, Wood DK, Briele HA, Greager JA, Walker MJ, Bork J, et al. Pelvic exenteration. J Surg Oncol 1985;30:231-4

Lobaton AT, Garcia MC, Mandujano M, Díaz Rodríguez LR. [Histerectomia radical en al tratamiento de1 cancer cervicouterino recurrente a radiacion.] Ginecol Obstet Mex 1983:51:7-12

Lopez MJ, Luna-Perez P. Composite pelvic exenteration: is it worthwhile? Ann Surg Oncol 2004:11:27-33

Lopez-Graniel C, Dolores R, Cetina L, Gonzalez A, Cantu D, Chanona J, et al. Preexenterative chemotherapy, a novel therapeutic approach for patients with persistent or recurrent cervical cancer. BMC Cancer 2005;5:118

Maenpaa JU, Kangasniemi K, Luukkaala T. Pelvic exenteration for gynecological malignancies: an analysis of 15 cases operated on at a single institution. Acta Obstet Gynecol Scand 2010;89:279-83

Magrina JF, Stanhope CR, Weaver AL. Pelvic exenterations: supralevator, infralevator, and with vulvectomy. Gynecol Oncol 1997:64:130-5

Marie G, Barjot P, Crouet H, De Ranieri J. [Place of pelvic exenteration in the treatment of recurrence of cancer of the uterine cervix.] J Chir 1993;130:165-9

Marnitz S, Köhler C, Müller M, Behrens K, Hasenbein K, Schneider A. Indications for primary and secondary exenterations in patients with cervical cancer. Gynecol Oncol 2006;103:1023-30

Martinez-Monge R, Jurado M, Cambeiro M, Valero J, Villafranca E, Alcázar JL. Perioperative high-dose-rate brachytherapy in locally advanced and recurrent gynecologic cancer: initial results of a phase II trial. Brachytherapy 2003;5:203-10

Matthews CM, Morris M, Burke TW, Gershenson DM, Wharton JT, Rutledge FN. Pelvic exenteration in the elderly patient. Obstet Gynecol 1992;79:773-7

Mayer M, Bobin JY, Colon J, Borg G. [Pelvic exenteration for carcinoma of the uterine cervix (authors' transl.).] Bull Cancer 1980;67:70-7

McGarry RC, Smith C, Seemayer TA. Treatment resistant small cell carcinoma of the cervix. Oncology 1999;57:293-6

Méndez L, Bernal A, Escudero P, González G, Fajardo A. [Pelvic exenteration, morbidity and survival.] Ginecol Obstet Mex 1994;62:161-5

Miller B, Morris M, Rutledge F, Mitchell MF, Atkinson EN, Burke TW, et al. Aborted exenterative procedures in recurrent cervical cancer. Gynecol Oncol 1993;50:94-9

Mirhashemi R, Averette HE, Estape R, Angioli R, Mahran R, Mendez L, et al. Low colorectal anastomosis after radical pelvic surgery: a risk factor analysis. Am J Obstet Gynecol 2000;183:1375-9

Monaghan JM. Surgical management of advanced and recurrent cervical carcinoma: the place of pelvic exenteration. Clin Obstet Gynaecol 1985;12:169-82

Monk BJ, Solh S, Johnson MT, Montz FJ. Radical hysterectomy after pelvic irradiation in patients with high risk cervical cancer or uterine sarcoma: morbidity and outcome. Eur J Gynaecol Oncol 1993;14:506-11

Morgan DJ, Hunter DC, McCracken G, McClelland HR, Price JH, Dobbs SP. Is laparoscopically assisted radical vaginal hysterectomy for cervical carcinoma safe? A case control study with follow up. BJOG 2007;114:537-42. [Erratum published in BJOG 2007;114:914.]

\section{Reason for exclusion}

Wrong study design - case report

Wrong population - different types of cancer

Waiting to be received

Wrong population - different types of cancer

Wrong intervention - preexenterative chemoradiotherapy

Wrong population - different types of cancer

Wrong population - different types of cancer

Wrong intervention - total pelvectomy, palliative treatment

Waiting to be received

Wrong population - different types of cancer, some patients underwent adjuvant $\mathrm{CH}$

Wrong population - different primary treatment

Wrong study design - literature review

Wrong study design - case report

Waiting to be received

Wrong study design - literature review

No relevant outcomes reported

Wrong study design - literature review

Wrong population - primary radiotherapy plus radical hysterectomy

Wrong population - open radical hysterectomy with other surgery 
TABLE 94 Effectiveness review list of excluded studies with reasons for exclusion (continued)

\section{Reference \\ Morley GW. Pelvic exenterative therapy and the treatment of recurrent carcinoma of the cervix. Semin Oncol 1982;9:331-40}

Morley GW, Lindenauer SM. Pelvic exenterative therapy in recurrent pelvic carcinoma. Am J Obstet Gynecol 1971;109:1175-86

Morley GW, Lindenauer SM. Pelvic exenterative therapy for gynecologic malignancy: an analysis of 70 cases. Cancer 1976;38:581-6

Morley GW, Hopkins MP, Lindenauer SM, Roberts JA. Pelvic exenteration, University of Michigan: 100 patients at 5 years. Obstet Gynecol 1989;74:934-43

Mourton SM, Chi DS, Sonoda Y, Alektiar KM, Venkatraman ES, Barakat RR, et al. Mesorectal lymph node involvement and prognostic implications at total pelvic exenteration for gynecologic malignancies. Gynecol Oncol 2006;100:533-6

Mourton SM, Sonoda Y, Abu-Rustum NR, Bochner BH, Barakat RR, Chi DS. Resection of recurrent cervical cancer after total pelvic exenteration. Int J Gynecol Cancer 2007:17:137-40

Moutardier V, Houvenaeghel G, Lelong B, Mokart D, Delpero JR. Colorectal function preservation in posterior and total supralevator exenteration for gynecologic malignancies: an 89-patient series. Gynecol Oncol 2003;89:155-9

Moutardier V, Houvenaeghel G, Martino M, Lelong B, Bardou VJ, Resbeut M, et al. Surgical resection of locally recurrent cervical cancer: a single institutional 70 patient series. Int J Gynecol Cancer 2004;14:846-51

Narayansingh GV, Cumming GP, Dighe S, Parkin DE, Millar I. Invasive adenocarcinoma of the vagina fallowing surgery for adenocarcinoma in situ of the cervix - recurrence or implantation? Int J Gynecol Cancer 2001;11:493-5

Norton JA, Javadpour N. Jejunal loop interposition in patients with ileal conduit failure after pelvic exenteration. Am J Surg 1977;134:404-7

Oliveira Poletto AH, Lopes A, Lopes Carvalho A, Ribeiro EA, Aloísio da Costa Vieira R, Mauro Rossi $B$, et al. Pelvic exenteration and sphincter preservation: an analysis of 96 cases. J Surg Oncol 2004;86:122-7

Orr JW Jr, Shingleton HM, Hatch KD, Taylor PT, Partridge EE, Soong SJ. Gastrointestinal complications associated with pelvic exenteration. Am J Obstet Gynecol $1983 ; 145: 325-32$

Osorio Gullón A, de Oca J, Lopéz Costea MA, Virgili J, Ramos E, del Rio C, et al. Double-barreled wet colostomy: a safe and simple method after pelvic exenteration. Int J Colorectal Dis 1997;12:37-41

Ota T, Takeshima N, Tabata T, Hasumi K, Takizawa K. Adjuvant hysterectomy for treatment of residual disease in patients with cervical cancer treated with radiation therapy. Br J Cancer 2008;99:1216-20

Palfalvi L, Ungar L. Extended Wertheim procedure: the laterally extended parameterectomy (LER), a new radical technique for pelvic side wall dissection. CME J Gynecol Oncol 2004;9:45-8

Papp Z, Csapó Z, Hupuczi P, Mayer A. Nerve-sparing radical hysterectomy for stage IA2-IIB cervical cancer: 5-year survival of 501 consecutive cases. Eur J Gynaecol Oncol 2006;27:553-60

Park JY, Choi HJ, Jeong SY, Chung J, Park JK, Park SY. The role of pelvic exenteration and reconstruction for treatment of advanced or recurrent gynecologic malignancies: analysis of risk factors predicting recurrence and survival. J Surg Oncol 2007;96:560-8

Pawlik TM, Skibber JM, Rodriguez-Bigas MA. Pelvic exenteration for advanced pelvic malignancies. Ann Surg Oncol 2006;13:612-23
Reason for exclusion

Waiting to be received

No relevant outcomes reported

Wrong population - different types of cancer

Wrong population - unclear primary treatment

Wrong population - different types of cancer

Wrong population - different types of cancer

Wrong population - different types of cancer

Wrong population - different primary treatment

Wrong study design - case report

Wrong study design - case report

Wrong population - different types of cancer

Wrong population - different primary treatment

No relevant outcomes reported

Wrong population radiotherapy plus adjuvant hysterectomy

Wrong study design description of the procedure

Wrong population - unclear primary treatment

Wrong population - different primary treatment

Wrong study design - literature review 
TABLE 94 Effectiveness review list of excluded studies with reasons for exclusion (continued)

\section{Reference}

Perches RD, Lobaton AT, Garcia MC. Radiotherapy combined with surgery as treatment for advanced cervical cancer. Int J Radiat Oncol Biol Phys 1983;9:1785-8

Perez-Mesa C. Persistent postirradiatioim carcinoma of cervix uteri: a pathologic study of 83 pelvic exenteration specimens. Arch Pathol 1963;75:462-74

Pikaart DP, Holloway RW, Ahmad S, Finkler NJ, Bigsby GE IV, Ortiz BH, et al. Clinicalpathologic and morbidity analyses of types 2 and 3 abdominal radical hysterectomy for cervical cancer. Gynecol Oncol 2007; 107:205-10

Plante M, Roy M. The use of operative laparoscopy in determining eligibility for pelvic exenteration in patients with recurrent cervical cancer. Gynecol Oncol 1995;59:401-4

Plante M, Roy M. Operative laparoscopy prior to a pelvic exenteration in patients with recurrent cervical cancer. Gynecol Oncol 1998;69:94-9

Plukker JT, Aalders JG, Mensink HJ, Oldhoff J. Total pelvic exenteration: a justified procedure. Br J Surg 1993;80:1615-17

Pomel C, Rouzier R, Pocard M, Thoury A, Sideris L, Morice P, et al. Laparoscopic total pelvic exenteration for cervical cancer relapse. Gynecol Oncol 2003;91:616-18

Pras E, Willemse PH, Boonstra H, Hollema H, Heesters MA, Szabó BG, et al. Concurrent chemo- and radiotherapy in patients with locally advanced carcinoma of the cervix. Ann Oncol 1996;7:511-16

Printz C. CancerScope: cervical cancer patients may benefit from new surgical technique. Cancer 2009;115:5131

Puntambekar S, Kudchadkar RJ, Gurjar AM, Sathe RM, Chaudhari YC, Agarwal GA, et al. Laparoscopic pelvic exenteration for advanced pelvic cancers: a review of 16 cases. Gynecol Oncol 2006;102:513-16

Puntambekar SP, Palep RJ, Puntambekar SS, Wagh GN, Patil AM, Rayate NV, et al. Laparoscopic total radical hysterectomy by the Pune technique: our experience of 248 cases. J Minim Invasive Gynecol 2007;14:682-9

Quigley MM, Knab DR, McMahon EB. Carcinoma of the cervix: a third treatment. Obstet Gynecol 1975;45:650-5

Reid GC, Morley GW, Schmidt RW, Hopkins MP. The role of pelvic exenteration for sarcomatous malignancies. Obstet Gynecol 1989;74:80-4

Richardson DL, Seamon LG, Gong MC, Chapman DM, Cohn DE. Panniculectomy concurrent with anterior pelvic exenteration for recurrent cervical cancer. Gynecol Oncol 2008;108:449-51

Rietbroek RC, Schilthuis MS, Bakker PJ, van Dijk JD, Postma AJ, González González D, et al. Phase II trial of weekly locoregional hyperthermia and cisplatin in patients with a previously irradiated recurrent carcinoma of the uterine cervix. Cancer 1997;79:935-43

Roberts WS, Cavanagh D, Bryson SC, Lyman GH, Hewitt S. Major morbidity after pelvic exenteration: a seven-year experience. Obstet Gynecol 1987;69:617-21

Rochard F, Michel G, Castaigne D, Lacour J. [Surgical treatment of carcinomas of the cervix stage III (authors' transl.).] Bull Cancer 1980;67:63-9

Rodriguez C, Torres A, De L, Hernandez D, Herrera L. Pelvic exenteration for carcinoma of the cervix: analysis of 252 cases. J Surg Oncol 1988;38:121-5

\section{Reason for exclusion}

Wrong population - patients with advanced cervical cancer and radiotherapy plus pelvic exenteration or radical hysterectomy

Wrong population - different primary treatment

Wrong population - primary treatment

Wrong intervention laparoscopy

No relevant outcomes reported

Wrong population - different types of cancer

Wrong study design - case report

Wrong population - advanced cervical cancer, primary cancer

Wrong study design - literature review

Wrong population - patients with locally advanced primary pelvic cancers

Wrong population - patients with primary cancers

Wrong intervention

- extraperitoneal

lymphadenectomy

Wrong population - different types of cancer

Wrong study design - case report

Wrong intervention radiotherapy plus chemotherapy or hyperthermia

Wrong population - different primary treatment

No relevant outcomes reported

Wrong population - different types of cancer 
TABLE 94 Effectiveness review list of excluded studies with reasons for exclusion (continued)

\section{Reference \\ Rose PG. Type II radical hysterectomy: evaluating its role in cervical cancer. Gynecol Oncol 2001;80:1-2}

Rouzier R, Morice P, De Crevoisier R, Pomel C, Rey A, Bonnet K, et al. Survival in cervix cancer patients treated with radiotherapy fallowed by radical surgery. Eur J Surg Oncol 2005;31:424-33

Rutledge FN, McGuffee VB. Pelvic exenteration: prognostic significance of regional lymph node metastasis. Gynecol Oncol 1987;26:374-80

Sahu L, Bupathy A, Badhe BA. Leiomyosarcoma of the uterine cervix in a young woman. J Obstet Gynaecol Res 2008;34:717-20

Saunders N. Pelvic exenteration: by whom and for whom? Lancet 1995;345:5-6

Schmitz HE, Smith CJ, Foley DV, Schack CB. Evaluation of surgical procedures employed fallowing the failure of irradiation therapy in cancer of the cervix. Am J Obstet Gynecol $1957 ; 74: 1165-73$

Schmitz RL, Schmitz HE, Smith CJ, Molitor JJ. Details of pelvic exenteration evolved during an experience with 75 cases. Am J Obstet Gynecol 1960;80:43-52

Schwarz $\mathrm{H}$. Total pelvic exenteration in the treatment of recurrent carcinoma of the cervix. West J Surg Obst Gynecol 1958;66:40-3

Shah K, Olson MH, Dillard EH. Carcinoma of the cervix: surgical staging and radiotherapy with 32 MeV betatron. Int J Radiat Oncol Biol Phys 1982;8:1601-6

Sharma DN, Chawla S, Chander S, Gairola M, Thulkar S, Singh MK. Cervical carcinoma recurring in an abdominal wall incision. Clin Oncol 2000;12:354-6

Sharma S, Odunsi K, Driscoll D, Lele S. Pelvic exenterations for gynecological malignancies: twenty-year experience at Roswell Park Cancer Institute. Int J Gynecol Cancer 2005;15:475-82

Shepherd JH, Ngan HY, Neven P, Fryatt I, Woodhouse CR, Hendry WF. Multivariate analysis of factors affecting survival in pelvic exenteration. Int J Gynecol Cancer 1994:4:361-70

Shepherd JH, Crawford RA, Christmas TJ, Hendry WF. Total pelvic reconstruction after exenteration for recurrent cervical cancer. Br J Urol 1997;80:79-81

Shiromizu K, Ogawa M, Kotake K, Koyama Y, Nakazono M, Hirao K. Reconstruction of sigmoid vagina and conduit in total pelvic exenteration for recurrent cervical carcinoma. Jpn J Clin Oncol 1989;19:170-2

Sloukgi JC, Guillemin F. [Repair surgery in the treatment of cancer of the cervical remnant.] Rev Fr Gynecol Obst 1991;86:336-40

Smith RR, Ketcham AS, Thomas LB. Carcinoma of the uterine cervix. Experience with radical surgery. Cancer 1963;16:1105-12

Soeiro Fidalgo de Matos C, Nogaret JM, Philippson C, Veys I, Van Velthoven R. The place for surgery in central recurrences of invasive cancer of cervix uteri. Acta Chir Belg 1995;95:38-43

Stallworthy J. Radical surgery fallowing radiation treatment for cervical carcinoma. Ann $R$ Coll Surg Engl 1964;34:161-78

Stanhope CR, Symmonds RE. Palliative exenteration - what, when, and why? Am J Obst Gynecol 1985:152:12-16

\section{Reason for exclusion}

Wrong study design - literature review

Wrong intervention

- radiotherapy or

chemoradiotherapy plus adjuvant extrafacial hysterectomy and pelvic and para-aortic lymphadenectomy

Wrong study design - literature review

Wrong study design - case report

Wrong study design - literature review

Waiting to be received

Waiting to be received

Waiting to be received

No relevant outcomes reported

Wrong study design - case report

Wrong population - primary SR

Wrong population - different types of cancer

Wrong population - primary radiotherapy plus radical hysterectomy

Wrong study design - case report

Wrong study design - case reports

Wrong population - different primary treatment

Wrong intervention - different types of SR

Wrong study design - literature review

Waiting to be received 
TABLE 94 Effectiveness review list of excluded studies with reasons for exclusion (continued)

\section{Reference}

Stelzer KJ, Koh WJ, Greer BE, Cain JM, Tamimi HK, Figge DC, et al. The use of intraoperative radiation therapy in radical salvage for recurrent cervical cancer: outcome and toxicity. Am J Obstet Gynecol 1995;172:1881-6

Symmonds RE. [The current role of exenteration for the treatment of malignant pelvic tumors.] Gynakologe 1981;14:170-6

Talledo OE. Pelvic exenteration. Medical callege of Georgia experience. Gynecol Oncol $1985 ; 22: 181-8$

Terai Y, Kanemura M, Sasaki H, Tsunetoh S, Tanaka Y, Yamashita Y, et al. Long-term fallow-up of neoadjuvant intraarterial chemotherapy using an original four-lumen double-balloon (4L-DB) catheter for locally advanced uterine cervical cancer. Int J Clin Oncol 2009; 14:56-62

Thompson $\sqcup$. Cancer of the cervix. Semin Oncol Nurs 1990;6:190-7

Torres Lobatón A, Rodríguez Cuevas H, Velázquez Venegas GJ, Díaz Rodríguez LR. [Pelvic exenteration in cancer of the cervix uteri. Analysis of 252 cases.] Ginecol Obstet Mex 1985:53:297-302

Torres-Lobatón A, González-Mendoza RL, Román-Bassaure E, Hernández-Aten D, RojoHerrera G. [Current status of frequency and complications of pelvic exenterations for recurrent cervico-uterine cancer after radiation.] Ginecol Obstet Mex 1996;64:538-43

Trelford JD, Goodnight J, Schneider P, Wolfe B, Sauder MT. Total exenteration, two or one ostomy. Surg Gynecol Obstet 1992;175:126-8

Trelford-Sauder M, Trelford JD, Matolo NM. Replacement of the peritoneum with amnion fallowing pelvic exenteration. Surg Gynecol Obstet 1977;145:699-701

Turko M, Benedet JL, Boyes DA, Nickerson KG. Pelvic exenteration: 1949-1971. Gynecol Oncol 1977;5:246-50

Turrini O, Guiramand J, Moutardier V, Viret F, Mokart D, Madroszyk A, et al. Perineal small bowel fistula after pelvic exenteration for cancer: technical guidelines for perineal fistula. Ann Surg Oncol 2006;13:1622-6

Twombly GH. The use of radical surgery in the treatment of cancer of the cervix. Am J Roentgenol 1959;8:115-19

Ulfelder $\mathrm{H}$. Extended radical surgery for recurrent and advanced cervical cancer. Clinical Obstet Gynecol 1967;10:940-57

Ungar L, Pálfalvi L, Siklós P, Csermely G, Szepesi J, Solt G. Orthotopic bladder replacement in irradiated female patients. Int J Gynecol Cancer 1998;8:307-9

Uzan C, Rouzier R, Castaigne D, Pomel C. [Laparoscopic pelvic exenteration for cervical cancer relapse: preliminary study.] J Gynecol Obstet Biol Reprod 2006;35:136-45

Vera MI. Quality of life fallowing pelvic exenteration. Gynecol Oncol 1981;12:355-66

Viswanathan AN, Lee $H$, Hanson E, Berkowitz RS, Crum CP. Influence of margin status and radiation on recurrence after radical hysterectomy in stage IB cervical cancer. Int $J$ Radiat Oncol Biol Phys 2006;65:1501-7

Walji N, Chue AL, Yap C, Rogers $\sqcup$, El-Modir A, Chan KK, et al. Is there a role for adjuvant hysterectomy after suboptimal concurrent chemoradiation in cervical carcinoma? Clin Oncol 2010;22:140-6

Webb GA. The role of ovarian conservation in the treatment of carcinoma of the cervix with radical surgery. Am J Obstet Gynecol 1975;122:476-84

Wrigley JV, Prem KM, Fraley EE. Pelvic exenteration: complications of urinary diversion. J Urol 1976:116:428-30

\section{Reason for exclusion}

Wrong population - different primary treatment

Wrong population - different types of cancer

Wrong population - different types of cancer

Wrong population - patients with locally advanced primary cervical cancer, neoadjuvant $\mathrm{CH}$

Wrong study design - literature review

Waiting to be received

Waiting to be received

No relevant outcomes reported

No relevant outcomes reported

Wrong population - different primary treatment

No relevant outcomes reported

Wrong population - primary treatment

Wrong population - different types of cancer

Wrong study design - case reports

Wrong study design - case reports

Waiting to be received

Wrong population - only some patients underwent radiotherapy for primary disease

Wrong population chemotherapy plus adjuvant hysterectomy

Wrong population - primary treatment

Wrong population - different types of cancer 
TABLE 94 Effectiveness review list of excluded studies with reasons for exclusion (continued)

Reference

Wydra D, Emerich J, Sawicki S, Ciach K, Marciniak A. Major complications fallowing exenteration in cases of pelvic malignancy: a 10-year experience. World J Gastroenterol 2005;12:1115-19

Wydra D, Emerich J, Ciach K, Sawicki S, Marciniak A. The role of pelvic exenteration for treatment of pelvic malignancy - a nine-year experience. Eur J Gynecol Oncol 2005:26:418-22

Xu H, Chen Y, Li Y, Zhang Q, Wang D, Liang Z. Complications of laparoscopic radical hysterectomy and lymphadenectomy for invasive cervical cancer: experience based on 317 procedures. Surg Endosc 2007;21:960-4

\section{Reason for exclusion}

Wrong population - different types of cancer

Wrong population - different types of cancer

Wrong population - patients after surgery in primary treatment 


\section{Appendix 14 Baseline characteristics of chemotherapy randomised controlled trials}




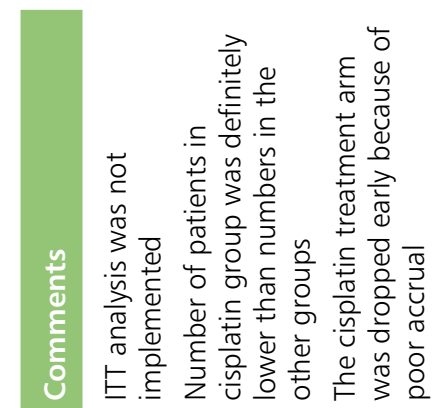

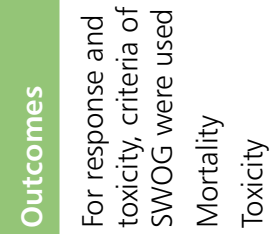

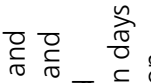

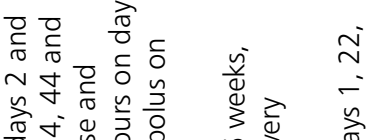

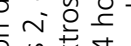

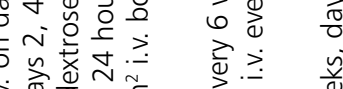

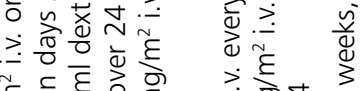

है ठ ह ठे के त्रेण

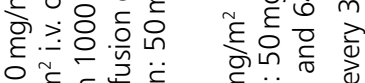

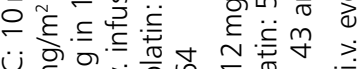

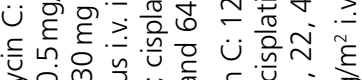

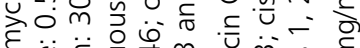

है

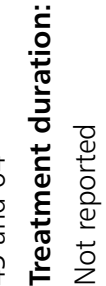

u.

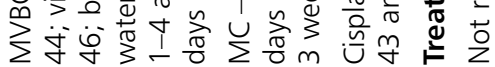

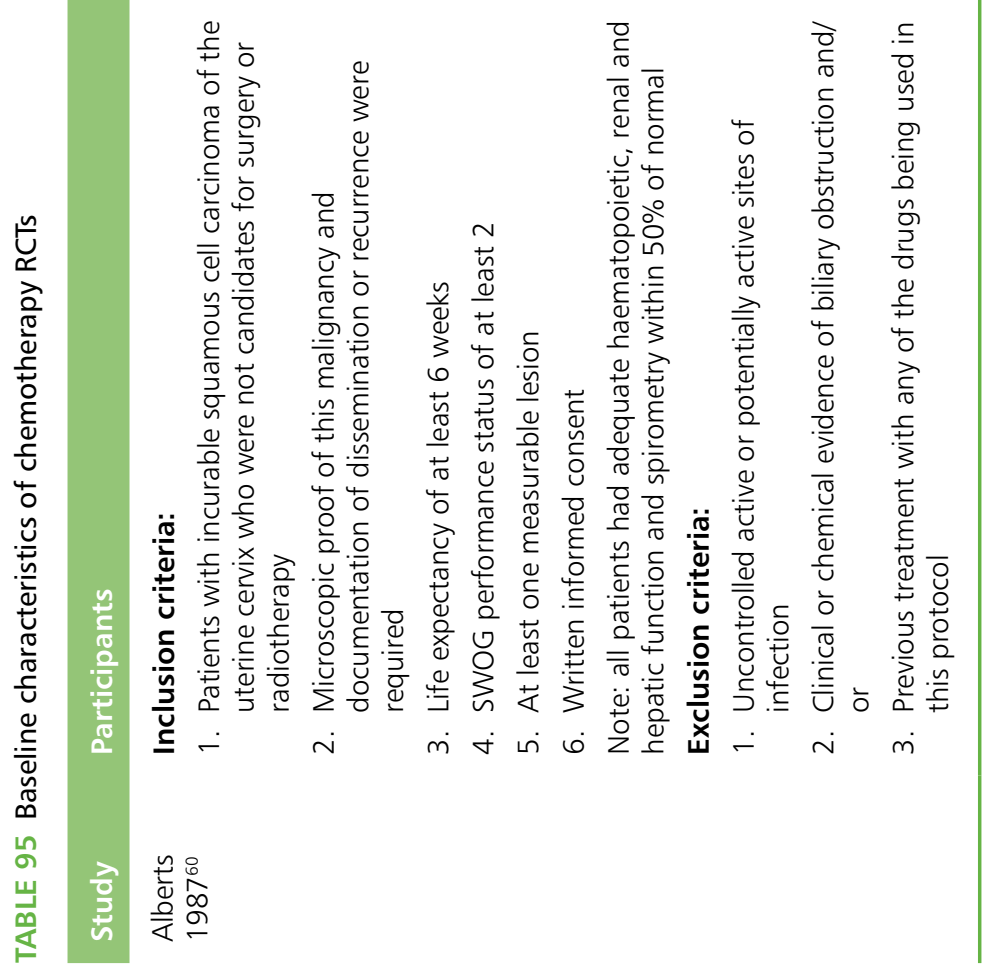


든

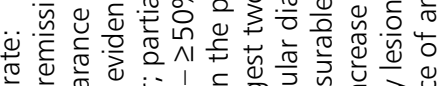

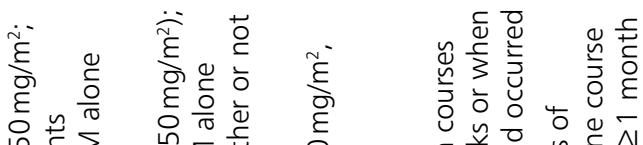

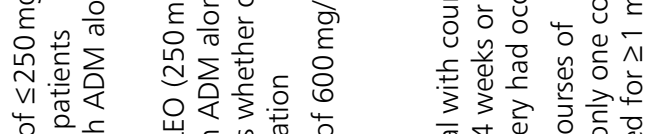

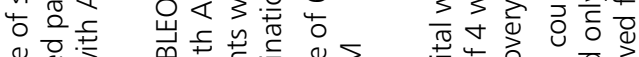

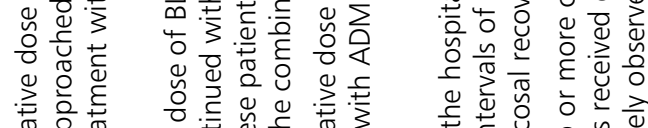

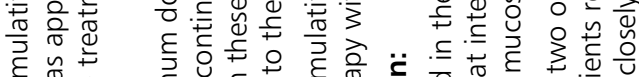

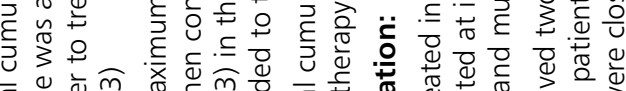

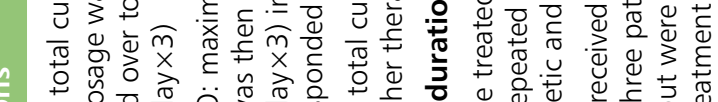
응잉

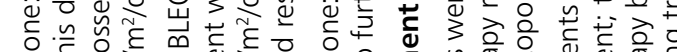

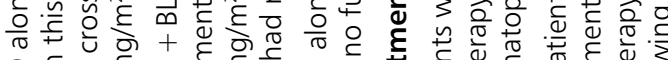

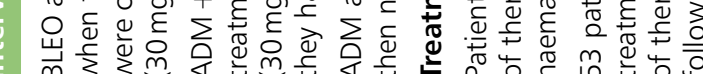




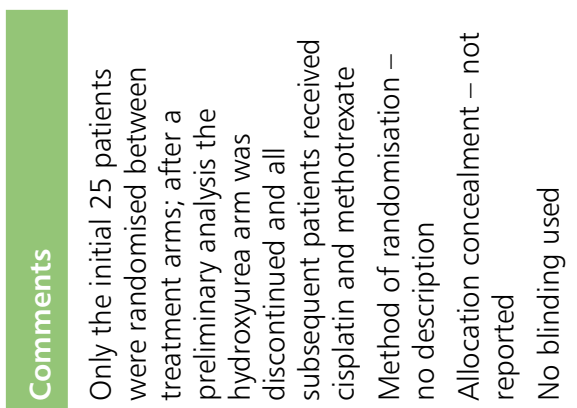

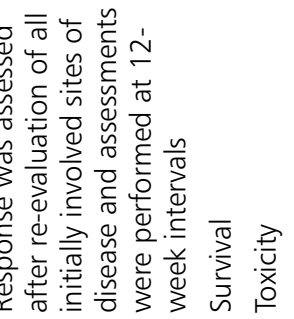

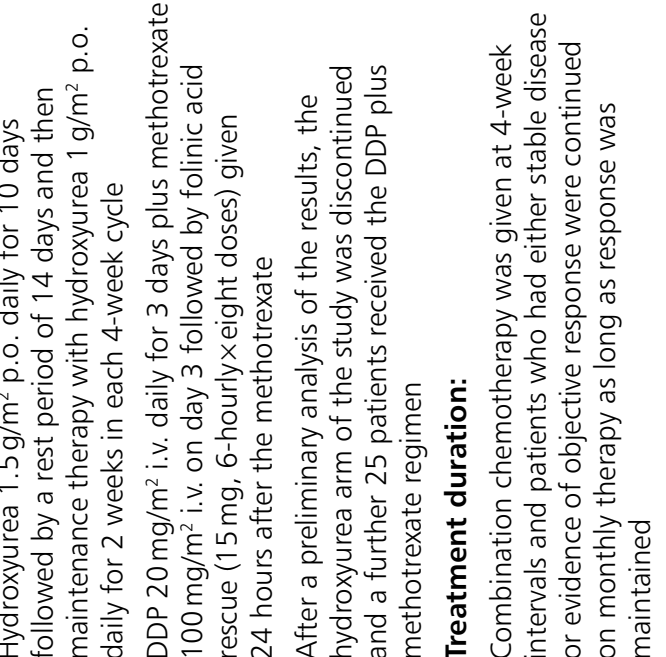




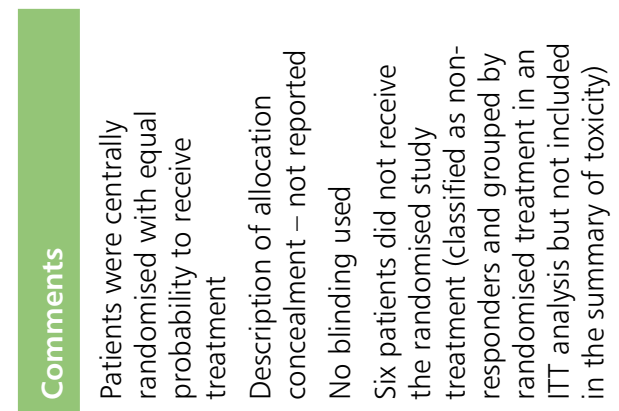

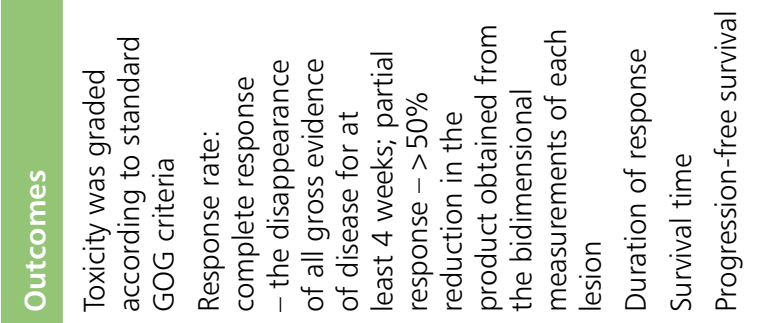

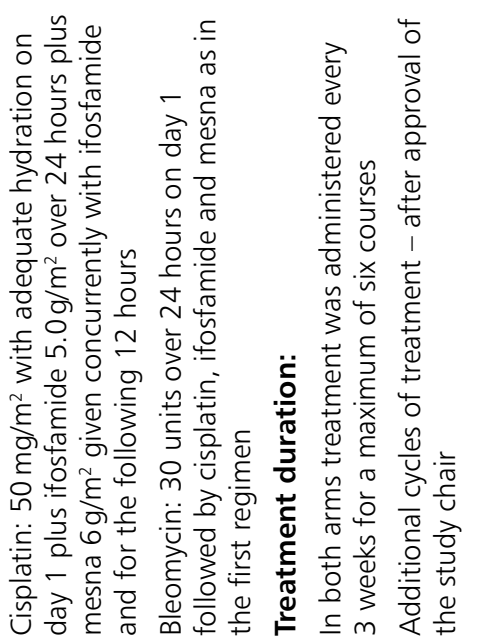

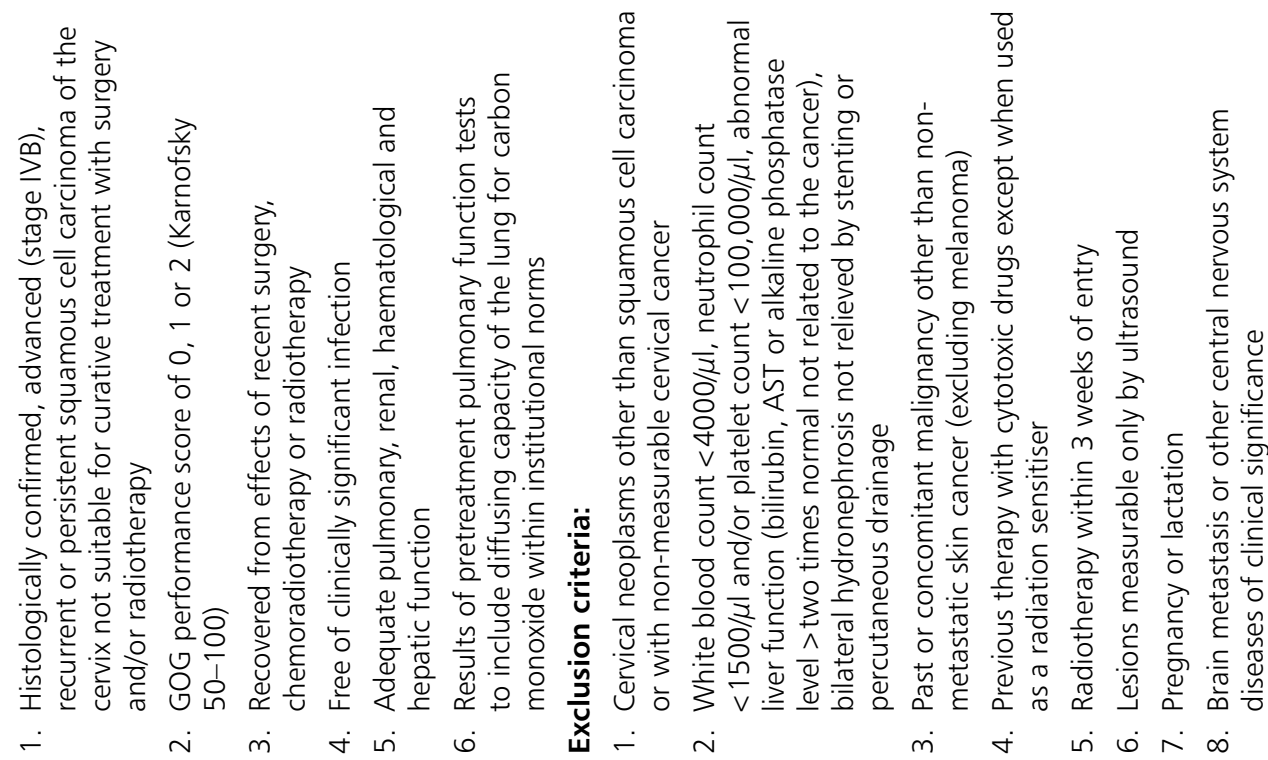

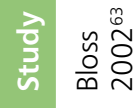




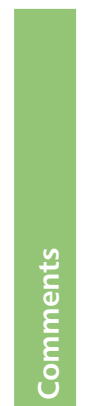

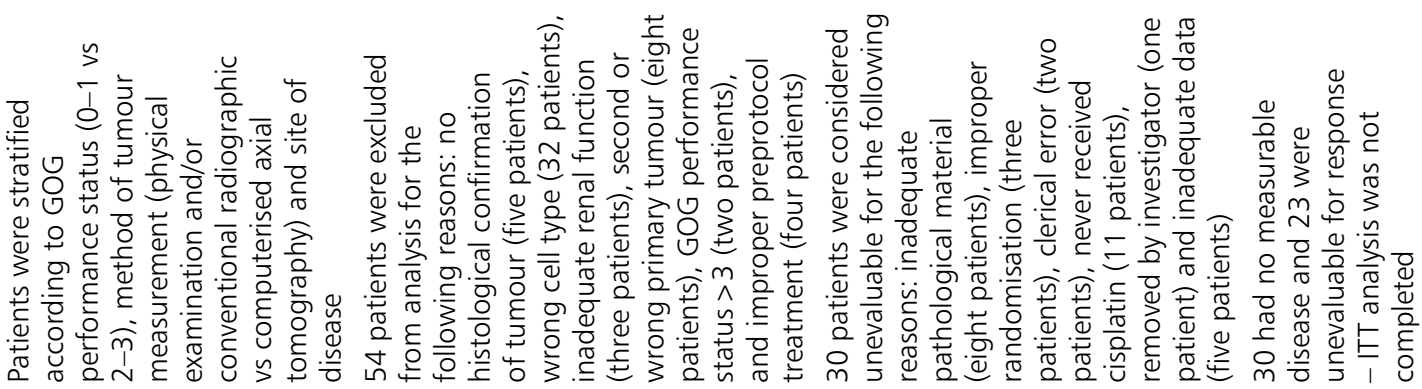
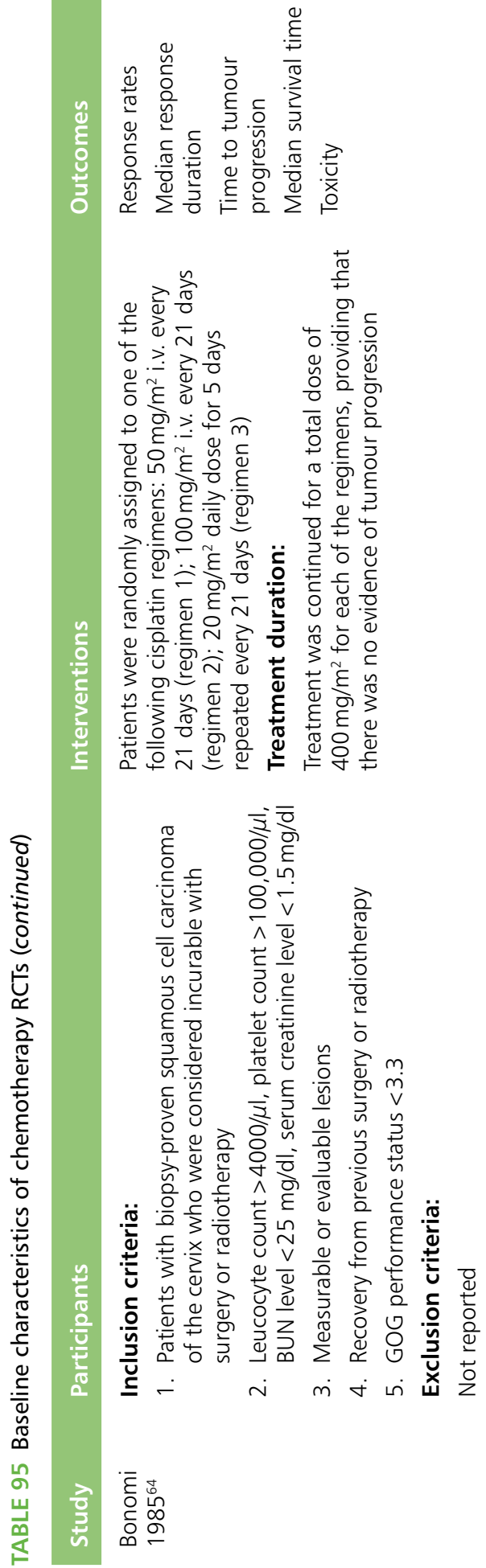

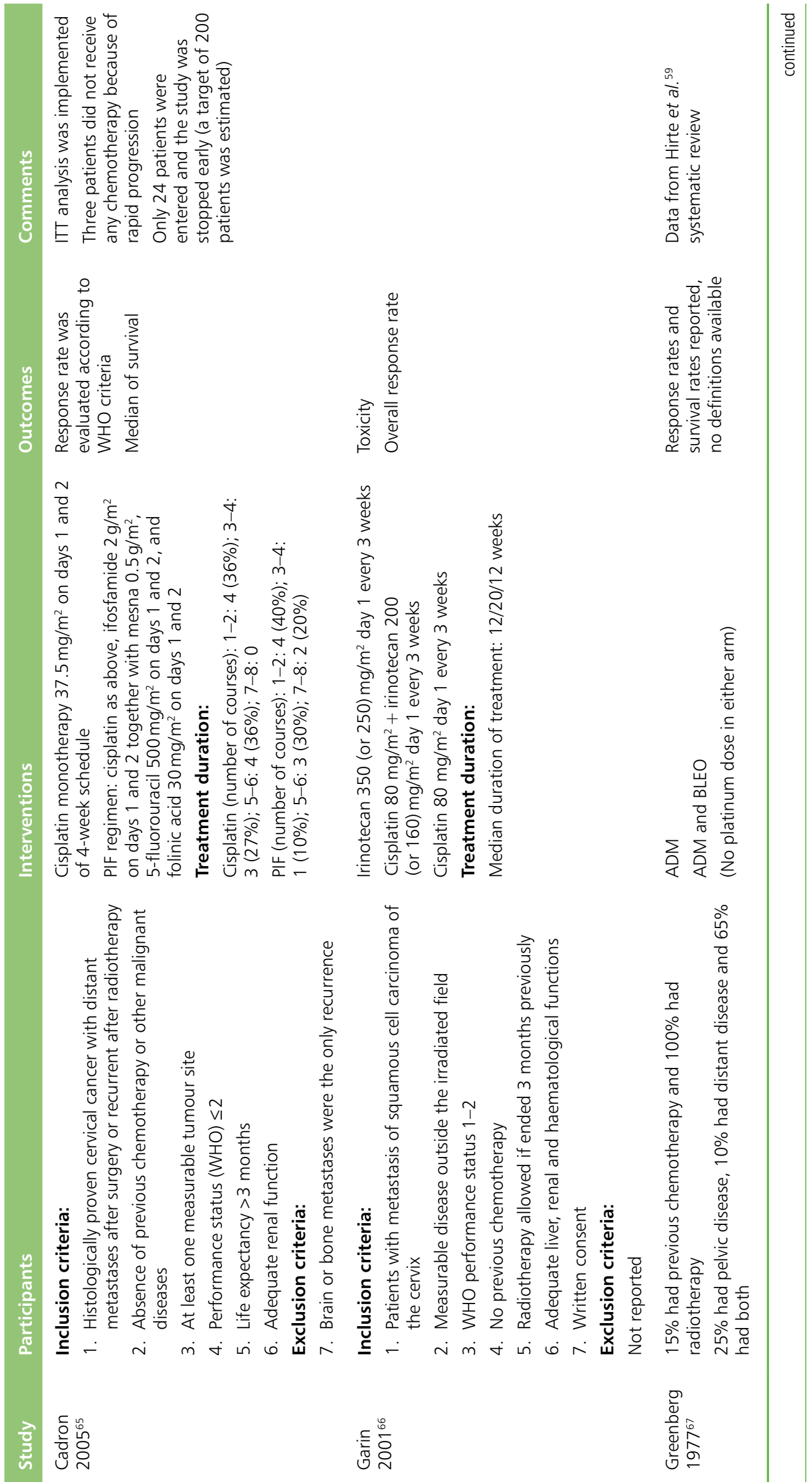


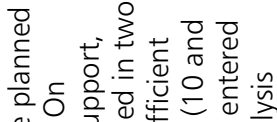

ठ

है.

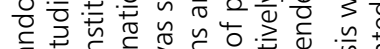

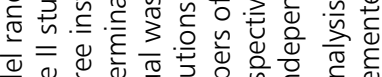

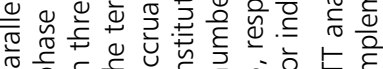

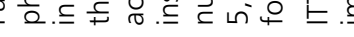

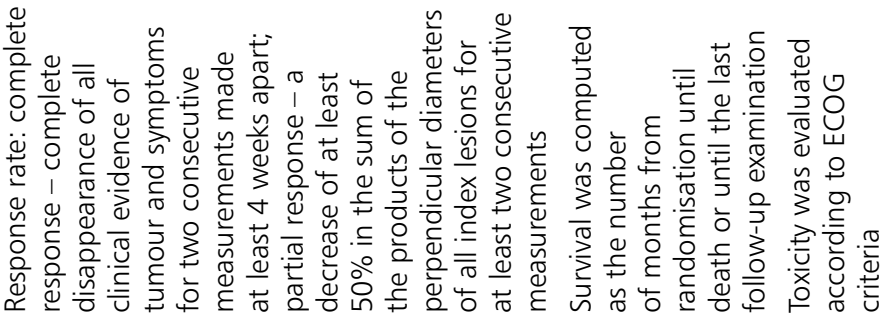

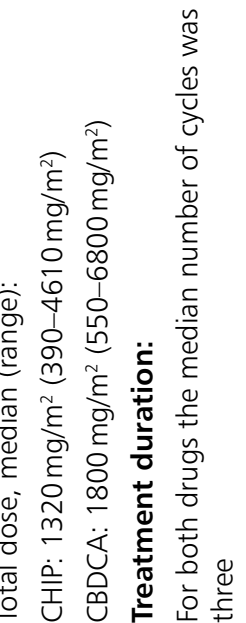

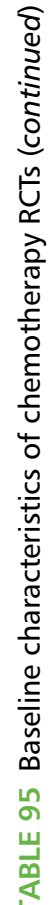

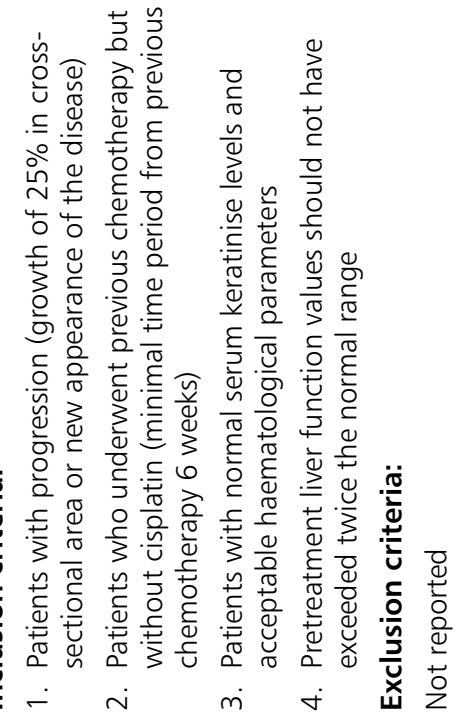




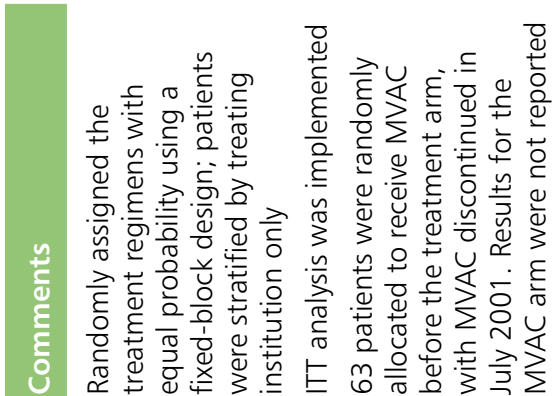

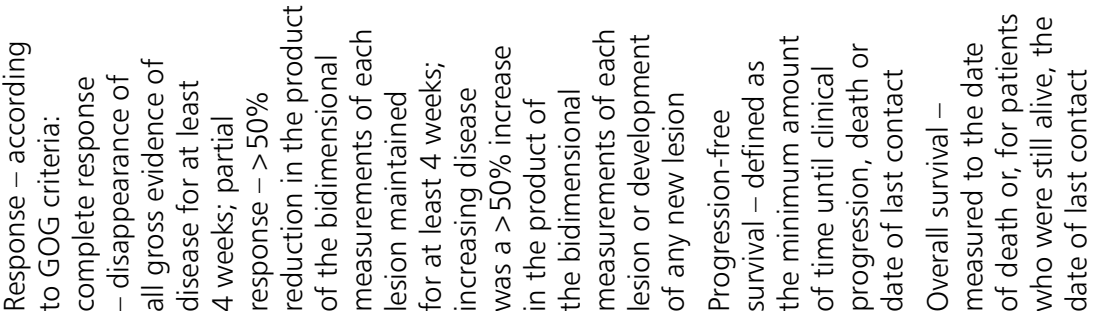

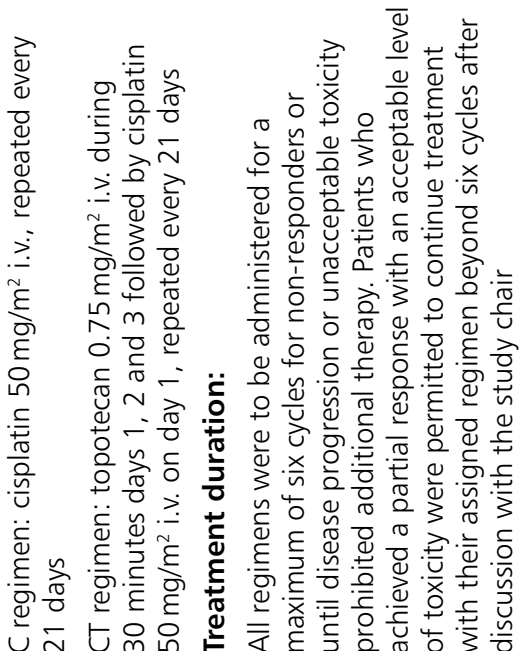
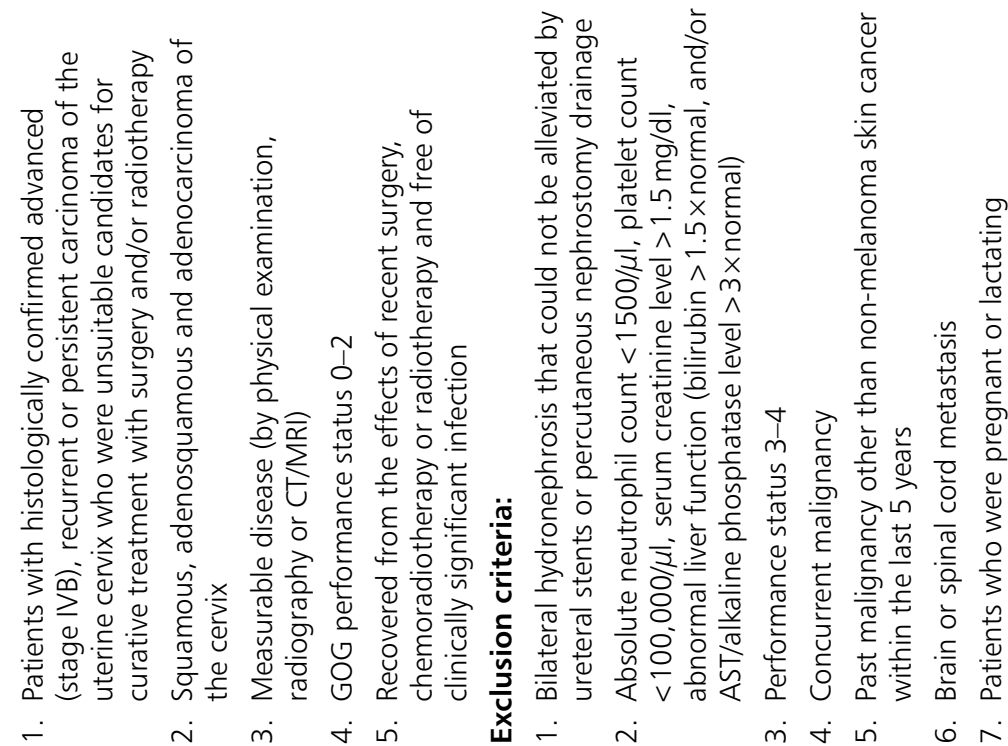

흘 옿윰

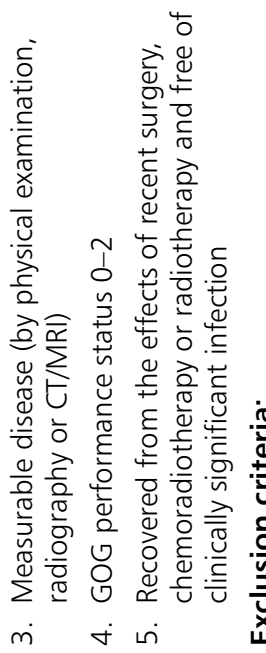




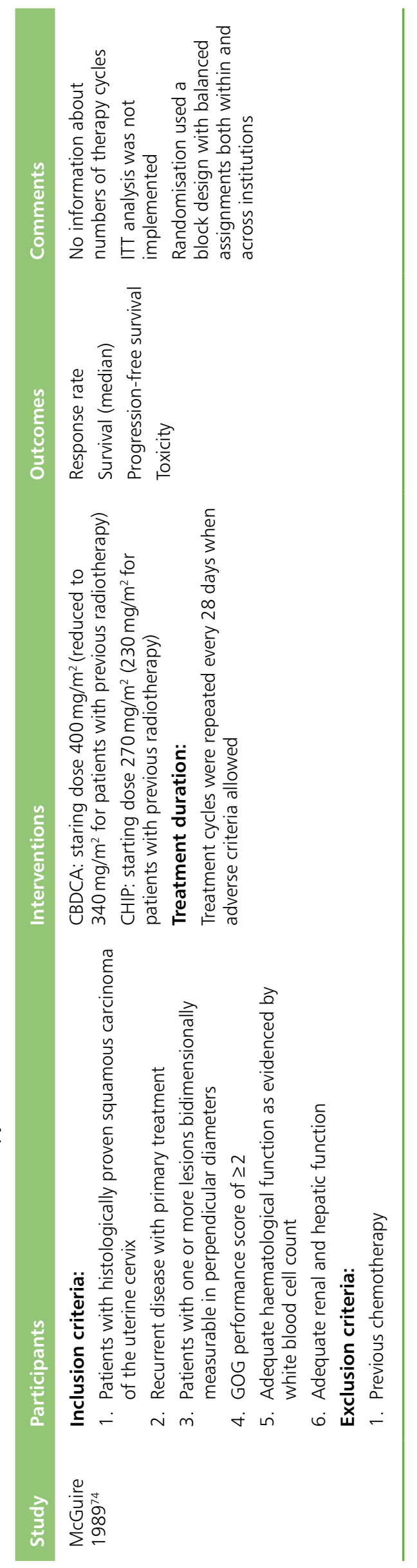



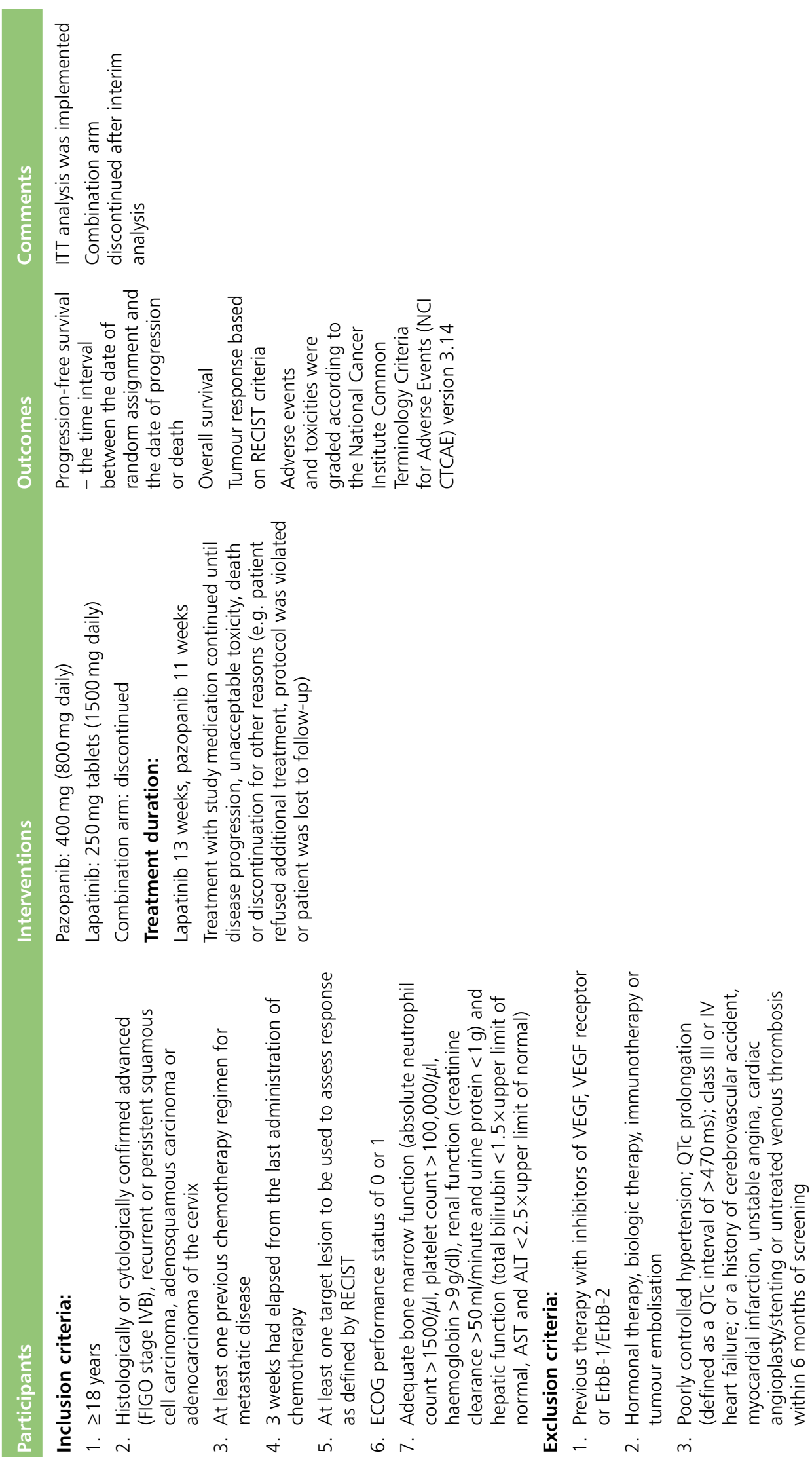

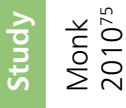




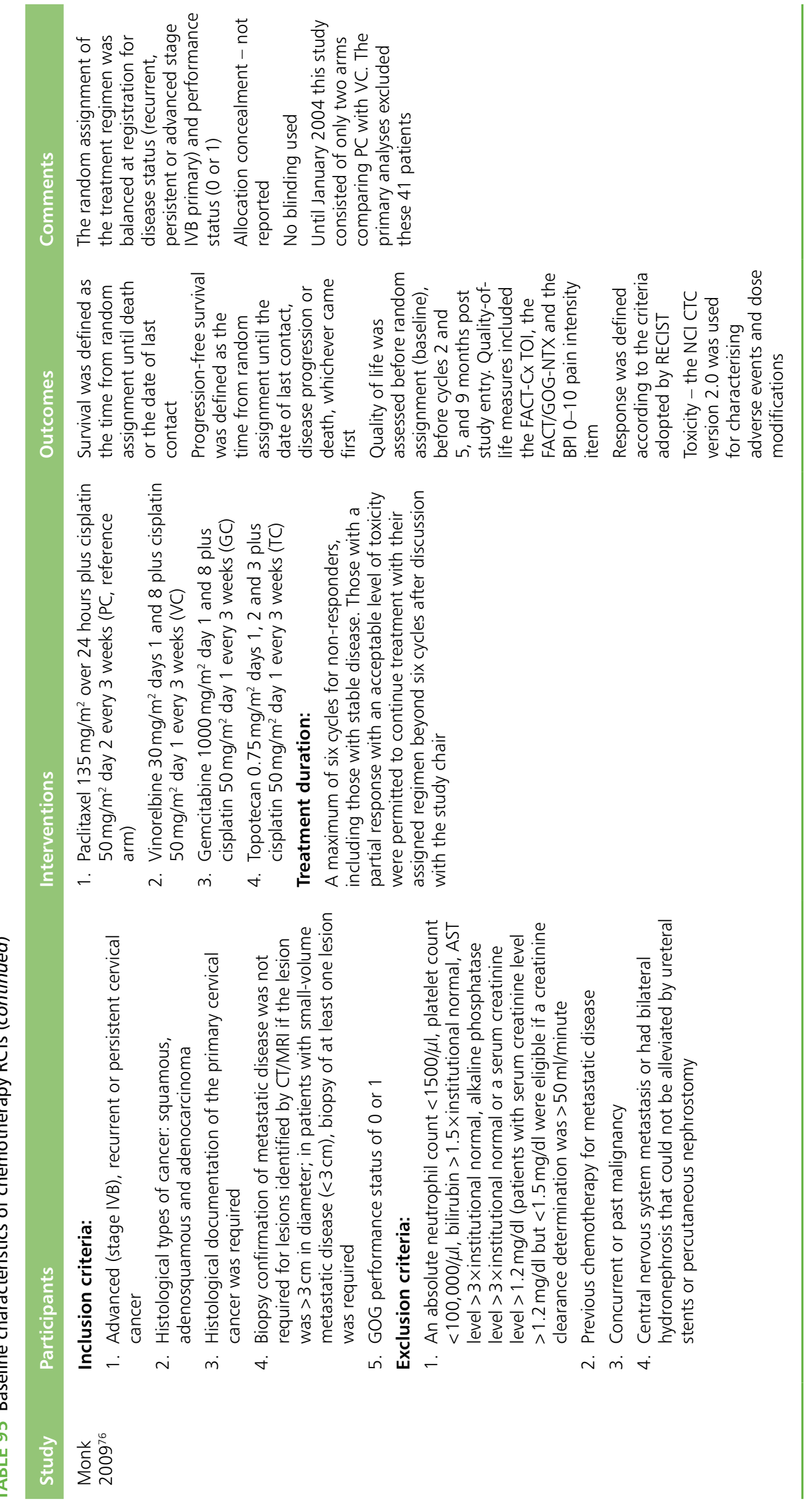



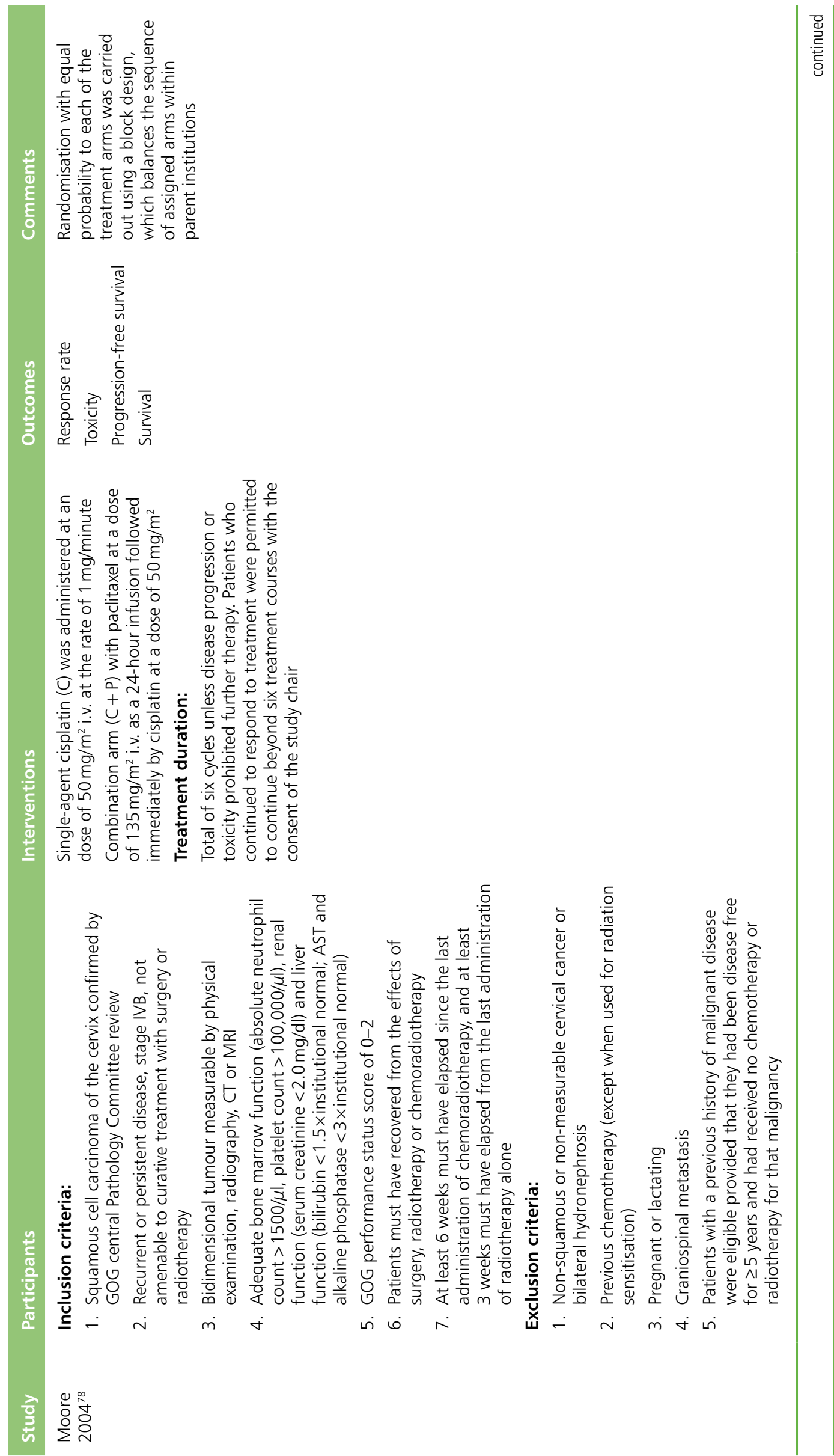


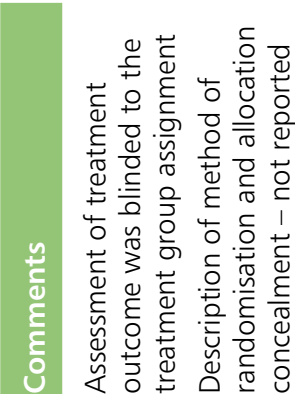

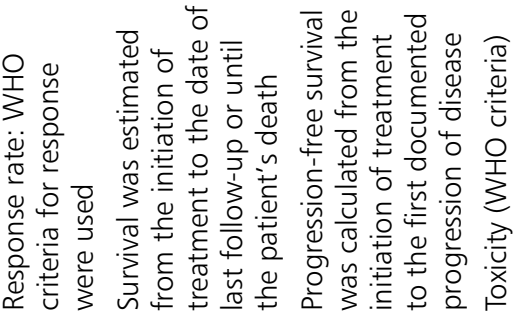

>-

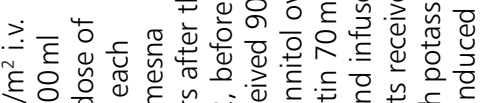

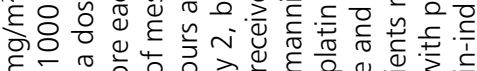

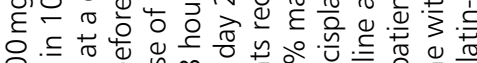

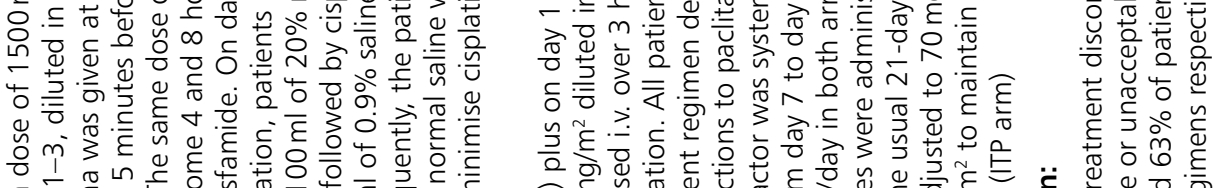

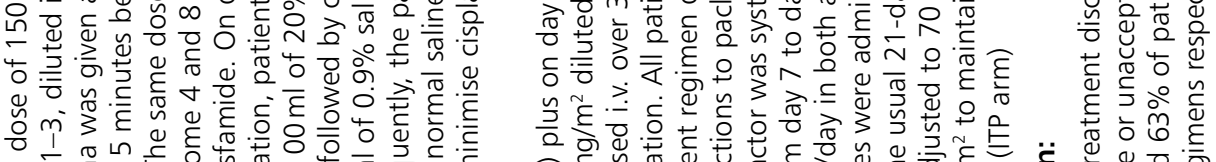

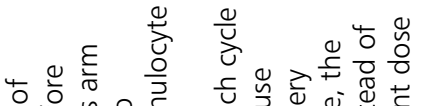

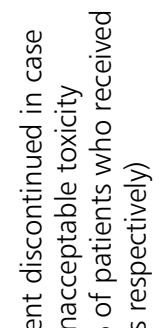

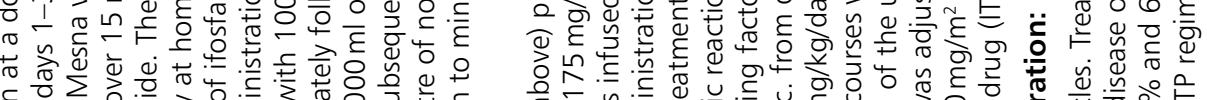

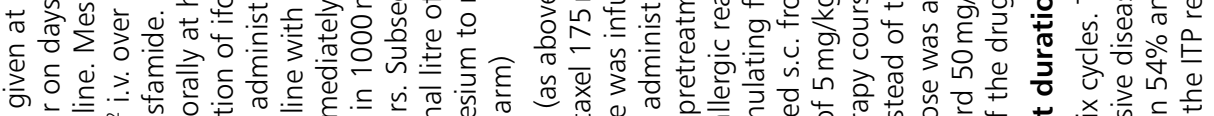
के

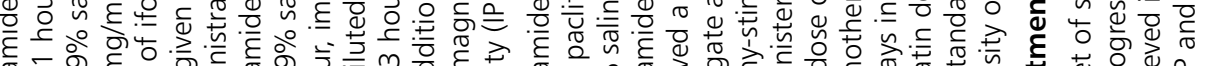

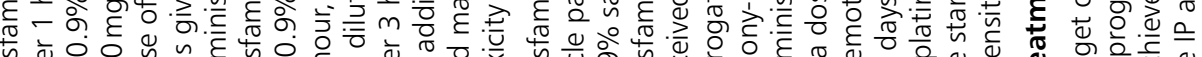

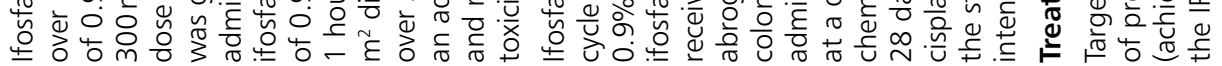

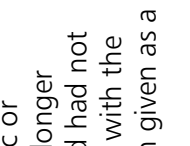

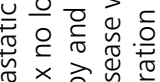

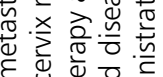

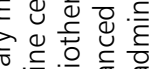

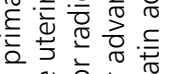

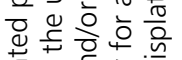

要

:

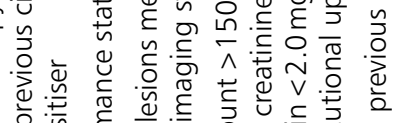

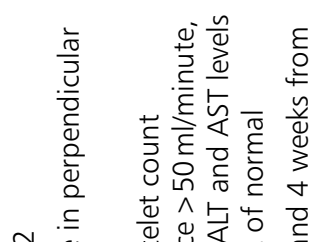

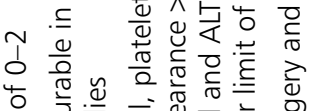

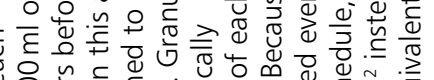
屯

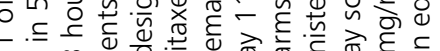

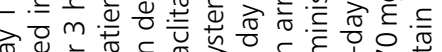

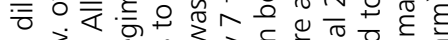




\section{है}

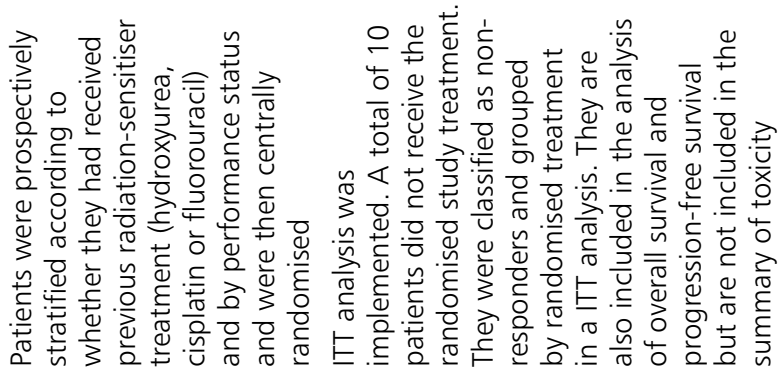

常

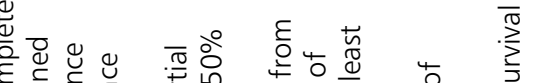

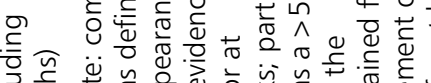

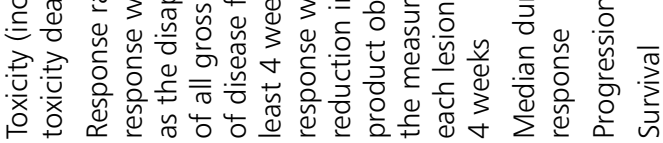

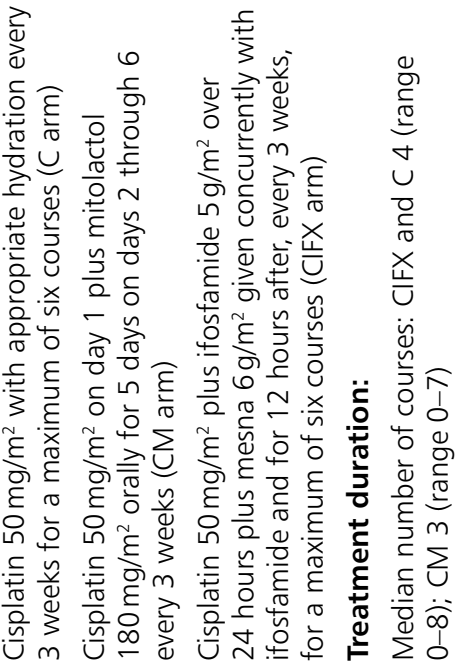

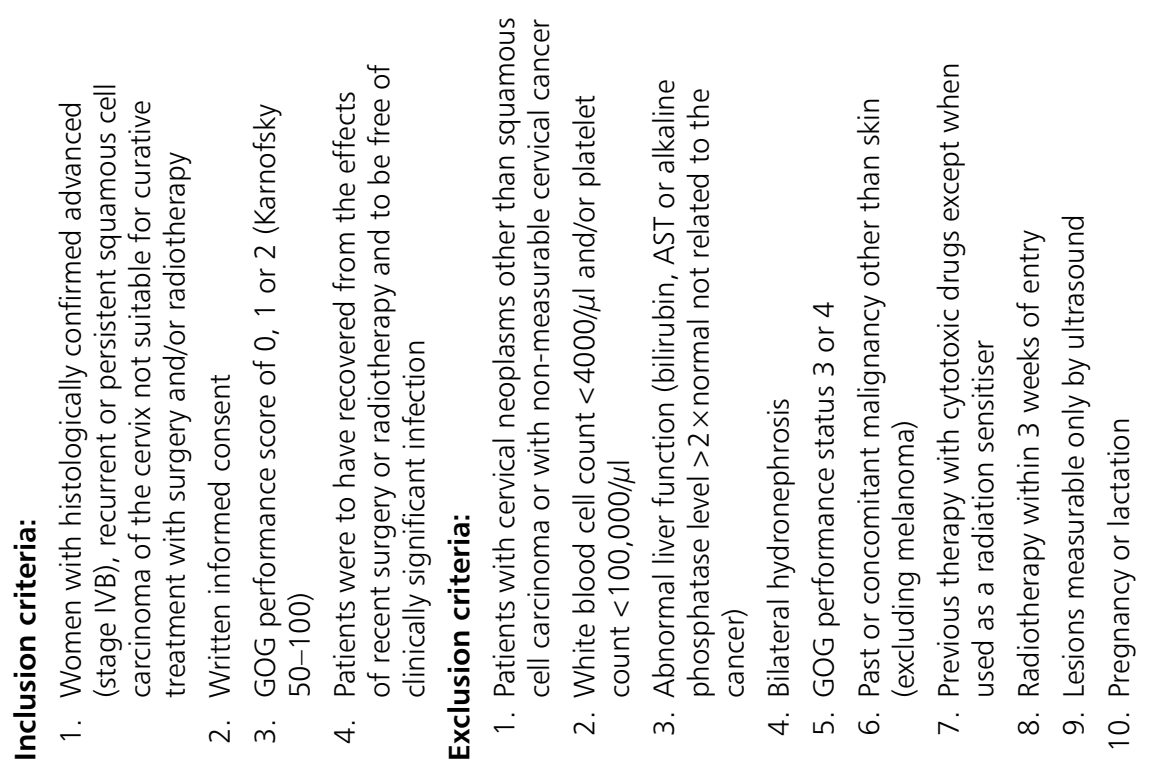

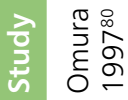




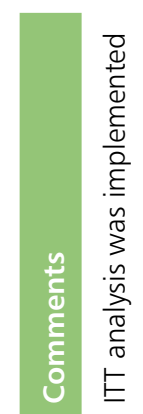

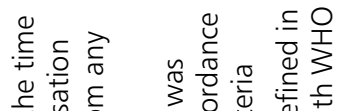

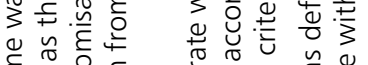

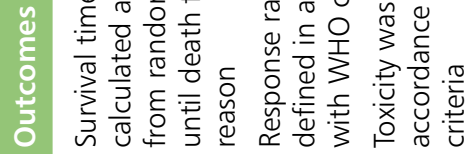

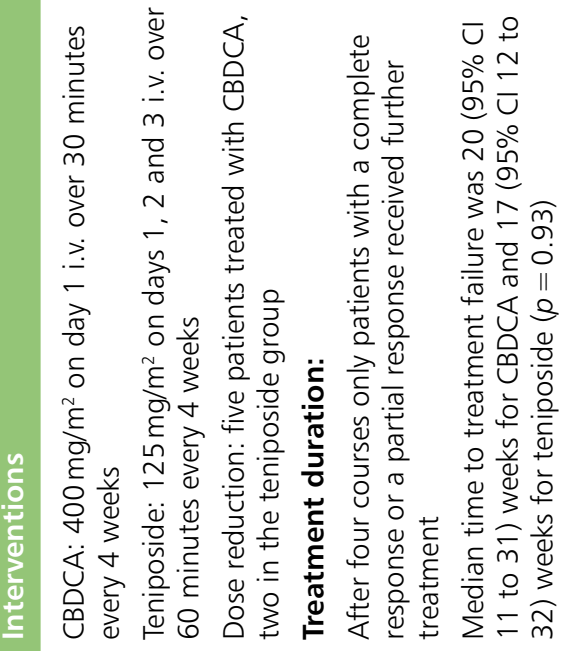

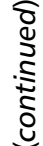

홓 울

बั $\frac{\pi}{\pi}$

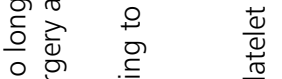

$\stackrel{n}{\underline{n}}$

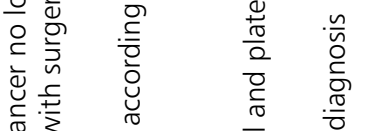




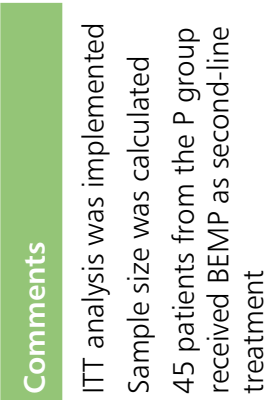

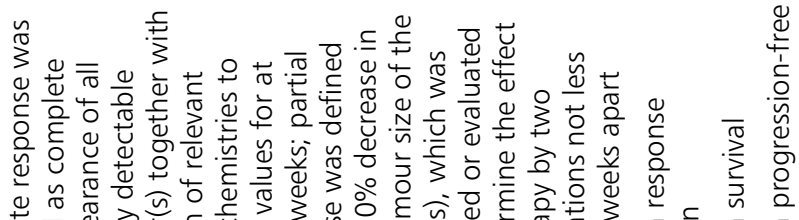

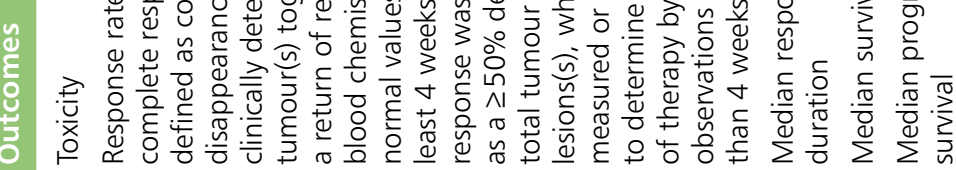

ำ

望荤

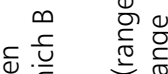

$\stackrel{-2}{-\infty}$

ते $\frac{\text { हे }}{0}$

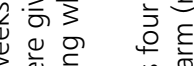

3 势

है $\sum$

केष

ㅇำ

这过岛

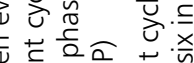

:

会 है

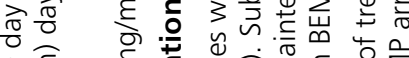

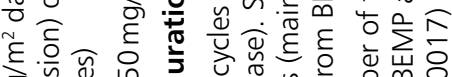

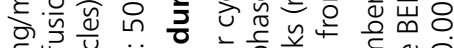

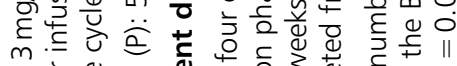

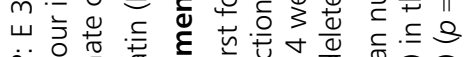

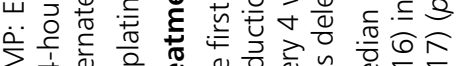

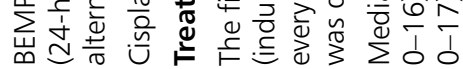

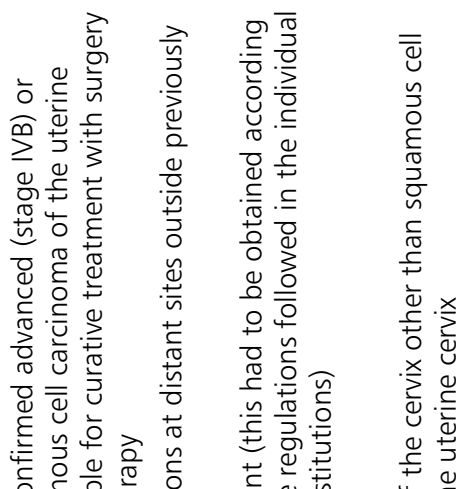

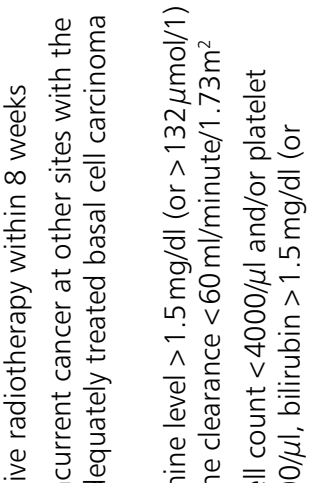

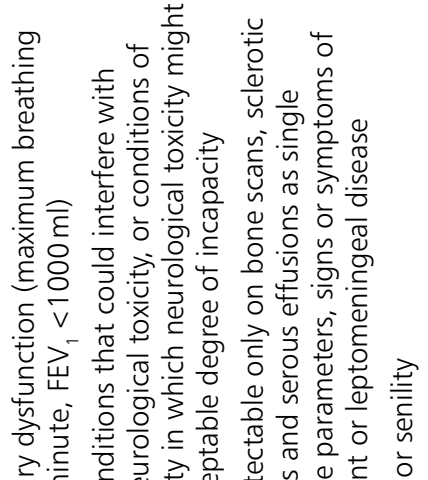

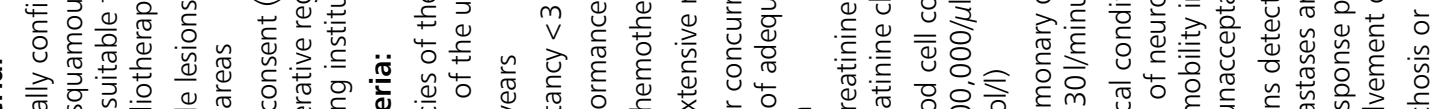

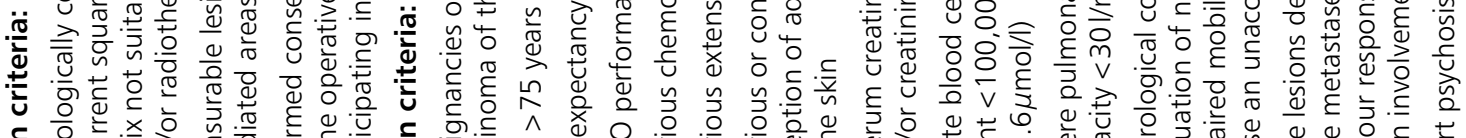

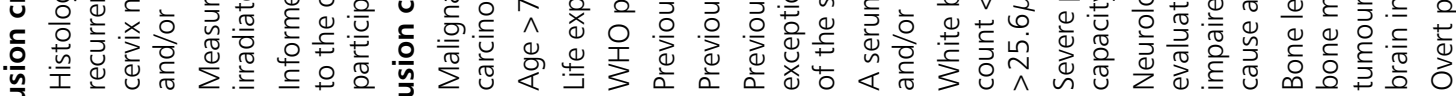

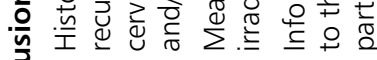
-

$\sim i \quad$ i

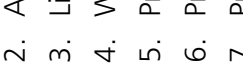

$\infty \quad 0$

$\therefore$

$\stackrel{1}{1}$

$\dot{m}$

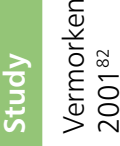




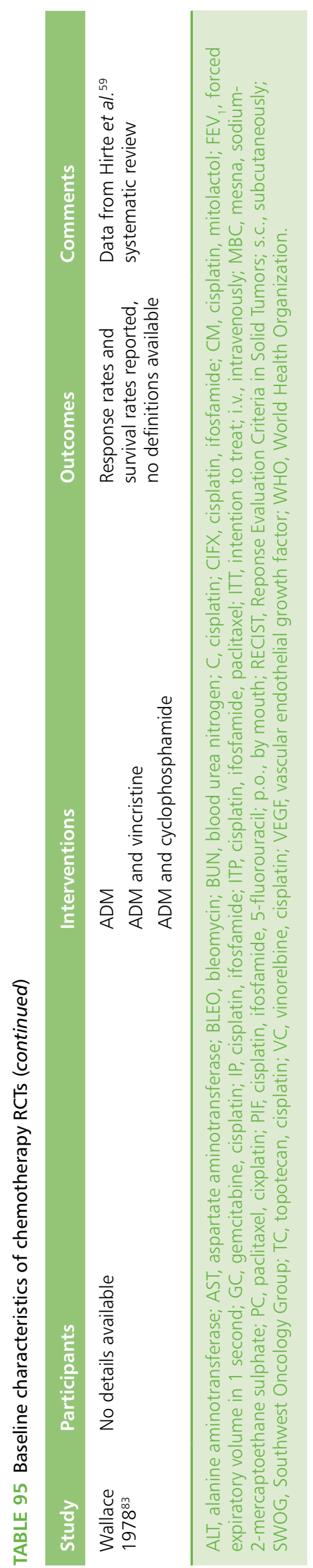




\section{Appendix 15 Quality assessment of case series: radiotherapy and chemoradiotherapy}




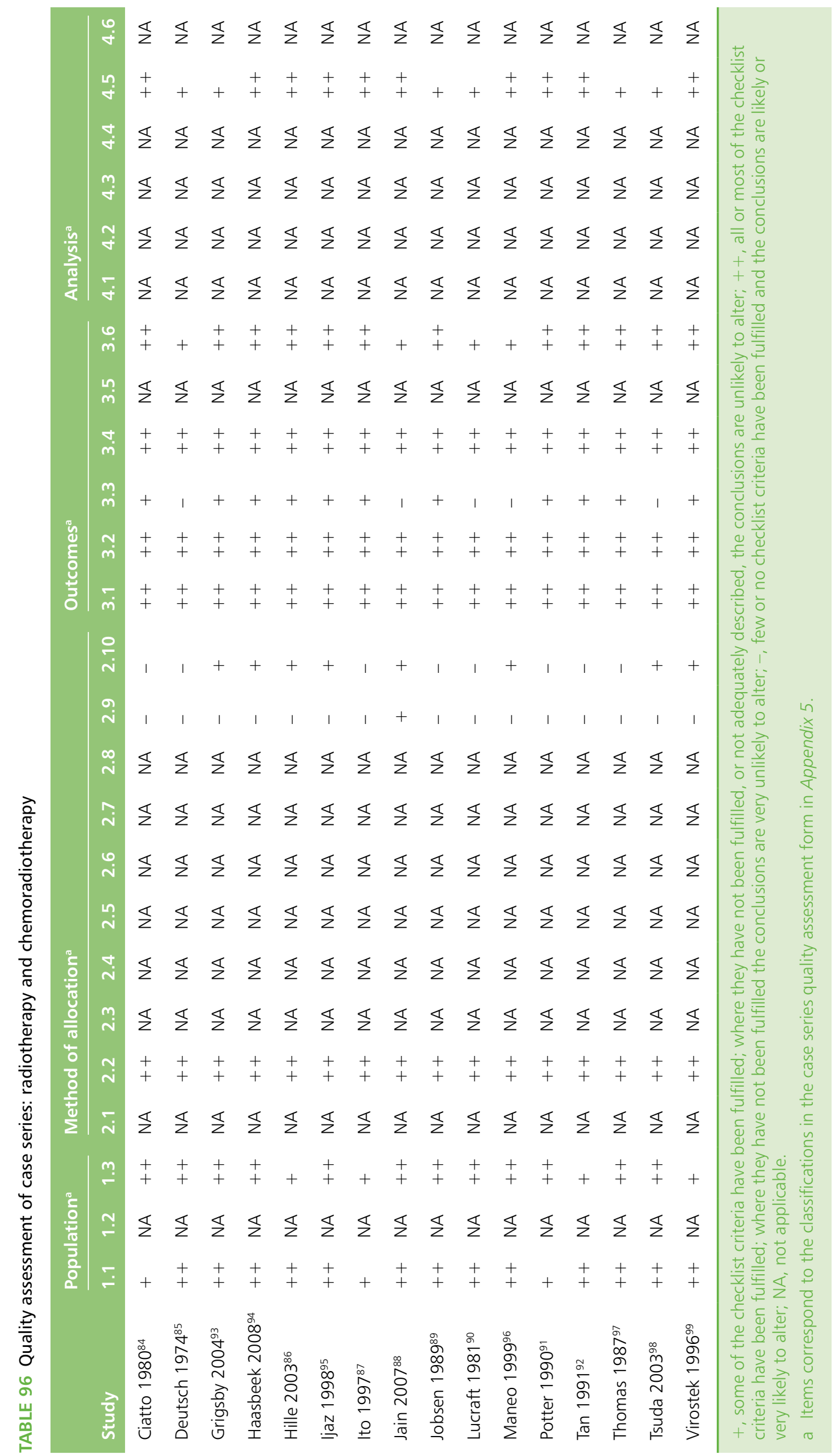




\section{Appendix 16 Quality assessment of case series: surgery}




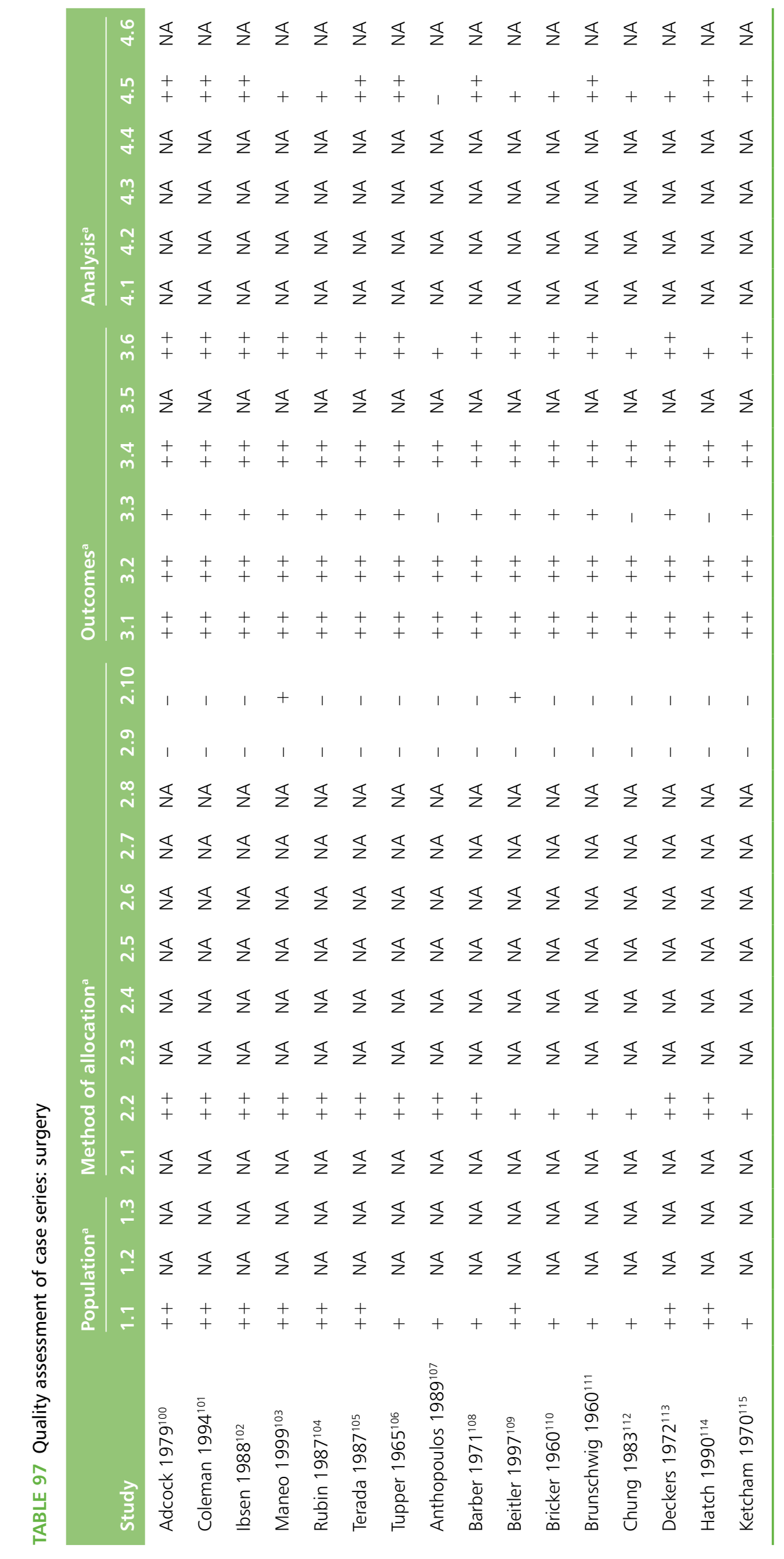




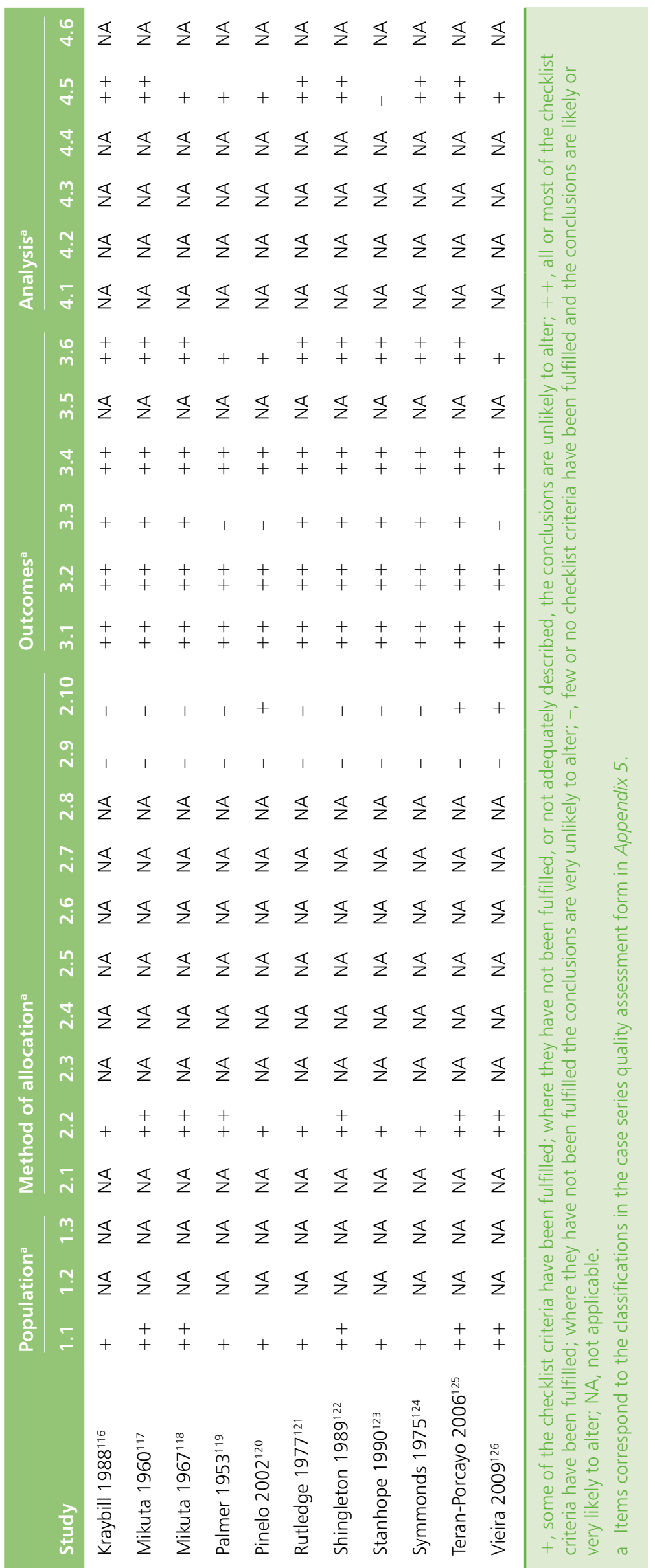





\section{Appendix 17 Systematic review of single cisplatin treatment in cervical cancer}

\section{Methods}

Searches were conducted in MEDLINE (Ovid) and the Cochrane database (CENTRAL; from inception to January 2012) for any studies evaluating cisplatin in cervical cancer. The search terms used were 'cisplatin' and 'cervical cancer'. Both MeSH terms and text words were used and a therapy clinical query maximising sensitivity was used in MEDLINE. Reference lists from relevant systematic reviews and guidelines were also searched. Included were any fully published RCTs in women with diagnosed cervical cancer (any stage, recurrent or primary) investigating cisplatin (including synonyms such as cis-platinum, cis-DDP) compared with no treatment and presenting any clinical outcomes but specifically interested in overall survival curves. The best-quality evidence for input into the economic model was sought. Preliminary and final inclusion decisions were made by one researcher (CM). Data extraction and quality assessment for the chosen $\mathrm{RCT}$ were performed by two researchers (CM, PA). If more than one large recent good-quality study was available meta-analysis would have been performed but this proved not to be necessary.

\section{Results}

Database searches yielded 1524 citations. Two published papers that nearly met the inclusion criteria were excluded: the study by Bonomi et al. ${ }^{64}$ is a RCT that evaluates three different doses of cisplatin but has no control arm, and the study by Thigpen et al. ${ }^{149}$ is a case series of cisplatin in recurrent cervical cancer and has no control arm without cisplatin. Three abstracts ${ }^{66,150,151}$ were also excluded. One paper ${ }^{152}$ was not available but as this did not give a sample size (as reported in Tzorias et al. ${ }^{146}$ ) it would be unlikely that this would be the best paper available. Four full papers ${ }^{127,153-155}$ were evaluated and all compared cisplatin plus radiotherapy with radiotherapy only. Pearcey et al. ${ }^{127}$ was chosen as the best paper for several reasons: participants were enrolled between 1991 and 1996 (i.e. after 1990), it had the largest sample size (259 patients), patients had a variety of FIGO stages including IVA and a survival curve for both arms was presented for up to 10 years. 



\section{Appendix 18 Health economics}


TABLE 98 Branch probabilities used in model 1

\section{Stage: $\quad$ Range \\ Label early $\quad(95 \% \mathrm{Cl}) \quad$ Probability distribution}

Surgical treatment for initial cervical cancer

Asymptomatic without cancer (A)

Surviving within 3 months having received clinical

a1

0.9993

Fixed

follow-up

Progressing to recurrent cervical cancer after having a a2

biopsy and survived within 3 months

$0.0103 \quad 0.0098$ to $\quad \operatorname{Beta}(506.35,48,654.06)$

a3 $\quad 0.9000$

0.0108

Recurrence being asymptomatic given recurrence occurred within 3 months

a4 $\quad 0.9000 \quad 0.8550$ to $\operatorname{Beta}(149.37,16.60)$

Remaining asymptomatic without cancer within

0.9450

\section{Asymptomatic cancer at 3 months (B)}

Surviving within 3 months after treatment

b1 $\quad 0.9307 \quad 0.8807$ to $\operatorname{Beta}(102.90,7.66)$

Surviving within 3 months if cancer is undetected

b2 $\quad 0.8406$

0.7986 to $\operatorname{Beta}(241.05,45.71)$ and untreated

b3 $\quad 0.9000$

0.8826

Remaining asymptomatic with untreated cancer conditional on surviving within 3 months

0.8550 to $\operatorname{Beta}(123.47,13.72)$ 0.9450

\section{Asymptomatic recurrence (C)}

Surviving within 3 months after treatment

C1

0.8807 to $\operatorname{Beta}(89.25,6.65)$

Surviving within 3 months if cancer is undetected

c2 $\quad 0.8406$

0.9807

and untreated

0.7986 to $\operatorname{Beta}(241.05,45.71)$

Remaining asymptomatic with untreated cancer

c3 $\quad 0.9000$

0.8826

conditional on surviving within 3 months

0.8550 to $\operatorname{Beta}(149.37,16.60)$ 0.9450

Symptomatic recurrence (D)

Surviving within 3 months after treatment

d1

0.9307

0.8807 to $\operatorname{Beta}(89.25,6.65)$

0.9807

Surviving within 3 months following undetected

d2

0.8406

0.7986 to $\operatorname{Beta}(241.05,45.71)$ cancer 0.8826

\section{Symptomatic cancer at 3 months (E)}

Surviving within 3 months after treatment

e1

e2

Surviving within 3 months following undetected cancer
0.8406
0.8807 to $\operatorname{Beta}(89.25,6.65)$

0.9807

0.7986 to $\operatorname{Beta}(241.05,45.71)$ 0.8826 
TABLE 98 Branch probabilities used in model 1 (continued)

\begin{tabular}{|c|c|c|c|c|}
\hline & Label & $\begin{array}{l}\text { Stage: } \\
\text { early }\end{array}$ & $\begin{array}{l}\text { Range } \\
(95 \% \mathrm{Cl})\end{array}$ & Probability distribution \\
\hline \multicolumn{5}{|l|}{ Symptomatic without cancer (F) } \\
\hline Surviving within 3 months following false symptoms & f1 & 0.9993 & & Fixed \\
\hline $\begin{array}{l}\text { Progressing to recurrent cervical cancer after having a } \\
\text { biopsy and survived within } 3 \text { months }\end{array}$ & $\mathrm{f} 2$ & 0.0103 & $\begin{array}{l}0.0094 \text { to } \\
0.0112\end{array}$ & $\operatorname{Beta}(506.35,48654.06)$ \\
\hline $\begin{array}{l}\text { Recurrence being asymptomatic given recurrence } \\
\text { occurred within } 3 \text { months }\end{array}$ & f3 & 0.4000 & $\begin{array}{l}0.3800 \text { to } \\
0.4200\end{array}$ & $\operatorname{Beta}(915.02,1372.53)$ \\
\hline $\begin{array}{l}\text { Remaining asymptomatic without cancer within } \\
3 \text { months given no recurrence }\end{array}$ & $\mathrm{f} 4$ & 0.9000 & $\begin{array}{l}0.8550 \text { to } \\
0.9450\end{array}$ & $\operatorname{Beta}(149.37,16.60)$ \\
\hline \multicolumn{5}{|l|}{ Post treatment: asymptomatic cancer at 3 months (G) } \\
\hline $\begin{array}{l}\text { Mean survival time following treatment for those } \\
\text { who were diagnosed with asymptomatic cancer at }\end{array}$ & g & 0.9307 & $\begin{array}{l}0.8807 \text { to } \\
0.9807\end{array}$ & $\operatorname{Beta}(89.25,6.65)$ \\
\hline
\end{tabular}

3 months

Post treatment: asymptomatic $(H)$

Mean survival time following treatment for those

h $\quad 0.9307 \quad 0.8807$ to $\operatorname{Beta}(89.25,6.65)$

who were diagnosed with asymptomatic cancer

0.9807

Post treatment: symptomatic cancer at 3 months (I)

Mean survival time following treatment for those who were diagnosed with symptomatic cancer at 3 months

Post treatment: symptomatic (J)

Mean survival time following treatment for those j $\quad 0.9307 \quad 0.8807$ to $\quad$ Beta $(89.25,6.65)$ who were diagnosed with symptomatic cancer

0.9807

\section{Dead (absorbing state)}


TABLE 99 Transition probabilities used in model 2

\section{$\begin{array}{llll} & \text { Stage: } & \text { Range } & \\ \text { Label } & \text { early } & (95 \% \mathrm{Cl}) & \text { Probability distribution }\end{array}$}

\section{Surgical treatment for initial cervical cancer}

Asymptomatic without cancer (A)

Surviving within 3 months having received clinical

a1

follow-up

Progressing to recurrent cervical cancer after having a2 a biopsy and survived within 3 months

Recurrence being asymptomatic given recurrence a3 occurred within 3 months

Remaining asymptomatic without cancer within a4

3 months given no recurrence

Asymptomatic cancer at 3 months (B)

Surviving within 3 months after treatment

Surviving within 3 months if cancer is undetected

and untreated

Remaining asymptomatic with untreated cancer conditional on surviving within 3 months

\section{Asymptomatic recurrence (C)}

Surviving within 3 months after treatment

c1 $\quad 0.9778$

Surviving within 3 months if cancer is undetected

c2

and untreated

0.8406

Remaining asymptomatic with untreated cancer conditional on surviving within 3 months

c3

$0.9000 \quad 0.8550$ to
Fixed

$\operatorname{eta}(506.35,48654.06)$

0.0108

0.8550 to $\quad \operatorname{Beta}(149.37,16.60)$

0.8550 to $\quad \operatorname{Beta}(149.37,16.60)$

0.9450

Symptomatic recurrence (D)

Surviving within 3 months after treatment

d1

0.9778

Surviving within 3 months following undetected cancer

\section{Symptomatic cancer at 3 months (E)}

Surviving within 3 months after treatment

e1

e2

Surviving within 3 months following undetected cancer d2 $\quad 0.8406 \quad 0.7986$ to

0.8826
0.8526 to

0.9968

0.7986 to

0.8826 0.9450

0.8526 to

0.9968

Converted from log-normal distribution for hazard rate

$\operatorname{Beta}(241.05,45.71)$

$\operatorname{Beta}(149.37,16.60)$

Converted from log-normal distribution for hazard rate

$\operatorname{Beta}(241.05,45.71)$

Converted from log-norma distribution for hazard rate

$\operatorname{Beta}(241.05,45.71)$

0.7986 to 0.8826

\section{Symptomatic without cancer (F)}

Surviving within 3 months following false symptoms

Progressing to recurrent cervical cancer after having a biopsy and survived within 3 months

Recurrence being asymptomatic given recurrence occurred within 3 months

Remaining asymptomatic without cancer within 3 months given no recurrence

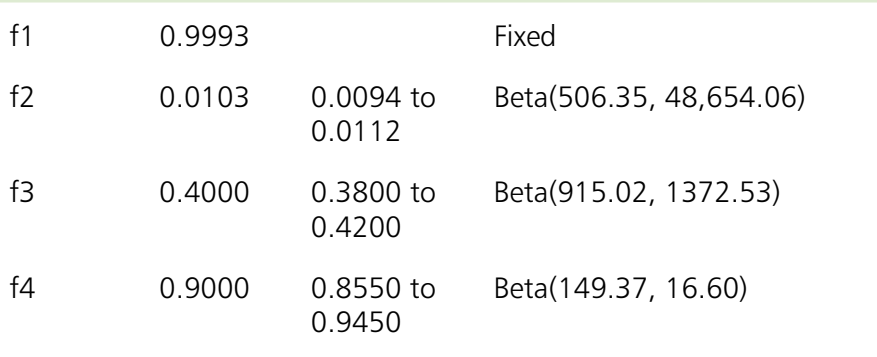


TABLE 99 Transition probabilities used in model 2 (continued)

\begin{tabular}{|c|c|c|c|c|}
\hline & Label & $\begin{array}{l}\text { Stage: } \\
\text { early }\end{array}$ & $\begin{array}{l}\text { Range } \\
(95 \% \mathrm{CI})\end{array}$ & Probability distribution \\
\hline \multicolumn{5}{|l|}{ Post treatment: asymptomatic cancer at 3 months (G) } \\
\hline $\begin{array}{l}\text { Mean survival time following treatment for those } \\
\text { who were diagnosed with asymptomatic cancer at } \\
3 \text { months }\end{array}$ & $g$ & 0.9778 & $\begin{array}{l}0.8526 \text { to } \\
0.9968\end{array}$ & $\begin{array}{l}\text { Converted from log-normal } \\
\text { distribution for hazard rate }\end{array}$ \\
\hline \multicolumn{5}{|l|}{ Post treatment: asymptomatic $(H)$} \\
\hline $\begin{array}{l}\text { Mean survival time following treatment for those } \\
\text { who were diagnosed with asymptomatic cancer }\end{array}$ & $\mathrm{h}$ & 0.9778 & $\begin{array}{l}0.8526 \text { to } \\
0.9968\end{array}$ & $\begin{array}{l}\text { Converted from log-normal } \\
\text { distribution for hazard rate }\end{array}$ \\
\hline \multicolumn{5}{|l|}{ Post treatment: symptomatic cancer at 3 months (I) } \\
\hline $\begin{array}{l}\text { Mean survival time following treatment for those } \\
\text { who were diagnosed with symptomatic cancer at } \\
3 \text { months }\end{array}$ & i & 0.9778 & $\begin{array}{l}0.8526 \text { to } \\
0.9968\end{array}$ & $\begin{array}{l}\text { Converted from log-normal } \\
\text { distribution for hazard rate }\end{array}$ \\
\hline \multicolumn{5}{|l|}{ Post treatment: symptomatic $(J)$} \\
\hline $\begin{array}{l}\text { Mean survival time following treatment for those } \\
\text { who were diagnosed with symptomatic cancer }\end{array}$ & j & 0.9778 & $\begin{array}{l}0.8526 \text { to } \\
0.9968\end{array}$ & $\begin{array}{l}\text { Converted from log-normal } \\
\text { distribution for hazard rate }\end{array}$ \\
\hline Dead (absorbing state) & & & & \\
\hline
\end{tabular}


TABLE 100 Transition probabilities used in model 3

\section{Stage: Range \\ Label late $\quad(95 \% \mathrm{Cl}) \quad$ Probability distribution}

Surgical treatment for initial cervical cancer

Asymptomatic without cancer (A)

Surviving within 3 months having received clinical a follow-up

Progressing to recurrent cervical cancer after having a biopsy and survived within 3 months

a2

Recurrence being asymptomatic given recurrence a3 occurred within 3 months

Remaining asymptomatic without cancer within a4

3 months given no recurrence

Asymptomatic cancer at 3 months (B)

Surviving within 3 months after treatment

b1

Surviving within 3 months if cancer is undetected and untreated

0.9993

Fixed

Remaining asymptomatic with untreated cancer conditional on surviving within 3 months

b3

\section{Asymptomatic recurrence (C)}

Surviving within 3 months after treatment

$\begin{array}{ll}\text { c1 } & 0.9779 \quad \begin{array}{l}0.8530 \text { to } \\ \end{array} \\ & 0.9969\end{array}$

Converted from log-normal distribution for hazard rate

Surviving within 3 months if cancer is undetected and untreated

c2 $\quad 0.8406$

0.7986 to

$\operatorname{Beta}(241.05,45.71)$

Remaining asymptomatic with untreated cancer conditional on surviving within 3 months

c3

$0.9000 \quad 0.8550$ to 0.9450

\section{Symptomatic recurrence (D)}

Surviving within 3 months after treatment

d1

0.9779

0.8530 to

0.9969

Surviving within 3 months following undetected cancer

$\mathrm{d} 2$

0.8406

0.7986 to 0.8826

Symptomatic cancer at 3 months (E)
Surviving within 3 months after treatment

e1

e2

Surviving within 3 months following undetected cancer

$\begin{array}{ll}0.9779 & 0.8530 \text { to } \\ & 0.9969\end{array}$

$0.8406 \quad 0.7986$ to 0.8826
Converted from log-normal distribution for hazard rate

$\operatorname{Beta}(241.05,45.71)$

Converted from log-normal distribution for hazard rate

$\operatorname{Beta}(241.05,45.71)$

Symptomatic without cancer (F)

Surviving within 3 months following false symptoms

Progressing to recurrent cervical cancer after having a biopsy and survived within 3 months

f1

0.9993

Fixed

Recurrence being asymptomatic given recurrence occurred within 3 months

Remaining asymptomatic without cancer within 3 months given no recurrence 
TABLE 100 Transition probabilities used in model 3 (continued)

\begin{tabular}{|c|c|c|c|c|}
\hline & Label & $\begin{array}{l}\text { Stage: } \\
\text { late }\end{array}$ & $\begin{array}{l}\text { Range } \\
(95 \% \mathrm{Cl})\end{array}$ & Probability distribution \\
\hline \multicolumn{5}{|c|}{ Post treatment: asymptomatic cancer at 3 months (G) } \\
\hline $\begin{array}{l}\text { Mean survival time following treatment for those } \\
\text { who were diagnosed with asymptomatic cancer at } \\
3 \text { months }\end{array}$ & $g$ & 0.9779 & $\begin{array}{l}0.8530 \text { to } \\
0.9969\end{array}$ & $\begin{array}{l}\text { Converted from log-normal } \\
\text { distribution for hazard rate }\end{array}$ \\
\hline \multicolumn{5}{|l|}{ Post treatment: asymptomatic $(H)$} \\
\hline $\begin{array}{l}\text { Mean survival time following treatment for those } \\
\text { who were diagnosed with asymptomatic cancer }\end{array}$ & $\mathrm{h}$ & 0.9779 & $\begin{array}{l}0.8530 \text { to } \\
0.9969\end{array}$ & $\begin{array}{l}\text { Converted from log-normal } \\
\text { distribution for hazard rate }\end{array}$ \\
\hline \multicolumn{5}{|l|}{ Post treatment: symptomatic cancer at 3 months (I) } \\
\hline $\begin{array}{l}\text { Mean survival time following treatment for those } \\
\text { who were diagnosed with symptomatic cancer at } \\
3 \text { months }\end{array}$ & i & 0.9779 & $\begin{array}{l}0.8530 \text { to } \\
0.9969\end{array}$ & $\begin{array}{l}\text { Converted from log-normal } \\
\text { distribution for hazard rate }\end{array}$ \\
\hline \multicolumn{5}{|l|}{ Post treatment: symptomatic $(J)$} \\
\hline $\begin{array}{l}\text { Mean survival time following treatment for those } \\
\text { who were diagnosed with symptomatic cancer }\end{array}$ & j & 0.9779 & $\begin{array}{l}0.8530 \text { to } \\
0.9969\end{array}$ & $\begin{array}{l}\text { Converted from log-normal } \\
\text { distribution for hazard rate }\end{array}$ \\
\hline Dead (absorbing state) & & & & \\
\hline
\end{tabular}


TABLE 101 Transition probabilities used in model 4

\begin{tabular}{|c|c|c|c|c|}
\hline & Label & $\begin{array}{l}\text { Stage: } \\
\text { early }\end{array}$ & $\begin{array}{l}\text { Range } \\
(95 \% \mathrm{Cl})\end{array}$ & Probability distribution \\
\hline \multicolumn{5}{|l|}{$\begin{array}{l}\text { Surgical treatment for initial cervical cancer } \\
\text { Asymptomatic without cancer }(A)\end{array}$} \\
\hline $\begin{array}{l}\text { Surviving within } 3 \text { months having received clinical } \\
\text { follow-up }\end{array}$ & a1 & 0.9993 & & Fixed \\
\hline $\begin{array}{l}\text { Progressing to recurrent cervical cancer after } \\
\text { having a biopsy and survived within } 3 \text { months }\end{array}$ & a2 & 0.0103 & $\begin{array}{l}0.0098 \text { to } \\
0.0108\end{array}$ & $\operatorname{Beta}(506.35,48,654.06)$ \\
\hline $\begin{array}{l}\text { Recurrence being asymptomatic given recurrence } \\
\text { occurred within } 3 \text { months }\end{array}$ & a3 & 0.9000 & $\begin{array}{l}0.8448 \text { to } \\
0.9442\end{array}$ & Beta(149.37, 16.60) \\
\hline $\begin{array}{l}\text { Remaining asymptomatic without cancer within } \\
3 \text { months given no recurrence }\end{array}$ & a4 & 0.9000 & $\begin{array}{l}0.8448 \text { to } \\
0.9442\end{array}$ & $\operatorname{Beta}(149.37,16.60)$ \\
\hline \multicolumn{5}{|l|}{ Asymptomatic cancer at 3 months $(B)$} \\
\hline Surviving within 3 months after treatment & b1 & 0.9778 & $\begin{array}{l}0.8526 \text { to } \\
0.9968\end{array}$ & $\begin{array}{l}\text { Converted from log-normal } \\
\text { distribution for hazard rate }\end{array}$ \\
\hline $\begin{array}{l}\text { Surviving within } 3 \text { months if cancer is undetected } \\
\text { and untreated }\end{array}$ & $\mathrm{b} 2$ & 0.8406 & $\begin{array}{l}0.7986 \text { to } \\
0.8826\end{array}$ & $\operatorname{Beta}(241.05,45.71)$ \\
\hline $\begin{array}{l}\text { Remaining asymptomatic with untreated cancer } \\
\text { conditional on surviving within } 3 \text { months }\end{array}$ & b3 & 0.9000 & $\begin{array}{l}0.8448 \text { to } \\
0.9442\end{array}$ & $\operatorname{Beta}(149.37,16.60)$ \\
\hline \multicolumn{5}{|l|}{ Asymptomatic recurrence (C) } \\
\hline Surviving within 3 months after treatment & $c 1$ & 0.9778 & $\begin{array}{l}0.8526 \text { to } \\
0.9968\end{array}$ & $\begin{array}{l}\text { Converted from log-normal } \\
\text { distribution for hazard rate }\end{array}$ \\
\hline $\begin{array}{l}\text { Surviving within } 3 \text { months if cancer is undetected } \\
\text { and untreated }\end{array}$ & $\mathrm{c} 2$ & 0.8406 & $\begin{array}{l}0.7986 \text { to } \\
0.8826\end{array}$ & $\operatorname{Beta}(241.05,45.71)$ \\
\hline $\begin{array}{l}\text { Remaining asymptomatic with untreated cancer } \\
\text { conditional on surviving within } 3 \text { months }\end{array}$ & c3 & 0.9000 & $\begin{array}{l}0.8448 \text { to } \\
0.9442\end{array}$ & $\operatorname{Beta}(149.37,16.60)$ \\
\hline \multicolumn{5}{|l|}{ Symptomatic recurrence (D) } \\
\hline Surviving within 3 months after treatment & d1 & 0.9778 & $\begin{array}{l}0.8526 \text { to } \\
0.9968\end{array}$ & $\begin{array}{l}\text { Converted from log-normal } \\
\text { distribution for hazard rate }\end{array}$ \\
\hline $\begin{array}{l}\text { Surviving within } 3 \text { months following undetected } \\
\text { cancer }\end{array}$ & $d 2$ & 0.8406 & $\begin{array}{l}0.7986 \text { to } \\
0.8826\end{array}$ & $\operatorname{Beta}(241.05,45.71)$ \\
\hline \multicolumn{5}{|l|}{ Symptomatic cancer at 3 months (E) } \\
\hline Surviving within 3 months after treatment & e1 & 0.9778 & $\begin{array}{l}0.8526 \text { to } \\
0.9968\end{array}$ & $\begin{array}{l}\text { Converted from log-normal } \\
\text { distribution for hazard rate }\end{array}$ \\
\hline $\begin{array}{l}\text { Surviving within } 3 \text { months following undetected } \\
\text { cancer }\end{array}$ & e2 & 0.8406 & $\begin{array}{l}0.7986 \text { to } \\
0.8826\end{array}$ & $\operatorname{Beta}(241.05,45.71)$ \\
\hline \multicolumn{5}{|l|}{ Symptomatic without cancer (F) } \\
\hline $\begin{array}{l}\text { Surviving within } 3 \text { months following false } \\
\text { symptoms }\end{array}$ & f1 & 0.9993 & & Fixed \\
\hline $\begin{array}{l}\text { Progressing to recurrent cervical cancer after } \\
\text { having a biopsy and survived within } 3 \text { months }\end{array}$ & $\mathrm{f} 2$ & 0.0103 & $\begin{array}{l}0.0094 \text { to } \\
0.0112\end{array}$ & $\operatorname{Beta}(506.35,48,654.06)$ \\
\hline $\begin{array}{l}\text { Recurrence being asymptomatic given recurrence } \\
\text { occurred within } 3 \text { months }\end{array}$ & f3 & 0.4000 & $\begin{array}{l}0.3800 \text { to } \\
0.4202\end{array}$ & $\operatorname{Beta}(915.02,1372.53)$ \\
\hline $\begin{array}{l}\text { Remaining asymptomatic without cancer within } \\
3 \text { months given no recurrence }\end{array}$ & $\mathrm{f} 4$ & 0.9000 & $\begin{array}{l}0.8448 \text { to } \\
0.9442\end{array}$ & $\operatorname{Beta}(149.37,16.60)$ \\
\hline
\end{tabular}


TABLE 101 Transition probabilities used in model 4 (continued)

\begin{tabular}{|c|c|c|c|c|}
\hline & Label & $\begin{array}{l}\text { Stage: } \\
\text { early }\end{array}$ & $\begin{array}{l}\text { Range } \\
(95 \% \mathrm{Cl})\end{array}$ & Probability distribution \\
\hline \multicolumn{5}{|l|}{ Post treatment: asymptomatic cancer at 3 months (G) } \\
\hline $\begin{array}{l}\text { Mean survival time following treatment for those } \\
\text { who were diagnosed with asymptomatic cancer at } \\
3 \text { months }\end{array}$ & $g$ & 0.9778 & $\begin{array}{l}0.8526 \text { to } \\
0.9968\end{array}$ & $\begin{array}{l}\text { Converted from log-normal } \\
\text { distribution for hazard rate }\end{array}$ \\
\hline \multicolumn{5}{|l|}{ Post treatment: asymptomatic $(H)$} \\
\hline $\begin{array}{l}\text { Mean survival time following treatment for those } \\
\text { who were diagnosed with asymptomatic cancer }\end{array}$ & $\mathrm{h}$ & 0.9778 & $\begin{array}{l}0.8526 \text { to } \\
0.9968\end{array}$ & $\begin{array}{l}\text { Converted from log-normal } \\
\text { distribution for hazard rate }\end{array}$ \\
\hline \multicolumn{5}{|l|}{ Post treatment: symptomatic cancer at 3 months (I) } \\
\hline $\begin{array}{l}\text { Mean survival time following treatment for those } \\
\text { who were diagnosed with symptomatic cancer at } \\
3 \text { months }\end{array}$ & i & 0.9778 & $\begin{array}{l}0.8526 \text { to } \\
0.9968\end{array}$ & $\begin{array}{l}\text { Converted from log-normal } \\
\text { distribution for hazard rate }\end{array}$ \\
\hline \multicolumn{5}{|l|}{ Post treatment: symptomatic $(J)$} \\
\hline $\begin{array}{l}\text { Mean survival time following treatment for those } \\
\text { who were diagnosed with symptomatic cancer }\end{array}$ & j & 0.9778 & $\begin{array}{l}0.8526 \text { to } \\
0.9968\end{array}$ & $\begin{array}{l}\text { Converted from log-normal } \\
\text { distribution for hazard rate }\end{array}$ \\
\hline Dead (absorbing state) & & & & \\
\hline
\end{tabular}

\section{Description of the pathways for the model structure}

\section{Asymptomatic without cancer}

At cycle 2 and onwards, that is, at 6 months' follow-up and onwards, these women, in accordance with the schedule, will receive standard practice together with PET-CT. Women with an abnormal examination will receive a biopsy. Women who survived 3 months following the biopsy can either become recurrent or remain without recurrence. For those women who become recurrent, the disease can be either asymptomatic or symptomatic. Those women who remain without recurrence can remain asymptomatic without cancer or symptomatic without cancer. Women who survived after normal examination or no examination can become recurrent or remain free of recurrent cervical cancer. Again, women in this group can also remain free of recurrence or remain asymptomatic without cancer or symptomatic without cancer. In the model structures below (specifically Figures 39 and 40) the term 'No examination' relates to cycles in the process in which the frequency of examination has dropped from every 3 months to every 6 months so at that point in the cycle there is no examination conducted.

\section{Symptomatic without cancer}

Women with symptoms that they suspect are related to recurrent cervical cancer will receive standard practice and PET-CT. Women who received an abnormal result will receive a biopsy. In this group the biopsy will confirm no recurrent cervical cancer and women will not receive any treatment. Women who survive at 3 months following biopsy can either become recurrent or remain without recurrence. For those women who are recurrent, the disease can be either asymptomatic or symptomatic. Those women who remained free of recurrence can remain asymptomatic without cancer or symptomatic without cancer. Women who survive after normal examination can become recurrent or remain free of recurrent cervical cancer.

\section{Asymptomatic cancer at 3 months}

At 3 months following treatment for initial cervical cancer, these women, in accordance with the schedule, will receive standard practice and PET-CT. Women with an abnormal examination will receive a biopsy. On confirmatory biopsy, women will receive treatment for persistent cancer. Women who survive 3 months following treatment will remain in the post-treatment asymptomatic with cancer state. Women with 
no examination or a normal examination who survive 3 months will become either asymptomatic or symptomatic recurrent in the next cycle of the model.

\section{Asymptomatic recurrence}

Women who have asymptomatic recurrence will receive standard practice and PET-CT. Women with an abnormal examination will receive a biopsy. On confirmatory biopsy, women will receive treatment for asymptomatic recurrent cancer. Women who survive for 3 months following treatment will remain in the post-treatment asymptomatic with cancer state. Women with no examination or a normal examination who survive 3 months will become either asymptomatic or symptomatic recurrent in the next model cycle.

\section{Symptomatic recurrence}

Women who have symptomatic recurrence will receive standard practice and PET-CT. Women with an abnormal examination will receive a biopsy. On confirmatory biopsy, women will receive treatment for symptomatic recurrent cancer. Women who survive 3 months following treatment will remain in the posttreatment symptomatic recurrence state. Women with a normal examination who survive 3 months will remain symptomatic in the next model cycle.

\section{Symptomatic cancer at 3 months}

Women who have symptomatic recurrence will receive standard practice and PET-CT. Women with an abnormal examination will receive a biopsy. On confirmatory biopsy, women will receive treatment for symptomatic persistent cancer. Women who survive 3 months following treatment will remain in the post-treatment symptomatic persistent state. Women with a normal examination who survive 3 months will remain symptomatic in the next model cycle and would be considered as recurrent once detected.

\section{Post-treatment asymptomatic cancer at 3 months}

Women will remain in this state until death.

Post-treatment symptomatic cancer at 3 months

Women will remain in this state until death.

Post-treatment asymptomatic recurrence

Women will remain in this state until death.

Post-treatment symptomatic recurrence

Women will remain in this state until death. 


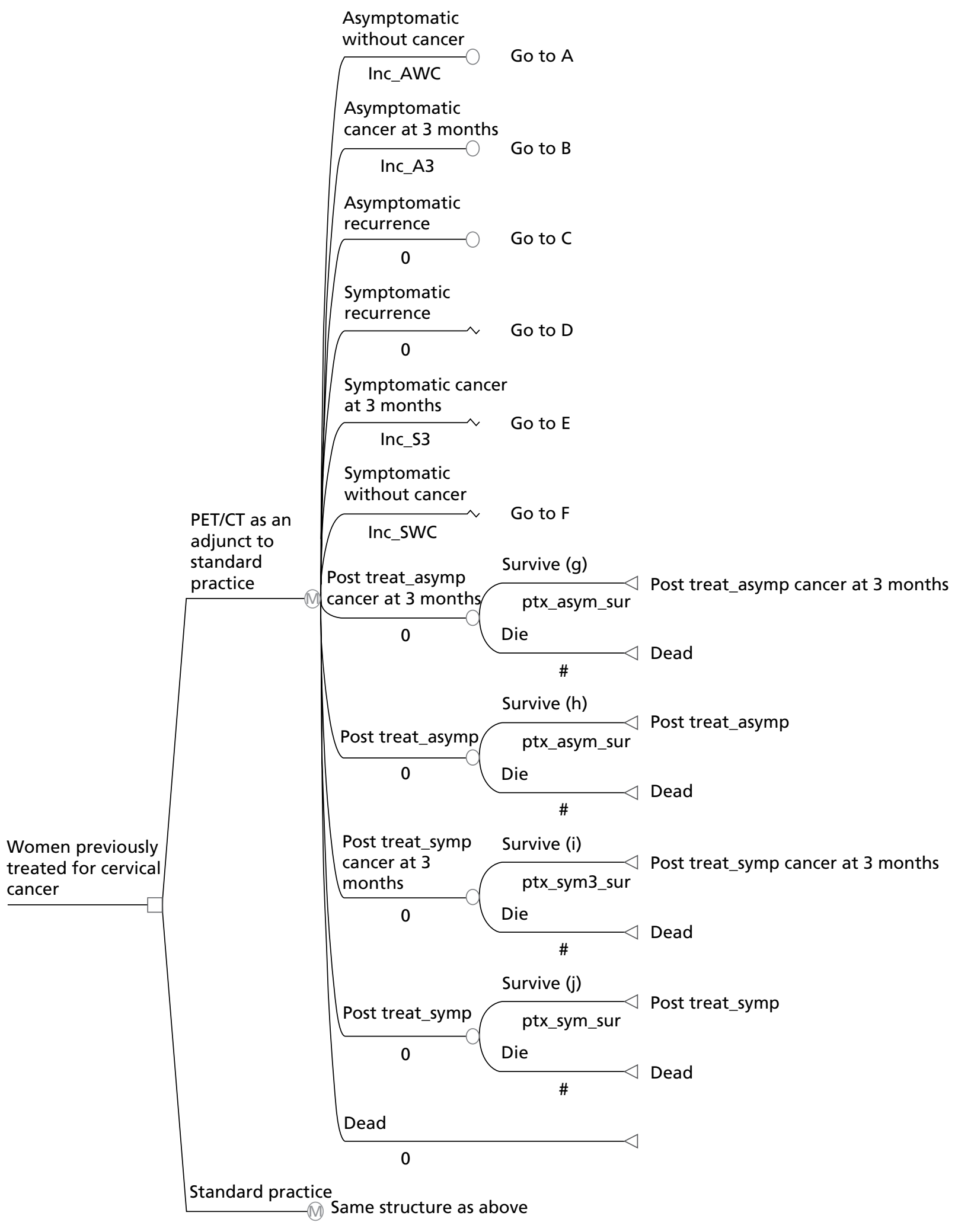

FIGURE 37 Decision tree-like model structure. 

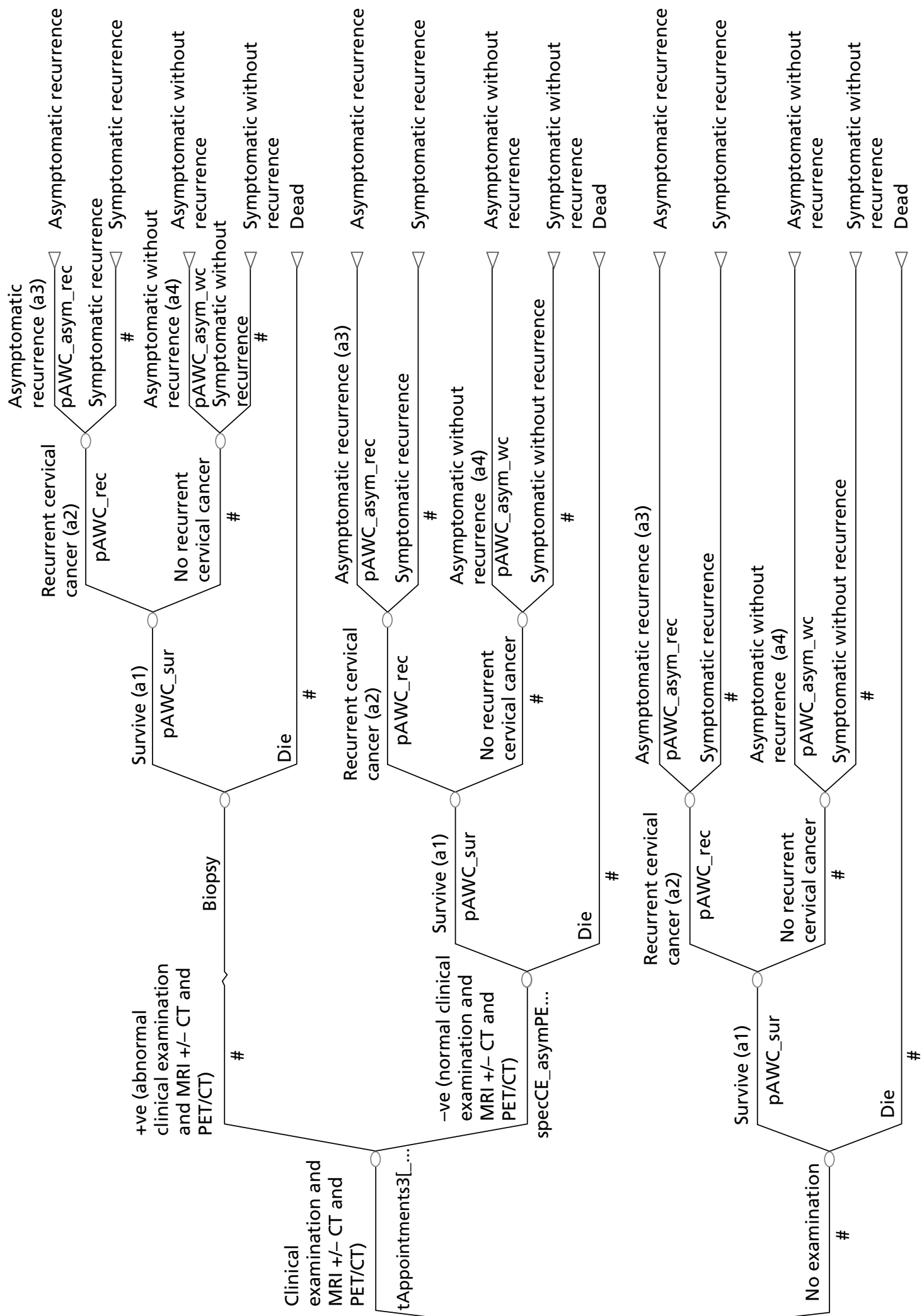


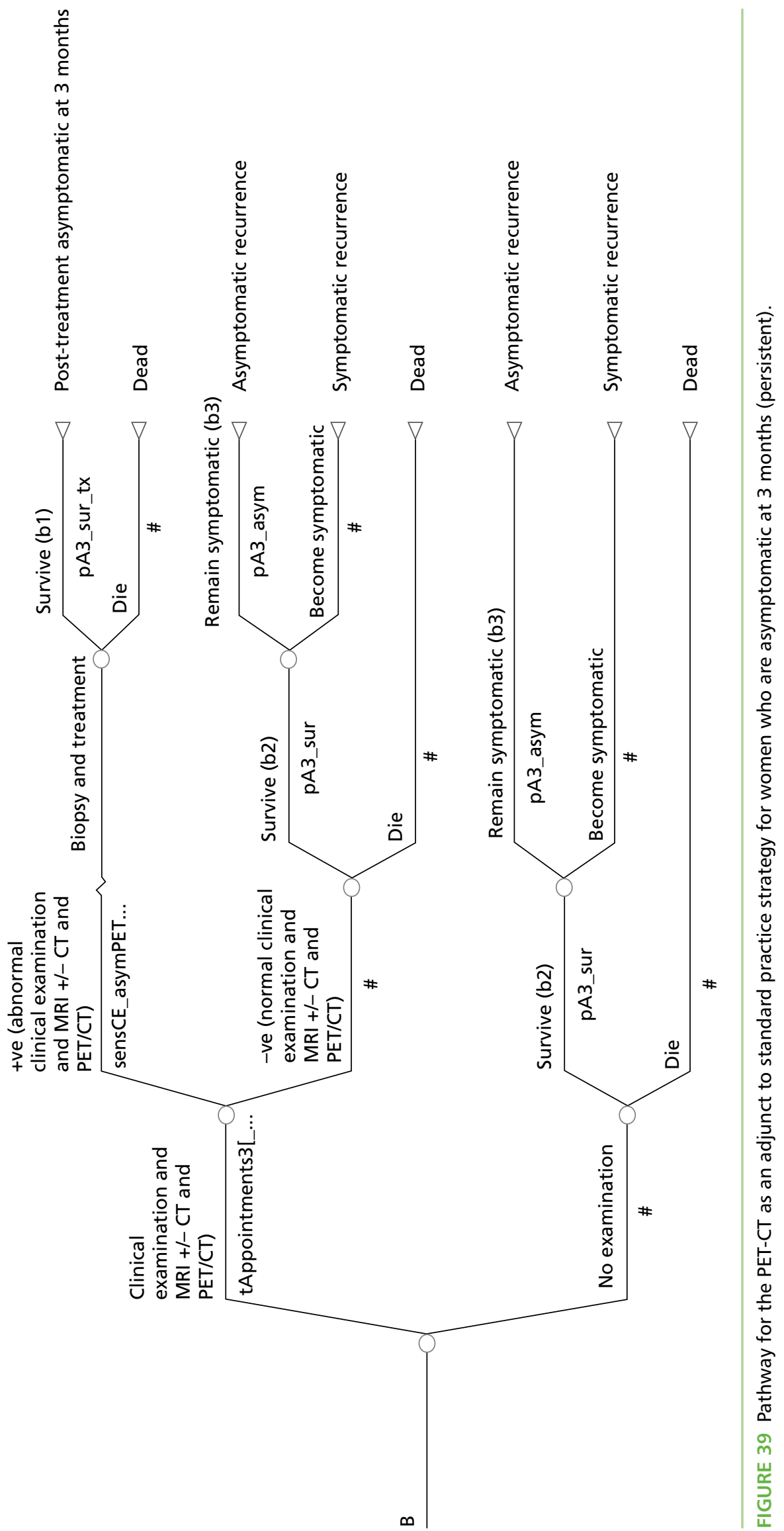



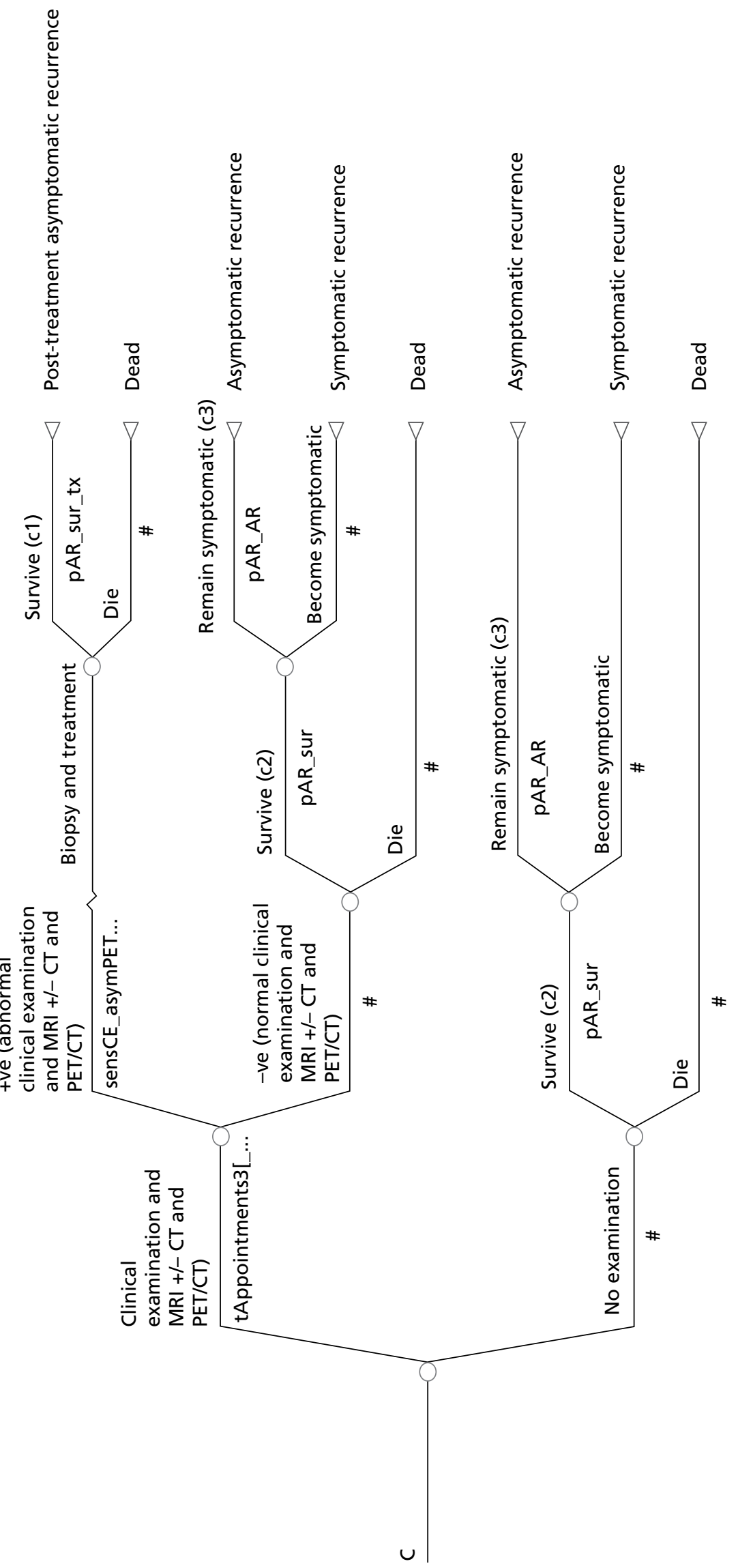


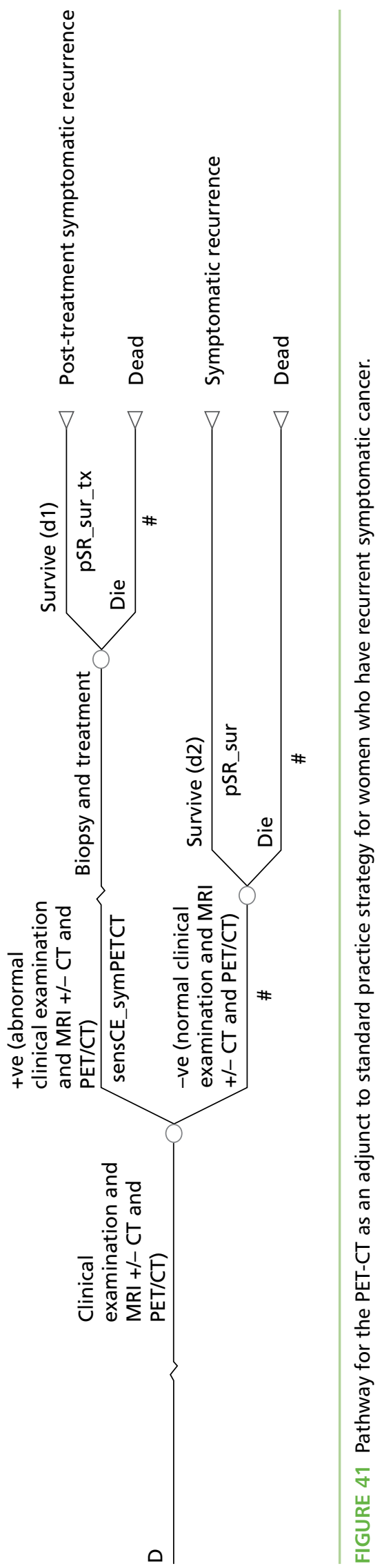




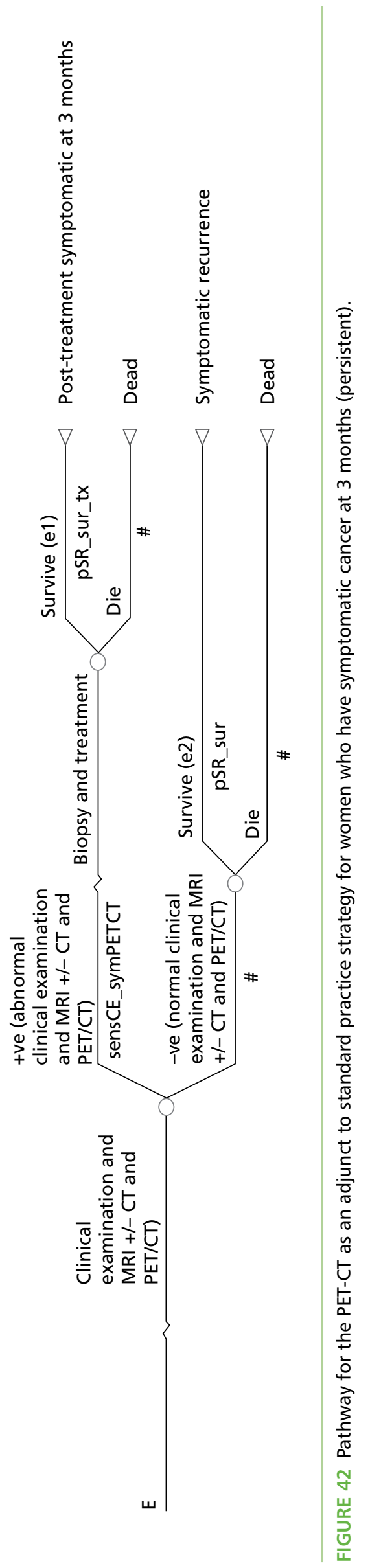




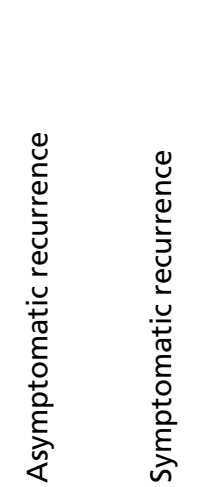

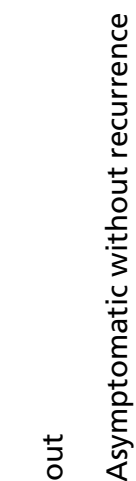

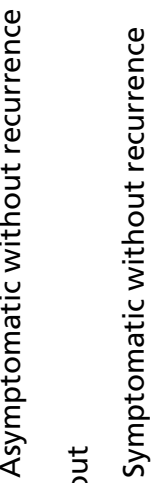

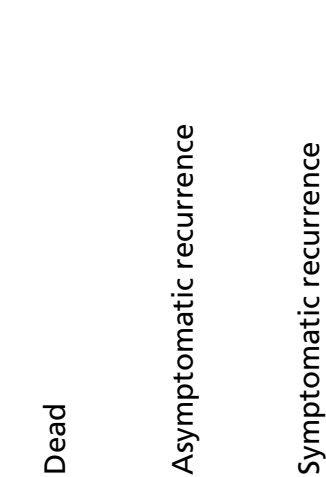

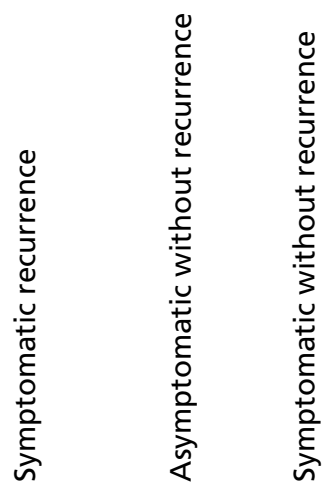

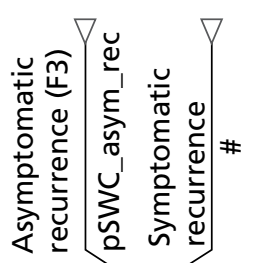

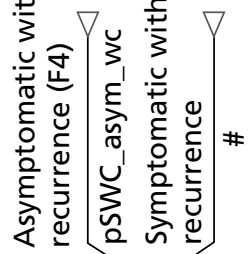

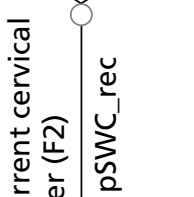

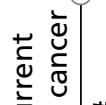

궝

일

幽

究

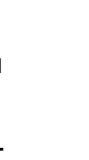

\section{.}

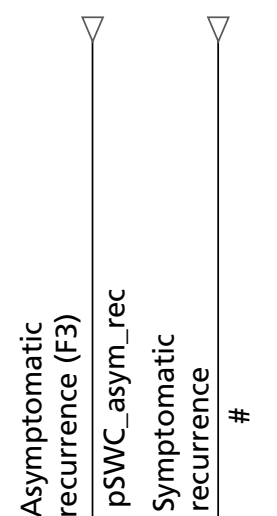

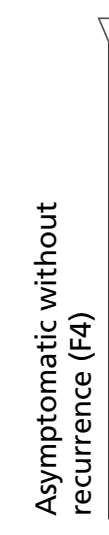

ס 




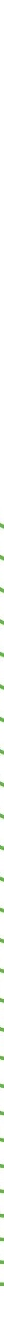

those of the NHS, the NIHR or the Department of Health 